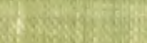

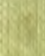

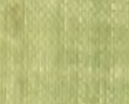

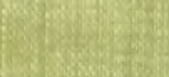

2.

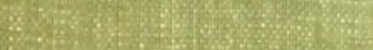

$-5$

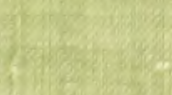

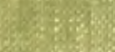

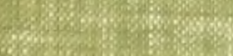

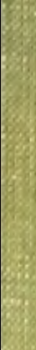

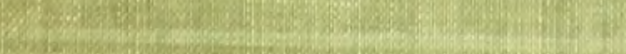

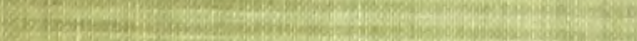

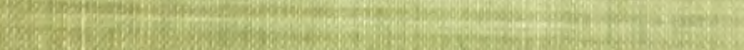
(n)

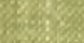

(x)

20.8.

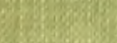

(2)

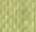

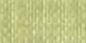

Digitized by Microsoft@ 


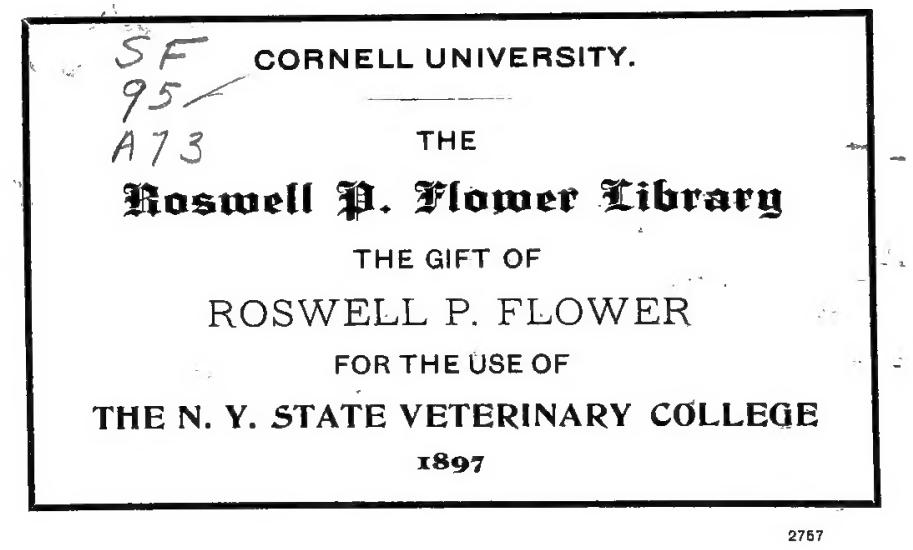




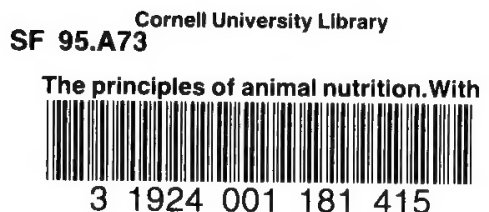

$\begin{array}{lllll}3 & 1924001 & 181415\end{array}$ 
This book was digitized by Microsoft Corporation in cooperation with Cornell University Libraries, 2007. You may use and print this copy in limited quantity for your personal purposes, but may not distribute or provide access to it (or modified or partial versions of it) for revenue-generating or other commercial purposes. 
Digitized by Microsoft巴 
Digitized by Microsoft巴 


\section{THE PRINCIPLES OF}

\section{ANIMAL NUTRITION.}

WITH SPECIAL REFERENCE TO THE NUTRITION OF FARM ANIMALS.

BY

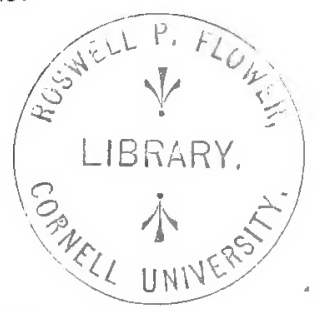

HENRY PRENTISS ARMSBY, PH.D.,

Director of The Pennsyivania State College Agrioultural Experiment Station; Expert in Animal Nutrition, United States Department of Agriculture.

FIRSTEDITION.

FIRST THOUSAND.

NEW YORK :

JOHN WILEY \& SONS.

LONDON: CHAPMAN \& HALL, LIMTTED.

1903. 
No.1994

Copyright, 1903,

BY

HENRY P. ARMSBY.

ROBERT DRUMMOND, PRINTER, NEW YORK.

Digitized by Microsoft巴 


\section{PREFACE.}

THE past two decades have not only witnessed great activity in the study of the various problems of animal nutrition, but they are especially distinguished by the new point of view from which these problems have come to be regarded. Speaking broadly, it may be said that to an increasing knowledge of the chemistry of nutrition has been added a clear and fairly definite general conception of the vital activities as transformations of energy and of the food as essentially the vehicle for supplying that energy to the organism. This conception of the function of nutrition has been a fruitful one, and in particular has tended to introduce greater simplicity and unity into thought and discussion. Much exceedingly valuable work has been done under its guidance, while it points the way toward even more important results in the future. The following pages are not a treatise upon stock-feeding, but are an attempt to present in systematic form to students of that subject a summary of our present knowledge of some of the fundamental principles of animal nutrition, particularly from the standpoint of energy relations, with special reference to their bearings upon the nutrition of farm animals. Should the attempt at systematization appear in some instances premature or ill-advised, the writer can only plead that even a temporary or tentative system, if clearly recognized as such, may be preferable. to unorganized knowledge. The scaffolding has its uses, even though it form no part of the completed building.

The attentive reader, should there be such, will not fail to note that the work is limited to those aspects of the subject included under the more technical term of "The Statistics of Nutrition," and that even in this restricted field some important branches of the subject have been omitted on account of what has seemed to 
the writer a lack of sufficient accurate scientific data for their profitable discussion. Moreover, many principles which are already familiar have been considered rather cursorily in order to allow a more full treatment of less well-known aspects of the subject, even at the expense of literary proportion.

The substance of this volume was given in the form of lectures before the Graduate Summer School of Agriculture at the Ohio State University in 1902, and has been prepared for publication at the request of instructors and students of that school. In thus presenting it to a somewhat larger public the author ventures to hope that it may tend in some degree to promote the rational study of stock-feeding and to aid and stimulate systematic investigation into both its principles and practice.

State College, PA., November, 1902. 


\section{CONTENTS.}

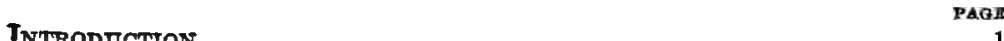

The Statistics of Nutrition. $\ldots \ldots \ldots \ldots \ldots \ldots \ldots \ldots \ldots \ldots \ldots \ldots \ldots \ldots \ldots \ldots,{ }_{3}$

PART I.
THE INCOME AND EXPENDITURE OF MATTER.

CHAPTER I.

The Food.

CHAPTER II.

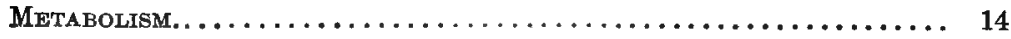

\& 1. Carbohydrate Metabolism.................... 17

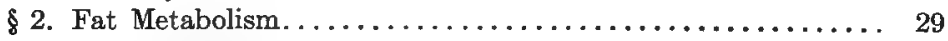

8 3. Proteid Metabolism.......................... 38

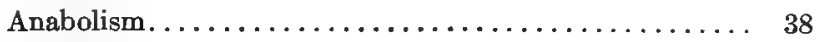

Katabolism.......................... 41

The Non-proteids...................... 52

CHAPTER III.

Methods of Investigation.

CHAPTER IV.

The Fasting Metabolism......................... 80

\$ 1. The Proteid Metabolism..................... 81

2. The Total Metabolism...................... 83

CHAPTER V.

The Relations of Metabolism to Food-supply............. 93

$\S 1$. The Proteid Supply....................... 94

Effects on Proteid Metabolism................. 94

Effects on Total Metabolism.................. 104

Formation of Fat from Proteids.................. 107 
\$2. The Non-nitrogenous Nutrients................... 114

Effects on Proteid Metabolism .................. 114

The Minimum of Proteids.................. 133

Effects on Total Metabolism.................... 144

Mutual Replacement of Nutrients............. 148

Utilization of Excess-Sources of Fat.......... 162

\section{CHAPTER VI.}

Tee Influence of Muscular Exertion upon Metabolism........ 185

\$1. General Features of Muscular Activity.............. 185

Muscular Contraction..................... 185

Secondary Effects of Muscular Exertion........... 191

\$2. Effects upon Metabolism. . . . . . . . . . . . . . . . . . 193

Upon the Proteid Metabolism..................... 194

Upon the Carbon Metabolism................. 209

\section{PART II.}

THE INCOME AND EXPENDITURE OF ENERGY.

CHAPTER VII.

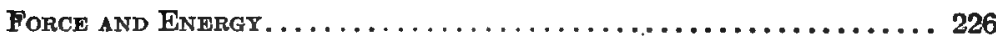

CHAPTER VIII.

METhods of Inveistigation ....................... 234

CHAPTER IX.

The Construation of Engrgy in the Animal Body......... 258

CHAPTER $X$.

The Food as a Source of Energy-Metabolizable Engrgy. . . . . 269

1. Experiments on Carnivora..................... 272

2. Experiments on Man......................... 277

3. Experiments on Herbivora........................ 281

Metabolizable Energy of Organic Matter.......... 284

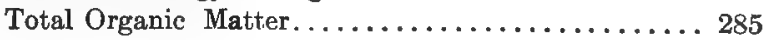

Digestible Organic Matter................ 297

Energy of Digestible Nutrients.............. 302

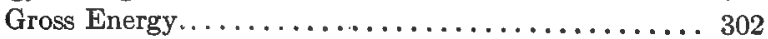

Metabolizable Energy................... 310 


\section{CHAPTER XI.}

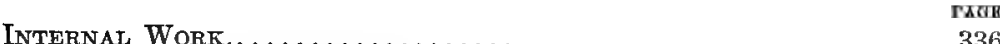

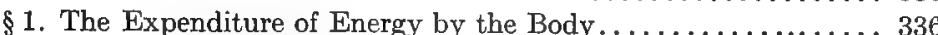

$\S 2$. The Fasting Metabolism....................... 340

Nature of Demands for Energy.................. 340

Heat Production......................... 344

Influence of Thermal Environment . . . . . . . . . 347

Influence of Size of Animal . ................ 359

§3. The Expenditure of Energy in Digestion and Assimilation.... . 372

\section{CHAPTER XII.}

Net Avatlable Energt-Maintenance. . . . . . . . . . . . . . . . . . . . 394

$\S 1$. Replacement Values............................. 396

\$2. Modified Conception of Replacement Values............. 405

\$ 3. Net Availability.............................. 412

Determinations of Net Availability ............ 413

Discussion of Results................... 430

Influence of Amount of Food .............. 430

Character of Food.....................4 431

The Maintenance Ration................. 432

\section{CHAPTER XIII.}

The Utilization of Energy......................... 444

$\S 1$. Utilization for Tissue Building. ................. 448

Experimental Results..................... 448

Discussion of Results................... 465

Influence of Amount of Food...............4466

Influence of Thermal Environment........... 471

Influence of Character of Food............... 472

The Expenditure of Energy in Digestion, Assimilation and Tissue Building. . . . . . . . . . . . 491

\$2. Utilization for Muscular Work. ................... 494

Utilization of Net Available Energy ............ 497

The Efficiency of the Animal as a Motor......... 498

Conditions determining Efficiency............ 511

Utilization of Metabolizable Energy . ........... 525

Wolff's Investigations. . . . . . . . . . . . . 528 
Digitized by Microsoft@ 


\section{THE PRINCIPLES OF ANIMAL NUTRITION.}

\section{INTRODUCTION.}

THi body of an animal, regarded from a chemical point of view, consists of an aggregate of a great variety of substances, of which water, protein, and the fats, with smaller amounts of certain carbohydrates, largely predominate. By far the greater portion of the substance of the body, aside from its water, consists of socalled "organic" compounds; i.e., compounds of carbon with hydrogen, oxygen, nitrogen, and, to a smaller extent, with sulphur and phosphorus. These compounds are in many cases very complex, and all of them have this in common, that they contain a considerable store of potential energy.

It is through these complex "organic" compounds that the phenomena of life are manifested. All forms of life with which we are acquainted are intimately associated with the conversion of complex into simpler compounds by a series of changes which, regarded as a whole, partake of the nature of oxidations. During this breaking down and oxidation more or less of the potential energy of these compounds is liberated, and it is this liberation of energy which is the essential end and object of the whole process and which, if not synonymous with life itself, is the objective manifestation of life. This is equally true of the plant and the animal, although masked in the case of green plants by the synthetic activity of the chlorophyl in the presence of light. The process is most manifest in the animal, however, both on account of the inability of the latter to utilize the radiant energy of the sun and on account of the greater intensity of the process itself.

Setting aside for the moment any storing up of material, and 
therefore of potential energy, in the body for the future use of the animal itself or of its offspring as being, from a physiological point of view, temporary and incidental, the sole useful product of the animal is energy. All the physical effect which we can produce, either through our own bodies or those of our domestic animals, is simply to move something, and moving something is equivalent to the exertion of energy. This motion may be the motion of visible masses of matter in the performance of useful work or the invisible molecular motion of heat, which is economically a waste product, but in either case the animal is a source of energy which is imparted to its surroundings. From this point of view, then, we may look upon the animal as a mechanism for transforming the stored-up energy of the sun's rays, contained in its tissues, into the active or "kinetic" forms of heat and motion. The various cells and tissues of the living animal body, in the performance of their several functions, break down and oxidize the proteids, fats, carbohydrates, and other materials of which they are composed or which are contained in them, seizing, as it were, upon the energy thus liberated and converting it, here into heat, there into motion, again into the energy of chemical change, as the needs of the organism demand.

The very definition of physical life, then, implies that the living animal is constantly consuming its own substance, rejecting the simpler compounds which result and giving off energy in the various forms characteristic of living beings. Obviously, this process, if unchecked, would soon lead to the destruction of the organism and the dissipation of its store of potential energy. To prevent this catastrophe is the object of the great function of nutrition.

This function, in its broader outlines, is familiar to us all through daily experience and observation. The living animal requires to be frequently supplied with certain substances which collectively constitute its food. This food contains a great variety of chemical ingredients, but much the larger part of it consists of "organic" compounds belonging to the three great groups already noted as making up the larger share of the organic matter of the body, viz., the proteids, the fats, and especially the carbohydrates, and while the individual members of these groups differ in the two cases, the ingredients of the food, like those of the body, contain a large store of potential energy. These and other "organic" substances, 
together with more or less mineral matter, are separated by the organism, in the processes of digestion and resorption, from the unessential or unavailable matters of the food. The latter are rejected from the body, while the former are used by it to take the place of the material broken down and excreted by its vital activities, and thus serve to maintain its capital of matter and of potential energy.

In other words, the food may be regarded as the vehicle by means of which a little portion of the "infinite and eternal energy from which all things proceed " is put for the time being at the service of the individual ; as being not so much a supply of matter to make good the waste of tissue as a supply of energy for the manjfestations of life.

The animal body, then, from our present standpoint, consists of a certain amount of matter which has been temporarily segregated from the rest of the universe and which represents a certain store or capital of potential energy. This aggregate of matter and energy is in a constant state of change or flux. On the one hand, its vital activities are continually drawing upon its capital. By the very act of living it expends matter and energy. On the other hand, by means of the function of nutrition, it is continually receiving supplies of matter and energy from its environment and adding them to its capital. Plainly, then, the growth, the maintenance, or the decay of the body depends upon the relation which it is able to maintain between the income and the expenditure of matter and energy. If the two are equal, the animal is simply maintained without increase or decrease; if the income is greater than the expenditure, the body adds to its capital of matter and energy, if the income is less than the expenditure, the necessary result is a diminution in the accumulated capital which: if continued, must ultimately result in death.

We thus reach an essentially statistical standpoint, and this aspect of the subject of nutrition, which has been designated by some writers as "The Statistics of Nutrition," forms the subject of the succeeding pages. The topic naturally divides itself into two distinct although closely related parts, viz:

1. The income and expenditure of matter.

2. The income and expenditure of energy. 
These topics will be considered in the above order, it being assumed that the reader is already familiar with the general nature of the nutritive processes included under the general heads of digestion, resorption, circulation, respiration, and excretion. 


\section{PART I.}

\section{THE INCOME AND EXPENDITURE OF MATTER.}

\section{CHAPTER I.}

\section{THE FOOD.}

The supply of matter to the body is, of course, contained in the food, including water and the oxygen taken up from the air. In a more limited and familiar sense, the term food is employed to signify the supply of solid matter, or dry matter, to the animal. It is proposed here simply to recall certain familiar facts relative to the composition and digestibility of the food in this narrower sense, taking up the subject in the barest outline.

Composition.-While a vast number of individual chemical compounds are found in common feeding-stuffs, the conventional scheme for their analysis unites these substances into groups and regards feeding-stuffs as composed, aside from water and mineral matter, essentially of protein, carbohydrates and related bodies, and fats. Or, setting aside the mineral ingredients, the "organic" ingredients may be divided into the nitrogenous, comprised under the term protein, and the non-nitrogenous, including the fats and carbohydrates.

Protein.-The name "protein" originated with Mulder, who used it to designate what he supposed to be a common ingredient of all the various proteids, but it has since come to be employed as a group name for the nitrogenous ingredients both of feeding-stuffs and of the animal body.

The amount of protein in feeding-stuffs we have at present no 
means of determining directly, but it is commonly estimated from the amount of nitrogen upon two assumptions: first, that all the substances of the protein group contain 16 per cent. of nitrogen, and second, that all the nitrogen of feeding-stuffs exists in the proteid form. On the basis of these assumptions, protein is, of course, equal to total nitrogen $\times 6.25$.

Although it was never claimed that this method of estimating protein was strictly accurate, it was for a long time assumed that the two sources of error involved were not serious. Later investigations, however, have dispelled this pleasing illusion. Further investigations of the true proteids, notably those of Ritthausen and of Osborne, have shown a very considerable variation in the percentage of nitrogen contained in them, while, on the other hand, the researches of Scheibler, E. Schulze, Kellner, and others have shown the presence in many feeding-stuffs of relatively large amounts of nitrogenous matters of non-proteid nature. The results of these latter investigations have made it necessary to subdivide the total nitrogenous matter of feeding-stuffs into two groups, called respectively "proteids" and "non-proteids," while the name "protein" has been retained in the sense of total nitrogen $\times 6.25$ or other conventional factor. For various classes of human foods, Atwater and Bryant* propose the following factors, based on the results indicated in the next two paragraphs, for the computation of protein from nitrogen:

Animal foods . . . . . . . . . . . . . . . 6.25

Wheat, rye, barley, and their manufactured products 5.70

Maize, oats, buckwheat and rice, and their manufactured products ....................6. 6.00

Dried seeds of legumes . . . . . . . . . . . . 6.25

Vegetables ........................ 5.65

Fruits .................... 5.80

Proteids.-In the absence of any adequate knowledge regarding the very complex molecular structure of the proteids, both the classification and the terminology of these bodies are in a very confused state. For convenience, however, we may adopt here those

* Storrs (Conn.) Ag. Ex. St., Rep. 12, 79 . 
tentatively recommended by the Association of American Agricultural Colleges and Experiment Stations,* viz.:

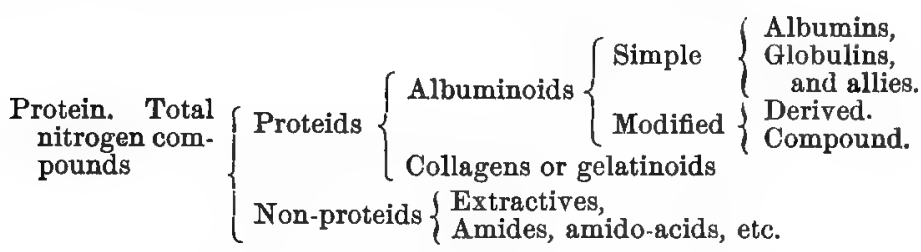

It is not necessary for our present purpose to enter into any discussion either of the properties of the proteids as a whole or of the differences between the different classes of proteids. One point, however, is of particular importance, namely, the elementary composition of these bodies. As noted above, this has been found to be more variable than was supposed earlier. In particular the percentage of nitrogen has been found to have a somewhat wide range. "Recent investigations with perfected methods show percentages of nitrogen in the numerous single proteid substances found in the grains ranging from 15.25 to 18.78. These are largest in certain oil seeds and lupines and smallest in some of the winter grains. Ritthausen, $\dagger$ a prominent German authority, concedes that the factor 6.25 should be discarded, and suggests the use of 5.7 for the majority of cereal grains and leguminous seeds, 5.5 for the oil and lupine seeds, and 6.00 for barley, maize, buckwheat, soja-bean, and white bean (Phaseolus) rape, and other brassicas. Nothing short of inability to secure greater accuracy justifies the longer continuance of a method of calculation which is apparently so greatly erroneous." (Jordan.)

Non-proteids.-This term is used as a convenient designation for all the nitrogenous materials of feeding-stuffs which are not proteid in their nature. It is an abbreviated form of non-proteid nitrogenous bodies. The substances of this class found in plants are chiefly the organic bases, amides, amido-acids, and similar bodies which are produced by the cleavage of the proteid molecule under the action of digestive and other ferments or of hydrating agents. They appear to exist in the plant partly as intermediate stages in the synthesis of the proteids and partly as products of

* U. S. Dept. Agr., Office of Experiment Station, Bul., 65, p. 117.

$\dagger$ Landw. Vers. Stat., 74, 391. 
their subsequent cleavage in the metabolism of the plant. They are chiefly soluble, crystalline bodies. The most common of them is asparagin, which has been, to a certain extent, regarded as typical of the group.

The non-proteids are commonly determined by determining as accurately as possible the non-proteid nitrogen and multiplying the latter by the factor 6.25 . In the case of asparagin, however, which contains 21.2 per cent. of nitrogen, the proper factor obviously should be 4.7, while the factor would vary for the different forms of non-proteids which have been observed in plants. It is no simple matter, therefore, either to determine directly the amount of nonproteids or to decide upon the proper nitrogen factor in any particular case. For the present, however, the factor 4.7 would seem to be at least a closer approximation to the truth than 6.25 .

In the animal body the group of non-proteids is represented by the so-called "extractives" or "flesh bases" of the muscle, chiefly creatin and creatinin.

FATS.--The fats of the plant, like those of the animal, consist chiefly of glycerin compounds of the so-called "fatty acids," or of similar bodies. These are accompanied in the plant, however, by other materials - wax, chlorophyl, etc.-which are extracted along with the fat by the common method of determination and constitute part of the "crude fat" or "ether-extract." The results, therefore, which have been obtained in feeding experiments with pure fats cannot be used with safety as a basis for estimating the nutritive value of the so-called "fat" of feeding-stuffs, particularly in the case of coarse fodders.

Carbohrdrates. - The well-characterized group of carbohydrates makes up a large proportion of the organic matter of our more common feeding-stuffs. This group of substances may be subdivided on the basis of molecular structure into hexosans and their derivatives (hexoses, bioses, trioses, etc.), on the one hand, whose molecules contain six atoms of carbon or a multiple of that number, and the pentosans and pentoses, or five-carbon series, on the other. In the grains and other common concentrated feeding-stuffs, and particularly in the food of man, the hexose group largely predominates, including starch, dextrin, the common sugars, and more or less cellulose. In the coarse fodders consumed by our domestic 
herbivorous animals, while the hexose group is also largely represented it is accompanied by no inconsiderable quantities of carbohydrates belonging to the pentose group. The individual members of this latter group are both less abundant and less well known chemically than the hexoses, and at present our knowledge of their actual nutritive value is somewhat scanty. Since the methods for their determination are based upon the fact that they yield furfural upon boiling with dilute hydrochloric acid, some recent analysts have proposed the term "furfuroids" as a more appropriate designation of these substances as determined by present methods.

In the conventional scheme for the analysis of feeding-stuffs, the carbohydrates are subdivided, not upon the basis of their chemical structure but upon the basis of their solubility. Those members of the group which can be brought into solution by boiling dilute acids and alkalies under certain conventional conditions are grouped together as "Nitrogen-free extract," while those ingredients which resist solution under these conditions are designated as "Crude fiber." The more common hexose carbohydrates, such as. starch, sugars, etc., are included in the nitrogen-free extract, while the larger part, although not all, of the cellulose is included under the crude fiber. At the same time, more or less of the pentose carbohydrátes or "furfuroids" are found in both these groups, while the crude fiber of coarse fodders contains also a variety of other illknown compounds, somewhat roughly grouped together under the general designation of ligneous material.

Digestibility.-A part of nearly all common food materials is incapable of digestion and is rejected in the feces. In the food of man and that of carnivorous animals this indigestible portion is usually small and may disappear entirely. In the food of herbivora, on the other hand, there are contained relatively large amounts of substances which are incapable of solution in the digestive tract, while varying proportions of materials which in themselves are capable of being digested may escape actual digestion under some circumstances. In the latter animals, therefore, it becomes particulary important to determine the digestible portion of the fond. The digestibility of a feeding-stuff is estimated indirectly by determining as accurately as possible the undigested matter eliminated from the body in the feces and subtracting it from the total amount 
contained in the food. This method may of course be applied either to the dry matter or the organic matter of the food as a whole or to any single determinable ingredient.

Metabolic Products.- The digestive tract of an animal, however, not only serves as a mechanism for the digestion of food but has excretory functions as well, and the rejected matter contains, besides the undigested portion of the food, these excreta and the metabolic products of intestinal action. In the case of food largely or completely digestible, these substances may make up the larger portion or even the whole of the feces, while, on the other hand, they constitute but a small proportion of the bulky excreta of herbivora.

It is obvious that these products must be taken account of if it is desired to learn the actual digestibility of the food. Unfortunately, however, we have at present no trustworthy method for their determination. In the past it has been customary to designate the difference between food and feces as digestible and, in the case of domestic animals at least, to assume that the error involved is not serious.

Apparent Digestibility-Availability.-Even with herbivorous animals, however, the presence of the so-called metabolic products in the feces may give rise to serious errors in the determination of the real digestibility of some ingredients of the food, notably fat and protein. With carnivora, or with the human subject, the case is for obvious reasons still worse, and it is scarcely possible to determine the digestibility of the food in the strict sense of the word.

The difference between food and feces does represent, however, the net gain of matter to the organism resulting from the digestion of the food. To express this conception, the use of the word available has been proposed by Atwater.* The "available nutrients" of a food, according to him, are the actually digestible nutrients minus the metabolic products contained in the feces and which may be regarded as representing the expenditure of matter, in the form of residues of digestive fluids, intestinal mucus, epithelium, etc., necessarily incident to the digestion of the food. The term has been

* Storrs (Conn.) Agr'] Expt. St., Rep., 12, 69. 
used chiefly in connection with human nutrition. In discussions of animal nutrition the terms digestible and digestibility have become so firmly established that it may be questioned whether the introduction now of a new term would not create more confusion than it would prevent, and whether it is not preferable, when strict accuracy of expression is required, to attach a modifying word and designate the difference between food and feces as apparently digestible, in distinction from the real digestibility, which we cannot as yet determine.

Determination of Apparent Digestibility.-The determination of the apparent digestibility of the nutrients of a feedingstuff in the above sense, or of their "digestibility" in the older sense, consists simply in determining the amount of the feces or of their separate ingredients and comparing them with the corresponding amounts in the food.

Aside from ordinary analytical precautions, the chief condition of accurate results is that the feces correspond to the food consumed. In animals with a comparatively simple digestive canal, like man and the carnivora, this is readily brought about by the ingestion of a small amount of some substance like powdered charcoal or infusorial earth, which is in itself indigestible and which serves to separate the feces of two successive periods. In the case of herbivora, on the other hand, the undigested residues of the food become mixed to a large extent with those of the previous period. In this case, therefore, it is essential that a preliminary feeding be continued for a sufficient length of time to remove the residues of previous foods from the digestive organs, and further that the experiment itself extend through a number of days in order to eliminate the influence of irregularity of excretion.

Significance of Results.-It is plain from what has just been said that what the results of such an experiment actually show is that a certain amount of material has disappeared from the food during its transit through the alimentary canal. This fact of itself. however, does not necessarily show that the missing material has been digested in any true sense. In the case of animals possessing a relatively short and simple digestive apparatus. we are probably justified in assuming that the difference between food and undigested matter represents material that has actually been 
digested. In the long and complicated digestive apparatus of herbivora, however, there is the possibility that a variety of processes may go on aside from a simple solution of nutrients by the digestive fluids. In particular, it has been shown, as will appear in greater detail later, that extensive fermentations, particularly of the carbohydrates, occur, and that relatively large amounts of these bodies may be destroyed in this way.

Furthermore, with our present conventional scheme for fodder analysis, we have to take account of the possibility of the conversion of members of one group of nutrients into those of another. For example, it seems not improbable that a portion of the crude fiber of feeding-stuffs may be so modified in the digestive tract, without being actually dissolved, that, in the feces, it is determined as nitrogen-free extract, thus diminishing the apparent digestibility of the latter group and increasing that of the crude fiber.*

Composition of Digested Food.-The proteids during the process of digestion are largely converted into proteoses and peptones, while the trypsin of the pancreatic juice, at least outside the body, carries the cleavage of the proteid molecule still further and gives rise to comparatively simple, crystalline bodies. It is not altogether clear to what extent this degradation of the proteids occurs in natural digestion, but the probability appears to be that it does not play a large part, and it has been generally believed that the proteids are resorbed chiefly as proteoses and peptones.

The non-proteids being largely crystalline bodies and readily soluble, we may presume that they are resorbed without material change except so far as they may serve as nitrogenous food for the micro-organisms of the digestive tract.

The fat of the food does not undergo any profound change in digestion, but appears to be resorbed largely in the form of an emulsion. A part of it, however, is undoubtedly saponified by the bile, although the extent to which this process takes place is a disputed point, while in some cases at least a cleavage into glycerin and free fatty acids appears to take place.

The carbohydrates, particularly the easily soluble members of the hexose group, are in the case of man and the carnivora, and

${ }^{*}$ Cf. Fraps, Jour. Am. Chem. Soc, 22, 543. 
probably. also to a large extent in the swine and horse, converted into sugars and resorbed in that form.

Fermentations.-Reference has already been made to the fermentations taking place in the digestive tract. In the herbivora, and especially in ruminants, these fermentations play an important part in the solution of the carbohydrates which make up so large a portion of the food of these animals. These bodies undergo a fermentation which was first studied by Tappeiner * in the case of cellulose, but which has since been shown by G. Kühn † to extend also to the more soluble carbohydrates. The products of this fermentation appear to be methane, carbon dioxide, and organic acids, chiefly, according to Tappeiner, acetic and butyric. Of these products, only the organic acids at best can be supposed to be of any value to the animal organism, and obviously it makes a very serious difference in our estimate of the nutritive value of starch, for example, whether it is resorbed chiefly or entirely in the form of sugar or whether in a ruminant more than half of it, as in some of Kühn's experiments, is fermented.

* Zeit. f. Biol, 20, $52 . \quad$ † Landw. Vers. Stat., 44, 569. 


\section{CHAPTER II.}

\section{METABOLISM.}

General Conception.-By the various processes of digestion and resorption the epithelium of the alimentary canal extracts from the crude materials eaten those ingredients which are fitted to nourish the animal and transmits them more or less directly to the general circulation which carries them to all the tissues of the body. While these ingredients are many in number and diverse in character, yet the vast mass of them, aside from the water in which most of them are dissolved, may be grouped under six heads, viz., ash ingredients, albuminoids or bodies related to the albuminoids, amides and other crystalline nitrogenous substances, fats, carbohydrates, and organic acids, and these, together with relatively small amounts of other materials, may be regarded as constituting the real food of the organism.

As was pointed out in the Introduction, the cells of which the living tissues of the animal body are composed are the seat of continual chemical change. On the one hand, the digested ingredients of the food which are brought to them by the circulation are being built up into the structure of the body. On the other hand, the material of the cells is undergoing a continual process of breaking down and oxidation, uniting with the oxygen supplied by the blood to form the waste products which are removed from the body through the organs of excretion. These excretory products are substantially carbon dioxide, water, and urea and similar nitrogenous substances.

The general term Metabolism is commonly used to designate the totality of the chemical and physical changes which the materials of the resorbed food, or of the tissues formed from them, undergo in being converted into the excretory products. Similarly, we may speak in a more restricted sense of the metabolism of a single ingre- 
dient of the food, as of the proteids, carbohydrates, or fats. Thus proteid metabolism signifies the chemical changes undergone by the proteids of the food in their conversion into the corresponding excretory products. In ordinary usage the chemical reactions undergone by the ash ingredients of the food are not included, the word metabolism being practically used to designate the chemical changes in the organic matter of food or tissue.

Metabolism a Process of Oxidation.-The process of metabolism as a whole is one of oxidation. While we must beware of being misled by analogy into regarding as a simple burning of food-materials that which is in reality a highly complex action of the living cells of the organism, still the final result is much the same in both cases. Starting with more or less complex organic substances and oxygen, we end either with the completely oxidized compounds carbon dioxide and water or with nitrogenous substances like urea more highly oxidized than the protein from which they are derived.

The oxidative character of the total metabolism is most simply illustrated by a comparison of the percentage of oxygen contained in the most prominent ingredients of the food, on the one hand, and in the chief excretory products, on the other hand, as in the,following statement:

In food:

\section{Percentage of Oxygen.}

Protein (average)............... 23.00

Fats ...................... 11.50

Dextrose ................... 53.33

In excreta:

Urea.................... 26.67

Carbon dioxide................. 72.72

Water..................... 88.89

Metabolism an Analytic Process.-From a slightly different point of view, metabolism may be described as an analytic process. The molecules of the food constituents are highly complex. The molecule of dextrose or lævulose, the forms in which the carbohydrates are chiefly resorbed, contains 24 atoms; the molecules of 
the three most common fats, respectively 155,167 , and 173 atoms. The molecular structure of the proteids has not yet been made out, but it is highly complex.* The molecules of the excretory products, on the contrary, are comparatively simple, those of carbon dioxide and water containing but three atoms each, that of urea eight, and even that of hippuric acid but twenty-two.

In metabolism, in other words, the complex molecules of the carbohydrates, fats, proteids, etc., which have been built up in the plant, by means of the energy contained in the sun's rays, out of carbon dioxide, water, and nitric acid or ammonia, gradually break down again into simpler compounds, their atoms reuniting with the oxygen from which they were separated in the plant.

Metabolism a Gradual Process.-The chemical changes included under the term metabolism take place gradually. As has already been indicated, metabolism is not a simple oxidation of nutrients, like the burning of fuel in a stove, but the nutrients enter, to a large extent at least, into the structure of the cells of which the various tissues are composed. Metabolism is really the sum of the chemical actions through which the nutrition and life of these cells is manifested. These actions, however, differ from tissue to tissue and from cell to cell, and even in the same cell from time to time, and the resulting metabolic products are correspondingly varied. Between the nutrients supplied to the cells by the blood and the final products of metabolism as excreted from the body there are innumerable intermediate products, a few of which we know but concerning most of which we are still ignorant. We know the first and last terms of the series and thus are able to measure, as it were, the algebraic sum of the changes, but of the single factors making up this sum. as well as of the specific tissues concerned in the changes, we are largely ignorant, although we know that they are numerous.

Anabolism and Katabolism.-While the process of metabolism as a whole is one of analysis and oxidation, with liberation of energy, it must not be supposed that each single step in the process is of this nature. As has been already pointed out, the chemical activities of the tissues possess a dual character. By the

* Osborne (Zeit. physiol. Chem., 33, 240) has recently obtained the number 14,500 as the approximate molecular weight of edestin. 
various processes of nutrition, ingredients of the food are first incorporated into the tissues of the body, to be subsequently broken down and oxidized. In this building-up process changes undoubtedly occur in the direction of greater complexity of molecular structure, involving the temporary absorption of energy. Thus it is known that fats may be formed from carbohydrates in the body. Many physiologists hold that the metabolism in the quiescent muscle results in the building up of a complex "contractile substance," whose breaking down furnishes the energy for muscular work. In general, we may regard it as highly probable that the molecules of the living substance of the body are much more complex than those of the nutrients of the food, and that the former are built up out of the latter by synthetic processes, carried on. at the expense of energy derived from the breaking down of other molecules. Such changes as this are called anabolic and the process anabolism, while the changes in the direction of greater simplicity of molecular structure are called katabolic, and the process katabolism. The metabolism of the living. body, then, consists of both anabolism and katabolism. By the former the food nutrients are built up into body material; by the latter they are broken down, yielding finally the comparatively simple excretory products. On the whole, however, the katabolism prevails over the anabolism, so that metabolism as a whole is, as already stated, an analytic and oxidative process. Neither the anabolism of tissue production nor the minor anabolic changes which seem to occur in various tissues alter the main direction of the metabolic changes in the body, but, from the standpoint of the statistics of nutrition, are simply eddies in the main current

\section{$\S 1$. Carbohydrate Metabolism.}

\section{HEXOSE CARBOHYDRATES.}

The hexose carbohydrates of the food appear to be resorbed chiefly by the capillary blood-vessels of the intestines. For the most part, they reach the blood in the form of dextrose, with smaller amounts of lævulose and with greater or less quantities of acetic, butyric, lactic, and other acids derived chiefly from the fermentation of the carbohydrates in the digestive tract. In the general circulation only dextrose is found. 
The percentage of dextrose in the blood is small, but remarkably constant, the limits of variation being from about 0.11 to about 0.20 per cent., and the average about 0.15 per cent. Its amount varies but slightly in different regions of the body, and in different classes of animals, and is scarcely at all affected by the nature or amount of the food. Not only so, but any excess of dextrose in the blood is promptly gotten rid of. It is a striking fact that if any considerable amount of this substance, which forms so large a part of the resorbed nutriment, be injected directly into the blood it is treated as an intruder and at once excreted through the kidneys. Evidently it is of the greatest importance to the organism that the supply of this substance to the tissues shall be constant.

Under ordinary conditions, however, the influx of sugar from the digestive tract is more or less intermittent. After a meal rich in easily digestible carbohydrates, an abundant supply of it is taken up by the intestinal capillaries, while on a diet poor in carbohydrates or in prolonged fasting, the supply sinks to a minimum. This is, of course, especially true of animals like man and the carnivora in which the process of digestion is comparatively rapid, but even in herbivorous animals, with their more complicated digestive apparatus, the rate of resorption of dextrose, and still more its absolute amount, must be more or less fluctuating. Evidently there must be some regulative apparatus which holds back from the general circuation any excess of dextrose, on the one hand, and prevents its. being excreted unused, and on the other, supplements any lack resulting from a deficiency of the food in carbohydrates. This regulation is accomplished by the liver.

\section{Functions of the Liver.}

The functions of the liver in this regard appear to be twofold: First, it manufactures dextrose and supplies it to the general circulation; and second, it serves as a reservoir, or a place of deposit, for any excess of carbohydrates supplied by the digestive apparatus.

The Liver as a Source of Dextrose.-The blood as it comes from the intestinal capillaries, bearing the digested carbohydrates and proteids, enters the liver through the portal vein and is distributed by means of the capillary blood-vessels into which this vein divides through all parts of that organ, reaching the general 
circulation again through the hepatic vein. In its passage through the capillaries of the liver, the blood is subjected to the action of the cells of the liver (hepatic cells). Our knowledge of the exact nature of this action is still more or less conjectural, in spite of a vast amount of experimental investigation, but certain general facts are pretty clearly established.

In the first place, the hepatic cells appear to serve as a source of dextrose when no carbohydrates are supplied in the food. If a carnivorous animal be given a diet as free as possible from carbohydrates, as, for instance, prepared lean meat, consisting substantially of proteids, its blood still contains a normal amount of dextrose and the blood in the hepatic vein is found to be richer in dextrose than that of the portal vein, showing that this substance is being formed in the liver. Moreover, while the percentage of dextrose in the blood is small, the total amount thus manufactured is very considerable. Seegen * estimates it at about one per cent. of the weight of the body in twenty-four hours. This is regarded by many physiologists as an overestimate, the considerable differences in sugar content between the portal and hepatic blood found by Seegen being regarded as in part the effect of the necessary operation. Indeed, it is questioned by some whether any actual difference in sugar content between the portal and hepatic blood under normal conditions has been satisfactorily established analytically, but the indirect evidence at least seems strongly in its favor.

In the second place, the same outflow of dextrose from the liver appears to take place when the animal consumes a mixed diet containing carbohydrates. In this case also, except shortly after a meal containing much carbohydrates, the blood of the hepatic vein shows an excess of dextrose over that of the portal vein. The amount of dextrose thus introduced into the circulation is substantially the same as in the first case, and its percentage in the blood is not perceptibly altered. The source of this dextrose, however, is not so simple a question, since it is possible that all or a considerable portion of it may be supplied directly or indirectly by the dextrose resorbed by the intestinal capillaries.

Granting the continual production of sugar by the liver, sub-

* Die Zuckerbildung im Thierkörper, p. 115. 
stantially two suppositions are open: On the one hand, we may consider that the resorbed carbohydrates of the food, after being temporarily stored up in the liver, as described below, are given off again without radical change and that the sugar-forming power of the hepatic cells is limited to the transformation of the proteids and perhaps the fats of the food. Or, on the other hand, we may suppose that the nutrients brought to the liver by the portal blood enter into the constitution of the protoplasm of the hepatic cells, and that the vital activity of this protoplasm gives rise to the dextrose found in the blood, to the glycogen found in the liver, and to other products of whose nature we are largely ignorant. The evidence at hand is doubtless insufficient for a final decision between these alternatives, but the latter hypothesis would seem more in accord with our general knowledge of cell activity. As relates to the carbohydrates, it is supported by the fact that while various sugars besides dextrose (lævulose, mannose, galactose, sorbinose, and, as Münch * has shown, certain artificial hexoses) may be converted into glycogen, the resulting glycogen is always the same and the product of its hydration is always dextrose. $\dagger$ In other words, the molecular structure of these sugars is altered in a manner suggesting an assimilation by the hepatic cells rather than anything resembling an enzyme action. The subject can be more intelligently considered, however, in the light of a discussion of the second function of the liver.

The Liver as a Reservolr of Carbohydrates.-When the food is rich in carbohydrates, the supply of dextrose to the blood through the intestinal capillaries is more or less intermittent. As a means of regulating this intermittent supply, the hepatic cells have the power of arresting the dextrose brought to them by the portal vein and converting it into an insoluble carbohydrate called "glycogen" or "animal starch" which is stored up in the liver. On the other hand, when the supply of carbohydrate food is cut off, and especially if all food be withdrawn, the glycogen of the liver rapidly diminishes, being apparently reconverted into dextrose. This latter phenomenon may be readily observed in the liver of a freshly killed animal. If the fresh liver, after removal from the

*Zeit. physiol Chem . 29, 493.

$†$ Compare Neumeister. Physiologische Chemie, p. 326 
body, be washed out by water injected through the portal vein till all sugar is removed, and if then, after standing for a time, the washing be renewed, the first portions of water that pass contain sugar. The same process may be repeated several times.

What is known as the glycogenic function of the liver was discovered by Claude Bernard in 1853, and has been the subject of a bewildering amount of discussion and controversy, both as to the origin of glycogen, its final fate, and its relations to the production of dextrose by the liver. Certain facts, however, may be regarded as established with at least a high degree of probability:

First-The liver produces glycogen from dextrose and other (not all) carbohydrates, as above described.

Second-The liver seems also to form glycogen from proteids, since this substance is found in considerable quantity in the livers of animals fed exclusively on meat.

Third-Glycogen largely disappears from the liver during fasting, and to a considerable degree also in the absence of carbohydrates from the food.

Fourth-The liver produces dextrose at an approximately constant rate, largely independent of the food-supply or the variations in the store of glycogen.

These facts seem to point unmistakably to the sugar-producing function of the liver as the primary factor in the whole matter. The general metabolism of the body requires a constant proportion of dextrose in the blood, and as this dextrose is consumed the liver furnishes a fresh supply. This supply it manufactures from the materials brought to it by the blood of the portal vein. When carbohydrates are lacking in this blood, it apparently has the power of breaking down the proteids and perhaps the fats, thus supplying the needful dextrose. Some authorities claim that the same process goes on when carbohydrates are present, and it seems not unlikely that this is true, but when the food-supply consists so largely of carbohydrates as it does in the case of our domestic herbivorous animals, the conclusion seems unavoidable that at least a considerable part of the dextrose consumed in the body must be derived from these substances. As already suggested, a very plausible view of the matter is to regard the resorbed nutrients of the portal blood as serving to feed the protoplasm of the hepatic cells and to look 
upon the dextrose as one of the products of the metabolism of those cells.

Since, however, the demands of the organism for dextrose and the supply of it, or of the materials for its manufacture, in the food do not keep pace with each other, sometimes one and sometimes the other being in excess, the liver has a second function. When the food-supply, of whatever kind, is in excess, instead of continuing to produce dextrose the metabolism in the liver takes a slightly different form and produces the insoluble glycogen, or perhaps the dextrose of the portal blood is simply converted into glycogen without entering into the structure of the hepatic protaplasm. When; on the other hand, the food-supply is deficient, the stored-up glycogen is converted into dextrose; whether by some sort of enzyme action or by again serving as food for the hepatic protoplasm is uncertain.

\section{Fate of the Dextrose of the Blood.}

The fact that the proportion of dextrose in the blood is approximately constant, notwithstanding the continual supply which is received from the liver, shows that there must be a continual abstraction of dextrose from the blood, which is as continually made good by the activity of the hepatic cells. In fact, the dextrose of the blood appears to play a very prominent part in the animal economy, and the function of the liver in preparing it from other ingredients of the food is a most important one.

Consumption in the Muscles.-From the point where it leaves the liver, our knowledge of the metabolism of the dextrose of the blood is scanty, but a large proportion of it undoubtedly takes place in the muscles. It was early shown by Chauveau that the proportion of dextrose in the blood diminishes in its passage through the capillaries of the body, so that the arterial blood contains more of this substance than the venous. In conjunction with Kaufmann * he has subsequently shown more specifically that in its passage through the muscular capillaries and through those of the parotid gland the blood is impoverished in dextrose, and to a much greater extent in the active than in the quiescent muscle. Coin-

* Comptes rend., 108, 974 and 1057; 104, 1126 and 1352. 
cident with this disappearance of dextrose, there is an increase in the carbon dioxide of the blood and a decrease of its oxygen.

The relations of the dextrose of the blood to the evolution of heat and work in the muscles and other tissues, so far as they are at present understood, will be considered in a subsequent chapter. For our present purpose it suffices to note the fact that it disappears in the capillaries with the ultimate production of carbon dioxide and water. That the dextrose is immediately oxidized to carbon dioxide and water, however, is extremely unlikely. It has been suggested that the lactic acid which is found in the muscle after muscular contraction is one of the intermediate products of the oxidation. Several considerations, however, seem to render it more probable that the dextrose first enters in some way into the constitution of the muscles, or in other words, that a synthetic or anabolic process precedes the katabolic one.

MUSCULAR GLYCOGEN.--Another fact, of much interest in this connection, is that the muscles (and other tissues also), as well as the liver, contain glycogen. Moreover, the muscular glycogen diminishes or disappears during work and reappears again after rest. It would appear, then, that the muscular tissue shares with the liver the ability to form glycogen. As in the case of the former organ, the simplest supposition is that this glycogen is produced from the dextrose supplied in the blood, and Kültz* and others have shown that subcutaneous injections of sugar give rise to a formation of muscular glycogen in frogs whose livers have been removed. On the other hand, of course, the considerations presented above relative to the sources of the liver glycogen apply, ceteris paribus, to the formation of glycogen in the muscles. Neither the source nor the exact functions of the muscular glycogen are yet beyond controversy, but the facts just stated strongly suggest a storing up of reserve carbohydrates during rest to be drawn upon when there is a sudden demand for rapid metabolism.

Fat Production.-In addition to its important relation to the muscles, the dextrose of the blood likewise supplies nourishment for the fat tissues of the body. Hitherto we have spoken as if the supply of dextrose to the blood were determined substantially by

* Neumeister, Physiologische Chemie, p. 322. 
the demands of the general metabolism for material to produce heat and motion. Plainly, however, the capacity of the muscles and the liver to store up carbohydrates is limited, and if the food-supply is permanently greater than the demands of the organism, some other provision must be made for the excess. Under these circumstances the superfluous dextrose which finds its way into the blood gives rise to a production of fat, which is stored up as a reserve in special tissues and apparently does not enter again into the general metabolism until a permanent deficiency in the food-supply occurs.

The experimental evidence of the production of fat from carbohydrates, as well as the quantitative relations of the process so far as they are known, will be considered subsequently. In its relations to the economy of the organism the process is analogous to the formation of glycogen in the liver, except that the storage capacity of the fat tissues is vastly greater, but as compared with the formation of glycogen it is distinctively an anabolic process, the fat molecule being more complex and containing more potential energy than that of dextrose. Hanriot,* assuming the formation of olein, stearin, and palmitin in molecular proportions, represents the process by the equation:

$$
13 \mathrm{C}_{6} \mathrm{H}_{12} \mathrm{O}_{6}=\mathrm{C}_{55} \mathrm{H}_{104} \mathrm{O}_{8}+23 \mathrm{CO}_{2}+26 \mathrm{H}_{2} \mathrm{O} \text {. }
$$

\section{PENTOSE CARBOHYDRATES.}

The facts of the foregoing paragraphs relate primarily to the hexose carbohydrates, particularly starch and sugar, and to a considerable extent to the metabolism of carnivorous animals. The food of herbivora, however, contains a great variety of carbohydrates and especially considerable quantities of the pentose or fivecarbon carbohydrates. That these substances are in part digestible, or that at least a considerable proportion of them disappears from the food during its transit through the alimentary canal, was first shown by Stone, $\uparrow$ and has since been fully confirmed by the investigations of Stone \& Jones $\ddagger$ and of Lindsey \& Holland, but of their further fate in the body relatively little is known.

* Archives de Physiol., 1893, 248.

$\dagger$ Amer. Chem. Jour., 14, 9.

+ Agricultural Science, 5, 6.

\$Ibid., 8, 172. 
Ebstein,* who was the first to investigate this subject, showed qualitatively the presence of pentose carbohydrates in the urine of man after the ingestion of arabinose and xylose even in very small doses, and concluded that these sugars are not assimilable.

Salkowski $\uparrow$ shortly afterward observed the appearance of pentoses in the urine of rabbits given arabinose after five or six days of fasting. He found in the urine, however, only about one-fifth of the amount ingested, together with small amounts in the blood and larger ones in the muscles, but there was a considerable increase of the glycogen of the liver. From the latter fact Salkowski concludes that arabinose may be, either directly or indirectly, a source of glycogen. The glycogen found in his experiment was the ordinary six-carbon glycogen.

Subsequent investigations by Cremer, $\ddagger$ Munk, $\S$ Frentzel, $\|$ Lindemann \& May, $\uparrow$ Fr. Voit,** Jacksch, † Münch, and othors have been directed largely to two questions, viz., whether the pentose carbohydrates are oxidized in the body and whether they serve as a source of glycogen.

Pentoses Oxidized IN THE BODY.-As the general result of these investigations, it may be stated that pentoses (in particular arabinose and xylose), whether administered by the stomach or injected into the blood, are at least partially oxidized in the body. In the human organism the power of oxidizing the pentoses, which do not normally constitute any considerable portion of its food, appears to be quite limited, and even when they are given in small quantities a portion (not all) is excreted in the urine. In the rabbit the pentoses seem to be more vigorously oxidized, only about twenty per cent. being excreted unaltered, even when comparatively large doses are given.

In these experiments the pentose sugars were administered in considerable amounts at once, and the excretion of a portion unaltered would seem to be a phenomenon similar to the temporary

\footnotetext{
*Virchow's Archiv, 129, 401; 132, 368. T Arch. klin. Med., 56, 283.

$\dagger$ Centralbl. med. Wiss., 1893, p. $193 . \quad$ ** Ibid., 58, 524.

†Zeit. f. Biol., 29, 536; 42, 428. †† Zeit. f. Heilk., 20, 195.

\$Centralbl. med. Wiss., 1894, p. 83. \$ Zeit. physiol. Chem., $29,493$.

\| Arch. ges. Physiol., 56, 273.

$\$ \S$ Ibid., 32, 393.
} 
glycosuria caused by large doses of the common sugars. The pentose carbohydrates in the ordinary food of herbivora, however, are largely or entirely the comparatively insoluble pentosans. As already stated, these bodies are partially digested-that is, they do not reappear in the feces. As to the manner of their digestion we are ignorant. If we are justified in assuming that the digested portion is converted, wholly or partially, into pentoses, then the conditions differ from those of the experiments above mentioned in that the production and assimilation of the pentoses is gradual. Under these circumstances we might be justified in anticipating a more complete oxidation of these bodies. To what extent this is true it is at present impossible to say. Weiske,* in connection with his investigations upon the digestibility of the pentosans, states that the urine of the sheep and rabbits experimented upon gave only a slight reaction for pentoses. The writer has not been able to find any records of other tests of the urine of domestic animals for pentoses.

Pentoses as a Source of Glycogen.-Most, although not all, investigators have found an increase in the glycogen of the liver consequent upon the ingestion of pentoses, but in every case it has been the ordinary six-carbon glycogen. This has been commonly and most naturally interpreted as showing that the pentoses are not themselves converted into glycogen in the body, but are simply oxidized in the place of some other material which is the true source of the observed gain of glycogen: In the light of known facts regarding the apparent power of the liver to produce glycogen from very diverse hexoses (see p. 20) it would seem, however, that the possibility of an actual assimilation of the pentoses by the hepatic cells should at least be borne in mind.

\section{THE ORGANIC ACIDS.}

In addition to such quantities of the organic acids, free and combined, as are contained in their food, relatively large amounts of these substances are, in the case of herbivorous animals and particularly of ruminants, produced by the fermentation of the carbohydrates in the alimentary canal. For this reason their meta-

* Zeit. physiol. Chem., 20, 489. 
bolism may properly be considered in connection with that of the carbohydrates themselves.

But little is known of the metabolism of the organic acids, however, beyond the fact that they are oxidized in the body, a portion of the resulting carbon dioxide appearing in the urine, in combination with sodium and potassium, rendering that fluid alkaline. Wilsing * and v. Knieriem $\dagger$ have shown that organic acids such as result from the fermentation of carbohydrates are not found to any appreciable extent in the excreta, while the researches of Munk $\ddagger$ and Mallevre, $\S$ which will be considered more particularly in another connection, have shown that the sodium salts of butyric and acetic acids when injected into the blood are promptly oxidized, and Nencki \& Sieber $\|$ have shown that lactic acid is readily oxidized, even by a diabetic patient.

\section{NON-NITROGENOUS MATTER OF THE URINE.}

It has been implied in the foregoing pages that the digested carbohydrates of the food, whatever the intermediate stages through which they may pass, are ultimately oxidized to carbon dioxide and water. Of the ordinary hexose carbohydrates this is doubtless true, but with some of the large variety of substances ordinarily grouped together, by the conventional scheme of feeding-stuffs analysis, as "carbohydrates and related bodies," or as "crude fiber" and "nitrogen-free extract," the case appears to be otherwise.

It has been shown that the urine, in addition to the nitrogenous products of proteid metabolism which will be considered in a subsequent section, contains also non-nitrogenous materials, presumably metabolic in their nature. In the urine of man and of the carnivora these non-nitrogenous substances are chiefly or wholly such as might be derived from the metabolism of proteids (phenols and other compounds of the aromatic series), and their amount is comparatively small. In the urine of herbivora, particularly of ruminants, however, their quantity is relatively very considerable, and it seems impossible to regard any large portion of them as derived from the proteid metabolism.

\footnotetext{
* Zeit. f. Biol., 21, 625 .

† Ibid., 21, 139.

$\ddagger$ Arch. ges. Physiol., 46, 322.

\$Ibid. , 49, 460.
}

\|Jour. pr. Chem., N. F., 26, 32. 
Henneberg * found that from 26.7 to 30.0 per cent. of the organic matter of sheep urine was neither urea nor hippuric acid, while from 95 to 100 per cent. of the total nitrogen was contained in these two substances. G. Kühn in his extensive respiration experiments on oxen, as reported by Kellner, $\nmid$ assuming that all the nitrogen of the urine was in the form either of hippuric acid or urea, found that from 40.05 to 67.64 per cent. of the total carbon of the urine was present in non-nitrogenous substances. The more recent investigations of Kellner, $\ddagger$ as well as those of Jordan $\S$ and of the writer, $\|$ have fully confirmed this fact.

Apparently these non-nitrogenous organic substances are derived in some way largely from the coarse fodders. Their proportion in the urine is relatively large when the ration consists exclusively of coarse fodder, and the addition of such fodders to a basal ration causes a marked increase in their amount; while, on the other hand, such concentrated feeding-stuffs as have been investigated do not produce this effect in any very marked degree. Furthermore, their amount seems to bear no fixed relation to the protein of the coarse fodder. When the amount of the latter ingredient is small, the total organic matter of the urine has in some cases exceeded the maximum amount that could have been derived from the protein of the food, thus demonstrating that a portion at least of the non-nitrogenous urinary constituents must have had some other source. As the proportion of protein in the food increases, the amount of nitrogenous products in the urine likewise increases, while that of the non-nitrogenous products appears to be more constant, so that the ratio of urinary nitrogen to carbon increases. The most plausible explanation of these facts seemis to be that the substances in question are derived from some of the non-nitrogenous ingredients of the coarse fodders, but from what ones, or what is the nature of the products, we are still ignorant. 1

* Neue Beiträge, etc., p. 119.

† Landw. Vers. Stat., 44, 348, 404, 474, 529.

$\ddagger I b i d$. 47,$275 ; 50,245 ; 53,1$.

$\S$ New York State Expt. Station, Bull. 197, p. 27.

|| Penna. Expt. Station, Bull. 42, p. 150.

If A further discussion of this subject in its relations to the energy of the food will be found in Part II. 


\section{§ 2. Fat Metabolism.}

Scarcely a tissue or portion of the animal body can be named in which more or less fat is not found. The muscular fibers, the epithelium, the nerves and ganglia, etc., all contain cells in which globules of fat may be recognized, so that the capacity to produce or store up fat seems to be common to almost all the cells of the body. It is particularly in certain cells of the connective tissue, however, that the large accumulations of visible fat in the body take place. At the outset these cells present no special characters, but in a well-nourished animal globules of fat begin to accumulate in them, the cells enlarge, the globules of fat coalesce into larger ones, and finally the cell substance is reduced to a mere envelope, the nucleus being pushed to one side and almost the whole volume of the cell occupied by fat. Masses of connective tissue thus loaded with fat constitute what is called adipose tissue. Large deposits of adipose tissue are met with surrounding various organs, particularly the kidneys, but the largest deposit of fat is usually in the connective tissue underlying the skin. In milk production, too, large amounts of fat appear in the epithelial cells of the milk glands.

Fat Manufactured in the Body.--The older physiologists held that all the ingredients of the body pre-existed in the food. Specifically, animal fat was regarded as simply vegetable fat which had escaped oxidation in the body and been deposited in the tissues. But while there is no doubt that the fat of the food can contribute to the fat supply of the body, the food of herbivorous animals usually contains a relatively small quantity of fat and the amount produced by a rapidly fattening animal or by a good dairy cow is usually much greater than that consumed in the food.

Deferring to subsequent pages a discussion of the sources of animal fat,* we may content ourselves here with anticipating the general results of the great amount of experimental inquiry which has been expended upon this question. These results may be briefly summarized in the following statements:

* For a very complete review of the literature of fat production up to 1894, see Soskin, Journ. f. Landw., 42, 157. 
1. The animal body produces fat from other ingredients of its food.

2. The carbohydrates and related bodies of the food serve as sources of fat.

3. It is probable that the proteids also serve as sources of fat.

So far, then, as that portion of the fat which is actually produced in the body from other substances is concerned, we may most readily conceive of its formation as consisting essentially of a manufacture of fat by the protoplasm of the fat cells, which are nourished by the carbohydrates, proteids, and other materials brought to them by the circulation.

Functions of the Food Fat.-The fat which is manufactured in the body from other ingredients of the food, however, often constitutes the larger portion of the total fat production, while but a relatively small proportion at most can be derived from the fat of the food. The question naturally arises whether this smaller portion contained in the food is simply deposited mechanically, so to speak, in the fat cells, or whether it too, like the carbohydrates and proteids, serves to nourish the fat cells and supply raw material out of which they may manufacture fat.

At first thought the former alternative might seem more probable. The fat of the food, so far as we are able to trace it, does not undergo any considerable chemical changes, such as the proteids do, e.g., in the process of digestion, but is largely resorbed in the form of apparently unaltered fat. Moreover, resorption of fat takes place largely through the lacteals and the resorbed fat reaches the general circulation without being subjected like the carbohydrates to the action of the liver.

Deposition of Foreign Fats.-The view just indicated is supported to a considerable extent by the results of experiments upon the fate of foreign fats introduced into the body.

Experiments by Radziejewsky* and Subbotin $\dagger$ were indecisive. but Lebedeff $\ddagger$ was later successful in obtaining positjve results. Two dogs, after prolonged fasting, received small amounts of almost fat-free meat together with, in the one case. linseed oil.

*Virchow's Archiv., 56, 211 ; 43, 268.

†Zeit. f. Biol. : 6, 73 .

$\ddagger$ Thier. Chem. Ber., 12, 425; Zeit. Physiol. Chem.. 6, 149; Centralbl. med. Wiss., 1882, 129. 
and in the other, mutton tallow. After three weeks, during which the animals recovered their original weights, the adipose tissue was found to contain, in the one case, fat fluid at $0^{\circ} \mathrm{C}$. and agreeing very closely with linseed oil in its chemical behavior, while in the other case the fat had a melting-point of over $50^{\circ} \mathrm{C}$, and was almost identical with mutton fat. On the other hand, the same author in experiments with tributyrine failed to obtain any noteworthy deposition of this substance.

Munk * fed large amounts of rape oil to a previously fasted dog for seventeen days and found in the body considerable amounts of fat differing markedly in appearance and properties and in the proportion of olein to solid fats from normal dog fat. He likewise succeeded in isolating from the fat eruic acid, the characteristic ingredient of rape oil. In a second experiment $\dagger$ the fatty acids prepared from mutton tallow were fed with similar results, the proportion of stearin and palmitin to olein being approximately reversed as compared with normal dog fat. The latter experiment is also of interest as showing that the fatty acids may be synthesized to fat in the body, the change taking place, according to Munk, in the process of resorption.

More recently Winternitz $\ddagger$ has experimented with the iodine addition products of fats. He observed the retention of a considerable proportion of iodine in the body (of hens and dogs) in organic form and also found iodine in the fat of the body at the close of the experiment. Similar experiments on a milking goat $\S$ showed that at least 6 per cent. of the fat fed passed into the milk.

Henriques and Hansen || fed two three-months-old pigs for about nine months with ground barley, to which was added, in one case linseed oil and in the other cocoanut oil, while in the succeeding three months the rations were exchanged. Samples of the subcutaneous fat of the back were taken (with the aid of cocaine) at four different times and the fat of the carcasses at the close of the experiment was also examined. The results showed an abundant deposition of the linseed oil (and cocoanut oil?). On the other

* Thier. Chem. Ber., 14, 411; Virchow's Archiv., 95, 407.

$\dagger$ Archiv. f. (Anat. u.) Physiol., 1883, p. 273.

$\$$ Zeit. physiol. Chem , 24, 425.

§ Thier. Chem. Ber., 27, 293.

ปIbid., 29. 68. 
hand, experiments with cows failed to show any passage of linseed oil as such into the milk.

Leube * made subcutaneous injections of melted butter on two dogs and found an abundant deposit of butter fat especially under the skin of the abdomen, the Reichert-Meissl number of the fat being 20.46 in the first case and 15.3 in the second. Rosenfelt $\uparrow$ fed fasted dogs with mutton fat and observed a large deposit of this fat in all parts of the body.

InFLUence of FeEding on Composition of Fat.-In addition to the more purely physiological experiments just cited, there are on record a not inconsiderable number of feeding experiments, especially upon swine, in which the feeding appears to have sensibly influenced the appearance, firmness, melting-point, or composition of the body fat.

While it is not impossible, however, that in some cases the peculiar fats of the food (e.g., the fat of maize or of the oil-meals) may have been deposited in the adipose tissue unchanged, it must be borne in mind that these experiments were made on mixed rations and that undoubtedly there was a considerable production of fat in the body from other ingredients of the food. This being the case, we are left in doubt as to whether the effect observed is due directly to the fat of the food or is to be explained as an effect of the food as a whole, or of some unknown ingredients of it, in modifying the nature of the metabolism in the fat cells. That such an explanation is at least possible would seem to be indicated by the wellestablished fact that marked changes of food do modify the metabolism in the milk gland sufficiently to materially affect the proportion of volatile fatty acids in butter fat.

A striking example of the possibility of such an effect upon the metabolism of the fat cells is afforded by the recent investigations of Shutt \$ into the causes of "soft" pork. On the average of a considerable number of animals, he finds that the shoulder and loin fat of pigs fed exclusively on maize shows a very low melting-point and a high iodine absorption number, indicating a large percentage of olein, and inclines to attribute this effect to the oil of the maize. When, however, he fed skim milk with the maize, he obtained pork

* Thier. Chem. Ber., 25, 45.

† Ibid., 25, 44.

‡ Canada: Dominion Experiment Station, Bull. 38 . 
of good quality, the fat having a melting-point and iodine number not widely different from those obtained with the most approved rations. While it is possible that part of this effect was due to a reduced consumption of maize oil, so that more fat was produced from the other ingredients of the food, the conclusion seems justified that the principal factor was the influence of the skim milk upon the nutrition of the fat cells. This influence may with some degree of probability be ascribed to its protein, and it is worthy of notice that in Shutt's experiments the rations which produced the highest grade of pork were composed of materials rich in protein.

Another fact warns us to be cautious in our interpretation of the results of this class of feeding experiments. Such experiments in most cases involve a comparison of the composition of the fat from animals differently fed. Albert* has found that both with swine and sheep the composition of the body fat is subject to very considerable individual variations as to melting-point, refractive index, and iodine number, the differences being, in his experiments, greater than the average differences which could be ascribed to the feeding.

Moreover, the fat of the same individual has not the same composition in different parts of the body. This point has recently been the subject of an elaborate investigation by Henriques \& Hansen, $\dagger$ whose results show a higher melting-point and a lower iodine number in the inner as compared with the outer layers of fat. This difference they ascribe to the difference in the temperature of the tissues and support this view by an experiment with three pigs. One animal was kept in a stall heated to about $30^{\circ} \mathrm{C}$. for two months, while the others were exposed to a temperature of $0^{\circ} \mathrm{C}$., one unprotected and the other partially enveloped in a sheeppelt. At the close of the experiment the fat immediately under the skin gave the following figures:

\begin{tabular}{|c|c|c|}
\hline Kept at $30^{\circ}-35^{\circ}$ C....... & $\begin{array}{l}\text { Todine } \\
\text { Number. } \\
\text {. } 69.4\end{array}$ & $\begin{array}{l}\text { Solidifying } \\
\text { Point. } \\
24.6^{\circ} \mathrm{C} .\end{array}$ \\
\hline Kept at $0^{\circ}$, in sheep pelt: & & \\
\hline Part under the pelt. & 67.0 & $25.4^{\circ} \mathrm{C}$ \\
\hline Part exposed........ & 69.4 & $24.1^{\circ} \mathrm{C}$ \\
\hline Kept at $0^{\circ}$, unprotected. & 72.3 & $23.3^{\circ} \mathrm{C}$ \\
\hline
\end{tabular}


Towards the interior of the body the differences became gradually less.

It is evident, then, that the sources of possible error in experiments upon the influence of food on the composition of body fat are considerable, and that not only is great care necessary to secure representative samples of fat for examination, but the effect of individuality must be eliminated so far as possible by the use of a considerable number of animals. When we add to this the other fact that the fat production of herbivorous animals is. largely at the expense of other nutrients than fat, we shall hardly incline to give the results of such investigations much weight as regards the question of the functions of food fat.

Quantitative Relations.- Some further light upon the point under discussion may perhaps be obtained from a consideration of the quantitative relations of food fat to fat production shown by respiration experiments and which will be considered more fully on subsequent pages (compare Chapter V). In scarcely any of these experiments has the food fat been deposited quantitatively in the tissue. In three out of five experiments by Rubner in which fat was given to a previously fasting animal, from 65.82 to 91.89 per cent. of the fat supplied in excess of the amount metabolized during fasting was stored up in the body. Similarly, in the experiments of Pettenkofer \& Voit, in which the fat was added to a ration already more than sufficient for maintenance, on the average 87.86 per cent. of the fat of the food was deposited in the tissues.

Kellner,* among his extensive respiration experiments upon cattle, reports the results of three in which peanut oil was added to a. basal ration more than sufficient for maintenance. The amounts of fat consumed in excess of the basal ration and the resulting gains by the animals were as follows, the slight variations in the amounts of the other nutrients being neglected:

\begin{tabular}{c|c|c|c|c}
\hline Animal. & $\begin{array}{c}\text { Additional Fat } \\
\text { Digested, } \\
\text { Grams. }\end{array}$ & \multicolumn{2}{|c|}{ Gain by Animal. } & $\begin{array}{c}\text { Gain of Fat } \\
\text { in Per Cent. of Fat } \\
\text { Digested. }\end{array}$ \\
\cline { 1 - 2 } Drotein, & $\begin{array}{c}\text { Fat, } \\
\text { Grams. }\end{array}$ & Grams. & \\
\hline F & 677 & 8 & 239 & 35.30 \\
G & 542 & 86 & 205 & 37.83 \\
& 458 & 44 & 279 & 60.91 \\
\hline
\end{tabular}

* Landw. Vers. Stat., 53, 112, 124, 199, 214. 
Computations of the proportion of the energy of the added fat which was recovered in the total gain of flesh and fat (compare Chapter XIII, § 1) showed, according to the method of computation employed, a loss of from 31 to 48 per cent.

The comparatively small losses observed in Rubner's and in Pettenkofer \& Voit's experiments may well be ascribed to a consumption of energy in the work of digestion (compare Chapter $\mathrm{XI}$ ), but it hardly seems possible to account in this way for the large losses observed by Kellner. Apparently the peanut oil in these experiments, after its digestion and resorption, must have been subjected to extensive molecular changes involving a considerable expenditure of potential energy, and if this be true, the suggestion of an assimilation by the fat cells and a construction of animal fat from the oil is obvious.

Constancy of Composition of Fats.-The relatively constant and characteristic composition of the fat of the same species of animal, notwithstanding differences in the food, has been urged in favor of the view that the fat of the animal is a product of the protoplasmic activity of the fat cells. "The fat of a man differs from the fat of a dog, even if both feed on the same food, fatty or otherwise" (M. Foster). The steer produces beef fat and the sheep mutton fat on identical rations. Unless, however, we are prepared to discredit the experimental results above cited, it would appear that this general and approximate uniformity of composition is largely due to a general uniformity of food, and that marked changes in the nature of the latter may result in altering the former. To this must be added, as already insisted upon, the fact that much of the fat found in the body, especially in the herbivora, is undoubtedly produced in the organism. We may fairly presume that this fat will be the characteristic fat of the species. If we may suppose further that a considerable share of the food fat is oxidized directly, and if we take into consideration the general uniformity of diet of our domestic animals and the relatively small total amount of fat which it often contains, we have at least a plausible explanation of the observed facts and one which does not preclude a direct deposition of food fat in the body and a consequent effect upon the composition of the body fat.

The Katabolism of Fat.-The proportion of the food fat which 
serves to increase the store of fat in the body depends largely upon the total food-supply. When the latter is more than sufficient to balance the total metabolism of the organism, the excess may give rise to a storage of fat, and under these circumstances the food fat or a part of it may, as we have seen, contribute to the increase of adipose tissue. On the other hand, when the food-supply is insufficient, not only is its fat in common with its other ingredients in effect consumed to support the vital processes, but the fat previously stored in the adipose tissue is drawn upon to make up the deficiency. Under these circumstances the fat disappears more or less rapidly from the fat cells, passing away gradually either into the lymphatics or the blood-vessels in some manner not as yet fully understood.

Fat, then, whether derived immediately from the food or drawn in the first instance from the adipose tissue of the body, passes into the circulation and serves to supply the demands of the body for oxidizable material and energy, the final products of its oxidation being carbon dioxide and water. Of the intermediate steps in this katabolic process we are comparatively ignorant, but one hypothesis regarding it has acquired so much importance in its bearings on the availability of the potential energy of the food as to require mention here.

Formation of Dextrose from Fat.-This hypothesis is, in brief, that the first step in the katabolism of fat takes place in the liver and consists in its conversion into sugar. In other words, it is held that the fat of the food or that drawn from the adipose tissue of the body supplies the liver with part of the material for its function of sugar production described in the previous section.

This hypothesis is advocated especially by those physiologists who, like Seegen in Vienna and Chauveau and his associates in Paris, look upon the carbohydrates, and particularly dextrose, as the immediate source of the energy exerted in muscular contraction or in the various other forms of physiological work. The evidence upon which this view is based will be considered in subsequent chapters. For the present it suffices to point out that, if we admit its truth, then the general metabolism of the body is essentially a carbohytrate metabolism. Whether we consider the case of a fasting animal, living upon its store of protein and fat, or that of an 
animal receiving food, the liver breaks down the proteids and fat supplied to the blood either by the food or from the tissues, producing dextrose. This dextrose, like that derived from the carbohydrates of the food, is then, as indicated in the previous section, oxidized in the tissues either directly or with previous conversion into glycogen.

As regards the katabolism of fat, in particular, Nasse * has brought forward reasons for believing that the liver is concerned in it. Seegen $\dagger$ submitted fat to the action of finely chopped, freshly excised liver suspended in defibrinated blood at a temperature of $35-40^{\circ} \mathrm{C}$., in a current of air and observed a considerable formation of sugar in five to six hours as compared with a control experiment without the fat. He likewise found $\ddagger$ in experiments upon dogs fed on fat with little or no meat that the blood of the hepatic vein was much richer in sugar than that of the portal vein. On the basis of the probable amount of blood circulating through the liver, he computes that the total amount of sugar thus produced was much greater than could have been supplied by the glycogen stored in the liver and the amount of proteids metabolized (as measured by the urinary nitrogen), and hence concludes that at least the difference was produced from fat. As was pointed out in the preceding section, however, many physiologists regard the large differences between the dextrose content of the portal and the hepatic blood observed by Seegen as being in large part the result of the necessary operation and thus abnormal, and the production of glycogen or dextrose from fat is not regarded as proven by the majority of physiologists.§ Thus Girard $\|$ and Panormow found the post-mortem formation of sugar in the liver to be strictly proportional to the disappearance of glycogen, and similar results were obtained by Cavazzani and Butte.**

Kaufmann, $\dagger \dagger$ who has developed this hypothesis in considerable

* v. Noorden, Pathologie des Stoffwechsels, p. 85.

† Die Zuckerbildung im Thierkörper, p. 151.

$\ddagger$ Ibid., p. 171.

\$ Cf. Neumeister, Physiologische Chemie, p. 368.

I Arch. ges. Physiol., 41, 294.

T Thier. Chem. Ber., 17, 304.

** Ibid., 24, 391 and 394.

t† Archives de Physiol., 1896, p. 331. 
detail, represents the two supposed stages in the katabolism of fat by the two following equations, proposed by Chauveau:*

First Stage: $2\left(\mathrm{C}_{57} \mathrm{H}_{110} \mathrm{O}_{6}\right)+67 \mathrm{O}_{2}=16\left(\mathrm{C}_{6} \mathrm{H}_{12} \mathrm{O}_{6}\right)+18 \mathrm{CO}_{2}+14 \mathrm{H}_{2} \mathrm{O}$. Second Stage : $16\left(\mathrm{C}_{6} \mathrm{H}_{12} \mathrm{O}_{6}\right)+96 \mathrm{O}_{2}=96 \mathrm{CO}_{2}+96 \mathrm{H}_{2} \mathrm{O}$.

Even, however, if we admit the formation of dextrose from fat in the body, it may fairly be doubted whether the process is as simple as these equations, even if regarded as simply schematic, would imply.

\section{§ 3. Proteid Metabolism.}

ANABOLISM.

Digestive Cleavage. - The digestion of the proteids is essentially a process of cleavage and hydrution under the influence of certain enzyms. By this process the complex proteid molecules are partially broken up into simpler ones. By the action of pepsin in acid solution we obtain albumoses and peptones, while the trypsin of the pancreatic juice, at least outside the body, carries the cleavage still further, producing crystalline nitrogenous bodies of comparatively simple constitution. Opinions are still more or less divided as to how far these processes of cleavage and hydration are carried in the actual process of digestion, where the products of the action are constantly being resorbed, but there are not wanting indications that it is both less extensive and less rapid than in artificial digestion. It likewise seems to have been demonstrated that some soluble proteids are capable of direct resorption without change, while others are not and some, notably casein, are promptly coagulated by the rennet ferment, apparently expressly in order that they may be subjected to the action of the digestive ferments. In a general way, the statement appears to be justified that the larger share of the proteid material of the food is resorbed as albumoses and peptones.

Purpose of the Cleavage.-The fact just mentioned that, on the one hand, some soluble proteids appear capable of direct resorption, while, on the other hand, some, like casein, are at once rendered insoluble as the first step in digestion, plainly necessitates a material modification of the old view that the object of the cleav-

* La Vie et l'Energie chez l'Animale. 
age and hydration of the proteids in digestion is to render them soluble. Undoubtedly this is an important function of the digestive fluids, but the fundamental object lies deeper and is found in the constitution of the proteids themselves.

Nature of the Proteids.-While we are still very far removed from any adequate knowledge of the molecular structure of the proteids, a study of the action upon them of various hydrolytic agents, and particularly of the proteolytic enzyms of the digestive fluids, has shown that they undergo cleavage along certain definite lines, giving rise to two series of products known as the hemi- and the anti-series. The primary products are the proteoses, or albumoses (hemi and anti). By further action of the ferment these give rise to the secondary or deutero-proteoses, and these in turn to peptones, while the peptones of the hemi-series, by the further action of trypsin, are broken up, as noted, into simpler bodies such as aspartic acid, glutaminic acid, and notably tyrosin and leucin. The two latter bodies belong to the aromatic series and contain the phenyl radicle, which is thus shown to be present in the bodies of the hemi group, while it is absent from the anti group. Without pursuing the subject further, enough has been said to show that the general result of the digestive proteolysis is to break up the proteid molecule into a considerable number of unlike fragments.*

Differences in Proteids. - Turning now to another phase of the subject, it is a familiar fact that the numerous proteids which have been studied differ quite markedly from each other in properties and in composition. To instance but a single characteristic difference, the investigations of Osborne and his associates at the Connecticut Agricultural Experiment Station have shown in detail what was to a certain extent known before, viz., that the nitrogen content of the vegetable proteids is notably higher than that of the animal proteids. We can only interpret these differences in composition and properties as the results of differences in molecular structure. We may fairly suppose that these differences in structure are brought about, in part at least, by differences in the relative proportions in the proteid molecule of the several molecular groupings whose presence is indicated to us by the results of proteolysis.

* For a full treatment of the subject, compare Chittenden, Digestive Proteolysis, 1894. 
Food Proteids and Body Proteids.-What is especially to be noted in this connection is that the food proteids are not identical with the body proteids. This is especially true of the vegetable proteids in the food of the herbivora, and of the casein of milk, but is measurably true in all cases. A simple resorption of unaltered protein, therefore, would not serve the purposes of the organism. The food proteids must be changed to body proteids. This means, however, that the proportions of those molecular groupings which have just been spoken of must be changed-that is, the molecules of the food proteid must be so far broken down into their constituent molecular groupings as to permit of a rearrangement and reproportioning of the latter into molecules of body proteid.

Such a partial breaking down of proteid material takes place in digestion, and indeed, as has been indicated above, it is the study of digestive proteolysis which has given us our general conception of the structure of the proteid molecule. The products of proteid digestion, then, as they are presented to the resorbent organs of the digestive tract, are no longer proteids, but the constituent molecular groupings out of which body proteids may be built up.

Rebuilding of Proteids.-But while the proteids of the food are resorbed in the form of cleavage products, apparently largely as peptones, no trace of these bodies is found in the blood or in the lymph, nor even in the walls of the digestive canal. Still further, peptones when injected into the blood are treated by the organism as foreign substances and excreted as rapidly as possible, while if added in any considerable amount they act as poisons. The reconstruction of the proteid molecule from the fragments produced by the digestive process has been thought to take place in the epithelial cells of the intestines, the first product being probably serum albumen, so that we may say that the first step in proteid metabolism is anabolic.

Recently, however, Okunew, working in Danilewsky's laboratory, has announced the discovery that the enzym of rennet (chymosin) has the power of synthesizing peptones to proteids, and Sawjalow * has published further studies on the same subject. The latter investigator finds the product to be a gelatinizing proteid which is identical whatever the original source of the peptones and which he calls plastein. He considers that this plastein is

*Arch. ges. Physiol., 85, 171. 
formed in the digestive canal and is the form in which the proteids of the food are resorbed, and points out that this hypothesis accounts for the hitherto puzzling fact of the occurrence of the milk-curdling ferment in animals, such as birds, fishes, and amphibia, which never consume milk. In further support of this view, Winogradow* finds the formation of chymosin in the stomach to be most active at the height of the digestive process, when peptones are being formed most freely.

The proteid or proteids first formed from the albumoses and peptones, whether in the epithelial cells or by the action of chymosin, is subject to still further changes in other portions of the body, inasmuch as all the various nitrogenous tissues of the body are formed from it. Some of these changes may be slight. but others, as, e.g., the formation of the collagens, must be profound, while the formation of the compound proteids like hæmoglobin, mucin, the nucleins, etc., is clearly synthetic and anabolic. As: to where and how these changes and syntheses take place, we are largely ignorant. We simply know the general fact that the food proteids are first partially broken down in the process of digestion and then that the fragments are built up again into body proteicls; first, probably, into some single form and later into still more complex bodies in the various tissues.

\section{KATABOLISM.}

Final Products. -The anabolic processes which have just been indicated might be characterized in general terms as a preparation of the food proteids for their diverse functions in the body. In the performance of those functions they, like all the organic ingredients of the body, undergo katabolic changes, liberating the energy which was originally contained in them or which may have been temporarily added in the preliminary anabolic changes. We have every reason to believe that the katabolism of proteids is a gradual process, passing through many intermediate stages, but we have very little actual knowledge of the steps which intervene between the proteids and bodies which are either excretory products themselves or closely related to them. Such information as has

* Arch. ges. Physiol., 87, 170. 
thus far been acquired upon this subject has resulted chiefly from attempts to trace back the excretory products to their antecedents.

The products of the complete breaking down and oxidation of proteids in the body are carbon dioxide and water, excreted through the lungs, skin, and kidneys, and urea and a number of other comparatively simple crystalline nitrogenous compounds found in the urine. To these are to be added the nitrogenous metabolic products of the feces, the sulphuric and phosphoric acids resulting from the oxidation of the sulphur of the proteids and the phosphorus of the nucleo-proteids, and the relatively minute amounts of nitrogenous matter found in the perspiration.

Excretion of Free Nitrogen.-The question whether any portion of the nitrogen of the proteids is excreted as free gaseous nitrogen is one which has been the subject of no little investigation and controversy in the past, the especial champions being, on the affirmative, Seegen in Vienna and, on the negative, Voit in Munich. It would lead us too far aside from our present purpose, however, to attempt even to outline the evidence, and it must suffice to say that the great majority of physiologists regard it as established that there is no excretion of gaseous nitrogen as a result of the katabolism of proteids, but that all the proteid nitrogen is excreted in the urine and feces with the exception of small amounts in the perspiration. In accordance with this view, we shall assume in subsequent pages that the urinary nitrogen (together with, strictly speaking, the metabolic nitrogen of the feces and perspiration) furnishes a measure of the total proteid katabolism of the body.

A brief consideration of some of the principal nitrogenous products of proteid katabolism will serve to indicate some of the main features of the process, so far as they have been made out.

UREA.- Urea, or dicarbamid, $\mathrm{CON}_{2} \mathrm{H}_{4}$, is the chief nitrogenous product of proteid metabolism in the carnivora and omnivora. In the urine of man, e.g., from 82 to 86 per cent. of the nitrogen is in the form of urea.*

Antecedents of Urea.-A vast amount of study has been expended upon this question without as yet leading to any general unanimity of views. It appears, however, to be fairly well made out that at

* v. Noorden, Pathologie des Stoffwechsels, p. 45. 
least a considerable part if not all of the urea is formed in the liver, and that its immediate antecedent is ammonium carbonate, to which it is closely related chemically. This theory of Schmiedeberg's is supported by the facts:

1st. That ammonium salts, and also the amid radicle $\mathrm{NH}_{2}$ in the amido acids of the fatty series, when administered in the food are converted into urea.

2 d. That ammonium carbonate or formiate injected into the portal vein is converted in the liver into urea which appears in the blood of the hepatic vein.

$3 \mathrm{~d}$. That the administration of inorganic acids to the dog and to man results in the excretion of ammonium salts in the urine, it being supposed that the acid displaces the weaker carbonic acid and that the resulting ammonium salt is incapable of conversion into urea in the liver.

4th. Severe disease of the liver has been observed to result in a decreased production of urea and an excretion of ammonium salts in the urine.

Later investigations by Minkowski* and others have followed the process of the formation of urea one step further back and rendered it highly probable that the ammonium salts out of which urea is formed reach the liver in the form of ammonium lactate. It has been shown that sarcolactic acid is one of the products of the metabolism of the muscles. It would appear that this acid unites with the ammonium radicle derived from the proteids to form ammonium lactate, and that the latter on reaching the liver is first oxidized to the carbonate, which is then converted into urea. If, by disease or surgical interference, this action of the liver is prevented, ammonium lactate appears in the urine, and the same effect may even be produced by excessive stimulation of the proteid metabolism, so that the production of ammonium lactate exceeds the capacity of the liver to convert it.

URIC ACID.-Uric acid is contained in small amounts in the urine of mammals. With birds it constitutes the chief nitrogenous product of the proteid metabolism. Of its antecedents in the organism scarcely anything is known. One theory regards it as a specific product of the metabolism of the nucleins, but this cannot

*Cf. Neumeister, Physiologische Chemie, pp. 313-318. 
be regarded as established, and appears difficuit to reconcile with its relation to the proteid metabolism of birds. Others regard it as an intermediate product in the production of urea, a small portion of which escapes further oxidation by being excreted by the kidneys.

HIPPURIC ACID.--This substance is a normal ingredient of the urine of mammals, but in that of man and the carnivora is found in but very small amounts. In the urine of herbivora, on the other hand, it occurs abundantly.

Light was thrown upon its origin by the well-known discovery by Wöhler, in 1824, that it is also found in large amount in the urine of man or of carnivora after the administration of benzoic acid. Chemically, hippuric acid is benzamido-acetic acid, or benzoyl glycocol. When the food contains benzoic acid the latter unites with glycocol resulting from the metabolism of the proteids and forms hippuric acid, while otherwise the glycocol would be further oxidized to simpler nitrogenous products. The synthesis of hippuric acid has been shown to occur only in the kidneys in the dog, but in the case of the rabbit and frog they appear to share this capacity with other organs.

In this action of benzoic acid we have the most familiar demonstration of the formation of metabolic products intermediate between the proteids and the comparatively simple nitrogenous substances found in the urine. Glycocol has never been detected in the body, obviously because as fast as it is formed it is again decomposed. Benzoic acid reveals its presence by seizing upon it and converting it into a compound which is incapable of further oxida. tion, and is therefore excreted. Other less familiar examples of the same fact might be cited did space permit.

The normal presence of small quantities of hippuric acid in the urine, even when no benzoic acid is contained in the food, arises from the fact that the putrefaction of the proteids in the intestines yields aromatic compounds, containing the benzoyl radicle, which are resorbed and combine with glycocol to form hippuric acid. The origin of the large quantities of hippuric acid ordinarily excreted by herbivora, however, or rather of its benzoyl radicle, is still more or less of a puzzle, notwithstanding the considerable amount of investigation which has been devoted to its study. The most natural supposition would be that the food of 
these animals contains substances of the aromatic series capable of yielding benzoic acid or its equivalent in the body, but in none of the feeding-stuffs known to be efficient in causing an excretion of hippuric acid have such compounds been discovered in quantity even remotely sufficient to account for the hippuric acid produced.

On the other hand, the hypothesis that the benzoyl radicle of the hippuric acid is derived to any large extent from the proteids of the food appears to be decisively negatived by several facts: First, the quantity of proteids in the ordinary rations of herbivora is relatively small, and even if it all underwent putrefaction the amount of aromatic products which could be formed, on any reasonable estimate, would account for only a small fraction of the hippuric acid actually found.* Second, in several instances it has been observed that variations in the extent of the putrefactive processes in the intestines, as measured by the amount of conjugated sulphuric acid in the urine (compare p. 46), bore no relation to the variations in the production of hippuric acid. Third, the addition of pure proteids or of foods very rich in proteids to a ration does not increase the production of hippuric acid, and in at least one case $\dagger$ was found to diminish it and even stop it altogether.

Apparently we must regard the non-nitrogenous ingredients of feeding-stuffs as the chief source of hippuric acid formation, but beyond this our knowledge is rather vague. It is well established that the coarse fodders are the chief producers of hippuric acid, while the concentrated feeding-stuffs give rise to little or none, and may even reduce the amount previously produced on coarse fodder, as may also starch. Among the coarse fodders, the gramineæ give rise to a markedly greater production of hippuric acid than the leguminosæ. This effect of the coarse fodders naturally led to the suspicion that the crude fiber contained in them in large amounts might be the source of the hippuric acid, and in fact numerous experiments seem to show that some relation exists between the two, although the results of various investigators are far from concordant.

Finally, the investigations of Groetze \& Pfeiffer, $\downarrow$ and of

* Compare Salkowski, Zeit. physiol. Chem., 9, 234.

$\uparrow$ Henneberg and Pfeiffer, Jour. f. Landw., 38, 239.

$\ddagger$ Landw. Vers. Stat., 47, 59 . 
Pfeiffer \& Eber,* have shown with a high degree of probability that the pentose carbohydrates of the feed have some connection with the production of hippuric acid. The former investigators observed a marked increase in the production of hippuric acid by a sheep after the administration of cherry gum (impure araban) and of arabinose, and the latter obtained the same effect, although in a less marked degree, by feeding cherry gum to a horse. They also call attention to the differences in the behavior of the pentose carbohydrates in the organism of the herbivora and in that of man and the carnivora, but do not attempt to give a final solution of the problem of the origin of the hippuric acid in the former case, while they freely admit that it is difficult, if not impossible, to explain some of the facts already on record on the hypothesis that the pentoses are the chief source of hippuric acid.

Creatin and Creatinin.-Among other nitrogenous constituents of the urine of man and the carnivora may be mentioned creatinin. This body is the anhydride of creatin, and the two together constitute the principal part of the so-called flesh bases which are contained in considerable quantity in muscular tissue. When meat is consumed, its creatin is converted into creatinin and excreted quantitatively in the urine, the creatinin content of which may be thus considerably increased. As to the physiological significance of the creatin of muscular tissue opinions are divided, but good authorities are inclined to regard it as an intermediate product of the metabolism of the proteids which is ultimately converted into urea, and to urge that the fate of creatin taken into the stomach is not necessarily the same as that of the creatin produced in the muscles.

Aromatic Compounds.-Besides the benzol radicle of hippuric acid, small amounts of other aromatic compounds are also found in the urine. These bodies, belonging chiefly to the phenol and indol groups, owe their origin exclusively to the putrefactive processes already mentioned as taking place in the intestines, and are found in the urine almost entirely in combination with sulphuric acid as the so-called conjugated sulphuric acids, so that the amount of the latter is employed as a measure of the extent of these putrefactive processes.

* Landw. Vers. Stat., 49, 97. 
Metabolic Products in Feces.--As already stated in Chapter $I$, the feces contain, in addition to undigested residues of the food, certain materials derived from the body of the animal. This fact was early recognized as true of both carnivora* and herbivora. $\dagger$ Of more recent investigations may be noted especially those of Müller, his associates on man, and those of Kellner,** Stutzer, $†$ Pfeiffer, and Jordan $\S \S$ on herbivora.

These "metabolic products" appear to consist of unresorbed or altered residues of the digestive fluids and of mucus and other materials excreted or otherwise thrown off by the walls of the intestines. Their production goes on even when the digestive tract is void of food, producing the so-called fasting feces which constitute a true excretory product. The consumption of highly digestible food-e.g., lean meat-does not seem to materially increase their amount, but when food containing indigestible matter is eaten it is believed that they increase in quantity.

It is presumed that these substances are largely nitrogenous in character, and it is known at any rate that not inconsiderable amounts of nitrogen may leave the body by this channel. In other words, these nitrogenous substances, derived from the proteids of the body, instead of undergoing complete conversion into the ordinary crystalline products have their katabolism interrupted as it were at an intermediate stage.

Many attempts have been made to determine the amount of these metabolic products, or of their nitrogen, in the feces, but without much success, and it may fairly be said that at present we have no method which can be depended upon to distinguish sharply between the nitrogen of undigested-food residues and that of metabolic products.

* Bischoff and Voit, Die Ernăhrung des Fleischfressers, p. 291.

$\dagger$ Henneberg, Beiträge, etc., 1864, p. 7.

+Zeit. f. Biol., 20, 327.

\$Ibid., 20, 378 .

|l Ibid., 35, 68 .

Tl Ibid., 35, 287; 39, 277; 42, 377.

** Landw. Vers. Stat., 24, 434; Bied. Centralbl., 9, 763.

†† Zeit. physiol. Chem., 9, 211.

草 Jour. f. Landw., 31, 221; 33, 149; Zeit. physiol. Chem., 10, 561.

§ Maine Expt. Station Rep., 1888, p. 196. 
Nitrogen in Perspiration.-The perspiration of such animals as secrete this fluid must be regarded as one of the minor channels by which nitrogen is excreted. In human perspiration there have been found, in addition to small amounts of proteids, urea, uric acid, creatinin, and other nitrogenous products of the proteid metabolism. In a recent investigation, Camerer * found about 34 per cent. of the total nitrogen of human perspiration to be in the form of urea, about 7.5 per cent. existed as ammonium salts, and the remainder in undetermined forms, including uric acid and traces of albumen.

The total quantity of nitrogen excreted in the insensible perspiration appears to be insignificant. Atwater \& Benedict $\dagger$ found it to amount to 0.048 gram per day for an adult man in a state of rest. Rubner \& Heubner $\ddagger$ obtained from the clothing of an infant $2.83 \mathrm{mgrs}$. of ammonia and $0.0205 \mathrm{mgr}$. of urea per day and estimated the total nitrogen of the perspiration at $39 \mathrm{mgrs}$.

When the secretion of sweat is stimulated by work or a high external temperature the amount of nitrogen excreted may be considerably increased as compared with a state of rest, although its absolute amount is still small. Atwater \& Benedict, $\$$ in a work experiment, observed an excretion of 0.220 gram of nitrogen per day in the perspiration of man.

The Non-nitrogenous Residue of the Proteids.-The various nitrogenous products found in the urine and other excreta, the most important of which have been noticed above, are believed to contain all the nitrogen of the metabolized proteids. This does not imply, however, that a quantity of proteids equivalent to this nitrogen, or even to that of the urine, has been completely oxidized to the final products of metabolism, viz., carbon dioxide, water, and urea and its congeners.

A comparison of the ultimate composition of the proteids with that of the nitrogenous products of their metabolism reveals the fact that an amount of the latter sufficient to account for all the nitrogen of the proteids contain but a relatively small part of their carbon, hydrogen, and oxygen. Taking urea as the chief and *Zeit. f. Biol., 41, 271.

$\nmid$ U. S. Dept. Agr., Office of Expt. Stations, Bull. 69, 73.

tZeit. f. Biol., 36, 34 .

§Loc. cit., p. 53. 
typical metabolic product, and using average figures for the composition of animal proteids, we have, omitting the sulphur of the proteids, the following:

\begin{tabular}{|c|c|c|c|}
\hline Carbon........ & $\begin{array}{c}\text { Proteids. } \\
53.0\end{array}$ & $\begin{array}{r}\text { Urea. } \\
6.86\end{array}$ & $\begin{array}{c}\text { Residue } \\
46.14\end{array}$ \\
\hline Hydrogen . . ....... & 7.0 & 2.29 & 4.71 \\
\hline Oxygen ......... & 24.0 & 9.14 & 14.86 \\
\hline Nitrogen ........... & 16.0 & 16.00 & \\
\hline & 100.0 & 34.29 & 65.71 \\
\hline
\end{tabular}

After abstracting the elements of urea, we have left considerably over half the hydrogen and oxygen of the proteid and the larger part of its carbon. A substantially similar result is reached in case of the other nitrogenous metabolic products. The splitting off of these products from the proteids leaves a non-nitrogenous residue.

Fate of the Non-nitrogenous Residue.-The foregoing statements and comparison must not be understood to mean that the proteids split up in the body into two parts, viz., urea, etc., on the one hand, and an unknown non-nitrogenous substance or substances on the other. As we have already seen, the processes of proteid metabolism are far more complicated than such a simple cleavage. Neither are we to assume that any substance or group of substances corresponding in composition to the "residue" of the above computation exists. The figures mean simply that while the nitrogenous bodies of the urine contain all the nitrogen of the proteids they do not account for all of the other elements, but that part of the latter must be sought elsewhere.

Ultimately, of course, the elements of this non-nitrogenous residue are converted into carbon dioxide and water. The conversion into these final products, however, is necessarily a process of oxidation, presumably yielding energy to the organism. It is a matter of some interest, then, to trace the steps of the transformation far as this is at present possible.

Formation of Sugar.-In discussing the functions of the liver in $\$ 1$ of this chapter, we have seen reason to believe that this organ continues to produce sugar when the diet consists largely or exclusively of proteids. In this case we are forced to the conclusion that this sugar is manufactured from the elements of the non-nitrogenous residue. 
This conclusion, based on what appears to be the normal function of the liver, is further strengthened by a large number of experiments and observations upon the metabolism in diabetes. This disease, whether arising spontaneously or provoked artificially, is characterized by the presence of large amounts of sugar in the urine. It has been shown that this production of sugar continues when all carbohydrates are withdrawn from the diet, and furthermore, that the amount of sugar excreted bears a quite constant relation to the amount of proteids metabolized, thus clearly indicating the latter as the source of the sugar. It is true that the formation of sugar from proteids is denied by some physiologists, * but by the majority it seems to be accepted as a well-established fact that sugar is one of the intermediate products of proteid metabolism.

Of the steps of the process, as well as of its quantitative relations, we are ignorant. In effect, it is a process of oxidation and hydration, since a residue of the composition computed above would require the addition of both hydrogen and oxygen to convert it into sugar, but that it is as simple a process as this statement would make it appear, or that the conversion is a quantitative one, may well be doubted.

In conclusion it may be stated that while recent investigations have shown the presence of a carbohydrate radicle in numerous (although by no means all) proteids, it does not appear that this fact stands in any direct relation to the physiological production of sugar from these substances. In the first place, the carbohydrate radicle constitutes a much smaller proportion of these proteids than corresponds to the amount of sugar which they are apparently capable of yielding in the body, and in the second place it appears to be a well-established (although not undisputed) fact that the organism can produce sugar from proteids which do not contain the carbohydrate radicle.

Formation of Fat.-Whether fat is formed from the elements of proteids in the animal body is at present a subject of controversy, but this question will be more profitably considered in a subsequent chapter. It is sufficient to remark here that while much of the earlier evidence bearing upon this point has been shown to be

*Cf. Schöndorf, Arch. ges. Physiol, , 82, 60. 
inconclusive, the formation of fat from proteids has not yet been disproved and has weighty direct evidence in its favor, while the facts that sugar may be formed from proteids, and that carbohydrates are certainly a source of fat to the animal organism are strong additional arguments in favor of its possibility.

Schematic Equations.-Chauveau and his associates * whose views regarding the functions of the carbohydrates in the body have already been mentioned, regard the katabolism of the proteids as taking place in three stages. The first consists of the splitting off of urea with production of carbon dioxide, water, and fat, according to the equation:

$$
\begin{aligned}
4\left(\mathrm{C}_{72} \mathrm{H}_{112} \mathrm{~N}_{18} \mathrm{O}_{22} \mathrm{~S}\right) & +139 \mathrm{O}_{2} \\
= & 2\left(\mathrm{C}_{57} \mathrm{H}_{110} \mathrm{O}_{8}\right)+36 \mathrm{CON}_{2} \mathrm{H}_{4}+138 \mathrm{CO}_{2}+42 \mathrm{H}_{2} \mathrm{O}+2 \mathrm{~S}_{2} .
\end{aligned}
$$

The resulting fat is then, according to Chauveau, further oxidized in the liver, yielding dextrose, in accordance with the equation already given on p. 38 , viz.,

$$
2 \mathrm{C}_{57} \mathrm{H}_{110} \mathrm{O}_{6}+67 \mathrm{O}_{2}=16 \mathrm{C}_{6} \mathrm{H}_{12} \mathrm{O}_{6}+18 \mathrm{CO}_{2}+14 \mathrm{H}_{2} \mathrm{O},
$$

and the dextrose is finally oxidized to carbon dioxide and water.

Another equation representing the katabolism of proteids is that proposed by Gautier, which regards the first step in the process as a combined hydration and cleavage with the production of urea, fat, dextrose, and carbon dioxide, as follows:

$$
\begin{aligned}
2\left(\mathrm{C}_{72} \mathrm{H}_{112} \mathrm{~N}_{18} \mathrm{O}_{22} \mathrm{~S}\right)+ & 28 \mathrm{H}_{2} \mathrm{O} \\
= & 18 \mathrm{CON}_{2} \mathrm{H}_{4}+2 \mathrm{C}_{51} \mathrm{H}_{98} \mathrm{O}_{6}+\mathrm{C}_{8} \mathrm{H}_{12} \mathrm{O}_{6}+18 \mathrm{CO}_{2}+\mathrm{S}_{2} .
\end{aligned}
$$

It may be assumed that these authors regard the above equations simply as schematic representations of the general course of proteid metabolism and do not intend to imply that there are no intermediate stages in the process. Interpreting them in this sense, we have good reasons for believing that the facts which they represent are qualitatively true. A chemical equation, however, expresses not merely qualitative but quantitative results. If the above equations have any significance beyond that of the mere verbal statement that fat and sugar are products of proteid meta-

* Cf. Kaufmann, Archives de Physiol., 1896, p. 341. 
bolism, they mean that from 100 grams of proteids there is produced, according to the first scheme, 27.61 grams of fat, and that from this, by the addition of oxygen, 44.67 grams of sugar are formed. Some of the evidence by which these equations are supported will be considered in another connection, but may be anticipated here in the statement that, in the judgment of the writer, it is far from sufficient to establish them as quantitative statements.

\section{THE NON-PROTEIDS.}

Under this comprehensive but somewhat vague term have been grouped all those numerous nitrogenous constituents of the food which are not proteid in their nature, the name being a contraction of non-proteid nitrogenous substances. It includes the extractives of meat, and in vegetable foods several groups of substances, of which, however, the amides and amido-acids are most abundant. Various substances of this class are produced by the splitting up of the reserve proteids in the germination of seeds and apparently also to some extent in the translocation of proteids in the growing plant, while some at least of them appear to be produced synthetically from inorganic materials and to be the forerunners of proteids. In young plants a considerable proportion of the so-called crude protein $(\mathrm{N} \times 6.25)$ often consists of these non-proteids, and considerable interest, therefore, attaches to their transformations in the body.

Amides Oxidized in the Body.-It has been shown by numerous investigators that various amides and amido-acids when added to the food are oxidized, giving rise to a production of urea. Shultzen \& Nencki* found that glycocol, leucin, and tyrosin were thus oxidized, while acetamid apparently was not. So far as glycocol is concerned, this result is what would have been expected. since, as we have seen (p. 44), this body appears to be normally formed in the body as an intermediate product of proteid metabolism. Similar results were obtained by v. Knierien $\nmid$ from trials with asparagin, aspartic acid, glycocol, and leucin. MIunk $\$$ likewise found that the ingestion of asparagin increased the pro-

\footnotetext{
* Zeit. f. Biol., 8, 124.

$\dagger$ Ibid., 10, 277; , 36.

†Virchow's Archiv. f. path. Anat., 94, 441.
} 
duction of urea in the dog, all the nitrogen of the asparagin together with an excess over that previously found in the urine being excreted. The sulphur in the urine also increased. Hagemann * has more recently fully confirmed this result. Salkowski $\dagger$ found that glycocol, sarkosin, and alanin were oxidized to urea and caused no gain of proteids. Apparently, then, this class of bodies, like ammonia, furnish material out of which the organism can construct urea.

Can Amides Replace Proteids?-Since the amides yield the same end products of metabolism as the proteids, it is natural to inquire whether they can perform any of the functions of those substances.

Amides not Synthesized to Proteids.-We have already seen that the albumoses and peptones resulting from the cleavage of the proteids during digestion are built up again into proteids in the process of resorption. The amides commonly found in vegetable feeding-stuffs are likewise simpler cleavage products of the proteids, and some of them are also formed in digestion by the proteolytic action of trypsin. Can proteids be regenerated from these simpler cleavage products?

If this is the case, then it should be possible, under suitable conditions, to cause a gain of proteids, or at least to maintain the stock of proteids in the tissues, on a food free from proteids but containing amides. Up to the present time, however, all attempts of this sort have failed. With the most abundant supply of nonnitrogenous nutrients and ash, the animals perished when supplied with amides (asparagin) but not with proteids. I What has thus been found to be true of asparagin we may regard as probably true of other amides and say that there is no evidence that the animal body can build proteids from amides.

Partial Replacement of Proteids.-But even if the amides cannot serve as a source of proteids to the animal, it seems not impossible that they may by their oxidation perform a part of the functions of the proteids, thus protecting a portion of the latter from oxidation and rendering it available for tissue production.

* Landw. Jahrb., 20, 264.

† Zeit. physiol. Chem., 4, 55.

$\ddagger$ Compare Politis, Zeit. f. Biol., 28, 492, and Gabriel, Ib., 29, 115. 
The earliest investigations upon this point are those of Weiske* and his associates upon the nutritive value of asparagin. The experiments were made upon rabbits, hens, geese, sheep, and goats, and in the case of the two latter species included experiments on milk production. While the experiments are open to criticism in some respects, as a whole they seemed to show that asparagin, especially when added to a ration poor in proteids, caused a gain of proteids by the body. Weiske accordingly concluded that asparagin, while not capable of conversion into proteids, was capable of partially performing their functions and thus acting indirectly as a source of proteids, and this view has been somewhat generally accepted. Subsequent experiments by Bahlmann, $\uparrow$ Schrodt, Potthast, $§$ Meyer, $\|$ and Chomsky 9 upon milch-cows, rabbits, and sheep gave results which tended to confirm Weiske's conclusions.

Not all of Weiske's experiments, however, gave positive results in favor of asparagin, and experiments upon carnivorous and omnivorous animals have failed to show any such effect. In addition to the experiments of Politis and of Gabriel, referred to above, Mauthner,** Munk, t† $^{*}$ and Hagemann $t \downarrow$ have failed to observe any gain of proteids by the body as a result of the ingestion of asparagin, but found simply an increase in the apparent proteid metabolism as measured by the urinary nitrogen.

Influence on Digestion.--It can hardly be assumed that the actual processes of metabolism in the body tissues are fundamentally different in different species of mammals, and investigators have therefore been led to seek an explanation of the striking difference in the effects of asparagin on herbivora and carnivora in the differences in the digestive processes of the two classes of animals.

Digestion in herbivora is a relatively slow process and, as pointed out in Chapter I, is accompanied by extensive fermentations par-

* Zeit. f. Biol., 15, 261 · 17, 415; 30, 254.

† Reported by Zuntz, Arch. f. (Anat. u.) Physiol., 1882, 424.

廿Jahresb، Agr. Chem., 26, 426.

$\S$ Arch. ges. Physiol., 32, 288.

\|Cf. Kellner, Zeit. f. Biol., 39, 324.

I Ber. physiol. Lab. Landw. Inst. Halle, 1898, Heft 13, p. 1.

** Zeit. f. Biol., 28, 507.

†† Virchow's Arch. f. path. Anat., 94, 441.

ł Landw. Jahrb., 20, 264. 
ticularly of the carbohydrates of the food, as is shown by the large amounts of gaseous hydrocarbons produced by these animals. In carnivora, on the contrary, digestion is relatively rapid and the $d o g$, as a representative of this class, excretes, according to Voit \& Pettenkofer,* but traces of hydrocarbons, and according to Tappeiner,$\dagger$ none.

Zuntz $\ddagger$ has therefore suggested that soluble amides introduced into the digestive canal of herbivora may be used as nitrogenous food by the micro-organisms there present in preference to the less soluble proteids, so that the latter are to a certain extent protected, and that it is even possible that the amides are synthesized to proteids by the organisms. Hagemann $\S$ has added the suggestion that the proteids possibly thus formed may be digested in another part of the alimentary canal and thus actually increase the proteid supply of the body.

If this explanation is correct, we should expect the effect of asparagin to be more marked when the proportion of proteids in the food is small, and precisely this appears to be the case. In Weiske's first experiments, which gave the most decided results, the nutritive ratio of the ration without asparagin was $1: 19-20$, while a later experiment with a nutritive ratio of $1: 9.4$ showed no effect of the asparagin upon the gain of protein. Chomsky's results, too, were obtained with rations poor in protein and rich in carbohydrates.

Later experiments on lambs by Kellner $\|$ have fully confirmed this anticipation. In his first experiment two yearling lambs were fed with a mixture of hay, starch, and cane-sugar, having a nutritive ratio of $1: 28$, until nitrogen equilibrium was reached, when fifty grams of the starch was replaced by asparagin. The result was a gain of protein by both animals as compared with a loss in the first period. In the third experiment asparagin was substituted for starch in a ration having a nutritive ratio of $1: 7.9$, and caused with one animal a slight gain and with the other a slight loss of protein. In the fourth experiment it was added to a ration

* Zeit. f. Biol., 7, 433; 9, 2 and 438.

$\dagger$ Ibid., 19, 318.

$\$$ Arch. ges. Physiol., 49, 483.

\$Landw. Jahrb., 20, 264.

|| Zeit. f. Biol., 39, 313. 
having a nutritive ratio of $1: 7.7$, and caused neither a gain nor a loss of any consequence.

Particular interest attaches to Kellner's second experiment in which ammonium acetate was added to a ration poor in protein (1:19), followed in a third period by a quantity of asparagin containing the same amount of nitrogen. The average amounts of protein $(\mathrm{N} \times 6.25)$ gained per day and head by the two lambs were as follows:

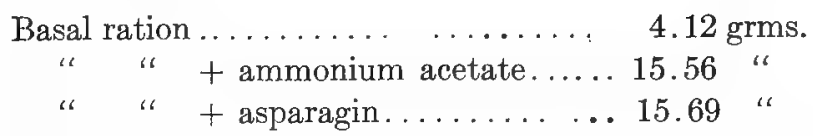

Although it is impossible to suppose that the ammonium acetate is capable of performing any of the functions of proteids in the body, it nevertheless caused as great a gain of protein by the body as did the asparagin. The only obvious explanation is that both these substances acted in the manner suggested by Zuntz to protect the small amount of protein in the food from the attacks of the organized ferments of the digestive tract. Accepting this explanation, we must suppose that when the contents of the alimentary canal contain a normal amount of proteids the micro-organisms find an abundant supply of nitrogenous food in their cleavage products and reach their normal development, so that an addition of soluble nitrogenous substances is a matter of indifference. When, on the other hand, the amount of protein present is abnormally low, as in Weiske's and Kellner's experiments, the organisms are limited in their food-supply and attack the food proteids themselves.

Kellner's results stand in apparent contradiction to the earlier ones of Weiske and Flechsig,* who report no gain of proteids as resulting from the addition on three days of a mixture of ammonium carbonate and acetate to a ration poor in protein. The excretion of sulphur in the urine was likewise unaffected. They assume, however, a long-continued after effect of the ammonium salts on the nitrogen excretion. If the comparison be limited to the three days on which the ammonium salts were given and the next following day, a gain of 1.15 grams of nitrogen per day results, but, as just stated, there was no corresponding gain of sulphur.

*Journ. f. Landw., 38, 137. 
Kellner's experiments afford indirect evidence that both the asparagin and the ammonium acetate actually did stimulate the development of the ferment organisms, in the fact that the apparent digestibility of the carbohydrates of the food was increased. On the basal rations starch could be readily recognized in the feces, but under the influence of the two substances mentioned it disappeared. In the second experiment the increase in the amounts of crude fiber and of nitrogen-free extract digested was as follows:

$$
\text { Crude Fiber. } \quad \text { Nitrogen-free }
$$

With ammonium acetate... 10.7 grms. $\quad 20.4$ grms.

With asparagin ........ 10.0 " 20.0 "

Since we know that large amounts of the nitrogen-free extract are attacked and decomposed by organized ferments in the herbivora, and that this is the chief if not the only method by which crude fiber is digested, we are justified in interpreting the above figures as demonstrating an increased activity of these organisms as a result of the more abundant supply of nitrogenous food. The bearing of this result upon the so-called depression of digestibility by starch and other carbohydrates is obvious, but is aside from our present discussion.

Tryniszewsky * experimented upon a calf weighing about 175 kgs., using in the second and fourth periods (the first period being preliminary) a ration of barley straw, sesame cake, starch and sugar, containing a minimum of non-proteids. In the third period onethird of the sesame cake was replaced by a mixture of asparagin, starch and sesame oil, computed to contain an equivalent amount of nitrogen, carbohydrates, and fat. Owing to differences in digestibility, however, the amounts of digested nutrients, and particularly of nitrogen, varied more or less. The results of the nitrogen balance per $100 \mathrm{kgs}$. live weight were:

\begin{tabular}{|c|c|c|c|c|c|}
\hline & \multicolumn{3}{|c|}{ Nitrogen Digested. } & \multirow{2}{*}{$\begin{array}{l}\text { Nitrogen } \\
\text { Metabolism, } \\
\text { Grms. }\end{array}$} & \multirow{2}{*}{$\begin{array}{l}\text { Gain of } \\
\text { Nitrogen } \\
\text { Grms. }\end{array}$} \\
\hline & $\begin{array}{l}\text { Proteid, } \\
\text { Gruns. }\end{array}$ & $\begin{array}{c}\text { Non-proteid, } \\
\text { Grus. }\end{array}$ & $\begin{array}{l}\text { Total, } \\
\text { Grms, }\end{array}$ & & \\
\hline 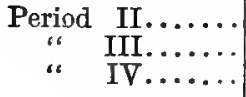 & $\begin{array}{l}72.16 \\
67.05 \\
90.86\end{array}$ & $\begin{array}{c}\ldots \ldots \ldots \\
23.68 \\
\ldots \ldots \ldots \ldots\end{array}$ & $\begin{array}{l}72.16 \\
90.73 \\
90.86\end{array}$ & $\begin{array}{l}56.86 \\
78.43 \\
76.36\end{array}$ & $\begin{array}{l}15.3 \\
12.3 \\
14.5\end{array}$ \\
\hline
\end{tabular}

*Jahresb. Aq. Ch., 43, 513. 
From the smaller gain in Period III, the conclusion is drawn that the asparagin has a lower nutritive value than the proteids. In this period the percentage digestibility of the crude fiber of the ration was found to be 64.88 , as compared with 43.96 and 33.33 in the second and fourth periods, an effect corresponding to that observed by Kellner, and which Tryniszewsky also ascribes to an increase in the micro-organisms of the digestive tract.

The results of the experiments which have been cited are, of course, valid, in the first instance, only for the particular nonproteids experimented with. If, however, the above interpretation of the results is correct, it is to be anticipated that other soluble nitrogenous substances in the food will be found to produce similar effects. If this anticipation proves to be correct, then we shall reach the following conclusions regarding the amides and similar bodies in feeding-stuffs.

1. That they do not serve as sources of proteids.

2. That in rations very poor in protein they have, in the herbivora, an indirect effect in protecting part of the food protein from fermentation in the digestive tract.

3. That in carnivora, and in herbivora on normal rations, they probably have no effect on the production of nitrogenous tissue. 


\section{CHAPTER III.}

\section{METHODS OF INVESTIGATION.}

An essential prerequisite for an intelligent study of the income and expenditure of matter by the animal body is a knowledge of the general nature of the current methods of investigation and of the significance of the results attained by means of them. It is not the purpose here to enter into technical details; this is not a treatise upon analytical or physiological methods. The present chapter will be confined to outlining the general principles upon which those methods are based and to pointing out the logical value of their results. It will be confined, moreover, mainly to those general methods by which the balance of income and expenditure of matter is determined.

Tissue.-The animal body has already been characterized as consisting, from the chemical point of view, of an aggregate of various substances, chiefly organic, representing a certain capital of matter and energy. These various substances are grouped together in the body to form the organized structures known as tissues. For the sake of brevity, then, it may be permissible to use the word tissue as a convenient general designation for the aggregate of all the organic matter contained in the tissues of the body, including both their organized elements and any materials present in the fluids of the body or in solution in the protoplasm of the cells. In this sense tissue is equivalent to the whole capital or store of organic matter in the body.

GaINS AND LossES.-The tissue of the body, as thus defined, is in a constant state of flux, the processes through which the vital functions are carried on constantly breaking it down and oxidizing it (katabolism), while the processes of nutrition are as constantly building it up again (anabolism). If the activity of nutrition 
exceeds that of destruction, material of one sort or another is stored up in the body, and such an addition to its capital of matter and energy we may speak of as a gain of tissue. Conversely, if the katabolic processes consume more material than the processes of nutrition can supply, the store of matter and energy in the body is diminished and a loss of tissue occurs. A simple comparison of the amount of matter supplied in the food (including, of course, the oxygen of the air) with that given off in the solid, liquid and gaseous excreta, therefore, will show whether the body is gaining or losing tissue.

The mere fact of a gain or loss of matter by the body, however, conveys but little useful information unless we know the nature of the material gained or lost. This we have no means of determining directly. The processes of growth or decrease are not accessible to immediate observation, while changes in the weight of the animal (even aside from the great uncertainties introduced, especially in the herbivora, by variations in the contents of the alimentary canal) represent simply the algebraic sum of the gains and losses of water, ash protein, fats, and other materials, and so give but a very slight clue if any to the real nature of the tissue-building. We are compelled, therefore, to have recourse to indirect methods, and to base our conclusions as to tissue-building upon a comparison of the income and outgo of the chemical elements of which the body is composed, particularly of nitrogen and carbon.

The Schematic Body.-The basis of this method of comparison is the conception of the schematic body, first introduced by Henneberg.* This conception regards the dry matter of the body of the animal as composed essentially of three groups of substances, viz., ash, fat, and protein, with at most comparatively small amounts of carbohydrates (glycogen), and assumes that the vast number of other compounds which it actually contains are present in such small and relatively constant proportions as not to materially affect the truth of this view. A knowledge of the ultimate composition of these three groups then affords the basis for a computation of the gain or loss of each from the income and outgo of their elements.

AsH.-The ash ingredients of the body form a well-defined * Neue Beiträge, etc., p. vii. 
group, and the determination of the gain or loss of each ingredient from a comparison of income and outgo is in principle a relatively simple matter and calls for no special consideration here.

FAT.-The elementary composition of the fat of the body has been shown to be remarkably similar not only in different animals of the same species, but likewise in different species. The classic investigations of Schulze \& Reinecke* upon the composition of animal fat gave the following results:

\begin{tabular}{|c|c|c|c|c|c|c|c|c|c|c|}
\hline & \multirow[b]{2}{*}{$\begin{array}{c}\text { No. of } \\
\text { Sam- } \\
\text { ples. }\end{array}$} & \multicolumn{3}{|c|}{ Carbon. } & \multicolumn{3}{|c|}{ Hydrogen. } & \multicolumn{3}{|c|}{ Oxygen. } \\
\hline & & \begin{tabular}{|c|} 
Aver- \\
age \\
Per \\
Cent.
\end{tabular} & $\left|\begin{array}{l}\text { Maxi- } \\
\text { mum } \\
\text { Per } \\
\text { Cent. }\end{array}\right|$ & $\mid \begin{array}{c}\text { Mini- } \\
\text { mum } \\
\text { Per } \\
\text { Cent. }\end{array}$ & $\mid \begin{array}{c}\text { Aver- } \\
\text { age } \\
\text { Per } \\
\text { Cent. }\end{array}$ & $\begin{array}{c}\text { Maxi- } \\
\text { mumm } \\
\text { Per } \\
\text { Cent. }\end{array}$ & $\begin{array}{l}\text { Mini- } \\
\text { mum } \\
\text { Per } \\
\text { Cent. }\end{array}$ & $\begin{array}{c}\text { Aver- } \\
\text { age } \\
\text { Per } \\
\text { Cent. }\end{array}$ & $\begin{array}{l}\text { Maxi- } \\
\text { mum } \\
\text { Per } \\
\text { Cent. }\end{array}$ & $\begin{array}{l}\text { Mini- } \\
\text { mum } \\
\text { Per } \\
\text { Cent. }\end{array}$ \\
\hline $\begin{array}{l}\text { Beef fat......... } \\
\text { Pork fat....... } \\
\text { Mutton fat..... }\end{array}$ & $\begin{array}{r}10 \\
6 \\
12\end{array}$ & $\mid \begin{array}{l}76.50 \\
76.54 \\
76.61\end{array}$ & $=\mid \begin{array}{l}76.74 \\
76.78 \\
76.85\end{array}$ & $\begin{array}{l}76.27 \\
76.29 \\
76.27\end{array}$ & $\begin{array}{l}11.91 \\
11.94 \\
12.03\end{array}$ & $\begin{array}{l}12.11 \\
12.07 \\
12.16\end{array}$ & $\left|\begin{array}{l}11.76 \\
11.86 \\
11.87\end{array}\right|$ & $\begin{array}{l}11.59 \\
11.52 \\
11.36\end{array}$ & $\begin{array}{l}11.86 \\
11.83 \\
11.56\end{array}$ & $\begin{array}{l}11.15 \\
11.15 \\
11.00\end{array}$ \\
\hline 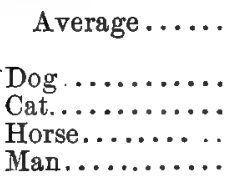 & 28 & $\begin{array}{l}76.50 \\
76.63 \\
76.56 \\
77.07 \\
76.62\end{array}$ & & & $\begin{array}{l}12.00 \\
12.05 \\
11.90 \\
11.69 \\
11.94\end{array}$ & & & $\begin{array}{l}11.50 \\
11.32 \\
11.44 \\
11.24 \\
11.44\end{array}$ & & \\
\hline
\end{tabular}

Benedict and Osterberg $\uparrow$ obtained the following results for the composition of human fat:

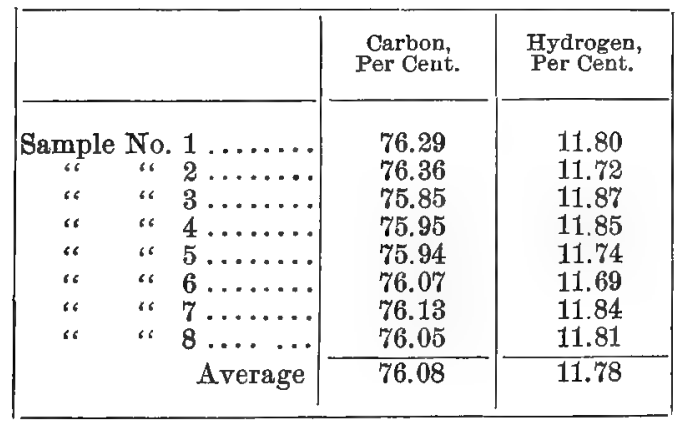

The fat of the body has been commonly regarded as containing 76.5 per cent. of carbon. A gain of 100 parts of fat by the body

* Landw. Vers. Stat., 9, 97. $\nmid$ Amer. Jour. Physiol., 4, 69. 
is accordingly equivalent to a gain of 76.5 parts of carbon, and conversely, if it be shown that the body has gained one part of carbon in the form of fat, this is equivalent to a gain of $1 \div 0.765=1.307$, or, in round numbers, 1.3 parts of fat. Benedict \& Osterberg's average corresponds to the factor 1.314 .

Protein.-As in the case of the food, the term protein is used to signify the whole mass of nitrogenous material in the body, including, besides the true albuminoids, the collagens or gelatinoids, the keratin-like bodies, the nitrogenous extractives, etc.

Neumeister* gives the following figures for the elementary composition of the simple albuminoids:

\begin{tabular}{|c|c|c|c|}
\hline & $\begin{array}{l}\text { Minimum, } \\
\text { Per Cent. }\end{array}$ & $\begin{array}{l}\text { Maximum, } \\
\text { Per Cent. }\end{array}$ & $\begin{array}{l}\text { Average, } \\
\text { Per Cent. }\end{array}$ \\
\hline \multirow[t]{2}{*}{ 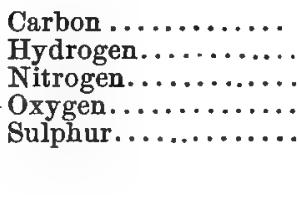 } & \multirow[t]{2}{*}{$\begin{array}{c}50 \\
6.5 \\
15 \\
19 \\
0.3\end{array}$} & \multirow[t]{2}{*}{$\begin{array}{l}55 \\
7.3 \\
17.6 \\
24 \\
2.4\end{array}$} & $\begin{array}{r}52 \\
7 \\
16 \\
23 \\
2\end{array}$ \\
\hline & & & 100 \\
\hline
\end{tabular}

Some of the compound albuminoids, particularly the nucleoalbuminoids, do not vary greatly in composition from the above figures, while others notably the mucins, which contain a carbohydrate group, show a higher percentage of oxygen and less carbon and nitrogen.

The gelatinoids, likewise, do not differ greatly in composition from the albuminoids. For collagen, Hofmeister $\dagger$ found the following averages:

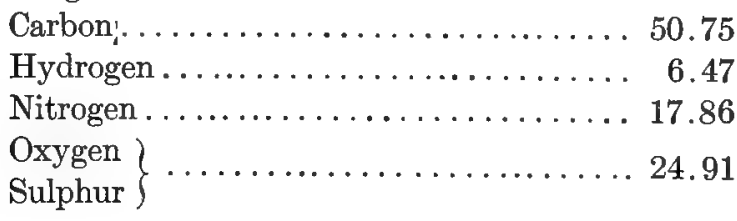

100.00

Keratin is distinguished by a relatively high proportion of sulphur (3 to 5 per cent.), but otherwise, according to Neumeister, $\ddagger$ does not differ materially in composition from the true albuminoids.

* Lehrbuch der Physiol. Chem., p. 22. †Zeit. physiol. Chem., 2, 322. $\ddagger$ Loc. cit., p. 493. 
Hoppe-Seyler* quotes the following figures for the composition of epidermis and some of the tissues derived from it:

\begin{tabular}{|c|c|c|c|c|c|}
\hline & $\begin{array}{l}\text { Epidermis } \\
\text { of Man. }\end{array}$ & Hair. & Nails. & $\begin{array}{l}\text { Horn of } \\
\text { Cow. }\end{array}$ & $\begin{array}{l}\text { Hoof of } \\
\text { Horse. }\end{array}$ \\
\hline 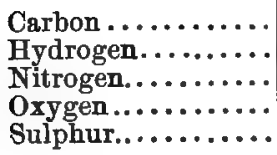 & $\begin{array}{r}50.28 \\
6.76 \\
17.21 \\
25.01 \\
0.74\end{array}$ & $\begin{array}{r}50.65 \\
6.36 \\
17.14 \\
20.85 \\
5.00\end{array}$ & $\begin{array}{r}51.00 \\
6.94 \\
17.51 \\
21.75 \\
2.80\end{array}$ & $\begin{array}{r}51.08 \\
6.80 \\
16.24 \\
22.51 \\
3.42\end{array}$ & $\begin{array}{r}51.41 \\
6.96 \\
17.46 \\
19.49 \\
4.23\end{array}$ \\
\hline & 100.00 & 100.00 & 100.00 & 100.00 & 99.55 (?) \\
\hline
\end{tabular}

Henneberg $\dagger$ obtained the following figures for the composition of two samples of pure and dry wool, calculated ash-free:

I.

Carbon.................. 49.67

Hydrogen ................ 7.26

Nitrogen................ 16.01

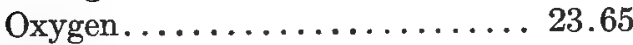

Sulphur................ 3.41

100.00
II.

49.89

7.36

16.08

23.10

3.57

100.00

The following analyses by Rubner, $\ddagger$ Stohmann \& Langbein, and Argutinsky\| show the ultimate composition of ash-free muscular tissue after prolonged extraction with ether: 9

\begin{tabular}{|c|c|c|c|c|c|c|}
\hline & $\begin{array}{l}\text { Carbon, } \\
\text { Per Cent. }\end{array}$ & $\begin{array}{c}\text { Hydro- } \\
\text { gen, } \\
\text { Per Cent. }\end{array}$ & $\begin{array}{l}\text { Nitrogen, } \\
\text { Per Cent. }\end{array}$ & $\begin{array}{l}\text { Sulphur, } \\
\text { Per Cent. }\end{array}$ & $\begin{array}{l}\text { Oxygen, } \\
\text { Per Cent. }\end{array}$ & $\begin{array}{c}\text { Heat of } \\
\text { Com. } \\
\text { bustion } \\
\text { perGram. } \\
\text { Cals. }\end{array}$ \\
\hline $\begin{array}{l}\text { Rubner ................. } \\
\text { Stohmann and Langbein. } \\
\text { Argutinsky............. }\end{array}$ & $\begin{array}{l}53.40 \\
52.02 \\
52.33\end{array}$ & $\begin{array}{l}7.30 \\
7.30\end{array}$ & $\begin{array}{l}16.30 \\
16.36 \\
16.15\end{array}$ & \multicolumn{2}{|c|}{$\begin{array}{l}24.32 \\
24.22\end{array}$} & $\begin{array}{l}5.6561 \\
5.6409\end{array}$ \\
\hline
\end{tabular}

* Physiol. Chem., p. 90.

$\ddagger$ Zeit. f. Biol., 21, 310.

$\|$ Arch. ges. Physiol, , 55, 345.

If It has since been shown by Dornmeyer (Arch. ges. Physiol., 65, 90) that such material is not fat-free.
+ Neue Beiträge, etc., p. 98.

§Jour. f. prakt. Chem., N. F., 44, 364. 
Köhler* has investigated the elementary composition of the muscular tissue of cattle, sheep, swine, horses, rabbits and hens. The material was prepared with much care, the fat being removed as fully as possible by prolonged extraction with ether. The residual fat which cannot be removed in this way was determined by Dornmeyer's digestion method, $\uparrow$ and a corresponding correction made in the analytical results. The following are his averages for the fat- and ash-free substance:

\begin{tabular}{|c|c|c|c|c|c|c|c|}
\hline & $\begin{array}{c}\text { No. of } \\
\text { Samples. }\end{array}$ & \begin{tabular}{|} 
Carbon, \\
Per Cent.
\end{tabular} & $\begin{array}{l}\text { Hydrogen, } \\
\text { Per Cent. }\end{array}$ & $\begin{array}{l}\text { Nitrogen, } \\
\text { Per Cent. }\end{array}$ & $\begin{array}{l}\text { Sulphur, } \\
\text { Per Cent. }\end{array}$ & $\begin{array}{l}\text { Oxygen, } \\
\text { Per Cent. }\end{array}$ & $\begin{array}{c}\text { Heat of } \\
\text { Combustion } \\
\text { per Gram, } \\
\text { Cals. }\end{array}$ \\
\hline Cattle. & 4 & 52.54 & 7.14 & 16.67 & 0.52 & 23.12 & 5.6776 \\
\hline Sheep. & 2 & 52.53 & 7.19 & 16.64 & 0.69 & 22.96 & 5.6387 \\
\hline Swine ...... & 2 & 52.71 & 7.17 & 1660 & 0.59 & 22.95 & 5.6758 \\
\hline Horse....... & 3 & 52.64 & 7.10 & 15.55 & 0.64 & 24.08 & 5.5990 \\
\hline Rabbit...... & 2 & 52.83 & 7.10 & 16.90 & & & 56166 \\
\hline Hen... & 2 & 52.36 & 6.99 & 16.88 & 0.50 & 23.28 & 5.6173 \\
\hline
\end{tabular}

All the samples were tested for glycogen, but only traces were found, except in the horseflesh, for the two samples of which an average of 3.65 per cent. was obtained, a result which accounts for the low figure for nitrogen.

In the classic investigation by Lawes \& Gilbert $\$$ into the composition of the whole bodies of animals, determinations were made of the total dry matter, the ash, the fat, and the total nitrogen. From these data Henneberg $\$$ has compared the total amount of dry matter other than ash and fat with the total amount of nitrogen. His results in a slightly altered form are given in the table opposite.

The average nitrogen content is $\mathbf{1 6 . 2 1}$ per cent. Lawes \& Gilbert extracted the fat with ether and hence, as above noted, the residue was not absolutely fat-free. Köhler's average results for the

* Zeit. physiol. Chem., 31, 479.

$\dagger$ Arch. ges. Physiol., 65, 102.

† Phil. Trans., 1859, II, 498.

$\S$ Neue Beiträge, etc., p. $\mathbf{x}$. 


\begin{tabular}{|c|c|c|c|c|c|c|}
\hline & \multicolumn{2}{|c|}{ Ox. } & \multicolumn{2}{|c|}{ Sheep. } & \multicolumn{2}{|c|}{ Swine. } \\
\hline & $\mid \begin{array}{l}\text { Half Fat } \\
\text { Per Cent. }\end{array}$ & $\begin{array}{l}\text { Fat, } \\
\text { Per Cent. }\end{array}$ & $\begin{array}{l}\text { Lean, } \\
\text { Per Cent }\end{array}$ & $\begin{array}{c}\text { Fat, } \\
\text { Per Cent. }\end{array}$ & $\begin{array}{l}\text { Leun, } \\
\text { Per Cent. }\end{array}$ & $\begin{array}{l}\text { Fat, } \\
\text { Per Cent. }\end{array}$ \\
\hline 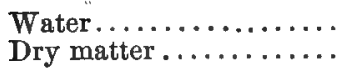 & $\begin{array}{l}56.1 \\
43.9\end{array}$ & $\begin{array}{l}48.6 \\
51.4\end{array}$ & $\begin{array}{l}61.0 \\
39.0\end{array}$ & $\begin{array}{l}46.2 \\
53.8\end{array}$ & $\begin{array}{l}58.2 \\
41.8\end{array}$ & $\begin{array}{l}42.9 \\
57.1\end{array}$ \\
\hline In the dry matter: & 100.00 & 100.00 & 100.00 & 100.00 & 100.00 & 100.00 \\
\hline 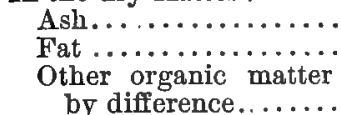 & $\begin{array}{r}5.1 \\
20.7\end{array}$ & $\begin{array}{r}4.1 \\
31.9\end{array}$ & $\begin{array}{r}3.4 \\
19.9\end{array}$ & $\begin{array}{r}2.9 \\
37.9\end{array}$ & $\begin{array}{r}2.8 \\
24.6\end{array}$ & $\begin{array}{r}1.7 \\
44.0 \\
11.4\end{array}$ \\
\hline & 43.9 & 51.4 & 39.0 & 53.8 & 41.8 & 57.1 \\
\hline $\begin{array}{l}\text { Total nitrogen ............ } \\
\text { Per cent. of nitrogen in }\end{array}$ & 3.0 & 2.4 & 2.55 & 2.1 & 2.3 & 1.9 \\
\hline "other organic matter" & 16.58 & 15.59 & 16.24 & 16.19 & 15.97 & 16.66 \\
\hline
\end{tabular}

flesh of cattle, sheep, and swine, after extraction with ether for 480 hours, computed ash-free, were:

Carbon....................... 52.84

Hydrogen................... 7.22

Nitrogen................... 16.46

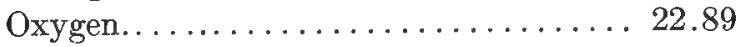

Sulphur................. 0.59

100.00

Considering the indirect method by which Henneberg's result was reached, the agreement as regards nitrogen, both with Köhler's results and with those of Rubner, Stohmann, and Argutinsky just cited, is remarkably close. Henneberg assumed the following round numbers to represent the average composition of the total protein of the body, and his example has been generally followed by subsequent investigators:

Carbon...................... 53 per cent.

Hydrogen.................... 7 " "

Nitrogen.................... 16 " "

Oxygen..................... 23 " "

Sulphur................... 1 " " 
Köhler's averages for dry, fat-free flesh are:

Carbon................. 52.60 per cent.

Nitrogen................. 16.67 " "

GLYCOGEN.-Of the substances other than ash, fat and protein, which are found in the animal body, only glycogen calls for special mention here. This body, as we have seen, may be stored up in considerable amounts in the liver, and is found also in the muscles, although not in large proportion, except in case of the horse. In the aggregate, however, the store of glycogen in the body is not inconsiderable, having been estimated to be in the neighborhood of 300 grams in the human body. Moreover, changes of food or conditions, as well as muscular activity, may materially alter the store of glycogen and thus, perhaps, appreciably affect the makeup of the schematic body.

So far as appears, however, the capacity of the body to store up glycogen is limited, as is indicated by the relatively small amount of it formed after even the most abundant feeding, and we may fairly assume that, at least on a ration equal to or exceeding the maintenance requirements, no long-continued change in the amount of glycogen in the body is likely to occur.

Summary.-We may sum up the foregoing paragraphs in the brief statement that for the purpose of investigating the statistics of nutrition we may consider the organic part of the animal body as composed essentially of fat and protein, with small amounts of glycogen, and that we may regard the permanent effect of a ration upon the body as consisting (aside from its effect on the ash ingredients) in an increase or decrease of its stores of fat and protein, these substances having the average compositon indicated above.

The Gain or Loss of Protein.--Since the term protein as here used is synonymous with total nitrogenous matter, the gain or loss of protein by the body is necessarily indicated by its gain or loss of nitrogen.

The supply of nitrogen to the body is contained in the protein of the food. The losses of nitrogen from the body are contained-

First, in that part of the protein of the food which fails of digestion and is excreted in the feces. 
Second, in the nitrogenous products of the proteid metabolism, contained chiefly in the urine but including also the small quantities of nitrogenous metabolic products contained in the feces and perspiration.

The nitrogen of urine and perspiration, then, together with the metabolic nitrogen of the feces, will indicate the extent of proteid katabolism, while the difference between total income and total outgo of nitrogen will show whether the body is gaining or losing protein. Finally, since the losses of metabolic nitrogen in feces and perspiration are relatively small, and often not readily determinable, in cases where the greatest accuracy is not required, and particularly in comparative experiments, we may regard the total urinary nitrogen as representing with a fair degree of accuracy the amount of protein broken down by the organism.

In the foregoing statements, however, it has been tacitly assumed that the protein of the food consists of true proteids. If, however, the latter are accompanied by amides and other non-proteid nitrogenous bodies, which do not appear to contribute to the formation of proteid tissue (compare p. 53), the corresponding amount of nitrogen will appear in the urine and be added to that derived from the actual katabolism of body or food proteids. This, howeyer, does not, of course, affect any conclusions as to the gain or loss of protein by the body.

FACtor for Protein.- It is thus comparatively easy to determine in terms of nitrogen both the proteid katabolism and the gain or loss of protein, the principal precaution necessary, aside from technical details, being that the experiment shall extend over a sufficient length of time to eliminate the influences of irregularities in ingestion and excretion.

Knowing approximately the ultimate composition of the protein of the body, we may take a step further and infer from the amounts of nitrogen determined the corresponding amounts of protein, the accuracy of the result depending, of course, upon the accuracy of the figures on which it is based. The composition commonly assumed for the body protein has been that given on page 65 , and the same conventional factor, 6.25 , has been used to convert nitrogen into protein which has been employed in case of feeding-stuffs. Köhler's investigations ( $p .64$ ) show that the 
nitrogenous organic matter of muscular tissue has a materially higher percentage of nitrogen, viz., about 16.67 per cent. This would reduce the factor for protein from 6.25 to 6.00. Köhler's samples, after extraction with ether for 480 hours, still contained from 0.27 to 1.61 per cent. of fat. If we assume the ash and fat-free substance of Lawes \& Gilbert's experiments (p. 65) to have still contained 1 per cent. of fat, the average nitrogen content of the fat-free substances would be 16.38 per cent. and the corresponding protein factor 6.11 , while the factor 6.00 would require the assumption of a fat-content of 2.7 per cent.

The factor 6.0 has been used by Kellner in computing the results of his extensive investigations upon cattle at Möckern. Strictly speaking, this assumes that all the gain of nitrogen takes place either in the form of muscular tissue or of material of the same average composition. To what extent such an assumption is justified it is difficult to say. Certainly a part of the protein of the food is applied to the production of epidermis, hair, horns, hoofs, etc., consisting largely of keratins. The data regarding the composition of these tissues given on p. 63 would seem to show that they are, on the average, richer in nitrogen than muscular tissue, a fact which would tend to lower the protein factor, but on the other hand, the amount of this growth is small as compared with the usual protein supply. On the whole, Köhler's factor would seem to afford the most trustworthy basis of computation which is at present available, especially in view of its close agreement with Lawes \& Gilbert's results.

Urea as a Measure of Proteid Metabolism.-In the earlier investigations upon this subject, the urea of the urine, as determined by Liebig's titration method, was commonly taken as the measure of proteid metabolism, one part of urea equaling 2.9 parts of protein, while in many cases the metabolism was also expressed in terms of "flesh" (muscular tissue) with its normal water content and an average of 3.4 per cent. of nitrogen. The errors incident to the use of this method are now generally recognized, while its inapplicability to herbivora was obvious from the first, and with the improvements in the methods of nitrogen determination, the latter has almost entirely replaced the old urea determination and 
the proteid metabolism is now almost exclusively expressed in terms of either nitrogen or protein.

The Gain or Loss of Fat.-As the balance between income and outgo of nitrogen serves to measure the gain or loss of protein by the schematic body, so the balance between income and outgo of carbon furnishes the means for estimating the gain or loss of fat.

The income of carbon is, of course, the carbon of the food. The outgo of carbon consists of-

First, the carbon of the undigested food contained in the feces.

Second, the carbon of the products of metabolism contained in feces, urine, and perspiration.

Third, the carbon of the gaseous excreta, including the carbon dioxide given off by the lungs and skin and the carbon dioxide and hydrocarbons resulting from fermentations in the digestive tract.

Respiration Apparatus.-The carbon of the visible excreta is readily determined by the ordinary analytical methods. The determination of the carbon of the gaseous excreta requires the use of a special apparatus, commonly called a respiration apparatus.

In early experiments upon respiration the animal was simply placed in a known confined volume of air which was analyzed before and after the experiment. By this method, however, the oxygen of the air is progressively diminished, while the respiratory products accumulate, both of which conditions are liable to disturb the normal respiratory exchange, although Kaufmann,* who has recently reverted to this primitive method, claims to have secured accurate results in rather short experiments.

The obvious desirability of renewing the oxygen and removing the products of respiration soon led to the construction of more complicated forms of apparatus of which three principal types may be distinguished.

The Regnault Apparatus.-The oldest of these is the Regnault $\dagger$ or closed circuit respiration apparatus. In this type of apparatus the subject breathes in a confined volume of air, the carbon dioxide being removed by suitable absorbents and weighed, while the oxygen consumed is replaced from a receiver containing pure oxygen, the amount admitted to the apparatus being measured. These

* Archives de Physiol., 1896, p. 329.

$\nmid$ Regnault \& Reiset, Ann. de Chim. et de Physique, 3d series, 26, 299. 
data, with the addition of analyses of the known volume of air contained in the apparatus at the beginning and end of the experiment, afford the means of computing both the carbon dioxide and other gases given off and the oxygen cousumed.*

In theory this is the most complete and satisfactory type of respiration apparatus, since it permits a determination of the total gaseous exchange. Serious practical difficulties have been found in its use, however, especially for the larger animals, among them the difficulty of maintaining the air reasonably pure, the difficulty of securing a uniform temperature and mixture of the gases in a large and complicated apparatus, and the liability to contamination of the oxygen used. Seegen \& Nowak $\dagger$ used an apparatus of this type for their experiments upon the excretion of gaseous nitrogen by animals (see p. 42). Laulanié $\ddagger$ has described a Regnault apparatus for small animals in which a continuous graphic measureunent of the oxygen admitted to the apparatus is made, HoppeSeyler $\&$ has constructed at Strasburg an apparatus of this type large enough to contain a man, and Bleibtreu $\|$ has recently made use of a small one to investigate the formation of fat in geese, but the apparatus has not come into general use. $\uparrow$

The Pettenkofer Apparatus.-The second type of respiration apparatus is that of $\mathrm{v}$. Pettenkofer. In this type the subject breathes in a closed chamber through which a measured current of air is maintained.

Scharling ** appears to have been the first to construct an apparatus of this sort. The ingoing air was freed from carbon dioxide by passing through potash solution, while the outcoming air, after drying, gave up its carbon dioxide to a weighed potash bulb. Various similar forms of apparatus were constructed, but it was found

\footnotetext{
* For a description of the apparatus, see also Hoppe-Seyler, Physiol. Chem., pp. 526 and 536.

$\dagger$ Sitzungsber. Wiener Akad., Math.-Naturwiss. Classe, 71, III, 329; Arch. ges. Physiol., 19, 349.

\$Archives de Physiol., 1890, p. 571.

$\S$ Zeit. physiol. Chem., 19, 574 .

\| Arch. ges. Physiol., 85, 366.

T See also Pfluger and Colasanti (Arch. ges. Physiol., 14, 92) and Schulz (Ib., p. 78).

**Ann. Chern. Pharm., 45, 214.
} 
to be impossible to secure complete absorption of the carbon dioxide and at the same time maintain adequate ventilation.

In 1862 v. Pettenkofer * introduced the important improvement of diverting a known aliquot of both the ingoing and outcoming air for analysis. The results of these analyses, calculated upon the whole volume of air used, show the amounts of carbon dioxide and other gases added by the subject of the experiment.

The Pettenkofer apparatus has the advantage of placing the subject under unquestionably normal conditions as to purity of air, of maintaining a practically uniform temperature and mixture of gases throughout the apparatus, and of dispensing with the extreme care necessary in the Regnault apparatus to prevent gaseous diffusion between the air outside and that inside the apparatus. Its great drawback is that it does not in practice permit the determination of the amount of oxygen consumed. $\dagger$ To this is to be added the magnification of experimental errors involved in computing the results obtained by the analysis of small samples upon the whole volume of air used.

Despite these drawbacks, however, the Pettenkofer apparatus in various forms has been widely used, especially in experiments upon domestic animals, and has shown itself capable of yielding very accurate results within its scope. Laulanié, $\downarrow$ by largely reducing the rate of ventilation, has been able to make determinations of the oxygen consumed which he regards as satisfactory, while Haldane $\$$ has constructed an apparatus for small animals, in which the entire air current is passed over absorbents before entering and after leaving the apparatus, which also permits of a satisfactory indirect determination of the oxygen consumed. Sondén and Tigerstedt \| have also constructed a modified Pettenkofer respira-

* Ann. Chem. Pharm., Suppl. Bd. II, p. 1. See also Atwater, U. S. Dep. Agr., Office of Experiment Stations, Bull. 21, p. 106.

† Such a determination is theoretically possible from a comparison of the oxygen content of ingoing and outcoming air, but the delicacy of the measurements and analyses required is so great as to render the method impracticable, while the determination by difference concentrates all the errors in this one quantity.

\$ Archives de Physiologie, 1895, p. 619.

$\S$ Jour. Physiol., 13, 419.

.I Skand. Arch. Physiol., 6, 1. 
tion apparatus of very large dimensions. Recently Atwater \& Rosa * have constructed a form of Pettenkofer apparatus for use as an animal calorimeter in which the method of measuring and sampling the air current has been materially improved and rendered more accurate.

When the Pettenkofer apparatus is employed for experiments upon herbivora, special provision is necessary for the determination of the gaseous hydrocarbons excreted in considerable quantities by these animals. This is accomplished by passing a sample of the air coming from the apparatus through a combustion-tube containing copper oxide, or preferably spongy platinum (platinized kaolin), heated to redness. The hydrocarbons are thus oxidized and the resulting carbon dioxide determined.

Pettenkofer \& Voit, $\uparrow$ in their earlier investigations, determined the excretion of combustible gases by a dog, with the following results per day:

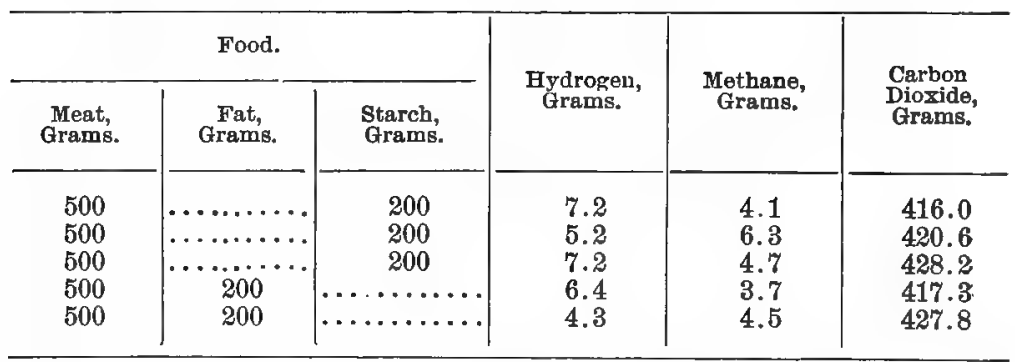

According to the above figures, a trifle less than 3 per cent., on the average, of the total carbon excretion was in the form of methane. No similar determinations seem to have been made by Pettenkofer \& Voit in their later experiments, and it appears to be generally assumed that they are unnecessary in investigations upon man and the carnivora.

The Zuntz Apparatus.-Both the Regnault and the Pettenkofer types of apparatus are calculated for the determination of the total gaseous excreta of lungs, skin, and digestive tract through considerable periods of time, and their use enables us to compare the total income and outgo of carbon.

* U. S. Dept. Agr., Office of Experiment Stations, Bulletins 44 and 63.

† Ann. Chem. Pharm., Supp. Bd. II, p. 66. 
The third type of respiration apparatus is best known by the name of Zuntz,* from the extensive development given it by this investigator, although it has assumed various forms in the hands of different experimenters. This apparatus is radically different from the other two types in that it is intended simply for the determination of the respiratory exchange in the lungs. For this purpose the expired air is collected, either by means of a mask or a tracheal cannula, its volume measured, and its content of carbon dioxide and of oxygen determined in an aliquot sample, the composition of the inspired air being assumed to be that of the normal atmosphere. The fundamental principle is really that of the Pettenkofer apparatus, but, owing to the fact that the excretory gases are not diluted with many times their volume of air, the results are much sharper and it is possible to determine the amount of oxygen consumed as well as of the carbon dioxide given off. In addition to this advantage, it permits the experimenter to follow the variations in the respiratory exchange in comparatively short periods. It is thus especially adapted for investigating such questions as the influence of muscular work upon metabolism, and it is in the study of this question that it has found its chief application. On the other hand, it is impracticable to continue its use through long periods-a day, e.g. - and it takes no account of the excretion through the skin and the alimentary canal. Only by indirect methods, therefore, is it possible to compute the total income and outgo of carbon by its use.

But while the Zuntz form of respiration apparatus is especially adapted for investigating the carbon metabolism during short periods, it is important that these periods be not made too short. What is actually determined by the use of any form of respiration apparatus is the excretion or absorption of carbon dioxide or oxygen. In an experiment extending over several hours, we may fairly assume that this is substantially a measure of the actual production or consumption of these gases going on in the tissues. In periods of a few minutes, however, there is always a possibility of an accumulation of oxygen or a partial retention of the products of metabolism in the tissues or the blood, while, on the other hand,

* Rohrig \& Zuntz, Arch. ges. Physiol., 4, 57; v. Mehring \& Zuntz, ib., 32, 173; Geppert \& Zuntz, ib., 42, 189. 
the products of previous metabolism may be added to those formed during the experiment. This is especially true of the carbon dioxide, particularly in work experiments, where the rate and volume of respiration are largely affected. During severe work, there may be more or less accumulation of this gas in the blood, while, on the other hand, the increased respiration in an immediately following period of rest may reduce the proportion in the blood below the normal. The oxygen is thought to be far less subject to this error than the carbon dioxide, and therefore to be a more accurate indicator of the total metabolism.

The Respiratory Quotient.-This name was given by Pflüger to the ratio of the volume of carbon dioxide excreted to the volume of oxygen consumed in the same time. It is frequently represented by the abbreviation R.Q., or by the symbol $\frac{\mathrm{CO}_{2}}{\mathrm{O}}$.

It is obvious that this ratio will vary with the nature of the material metabolized. Thus the oxidation of a carbohydrate, e.g. dextrose, will give rise to a volume of carbon dioxide equal to that of the oxygen consumed, since, as the following equation shows, each molecule of oxygen gives rise to a molecule of carbon dioxide:

$$
\mathrm{C}_{6} \mathrm{H}_{12} \mathrm{O}_{6}+6 \mathrm{O}_{2}=6 \mathrm{CO}_{2}+6 \mathrm{H}_{2} \mathrm{O} \text {. }
$$

In this case the respiratory quotient is equal to unity. On the other hand, when fat is oxidized, a portion of the oxygen combines with the hydrogen of the fat to form water, and the volume of carbon dioxide produced is less than that of the oxygen employed. Representing the process by the equation used by Chauveau, * viz.,

$$
2 \mathrm{C}_{57} \mathrm{H}_{110} \mathrm{O}_{0}+163 \mathrm{O}_{2}=114 \mathrm{CO}_{2}+110 \mathrm{H}_{2} \mathrm{O},
$$

the respiratory quotient is $\frac{114}{163}=0.6993$. Computed from the average percentage composition of animal fat as given on p. 61, it equals 0.7069 .

The proteids of the food, as we have seen, are not completely oxidized in the body, a portion of their carbon, along with all their nitrogen, being excreted in the form of urea and other organic

* La Vie et l'Energie chez I'Animale. 
compounds in the urine. Chauveau \& Kaufmann,* starting with an empirical formula for albumin, represent its complete metabolism in the body by the equation

$$
2 \mathrm{C}_{72} \mathrm{H}_{112} \mathrm{~N}_{18} \mathrm{O}_{22} \mathrm{~S}_{2}+151 \mathrm{O}_{2}=18 \mathrm{CH}_{4} \mathrm{~N}_{2} \mathrm{O}+126 \mathrm{CO}_{2}+76 \mathrm{H}_{2} \mathrm{O}+\mathrm{S}_{2},
$$

thus obtaining the respiratory quotient $\frac{126}{151}=0.8344$, neglecting the oxygen required to oxidize the sulphur.

The urine, however, always contains greater or less quantities of nitrogenous compounds richer in carbon than urea, and in herbivorous animals in particular such compounds are abundant. The respiratory quotient of the proteids is therefore variable, depending upon the extent to which their carbon is completely oxidized. Thus Zuntz and Hagemann, $\dagger$ in an experiment upon the horse in which approximately 15 per cent. of the total nitrogen of the urine was contained in hippuric acid, compute it at 0.765 .

Deductions from Respiratory Quotient. - The value of a determination of the respiratory quotient lies in the clue which it affords to the nature of the substances which are being oxidized in the body. Assuming that the materials available for oxidation in the schematic body are substantially proteids, carbohydrates and fat it is evident that when the quotient approaches 1.0 the material consumed must consist largely of carbohydrates, while if it falls to the neighborhood of 0.7 it is clear that the oxygen is combining chiefly with fat. An intermediate value, on the other hand, would be more am. biguous, since it might result from the oxidation of proteids, carbohydrates and fat in several proportions.

If, however, the amounts of oxygen consumed and of carbon dioxide produced in the oxidation of any one of the three groups be known, it is a simple matter to compute the proportion in which the other two enter into the reaction. For the amount of protejds metabolized, we have an approximate measure in the total urinary nitrogen. If we can also determine the amounts of carbon, hydrogen and oxygen contained in these nitrogenous urinary products, we can compute the quantity of oxygen required to oxidize the nonnitrogenous residue of the proteids and the amount of carbon dioxide resulting from it upon the assumption of complete oxidation.

* Compare p. 51. † †andw. Jahrb., 27, Supp. III, 240. 
As a matter of fact, however, it is not easy to determine satisfactorily the proportion of the respiratory exchange due to the proteids, both because the nitrogenous products of their metabolism are numerous and occur in varying proportions in the urine, and because we may not always be justified in assuming complete oxidation of the non-nitrogenous residue. Computations of the nature indicated above, therefore, must be accepted with some reserve.

A simpler case, and one which has been extensively investigated, is the nature of the increased metabolism arising from muscular exertion. As we shall see in a succeeding chapter, such exertion causes a marked increase in the respiratory exchange while producing at most but a slight effect upon the proteid metabolism. If we neglect altogether this latter effect, the ratio between the increments of carbon dioxide and oxygen will indicate whether the additional material consumed during the performance of the work consisted of fat or carbohydrates or a mixture of the two, of course on the same assumption as before, viz., that substantially only these two classes of substances are available in the schematic body.

For example, in an investigation by Zuntz, cited on a subsequent page, the performance of one kilogram-meter of work of draft by a dog caused the following increments in the respiratory exchange:

Oxygen....................... 1.6704 c.c.

Carbon dioxide................ 1.4670 "

Respiratory quotient............ 0.878

Assuming, as above, that these amounts arise from the oxidation of fat and carbohydrates only, let $x$ equal the amount of oxygen consumed in the oxidation of fat and $1.6704-x$ the amount consumed in the oxidation of carbohydrates. Since the respiratory quotient of fat is 0.7069 , the $x$ cubic centimeters of oxygen would yield $0.7069 x$ cubic centimeters of carbon dioxide, while the $1.6704-x$ cubic centimeters of oxygen used to oxidize the carbohydrates would yield an equal volume of carbon dioxide. We therefore have-

whence

$0.7069 x+(1.6704-x)=1.4670$, $x=0.6939$. 
The division of the increments of the respiratory gases was accordingly-

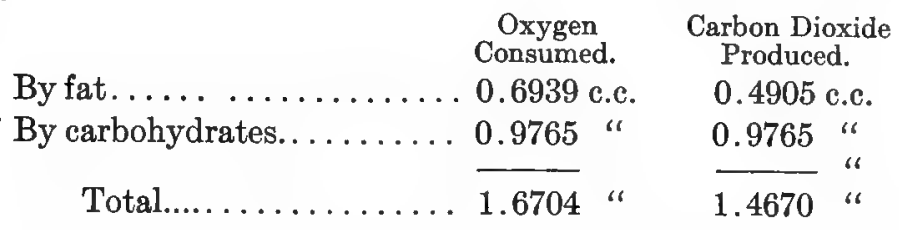

From these data the actual amounts of fat and carbohydrates metabolized can be readily computed, one gram of fat requiring for its oxidation 2.8875 grams (2.028 liters) of oxygen and producing 1.434 liters of carbon dioxide, while one gram of a carbohydrate of the composition of starch requires 1.185 grams ( 0.832 liter) of oxygen and produces the same volume of carbon dioxide.

Computation of Fat from Carbon Balance.-While the use of the Zuntz type of respiration apparatus may afford invaluable information regarding the nature of the chemical changes going on in the body, a satisfactory determination of the gain or loss of carbon by the body usually requires the employment of one of the other types of apparatus.* Having by such means added a determination of the carbon balance to that of the nitrogen balance, we have the data necessary for computing the gain or loss of fat as well as of protein by the schematic body.

For this purpose we first compute the gain or loss of protein in the manner already described. Using Köhler's factor for protein (p. 67), a gain of 16.67 grams of nitrogen is equivalent to a gain of 100 grams of protein. This 100 grams of protein will contain, according to Henneberg, 53 grams, or according to Köhler, 52.6 grams of carbon. Any gain of carbon in excess of this amount must therefore be in the form of non-nitrogenous organic matter, while if less than this amount of carbon has been gained the nonnitrogenous matter of the body must have been drawn upon to supply the difference. The only non-nitrogenous organic substance assumed to be present in the schematic body, however, is fat, containing on the average 76.5 per cent. of carbon (p. 61). Neces-

* For a direct comparison of results obtained upon the horse by the Zuntz and the Pettenkofer forms of apparatus, see Lehmann, Zuntz, \& Hagemann, Landw. Jahrb., 23, 125. 
sarily, then, on this assumption, each gram of carbon gained in excess of that stored in the form of protein will represent 1.3 grams of fat stored.

Formation of Glycogen.-Granting the substantial accuracy of the computation of the gain or loss of protein, the only serious criticism to which the above method of computing the gain or loss of fat is subject is that it does not take account of the possible storage of carbon in other forms, and particularly as glycogen. In other words, it may be contended that the schematic body should be regarded as consisting of water, ash, fat, and carbohydrates. There is undoubtedly a certain degree of justification for this contention, and the significance of small gains of carbon, or of gains observed during short periods, is by no means unambiguous. But when such a gain is observed to continue day after day for weeks on an unchanged ration, as in some of the experiments cited on subsequent pages, the objection loses all force.

Computation of Total Metabolism.-The same principle may be applied to the computation of the total amount of protein and fat metabolized. From the urinary nitrogen (plus that of the feces if the latter be regarded as a metabolic product) by multiplication by the conventional factor we obtain, as already explained, the total proteid metabolism. Subtracting the amount of carbon corresponding to this quantity of protein from the total carbon excretion leaves a remainder which must have been derived from non-nitrogenous material. If carbohydrates are absent from the food, this material, in an experiment of any length, must be substantially fat, and the amount of the latter can be computed from the carbon by the use of the factor 1.3. In the presence of any considerable amount of carbohydrates, however, the results are ambiguous unless we know also the quantity of oxygen consumed.

Other Determinations.-The great majority of investigations upon the metabolism of matter have been confined to determinations of the nitrogen and carbon balance. Occasionally, however, other determinations have been made.

Hydrogen BaLANCE.-Determinations of water and of hydrogen in organic combination in food and excreta enable us, after making 
allowances for the hydrogen gained or lost in protein and fat, to compute the gain or loss of water by the body.

With the earlier forms of respiration apparatus, great difficulty was experienced in obtaining satisfactory results for the water, ${ }^{*}$ and Stohmann $\dagger$ has traced the difficulty to an invisible condensation of water on the walls of the chamber and connections. More recently Rubner $\ddagger$ has been able to make satisfactory determinations of water with a Pettenkofer apparatus by avoiding as much as possible differences of temperature between different parts of the apparatus and by taking the sample of the outcoming air for analysis as close to the respiration chamber as possible. Atwater \& Rosa have shown that their form of Pettenkofer apparatus (p. 72) permits of very accurate determinations of water.

OXYGen BaLANCE.-Owing to the technical difficulties already indicated in considering the different types of respiration apparatus, direct determinations of the oxygen balance have rarely been made. This is the more to be regretted since such a determination would erve to check those of nitrogen, carbon, and hydrogen, and would be a test of the accuracy of our deductions from those determinations as to the nature of the material gained or lost by the body.

AsH INGREDIENTS. - The gain or loss of ash ingredients can of course be readily determined, but the subject as yet has hardly received the attention which it deserves.

Sulphur And Phosphorus.-Sulphur forms an essential constituent of the proteids, while phosphorus enters into the composition of the nucleins and also of lecithin. The determination of the income and outgo of these two elements is often of value in relation to special physiological questions, but from the somewhat general standpoint of this work may be considered as of rather minor importance.

* Zeit. f. Biol., 11, 126.

$\$$ Landw. Vers. Stat., 19, 81.

$\ddagger$ Arch. f. Hygiene, 11, 160. 


\section{CHAPTER IV.}

\section{THE FASTING METABOLISM.}

THE matter which the animal organism derives from its food is applied substantially in three general directions: first, to the maintenance of those vital activities, such as circulation, respiration, secretion, the metabolic activity of the various tissues, etc., and probably to some extent the direct production of heat, which in their entirety make up the physical life of the organism; second, to the support of those functions by which the crude materials ingested are prepared to nourish the body, that is, to the work of digestion and assimilation; third, to the production of external mechanical work or to the storage of surplus material in the form of growth of tissue.

Of these three general functions of the food, the one first named is obviously of fundamental significance, and a determination of the nature and amount of its demands constitutes the natural first step in a study of the laws of nutrition. For this purpose we can eliminate the influence of the other two factors by keeping the animal as nearly as possible in a state of absolute rest and by withholding food. Under these circumstances the expenditure of matter from the tissues of the body may be taken as representing the miminum demands of the vital functions. It will therefore be both logical and convenient to consider first, in the present chapter, the fasting metabolism of the quiescent animal, while in succeeding chapters we take up the influence respectively of the food-supply and of external work upon metabolism. The protein of the food has such peculiar and distinct functions in the animal economy that it will be a matter of practical convenience to follow the historical order of investigation and consider first the proteid metabolism by itself 
and subsequently the total metabolism as shown by the combined nitrogen and carbon balance.

\section{$\S$ I. The Proteid Metabolism.}

Tends to Become Constant.-When food is withheld from a well-nourished animal, particularly a carnivorous animal, the proteid metabolism usually diminishes, at first rapidly and more slowly later, until within a few days it reaches a minimum value which may then remain nearly unchanged for a considerable time. This was first shown by the investigations of Carl Voit, in conjunction with Bischoff and later with v. Pettenkofer, and has been fully confirmed by later results.

The following table shows the results obtained by Voit* in several experiments upon a dog weighing about $35 \mathrm{kgs}$., the proteid metabolism being expressed in grams of urea per day. As noted in Chapter III, such results are not absolutely accurate and do not represent the total proteid metabolism, but the fact that they are comparable is sufficient for our present purpose.

\begin{tabular}{|c|c|c|c|c|c|c|c|c|}
\hline & & & & \multicolumn{5}{|c|}{ Previous Food per Day. } \\
\hline & & & & $\begin{array}{c}2500 \text { Grms. } \\
\text { Meat. }\end{array}$ & \begin{tabular}{|c|}
1800 Grms. \\
Meat; \\
250 Grms. \\
Fat.
\end{tabular} & $\begin{array}{c}1500 \text { Grms. } \\
\text { Meat. }\end{array}$ & $\begin{array}{l}1500 \text { Grms. } \\
\text { Meat. }\end{array}$ & Nothing. \\
\hline \multicolumn{4}{|c|}{ Urea per day: } & $\begin{array}{l}\text { Grms. } \\
180.8\end{array}$ & $\begin{array}{l}\text { Grms. } \\
130.0\end{array}$ & $\begin{array}{l}\text { Grms. } \\
110.8\end{array}$ & $\begin{array}{l}\text { Grms. } \\
110.8\end{array}$ & $\underset{24.7}{\text { Grms. }}$ \\
\hline 1st & "6 & “ & fasting..... & 60.1 & 37.5 & 29.7 & 26.5 & 19.6 \\
\hline $2 \mathrm{~d}$ & $“^{\circ}$ & "6 & " & 24.9 & 23.3 & 18.2 & 18.6 & 15.6 \\
\hline $3 \mathrm{~d}$ & ، & & $\ldots$ & 19.1 & 16.7 & 17.5 & 15.7 & 14.9 \\
\hline 4 th & sc & & $\ldots$. & 17.3 & 14.8 & 14.9 & 14.9 & 13.2 \\
\hline 5th & "s & & .... & 12.3 & 12.6 & 14.2 & 14.8 & 12.7 \\
\hline 6 th & " & & ..... & 13.3 & 12.8 & 13.0 & 12.8 & 13.0 \\
\hline 7th & "6 & ، & $\ldots .$. & 12.5 & 12.0 & 12.1 & 12.9 & \\
\hline 8 th & "6 & & $\ldots .$. & 10.1 & $\ldots \ldots$ & 12.9 & 12.1 & \\
\hline 9th & "، & "6 & $\ldots .$. & $\ldots \ldots \ldots$ & $\ldots \ldots \ldots$ & ......... & 11.9 & \\
\hline 10 th & $"$ & “ & "6 & o & ........ & $\ldots \ldots \ldots$ & 11.4 & \\
\hline
\end{tabular}

Two Factors of Proteid Metabolism.-In these, as in many similar experiments, the proteid metabolism was quite unequal on the last day of the feeding and on the first fasting day, but in a

* Zeit. f. Biol., 2, 311. 
comparatively short time it sank to a minimum which was practically the same in all the experiments upon this particular animal, viz., the equivalent of about 12 grams of urea per day. This minimum we may fairly regard as representing the necessary and inevitable destruction of proteids involved in the vital processes of the organism, and therefore may consider as taking place also when the animal was fed. If, now, we subtract from the total urea excreted the 12 grams corresponding to the minimum demand of the body, there is revealed the second and variable factor of the proteid metabolism, which is large in the well-fed animal but rapidly disappears during fasting, as the following table shows:

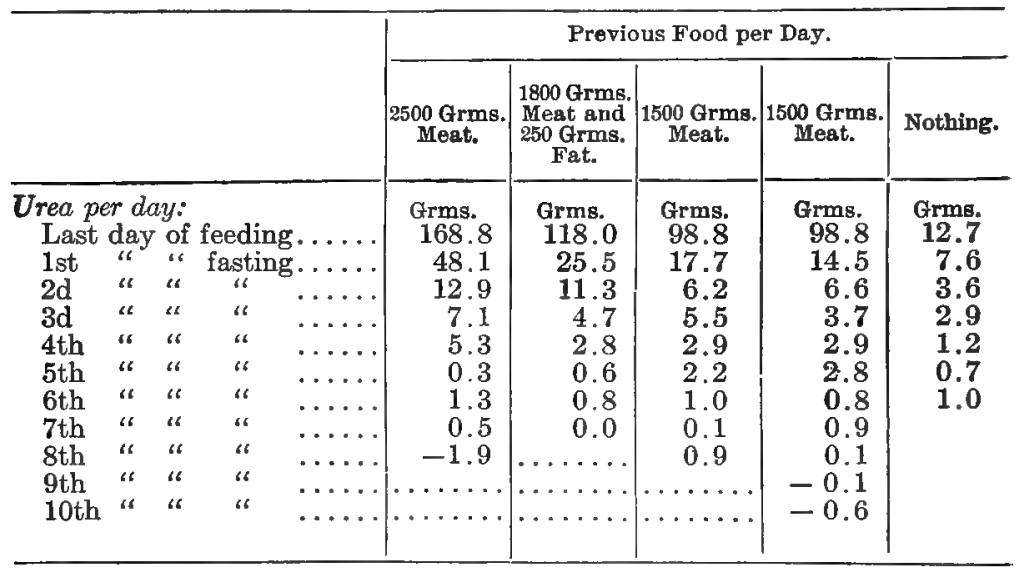

Organized and Circulatory Protein.-It is evident from the above results, and will appear still more clearly when we come to consider the influence of the food-supply upon proteid metabolism, that in addition to the great mass of proteid tissue in the body, whose metabolism results in the excretion of a relatively small and constant amount of nitrogenous products, the wellnourished organism may also contain variable amounts of nitrogenous matter which is subject to rapid metabolism and which speedily disappears during fasting. Voit employed the term circulatory protein (Zirkulationseiweiss) to designate this variable store of rapidly metabolized nitrogenous matter, which he regards as being substantially the dissolved protein which penetrates from 
the blood and lymph into the cells of the tissues, while he termed the protein of the organized tissues, which is relatively stable and but slowly metabolized, organized protein (Organeiweiss). The amount of the circulatory protein is small in all cases as compared with that of the organized protein, its absolute amount being dependent, as the above tables indicate and as will appear more clearly in the next chapter, upon the supply of proteids in the food. Owing to its rapid metabolism, however, it furnishes by far the larger part of the nitrogenous waste products in the liberally fed animal.

That the anatomical distinctions implied in the terms used by Voit correspond to the actual facts of the case has been disputed and may be open to question, but for our present purpose this does not particularly concern us. The fact of the existence of the two factors of proteid metabolism, viz., a variable one, depending upon the previous food-supply and a relatively constant one independent of the latter is fully established, by whatever names we may choose to call them.

A Minimum of Protein Indispensable.-While the proteid metabolism of the fasting animal is speedily reduced to relatively small proportions, it is never entirely suspended as long as the animal lives. Moreover, to anticipate a portion of the following chapter, even the most liberal supply of non-nitrogenous nutrients is powerless to suspend or very greatly reduce the proteid metabolism of a fasting animal. A certain amount of proteid metabolism is indissolubly associated with the continuance of life, and neither the fat of the body nor the non-nitrogenous ingredients supplied in the food can perform these special functions of protein in the body.

\section{§. Total Metabolism.}

Constant Loss of Tissue.-Common observation, no less than scientific investigation, teaches that a fasting animal suffers a continual loss of tissue. Such an animal derives the energy required for its vital activities from the metabolism of its store of proteids and of fat. As regards the former, we have just seen that in a short time, or as soon as the influence of the previous supply of 
proteids in the food is exhausted, the proteid metabolism reaches a minimum and thereafter remains nearly constant for a considerable time, and subsequent investigations have shown that this constancy is still more marked when the proteid metabolism is computed per unit of live weight.

What has thus been found to be true of the proteid metabolism has also been shown to hold good of the total metabolism of proteids plus body fat. As soon as the influence of the previous food has disappeared, the rate of metabolism of both proteids and fat shows but slight variations throughout a considerable time. Of the early experiments of Pettenkofer and Voit, the following * may be cited as illustrating approximately this constancy:

\begin{tabular}{|c|c|c|c|c|c|}
\hline & \multicolumn{2}{|c|}{ Series $a, 1862$} & \multicolumn{3}{|c|}{ Series $b, 1861$. } \\
\hline & $\begin{array}{l}\text { March 10, } \\
\text { 6th Day. }\end{array}$ & $\begin{array}{l}\text { March 14, } \\
\text { 10th Day. }\end{array}$ & $\begin{array}{l}\text { April 5, } \\
\text { 2d Day. }\end{array}$ & $\begin{array}{l}\text { April 8, } \\
\text { 5th Day. }\end{array}$ & $\begin{array}{l}\text { April 11, } \\
\text { 8th Day. }\end{array}$ \\
\hline Live weight ... & $\begin{array}{l}\text { Kgs. } \\
31.21\end{array}$ & $\begin{array}{l}\text { Kgs. } \\
30.05\end{array}$ & Kgs. & $\begin{array}{l}\text { Kgs. } \\
\mathbf{3 1 . 6 7}\end{array}$ & $\begin{array}{l}\mathrm{Kgs} . \\
30.54\end{array}$ \\
\hline $\begin{array}{l}\text { Carbon of excreta } . . . . . . \\
\text { Nitrogen of excreta....... }\end{array}$ & $\begin{array}{c}\text { Grms. } \\
104.1 \\
5.95\end{array}$ & $\begin{array}{c}\text { Grms. } \\
82.4 \\
5.23\end{array}$ & $\begin{array}{c}\text { Grms. } \\
108.7 \\
11.6\end{array}$ & $\begin{array}{c}\text { Grms. } \\
100.0 \\
5.7\end{array}$ & $\begin{array}{c}\text { Grms. } \\
93.2 \\
4.7\end{array}$ \\
\hline Total loss: & 0.80 & 0.20 & 11.0 & 0.8 & 4.1 \\
\hline Proteids .. & 37.18 & 32.69 & 72.51 & 35.63 & 29,38 \\
\hline Foss per ${ }_{\text {Lg. live weight: }}$. & 107. & 83. & 86. & 103. & 99.2 \\
\hline Proteids ................ & 1.19 & 1.09 & 2.21 & 1.13 & 0.96 \\
\hline Fat...$\ldots$ & 3.43 & 2.76 & 2.62 & 3.25 & 3.25 \\
\hline
\end{tabular}

Finkeler $\dagger$ determined the respiratory exchange of fasting guinea-pigs in two-hour periods. Upon the highly probable assumption that their proteid metabolism was relatively small and constant, the results of such experiments would furnish a measure of the relative intensity of the total metabolism. Finkeler's average results are contained in the table on the opposite page.

But a slight decrease in the amount of oxygen consumed is observed in the different stages of the fasting, while there is a marked decrease in the amount of carbon dioxide produced. The relation between these two quantities, as expressed by the respiratory quotient, $\ddagger$ shows us that at the beginning of the fasting the metabolism was largely at the expense of the carbohydrates of the

* Zeit. f. Biol., 5, 369. † Arch. ges. Physiol., 23, $175 . \quad \ddagger$ Compare p. 74. 


\begin{tabular}{c|c|c|c}
\hline & \multicolumn{2}{|c|}{ Per Hour and Kg. Live Weight. } & \\
\cline { 2 - 4 } $\begin{array}{c}\text { Length of Fasting, } \\
\text { Minutes. }\end{array}$ & $\begin{array}{c}\text { Oxygen Consumed, } \\
\text { c.c. }\end{array}$ & $\begin{array}{c}\text { Carbon Dioxide } \\
\text { Excreted, } \\
\text { c.c. }\end{array}$ & $\begin{array}{c}\text { Respiratory } \\
\text { Quotient. }\end{array}$ \\
\hline 0 & 1202.19 & 1111.80 & 0.93 \\
1468 & 1154.53 & 923.75 & 0.80 \\
2950 & 1146.76 & 811.12 & 0.71 \\
\hline 0 & 1250.28 & & \\
1575 & 1226.18 & & \\
3543 & 1241.78 & & \\
5940 & 1192.50 & & \\
\hline 334 & 1959.45 & 1494.68 & 0.76 \\
1712 & 1850.02 & 1318.19 & 0.71 \\
\hline 3233 & 1809.85 & 1289.63 & \\
\hline
\end{tabular}

body, while as the experiment progressed the store of carbohydrates (glycogen) in the body was gradually, exhausted and the metabolism finally became a fat metabolism. Since, now, as will be shown in Chapter VIII, the consumption of equal amounts of oxygen results in the liberation of approximately equal amounts of energy whether that oxygen is employed to oxidize carbohydrates or fats, Finkeler concludes that the total metabolism, as measured in terms of energy, was nearly constant.

Lehmann and Zuntz * have observed a similar constancy of the respiratory exchange per unit of weight in the case of two men fasting for eleven and six days respectively, while Munk $\dagger$ found their urinary nitrogen to be also approximately constant. Magnus-Levy $\ddagger$ has likewise observed a similar constancy in the respiratory exchange of the dog and of man during fasting, as have also Johansson, Landgren, Sondén, \& Tigerstedt § for man.

Rubner,\| as a preliminary to his investigations upon the replacement values of the nutrients, discusses this question at some length and gives the results of experiments upon dogs, rabbits, guinea-pigs and fowls, in which the excretion of nitrogen and carbon per unit weight shows a marked degree of constancy through considerable periods.

* Virchow's Archiv, 131, Supp.

$\dagger$ Ibid.

$\ddagger$ Arch. ges. Physiol., 65, 1.

§Skand. Arehiv. f. Physiol., 7, 29.

|| Zeit. f. Biol., 17, 214; 19, 313; Biologische Gesetze, p. 15. 
Metabolism Proportional to Active Tissue.-In a critical discussion of these and other results on fasting animals, to which we shall have occasion to refer again in Part II, E. Voit* shows that a still more constant relation is obtained when either the proteid or the total metabolism is compared with the total mass of proteid tissue estimated to be contained in the body on the several days of the experiment. The total protein of the body, however, may be regarded as at least an approximate measure of the active cell mass, as distinguished from the relatively inactive cells of adipose tissue. It is the vital activities of the former, in the fasting animal, that mainly determine the amount of the total metabolism, the energy liberated being supplied in part by the relatively small amount of proteid metabolism which goes on in the cells of the fasting animal, but largely by the metabolism of fat supplied to the active cells from the adipose tissue.

Ratio of Proteid to Total Metabolism.-In the preceding paragraph it was implied that the proteid metabolism constitutes but a small portion of the total metabolism of the fasting animal, the remainder of the necessary energy being supplied, after the small store of glycogen in the body is exhausted, by the metabolism of body fat. Rubner $\dagger$ appears to have been the first to call specific attention to this aspect of the question. In his investigations upon the relation of size of animal to total metabolism he adduces experimental results to prove that this ratio is not materially different in large and in small animals. The question has, however, been more recently discussed by E. Voit $\ddagger$ from a general point of view, the results of numerous investigators being summarized. In discussing these results, Voit has computed from the nitrogen and carbon balance, when these data were available, in substantially the manner described in Chapter VIII, the amount of energy liberated by the metabolism of the protein and fat lost by the body. In those instances in which only the nitrogen balance was determined, he estimates the amount of energy liberated in the body from the computed surface on the basis of average results with similar animals. (Compare Chapter XI, \& 2.) Taking this amount, expressed in calories, as the measure of the total metabolism, and including only experiments in which the animals *Zeit. f. Biol., 41, 113. †Ibid., 19, 557. †Ibid., 41, 167. 
are believed to have been in good bodily condition (well nourished) at the beginning of the trials, he obtains the following average results:

\begin{tabular}{|c|c|c|c|c|}
\hline & \multirow{2}{*}{$\begin{array}{c}\text { Live Weight, } \\
\text { Kgs. }\end{array}$} & \multicolumn{2}{|c|}{ Nitrogen Excretion per Day. } & \multirow{2}{*}{$\begin{array}{c}\text { Proteid } \\
\text { Metabolism } \\
\text { in } \% \text { of Total } \\
\text { Metabolism. }\end{array}$} \\
\hline & & $\begin{array}{l}\text { Total, } \\
\text { Grms. }\end{array}$ & $\begin{array}{l}\text { Per Kg. } \\
\text { Live Weight, } \\
\text { Grms. }\end{array}$ & \\
\hline 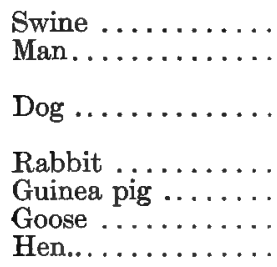 & $\begin{array}{r}115.0 \\
63.7 \\
28.6 \\
18.7 \\
7.2 \\
2.7 \\
0.6 \\
3.3 \\
2.1\end{array}$ & $\begin{array}{r}6.8 \\
12.6 \\
5.1 \\
3.8 \\
2.2 \\
1.2 \\
0.4 \\
0.8 \\
0.7\end{array}$ & $\begin{array}{l}0.06 \\
0.20 \\
0.18 \\
0.20 \\
0.30 \\
0.46 \\
0.65 \\
0.23 \\
0.34\end{array}$ & $\begin{array}{r}7.3 \\
15.6 \\
13.2 \\
10.7 \\
13.5 \\
16.5 \\
10.8 \\
7.4 \\
10.0\end{array}$ \\
\hline
\end{tabular}

As will appear later, the total metabolism of a small animal is greater per unit of weight than that of a large animal. The above figures show that the same thing is true of the proteid metabolism. When, however, the proteid metabolism is computed as a percentage of the total metabolism, as in the last column of the table, this dependence upon the live weight disappears. While the figures still show considerable variations, these are much reduced and show no connection with the live weight. In other words, the proteid metabolism tends to be a somewhat uniform percentage of the total metabolism, ranging in these experiments, aside from two apparently exceptional results, between 10 and 16 per cent.

The individual experiments cited by Voit show a similar general uniformity, both in the same animal on successive days of fasting and in case of different animals. Thus twenty-seven experiments on the dog gave the following:

\begin{tabular}{|c|c|c|}
\hline \multirow{2}{*}{$\begin{array}{c}\text { Range of Proteid Metabolism in Per Cent. } \\
\text { of Total Metabolism. }\end{array}$} & \multicolumn{2}{|c|}{ Number of Cases. } \\
\hline & Absolute. & Per Cent. \\
\hline 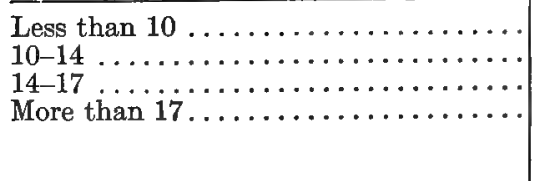 & $\begin{array}{r}4 \\
15 \\
5 \\
3 \\
27\end{array}$ & $\begin{array}{r}14.8 \\
55.6 \\
18.5 \\
11.1 \\
100.0\end{array}$ \\
\hline
\end{tabular}


The great majority of cases gave values lying between 10 and 17 per cent.

EFFECT OF Body FAT.-Both from the summary on p. 87 and from the individual results cited by Voit, it is evident that while the proportion of energy supplied by the metabolism of proteids in the fasting animal is normally small and varies only within rather narrow limits, it is still subject to relatively considerable variations. The most important cause of these variations in the fasting animal under uniform external conditions appears to be the ratio of fat to protein in the body.

C. Voit* appears to have first noted that when fasting is prolonged sufficiently to nearly exhaust the reserve of visible fat in the body, the proteid metabolism, after remaining nearly constant or decreasing slightly for some days, as in the examples just given, begins to increase somewhat rapidly. This increase Voit attributed to the exhaustion of the fat, the oxidation of which had hitherto partially protected the organized proteids of the body. Subsequent investigations; particularly Rubner's, $\dagger$ have in general confirmed Voit's observation, while giving it a somewhat more general form.

E. Voit $\ddagger$ has recently reviewed the available experiments upon fasting metabolism in their bearing on this question. From the experimental data he computes or estimates, first the ratio of proteid to total metabolism (expressed in terms of energy), and second the ratio of proteids to fat in the body on the several days of each experiment. A comparison of these ratios shows a very marked correspondence, a high ratio of proteids to fat in the body coinciding with a large proteid metabolism compared with that of fat, and vice versa. The graphic representations of the relations as given by Voit are especially convincing. Moreover, the results show that the extent of the proteid metabolism does not depend directly upon the duration of the fasting. With different animals, or with the same animal under different conditions, a certain ratio of proteid to total metabolism is attained whenever the corresponding ratio of proteid tissue to fat in the body is reached, whether this be early or late in the experiment.

The growing ratio of proteid to total metabolism in the fasting *Zeit. f. Biol., 2, 326. † Loc. cit. See p. $86 . \quad \ddagger$ Zeit. f. Biol., 41, 502. 
animal is explained by Voit to be due to an increasing difficulty in transferring the reserve fat from the adipose tissues, thus resulting in a diminution of the amount of fat (or its cleavage products?) circulating in the organism. If the body is well supplied with fat at the outset this phenomenon does not at first appear, and the ratio of proteid to total metabolism remains nearly constant for a time.

With continued fasting the store of body fat is, as has just been shown, drawn upon much more rapidly than that of protein, while at the same time the total amount of the former present at the beginning of fasting is often less than that of the latter. As a necessary result, the ratio of fat to protein in the body decreases. When this decrease passes a certain point, the fat of the adipose tissue is drawn upon with more and more difficulty for material to supply the demand for energy, and as a result additional protein is metabolized to make good the deficiency of available fat. From this time on, the ratio of proteid to total metabolism shows a continually accelerated increase. The time when the increase in the proteid metabolism becomes marked depends upon the original condition of the body. If the animal is well nourished, and especially if it contains large reserves of fat, the increase may be long deferred or even fail to appear at all. If, on the other hand, it is poorly nourished and contains little fat, an increase of the proteid metabolism may take place almost from the outset. The following three examples, cited by E. Voit from Rubner's experiments, may serve to illustrate these three types of fasting metabolism:

\begin{tabular}{|c|c|c|c|c|c|}
\hline \multicolumn{2}{|c|}{ Guinea Pig. } & \multicolumn{2}{|c|}{ Dog. } & \multicolumn{2}{|c|}{ Rabbit. } \\
\hline $\begin{array}{c}\text { Day of } \\
\text { Fasting. }\end{array}$ & $\begin{array}{c}\text { Proteid } \\
\text { Metabolism } \\
\text { in \& of Total } \\
\text { Metabolism. }\end{array}$ & $\begin{array}{c}\text { Day of } \\
\text { Fasting. }\end{array}$ & $\begin{array}{l}\text { Proteid } \\
\text { Metabolism } \\
\text { in \% of Total } \\
\text { Metabolism. }\end{array}$ & $\begin{array}{c}\text { Day of } \\
\text { Fasting. }\end{array}$ & $\begin{array}{l}\text { Proteid } \\
\text { Metabolism } \\
\text { in \% of Total } \\
\text { Metabolism. }\end{array}$ \\
\hline $\begin{array}{l}2 \ldots \ldots \\
3 \ldots \ldots \\
4 \ldots \ldots \\
5 \ldots \ldots \\
6 \ldots \ldots \\
7 \ldots \ldots \\
8 \ldots \ldots \\
9 \ldots \ldots \ldots\end{array}$ & $\begin{array}{r}10.4 \\
11.1 \\
11.0 \\
11.9 \\
11.8 \\
6.9 \\
11.2 \\
10.9\end{array}$ & $\begin{array}{l}2-4 \\
10-11 \\
12 \\
13 \\
14\end{array}$ & $\begin{array}{l}16.3 \\
13.1 \\
15.5 \\
17.4 \\
20.0\end{array}$ & $\begin{array}{l}3 \\
5-7 \\
9-12 \\
13-15 \\
16 \\
17-18\end{array}$ & $\begin{array}{l}16.5 \\
23.6 \\
26.5 \\
29.8 \\
50.1 \\
96.4\end{array}$ \\
\hline
\end{tabular}


Schulze * claims that this increase in the proteid metabolism of the fasting animal is not, in all cases at least, due to lack of fat or other non-nitrogenous material to protect the protein from destruction. He advances the hypothesis that the loss of protein incident to the fasting so injures the cells that finally many of them die and the protein of their protoplasm becomes part of the circulatory protein of the body and is rapidly decomposed, thus giving rise to an increased excretion of nitrogen.

While it is not impossible that this ingenious hypothesis has some basis of fact, Kaufmann, $\uparrow$ in a quite full review of the literature of the subject, together with original experiments, shows that it can by no means supplant Voit's explanation. He points out in particular that the time when the increase in the proteid metabolism begins seems to bear no relation to the loss of protein which the body has sustained, while, on the other hand, it coincides quite closely with the time when the supply of visible fat is nearly exhausted.

SUMmary.-In the light of the facts set forth in the foregoing paragraphs we may sketch the general outlines of the fasting metabolism somewhat as follows:

In the early stages of fasting, particularly if the previous food has contained an abundance of proteids, the proteid metabolism may be considerable. As the effect of the previous food disappears, however, and the store of "circulatory protein" in the body is exhausted, the proteid metabolism speedily falls to the minimum amount required for the vital activities of the protoplasm, and the remaining demands of the body for energy are supplied by the metabolism of the stored-up fat. If the latter is fairly abundant, this stage may last several days, the total metabolism remaining nearly constant and the proteids supplying a nearly constant proportion of the necessary energy (according to E. Voit about 15-16 per cent.). Sooner or later, however (unless in a very fat animal), the supply of fat from the adipose tissue begins to flag. The demand for energy, however, remains unabated, and as the fat-supply falls off, more and more protein is metabolized in its place, until at

* Arch. ges. Physiol., 76, 379. †Zeit. f. Biol., 41, 75.

$\ddagger$ Compare also E. Voit's critique of Schulze's investigations. (Zeit. f. Biol., 41, 550.) 
last the metabolism may even become almost entirely proteid in its character. We have in these facts the first of the numerous illustrations which we shall meet in the course of this discussion of the plasticity of the organism in adapting itself to differences in the food-supply, and of the controlling influence exerted upon the course of its metabolism by the demand for energy.

The Intermediary Metabolism.-The prime object of the metabolism of the quiescent fasting animal is, as already pointed out, to supply energy for the performance of the vital functions.

Mention has already been made in Chapter II of the hypothesis that the immediate source of energy to. the cells of both muscles and glands is the metabolism of carbohydrate material. This hypothesis in effect regards the metabolism of the fasting animal as divisible into three processes: first, the splitting up of the proteids, yielding urea and fat; second, the partial oxidation of fat, whether derived from the proteids or from the adipose tissue, yielding dextrose; third, the oxidation of the resulting dextrose in the tissues.

So far as the kind and amount of excretory products are concerned, it of course makes no difference whether the metabolism takes place in accordance with this hypothesis or whether the proteids and fat are oxidized directly in the tissues. In either case the fasting animal lives upon its store of proteids and fat, and the resulting excretory products, as well as the amount of heat produced, are qualitatively and quantitatively the same, so that the coincidence observed by Kaufmann * between the observed results and those computed from his equations is without special significance in this case.

There is, nevertheless, an important and essential difference in the two views. If we regard the proteids and fat as yielding up their energy directly for the vital activities, then all the energy thus liberated is available for this purpose. If, on the contrary, we suppose these substances to be first partially metabolized in the liver or elsewhere in the organism, then only that portion of their potential energy which is contained in the resulting dextrose is available directly for the general purposes of the body. The remainder of their energy is liberated as heat during the preliminary

*Archives de Physiologie, 1896, pp. 329 and 352. 
metabolism, and while contributing its quota towards maintaining the normal temperature of the body is not directly available for other purposes. In other words, the question is not one as to the total energy liberated, but as to its form and distribution. As regards the fasting animal itself, the question is of minor importance; but, as will appear in subsequent chapters, it materially affects our views as to the relative values of the several nutrients of the food. 


\section{GHAPTER V.}

\section{THE RELATIONS OF METABOLISM TO FOOD-SUPPLY.}

THE metabolism of the fasting animal was regarded in the preceding chapter as representing the essential demands of the vital functions for a supply of matter as a vehicle of potential energy. Under these conditions, as we have seen, the total metabolism bears a close relation to the mass of active tissue, while the qualitative character of the metabolism, that is the ratio of proteid to nonproteid matter consumed, appears to be likewise constant for any given condition of the body, depending upon the relative supply of proteids and non-nitrogenous matters to the active cells. When food is given to such an animal the conditions are modified in essentially three ways:

First, to the metabolism incident to the fasting state is added that required to supply the energy consumed in the digestion and assimilation of the food.

Second, the food-supply may alter the proportions in which the various nutrients are supplied to the active cells, and thus affect the metabolism qualitatively, giving rise to a relatively greater or less metabolism of proteids, fats, carbohydrates, etc.

Third, the food-supply may be in excess of the requirements of the body and lead to a storage of matter of one sort or another.

The quantitative relations of the food-supply to the total metabolism and to the storage of matter and energy in the body may be most satisfactorily considered upon the basis of the amounts of energy involved. Accordingly we may content ourselves here with a simple mention of this side of the question, deferring a discussion of it to Part II and confining the present chapter largely to a study of the qualitative changes in the metabolism brought about by variations in the food-supply. As in the previous chapter, it will be convenient to consider the relations of the proteids of the 
food and of the body separately from those of the non-nitrogenous nutrients.

\section{$\S$ I. The Proteid Supply.}

The effects of the proteid supply upon metabolism may be most readily and clearly traced in experiments in which the food consists solely, or nearly so, of proteids, deferring to the next section a consideration of the modifications introduced by the presence of non-nitrogenous nutrients in the food.

\section{Effects on Proteid Metabolism.}

Our knowledge of the relations between proteid supply and proteid metabolism in the animal body is based upon the fundamental investigations of Bischoff \& Voit,* Carl Voit, $\dagger$ and Pettenkofer \& Voit, $\dagger$ at Munich. The results of these researches have been so fully confirmed by subsequent investigators and have become so much the common property of science that it is unnecessary to do more than summarize them here, with the addition of such examples as may seem best adapted to illustrate them.

Amount Required to Reach Nitrogen Equilibrium.-As we have seen, the proteid metabolism of a fasting animal speedily reaches a minimum which we may probably regard as representing, at least approximately, the amount of proteids necessarily broken down and oxidized in the vital activities of the tissues of the body. If we supply proteid food to such an animal, we might naturally be inclined to expect that the first use to which the proteids of the food would be put would be to stop the loss of proteid tissue, and that if as much proteid was supplied in the food as was being metabolized in the body, nitrogen equilibrium would be reached.

Experiment shows, however, that this is very far from being the case. Even the least amount of proteids causes a prompt increase in the urinary nitrogen, and each successive addition of proteids results in a further increase, so that it is not until the food proteids largely exceed the amount metabolized during fasting that nitrogen equilibrium is reached. Thus Bischoff \& Voit, $\$$

* Gesetze der Ernährung des Fleischfressers, 1860.

† Published chiefly in the Annalen der Chemie und Pharmacie and the Zeitschrift für Biologie. See also Voit, "Physiologie des Stoffwechsels," in Herman's Handbuch der Physiologie.

$\ddagger$ Zeit. f. Biol., 3, 29 and 33. 
in a series of experiments upon a dog fed exclusively on lean meat, obtained the results shown in the following table, the proteid metabolism being expressed in terms of flesh with its normal water content $(\mathrm{N} \times 29.4)$ instead of dry proteids:

\begin{tabular}{|c|c|c|c|}
\hline Date. & Meat Fed. & $\begin{array}{c}\text { "Flesh" } \\
\text { Metabolized. }\end{array}$ & $\begin{array}{c}\text { Gain or Loss } \\
\text { of Flesh. }\end{array}$ \\
\hline 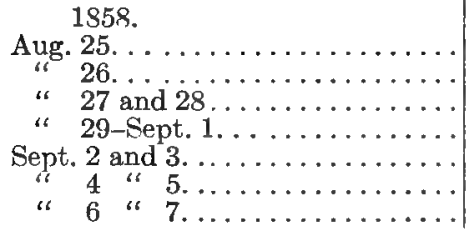 & $\begin{array}{r}0 \\
0 \\
300 \\
600 \\
900 \\
1200 \\
1500\end{array}$ & $\begin{array}{r}223 \\
190 \\
379 \\
665 \\
941 \\
1180 \\
1446\end{array}$ & $\begin{array}{r}-223 \\
-190 \\
-\quad 79 \\
-\quad 65 \\
-\quad 41 \\
+\quad 20 \\
+\quad 54\end{array}$ \\
\hline 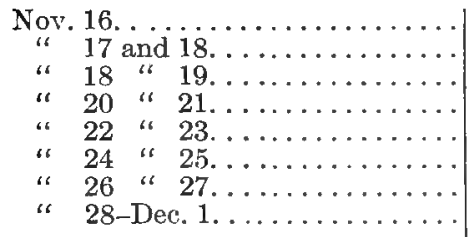 & $\begin{array}{r}1800 \\
1500 \\
1200 \\
900 \\
600 \\
300 \\
176 \\
0\end{array}$ & $\begin{array}{r}1764 \\
1510 \\
1234 \\
945 \\
682 \\
453 \\
368 \\
226\end{array}$ & $\begin{array}{l}+36 \\
-10 \\
-34 \\
-\quad 45 \\
-82 \\
-153 \\
-192 \\
-226\end{array}$ \\
\hline
\end{tabular}

- A much later series by E. Voit \& Korkunoff,* in which the results were determined in terms of nitrogen, may 'je cited to illustrate the same point. The food was lean meat from which the extractives had been removed by treatment with cold water. It contained 1.25 to 1.96 per cent. of fat.

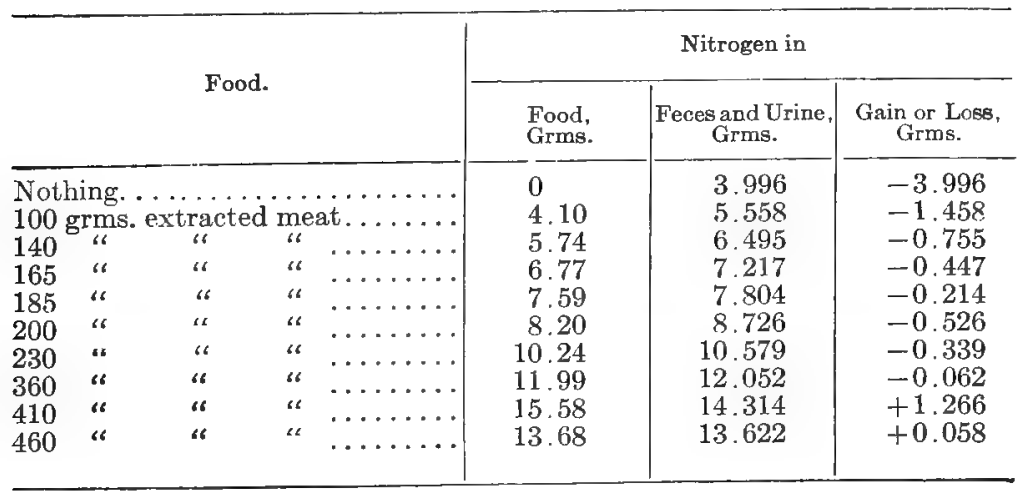

* Zeit. f. Biol., 32, 67. 
The proteid supply gradually overtakes the proteid metabolism, but when only proteids are fed the supply must largely exceed the fasting metabolism in order to attain nitrogen equilibrium. E. Voit has endeavored to obtain a numerical expression for this relation by taking as the basis of comparison the fasting metabolism. He estimates (loc. cit., p. 101) that of the total nitrogen excretion of a fasting animal 81.55 per cent. is derived from true proteids and 18.45 per cent. from the extractives of the muscles. Since the food in his experiments consisted substantially of true proteids, he compares its nitrogen with 81.55 per cent. of the nitrogen of the excreta and thus finds that the minimum supply of proteid nitrogen required to reach nitrogen equilibrium was between 3.67 and 4.18 times that metabolized during fasting, the true value being estimated at 3.68. Five other less exact experiments gave confirmatory results and similar confirmation is found in the experimental results of $\mathrm{C}$. Voit.

Effect of Excess of Proterds. - If the supply of proteids to a mature animal be still further increased after nitrogen equilibrium is reached, the excess of proteids is promptly metabolized, its nitrogen reappearing in the excreta. In other words, the excretory nitrogen keeps pace with the supply of nitrogen in the food. The experiments by Bischoff \& Voit just cited serve to illustrate this fact also. Approximate nitrogen equilibrium was reached on 1200-1500 grams of meat, but in other trials even double this supply caused but a slight apparent gain of nitrogen, and it is probable that if the total urinary nitrogen had been determined instead of the urea, and account taken of the nitrogen of the feces, even this small difference would have disappeared.

It is needless to multiply examples of this perfectly well-established fact. The animal body puts itself very promptly into equilibrium with its nitrogen supply and no considerable or long-continued gain of proteid tissue can be produced in the mature animal by even the most liberal supply of proteid food.

Transtrory Storage of Proteids.-But while no continued gain of protein by the body can be brought about by additions to the proteid food, nevertheless, during the first few days following such an increase in the proteid supply a transitory storage of nitrogen takes place. Conversely, too, a decrease in the proteid supply 
causes at first a loss of nitrogen from the body, which, however, unless the new supply of proteids falls below a certain minimum is as transitory as the gain in the other case. In other words, while the nitrogen excretion of the mature animal is in the long run equal to the supply in the food, when the amount of the latter is changed the full effect on the excretion is not realized at once.

This fact is well illustrated by the following selection from C. Voit's investigations upon the dog,* the results being expressed in terms of "flesh":

\begin{tabular}{|c|c|c|c|c|c|c|c|c|c|}
\hline \multirow{3}{*}{$\begin{array}{c}\text { Previous } \\
\text { Ration. } \\
\text { Grms. } \\
\text { Meat. }\end{array}$} & \multirow{3}{*}{$\begin{array}{l}\text { New } \\
\text { Ration. } \\
\text { Grms. } \\
\text { Meat. }\end{array}$} & \multicolumn{8}{|c|}{ "Flesh" Metabolized per Day.' } \\
\hline & & \multirow{2}{*}{$\begin{array}{c}\text { On } \\
\text { Previous } \\
\text { Ration. } \\
\text { Grms. }\end{array}$} & \multicolumn{7}{|c|}{ On New Ration. } \\
\hline & & & $\begin{array}{l}\text { 1st Day. } \\
\text { Grms. }\end{array}$ & $\begin{array}{l}\text { 2d Day. } \\
\text { Grms. }\end{array}$ & $\begin{array}{l}\text { 3d Day } \\
\text { Grms. }\end{array}$ & $\begin{array}{l}\text { 4th Day } \\
\text { Grms. }\end{array}$ & $\begin{array}{l}\text { 5th Day. } \\
\text { Grma. }\end{array}$ & $\begin{array}{l}\text { 6th Day } \\
\text { Grms. }\end{array}$ & $\begin{array}{l}\text { 7th Day. } \\
\text { Grms. }\end{array}$ \\
\hline 1800 & 2500 & 1800 & 2153 & 2480 & 2532 & & & & \\
\hline 500 & 1500 & 547 & 1222 & 1310 & 1390 & 1410 & 1440 & 1450 & 1500 \\
\hline & 1500 & 176 & 1267 & 1393 & 1404 & & & & \\
\hline 2500 & 2000 & 2500 & 222 & 1970 & & & & & \\
\hline 1500 & 100 & 1500 & 1153 & 1086 & 1088 & 1080 & 1027 & & \\
\hline 1000 & 5000 & 1000 & 706 & 610 & 623 & 560 & & & \\
\hline
\end{tabular}

An example of the same fact is found in the experiments cited on p. 81 , in which $\mathbf{s i}^{;}$proteid food was withdrawn, the nitrogen excretion falling rapidly, but reaching its minimum only after three or four days.

Voit explained the facts just adduced as the consequence of the difference between organized and circulatory proteids already noted on p. 82. According to this hypothesis, the amount of the proteid metabolism is chiefly determined by the store of circulatory proteids in the body. The ingestion of additional proteids increases the amount of these circulatory proteids in the body, and as a consequence the proteid metabolism increases until the nitrogen excretion overtakes the supply. Similarly, a decrease in the proteid food has the converse effect.

Proteid Metabolism and Nitrogen Excretion.-Up to this point, following common usage, the terms nitrogen excretion and proteid metabolism have been employed as practically synonymous. In one sense this usage is correct, but it is liable to give * E. v. Wolff, Ernährung Landw. Nutzthiere, p 271. 
rise to a misconception. It is perfectly true that the presence of one gram, e.g., of nitrogen in the urine, implies that about six grams. of protein have yielded up their nitrogen in the form of urea or other metabolic products and therefore have ceased to exist as protein. It by no means follows from this, however, that this protein has been completely oxidized to carbon dioxide and water. We have already seen (Chapter II, p. 48) that the abstraction of the elements of urea from protein leaves a non-nitrogenous residue equal to nearly two-thirds of the protein, and that there is reason to believe that this residue may, according to circumstances, be oxidized to supply energy or give rise to a production of glycogen or of fat. In other words, the separation of its nitrogen from protein and the complete oxidation of its carbon and hydrogen are two distinct things. When, therefore, we assert, on the basis of the evidence noted above, that the proteid metabolism of the mature animal is determined by the supply of proteids in the food, what we really mean is that the cleavage of proteids and the excretion of their nitrogen is so determined.

Rate of Nitrogen Excretion.-A consideration of the course of the nitrogen excretion after a meal of proteids is calculated to throw light upon the relations of nitrogen cleavage to the total metabolism of the proteids. The early investigations of Becher, Voit, Panum, Forster, and Falck showed that when proteids are given to a fasting animal the rate of nitrogen excretion shows a rapid increase, reaching a maximum within a few hours.

Feder* observed the maximum rate of nitrogen excretion by dogs in different experiments between the fifth and eighth hour after a meal of meat. From this point the rate of excretion decreased less rapidly than it had increased and continued to decrease until about thirty-six hours after the meal.

Graffenberger,$\dagger$ experimenting upon himself, obtained similar results after the consumption of fibrin, gelatin, and asparagin, while the results with a commercial "meat peptone" were markedly different; and Rosemann, in studies upon the rate of nitrogen excretion by man, traces clearly a similar influence of the ingestion of nitrogenous food, while Krummacher's $\S$ results on dogs fully

* Zeit. f. Biol., 17, 531; Thier Chem. Ber., 12, 402. † Zeit. f. Biol., 28, 318.

$\$$ Arch. ges. Physiol., 65, 343.

§ Zeit. f. Biol., 35, 481 . 
confirm those of Feder. Sherman and Hawk * have likewise found the curve of nitrogen excretion by man after the ingestion of lean meat to show the same general form observed by Feder and by Graffenberger.

Nitrogen Cleavage Independent of Total Metabolism. Kaufmann, $\uparrow$ by the method outlined in Chapter VIII, has made a series of determinations of the nitrogen excretion, respiratory exchange, and heat production of dogs during the time when nitrogen cleavage is most active, i.e., from the second to the seventh hour after a full meal of meat. From his theoretical equations for the complete metabolism of proteids (pp. $51 \& 75$ ) he computes the respiratory exchange and heat production corresponding to the observed excretion of urinary nitrogen and compares them with the actual results per hour as follows:

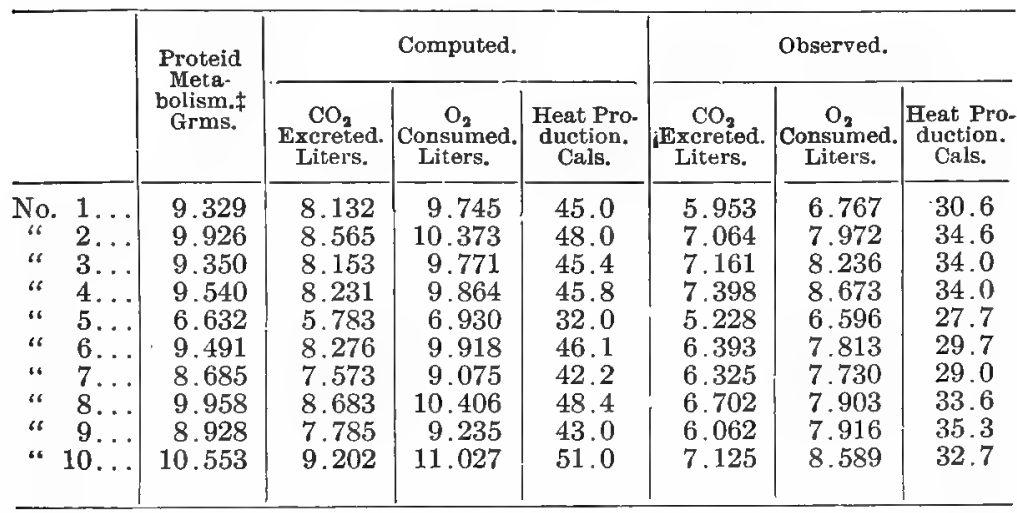

But a glance is needed to show that the total metabolism, whether measured by the gaseous exchange or by the heat production, is much less than that computed, which is equivalent to saving that the non-nitrogenous residue of the proteids was not completely oxidized. Gruber, $\S$ whose experimental results upon the rate of nitrogen excretion fully confirm those above cited, has shown very clearly the bearing of these facts. He points out that if we

* Amer. Jour. Physiol., 4, 25.

$\uparrow$ Archives de Physiologie, 1896, pp. 346 and 768. is 6.39 .

$\$$ Kaufmann's factor for proteids, derived from the formula $\mathrm{C}_{72} \mathrm{H}_{112} \mathrm{~N}_{18} \mathrm{O}_{20} \mathrm{~S}$,

§ Zeit. f. Biol., 42, 407. 
regard the nitrogen excretion as denoting the complete metabolism to carbon dioxide, water, urea, etc., of a corresponding amount of proteids, we get figures for the total evolution of energy (heat) in the organism. which are entirely incompatible with those derived from other considerations. For example, a daily diet of 1500 grams of lean meat given to a dog not only sufficed to supply the demands for energy but produced a storage of fat in the body. The total daily production of heat, computed from the results of respiration experiments (see Chapter VIII), was 1060.2 Cals., equivalent to 88.3 Cals. in two hours, which must have been derived essentially from the metabolism of proteids. If, however, we compute the evolution of energy from the results of the nitrogen excretion as determined in two-hour periods, we get strikingly variable results.

\begin{tabular}{|c|c|c|}
\hline Hour. & $\begin{array}{c}\text { Urinary Nitrogen, } \\
\text { Grams. }\end{array}$ & $\begin{array}{l}\text { Equivalent Energy,* } \\
\text { Cals. }\end{array}$ \\
\hline 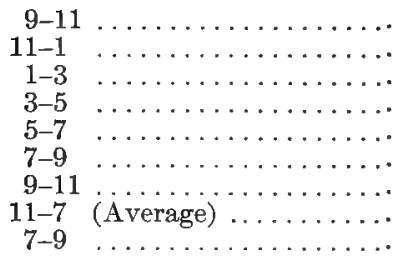 & $\begin{array}{l}3.11 \\
5.71 \\
6.62 \\
6.98 \\
6.35 \\
6.04 \\
5.08 \\
2.65 \\
1.24\end{array}$ & $\begin{array}{r}80.6 \\
148.2 \\
171.6 \\
181.2 \\
165.1 \\
156.0 \\
132.6 \\
68.9 \\
32.5\end{array}$ \\
\hline
\end{tabular}

The heat production as thus computed varies from over twice the average two-hour rate to an amount equal to scarcely more than one half of the average fasting metabolism of the same animal (62 Cals. per two hours). Such fluctuations are entirely inconsistent with all data as to the heat production of the body, which, as we shall see later, appears to go on with a remarkable degree of uniformity under uniform conditions. The only reasonable conclusion, then, appears to be that the nitrogen cleavage and the total oxidation of the proteids are distinct and at least largely independent processes.

Gruber's explanation of these facts is substantially as follows: It is well established that a relatively constant composition of the blood and of the fluids of the body generally is an essential condi-

* One gram $\mathrm{N}$ equivalent to $26 \mathrm{Cals}$. See Chapter VIII. 
tion of normal physiological activity. It has been repeatedly demonstrated, however, that when the period of growth is past, the animal body has not the ability to produce any material amount of proteid tissue. A large supply of proteid food, then, necessarily tends to alter the composition of the blood and other fluids of the body, and the nitrogen cleavage is evidently an effort on the part of the organism to counteract this effect by splitting off from the proteids a nitrogenous group which can be rapidly excreted, leaving a non-nitrogenous residue which, so far as it is not immediately needed to supply energy, is capable of storage in the relatively inert and insoluble forms of glycogen and of fat.

According to Rosemann,* the rapid increase in the nitrogen excretion after a meal arises from two concurrent causes: first, a direct stimulus to the proteid metabolism, due to the rapid increase of proteids and their digestion products in the blood, which is somewhat transitory in character; and, second, the effect of a larger relative supply of proteids in causing, according to wellknown physico-chemical laws, a relatively larger number of molecules of these substances to enter into reactions with the cell protoplasm. The accompanying graphic representation by Gruber $\dagger$ of the course of the nitrogen excretion of a dog on the second day of

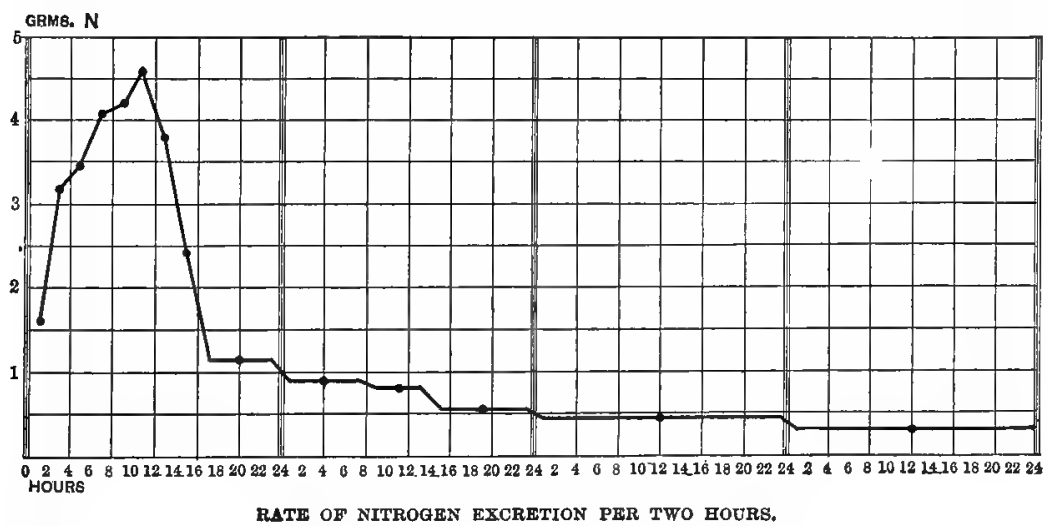

feeding with 1000 grams of lean meat and on the three following fasting days shows plainly the sudden stimulation of the excretion

* Loc. cit. $\dagger$ Loc. cit., p. 421. 
at first and the fall, rapid at first, and then very gradual, until the minimum of the fasting excretion is reached about the third day.

On the other hand, Rjasantzeff $*$ and Shepski $\nmid$ ascribe the increase in the nitrogen cleavage after a meal to the increase in the digestive work rather than to the proteids as such. They find it possible, by stimulating the activity of the digestive organs without introducing food, to considerably increase the nitrogen excretion in the urine, while, on the other hand, the introduction of proteid food through a gastric fistula produced little or no effect. They also find the increase with the same amount of food nitrogen to be proportional to the (estimated?) amount of digestive work, but seem to offer no explanation of the equality of nitrogen cleavage and nitrogen supply.

Cause of Transitory Storage.-As already noted (p. 96), any change in the rate of proteid supply in the food, while resulting ultimately in a corresponding change in the rate of nitrogen excretion, gives rise to a transitory gain or loss of nitrogen by the body, which was interpreted by Voit as consisting in a corresponding change in the stock of "circulatory protein" in the body. The facts which we have just been considering permit us to trace somewhat more fully the details of the phenomenon. Gruber points out that while the larger part of the nitrogen cleavage consequent upon a single meal of proteids takes place within a few hours, the remainder is prolonged over two or three days, as in the case illustrated above, while he likewise shows experimentally that this effect is not due to a retention of the nitrogenous metabolic products, but represents the actual course of nitrogen cleavage.

Such being the case, the transitory gain or loss incident to a change in the rate of proteid supply is most simply explained as the result of a superposition of the daily curves. Let it be assumed, for example, that 80 per cent. of the nitrogen cleavage incident to a single meal of proteids takes place on the first day, 13 per cent. on the second, 5 per cent. on the third, and 2 per cent. on the fourth. Then if we give to a fasting animal an amount of proteids containing 100 grams of nitrogen for five successive days and then withdraw the food, the food nitrogen will be excreted as follows on the several days:

* Jahr. Thier Chem., 26, $349 . \quad$ † Ibid., 30, 711. 


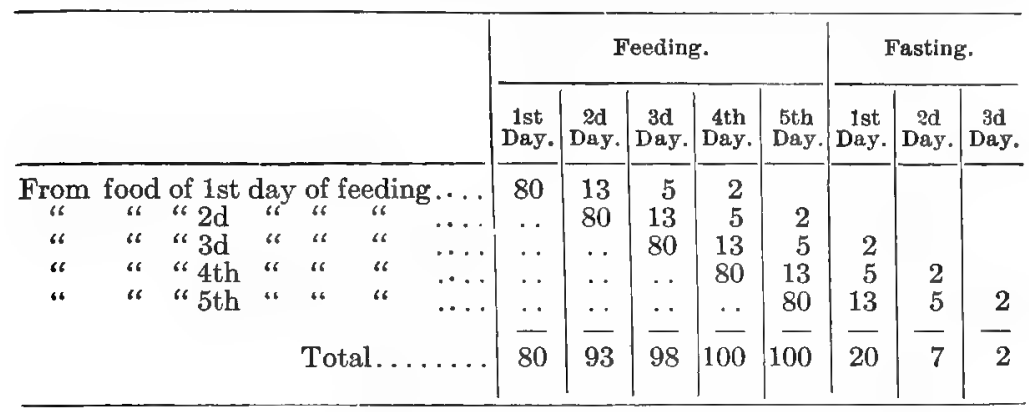

On the above assumptions, there remained in the body at the end of the first day 20 grams of nitrogen in the form of unmetabolized proteids. At the end of the second day this had increased to 27 grams, and at the end of the third day had reached the maximum of 29 grams. At the end of the first day's fasting it had fallen to 9 grams, at the end of the second day to 2 grams, and at the end of the third day to zero. In other words, the transitory storage of proteids observed by Voit and others is explained by Gruber as due to the fact that the nitrogen cleavage extends over more than a single day.

In reality, of course, the excretion does not take place with any such mathematical exactness as in this schematic example, and after long fasting in particular a certain rebuilding of proteid tissue may occur, but the assumed figures may serve to give a general notion of the relations of food-supply and excretion.

In brief, then, we may suppose that when proteid food is given to a fasting animal the stimulating effect upon the nitrogen cleavage anticipates the use of the proteids for constructive purposes and that a large proportion of them is thus destroyed as proteids before it can be used to make good the loss of proteids by the organized tissues. In other words, the proteids actually available for the tissues are much less than the amount supplied in the food. In this view of the matter we can readily see why the proteid supply overtakes the nitrogen excretion so slowly and why two or three times the amount metabolized in fasting is necessary to make good the loss from the body and ensure nitrogen equilibrium. 


\section{Effects on Total Metabolism.}

In the preceding paragraphs the effects of an exclusive proteid diet upon the proteid metabolism have been discussed. There remain to be considered its effects upon the metabolism of fat.

Proteids Substituted for Body Fat.-When proteids are given to a fasting animal the proteid metabolism is increased, as we have seen, but at the same time the loss of body fat is diminished.

Pettenkofer \& Voit * fed a dog with varying amounts of lean meat, which may be regarded as consisting chiefly of proteids together with small amounts of fat, with the following average results in terms of nitrogen:

\begin{tabular}{c|c|c|c|c}
\hline $\begin{array}{c}\text { Meat Fed, } \\
\text { Grms. }\end{array}$ & $\begin{array}{c}\text { Nitrogen } \\
\text { of Food, } \\
\text { Grms. }\end{array}$ & $\begin{array}{c}\text { Nitrogen } \\
\text { Metabolized, } \\
\text { Grms. }\end{array}$ & $\begin{array}{c}\text { Gain or Loss } \\
\text { of Nitrogen, } \\
\text { Grms. }\end{array}$ & $\begin{array}{c}\text { Gain or Loss } \\
\text { of Fat, } \\
\text { Grms. }\end{array}$ \\
\hline $0 \dagger$ & 0 & 5.6 & -5.6 & -95 \\
500 & 17.0 & 20.4 & -3.4 & -47 \\
1000 & 34.0 & 36.7 & -2.7 & -19 \\
$1500 \ddagger$ & 51.0 & 51.0 & 0 & +4 \\
\hline
\end{tabular}

Rubner $\S$ has obtained a similar result by the use of the proteid mixture resulting from the extraction of lean meat with water, and which still contained some fat. As compared with the fasting state, the consumption of 740 grams of the moist material (containing 72.2 per cent. of water) produced the following effect:

\begin{tabular}{|c|c|c|c|}
\hline & $\begin{array}{c}\text { Nitrogen of Food, } \\
\text { Grms. }\end{array}$ & $\begin{array}{l}\text { Nitrogen } \\
\text { Metabolized, } \\
\text { Grms. }\end{array}$ & $\begin{array}{c}\text { Fat } \\
\text { Metabolized, } \\
\text { Grms. }\end{array}$ \\
\hline $\begin{array}{l}\text { Fasting } \ldots \ldots \ldots \ldots \ldots \ldots \ldots \ldots \\
\text { Fed } \ldots \ldots \ldots \ldots \ldots \ldots\end{array}$ & $\begin{array}{c}0 \\
35.22\end{array}$ & $\begin{array}{r}5.25 \\
26.37\end{array}$ & $\begin{array}{l}84.39 \\
28.37\end{array}$ \\
\hline Difference...... & $\cdots \cdots$ & $+\overline{21.12}$ & -56.02 \\
\hline
\end{tabular}

The increased nitrogen cleavage resulting from an increase in the proteid supply liberates a certain amount of energy for the vita] activities of the body, while the non-nitrogenous residue of the cleav -

* Zeit f. Biol, , 7, 489.

† Average of first two experiments, p. 84, Chapter IV.

‡ Series I only. The others showed a greater gain of fat and of nitrogen.

\& Zeit. f. Biol., 22, 51. 
age becomes available also as a source of energy to the organism, and the metabolism of fat is correspondingly diminished. In effect, then, the proteids are simply substituted for more or less of the body fat as a source of energy, and Rubner, in a series of experiments which will be considered in Part II, has shown that the substitution takes place, under the condition of these experiments, approximately in proportion to the amount of available potential energy contained in the proteids and fats respectively. That is, if the extra proteids metabolized can supply a certain amount, 100 Cals., e.g., of energy to the organism, the fat metabolism is diminished by a corresponding amount, so that the total expenditure of energy by the body remains unchanged, being simply drawn from different sources in the two cases.

Amount Required to Produce Carbon Equilibrium.-In the experiments by Pettenkofer \& Voit cited above, the quantity of food proteids which resulted on the average in nitrogen equilibrium produced substantially an equilibrium also between the supply and excretion of carbon. The earlier experiments of Bidder \& Schmidt* gave similar results. Later experiments, however, have given divergent results, nitrogen equilibrium appearing to be reached with an amount of proteids which is far from supplying sufficient energy for the organism, so that while the stock of proteids in the body is maintained, its store of fat is still drawn upon.

We have seen that the proteid metabolism in the normal fasting animal amounts to $10-14$ per cent. of the total metabolism, while according to E. Voit (p. 96) the food proteids required for nitrogen equilibrium are, roughly, $2 \frac{1}{2}$ to 3 times the fasting proteid metabolism. It follows, then, that an amount of proteids containing from 25 to 42 per cent. of the total available energy expended by the fasting organism will maintain its store of proteids, and this being so, the remaining $58-75$ per cent. must necessarily be supplied by the metabolism of body fat. Thus with the dog on which E. Voit's main experiment was made, nitrogen equilibrium was approximately reached with 12.05 grams of nitrogen in the food, $\dagger$ equivalent to 75.31 grams of protein $(\mathrm{N} \times 6.25)$ and containing

* Compare Atwater \& Langworthy; Digest of Metabolism Experiments;

U. S. Dept. of Agr., Office of Experiment Stations, Bul. 45, 388.

$\dagger$ Loc. cit., p. 69 . 
approximately, according to Rubner (see Chapter X), 321 Cals. of available energy. The actual expenditure of energy by the animal was not determined, but is estimated by the author on the basis of Rubner's investigations at about 1280 Cals.

Several experiments by Rubner* lead to the same conclusion. In these experiments the carbon and nitrogen of the excreta were determined and the nitrogen of the food estimated from average figures. The proteid metabolism having been computed from the total excretory nitrogen, the corresponding amount of carbon is computed from the average composition of the proteicls and any excess in the excreta is assumed to be derived from the metabolism of fat. (Compare p. 78.) The following are the results in brief, including the one cited above (p. 104):

\begin{tabular}{|c|c|c|c|c|}
\hline Food. & $\begin{array}{l}\text { Nitrogen } \\
\text { of Food, } \\
\text { Grms. }\end{array}$ & $\begin{array}{c}\text { Nitrogen } \\
\text { of } \\
\text { Excreta, } \\
\text { Grms. }\end{array}$ & $\begin{array}{l}\text { Fat } \\
\text { Metab- } \\
\text { olized, } \\
\text { Grms. }\end{array}$ & Remarks. \\
\hline $\begin{array}{l}\text { Nothing } . . . . . . . . . . \\
415 \text { grms. lean meat ... }\end{array}$ & 14.11 & $\begin{array}{r}4.38 \\
13.72\end{array}$ & $\begin{array}{l}49.33 \\
25.44\end{array}$ & Average of several days. \\
\hline $\begin{array}{l}\text { Nothing . . . . . . . . . } \\
740 \text { grms. lean meat . }\end{array}$ & 25.16 & $\begin{array}{r}2.80 \\
20.63\end{array}$ & $\begin{array}{l}79.94 \\
30.73\end{array}$ & 1st two days of feeding. \\
\hline $\begin{array}{l}\text { Nothing } \ldots . . . \ldots \ldots \ldots \\
740 \text { grms. extracted lean } \\
\text { meat } \ldots . . . \ldots \ldots \ldots\end{array}$ & $\begin{array}{l}\cdots \\
35.22\end{array}$ & 26.37 & 28.37 & 1st to 4 th day of feeding. \\
\hline 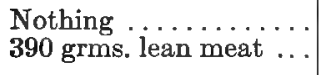 & 13.26 & $\begin{array}{l}1.08 \\
8.53\end{array}$ & $\begin{array}{l}22.88 \\
11.42\end{array}$ & 1st day of feeding. \\
\hline $\begin{array}{l}\text { Nothing . . . . . . . . } \\
\mathbf{3 5 0} \text { grms. lean meat }\end{array}$ & 11.90 & $\begin{array}{r}1.08 \\
10.10\end{array}$ & $\begin{array}{l}22.88 \\
11.79\end{array}$ & $3 \mathrm{~d}$ to 6 th day of feeding. \\
\hline $\begin{array}{l}\text { Nothing } \ldots \ldots . . . . . \\
580 \text { grms. lean meat }\end{array}$ & 19.72 & $\begin{array}{r}3.50 \\
18.47\end{array}$ & $\begin{array}{l}37.24 \\
21.45\end{array}$ & 1st to 7 th day of feeding. \\
\hline
\end{tabular}

While some of the experiments were hardly continued long enough to absolutely establish the sufficiency of the proteid supply, nevertheless we see in all cases a material loss of fat on rations which apparently are sufficient to prevent a loss of nitrogen from the body.

It should perhaps be noted that in Pettenkofer \& Voit's experiments 1000 grams of meat nearly prevented a loss of nitrogen *Zert. f. Biol., 22, 43-48; 30, 122-134. 
from the body. It appears possible, then, that nitrogen equilibrium might have been reached with a less amount than 1500 grams, and that with this less amount there might still have been a loss of fat-from the body. Whether this possibility is sufficient to explain the apparent discrepancy between these and later results must, however, remain a matter of conjecture.

Utilization of Excess of Proteins.-We have seen that no very considerable or long-continued storage of protein takes place in the body of the mature animal. However large the supply of food proteids, the body very soon reaches the condition of nitrogen equilibrium, the outgo of this element in the excreta equaling the supply in the food. This fact, as has been pointed out, does not necessarily prove that the elements of the food proteids are completely oxidized in the organism. As was shown in Chapter II, the abstraction from proteid matter of the elements of urea (or, more strictly speaking, of the elements found in the urine) leaves a very considerable non-nitrogenous residue available for the purposes of the organism. It was there stated that this residue could serve as a source of energy, and likewise that there was good reason to believe that sugar was formed from it, while finally the question of its ability to serve as a source of fat was reserved for discussion in the present connection.

\section{Formation of Fat from Proteids.}

Mention has already been made in Chapter II (p. 29) of the fact, first asserted by Liebig,* that the animal body manufactures fat from other ingredients of its food. As a result of the investigations incited by the publication of his views regarding the origin of animal fat, Liebig's classification of the nutrients into "plastic" and "respiratory" was generally accepted. The proteids were regarded as the material for the growth and repair of the muscles and the force exerted by the latter was considered to arise from their oxidation, while the non-nitrogenous ingredients of the food, especially the carbohydrates, were the source of the animal heat, and when present in excess gave rise to a production of fat.

As time went on, however, observations began to accumulate *Compare p. 163. 
tending to show that the proteids were not without influence on fat-production.

As early as $1745 \mathrm{R}$. Thomson,* in experiments on milch cows, noted an apparent connection between the supply of proteids in the food and the production of butter.

Hoppe $\dagger$ in 1856 interpreted the results of an experiment in which a dog was fed lean meat with and without the addition of sugar as showing a formation of fat from proteids. The same author $\ddagger$ in 1859 claimed to have shown a slight formation of fat from casein in milk exposed to the air, and this was confirmed later by Szubotin.\& The latter author, and also Kemmerich, $\|$ and later Voit, $\uparrow$ experimented upon the production of milk-fat by dogs. Their results, while indicating the possibility of a formation of fat from proteids, were indecisive.

Pettenkofer \& Vort's Experiments.-Carl Voit, however, was the first to distinctly champion the new theory, and aside from certain confirmatory facts, ${ }^{* *}$ such as the formation of fatty acids in the oxidation of proteids, the formation of adipocere, the alleged formation of fat from proteids in the ripening of cheese and in the fatty degeneration of muscular tissue, especially in cases of phosphorous poisoning,-facts not all of which are fully established and whose importance in this connection has probably been overestimated, - the evidence bearing on the question of the formation of fat from proteids has been until recently largely that supplied by the famous researches of Pettenkofer \& Voit †† at Munich.

In these experiments a dog weighing about $30 \mathrm{kgs}$. was fed varying amounts of prepared lean meat from which fat, connective tissue, etc., had been removed as completely as was possible by mechanical means. The material thus prepared, while still containing small amounts of fat, etc., was as near an approach to an exclusively proteil diet as was practicable, it having been found impossible to successfully carry out feeding experiments with pure

* Ann. Chem. Pharm., 61, 228.

† Virchow's Archiv, 10, 144

‡ Virchow's Archiv, 17, 417 .

\| Wolff, Ernahrung Landw. Nutzthiere, p. 351.

ๆ Zeit. f. Biol., 5, 136.

** Compare Voit's summary in 1869, Zeit. f. Biol., 5, 79-169.

†† Am. Chem. Pharm., II, Suppl. Bd., pp. 52 and 361 ; Zeit. f. Biol., 5, $106 ; 7,433$. 
proteids. The experiments were conducted with the aid of a respiration apparatus, the gain or loss of proteids and fat being computed from the nitrogen and carbon balance in the manner described in Chapter III.

The following is a condensed summary of the average results of these experiments, as given by the authors, ${ }^{*}$ but includes also the average of all the experiments with 1500 grams of meat. On

\begin{tabular}{|c|c|c|c|}
\hline \multirow{2}{*}{$\begin{array}{l}\text { Number of } \\
\text { Experiments. }\end{array}$} & \multirow{2}{*}{$\begin{array}{l}\text { Meat Eaten per Day, } \\
\text { Grms. }\end{array}$} & \multicolumn{2}{|c|}{ Gain (+) or Loss (-) by Animal } \\
\hline & & $\begin{array}{l}\text { Flesh. } \\
\text { Grms. }\end{array}$ & $\begin{array}{c}\text { Fat. } \\
\text { Grms. }\end{array}$ \\
\hline $\begin{array}{c}3 \dagger \\
22 \\
1 \\
2 \\
1\end{array}$ & $\begin{array}{r}0 \\
500 \\
1000 \\
1500 \\
1500 \\
1800 \\
2000 \\
2500\end{array}$ & $\begin{array}{r}-165 \\
-\quad 99 \\
-\quad 79 \\
0 \\
+\quad 18 \\
+\quad 43 \\
-\quad 44 \\
-\quad 12\end{array}$ & $\begin{array}{l}-95 \\
-47 \\
-19 \\
+4 \\
+9 \\
+1 \\
+58 \\
+57\end{array}$ \\
\hline
\end{tabular}

the smaller rations, which were obviously insufficient for maintenance, the animal lost both flesh and fat. A ration of 1500 grams of meat per day sufficed approximately to maintain the animal as regards flesh and to cause a small gain of fat. On the heavier rations the excretion of nitrogen kept pace with the supply in the food in the manner illustrated on pp. 94-96 but the excretion of carbon fell considerably below the supply, indicating a production of fat.

It is to be noted that only the last three experiments in the above table actually show any very considerable production of fat. The insufficient rations naturally do not, and while among the twentytwo trials with 1500 grams of meat the majority appear to show a formation of fat, the amount is usually comparatively small, and in two cases a loss was observed. On the whole, however, the evidence of this series of experiments has been generally accepted as conclusive in favor of the formation of fat from proteids.

PFLÜGeR's ReCalCULations.-One very important point, however, has until recently been overlooked. The evidence is based on

*Zeitschr. f. Biol, 7, 489.

$\dagger$ Series 1 only. 
a comparison of the income and outgo of carbon and nitrogen. Pflüger,* however, has called attention to the fact that while Pettenkofer \& Voit made direct determinations of the outgo of these elements, or at least of the principal factors of it, the income is not computed from actual analyses of the meat used, but upon the assumption of average composition. According to Pflüger, not only are the possible variations from the average in individual experiments a serious source of error, but the average itself is erronenus, the percentage of carbon assumed in the meat being too high. Pettenkofer \& Voit estimate the ratio of nitrogen to carbon in lean meat $\dagger$ as $1: 3.684$, while according to Pflüger it is not higher than $1: 3.28$, and probably lower. Moreover, Pettenkofer \& Voit failed to take due account of the fact that a part of the gain of carbon which they observed could be ascribed to the fat still contained in the prepared "lean" meat. Another, although slight, source of error, according to Pflüger, lies in the fact that the carbon in the urine was estimated from the amount of nitrogen found by analysis on the assumption of a ratio of $1: 0.60$, while it should be $1: 0.67$.

Using the above corrections, Pflüger has recalculated twentyfour of the experiments by Pettenkofer \& Voit, which have been generally accepted as demonstrating the formation of fat from proteids, with the results shown on the opposite page.f

In the great majority of cases the experiments as recalculated show a loss instead of a gain of fat, and in three of the four cases in which a gain still appears it is small in amount, and, as Pflüger believes, within the limits of experimental error. Naturally such calculations as the above can neither prove nor disprove the hypothesis that the proteids serve as a source of fat. They simply show that the experiments which have served as the principal support for that hypothesis do not demonstrate what they were supposed to. The question turns largely upon the elementary composition of the meat used by Pettenkofer \& Vnit, which they failed to determine. It is manifestly impossible to repair this error now,

* Arch. ges. Physiol., 51, 229.

† Including such fat as cannot be removed by mechanical means.

$\ddagger$ Loc. cit., p. 267 . The experiments which showed a loss of fat as originally computed are omitted. 


\begin{tabular}{|c|c|c|c|}
\hline \multirow{2}{*}{ Date of Experiment. } & \multirow{2}{*}{$\begin{array}{l}\text { Meat Eaten } \\
\text { per Day, } \\
\text { Grms. }\end{array}$} & \multicolumn{2}{|c|}{ Gain $(+)$ or Loss $(-)$ of Fat. } \\
\hline & & $\begin{array}{l}\text { According to Pet- } \\
\text { tenkofer \& Voit. } \\
\text { Grms. }\end{array}$ & $\begin{array}{l}\text { According to } \\
\text { Pfliger. } \\
\text { Grans. }\end{array}$ \\
\hline 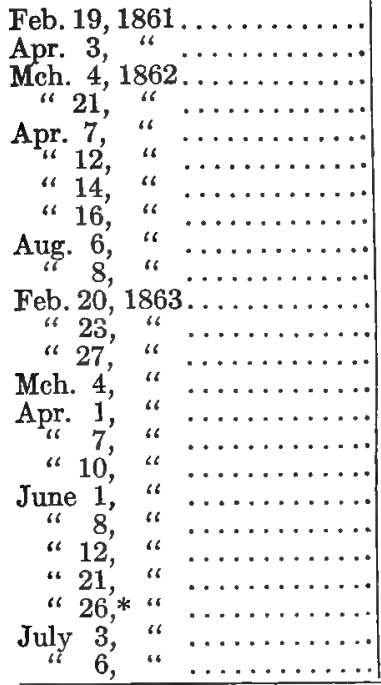 & $\begin{array}{l}1800 \\
2500 \\
1500 \\
1500 \\
1500 \\
1500 \\
1500 \\
1500 \\
1500 \\
1500 \\
1500 \\
1500 \\
1500 \\
1500 \\
1500 \\
1500 \\
1500 \\
1500 \\
1500 \\
1500 \\
2000 \\
2000 \\
1500 \\
1500\end{array}$ & $\begin{array}{l}+1.4 \\
+56.7 \\
+3.4 \\
+7.3 \\
+34.4 \\
+20.7 \\
+35.9 \\
+22.9 \\
+8.7 \\
+17.4 \\
0.0 \\
+9.9 \\
+2.1 \\
+14.3 \\
0.0 \\
+13.8 \\
+9.0 \\
+12.7 \\
+26.3 \\
+29.1 \\
+55.9 \\
+58.5 \\
+11.9 \\
+9.4\end{array}$ & $\begin{array}{l}-35.8 \\
+3.93 \\
-29.3 \\
-23.4 \\
+3.7 \\
-11.1 \\
+3.8 \\
-8.4 \\
-13.5 \\
-8.3 \\
-31.6 \\
-22.1 \\
-24.4 \\
-16.9 \\
-31.8 \\
-17.5 \\
-22.0 \\
-13.0 \\
-5.4 \\
-2.9 \\
+13.6 \\
+1.6 \\
-20.6 \\
-23.7\end{array}$ \\
\hline
\end{tabular}

and since Pflüger's estimates seem to be at least as trustworthy as Pettenkofer \& Voit's, and lead to exactly the opposite results, the only verdict possible, so far as these experiments are concerned, is "Not proven."

Later Experiments. - Shortly after the publication of Pflüger's critique, E. Voit, $\uparrow$ in a preliminary communication, presented the results of investigations upon this question undertaken in the Munich laboratory. So far as the writer is aware, no complete account of these experiments has yet appeared, but the data given by Voit in the preliminary account show a retention in the body of 8 to 10 per cent. of the carbon of the metabolized proteids, and to this extent confirm the earlier results obtained by his father. He believes the observed gain of carbon to be too great to be accounted for by the storage of glycogen and interprets it as showing a production of fat from proteids.

* Includes a correction of Pettenkofer \& Voit's figures for the urinary nitrogen: Loc. cit. p. 263.

† Thier. Chem. Ber., 22, 34. 
Kaufmann likewise interprets the results of the respiration experiments cited in another connection on p. 99 as demonstrating the production of fat from proteids, but in view of the brevity of the experiments (five hours), and the fact that they covered the period of most active nitrogen cleavage, this conclusion seems hardly justified.

Cremer * has reported the results of an experiment upon a cat which he regards as showing a formation of fat from proteids. The animal, weighing $3.7 \mathrm{kgs}$., passed eight days continuously in the respiration apparatus and received per day 450 grams of lean meat. No complete nitrogen and carbon balance is reported. The average daily excretion of nitrogen was 13 grams. Assuming the ratio of nitrogen to carbon in fat and glycogen-free flesh to be $1: 3.2, \dagger$ this corresponds to 41.6 grams of carbon in the form of proteids, while the total excretion of this element was only 34.3 grams, thus showing a retention by the organism of 7.3 grams per day. The body of the animal at the close of the experiment was found to contain not more than 35 grams of glycogen and sugar, while the observed gain of carbon during the eight days was equivalent to about 130 grams of glycogen. It is therefore concluded that fat was formed from proteids. In three other experiments, with an abundant meat diet, it is computed that from 12.6 to 17.0 per cent. of the carbon of the metabolized proteids was stored in the body.

Gruber $\ddagger$ has recently reported two experiments, dating from the year 1882, in which a dog was fed 1500 grams per day of lean meat. The nitrogen of feces and urine were determined daily for six and eight days respectively and the carbon dioxide of the respiration on five days in each experiment; the carbon of urine and feces and of the metabolized proteids was computed from the nitrogen, using for the carbon of the proteids the factor 3.28. The excretion of nitrogen approximately equaled the supply, especially on the later days of the experiments, but from 10 to 15 per cent. of the carbon was unaccounted for in the excreta. The total retention of carbon during the experiments, together with the equivalent quantities of glycogen, were:

* Jahresb. Agr. Chem., 40. 538; Zeit. f. Biol., 38, 309.

$\dagger$ Köhler (Zeit. f. physiol. Chem., 31,479) found an average of 1:3.16 for the fat-free flesh of cattle, swine, sheep, rabbits, and hens. See p. 64 .

$\ddagger$ Zeit f Biol., 42, 409 . 


\begin{tabular}{|c|c|c|}
\hline & Carbon. & Equivalent Glycogen. \\
\hline $\begin{array}{c}\text { Experiment } \\
\text { 1, } \\
\text { 2, eight }\end{array}$ & $\begin{array}{l}113.9 \text { Grms. . } \\
195.9\end{array}$ & $\begin{array}{l}256.3 \text { Grms. } \\
441.0\end{array}$ \\
\hline
\end{tabular}

These amounts of glycogen are much greater than have ever been found in the body of a dog of this weight (about $20 \mathrm{kgs}$.), and the larger part of the storage of carbon must therefore have been in the form of fat.

In addition to the above results on normal animals, Polimanti * has reported experiments apparently showing a formation of fat from proteids in phosphorous poisoning. The latter investigation, as well as those of Cremer and of E. Voit, have been the subjects of searching criticism by Pflüger, $\dagger$ who claims to have shown the insufficiency of the experimental evidence adduced, but it is impossible here to enter into the details of these controversial articles. Negative results have also been reported by Kumagawa \& Kaneda, $\ddagger$ Rosenfelt, $\$$ Taylor, „| Athanasiu, $\uparrow$ and Lindemann, ${ }^{* *}$ but in a matter of this sort negative evidence naturally carries much less weight than positive, and on the whole there would appear to be good reason for still regarding the proteids as a possible source of fat.

Difficulty of Proof.-A serious difficulty in the way of an unquestionable demonstration of this possibility lies in the limited amount of proteids which an animal can consume. As we have seen, a relatively large supply of them is necessary even to produce nitrogen equilibrium, and a still further large addition is required to supply the demands of the organism for energy. Only the proteids supplied in excess of this latter amount are available for fat production, and thus it comes about that the limit of consumption and digestion by the animal is reached before any very large production of fat can take place.

On the other hand, if non-nitrogenous nutrients (carbohydrates

* Arch. ges. Physiol., 70, 349.

† Ibid., 68, 176; 71, 318.

$\ddagger$ U. S. Dept. Agr., Expt. Station Record, 8, 71.

\&Jahresb. Physiol., 6, 260.

|| Ibid. . 8, 249, Jour Exper. Medicine, 4, 399.

T Arch. ges. Physiol., 74, 511.

** Zeit. f. Biol., 39, 1. 
for example) are employed to supply a part of the necessary energy, a more abundant fat production may be caused but the results are ambiguous, sinçe it is possible that the non-nitrogenous residue of the proteids may be metabolized to furnish energy otherwise supplied by the non-nitrogenous nutrients and that the actual material for the formation of fat may come from the latter.

That proteids added to a mixed ration may give rise to a large amount of fat has been strikingly shown by Kellner* in experiments on oxen in which wheat gluten was added to a fattening ration. Approximately 198 grams of fat were produced for each kilogram of protein fed, but to the writer the reasoning by which Kellner seeks to prove that this fat must have been derived directly from the proteids seems inconclusive.

Finally, as was indicated in Chapter II (p. 50), the apparently well-established fact that the metabolism of proteids in the body gives rise to the formation of carbohydrates (or at least may do so), together with the further fact that fat is undoubtedly formed from carbohydrates, renders it difficult to assign any reason why the nonnitrogenous residue of the proteids should not supply material to the cells of the adipose tissue for the production of fat.

\section{$\S 2$. The Non-nitrogenous Nutrients.}

Effects on the Proteid Metabolism.

The relations between proteid metabolism and proteid supply which have been outlined in the preceding section, while deduced mainly from experiments in which the food consisted substantially of proteids only, are of general applicability, yet are subject to important modifications in the presence of non-nitrogenous nutrients.

Tend to Diminish Proteid Metabolism.-As was first shown by $\mathrm{C}$. Voit, the addition of non-nitrogenous nutrients to a ration consisting of proteids tends to render the proteid metabolism less than it otherwise would be. The effect is common to the fats and carbohydrates, although with some differences in details.

FATS.-The following example, taken from Voit's experiments, $\uparrow$ illustrates in a somewhat marked way the influence of the addition of fat to proteid food upon the excretion of nitrogen. A dog consuming daily 1000 grams of lean meat received in addition on two days 100 and 300 grams of fat, with the following results:

* Landw. Vers. Stat., 53, 456.

$\dagger$ Zeit. f. Biol., 5, 334 . 


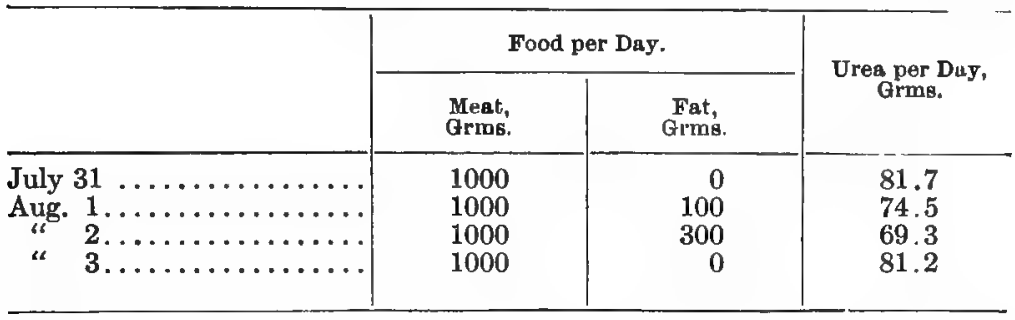

In the whole series of eight experiments with varying amounts of meat and fat the decrease in the excretion of urea ranged from 1 per cent. to 15 per cent. of the amount supplied in the food, averaging about 7 per cent. With the same amount of fat in the food the decrease in the excretion of urea was not, as a rule, greater with large than with moderate rations of meat. On the other hand, with a small proteid supply in the food the production of urea was sometimes increased slightly by the addition of much fat, and the same result was observed to a more marked extent when fat alone was given to fasting animals. With medium rations of meat, increasing the fat supply had usually little effect, but with heavy meat rations it tended to further diminish the excretion of urea.

Subsequent investigation has fully established this tendency of fat to diminish the proteid metabolism, and the fact is too well known to require extended illustration here. As a recent instance may be cited the following results obtained by Kellner * in experiments upon oxen, in which oil was added to a basal ration:

\begin{tabular}{|c|c|c|c|c|}
\hline & \multicolumn{2}{|c|}{ Nitrogen Digested. } & \multicolumn{2}{|c|}{ Nitrogen in Urine. } \\
\hline & $\begin{array}{c}\text { Basal Ration, } \\
\text { Grms. }\end{array}$ & $\begin{array}{c}\text { Basal Ration }+ \text { Oil, } \\
\text { Grms. }\end{array}$ & $\begin{array}{c}\text { Basal Ration, } \\
\text { Grms. }\end{array}$ & $\begin{array}{c}\text { Basal Ration + Oil, } \\
\text { Grms. }\end{array}$ \\
\hline 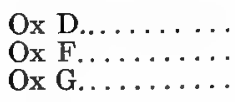 & $\begin{array}{r}135.30 \\
111.67 \\
86.27\end{array}$ & $\begin{array}{r}134.55 \\
109.17 \\
87.08\end{array}$ & $\begin{array}{r}122.54 \\
106.03 \\
86.30\end{array}$ & $\begin{array}{r}120.38 \\
89.27 \\
79.83\end{array}$ \\
\hline
\end{tabular}

CARBohydrates.-The effects of the readily soluble hexose carbohydrates (starch and the sugars) have been quite fully investigated, while as to those of the less soluble carbohydrates, particularlv of the five-carbon series, considerable diversity of opinion still prevails.

* Landw. Vers. Stat., 53, 121 and 210. 
Starch and Sugars.-The investigations of C. Voit* show that starch or sugar added to a proteid diet causes, as does fat, a decrease in the elimination of urea. Voit found an average decrease of about 9 per cent. in the proteid metabolism, the extremes being 5 and 15 per cent. with varying amounts of carbohydrates. An increase in the carbohydrates, the proteid food remaining the same, tended to further diminish the excretion of urea. The following examples illustrate this effect of the carbohydrates. When given to a fasting animal, carbohydrates did not, as in the case of fat, cause an increase in the proteid metabolism.

\begin{tabular}{|c|c|c|c|}
\hline & \multicolumn{2}{|r|}{ Food. } & \multirow{2}{*}{$\begin{array}{l}\text { Urea } \\
\text { per Day, } \\
\text { Grms. }\end{array}$} \\
\hline & $\begin{array}{l}\text { Meat, } \\
\text { Grms. }\end{array}$ & $\begin{array}{c}\text { Carbohydrates, } \\
\text { Grms. }\end{array}$ & \\
\hline 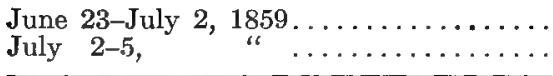 & $\begin{array}{l}500 \\
500\end{array}$ & $\begin{array}{c}300-100 \\
0\end{array}$ & $\begin{array}{l}35.4 \\
39.9\end{array}$ \\
\hline 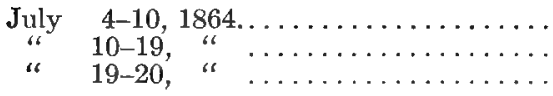 & $\begin{array}{l}800 \\
800 \\
800\end{array}$ & $\begin{array}{c}0 \\
100-400 \\
0\end{array}$ & $\begin{array}{l}59.1 \\
54.5 \\
63.8\end{array}$ \\
\hline 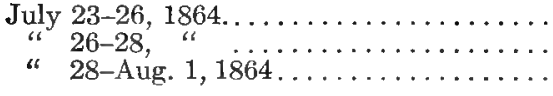 & $\begin{array}{l}1000 \\
1000 \\
1000\end{array}$ & $\underset{0}{100-400}$ & $\begin{array}{l}73.5 \\
64.4 \\
79.6\end{array}$ \\
\hline $\begin{array}{l}\text { June } 29-J u l y \\
\text { July } 8,1863 \ldots \ldots \ldots \ldots \ldots \ldots \ldots\end{array}$ & $\begin{array}{l}1500 \\
1500\end{array}$ & $\begin{array}{c}0 \\
200\end{array}$ & $\begin{array}{l}114.9 \\
103.3\end{array}$ \\
\hline 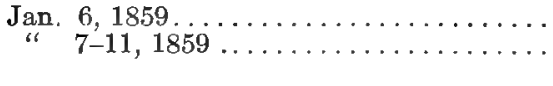 & $\begin{array}{l}2000 \\
2000\end{array}$ & $\begin{array}{c}0 \\
200-300\end{array}$ & $\begin{array}{l}143.7 \\
131.0\end{array}$ \\
\hline
\end{tabular}

This effect of the carbohydrates, like that of fat, has been abundantly confirmed by later investigators and is one of the well-established facts of physiology. Weiske $\uparrow$ in particular has investigated the effect of the non-nitrogenous nutrients upon the metabolism of sheep, while Miura $\ddagger$ and Iusk \& have shown that the abstraction of carbohydrates from the diet of a man results in a marked increase in the proteid metabolism. The following data, taken from Kellner's extensive respiration experiments at Möckern, illustrate the same effect of starch in the case of cattle:

* Zeit. f. Biol., 5, 434.

$\dagger$ Zeit. physiol. Chem. 21, 42; 22, 137 and 265.

¥ v. Noorden, Pathologie des Stoffwechsels, p. 117.

\& Zeit. f. Biol., 2T, 459. 


\begin{tabular}{|c|c|c|c|c|}
\hline & \multicolumn{2}{|c|}{ Nitrogen Digested. } & \multicolumn{2}{|c|}{ Nitrugen in Urine. } \\
\hline & $\begin{array}{c}\text { Basal Ration, } \\
\text { Grms. }\end{array}$ & $\begin{array}{c}\text { Basal Ration } \\
\text { + Stareh, } \\
\text { Grms. }\end{array}$ & $\begin{array}{l}\text { Basal Ration } \\
\text { Grms. }\end{array}$ & $\begin{array}{c}\text { Basal Ration } \\
\text { + Starch } \\
\text { Grms. }\end{array}$ \\
\hline 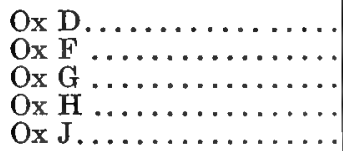 & $\begin{array}{r}135.30 \\
111.67 \\
86.27 \\
116.51 \\
128.11\end{array}$ & $\begin{array}{r}118.40 \\
107.55 \\
80.92 \\
94.66 \\
118.18\end{array}$ & $\begin{array}{r}122.54 \\
106.03 \\
86.30 \\
109.28 \\
122.62\end{array}$ & $\begin{array}{r}104.69 \\
81.18 \\
63.83 \\
81.71 \\
103.13\end{array}$ \\
\hline
\end{tabular}

Since the addition of starch to the basal ration diminished the apparent digestibility of the protein, the effect is most clearly seen by comparing the daily gains of nitrogen by the animals on the two rations, as follows:

\begin{tabular}{|c|c|c|c|}
\hline & $\begin{array}{c}\text { On Basal Ration, } \\
\text { Grms. }\end{array}$ & $\begin{array}{l}\text { With Addition } \\
\text { of Starch, } \\
\text { Grms. }\end{array}$ & Difference. \\
\hline 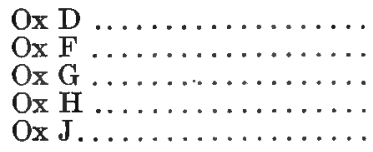 & $\begin{array}{r}12.76 \\
5.64 \\
-0.03 \\
7.23 \\
5.49\end{array}$ & $\begin{array}{l}13.71 \\
26.37 \\
17.09 \\
12.95 \\
15.05\end{array}$ & $\begin{array}{l}+0.95 \\
+20.73 \\
+17.12 \\
+5.72 \\
+9.56\end{array}$ \\
\hline
\end{tabular}

Cellulose.-The peculiar position occupied by cellulose, as the essential constituent of the "crude fiber" of feeding-stuffs, in the nutrition of domestic animals causes much interest to attach to the study of its effects upon metabolism. We shall consider here only its effects upon the proteid metabolism.

The first to take up this subject appears to have been v. Knieriem,* who experimented upon rabbits. In a preliminary experiment the addition of prepared "crude fiber" to a basal fiber-free ration in which the necessary bulk was obtained by the use of horndust $\nmid$ gave the following results for the urinary nitrogen per day:

I. Without fiber.... ....... . 0.9034 grams

II. With 9.284 grams fiber..... . . 0.7618

III. Without fiber. . . . . . . . . 0.7560

The low figure for the third period is ascribed to the effect of the crude fiber still remaining in the digestive tract. In a following series, in which respiration experiments were also made, the following results per day were obtained for the nitrogen:

* Zeit. f. Biol., 21, 67. † Shown to have been entirely indigestible. 


\begin{tabular}{|c|c|c|c|c|c|}
\hline & Period. & Food per Day. & $\begin{array}{l}\text { Nitrogen } \\
\text { of Food, * } \\
\text { Grms. }\end{array}$ & $\begin{array}{c}\text { Nitrogen } \\
\text { of Excreta. } \\
\text { Grms. }\end{array}$ & $\begin{array}{c}\text { Gain of } \\
\text { Nitrogen, } \\
\text { Grms. }\end{array}$ \\
\hline $\begin{array}{l}\text { I. } \\
\text { II. } \\
\text { III. } \\
\text { IV. } \\
\text { V. }\end{array}$ & $\begin{array}{l}9 \text { days. } \\
10 \text { days. } \\
5 \text { days. } \\
4 \text { days. } \\
3 \text { days. } \dagger\end{array}$ & 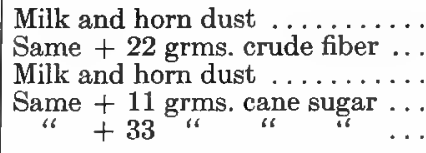 & $\begin{array}{l}2.75 \\
2.75 \\
2.70 \\
2.70 \\
2.70\end{array}$ & $\begin{array}{l}3.35 \\
2.65 \\
3.03 \\
3.02 \\
2.73\end{array}$ & $\begin{array}{l}-0.60 \\
+0.10 \\
-0.33 \\
-0.32 \\
-0.03\end{array}$ \\
\hline
\end{tabular}

Weiske $\ddagger$ disputes v. Knieriem's conclusion that cellulose diminishes the proteid metabolism. He experimented upon a sheep, which was fed in a first period exclusively on beans. In succeeding periods the effect upon the proteid metabolism of adding to this ration, first, inferior oat straw, and second, starch was tested, the bean ration being diminished slightly in these periods in order to keep the total digestible protein of the ration as nearly uniform as possible. On the basis of a preliminary digestion trial with the straw, the quantity of starch was so adjusted as to supply, in Period III, according to computation, an amount of digestible carbohydrates equal to the digested fiber and nitrogen-free extract of the straw of Period II, while in Period V it equalled the digested nitrogen-free extract only. Actual determinations of the digestibility of the mixed rations showed that this equality was approximately, although not exactly, reached, the amount of digested starch being rather less than the computed amount.

The results as regards the proteid metabolism as originally reported by Weiske are given in the first portion of the table on the opposite page. $\quad$ v. Knieriem, $\S$ having criticised the results on the ground that the metabolic nitrogen of the feces was not taken into account, and that when this was done the experiments made a more favorable showing for the digested crude fiber, Weiske $\|$ has recalculated his results on the assumption that the feces contained 0.4 grams of metabolic nitrogen for each 100 grams of dry matter digested, with the results shown in the second half of the table.

* Not including that of the horn dust.

$\uparrow$ Resuits regarded by the author as of doubtful value

$\ddagger$ Zeit. f. Biol., 22, 373.

§Ibid., 24, 293.

॥I Ibid., 24, 553.

T Compare Kellner, Landw. Vers. Stat., 24, 434; and Pfeiffer, Jour. f. Landw., 33, 149. 


\begin{tabular}{|c|c|c|c|c|c|c|c|c|}
\hline \multirow[b]{2}{*}{ 宫 } & \multirow{2}{*}{\multicolumn{2}{|c|}{ Ration. }} & \multicolumn{3}{|c|}{ Uncorrected. } & \multicolumn{3}{|c|}{ Corrected. } \\
\hline & & & $\begin{array}{c}\text { Nitro- } \\
\text { gen } \\
\text { Appar- } \\
\text { ently } \\
\text { Di- } \\
\text { gested. }\end{array}$ & $\begin{array}{c}\text { Nitro- } \\
\text { gen } \\
\text { of } \\
\text { Urine. }\end{array}$ & Gain, & $\begin{array}{c}\text { Com- } \\
\text { puted } \\
\text { Nitro- } \\
\text { gen } \\
\text { Di- } \\
\text { gested. }\end{array}$ & $\begin{array}{c}\text { Nitro- } \\
\text { gen } \\
\text { of } \\
\text { Urine. }\end{array}$ & Gain. \\
\hline I. & 500 grms. & beans. & $\begin{array}{l}\text { Grms. } \\
20.51\end{array}$ & $\begin{array}{l}\text { Grms. } \\
20.93\end{array}$ & $\begin{array}{l}\text { Grms, } \\
-0.42\end{array}$ & $\begin{array}{l}\text { Grins. } \\
22.02\end{array}$ & $\begin{array}{l}\text { Grrms. } \\
20.93\end{array}$ & $\begin{array}{l}\text { Grms. } \\
+1.09\end{array}$ \\
\hline II. & 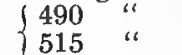 & $\begin{array}{l}\text { beans } \\
\text { straw }\end{array}$ & 19.58 & 16.82 & +2.76 & 21.78 & 16.82 & +4.96 \\
\hline IV. & $\left\{\begin{array}{l}490 \\
515\end{array}\right.$ & $\left.\begin{array}{l}\text { beans } \\
\text { straw }\end{array}\right\}$ & 18.81 & 17.26 & +1.55 & 21.09 & 17.26 & +3.83 \\
\hline & $\begin{array}{l}\text { Average of I] } \\
\text { (510 grms }\end{array}$ & $\begin{array}{l}\text { I. and IV. } \\
\text { beans }\end{array}$ & & & & & & +4.40 \\
\hline III. & $\left\{\begin{array}{r}180 \\
20 \\
500\end{array}\right.$ & $\left.\begin{array}{l}\text { starch } \\
\text { sugar }\end{array}\right\}$. & 20.03 & 14.94 & +5.09 & 22.16 & 14.94 & +7.22 \\
\hline V. & $\left\{\begin{array}{l}90 \\
10\end{array}\right.$ & $\left.\begin{array}{l}\text { starch } \\
\text { sugar }\end{array}\right\}$. & 20.64 & 17.75 & +2.89 & 22.43 & 17.75 & +4.68 \\
\hline
\end{tabular}

It will be seen that the experiments make substantially the same showing for the relative effects of cellulose and starch whether we take the uncorrected results or eliminate so far as possible the effects of the greater amount of food in the later periods upon the excretion of metabolic products in the feces. The addition of starch and sugar in Period III produced about twice as great an effect in reducing the proteid metabolism as did a somewhat larger amount of digestible fiber and nitrogen-free extract from straw in Periods II and IV. In Period V the starch added was only equal to the digested nitrogen-free extract of the straw in Periods II and IV. Since the effect upon the proteid metabolism is substantially the same, Weiske concludes that the nitrogen-free extract of the straw, which has the elementary composition of starch, is equal to it in its effect upon the proteid metabolism, and that the digested crude fiber is valueless in this respect. It must be said, however, that this latter conclusion is not warranted by the facts, since it rests upon the unproved assumption of equality of nutritive value (in respect of the proteid metabolism, at least) of starch and the nitrogen-free extract of the straw. Weiske also experimented with rabbits, finding in one case no effect upon the proteid metabolism and in the second an increase of it, as a result of adding crude fiber to a fiber-free ration. 
Lehmann * experimented upon a sheep by adding respectively crude fiber, prepared from wheat straw, and starch to a basal ration. The results were not entirely sharp but showed plainly a decrease of the proteid metabolism on the crude fiber ration which was equal approximately to 61 per cent. of that secured by the use of starch. In a second series of experiments, Lehmann and Vogel $\dagger$ compared the effects upon the proteid metabolism of sheep of canesugar and of the digestible non-nitrogenous matters of oat straw. On the basis of a very careful discussion of the experimental errors, they show that the latter substances have a marked effect in diminishing the proteid metabolism, and in particular that if we ascribe this effect exclusively to the digested nitrogen-free extract, as Weiske does, we must admit that the latter produced an effect from two to nine times as great as that of cane-sugar. They therefore conclude that their results show qualitatively an effect of the digested cellulose upon the proteid metabolism. Reckoning the digested nitrogen-free extract of the straw as equivalent to sugar, they compute from the average of all their experiments that the cellulose produced 75.7 per cent. as great an effect as the sugar, but they do not regard this quantitative result as well established.

Holdefleiss $\ddagger$ experimented upon two sheep, feeding in a first. period meadow hay exclusively. In the second period one half of the hay was replaced by a mixture of peanut cake, starch, and a little sugar, while in the third period the starch was replaced by paper pulp. In one case a fourth period was added in which the paper pulp and sugar were simply omitted from the ration. The digested nutrients and the proteid metabolism per day are tabulated on p.121.

Converting the small differences in the amount of crude fat digested into their equivalent in nitrogen-free extract by multiplication by the factor 2.5, Holdefleiss computes from a comparison of the second and third periods that the digested crude fiber produced on the first animal 80.1 per cent. and on the second animal 84.2 per cent. of the effect of the starch. A somewhat higher value would be obtained from a comparison of the first and second periods in the case of Sheep II, while on the other hand a comparison of the corresponding periods with Sheep I gives a much lower value, and is

* Jour. f. Landw., 37, 267.

$\ddagger$ Bied. Centr. Bl. Ag. Chem., 25, 372. 


\begin{tabular}{|c|c|c|c|c|c|c|c|}
\hline & \multirow[b]{2}{*}{ Ration. } & \multicolumn{4}{|c|}{ Apparently Digested. } & \multirow{2}{*}{$\begin{array}{l}\text { Nitro- } \\
\text { gen of } \\
\text { Urine, } \\
\text { Grms. }\end{array}$} & \multirow{2}{*}{$\begin{array}{l}\text { Gain } \\
\text { of } \\
\text { Nitro- } \\
\text { gen, } \\
\text { Grms. }\end{array}$} \\
\hline & & $\begin{array}{l}\text { Crude } \\
\text { Fat, } \\
\text { Grms. }\end{array}$ & $\begin{array}{l}\text { Crude } \\
\text { Fiber, } \\
\text { Grms. }\end{array}$ & $\begin{array}{c}\text { N. fr. } \\
\text { Extract, } \\
\text { Grms. }\end{array}$ & $\begin{array}{l}\text { Nitro- } \\
\text { gen, } \\
\text { Grms. }\end{array}$ & & \\
\hline \multirow{3}{*}{$\begin{array}{l}\text { Sheep } I . \\
\text { Period } 1 \\
6\end{array}$} & & & & \multirow[b]{2}{*}{470.85} & \multirow[b]{2}{*}{15.02} & \multirow[b]{2}{*}{13.83} & \multirow[b]{2}{*}{1.19} \\
\hline & Hay only ............. & 13.55 & & & & & \\
\hline & and starch ............... & 15.27 & 134.11 & 560.71 & 13.55 & 11.31 & 2.24 \\
\hline " 3 & $\begin{array}{l}\text { Hay, peanut cake, sugar, } \\
\text { and paper pulp ........ }\end{array}$ & 13.67 & & & & & 2.50 \\
\hline “ & Hay and peanut cake..... & 18.57 & 171.12 & 345.92 & 16.25 & 14.45 & 1.80 \\
\hline $\begin{array}{l}\text { Sheep II. } \\
\text { Period } 1\end{array}$ & Hay only & 1176 & 17192 & $|27649|$ & 1088 & $8 \quad 45$ & 94 \\
\hline & Hay, peanut cake, sugar, & & & & & & \\
\hline 3 & and starch $\ldots \ldots \ldots \ldots$ & $\mid 13.07$ & 77.48 & 336.62 & 9.54 & 7.85 & 1.69 \\
\hline & and paper pulp ........ & 15.14 & 235.31 & 198.46 & 8.82 & 7.62 & 1.20 \\
\hline
\end{tabular}

even consistent with the view that cellulose has no effect upon the proteid metabolism. In other words, the results on Sheep I, in the first period, appear inconsistent with the other results.

Kellner * has experimented with rye straw extracted with an alkaline liquid under pressure in the same manner as in papermaking and containing 76.78 per cent. of "crude fiber" and $\mathbf{1 9 . 9 6}$ per cent. of nitrogen-free extract. The results as regards the proteid metabolism, compared with those on starch, are given in the upper table of p. 122.

Taking the figures as they stand, and attempting no correction for the marked depression in the apparent digestibility of the nitrogen resulting from the addition of the extracted straw or starch, they show a considerable effect by both in diminishing the proteid metabolism relatively to the supply in the food and thus causing an increased gain of nitrogen by the body. Any correction for the metabolic nitrogen of the feces, as in Weiske's experiments, would, of course, tend to make the effect appear still greater. With the first animal, after taking account as well as possible of the slight differences in the fat digested in both periods and of the slight effect of the starch upon the digestibility of the fiber of the basal ration, the digestible matter of the extracted straw, five sixths of which was cellulose, appears to have produced more than twice as great an effect as an equal amount of starch. With the second * Landw. Vers. Stat., 53, 278. 


\begin{tabular}{|c|c|c|c|c|c|c|c|}
\hline & & \multicolumn{4}{|c|}{ Apparently Digested. } & \multirow{2}{*}{$\begin{array}{c}\text { Nitrogen } \\
\text { of Urine, } \\
\text { Grms. }\end{array}$} & \multirow{2}{*}{$\begin{array}{l}\text { Gain of } \\
\text { Nitroger } \\
\text { Grms. }\end{array}$} \\
\hline & & $\begin{array}{l}\text { Crude } \\
\text { Fat, } \\
\text { Grms. }\end{array}$ & $\begin{array}{l}\text { Crude } \\
\text { Fiber, } \\
\text { Grms. }\end{array}$ & $\begin{array}{l}\text { N. fr. } \\
\text { Extract } \\
\text { Grms. }\end{array}$ & $\begin{array}{l}\text { Nitrogen, } \\
\text { Grms. }\end{array}$ & & \\
\hline \multirow[t]{2}{*}{$\underset{\text { Period }}{O x}$} & \multirow{2}{*}{$\begin{array}{r}\text { Extracted straw.. } \\
\text { Basal ration...... } \\
\text { Difference...... }\end{array}$} & $\begin{array}{l}116 \\
101\end{array}$ & $\begin{array}{l}3129 \\
1083\end{array}$ & $\begin{array}{l}3351 \\
2912\end{array}$ & $\begin{array}{l}102.47 \\
116.51\end{array}$ & $\begin{array}{r}76.31 \\
109.28\end{array}$ & $\begin{array}{r}26.16 \\
7.23\end{array}$ \\
\hline & & 15 & 2047 & 439 & -14.04 & -32.97 & 18.93 \\
\hline \multirow[t]{2}{*}{ " 3} & Starch .............. & $\begin{array}{r}92 \\
101\end{array}$ & $\begin{array}{l}1057 \\
1083\end{array}$ & $\begin{array}{l}4773 \\
2912\end{array}$ & $\begin{array}{r}94.66 \\
.116 .51\end{array}$ & $\begin{array}{r}81.71 \\
109.28\end{array}$ & $\begin{array}{r}12.95 \\
7.23\end{array}$ \\
\hline & Difference..... . & -9 & -26 & 1861 & -21.85 & -27.57 & 5.72 \\
\hline \multirow[t]{2}{*}{$\begin{array}{l}O x J \\
\text { Period } 5 \\
\end{array}$} & $\begin{array}{l}\text { Extracted straw. } \\
\text { Basal ration..... }\end{array}$ & $\begin{array}{l}110 \\
107\end{array}$ & $\begin{array}{l}3101 \\
1114\end{array}$ & $\begin{array}{l}3344 \\
2895\end{array}$ & $\begin{array}{l}112.19 \\
128.11\end{array}$ & $\begin{array}{r}95.80 \\
122.62\end{array}$ & $\begin{array}{r}16.39 \\
5.49\end{array}$ \\
\hline & Difference...... & 3 & 1987 & 449 & -15.92 & -26.82 & 10.90 \\
\hline \multirow[t]{2}{*}{ “ } & 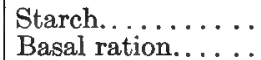 & $\begin{array}{r}85 \\
107\end{array}$ & $\begin{array}{l}1105 \\
1114\end{array}$ & $\begin{array}{l}4396 \\
2895\end{array}$ & $\begin{array}{l}118.18 \\
128.11\end{array}$ & $\begin{array}{l}103.13 \\
122.62\end{array}$ & $\begin{array}{r}15.05 \\
5.49\end{array}$ \\
\hline & Difference. & -22 & -9 & 1501 & -9.93 & -19.49 & 9.56 \\
\hline
\end{tabular}

animal, on the contrary, the effect of the digested matter of the extracted straw was but little more than two thirds that of the starch.

Ustjantzen * has recently reported the results of an experiment upon a sheep substantially like those of Weiske (p. 118), a basal ration of beans receiving, in succeeding periods, additions of meadow hay, rice, or sugar, the two latter being computed to supply an amount of digestible carbohydrates equal to the digestible nitrogenfree extract supplied by the hay. The increased amounts of crude fiber and nitrogen-free extract digested and the resulting increases in the gain of nitrogen by the animal were as follows:

\begin{tabular}{|c|c|c|c|c|}
\hline & & $\begin{array}{l}\text { Crude Fiber, } \\
\text { Grms. }\end{array}$ & $\begin{array}{l}\text { Nitrogen-free } \\
\text { Extract, } \\
\text { Grms. }\end{array}$ & $\begin{array}{c}\text { Gain of } \\
\text { Nitrogen, } \\
\text { Grms. }\end{array}$ \\
\hline $\begin{array}{c}\text { From hay ration } \\
\text { " rice " } \\
\text { sugar " }\end{array}$ & $\begin{array}{l}\ldots \ldots \ldots \ldots \ldots \\
\cdots \ldots \ldots \ldots \\
\cdots \ldots \ldots \ldots\end{array}$ & $\begin{array}{r}108.60 \\
-2.53 \\
5.07\end{array}$ & $\begin{array}{r}95.55 \\
107.15 \\
109.20\end{array}$ & $\begin{array}{l}3.33 \\
2.90 \\
2.59\end{array}$ \\
\hline
\end{tabular}

It appears that, as in Weiske's experiments, the carbohydrates of the rice and sugar produced nearly as great an effect upon the * Landw. Vers. Stat., 56, 463. 
gain of nitrogen as the total non-nitrogenous matter digested from the hay, and the author follows Weiske in concluding that the digested crude fiber producd but little effect on the proteid metabolism.

The same author also reports experiments upon a rabbit similar to those of v. Knieriem, crude fiber prepared from hay being added to a basal ration of peas, with the following results, which show practically no effect of the crude fiber upon the proteid metabolism:

\begin{tabular}{|c|c|c|c|c|c|}
\hline \multirow{2}{*}{ Food. } & \multirow{2}{*}{$\begin{array}{l}\text { Nitrogen } \\
\text { of Food, } \\
\text { Grms. }\end{array}$} & \multicolumn{3}{|c|}{ Nitrogen Excreted. } & \multirow{2}{*}{$\begin{array}{c}\text { Gain of } \\
\text { Nitrogen } \\
\text { Grms. }\end{array}$} \\
\hline & & $\begin{array}{l}\text { Urine, } \\
\text { Grms. }\end{array}$ & $\begin{array}{l}\text { Feces, } \\
\text { Grms. }\end{array}$ & $\begin{array}{l}\text { Total, } \\
\text { Grms. }\end{array}$ & \\
\hline 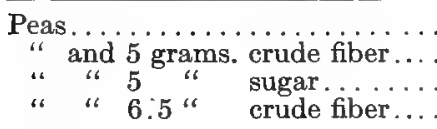 & $\begin{array}{l}0.845 \\
0.857 \\
0.845 \\
0.860\end{array}$ & $\begin{array}{l}0.855 \\
0.821 \\
0.701 \\
0.899\end{array}$ & $\begin{array}{l}0.016 \\
0.120 \\
0.080 \\
0.170\end{array}$ & $\begin{array}{l}0.871 \\
0.941 \\
0.781 \\
1.069\end{array}$ & $\begin{array}{r}-0.026 \\
-0.084 \\
+0.064 \\
-0.209\end{array}$ \\
\hline
\end{tabular}

While it is obviously unsafe to draw any positive conclusions regarding the relative effect of cellulose and the more soluble carbohydrates from the various experiments cited above, the balance of evidence seems clearly to show that their influence upon the proteid metabolism is qualitatively the same, while it appears on the whole probable that digested cellulose is at least not greatly inferior quantitatively to digested starch.

Organic Acids.-Certain methods of preparing or preserving fodder, notably ensilage, result in the formation of not inconsiderable amounts of organic acids. Moreover, it appears that these acids are normally produced in considerable quantities in the herbivora by the fermentation of cellulose and other carbohydrates, and that fact naturally leads to a consideration of their effects upon metabolism as compared with the latter substances.

We have seen (p. 27) that the organic acids are oxidized in the body, and it therefore seems natural to suppose that they may influence the proteid metabolism. This question has been investigated by Weiske \& Flechsig.* After some only partially successful experiments on a rabbit, they fed a sheep with a basal ration (of hay, starch, cane-sugar and peanut cake) containing a liberal supply of protein and having a nutritive ratio of $1: 3.4$. To this ration there was added in succeeding periods lactic acid as calcium lactate, acetic acid as sodium acetate, and for comparison dextrose. * Jour. f. Landw., 37, 199. 
Disregarding for our present purpose the slight effect of these substances upon the digestibility of the non-nitrogenous ingredients of the ration the results were:

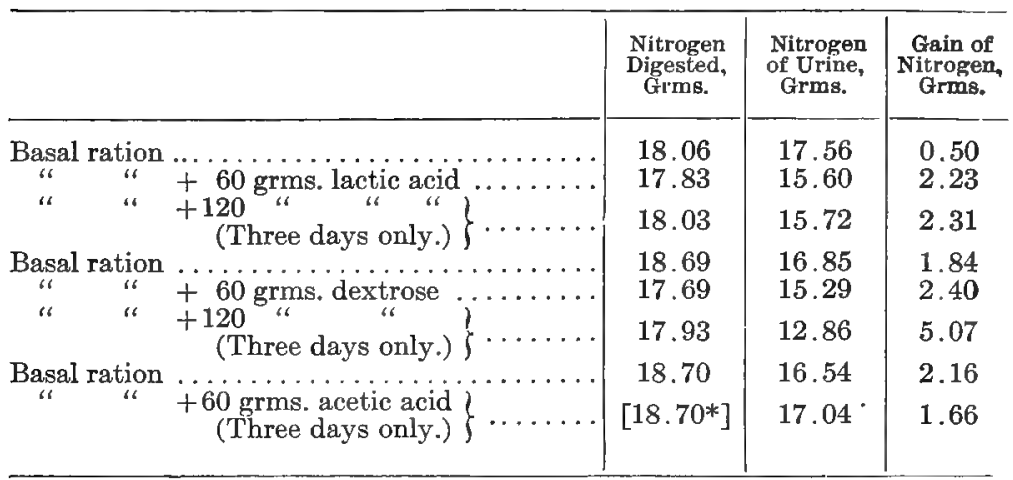

The smaller amount of lactic acid seems to have produced as great an effect in reducing the proteid metabolism as an equal weight of dextrose, but no further effect was noted from an increase in its amount, as was the case with the dextrose. The acetic acid, on the contrary, seems to have had a tendency to increase rather than to diminish the proteid metabolism, and the same effect was indicated in one of the experiments on a rabbit. It is to be remarked, however, that the sodium acetate appeared to be particularly obnoxious to the animals. In the case of the sheep it was introduced into the stomach in solution by means of a funnel, and besides causing the animal considerable discomfort had a very marked diuretic action. It may perhaps be questioned whether the results obtained under such conditions represent the normal effects of acetic acid.

Pentose Carbohydrates.- While the fate of the pentose carbohydrates in the body has been the subject of considerable research (compare Chapter II, p. 24), their effect upon the proteid metabolism does not seem to have been specifically investigated, although Pfeiffer \& Eber, $\dagger$ in the course of experiments upon the origin of hippuric acid, observed that after the consumption of 500 grams of

* Assumed to be the same as with the basal ration. The actual nitrogen of the feces for these three days was 4.78 grms., making the apparently digested nitrogen $19.33 \mathrm{grms}$.

+Landw. Vers. Stat., 49, 137. 
cherry gum, containing 41.98 per cent. of pentose carbohydrates, the urinary nitrogen of a horse decreased by over 6 per cent. They leave it uncertain, however, whether the effect was due to the pentosans or to other ingredients of the gum.

Among the early experiments of Grouven * are also four in which gum arabic, added to an exclusive straw ration, materially reduced the proteid metabolism, but the methods of these early experiments were naturally somewhat defective. On the other hand, Cremer's experiments $\dagger$ with rhammose on rabbits showed no marked effect of this substance upon the proteid metabolism.

Total Non-nitrogenous Matter of Feeding-stuffs.-The digestible non-nitrogenous matters of feeding-stuffs, aside from a small proportion of fat, are commonly although loosely grouped together as carbohydrates. They include both hexose and pentose carbohydrates, such organic acids as may be present or as are formed during digestion, and a variety of other less well-known substances.

As has already appeared in discussing the effect of crude fiber, the mixture of material included in the digestible crude fiber and nitrogen-free extract shows the same tendency as starch and sugar to diminish the proteid metabolism. In other words, while the common designation of digestible carbohydrates may be of questionable accuracy from a chemical point of view, nevertheless the somewhat heterogeneous mixture to which it is applied behaves, in this respect at least, qualitatively like the pure hexose carbohydrates. Numerous instances of this are cited by $\mathrm{v}$. Wolff $\ddagger$ in his discussion of the data prior to 1876 . Of more recent results, attention may be specially called to those of Kellner, some of which have been cited above. The results upon coarse fodders are those which are of particular interest, since it is these whose ingredients are least known chemically. They are presented on the following page in the same form as those upon extracted straw above.

Although the addition of hay or straw to the basal ration increased the supply of digestible nitrogenous matter, the proteid metabolism was not proportionately increased, but in every instance

*Wolff, Ernährung Landw. Nutzthiere, p. 289.

$\dagger$ Zeit. f. Biol., 42, 451 .

\$ Ernährung Landw. Nutzthiere, pp. 288-309. 


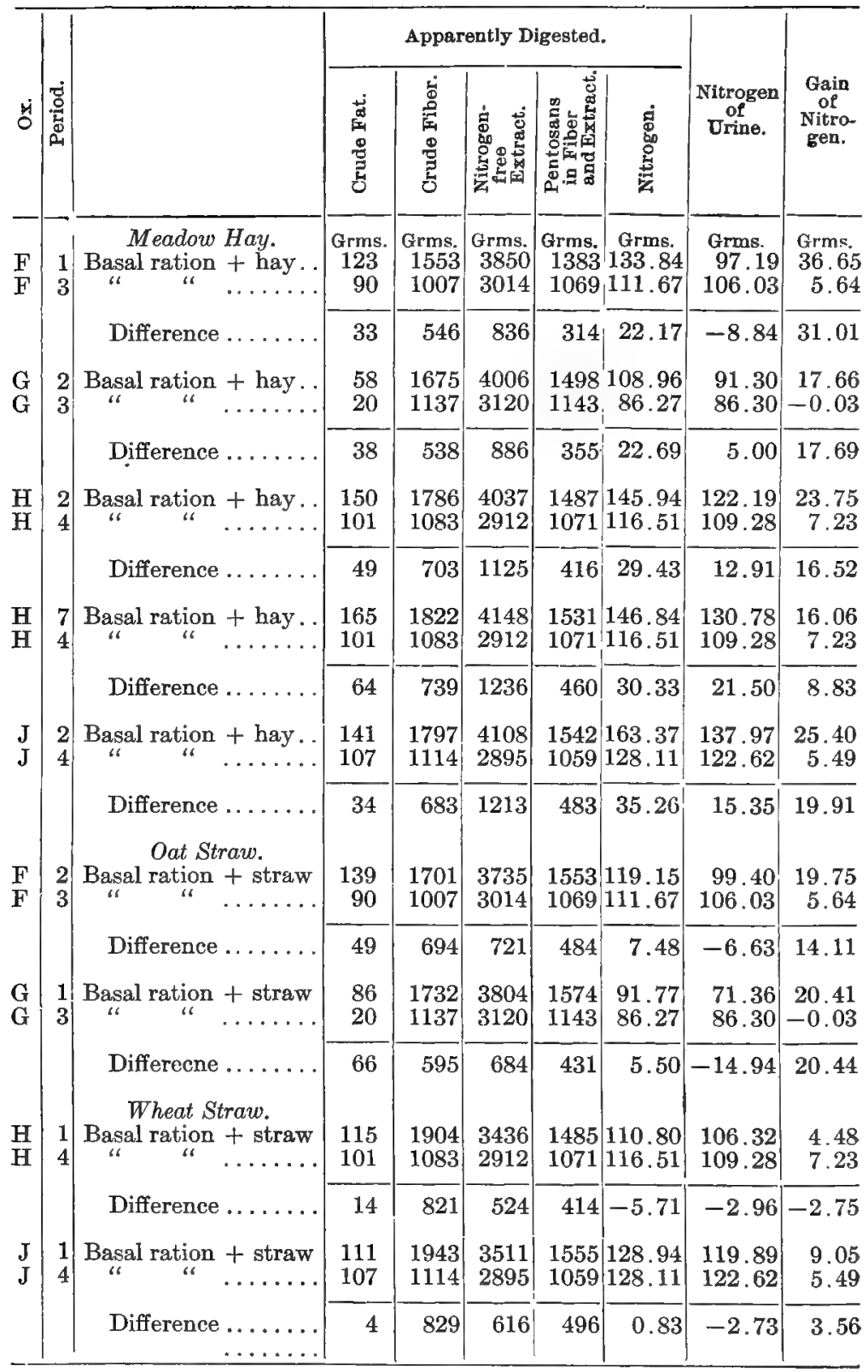


save one the gain of nitrogen by the body showed a marked increase, and this, it is to be noted, after the feeding had been continued for a considerable time. The one exceptional case, on wheat straw, is readily explained by the obvious effect of this material in increasing the metabolic nitrogen of the feces and thus diminishing the apparent digestibility of the protein of the ration. Had account been taken of these metabolic products, the increased gain of nitrogen by the animals would doubtless have been more marked in all cases. This gain, it would seem, may fairly be ascribed to the large additions of digestible non-nitrogenous matter derived from the hay or straw added.

Comparative Effects of Fat and Carbohydrates.-C. Voit* found the hexose carbohydrates to be superior to fat in diminishing the proteid metabolism. He gives the following comparisons:

\begin{tabular}{|c|c|c|c|}
\hline \multirow{2}{*}{ Date. } & \multicolumn{2}{|r|}{ Food per Day. } & \multirow{2}{*}{$\begin{array}{l}\text { Urea } \\
\text { per Day, } \\
\text { Grms. }\end{array}$} \\
\hline & $\begin{array}{l}\text { Meat, } \\
\text { Grms. }\end{array}$ & $\begin{array}{c}\text { Carbohydrates or Fat, } \\
\text { Grms. }\end{array}$ & \\
\hline $\begin{array}{r}\text { Nov. } 16-22,1857 \ldots \ldots \ldots \ldots \ldots \\
22-D e c .2,1857 \ldots \ldots \ldots \ldots\end{array}$ & $\begin{array}{l}150 \\
150\end{array}$ & $\begin{array}{l}\text { 150-350 sugar } \\
250 \text { fat }\end{array}$ & $\begin{array}{l}13.4 \\
15.6\end{array}$ \\
\hline $\begin{array}{l}\text { Oct. } 28-N o v .8,1857 \ldots \ldots \ldots \ldots \\
\text { Nov. } 8-15, \quad{ }^{2}, \ldots \ldots \ldots \ldots\end{array}$ & $\begin{array}{l}176 \\
176\end{array}$ & $\begin{array}{c}100-364 \text { starch } \\
250 \text { fat }\end{array}$ & $\begin{array}{l}15.1 \\
16.2\end{array}$ \\
\hline $\begin{aligned} \text { Feb. } & 23-25,1861, \ldots \ldots \ldots \ldots \ldots \\
\text { " } & 25-28, \\
& 28-M c h .3,1861 \ldots \ldots \ldots \ldots\end{aligned}$ & $\begin{array}{l}400 \\
400 \\
400\end{array}$ & $\begin{array}{l}200 \text { fat } \\
250 \text { starch } \\
250 \text { sugar }\end{array}$ & $\begin{array}{l}31.9 \\
30.5 \\
30.3\end{array}$ \\
\hline $\begin{array}{c}\text { June } 19-23,1859 \ldots \ldots \ldots \ldots \ldots \ldots \\
\text { “ } 23-26, \text { “ } \ldots \ldots \ldots \ldots \ldots \ldots \ldots \\
\text { “ } 26-29, \text { “ } 29 \ldots \ldots \ldots \ldots \ldots\end{array}$ & $\begin{array}{l}500 \\
500 \\
500 \\
500\end{array}$ & $\begin{array}{l}250 \text { fat } \\
300 \text { sugar } \\
200 \text { " } \\
100\end{array}$ & $\begin{array}{l}38.5 \\
32.7 \\
35.6 \\
37.9\end{array}$ \\
\hline 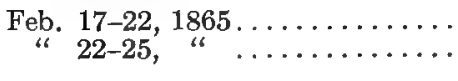 & $\begin{array}{l}800 \\
800\end{array}$ & $\begin{array}{l}250 \text { starch } \\
200 \text { fat }\end{array}$ & $\begin{array}{l}52.8 \\
54.7\end{array}$ \\
\hline 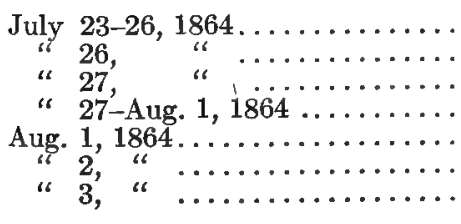 & $\begin{array}{l}1000 \\
1000 \\
1000 \\
1000 \\
1000 \\
1000 \\
1000\end{array}$ & $\begin{array}{l}0 \\
100 \text { starch } \\
400 \\
0 \\
100 \text { fat } \\
300 " \\
0\end{array}$ & $\begin{array}{l}73.5 \\
68.5 \\
60.2 \\
79.6 \\
74.5 \\
69.3 \\
80.2\end{array}$ \\
\hline Jan $7-12,1859 \ldots \ldots \ldots \ldots \ldots \ldots$ & $\begin{array}{l}2000 \\
2000\end{array}$ & $\begin{array}{c}200-300 \text { starch } \\
250 \text { fat }\end{array}$ & $\begin{array}{l}128.4 \\
135.9\end{array}$ \\
\hline
\end{tabular}

* Zeit. f. Biol., 5, 447. 
Subsequent investigations have substantially confirmed this conclusion. Thus Kayser * in an experiment upon himself found that the replacements of the carbohydrates of his diet by an amount of fat equivalent to them in heat value caused a marked increase in the urinary nitrogen, resulting in a loss of this element by the body in place of the previous small gain. The possible effect upon the apparent digestibility of the proteids of the food does not appear to have been considered.

Wicke \& Weiske † report two series of experiments upon sheep in which equivalent ("isodynamic") quantities of fat and of starch were added to a basal ration. In the first series the basal ration was comparatively poor in proteids and fat, having a nutritive ratio of about 1:8.3; in the second series it was richer in both these substances and had a nutritive, ratio of $1: 5.1$ and $1: 6.3$ for the two animals respectively. As is usually the case, the starch diminished the apparent digestibility of the protein of the basal ration, while the fat produced but a slight effect in this direction. Notwithstanding this complication, however, the effect of the starch in diminishing the proteid metabolism was clearly greater than that of the fat, and if the results were corrected for the increase in the nitrogenous metabolic products in the feces they would be still more decisive.

The investigations of E. Voit \& Korkunoff upon the minimum of proteids, which will be considered in a subsequent paragraph, also show a superiority in this respect of the carbohydrates over the fats which these authors ascribe to the greater lability of their molecular structure which enables them to enter into reactions in the body more readily than the fats.

Magnitude and Duration of the Effect.-The pre-eminent position of the proteids in nutrition has perhaps led investigators to attach undue importance to this power of the non-nitrogenous nutrients to diminish the proteid metabolism. It is well to note that it is relatively small. C. Voit, as already stated, found an average decrease of about 7 per cent. with fats and about 9 per cent. with carbohydrates, and subsequent investigators have obtained results entirely comparable with these.

Proteid Metabolism Determined by Supply.-In the presence *v. Noorden, Pathologie des Stoffwechsels, p. 117.

†Zeit. physiol. Chem., 21, 42; 22, 137. 
of non-nitrogenous nutrients it is still true that the proteid metabolism, or more exactly the excretion of nitrogen, is mainly determined by the supply of it in the food just as it is upon an exclusive. proteid diet. Fat or carbohydrates simply produce a relatively small, and probably more or less transitory, diminution of it without affecting the substantial truth of the above statement.

Lawes \& Gilbert,* in discussing the results of their fattening. experiments upon sheep and pigs, called attention to the very wide variations in the amount of protein consumed, both per unit of weight and especially per unit of gain, and concluded that the apparent excess of protein in some cases must have served substantially for respiratory purposes. The subsequent investigations of Bischoff, Voit, and v. Pettenkofer upon the proteid metabolism of carnivora showed clearly that the dependence of the latter upon the proteid supply, which is so marked upon a purely proteid diet, is equally evident upon a mixed diet, and thus supplied a scientific explanation of the facts observed by laawes \& Gilbert. The effect of the proteid supply upon the nitrogen excretion is clearly shown by the following summary of Voit's experiments: $\dagger$

\begin{tabular}{|c|c|c|}
\hline \multicolumn{2}{|c|}{ Food. } & \multirow{2}{*}{$\begin{array}{c}\text { Urea Excreted, } \\
\text { Grms. }\end{array}$} \\
\hline $\begin{array}{l}\text { Fat. } \\
\text { Grms. }\end{array}$ & $\begin{array}{l}\text { Lean Meat. } \\
\text { Grms. }\end{array}$ & \\
\hline 250 & 150 & 17.0 \\
\hline 300 & 176 & 18.9 \\
\hline 250 & 250 & 19.7 \\
\hline 200 & 500 & 36.6 \\
\hline 200 & 800 & 56.7 \\
\hline 250 & 1500 & 100.7 \\
\hline
\end{tabular}

Since Voit's researches, very many experiments, among the earliest of which were those of Henneberg \& Stohmann $\ddagger$ upon cattle, have confirmed his results, both for carnivora, herbivora and omnivora. A somewhat striking example is afforded by Stohmann's $\S$ experiments upon milch goats which are summarized in the following table:

* Rep. Brit Asso. Adv. Sci., 1852; Rothamsted Memoirs, Vol. II.

$\uparrow$ Zeit. f. Biol , 5, 329.

$\ddagger$ Beiträge, etc., Heft 2, p 412.

$\S$ Biologische Studien, 121. 


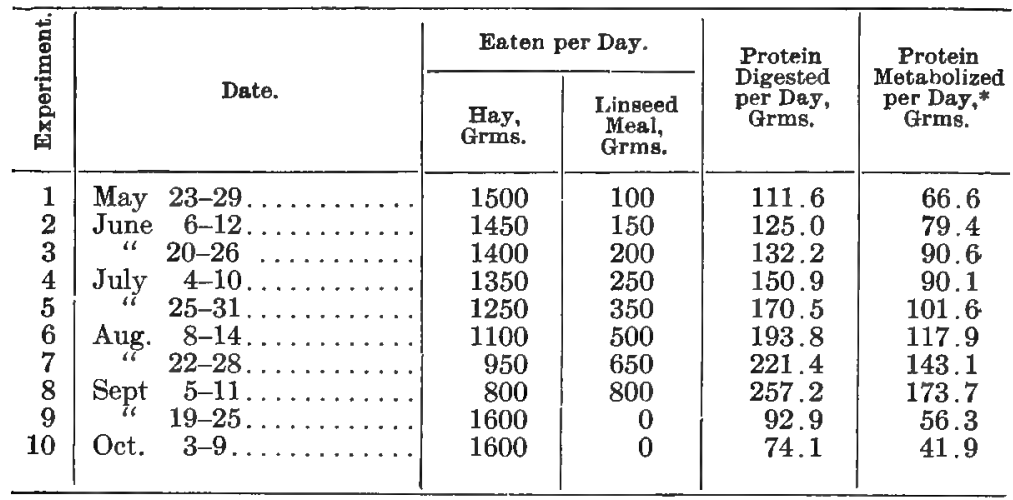

A full compilation of these earlier results has been made by $v$. Wolff, $\uparrow$ and the fact is now so well established that further citations would be superfluous.

Rate of Nitrogen Excretion.-Some interesting hints as to the manner in which the non-nitrogenous nutrients produce the effect upon the proteid metabolism which has just been described are afforded by a consideration of the rate of nitrogen excretion under their influence.

It was shown in the preceding section that the effect of a meal of proteids was a sudden, almost explosive, increase in the nitrogen cleavage and excretion, reaching its maximum within a few hours after the meal. If, however, non-nitrogenous nutrients are given along with the proteids, the character of the curve is essentially altered, the maximum rate of excretion being less and being reached somewhat later, while the fall from this maximum is less rapid. In other words, the rate of excretion becomes more uniform-the curve is flattened out. The influence of fat in this respect is clearly shown in the experiments of Panum $\ddagger$ and of Feder $\ddagger$ cited previously, and appears evident also in those of Graffenberger. § In the latter experiments the nitrogenous substances to be tested were added to a mixed diet. The results show a distinct maximum, but the rate of decrease after the maximum was reached was not rapid,

* Exclusive of the protein of the milk.

† Ernährung Landw. Nutzthiere, pp. 285-309

$\ddagger$ Thier. Chem. Ber., 12, 402.

§Zeit. f. Biol., 28, 318. 
and only a part of the nitrogen appeared during the twenty-four hours following its ingestion, viz.:

With fibrin................ 49.2 per cent.

With gelatin................... 37.6" "

With peptone................667.6 " "

With asparagin .............. 79.0 " "

Rosemann's * results upon the rate of nitrogen excretion by man, likewise cited above, indicate a similar effect of the nonnitrogenous nutrients, the fluctuations due to the ingestion of mixed food being much less sharp than those found by other experimenters with proteids alone.

If we accept Rosemann's view (p. 101), that the sudden increase in the nitrogen cleavage is due, in part at least, to a direct stimulus to the metabolic activity of the cells, arising from the presence in the fluids of the body of an increased percentage of proteids, we may perhaps suppose that the simultaneous resorption of nonnitrogenous matter renders this stimulus less and so reduces the maximum rate of nitrogen cleavage. This conjecture possibly receives some support also from the results of Krummacher, $\dagger$ who, contrary to Adrian and Munk, finds that the division of the proteid ration into several meals not only renders the rate of nitrogen excretion more uniform, but reduces somewhat the total amount excreted. Gebhardt $\ddagger$ has also obtained similiar results.

There is also the possibility, however, that the non-nitrogenous nutrients may modify the rate at which the proteids are resorbed, or perhaps, as has been suggested by various investigators, the extent to which the proteids are converted into amide-like bodies by the pancreatic juice or the extent of proteid putrefaction in the intestines. Suggestive in this regard is the fact found by Gruber $\S$ that common salt, which acts as a stimulant to thesecretion of hydrochloric acid by the stomach, and would thus tend to favor gastric as compared with intestinal digestion of the proteids, produces an effect on the nitrogen excretion similar to that of the non-nitrogenous nutrients.

*Arch. ges. Physiol., 65, 343.

$\uparrow$ Zeit. f. Biol., 35, 481 .

$\ddagger$ Arch. ges. Physiol., 65, 611.

$\S$ Zeit. f. Biol., 42, 425 . 
Extent of Protein Storage.-Whatever may be the explanation of the action of the non-nitrogenous nutrients, its effect is obvious. Attention has already been called (p. 102) to Gruber's hypothesis that the transitory storage of nitrogen following an increase in the proteid supply is the result of a superposition of the daily curves of nitrogen excretion. The effect of the non-nitrogenous nutrients appears to be to diminish the rate of nitrogen cleavage. and to protract it, in the case of a single meal of proteids, over a longer time. Evidently, then, an increase of the proteid supply in a mixed diet, or the addition of non-nitrogenous nutrients to a proteid diet, will extend its effect over a considerably longer period than in case of an exclusive proteid diet-that is, nitrogen equilibrium will be reached more slowly, and there will be a longer or shorter time after the change during which the nitrogen excretion will be less than in the absence of the non-nitrogenous matters.

This explanation also implies, however, that the storage of nitrogenous matter in the body of the mature animal is of limited duration and that no long-continued gain of protein can occur; in other words, that it is impossible to materially increase the proteid tissue (lean meat) of a mature animal.

Numerous comparative fattening experiments with domestic animals, notably those of Henneberg, Kern, \& Wattenberg* upon sheep, fully sustain this conclusion. On the other hand, metabolism experiments with domestic animals rarely show an equality between the income of nitrogen and its outgo in feces and urine, but almost always indicate a gain of nitrogenous matter by the body. As regards the significance of this fact, however, several considerations must be borne in mind.

First, the normal growth of the epidermal tissues-hair or wool, hoofs, horns, etc.-as pointed out in Chapter III, consumes a portion of the nitrogen of the food and contributes its share to the storage of nitrogen in the body.

Second, the adipose tissue itself contains a small percentage of proteid matter, and a storage of fat in considerable amounts involves the production of new adipose tissue in which to store it.

Third, in many cases the metabolism experiments which show a storage of nitrogen have been made within a rather short time

$$
\text { *Jour. f. Landw., 26, } 549 .
$$


after a change in the ration, and can therefore be interpreted as showing simply that sufficient time had not elapsed to reach nitrogen equilibrium.

If we consider also the somewhat indefinite nature of the term mature, and likewise the possibilities of error due to mechanical losses of excreta and to escape of nitrogen from the latter by fermentation and decomposition, we can readily see why the results of a short metabolism experiment may not agree with those of a long fattening experiment; yet, nevertheless, it must be confessed that the impression left by a comparison of the whole mass of evidence is that the discrepancy is as yet but partially explained.

In conclusion, we may anticipate a discussion in Chapter VI, and call attention to the fact that muscular exertion may, to a limited extent at least, stimulate those constructive processes which result in a storage of protein in the body.

The Minimum of Proteids.-In the preceding section it appeared that the administration of proteid food to a previously fasting animal caused a prompt and large increase in the nitrogen cleavage and excretion, while but a comparatively small portion of the proteids was applied to constructive purposes, the result being that two to three times as much proteids must be given as are metabolized during fasting before nitrogen equilibrium is reached. This effect was there ascribed to the stimulating effect of the rapid digestion and resorption of the proteids upon the nitrogen cleavage, much of the proteids being apparently destroyed as such before they can serve for tissue-building.

We have just seen that the effect of the non-nitrogenous nutrients is to diminish somewhat the nitrogen cleavage, apparently by moderating this stimulating effect. The necessary result is that, as the nitrogen supply is increased, it and the nitrogen excretion will start more nearly together and approach each other more rapidly upon a mixed diet than upon one consisting of proteids only. Consequently, while the percentage decrease in the proteid metabolism is, as we have seen, relatively small, nitrogen equilibrium may be reached with a much smaller supply of proteids than is the case in the absence of the non-nitrogenous nutrients. Indeed, it is conceivable that a sufficient supply of carbohydrates or fats in the diet should practically destroy the stimulative effects of the proteids. in 
which case we might expect a proteid supply equal to the fasting proteid metabolism to be sufficient to produce nitrogen equilibrium. Seen in this light, the apparently insignificant effect of the nonnitrogenous nutrients becomes a very important factor in nutrition.

The effect of the non-nitrogenous nutrients in largely diminishing the necessary proteid supply was pointed out by C. Voit* and appears clearly in many of his experimental results. Thus from the summary on p. 95 it appears that from 1200 to 1500 grams of lean meat per day was required to maintain the animal experimented upon in nitrogen equilibrium. When fat or carbohydrates were added to the ration, however, strikingly different results were reached, as appears from the following comparative statement, the results being expressed as "flesh" with 3.4 per cent. of nitrogen:

\begin{tabular}{|c|c|c|c|c|}
\hline & \multicolumn{2}{|c|}{ Food. } & \multirow{2}{*}{$\begin{array}{l}\text { Flesh. } \\
\text { Meta- } \\
\text { bolized. }\end{array}$} & \multirow{2}{*}{$\begin{array}{l}\text { Gain of } \\
\text { Flesh. }\end{array}$} \\
\hline & Meat. & $\begin{array}{l}\text { Fat or Carbo- } \\
\text { hydrates. }\end{array}$ & & \\
\hline Meat only (average of both series). \{ & $\begin{array}{r}300 \\
600 \\
900 \\
1200 \\
1500\end{array}$ & $\begin{array}{l}\ldots \ldots \\
\cdots \ldots \\
\cdots \cdots \\
\cdots \cdots \\
\cdots \cdots \\
\cdots\end{array}$ & $\begin{array}{r}416 \\
674 \\
943 \\
1207 \\
1478\end{array}$ & $\begin{array}{r}-116 \\
-74 \\
-\quad 43 \\
-\quad 7 \\
+\quad 22\end{array}$ \\
\hline Meat and fat $\ldots \ldots \ldots \ldots \ldots$ & $\begin{array}{r}500 \\
800 \\
1000\end{array}$ & $\begin{array}{l}250 \\
200 \\
250\end{array}$ & $\begin{array}{l}444 \\
720 \\
875\end{array}$ & $\begin{array}{l}+56 \\
+80 \\
+125\end{array}$ \\
\hline $\begin{array}{l}\text { Meat and carbohydrates (compare } \\
\quad \text { p. } 116) \ldots \ldots \ldots \ldots \ldots \ldots \ldots\end{array}$ & $\begin{array}{r}500 \\
800 \\
1000\end{array}$ & $\begin{array}{l}300-100 \\
100-400 \\
100-400\end{array}$ & $\begin{array}{l}502 \\
763 \\
902\end{array}$ & $\begin{array}{l}-2 \\
+37 \\
+88\end{array}$ \\
\hline
\end{tabular}

In the presence of non-nitrogenous nutrients, nitrogen equilibrium was reached with quantities of proteids from one third to one half as great as the amount required when fed alone. In other words, the non-nitrogenous nutrients materially reduced the minimum of food proteids required to maintain the proteid tissues of the body.

In view of the peculiar importance of the proteids in nutrition, as well as of their relative scarcity and high cost, particularly in the food of our domestic animals, great interest attaches to a determination of the least amount required to sustain a mature * Zeit. f. Biol., 5. 
animal. The results obtained by F. Voit \& Korkunoff * regarding the minimum requirement upon an exclusive proteid diet have already been stated in the first section of this chapter (p. 95). The same investigators have also studied the more interesting question of how far the necessary proteid supply can be reduced in the presence of non-nitrogenous nutrients.

Proteids and Fat.-The experiments were upon the same general plan as those just referred to on proteids alone. Beginning with an insufficient quantity of proteids, the amount was gradually increased, that of the fat remaining constant, until nitrogen equilibrium was reached. As in those experiments, too, the nitrogen of the food was practically all in the proteid form, and its amount is compared with the proteid nitrogen excreted, it being assumed that 18.45 per cent. of the urinary nitrogen was derived from the extractives of the flesh metabolized in the body. To the writer it would seem that a more suitable unit would be the total excretory nitrogen, since the proteids of the food had to make good the loss of extractives as well as of true proteids from the body, and the former loss is as unavoidable as the latter. Accordingly, the results have been stated in the table below in both ways.

Two series of experiments were made: one in which the total food-supply was less than was required to supply the estimated demands of the body for energy, and one in which it considerably exceeded that demand, with the following results:

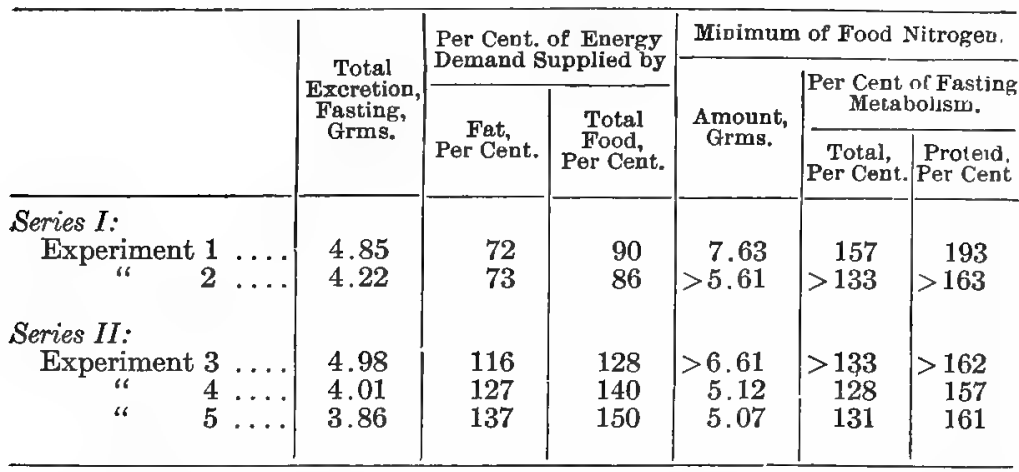

The authors also compute from experiments by C. Voit and by Rubner percentages lying between 162 and 207, and state as their

$$
\text { *Zeit. f. Biol., 32, } 58 .
$$


final result that the minimum of proteid nitrogen on a diet of proteids and fat lies between 160 and 200 per cent. of the proteid nitrogen excreted during fasting. These figures when computed on the total excretory nitrogen would become 131 per cent. and 163 per cent. respectively.

Proteids and Carbohydrates.-We have seen (p. 127) that the carbohydrates diminish the proteid metabolism to a greater extent than the fats. The results which have been reached as to their effect in lowering the minimum demand for proteids are on the whole in accord with this fact. With a liberal supply of carbohydrates in the food, a much smaller quantity of proteids would seem to suffice to maintain nitrogen equilibrium than when the non-nitrogenous matter of the ration consists of fat. Indeed, according to some investigators, the proteid metabolism may ever. be thus reduced much below that during fasting.

Munk * appears to have been the first to advance the view last mentioned. In an investigation upon the formation of fat from carbohydrates a dog was fasted for thirty-one days and then received a diet consisting of a little meat with large amounts of carbohydrates (starch and sugar) and also, during the first twelve days, gelatin. Omitting these twelve days and also the earlier days of the fasting period, the average daily excretion of nitrogen in the urine was

Twelfth to thirty-first days of fasting....... 5.38 grams

Thirteenth to twenty-fourth days of feeding (200 grams meat, 500 grams carbohydrates) .... 5.79 "

On the seventeenth day of the feeding the urinary nitrogen reached the minimum of 4.133 grams, and Munk regards this as showing the possibility of a reduction of the proteid metabolism considerable below the fasting level. It is to be noted, however, that the nitrogen excretion varied considerably from day to day, and a selection of a single day for comparison seems hardly justified.

Hirschfeld $\dagger$ and Kumagawa $\ddagger$ found that the nitrogen, equili-

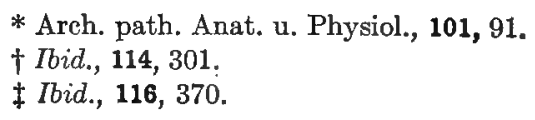


brium of man could be maintained on a diet containing little nitrogen but abundance of non-nitrogenous nutrients. Tnder these conditions the urinary nitrogen was reduced to 5.87 grams and 6.07 grams per day respectively, and the total nitrogen excretion to 7.45 grams and 8.10 grams, amounts much lower than have been observed for fasting men. Thus in the extensive investigations by Lehmann, Müller, Munk, Senator, \& Zuntz * of the metabolism of two fasting men, much higher figures than the above were obtained for the urinary nitrogen, and Munk (loc. cit., p. 225) calls attention to the fact that in one case the urinary nitrogen on the second day succeeding the fasting period was materially less than on the last day of the fasting, viz., 8.26 grams as compared with 9.88 grams.

In a subsequent series of experiments upon dogs, Munk $\uparrow$ showed that by very liberal feeding with food poor in proteids (rice with small amounts of meat) the nitrogen balance could be maintained for a considerable time at an amount very much lower than previous observers had found for the proteid metabolism of fasting dogs of similar weight.

\begin{tabular}{|c|c|c|c|c|c|c|c|}
\hline & & \multirow{2}{*}{$\begin{array}{l}\text { Length } \\
\text { of Exper- } \\
\text { iment, } \\
\text { Days. }\end{array}$} & \multirow{2}{*}{$\begin{array}{c}\text { Average } \\
\text { Live } \\
\text { Weight, } \\
\text { Kgg. }\end{array}$} & \multicolumn{3}{|c|}{ Food per Day. } & \multirow{2}{*}{$\begin{array}{l}\text { Urinary } \\
\text { Nitro- } \\
\text { gen, } \\
\text { Grms. }\end{array}$} \\
\hline & & & & $\begin{array}{c}\text { Fat, } \\
\text { Grms. }\end{array}$ & $\begin{array}{l}\text { Starch, } \\
\text { Grms. }\end{array}$ & $\begin{array}{l}\text { Nitrogen, } \\
\text { Grms. }\end{array}$ & \\
\hline With food: & $\begin{array}{l}\text { I } \\
\text { II } \\
\text { III } \\
\text { IV }\end{array}$ & $\begin{array}{l}5 \\
5 \\
4 \\
4\end{array}$ & $\begin{array}{r}11.20 \\
10.21 \\
9.88 \\
8.25\end{array}$ & $\begin{array}{l}55 \\
38 \\
53 \\
47\end{array}$ & $\begin{array}{r}116 \\
96 \\
108 \\
100\end{array}$ & $\begin{array}{l}2.63 \\
2.48 \\
2.66 \\
2.60\end{array}$ & $\begin{array}{l}2.61 \\
2.40 \\
2.67 \\
2.62\end{array}$ \\
\hline Fasting: & $\begin{array}{l}\text { Ink. } \\
\text { lck. }\end{array}$ & 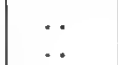 & $\begin{array}{r}14.4 \\
8.9\end{array}$ & $\begin{array}{l}\cdots \\
\ldots\end{array}$ & $\begin{array}{l}\ldots \\
\ldots\end{array}$ & 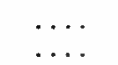 & $\begin{array}{l}3.65 \\
5.10\end{array}$ \\
\hline
\end{tabular}

Munk also cites in support of his conclusions Rubner's results on a $\operatorname{dog}$ fed exclusively on carbohydrates. A reference to these results as tabulated on a subsequent page does in fact show in most cases a decrease in the proteid metabolism as compared with the fasting values, but how much of this is due to the normal decrease during the first few days of abstinence from proteid food it is dif-

* Arch. path. Anat. u. Physiol., 131, Supp.

† Ibid., 132, 91. 
ficult to decide. Munk also cites results obtained by Salkowski,* who observed the nitrogen excretion of a dog on a light ration containing but little proteids to be scarcely greater than in the absence of all food.

E. Voit \& Korkunoff (loc. cit.) also included the carbohydrates in their investigation upon this subject, following the same general method as in the experiments with fat. The following are their results compared with the fasting proteid metabolism exactly as in the former case:

\begin{tabular}{|c|c|c|c|c|c|c|c|}
\hline & \multirow{3}{*}{$\begin{array}{c}\text { Live } \\
\text { Weight, } \\
\text { Kgs. }\end{array}$} & \multirow{3}{*}{$\begin{array}{c}\text { Total } \\
\text { Nitrogen } \\
\text { Excre- } \\
\text { tion, } \\
\text { Fasting, } \\
\text { Grms. }\end{array}$} & \multirow{2}{*}{\multicolumn{2}{|c|}{$\begin{array}{c}\text { Per Cent. of } \\
\text { Energy Demand } \\
\text { Supplied by }\end{array}$}} & \multicolumn{3}{|c|}{ Minimum of Food Nitrogen. } \\
\hline & & & & & \multirow[b]{2}{*}{$\begin{array}{l}\text { Amount, } \\
\text { Grms. }\end{array}$} & \multicolumn{2}{|c|}{$\begin{array}{c}\text { Per Cent.of Fastin } \\
\text { Metabolism. }\end{array}$} \\
\hline & & & $\begin{array}{c}\text { Carbo- } \\
\text { hydrates, } \\
\text { Per Cent. }\end{array}$ & $\left|\begin{array}{c}\text { Total } \\
\text { Food, } \\
\text { Per } \\
\text { Cent. }\end{array}\right|$ & & $\mid \begin{array}{c}\text { Total, } \\
\text { Per Cent. }\end{array}$ & $\begin{array}{l}\text { Proteid, } \\
\text { Per Cent. }\end{array}$ \\
\hline $\begin{array}{l}\text { Series I: } \\
\underset{\star}{\text { Experiment }} \underset{2}{3}\end{array}$ & $\begin{array}{l}24.0 \\
24.6\end{array}$ & $\begin{array}{l}4.93 \\
4.94\end{array}$ & $\begin{array}{l}78 \\
79\end{array}$ & $\begin{array}{l}91 \\
92\end{array}$ & $\begin{array}{r}>5.43 \\
5.00\end{array}$ & $\begin{array}{r}>110 \\
101\end{array}$ & $\begin{array}{r}>133 \\
124\end{array}$ \\
\hline $\begin{array}{cc}\text { Series II: } & \\
\text { Experiment } & 5 \\
\text { " } & 1 \\
\text { " } & 2 \\
\text { " } & 4 \\
& 3 b\end{array}$ & $\begin{array}{l}27.7 \\
24.1 \\
24.7 \\
30.0 \\
24.0 \\
-\end{array}$ & $\begin{array}{l}4.98 \\
5.25 \\
4.94 \\
4.08 \\
4.93\end{array}$ & $\begin{array}{l}111 \\
115 \\
118 \\
122 \\
155\end{array}$ & $\begin{array}{l}122 \\
126 \\
131 \\
136 \\
168\end{array}$ & $\begin{array}{r}5.11 \\
>4.91 \\
<4.35 \\
<4.47 \\
<4.48\end{array}$ & $\begin{array}{r}103 \\
>94 \\
<88 \\
<110 \\
<91\end{array}$ & $\begin{aligned} & 126 \\
&> 123 \\
&<108 \\
&<134 \\
&<111\end{aligned}$ \\
\hline
\end{tabular}

The authors also compute from a few experiments by C. Voit and by Rubner values not inconsistent with the above.

When compared with the total nitrogen excretion, the results of Voit \& Korkunoff show in but a single case a minimum unmistakably greater than the fasting proteid metabolism. In three cases the minimum falls below this amount, while in the remaining cases it is either substantially equal to it or doubtful. Regarded in this way, they seem on the whole in accord with Munk's claim that the proteid metabolism may be reduced below the fasting limit. Voit \& Korkunoff, however, dispute this and subject Munk's experiments to a detailed criticism, the principal points of which are that in the earlier experiments, as noted above, the nitrogen excretion was irregular and that the result of a single day is arbitrarily selected for comparison, while in the later experiments no

\footnotetext{
*Zeit. physiol. Chem., 1, 44.
} 
determinations of the fasting metabolism of the animals actually used for the experiments were made. By a re-computation of Munk's experiments they obtain results varying but little from 100 per cent. A computation from the average figures given on p. 136, assuming 3.4 per cent. of nitrogen in the meat and 0.51 grams of nitrogen per day in the feces, shows that the minimum is probably less than 107 per cent. of the fasting nitrogen excretion.

Much depends, however, upon whether we take as the unit of comparison the total nitrogen excretion or, like Voit \& Korkunoff, eliminate that portion derived from the extractives. If we select the former, then it appears that with a liberal supply of carbohydrates in the food the supply of proteids certainly need not exceed the fasting metabolism in order to maintain nitrogen equilibrium, and perhaps may be reduced materially below it.

Finally, it must be remembered that the fasting proteid metabolism itself is not a constant. In Chapter IV it was shown that as the store of fat in the body of a fasting animal becomes depleted the body proteids are drawn upon to an increasing extent to supply energy to the animal. It is not possible to show that the experimental results which have been cited are materially affected by this variability of the fasting proteid metabolism-indeed, it seems doubtful whether they are-but the fact that the demands of the organism for energy may affect the proteid metabolism is of itself sufficient to show that our unit of comparison, while practically convenient and perhaps sufficiently accurate, is not invariable.

Amount of Non-nitrogenous Nutrients ReQuired.-In most of the experiments which have been cited, the very low figures for the necessary proteid supply have been obtained by the employment of. an amount of non-nitrogenous nutrients materially in excess of the estimated requirements of the animal for energy, although in no case was this latter factor actually determined.

Sivén,* however, experimenting upon himself with a diet equal in amount to that ordinarily required to maintain his weight, was able to gradually reduce the total nitrogen of his food to 4.52 grams and maintain nitrogen equilibrium. He did not determine his fasting metabolism, but the above figure, which is equivalent to 0.08 gram of nitrogen per kilogram live weight, is lower than the low-

* Skand. Arch. f. Physiol., 10, 91. 
est fasting values previously obtained, Moreover, much of the nitrogen of his food was in the non-proteid form, the proteid nitrogen being estimated at only 0.03 gram per kilogram live weight.

Cremer \& Henderson * have attempted to reproduce Sivén's results in two experiments upon a dog, the total amount of food being equal to or slightly less than the estimated requirements of the animal. Under these conditions they were unable to reach even as low a minimum as did Voit \& Korkunoff. On the other hand, Jaffa, $\nmid$ in a dietary study of a child on a diet of fruits and nuts (so-called frutarian diet), observed a gain of nitrogen by the subject with only 0.041 gram of food nitrogen per kilogram body weight.

The Minimum for Herbivora.-The ordinary food of our domestic herbivora contains an abundance of non-nitrogenous matter and relatively little protein. It is impossible, for obvious reasons, to determine the fasting metabolism of ruminants, and the basis for comparisons like those made above is therefore largely lacking. There is, however, abundant evidence to show that only a comparatively small amount of proteids is necessary to maintain the nitrogen equilibrium of cattle in particular, although exact data as to the least amount required are still lacking.

The early experiments of Henneberg \& Stohmann $\$$ upon the maintenance ration of cattle furnish the following examples of the sufficiency of a very small proteid supply, the results being computed per $500 \mathrm{kgs}$. live weight per day:

\begin{tabular}{|c|c|c|c|}
\hline & \multicolumn{2}{|c|}{ Digested. } & \multirow{2}{*}{$\begin{array}{l}\text { Gain of } \\
\text { Nitrogen } \\
\text { by Animal } \\
\text { Grms. }\end{array}$} \\
\hline & $\begin{array}{l}\text { Protein, } \\
\text { Grms. }\end{array}$ & $\begin{array}{c}\text { Non-nitrogenous } \\
\text { Nutrients, } \\
\text { Grms. }\end{array}$ & \\
\hline 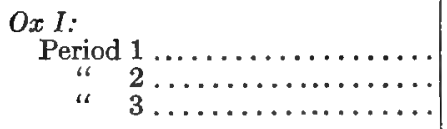 & $\begin{array}{l}178 \\
259 \\
209\end{array}$ & $\begin{array}{l}4247 \\
3546 \\
3926\end{array}$ & $\begin{array}{r}4.0 \\
21.0 \\
11.0\end{array}$ \\
\hline $\begin{array}{l}O x I I: \\
\quad \text { Period } 2 \ldots \ldots \ldots \ldots \ldots \ldots \ldots\end{array}$ & 278 & 3607 & 19.5 \\
\hline
\end{tabular}

* Zeit. f. Biol., 42, 612 .

$\dagger$ U. S. Dept Agr., Office of Expt. Stations, Bull. 107, 21.

$\ddagger$ Beiträge zur Begründung einer rationellen Fütterung der Wiederkaüer, Heft I. 
The following figures, obtained by the same investigators * in later experiments, are taken from Wolff's compilation: $\dagger$

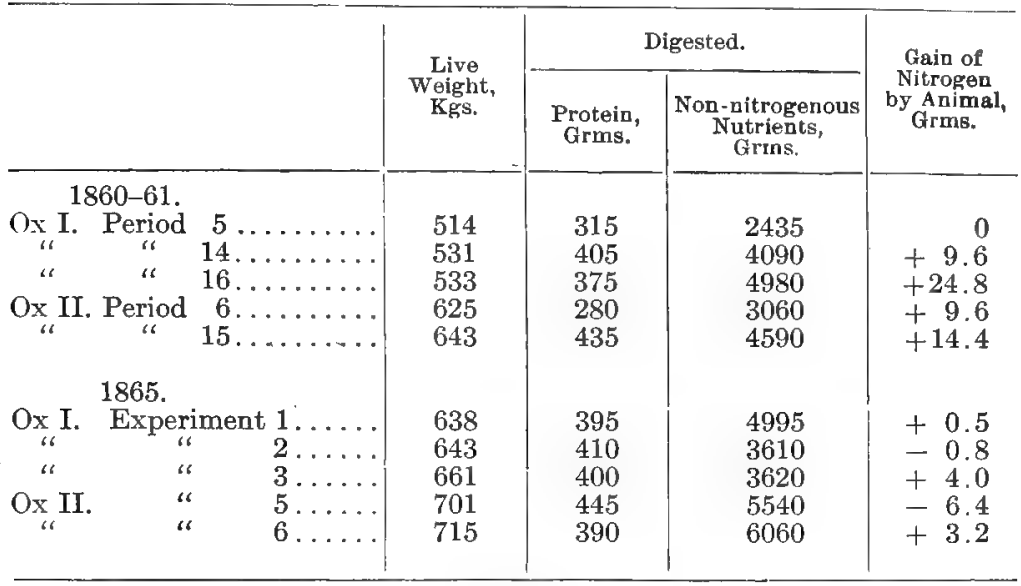

G. Kühn's extensive investigations at Möckern. together with subsequent ones by Kellner, $\S$ afford the following data for the periods in which the ration was approximately a maintenance ration:

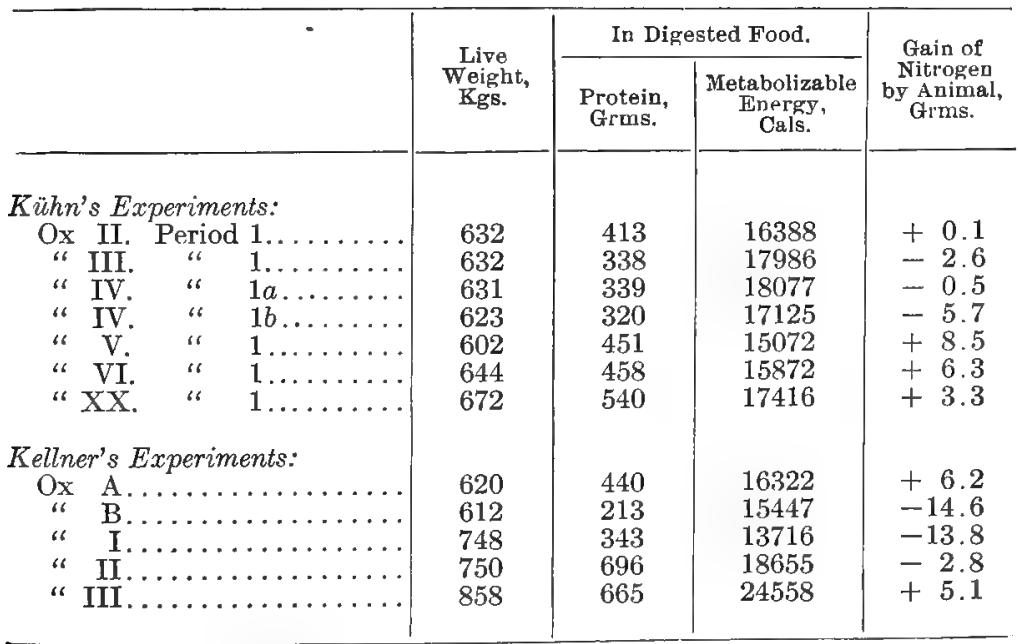

* Beiträge, etc., Heft II., and Neue Beiträge, etc.

† Ernährung landw. Nutzthiere, pp. 406-410.

\$ Landw. Vers. Stat., 44, 257.

§Ibid., 47, 275; 50, 245. 
Experiments by the writer * have shown that nitrogen equilibrium may be maintained, for a time at least, on even smaller amounts of protein than the above figures would indicate. The figures in the first column of the following table signify the proteid nitrogen only of the food multiplied by 6.25 :

\begin{tabular}{|c|c|c|c|c|c|}
\hline & $\begin{array}{l}\text { Digested Pro- } \\
\text { teida per Day } \\
\text { and } 500 \mathrm{Kgs} \text {. } \\
\text { Live Weight, } \\
\text { Grms. }\end{array}$ & $\begin{array}{l}\text { Meta- } \\
\text { bolizable } \\
\text { Energy } \\
\text { of Food, } \\
\text { Cals. }\end{array}$ & $\begin{array}{c}\text { Average } \\
\text { Live } \\
\text { Weight, } \\
\text { Kgs. }\end{array}$ & $\begin{array}{c}\text { Gain or Loss } \\
\text { of Nitrogen } \\
\text { by Body, } \\
\text { Grms. }\end{array}$ & $\begin{array}{c}\text { Nutritive } \\
\text { Ratio } \\
\text { 1: }\end{array}$ \\
\hline $\begin{array}{l}\text { Experiment } 1: \\
\text { Steer } 1 \ldots \ldots \ldots \ldots \\
\text { " } \quad 2 \ldots \ldots \ldots \ldots \\
" \quad 3 \ldots \ldots \ldots\end{array}$ & $\begin{array}{l}129 \\
113 \\
133\end{array}$ & $\begin{array}{l}7956 \\
7588 \\
7191\end{array}$ & $\begin{array}{l}420 \\
450 \\
400\end{array}$ & $\begin{array}{l}-2.51 \\
-0.39 \\
-1.08\end{array}$ & $\begin{array}{l}20.1 \\
20.4 \\
18.6\end{array}$ \\
\hline 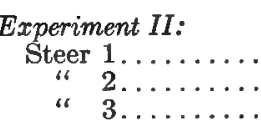 & $\begin{array}{l}192 \\
202 \\
209\end{array}$ & $\begin{array}{l}8144 \\
9590 \\
8084\end{array}$ & $\begin{array}{l}420 \\
450 \\
400\end{array}$ & $\begin{array}{l}+1.76 \\
+4.23 \\
+4.62\end{array}$ & $\begin{array}{l}13.4 \\
13.6 \\
12.8\end{array}$ \\
\hline $\begin{array}{c}\text { Experiment } V I: \\
\text { Steer } 1 \ldots \ldots \ldots \\
" \quad 2 \ldots \ldots \ldots \\
“ \quad 3 \ldots \ldots \ldots\end{array}$ & $\begin{array}{l}297 \\
277 \\
314\end{array}$ & $\begin{array}{l}11130 \\
11318 \\
11324\end{array}$ & $\begin{array}{l}450 \\
490 \\
430\end{array}$ & $\begin{array}{l}+4.67 \\
+6.47 \\
+2.65\end{array}$ & $\begin{array}{l}10.9 \\
10.9 \\
10.6\end{array}$ \\
\hline $\begin{array}{c}\text { Experiment VII: } \\
\text { Steer } 1 \ldots \ldots \ldots \\
\text { " } \quad 2 \ldots \ldots \ldots \\
" \quad 3 \ldots \ldots \ldots\end{array}$ & $\begin{array}{l}156 \\
131 \\
152\end{array}$ & $\begin{array}{l}11955 \\
11904 \\
11557\end{array}$ & $\begin{array}{l}450 \\
490 \\
430\end{array}$ & $\begin{array}{l}+5.68 \\
+3.98 \\
+4.15\end{array}$ & $\begin{array}{l}23.0 \\
25.3 \\
23.9\end{array}$ \\
\hline 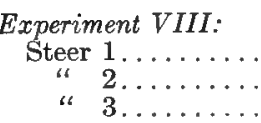 & $\begin{array}{l}258 \\
242 \\
275\end{array}$ & $\begin{array}{l}11634 \\
12976 \\
12030\end{array}$ & $\begin{array}{l}543 \\
629 \\
516\end{array}$ & $\begin{array}{l}+0.26 \\
-0.20 \\
-2.31\end{array}$ & $\begin{array}{l}10.4 \\
10.7 \\
10.6\end{array}$ \\
\hline
\end{tabular}

While the above data are hardly sufficient to fix absolutely the minimum of proteids for cattle on a maintenance ration, they indicate clearly that from 200 to 300 grams of digestible protein per day is at least sufficient for a steer weighing $500 \mathrm{kgs}$., and there is a possibility that the amount may be somewhat further reduced. Although we are unable to compare this with the fasting metabolism, a comparison on the basis of live weight with some of the results previously cited shows that the minimum demand for proteids on the part of cattle is relatively much less than on the part of carnivora. Thus the results obtained by Lehmann et. al. and Munk (p. 137), and by Voit \& Korkunoff (p. 138), computed in * Penna. Expt. Station, Bull. 42, 165. 
grams of food nitrogen per kilogram live weight, give the following figures for the minimum nitrogen requirements of the dog and of man as compared with cattle:

Experiments on Dogs.
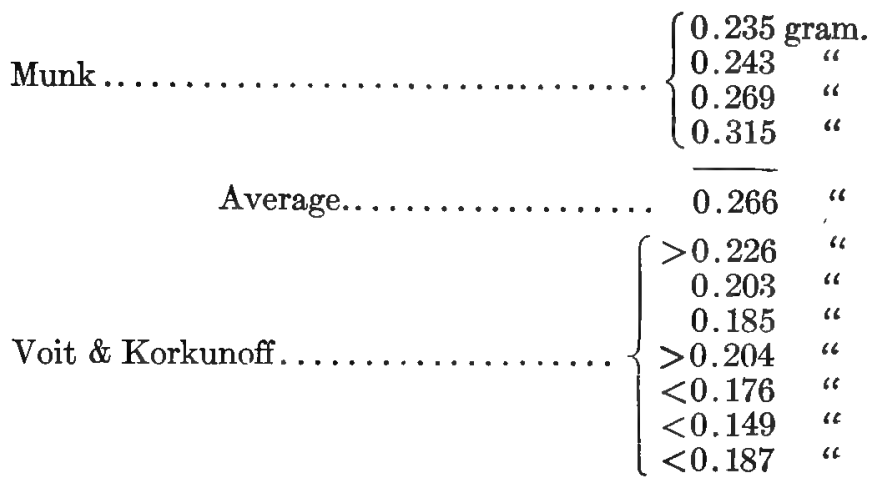

Experiments on Man.

Lehmann et al. .................. $\begin{cases}0.190 & \text { " } \\ 0.180 & \text { " } \\ 0.090 & \text { " } \\ 0.180 & \text { " }\end{cases}$

Experiments on Cattle.

Range of experiments cited ...... 0.064-0.098 gram.

Only one of the results on man, together with the very low figure obtained by Siven (p. 139), is comparable with those reached with cattle. Whether we are to ascribe the small demand of the latter for proteids to a specific difference in their rate of metabolism or to the large amounts of carbohydrate material which they habitually consume does not clearly appear.

EFFECTS UPON HEALTH.-Munk, in his experiments with rations very poor in proteids, made the observation that while such raticns were adequate to maintain the nitrogen balance of the body they nevertheless appeared to produce, in time, profound functional disturbances, sometimes ending in death. Similar observations have also been made by Rosenheim.* These experımenters ascribe

* Arch. ges. Physiol., 54, 61 
the ill effects directly to the small supply of proteids, but some other writers appear inclined to explain them as due to the long continuance of a uniform and rather artificial diet. The writer's experiments, cited above, showed no evidence of any ill effect in the case of cattle upon a ration containing but about 200 grams digestible protein per day and continued for seventy days, and subsequent observations, as well as the common experience of farmers in wintering cattle upon such feeding-stuffs as inferior hay, straw, etc., fully confirm this result.

\section{Effects on Total Metabolism.}

Substitution for Body Fat.-We have seen in the preceding section that proteid food, or rather the non-nitrogenous residue arising from its cleavage in the body, may be utilized as a source of energy in place of the body fat which would otherwise be metabolized. Similarly, the non-nitrogenous nutrients supplied in the food may be thus substituted for body fat in the metabolism of the animal. The substitution is shown most clearly in experiments upon fasting animals, although it appears also in those in which these nutrients are added to an insufficient ration.

FAT.-The following averages of Pettenkofer \& Voit's experiments, * computed from Atwater \& Langworthy's digest, $\dagger$ illustrate this substitution of food fat for body fat:

\begin{tabular}{|c|c|c|c|}
\hline \multirow{2}{*}{$\begin{array}{l}\text { Food, } \\
\text { Grms. }\end{array}$} & \multirow{2}{*}{$\begin{array}{c}\text { Number of } \\
\text { Experiments. }\end{array}$} & \multicolumn{2}{|c|}{ Gain or Loss by Body. } \\
\hline & & $\begin{array}{l}\text { Nitrogen, } \\
\text { Grms. }\end{array}$ & $\begin{array}{c}\text { Fat, } \\
\text { Grms. }\end{array}$ \\
\hline $\begin{array}{l}\text { Nothing } \\
100 \text { fat } \\
350 \text { " }\end{array}$ & $\begin{array}{l}5 \\
2 \\
1\end{array}$ & $\begin{array}{l}-6.64 \\
-4.90 \\
-7.70\end{array}$ & $\begin{array}{r}-97.76 \\
-16.25 \\
+113.60\end{array}$ \\
\hline
\end{tabular}

The smaller amount of fat not only diminished the proteid metabolism but also largely reduced the loss of fat from the body. The larger amount of fat showed the tendency noted on p. 115 to increase the proteid metabolism, but at the same time it not only suspended the loss of body fat but caused a storage of fat in the organism. Of course we have no means of distinguishing in such a case between

* Zeit. f. Biol., 5, 370.

$\dagger$ U.S. Dept. Agr., Office of Experiment Stations, Bull. 45. 
food fat and body fat, but it is most natural to suppose that the resorbed fat of the food, being already in circulation in the body, is more easily accessible to the active cells than the stored-up fat of the adipose tissue and is, therefore, metabolized in preference to the latter.

Rubner,* in his study of the replacement values of the several nutrients, has demonstrated the same effect of food fat. Fat supplied in the food is utilized as a source of energy to the body and a corresponding quantity of body fat escapes oxidation, while if supplied in excess fat is stored up in the body. The experiments were made in the same manner and are computed on the same assumptions as those upon proteids recorded on p. 106. All were on dogs except the third, which was on a rabbit.

\begin{tabular}{|c|c|c|c|}
\hline Food. & $\begin{array}{l}\text { Total Nitrogen } \\
\text { of Excreta, } \\
\text { Grms. }\end{array}$ & $\begin{array}{c}\text { Fat } \\
\text { Metabolized, } \\
\text { Grms. }\end{array}$ & $\begin{array}{l}\text { Gain or Loss } \\
\text { of Fat, } \\
\text { Grms. }\end{array}$ \\
\hline 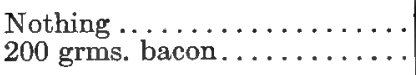 & $\begin{array}{l}1.69 \\
1.68\end{array}$ & $\begin{array}{l}60.47 \\
71.80\end{array}$ & $\begin{array}{l}-60.47 \\
+128.20\end{array}$ \\
\hline $\begin{array}{l}\text { Nothing ..... . . . . . ......... } \\
39.75 \text { grms. of butter fat.... }\end{array}$ & $\begin{array}{l}2.14 \\
2.44\end{array}$ & $\begin{array}{l}33.78 \\
33.48\end{array}$ & $\begin{array}{l}-\quad 33.78 \\
+\quad 6.27\end{array}$ \\
\hline $\begin{array}{l}\text { Nothing....................... } \\
26.1 \text { grms. bacon } \uparrow \ldots \ldots \ldots\end{array}$ & $\begin{array}{l}0.778 \\
1.045\end{array}$ & $\begin{array}{l}7.18 \\
6.44\end{array}$ & $\begin{array}{r}7.18 \\
+19.63\end{array}$ \\
\hline 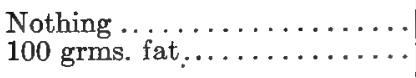 & $\begin{array}{l}2.56 \\
2.48\end{array}$ & $\begin{array}{l}42.40 \\
47.73\end{array}$ & $\begin{array}{l}-42.40 \\
+\quad 52.27\end{array}$ \\
\hline $\begin{array}{l}\text { Nothing } \ldots \ldots \ldots \ldots \ldots \ldots \\
40 \text { grms. bacon } . \ldots \ldots \ldots \ldots\end{array}$ & $\begin{array}{l}1.08 \\
1.32\end{array}$ & $\begin{array}{l}22.88 \\
28.73\end{array}$ & $\begin{array}{l}-22.88 \\
+\quad 11.27\end{array}$ \\
\hline
\end{tabular}

In nearly every case there was a slight increase in the proteid metabolism, as in Pettenkofer \& Voit's experiments, and a somewhat greater, although still not very considerable, increase in the fat metabolism. In the main, however, the food fat was metabolized in place of the body fat.

In those of Pettenkofer \& Voit's experiments in which fat was added to an insufficient ration of meat the same effect was produced, as appears when we compare the results upon a ration of meat

* Zeit. f. Biol., 19, 328-334; 30, 123.

$\dagger$ Results approximate only. 
and fat given on p. 150 with those upon the same ration of meat without the fat, as in the table below:

\begin{tabular}{|c|c|c|c|c|c|}
\hline & \multirow{2}{*}{$\begin{array}{l}\text { Number } \\
\text { of } \\
\text { Experi- } \\
\text { ments. }\end{array}$} & \multicolumn{2}{|c|}{ Food per Day. } & \multicolumn{2}{|c|}{ Gain or Loss by Body. } \\
\hline & & $\begin{array}{l}\text { Meat, } \\
\text { Grms. }\end{array}$ & $\begin{array}{c}\text { Fat, } \\
\text { Grms. }\end{array}$ & $\begin{array}{c}\text { Nitrogen, } \\
\text { Grms. }\end{array}$ & $\begin{array}{l}\text { Carbon, } \\
\text { Grms. }\end{array}$ \\
\hline $\begin{array}{c}\text { Proteids alone } . . \\
، \quad \text { and fat }\end{array}$ & $\begin{array}{l}6 \\
1 \\
5\end{array}$ & $\begin{array}{l}500 \\
500 \\
500\end{array}$ & $\begin{array}{l}\dot{100} \\
200\end{array}$ & $\begin{array}{l}-3.4 \\
+0.3 \\
-0.6\end{array}$ & $\begin{array}{l}-49.1 \\
+27.1 \\
+67.3\end{array}$ \\
\hline
\end{tabular}

Carbohydrates. - The more soluble hexose carbohydrates when given to a fasting animal serve, like the fats, as a source of energy for the organism in place of the body fat which would otherwise be oxidized.

The following is a summary of the average results obtained by Pettenkofer \& Voit* by feeding starch with a small amount of fat, the fasting metabolism being the same as that just given on p. 144. The averages are computed as before from Atwater \& Langworthy's digest (loc. cit.):

\begin{tabular}{|c|c|c|c|c|c|}
\hline & \multirow{2}{*}{$\begin{array}{l}\text { Number } \\
\text { of } \\
\text { Experi- } \\
\text { ments. }\end{array}$} & \multicolumn{2}{|c|}{ Food per Day. } & \multicolumn{2}{|c|}{ Gain or Loss by Body. } \\
\hline & & $\begin{array}{l}\text { Starch, } \\
\text { Grms. }\end{array}$ & $\begin{array}{c}\text { Fat, } \\
\text { Grms. }\end{array}$ & $\begin{array}{l}\text { Nitrogen, } \\
\text { Grms. }\end{array}$ & $\begin{array}{l}\text { Carbon, } \\
\text { Grms. }\end{array}$ \\
\hline $\begin{array}{l}\text { Fasting } \ldots \ldots \ldots \ldots \ldots \\
\text { Starch } \ldots \ldots \ldots \ldots \ldots\end{array}$ & $\left\{\begin{array}{l}5 \\
1 \\
1 \\
3\end{array}\right.$ & $\begin{array}{l}450 \\
597 \\
700\end{array}$ & $\begin{array}{l}16.9 \\
21.2 \\
20.2\end{array}$ & $\begin{array}{l}-6.64 \\
-7.20 \\
-9.40 \\
-6.20\end{array}$ & $\begin{array}{l}-97.76 \\
+19.40 \\
-28.50 \\
+61.30\end{array}$ \\
\hline
\end{tabular}

The fasting metabolism in this case represents a series of experiments antedating by a year or two that upon starch. In only one case were the respiratory products of the fasting animal determined during the latter series. That determination immediately followed a day on which a large amount of starch was consumed, and the results are believed by the authors to be affected thereby. No very strict comparison is therefore possible, but the general effect of the starch in diminishing the loss of body fat is evident.

*Zeit. f. Biol., 9, 485. 
The experiments by Rubner,* which have been already several times referred to, include trials in which sugar or starch was fed alone. The results are computed as previously described, with the additional assumption that all the carbohydrates digested (with the exception of small amounts of sugar found in the urine in some cases) were completely oxidized in the body. The gain or loss of carbon as fat is therefore computed by subtracting from the total excretory carbon, first, the carbon due to the protein metabolized, and second, that assumed to be derived from the carbohydrates. On this basis the results are as follows, the amounts of carbohydrates given in the table being those believed to have been actually oxidized:

\begin{tabular}{|c|c|c|c|}
\hline Food. & $\begin{array}{l}\text { Total Nitrogen } \\
\text { of Excreta, } \\
\text { Grms. }\end{array}$ & $\begin{array}{c}\text { Total Carbon } \\
\text { of Excreta, } \uparrow \\
\text { Grms. }\end{array}$ & $\begin{array}{l}\text { Gain or Loss } \\
\text { of Fat, } \\
\text { Grms }\end{array}$ \\
\hline $\begin{array}{l}\text { Nothing } \ldots \ldots \ldots \ldots \ldots \ldots \\
76.12 \text { grms. cane-sugar } \ldots \ldots \ldots \ldots \\
104.97\end{array}$ & $\begin{array}{l}1.94 \\
1.45 \\
1.07\end{array}$ & $\begin{array}{l}38.18 \\
43.19 \\
47.78\end{array}$ & $\begin{array}{rr}- & 40.99 \\
-\quad 8.41 \\
+\quad 0.51\end{array}$ \\
\hline 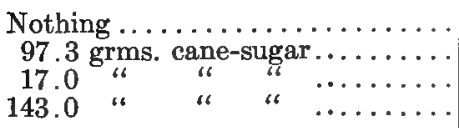 & $\begin{array}{l}1.86 \\
1.92 \\
1.41 \\
1.22\end{array}$ & $\begin{array}{l}39.22 \\
50.69 \\
39.52 \\
46.45\end{array}$ & $\begin{array}{l}-\quad 42.72 \\
-\quad 2.95 \\
-\quad 35.80 \\
+\quad 23.32\end{array}$ \\
\hline 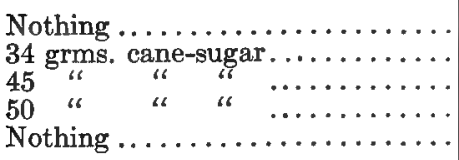 & $\begin{array}{l}1.32 \\
1.41 \\
1.25 \\
1.57 \\
1.39\end{array}$ & $\begin{array}{l}21.36 \\
26.18 \\
29.14 \\
27.68 \\
25.79\end{array}$ & $\begin{array}{l}-21.88 \\
-\quad 9.10 \\
-\quad 7.46 \\
=\quad 1.64 \\
-27.86\end{array}$ \\
\hline 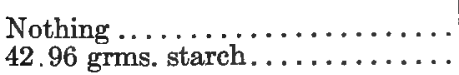 & $\begin{array}{l}1.42 \\
1.53\end{array}$ & $\begin{array}{l}26.47 \\
33.28\end{array}$ & $\begin{array}{l}-28.10 \\
-10.54\end{array}$ \\
\hline 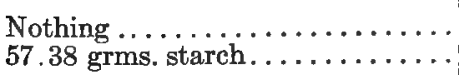 & $\begin{array}{l}2.00 \\
1.52\end{array}$ & $\begin{array}{l}31.53 \\
39.67\end{array}$ & $\begin{array}{l}-32.10 \\
-10.74\end{array}$ \\
\hline $\left.\begin{array}{rl}\text { Nothing (second day) } \ldots . . . & \ldots \ldots \\
94.36 \text { grms. cane-sugar } \\
67.96 \text { " } & \begin{array}{l}\text { starch } \\
4.70 \text { " }\end{array} \\
\text { fat }\end{array}\right\} \ldots \ldots \ldots$ & $\begin{array}{l}2.64 \\
1.23\end{array}$ & 38.94 & $\begin{array}{l}-24.97 \\
+116.35 t\end{array}$ \\
\hline
\end{tabular}

In place of the slight increase in the proteid metabolism frequently noticed when fat is consumed, the tendency of the carbo-

* Zeit. f. Biol., 19, 357-379; 22, 273.

$\uparrow$ Not including the carbon of the carbohydrates found in feces and urine. $\ddagger$ Total gain of carbon, computed as fat. Compare, loc. cit., 22, 279. 
hydrates seems to be to cause a slight decrease, but the chief effect is upon the carbon metabolism, increasing rations of carbohydrates resulting in a progressive reduction of the amount of body fat metabolized.

The effect of starch or sugar when added to an insufficient proteid diet may be illustrated, as in the case of fat, by a comparison of Pettenkofer \& Voit's results, cited on p. 150, with those on proteids alone:

\begin{tabular}{|c|c|c|c|c|c|c|c|}
\hline & \multirow{2}{*}{$\begin{array}{c}\text { Number } \\
\text { of } \\
\text { Experi- } \\
\text { ments. }\end{array}$} & \multicolumn{4}{|c|}{ Food per Day. } & \multicolumn{2}{|c|}{$\begin{array}{l}\text { Gain or Loss } \\
\text { by Body. }\end{array}$} \\
\hline & & $\begin{array}{l}\text { Meat, } \\
\text { Grms. }\end{array}$ & $\begin{array}{l}\text { Fat, } \\
\text { Grms. }\end{array}$ & $\begin{array}{l}\text { Starch, } \\
\text { Grms. }\end{array}$ & $\begin{array}{c}\text { Dextrose, } \\
\text { Grms. }\end{array}$ & $\begin{array}{c}\text { Nitrogen, } \\
\text { Grms. }\end{array}$ & $\begin{array}{l}\text { Carbon, } \\
\text { Grms. }\end{array}$ \\
\hline $\begin{array}{l}\text { Proteids alone } \ldots . . . . \\
\text { " and starch... }\end{array}$ & $\begin{array}{l}6 \\
8 \\
3\end{array}$ & $\begin{array}{l}500 \\
500 \\
500\end{array}$ & $\begin{array}{l}\ddot{5.3} \\
\ldots\end{array}$ & $\begin{array}{l}200 \\
\ldots\end{array}$ & $\ddot{2} 0 \dot{0}$ & $\begin{array}{l}-3.4 \\
-1.8 \\
-1.3\end{array}$ & $\begin{array}{l}-49.1 \\
+9.0 \\
+7.2\end{array}$ \\
\hline
\end{tabular}

Mutual Replacement of Nutrients.-The facts which have been considered in the foregoing pages show a remarkable degree of flexibility in the animal organism as regards the nature of the material consumed in its vital processes. The amount of proteid material necessarily required for the metabolism of the mature animal we have seen to be relatively small. Aside from this minimum, the metabolic activities of the body may be supported, now at the expense of the stored body fat, now by the body proteids, and again by the proteids, the fats, or the carbohydrates of the food. Whatever may be true economically, physiologically the welfare of the mature animal is not conditioned upon any fixed relation between the classes of nutrients in its food-supply, apart from the minimum requirement for proteids. The possibility of a mutual replacement of the several classes of nutrients in the food follows almost necessarily from the power of the organism to utilize them all indifferently (in a qualitative sense at least).

Replacement of Proteids.- - It has been shown that proteids in excess of the minimum demand can be used by the organism to take the place of body fat previously metabolized. Furthermore, as we have just seen, the non-nitrogenous nutrients of the food may likewise be substituted for body fat. It is natural to suppose, therefore, that that portion of the proteid supply which serves substan- 
tially as a source of energy only may be replaced either by body fat or by other food nutrients, and this supposition is borne out by the observed facts.

By Body Fat.-In considering the total metabolism of the fasting animal in Chapter IV, we saw that the fat of the body has a marked effect in protecting the body proteids from metabolism, and that with the progressive impoverishment of the body in fat, more and more of the proteids are substituted for the latter as a source of energy. In $\S 1$ of the present chapter it was further shown that the food proteids, or their non-nitrogenous residue, may be oxidized in the organism in place of the stored fat of the body.

It is clear, however, that the same experiments may equally well be regarded from the converse point of view as showing that the body fat may be oxidized and serve as a source of energy in place of the proteids of the food or of the body. In other words, it is possible, within quite wide limits, for the animal organism to draw its supply of energy, according to circumstances, either from food or body proteids or from its stored-up fat.

By Fats and Carbohydrates of Food.-When, in addition to its reserve of fat, a supply of non-nitrogenous nutrients is afforded in its food, this range of choice by the organism is still further widened. In considering the effects of non-nitrogenous nutrients upon the proteid metabolism, and particularly in the discussion of the minimum of proteids, it became evident incidentally that fat or carbohydrates may to a large extent be substituted for proteids in the food. A certain minimum of proteids was shown to be essential to the maintenance of the proteid tissues of the body, but proteids supplied in excess of this amount undergo nitrogen cleavage and serve substantially as a source of energy. This excess of proteids, as we have seen, can be replaced in the food by non-nitrogenous nutrients, particularly the carbohydrates, at least without damage to the proteid nutrition, as is shown by Voit's results there cited (p. 134). The later respiration experiments of Pettenkofer \& Voit show that this is true also as regards the total metabolism. As appears from the table on p. 109, a ration of 1500 grams of lean meat sufficed to maintain the dog experimented upon approximately in equilibrium as regards the income and outgo of both nitrogen and carbon. When, however, a con- 
siderable proportion of this meat was replaced by fat, starch, or sugar, not only was the nitrogen equilibrium maintained but the same was true of the carbon, as appears from the following averages computed from Atwater \& Langworthy's "Digest of Metabolism Experiments." * The results of Pettenkofer \& Voit's first series with 1500 grams of lean meat as given by them are also included in the table:

\begin{tabular}{|c|c|c|c|c|c|c|}
\hline & \multicolumn{4}{|c|}{ Food per Day. } & \multicolumn{2}{|c|}{$\begin{array}{l}\text { Gain or Lnss } \\
\text { by Body. }\end{array}$} \\
\hline & $\begin{array}{l}\text { Meat, } \\
\text { Grms. }\end{array}$ & $\begin{array}{l}\text { F'at, } \\
\text { Grms. }\end{array}$ & \begin{tabular}{|l|} 
Starch, \\
Grms.
\end{tabular} & \begin{tabular}{|l} 
Grape- \\
sugar, \\
Grms.
\end{tabular} & Nitrogen, & $\begin{array}{l}\text { Carbon, } \\
\text { Grms. }\end{array}$ \\
\hline $\begin{array}{l}\text { Proteids only: } \\
\quad \text { Series I ......................... } \\
\quad \text { Average of all }(22 \text { experiments })\end{array}$ & $\begin{array}{l}1500 \\
1500\end{array}$ & $\ldots$ & $\cdots$ & $\cdots$ & $\begin{array}{c}0 \\
+0.6\end{array}$ & $\begin{array}{r}+3.3 \\
+8.7\end{array}$ \\
\hline $\begin{array}{l}\text { Proteids and fat: } \\
\quad 100 \text { grms. fat ( } 1 \text { experiment }) . \\
200 \text { " }(5 \text { experiments })\end{array}$ & $\begin{array}{l}500 \\
500\end{array}$ & \begin{tabular}{|l|}
100 \\
200
\end{tabular} & $\begin{array}{l}\cdots \\
\cdots\end{array}$ & $\cdots$ & $\begin{array}{l}+0.3 \\
-0.6\end{array}$ & $\begin{array}{l}+27.1 \\
+67.3\end{array}$ \\
\hline $\begin{array}{l}\text { Proteids and carbohydrates : } \\
\quad \text { Starch (8 experiments)........ } \\
\text { Grape-sugar ( } 3 \text { experiments) . }\end{array}$ & $\begin{array}{l}500 \\
500\end{array}$ & $\begin{array}{l}5.3 \\
\ldots\end{array}$ & $\begin{array}{c}200 \\
\ldots\end{array}$ & 200 & $\begin{array}{l}-1.8 \\
-1.3\end{array}$ & $\begin{array}{r}+9.0 \\
+7.2\end{array}$ \\
\hline
\end{tabular}

While it is true, as was stated on page 109, that there is reason to suppose the carbon balance as computed by Pettenkofer \& Voit to be somewhat in error, this in no way affects the general showing of the above averages. The introduction into the diet of 100-200 grams of fat or carbohydrates made it possible to dispense with two thirds of the proteids previously required to maintain the animal, the remaining 500 grams of meat being nearly or quite suffcient to maintain nitrogen equilibrium. The fat or carbohydrates added were obviously used by the organism as sources of energy in place of the proteids (or their non-nitrogenous residue) oxidized for this purpose on a purely proteid diet, since the stored fat of the body was not only conserved but even shows a gain.

Rubner's investigations upon the source of animal heat $\dagger$ afford

* U. S. Dept. Agr., Office of Expt. Stations, Bull. 45. Compare Zeit. f. Biol., 7, 450-480; 9, 6-13 and 450-467.

$\dagger$ Zeit. f. Biol., 30, 125-132. 
a similar illustration of this effect of non-nitrogenous nutrients. Assuming average figures for the nitrogen and carbon content of the food materials used, he obtained the following results:

\begin{tabular}{|c|c|c|c|c|}
\hline & \multicolumn{2}{|c|}{ Food per Day. } & \multicolumn{2}{|c|}{ Gain or Loss by Body. } \\
\hline & $\begin{array}{l}\text { Meat, } \\
\text { Grms. }\end{array}$ & $\begin{array}{l}\text { Fat, } \\
\text { Grms. }\end{array}$ & $\begin{array}{l}\text { Nitrogen, } \\
\text { Grms. }\end{array}$ & $\begin{array}{l}\text { Carbon, } \\
\text { Grms. }\end{array}$ \\
\hline $\begin{array}{l}\text { Proteids alone ( } 1 \text { experiment) } \ldots \text { and fat ( } 2 \text { experiments). }\end{array}$ & $\begin{array}{r}350 \\
80\end{array}$ & 30 & $\begin{array}{l}+1.66 \\
-0.08\end{array}$ & $\begin{array}{l}-2.69 \\
+4.46\end{array}$ \\
\hline
\end{tabular}

The possibility of such a substitution of non-nitrogenous nutrients for the food proteids as is illustrated in the foregoing experiments seems, indeed, almost a necessary corollary of the facts concerning proteid metabolism considered on previous pages. We have seen that, beginning with the fasting metabolism, the effect of successive additions of proteids to the food is to stimulate the proteid metabolism. Only a relatively small proportion of the added proteids is employed by the organism for constructive purposes, the larger part of it undergoing very promptly nitrogen cleavage and thus constituting, to all intents and purposes, an addition to the supply of non-nitrogenous material available for metabolism. It appears quite natural, then, that the portion of the proteid supply which thus serves substantially as fuel to the organisen should be replaceable in the food by non-nitrogenous materials which are capable of serving the same purpose.

Fats and Carbohydrates. - The apparent identity of the functions of the fats and carbohydrates as sources of energy which has been shown in the preceding paragraphs necessarily implies the possibility of their mutual replacement in the food. Rubner* has completed the chain of evidence by showing experimentally that fat and dextrose may thus replace each other. A dog received for twelve days a ration of 300 grams of lean meat and 42 or 50 grams of fat, with the exception of three days, on which varying amounts of dextrose were substituted for the fat. On six days the respiratory products were determined. Averaging the results for all the days on which the food was the same, and assuming the lean

* Zeit. f. Biol., 19, 370. 
meat used to have contained 3.4 per cent. of nitrogen and 12.51 per cent. of carbon, and the fat 76.5 per cent. of carbon, we have:

\begin{tabular}{|c|c|c|c|c|c|}
\hline & \multicolumn{3}{|c|}{ Food per Day. } & \multicolumn{2}{|c|}{ Gain or Loss by Animal. } \\
\hline & $\begin{array}{l}\text { Meat, } \\
\text { Grims. }\end{array}$ & $\begin{array}{c}\text { Fat, } \\
\text { Grms. }\end{array}$ & $\begin{array}{c}\text { Dextrose, } \\
\text { Grms. }\end{array}$ & $\begin{array}{c}\text { Nitrogen, } \\
\text { Grms. }\end{array}$ & $\begin{array}{l}\text { Carbon, } \\
\text { Grms. }\end{array}$ \\
\hline $\begin{array}{l}\text { Meat and fat........ } \\
\text { Meat and dextrose... }\end{array}$ & $\begin{array}{l}\left\{\begin{array}{l}300 \\
300\end{array}\right. \\
\left\{\begin{array}{l}300 \\
300 \\
300\end{array}\right.\end{array}$ & $\begin{array}{l}42 \\
50 \\
\cdots \\
\cdots\end{array}$ & $\begin{array}{r}\cdots \\
63.7 \\
79.7 \\
115.5\end{array}$ & $\begin{array}{l}+1.81 \\
+0.10 \\
+1.78 \\
+2.28 \\
+1.98\end{array}$ & $\begin{array}{l}+1.27 \\
+9.31 \\
-7.44 \\
-8.15 \\
+6.21\end{array}$ \\
\hline
\end{tabular}

The averages of Pettenkofer \& Voit's results as tabulated on p. $\mathbf{1 5 0}$ may likewise be regarded in this light.

Relative Values. - The close similarity in the functions of the several non-nitrogenous nutrients is too obvious to have escaped early notice, and the investigations of the Munich school of physiologists served both to emphasize the similarity and to follow it into details. To Rubner, a pupil of Voit, is generally ascribed the credit of having first placed in a clear light the quantitative relations of the subject, although v. Hösslin* and Danilewsky $\dagger$ enunciated similar ideas at about the same time, which, however, were not based on their own experiments.

As the result of his investigations upon the replacement values of the nutrients, $\ddagger$ Rubner announced the law of "isodynamic replacement." This law is, in brief, that the several nutrients can replace each other in amounts inversely proportional to their physiological heat values, that is, to the amounts of heat which they would liberate if oxidized to the same final products which result from their metabolism in the body. In other words, aside from the minimum of proteids the nutrients are of value to the organism in proportion to the amount of energy which their metabolism liberates-they are "the fuel of the body." One gram of fat, for example, when oxidized to carbon dioxide and water, liberates about 9.5 Cals. of energy, while one gram of starch similarly

* Arch. path. Anat. u. Pbysiol., 89, 333.

$\uparrow$ Die Kraftrorräte der Nahrungsstoffe

† Zeit. f. Biol., 19, 313. 
oxidized liberates but about 4.2 Cals. The relative values of fat and starch, then, are as $9.5: 4.2$ or as $2.26: 1$. Similarly, one gram of proteids oxidized to carbon dioxide, water, and the nitrogenous metabolic products of feces and urine liberates (in the dog) about 4.4 Cals. of energy. So far, therefore, as they are used as a source of energy simply and not for constructive purposes, their value, compared with starch, would be as $4.4: 4.2$ or as $1.05: 1$.

A rival theory of "isoglycosic values," the basis of which has already been indicated in Chapter II, has been advanced by Chauveau* and his school in Paris. According to this school, dextrose (or glycogen) constitutes the material which is consumed in the vital activities of the organism. The various nutrients, then, will be of value to the organism in proportion to the amount of glycogen or dextrose which they can supply, and the chemical equations already given on pp. 38 and 51 are claimed to show substantially what that amount is. The carbohydrates, according to this theory, yield practically their entire store of energy to the organism, while if the equations mentioned are interpreted literally the sugar produced from one gram of proteids would, according to Chauveau's equation, contain but about 1.83 Cals. of potential energy in place of the 4.4 Cals. available from the proteids according to Rubner. If the proteids are assumed to be split up in accordance with Gautier's equation the resulting dextrose would contain about 80 per cent. of their potential energy, and this figure is used in computing their isoglycosic value. Similarly, the sugar derived from one gram of fat would contain about 6.07 Cals. out of the 9.5 Cals. contained in the original fat. In other words, while Chauveau does not question that the actual food of the living cells is of value in proportion as it supplies energy, he holds that in the complex organism of the higher animals a considerable share of the original potential energy of fats and proteids is lost during their conversion into material (carbohydrates) which the cells can use.

The conception of the mutual replacement of the nutrients on the basis of the amounts of energy they are capable of liberating for the use of the organism has proved a fruitful one and been the basis of much subsequent research. A full discussion of it and

\section{* La Vie et l'Energie chez l'Animale.}


of the modifications which later investigation has made necessary in Rubner's original conclusions, is possible only in connection with a general study of the energy relations of the food, the animal, and the environment such as forms the subject of Part II. For the present we may content ourselves with accepting the general idea that the relative values of the nutrients depend in very large measure upon their ability to furnish energy for the vital activities, deferring until later the consideration of quantitative relations.

The Non-nitrogenous Ingredients of Feeding-stuffs.The discussions of the foregoing paragraphs have had reference to the effects produced by pure or approximately pure nutrients upon the metabolism of carnivora. By reason of the simplicity of conditions which is possible in such experiments they are indispensable in a study of the fundamental laws of nutrition. We must presume also that the general principles, established by such experiments are applicable to all warm-blooded animals, since we know of no radical differences in their vital processes.

In making such an application to the nutrition of our domestic herbivorous animals, however, much caution is necessary to avoid unwarranted assumptions and conclusions. Two points need especially to be borne in mind:

First, the food of these animals is, from a chemical point of view, very heterogeneous. In addition to true proteids, there are present, especially in coarse fodders, various non-proteid nitrogenous substances, while the non-nitrogenous nutrients, besides hexose carbohydrates and true fats, include, on the one hand. pentosans and pentoses, lignin, and all the variety of unknown substances comprised under the conventional terms "nitrogen-free extract" and "crude fiber," and on the other the waxes, resins, coloring matters, etc., contained in the "crude fat."

Second, the process of digestion in herbivora, and especially in the ruminants, as was pointed out in Chapter I. differs materially from that in carnivora as regards the part played by fermentative processes, particularly in the solution of the carbohydrates and related bodies which are so abundant in vegetable materials.

It has been more or less customary to regard the digested portions of the crude fiber and nitrogen-free extract of feeding-stuffs 
as consisting essentially of carbohydrates. The basis for this assumption is the demonstration by Henneberg that the ultimate composition of that portion of these two groups of substances which is not recovered in the feces is 'substantially that of starch' or cellulose, while Kellner * has more recently demonstrated their equality in energy value. This fact of itself, however, does not justify the inference of equal nutritive value, as may be readily seen in the case of starch. It is obviously not a matter of indifference whether a given amount of this substance is resorbed from the digestive canal of a stcer in the form of sugar or whether, as in some of Kühn's experiments, 65 per cent. of it is converted into methane, carbon dioxide, and organic acids, yet the elementary composition of the "digested" portion would be the same in either case.

The fact is that while the resorbed food of herbivora contains proteids, carbohydrates, and fats, whose functions in nutrition must be assumed to be the same as in the carnivora, it is very far from consisting entirely of them, but contains also a variety of other substances of whose exact nature and proportions we are comparatively ignorant. We know, of course, that the digested non-nitrogenous ingredients of feeding-stuffs, taken as a whole, do serve as sources of energy. When an ox or a sheep is fed exclusively on ordinary coarse fodders such as hay, straw, or corn stover, the small supply of proteids that he receives is likely to be little if any in excess of the minimum demand, and the requirements of the body for energy must be satisfied very largely by the non-nitrogenous materials. Moreover, the supply of such substances as starch, sugars, and true fats in such a case is so small relatively that it appears difficult to suppose that these alone are sufficient for the needs of the organism, and one is forced to the conclusion that the ill-known ingredients of the "crude fiber" and "nitrogen-free extract" are also utilized.

The separation and identification of these various substances and the study of their physiological effects presents a problem at once attractive and laborious and one whose complete solution we cannot hope soon to reach. Some few data as to certain classes, however, are available.

* Compare Part II, Chapter X. 
Pentose Carbohydrates.-It has already been shown in Chapter II (p. 24) that such of the pentose carbohydrates as have been experimented upon are at least partially oxidized in the body, and that this appears to be especially the case with herbivora, the urine of these animals seldom containing pentoses.

It is of course conceivable that a substance may be oxidized in the body without producing any useful effect except in so far as the resulting heat may be of value to the organism, but it seems more consonant with our general conceptions of the nature of metabolism to suppose that the potential energy of any substance which is capable of entering into the metabolism of the cells may be utilized as a source of energy for their functions. In the case of the pentoses, moreover, we have the additional fact, seemingly well established, that pentoses may give rise, directly or indirectly, to a production of glycogen. (Compare p. 26.) If we suppose the latter body to be formed directly from the pentoses, then their nutritive value is established, since that of glycogen is unquestionable. If, on the other hand, we suppose that the pentoses enable glycogen to be produced by protecting other materials from oxidation, then their nutritive value is likewise established, since they serve as a source of energy to the organism.

Recent respiration experiments by Cremer * seem to fully confirm this conclusion. In addition to an only partially successful trial with a dog, four experiments were made in which the urinary nitrogen and the respiratory carbon of rabbits were determined on a diet of varying quantities of rhamnose as compared with a preceding and succeeding day of fasting. No examination of the feces was made, except to determine the amount of rhamnose contained in them. Small amounts of this substance were also found in the urine. Neglecting the carbon and nitrogen of the feces and estimating the urinary carbon from the nitrogen by the use of Rubner's factor, $\uparrow 0.7462$, the following results have been computed, the two or three fasting days in each experiment being averaged. The amount of rhamnose stated is exclusive of that found in feces and urine.

* Zeit f. Biol., 42, 451.

$\dagger$ Ibid., 19, 318. 


\begin{tabular}{l} 
\\
\hline
\end{tabular}

The conditions in the first experiment were not regarded as satisfactory. In the other three the loss of fat from the body was notably diminished by the administration of rhamnose, precisely as in the experiments of Pettenkofer \& Voit and of Rubner (pp. 147 and 148) with the hexose carbohydrates. The quantitative results vary considerably in the individual experiments, but in the second and fourth correspond quite closely to the law of isodynamic replacement.

Kellner * computes from the results of respiration experiments in which extracted rye straw was added to a basal ration that the furfuroids (presumably pentosans) of this material must have contributed to the production of fat to as great an extent as starch or cellulose. (Compare p. 183.) A fortiori, therefore, they must be capable of protecting the body fat from oxidation.

Organic Acids.-Mention was made in Chapter II of the fact that the organic acids, which are found to some extent in the food and which are produced in large amounts by the fermentation of the carbohydrates in the digestive apparatus of herbivora, are oxidized in the body. From this latter fact we should anticipate that they might serve as sources of energy to the organism, and this anticipation apparently has been confirmed by several investigators.

Zuntz \& v. Mehring $†$ determined the amount of oxygen consumed by fasting rabbits before, during, and after the injection

* Landw. Vers. Stat., 53, 457.

† Arch. ges Physiol., 32, 173. 
into the circulation of sodium lactate. The results per kilogram and quarter hour were as follows:

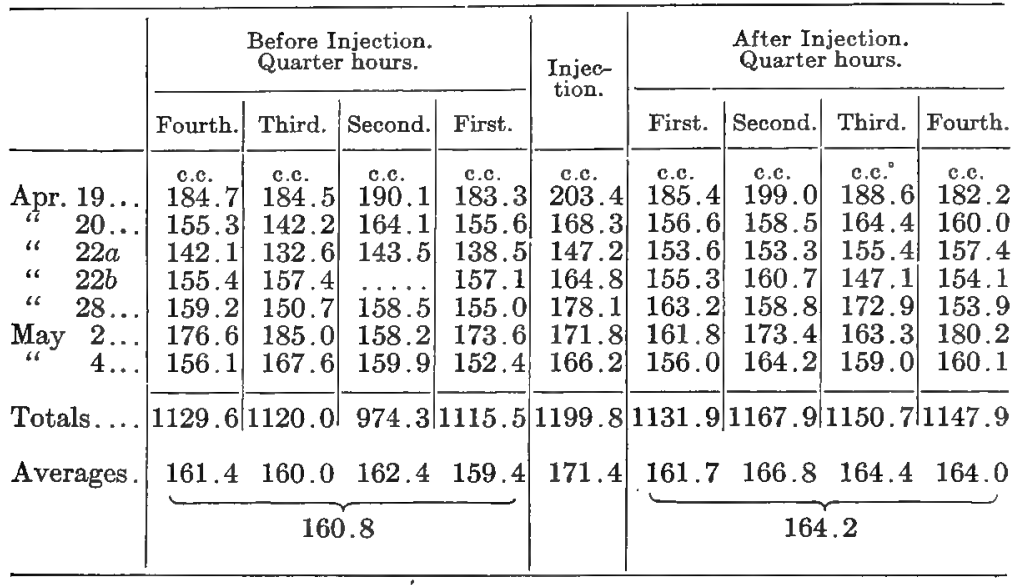

It being well established that lactic acid is readily oxidized in the body (compare p. 27), it is evident that in these experiments it must have protected the body fat from being metabolized, since otherwise the consumption of oxygen would have increased. Similar, although not decisive, results were obtained with sodium butyrate. On the other hand, sodium lactate administered by the mouth caused more or less increase in the oxygen cousumption. Wolfers * has reported confirmatory results with sodium lactate. Munk $\dagger$ injected sodium butyrate into the veins of fasting rabbits curarized to eliminate the effects of muscular activity and secure uniform metabolism, and determined the respiratory exchange by the Zuntz method ( $p .72$ ). The oxidation of sodium butyrate according to the equation.

$$
\mathrm{C}_{4} \mathrm{H}_{7} \mathrm{NaO}_{2}+5 \mathrm{O}_{2}=3 \mathrm{CO}_{2}+3 \mathrm{H}_{2} \mathrm{O}+\mathrm{NaHCO}_{3}
$$

corresponds to a respiratory quotient of 0.6 , which is less than that

* Arch. ges. Physiol., 32, 222.

$\dagger$ Ibid., 46, 322. 
of the fasting animal. The material lowering of the quotient which was observed was therefore interpreted as showing that the sodium butyrate was oxidized, and this conclusion was confirmed by the strongly alkaline character of the urine and the absence from it of butyric acid. The amount of sodium butyrate injected during $1 \frac{1}{4}$ to $1 \frac{1}{2}$ hours was sufficient in the several experiments to supply from 60 to 100 per cent. of the respiratory demand of the fasting animal. If this had been oxidized uselessly - that is, if the energy liberated had not been of use to the organism-then the consumption of oxygen and elimination of carbon dioxide should have increased correspondingly. This, however, was far from being the case, as the following fifteen-minute averages for the periods before, during, and after the injection show:

\begin{tabular}{|c|c|c|c|c|}
\hline & $\begin{array}{c}\text { Acid } \\
\text { Injected, } \\
\text { Grms. }\end{array}$ & $\begin{array}{c}\text { Oxygen } \\
\text { Consumed, } \\
\text { c.c. }\end{array}$ & $\begin{array}{c}\text { Carbon } \\
\text { Dioxide } \\
\text { Excreted, } \\
\text { c.c. }\end{array}$ & $\begin{array}{c}\text { Respira- } \\
\text { tory } \\
\text { Quotient. }\end{array}$ \\
\hline 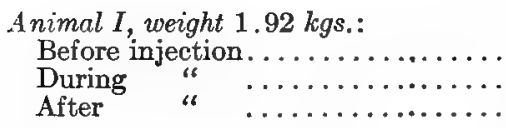 & $\begin{array}{c}0.133 \\
\ldots \ldots\end{array}$ & $\begin{array}{l}260.9 \\
280.0 \\
253.3\end{array}$ & $\begin{array}{l}196.1 \\
190.5 \\
181.2\end{array}$ & $\begin{array}{l}0.75 \\
0.68 \\
0.71\end{array}$ \\
\hline 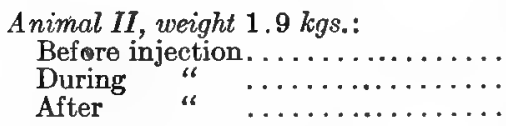 & $\begin{array}{c}\dot{0} \dot{199} \\
\ldots\end{array}$ & $\begin{array}{l}290.9 \\
325.2 \\
299.4\end{array}$ & $\begin{array}{l}228.3 \\
214.6 \\
230.9\end{array}$ & $\begin{array}{l}0.78 \\
0.66 \\
0.78\end{array}$ \\
\hline $\begin{array}{l}\text { Animal III, weight } 1.82 \mathrm{kgs} . \\
\quad \text { Before injection. } \ldots \ldots \ldots \ldots \ldots \ldots \ldots \ldots \ldots \ldots \\
\text { During " } \quad \ldots \ldots \ldots \ldots \ldots \ldots \ldots\end{array}$ & 0.206 & $\begin{array}{l}305.3 \\
330.9 \\
306.6\end{array}$ & $\begin{array}{l}243.4 \\
238.0 \\
235.3\end{array}$ & $\begin{array}{l}0.79 \\
0.72 \\
0.77\end{array}$ \\
\hline 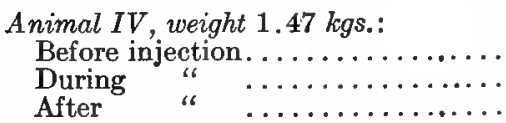 & 0.186 & $\begin{array}{l}278.9 \\
297.6 \\
278.1\end{array}$ & $\begin{array}{l}201.0 \\
197.9 \\
205.2\end{array}$ & $\begin{array}{l}0.72 \\
0.68 \\
0.73\end{array}$ \\
\hline
\end{tabular}

In place of an increase of 60 to 100 per cent. in the respiratory exchange under the influence of the sodium butyrate, there was an increase of only 7 to 8 per cent. in the oxygen and none at all in the carbon dioxide. It is evident, therefore, that the loss of fat from the body must have been largely diminished, the butyric acid serving as a source of energy in its place. A stimulating effect upon 
the heart's action was noticed, and Bókai is quoted as having shown a similar action on the peristaltic movements of the intestines, and these facts perhaps account for some of the increase of the oxygen, but Munk shows another reason for most of it. To produce $1 \mathrm{Cal}$. of energy by the oxidation of sodium butyrate he computes to require 0.324 gram of oxygen, while to produce $1 \mathrm{Cal}$. by the oxidation of fat requires, according to Zuntz \& Hagemann (Chapter VIII), $\mathbf{0 . 3 0 2}$ gram or 6.2 per cent. more in the first case. It would thus appear that the replacement of fat by sodium butyrate was substantially isodynamic.

Mallèvre * experimented with sodium acetate, whose respiratory quotient is 0.5 , by the same method as Munk, the amount injected equaling 85-100 per cent. of the respiratory demand. The results per quarter hour were:

\begin{tabular}{|c|c|c|c|c|c|}
\hline Weight and Condition. & & $\begin{array}{l}\text { Sodium } \\
\text { Acetate } \\
\text { per Kg. } \\
\text { Weight, } \\
\text { Grms. }\end{array}$ & $\begin{array}{l}\text { Oxygen } \\
\text { Con- } \\
\text { sumed. } \\
\text { c.c. }\end{array}$ & $\begin{array}{c}\text { Carbon } \\
\text { Dioxide } \\
\text { Excreted. } \\
\text { c.c. }\end{array}$ & $\begin{array}{l}\text { Respi- } \\
\text { ratory } \\
\text { Quotient. }\end{array}$ \\
\hline $\begin{array}{l}\text { Weight, } 1.44 \text { kgs. } \\
\text { Just after eating.. }\end{array}$ & $\begin{array}{l}\text { I. } \\
\text { Before injection.... } \\
\text { During } \quad . . . \\
\text { Residual effect.... } \\
\text { After injection.... } \\
\quad \text { II. }\end{array}$ & $\begin{array}{l}0.201 \\
\cdots \cdots \\
\cdots \cdots\end{array}$ & $\begin{array}{l}176.1 \\
193.8 \\
197.7 \\
178.0\end{array}$ & $\begin{array}{l}183.6 \\
167.1 \\
152.6 \\
171.2\end{array}$ & $\begin{array}{r}1.04 \\
0.86 \\
0.76 \\
.0 .96\end{array}$ \\
\hline $\begin{array}{l}\text { Weight, } 1.5 \text { kgs. } \\
\text { After two days' } \\
\text { fasting........ }\end{array}$ & $\begin{array}{l}\text { Before injection... } \\
\text { During " } \quad \ldots \\
\text { After }\end{array}$ & $\begin{array}{l}0.231 \\
\ldots \ldots\end{array}$ & $\begin{array}{l}195.3 \\
231.8 \\
211.2\end{array}$ & $\begin{array}{l}149.6 \\
168.2 \\
163.3\end{array}$ & $\begin{array}{l}0.77 \\
0.71 \\
0.77\end{array}$ \\
\hline $\begin{array}{l}\text { Weight, } 1.82 \mathrm{kgs} \text {; } \\
\text { After two days } \\
\text { fasting........ }\end{array}$ & 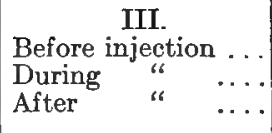 & $\begin{array}{l}0.127(?) \\
\ldots \ldots\end{array}$ & $\begin{array}{l}214.7 \\
244.8 \\
217.1\end{array}$ & $\begin{array}{l}165.9 \\
169.5 \\
166.6\end{array}$ & $\begin{array}{l}0.77 \\
0.69 \\
0.77\end{array}$ \\
\hline $\begin{array}{l}\text { Weight, } 1.7 \mathrm{kgs} . \\
\text { After one day's } \\
\text { fasting....... }\end{array}$ & $\begin{array}{l}\text { IV. } \\
\text { Before injection .... } \\
\text { During . } . . . \\
\text { Residual effect.... } \\
\text { After injection. .... }\end{array}$ & $\begin{array}{l}0.152 \\
\cdots \cdots \\
\cdots\end{array}$ & $\begin{array}{l}183.4 \\
208.1 \\
209.5 \\
194.7\end{array}$ & $\begin{array}{l}160.5 \\
167.0 \\
164.7 \\
164.7\end{array}$ & $\begin{array}{l}0.87 \\
0.80 \\
0.79 \\
0.85\end{array}$ \\
\hline
\end{tabular}

The decrease in the respiratory quotient. as well as the results of the examination of the urine, showed that the sodium acetate

* Arch. ges. Physiol., 49, 460. 
was oxidized in the body. The increase in the amount of oxygen consumed is much more marked than in Munk's experiments, ranging from 10.4 to 14 per cent. Moreover, as Mallèvre points out, in the oxidation of sodium acetate about the same volume of oxygen is required to produce a unit of heat as in the case of fat. Apparently, then, while the sodium acetate, like the sodium butyrate in Munk's experiments, must have largely diminished the metabolism of the body fat, it also stimulated the total metabolism and was substituted for the fat in less than the isodynamic ratio. As in Munk's experiments, a stimulation of the heart action and also an increased peristalsis of the intestines was observed.

It would seem, then, that lactic and butyric acids, when introduced into the circulation of the fasting animal, protect the body fat from oxidation, and replace other nutrients in isodynamic proportions. Acetic acid, on the contrary, seems inferior to the other two in this respect, and it is of interest to recall that according to Weiske \& Flechsig (p. 123) it apparently has also less effect in diminishing the proteid metabolism.

Crude Fiber.-As was stated on p. 117, the early experiments by v. Knieriem * upon the nutritive value of cellulose comprised respiration experiments as well as determinations of the proteid metabolism. Combining the results for nitrogen already given with those for carbon, we have the following:

\begin{tabular}{|c|c|c|c|c|}
\hline \multirow{2}{*}{ Period. } & \multirow{2}{*}{$\begin{array}{c}\text { Number } \\
\text { of } \\
\text { Days. }\end{array}$} & \multirow{2}{*}{$\begin{array}{l}\text { Food per Day. } \\
\text { (Two Animals.) }\end{array}$} & \multicolumn{2}{|c|}{ Gain or Loss of } \\
\hline & & & $\begin{array}{l}\text { Nitrogen, } \\
\text { Grms. }\end{array}$ & $\begin{array}{l}\text { Carbon, } \\
\text { Grms. }\end{array}$ \\
\hline $\begin{array}{l}\text { II } \cdots \\
\operatorname{III} \cdots \\
\operatorname{IV} \ldots \\
\operatorname{IV} \ldots\end{array}$ & $\begin{array}{r}9 \\
10 \\
5 \\
4 \\
3\end{array}$ & $\begin{array}{l}\text { Milk and horn dust ........... } \\
\text { Same }+18.63 \text { grms. crude fiber } \\
\text { Milk and horn dust ............ } \\
\text { Same }+11 \text { grms. cane-sugar..... } \\
\text { " }+33 \text { " }\end{array}$ & $\begin{array}{l}-0.599 \\
+0.104 \\
-0.330 \\
-0.318 \\
-0.023\end{array}$ & $\begin{array}{l}-4.521 \\
-0.434 \\
-4.868 \\
-1.673 \\
+5.653\end{array}$ \\
\hline
\end{tabular}

* Water-free.

$\uparrow$ Results regarded by the author as of doubtful value.

In addition to its effect in diminishing the proteid metabolism, the crude fiber in these experiments seems to have been fully as efficient as the cane-sugar as a substitute for body fat.

* Zeit. f. Biol., 21, 67. 
As we have seen, there has been considerable study of the effects of crude fiber on the proteid metabolism, but no other comparative experiments appear to have been made regarding the replacement values of cellulose and other carbohydrates in a maintenance ration.

The somewhat lower value which seems to be indicated for the organic acids by the experiments cited in the previous paragraph has been made the basis of conclusions as to the inferior nutritive value of cellulose, and Zuntz, ${ }^{*}$ in some comments on Mallèvre's experiments, remarks that the apparent equality between cellulose and starch observed in experiments on ruminants is to be explained by the fact that in these animals the starch also undergoes fermentation, a fact which the researches of G. Kühn at Möckern have since established. In other words, he would say that in case of ruminants the starch has as low a value as the cellulose rather than that the cellulose has as high a value as the starch.

Kellner has recently obtained results, to be discussed a little later, which seem to prove a participation by the digested cellulose in actual fat production to as great an extent as by starch, and which therefore seem to put the nutritive value of the form of cellulose used by him beyond dispute.

Utilization of Excess of Non-nitrogenous Nutrients. - No elaborate scientific investigation is needed to teach us that food supplied in exceess of the immediate demands of the organism results in a greater or less storage of material in the body, this material consisting, in the mature animal, largely of fat. But while the fact of fat formation is obvious, the exact source of the fat has been the subject of as much controversy as almost any physiological question. As we have seen in the previous section, opinions are still far from being unanimous as to the production of fat from proteids, while until quite recently the same might have been said regarding the carbohydrates as a source of fat. A very complete critical review of the literature of the subject of fat formation in the animal body was published by Soskin $\dagger$ in 1894, and to this the writer is indebted for a considerable number of the statements and references on the succeeding pages.

As was stated on p. 29, the older physiologists looked upon the

* Arch. ges. Physiol. 49, 447.

† Jour. f Landw., 42, 157. 
fat of the food as the sole source of the body fat. The contrary view was first propounded by Liebig * in 1843. After drawing the distinction between "plastic materials" (proteids), which serve to build up the tissue, and "respiratory materials" (non-nitrogenous substances), which serve as sources of heat, he asserts that any excess of the latter over the immediate needs of the organism is converted into fat. This proposition, which was based upon observation and general knowledge rather than upon specific experiments, led to an active controversy with the adherents of the older view and to much direct experimental work.

Liebig, while not denying that the food fat was a source of body fat, maintained that the amount contributed by it was insignificant and regarded the carbohydrates as the chief source of animal fat. The controversy turned upon the question of the possibility of accounting for the body fat by the food fat, both parties tacitly agreeing that any excess was to be credited to the carbohydrates. The principal champions of the older view were Boussingault, Dumas, and Payen. $\dagger$ Boussingault, in particular, brought forward the results of experiments on milch cows, according to which the fat of the food fully sufficed to account for that in the milk. They all, however, ultimately came to acknowledge the substantial accuracy of Liebig's view. Thus Dumas \& Milne-Edwards $\ddagger$ confirmed the results of Huber \& Gundlach, $\S$ cited by Liebig, according to which bees can produce wax from honey or sugar. Boussingault \| published the results of new experiments on milch cows as sustaining his previous view of the question, but later $\uparrow$ convinced himself by careful and laborious experiments on the fattening of swine and geese of its untenability and of the correctness of Liebig's position. Thus in one of his experiments nine pigs gained $103.2 \mathrm{kgs}$. of fat in ninety-eight days, while the food contained but $67.6 \mathrm{kgs}$, of which about $8 \mathrm{kgs}$. was excreted undigested in the feces. Persoz ** likewise, in experiments with geese, obtained similar

* Ann. Chem. Pharm., 45, 112; 48, 126; 54, 376.

$\dagger$ Annal. de Chim. et de Physique., 3d ser. 8, 63.

I Ibid., 14, 400 .

$\S$ Naturgeschichte der Bienen, Kassel, 1842.

|l Annal. de Chim. et de Physique., 3d ser., 12, 153

Il Loc. cit., 14, 419.

** Annal. de Chim. et de Physique., 14, 408. 
results and also observed a production of fat by these animals when fed on food from which all fat had been removed.

FAT.-That the fat of the food may serve directly as a source of body fat has been shown by Hofmann, ${ }^{*}$ who fasted a dog for thirty days, thus rendering the body almost fat-free, and then fed for five days large amounts of fat bacon containing as little lean meat as possible, and from which there were digested daily 370.8 grams of fat and 49.4 grams of protein. At the end of the five days the body of the animal contained 1352.7 grams of fat. Estimating its fat content at the close of the fasting period at 150 grams, there was produced daily about 240 grams of body fat. According to the highest recorded estimates not over 26 grams of this could possibly have been formed from the protein of the food. Hofmann also shows from the result of one of Pettenkofer \& Voit's respiration experiments, in which meat and fat were fed, that part of the observed gain of fat must have had its source in the fat of the food.

The latter investigators also showed in the last of the experiments cited on p. 144 that a large ration of fat alone may result in a considerable storage of fat. Most of the experiments by the same investigators in which lean meat and fat were fed show not merely a diminution of the loss of body fat but an actual increase in its amount. (Compare the averages on page 150.) The fact is most strikingly shown, however, in a series in which increasing amounts of fat were added to a uniform ration of meat which was itself sufficient to maintain both nitrogen and carbon equilibrium. The results as given by Pettenkofer \& Voit $\uparrow$ are contained in the table at the top of p. 165, those on the basal ration of meat being the same as those given also on p. 109 for the first series.

It is of course possible to interpret these results as showing that the fat of the food was oxidized and protected an equivalent amount of the non-nitrogenous residue of the proteids from oxidation and that the latter were the real source of the fat gained. No necessity for such an interpretation is apparent, however, and the direct explanation appears the simpler and more natural.

The results of experiments upon the deposition of foreign fats

* Zeit. f. Biol., 8, 153.

$\dagger$ Ibid., 9, 30 . 
THE RELATIONS OF METABOLISM TO FOOD-SUPPLY.

\begin{tabular}{|c|c|c|c|c|c|c|}
\hline \multirow{2}{*}{$\begin{array}{c}\text { Number } \\
\text { of } \\
\text { Trials. }\end{array}$} & \multicolumn{2}{|c|}{ Food. } & \multirow{2}{*}{$\begin{array}{l}\text { Nitrogen } \\
\text { of } \\
\text { Excreta. }\end{array}$} & \multirow{2}{*}{$\begin{array}{c}\text { Total Carbon } \\
\text { of } \\
\text { Excreta. }\end{array}$} & \multicolumn{2}{|c|}{ Gain or Loss of } \\
\hline & Meat. & Fat. & & & $\begin{array}{c}\text { Flesh. } \\
(\mathrm{N}+0.034 .)\end{array}$ & Fat. \\
\hline $\begin{array}{l}3 \\
2 \\
1 \\
2 \\
1 \\
2\end{array}$ & $\begin{array}{l}1500 \\
1500 \\
1500 \\
1500 \\
1500 \\
1500\end{array}$ & $\begin{array}{r}0 \\
30 \\
60 \\
100 \\
100 \\
150\end{array}$ & $\begin{array}{l}51.0 \\
49.6 \\
51.0 \\
47.7 \\
49.3 \\
49.5\end{array}$ & $\begin{array}{l}184.5 \\
180.6 \\
203.6 \\
182.4 \\
174.4 \\
193.1\end{array}$ & $\begin{array}{r}0 \\
+42.8 \\
-\quad 0.6 \\
+97.8 \\
+49.4 \\
+44.8\end{array}$ & $\begin{array}{l}+\quad 4.3 \\
+\quad 32.4 \\
+\quad 39.4 \\
+91.1 \\
+109.5 \\
+135.7\end{array}$ \\
\hline
\end{tabular}

in the body which were considered in Chapter II, p. 30, also testify to the direct formation of body fat from food fat.

CARBohydrates.-Among the experiments of Pettenkofer \& Voit which have been cited in the foregoing pages are several which show a production of fat upon a ration of lean meat with the addition of starch or dextrose or of starch alone. A more complete summary of these experiments $*$ is given below:

\begin{tabular}{|c|c|c|c|c|c|c|}
\hline & \multirow{2}{*}{$\begin{array}{c}\text { vimber } \\
\text { of } \\
\text { Fxpert- } \\
\text { ments. }\end{array}$} & \multicolumn{3}{|c|}{ Food per Day. } & \multicolumn{2}{|c|}{ Gain or Loss of } \\
\hline & & $\begin{array}{l}\text { Meat } \\
\text { Grms. }\end{array}$ & $\begin{array}{l}\text { Fat. } \\
\text { Grms. }\end{array}$ & $\begin{array}{c}\text { Carbo- } \\
\text { hy Irates. } \\
\text { Grms. }\end{array}$ & $\begin{array}{c}\text { Proteids, } \\
\text { Grms. }\end{array}$ & $\begin{array}{l}\text { Fat, } \\
\text { Grms. }\end{array}$ \\
\hline $\begin{array}{l}\text { Starch } \ldots \ldots \ldots \ldots \ldots \\
\text { Proteids and dextrose } \ldots \ldots \\
\text { Proteids and starch } \ldots \ldots\end{array}$ & $\begin{array}{l}1 \\
1 \\
3 \\
3 \\
1 \\
8 \\
1 \\
2 \\
1\end{array}$ & $\begin{array}{r}\cdots \\
\cdots \\
50 \\
500 \\
400 \\
500 \\
800 \\
1500 \\
1800\end{array}$ & $\begin{array}{r}16.9 \\
21.2 \\
20.2 \\
. .2 \\
5.6 \\
5.3 \\
13.7 \\
4.5 \\
10.1\end{array}$ & $\begin{array}{l}450 \\
597 \\
700 \\
200 \\
400 \\
200 \\
450 \\
200 \\
450\end{array}$ & $\begin{array}{r}-45.0 \\
-58.8 \\
-38.8 \\
-8.1 \\
-3.1 \\
-11.3 \\
+40.6 \\
+\quad 6.3 \\
+70.6\end{array}$ & $\begin{array}{r}+\quad 56.2 \\
+\quad 3.4 \\
+106.4 \\
+\quad 15.0 \\
+109.9 \\
+\quad 19.5 \\
+\quad 71.5 \\
+\quad 18.1 \\
+126.5\end{array}$ \\
\hline
\end{tabular}

Pettenkofer \& Voit's Conclusions.-In discussing these results Pettenkofer \& Voit assumed that, as computed by Henneberg, $† 100$ grams of proteids can give rise to a maximum of 51.4 parts of fat. On this basis they found that, with two apparent exceptions, the fat of the food, together with that which could be derived from

* Zeit. f. Biol., 9, 435.

$\dagger$ Landw. Vers. Stat., 10, 455, foot-note. 
the amount of proteids metabolized, was sufficient to account for the gain of fat. They therefore concluded that the carbohydrates simply protected these materials from oxidation and regarded the formation of fat from the former as improbable, being confirmed in this belief by the observation that the amount of fat produced was proportional to the proteids rather than to the carbohydrates of the food. The apparent exceptions they regarded as due to a retention of undigested starch in the alimentary canal. In brief, Pettenkofer \& Voit, while not denying that carbohydrates aid in the production of fat, regarded their action as an indirect one.

It should be added that, contrary to the general impression, Voit did not absolutely deny the formation of fat from carbohydrates, but regarded it as improbable and unproved. Moreover, he came later to admit the truth of the opposite view, and even furnished from his laboratory experimental evidence in its support.

At an earlier date Voit* had likewise made experiments on a milch cow, the result of which was that not only all the fat of the milk, but most of the milk-sugar as well, could be accounted for by the proteids and fat of the food. Voit also examined the numerous experiments of Dumas, Persoz, Boussingault, and others (p. 163) upon the origin of animal fat and satisfied himself that, although they undoubtedly showed, as their authors claimed, a formation of fat from other ingredients of the food, the amount produced could at least in the great majority of cases be accounted for by the proteids of the latter.

It is important to observe that the evidence supporting Voit's view was negative evidence. The results could be explained on the hypothesis that the carbohydrates did not contribute to fat production, but while a large number of such results might render the hypothesis very probable, they could not demonstrate its truth. On the other hand, even a single well-authenticated case in which the fat and proteids of the food did not suffice to account for the amount of fat formed in the body would suffice to establish the possibility of its formation from other materials. A few apparent cases of this sort among earlier experiments Voit was able to explain plausibly, but there was one important exception, viz., the experiments of

* Zeit. f. Biol., 5, 79-169. 
Lawes \& Gilbert* at Rathamsted, in 1850 , on the fattening of swine.

Lawes \& Gilbert's Investigations.-These experiments constituted part of a series of feeding trials with fattening sheep and pigs, undertaken to test the then current view of Boussingault, according to which the feeding value of stock foods was proportional to their content of nitrogen. From the results of their extensive experiments, Lawes \& Gilbert concluded that in fattening animals both the amount of food consumed by a given weight of animal within a given time and the increase in weight obtained are measurcd rather by the supply of non-nitrogenous than of nitrogenous constituents in the food. This fact of itself strongly suggests a production of fat from carbohydrates.

In connection with these feeding trials investigations were also made into the composition of the increase in live weight during fattening. $\dagger$ By a comparison of the weight and composition of one of the fattened pigs with those of an animal supposed to be precisely similar at the beginning of the fattening the percentage composition of the increase was found to be approximately:

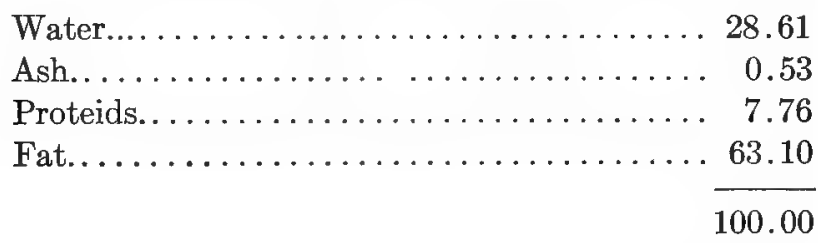

During the ten weeks of the fattening the animal gained 88 pounds, containing according to the above figures 55.5 pounds of fat, while the total food consumed contained but 13.7 pounds. In other words, over three fourths of the fat was formed from other ingredients of the food.

After the publication of Voit's first paper, Lawes \& Gilbert \$ presented the results of this and eight other experiments in their

* Report British Association Adv. Sci., 1852; Jour. Roy. Agr. Soc., 14, 459; Rep. British Asso. Adv. Sci., 1854; Rothamsted Memoirs, Vol. II.

$\dagger$ Jour. Roy. Agr. Society, 21, 465; Phil. Trans., Part II, 1859, p. 493.

$\ddagger$ Rep. British Asso. Adv. Sci., 1866; Phil. Mag., Dec., 1866; Rothamsted Memoirs, Vol. IV. 
bearing on the origin of the fat. Nos. 2 to 5 were selections from the first two series of the experiments of 1850 (designated as I and II in the table below) and Nos. 6 to 9 were experiments upon the equivalency of starch and sugar in food reported in $1854 *$ (designated below by S). The following table shows the original numbers of the several experiments and the character of the food consumed:

\begin{tabular}{|c|c|c|c|}
\hline \multirow{2}{*}{ No. } & \multicolumn{2}{|c|}{ Original Designation. } & \multirow{2}{*}{ Food. } \\
\hline & Series. & Number. & \\
\hline $\begin{array}{l}1 \\
2 \\
3 \\
4 \\
5 \\
6 \\
7 \\
8 \\
9\end{array}$ & $\begin{array}{l}\text { I } \\
\text { I } \\
\text { I } \\
\text { S } \\
\text { S } \\
\text { S } \\
\text { S }\end{array}$ & $\begin{array}{r}12 \\
1 \\
5 \\
5 \\
1 \\
2 \\
3 \\
4\end{array}$ & $\begin{array}{l}\text { Bean meal, lentil meal, bran, and barley meal ad lib. } \\
\text { Bean meal, lentil meal, bran, and corn meal ad lib. } \\
\text { Bean meal and lentil meal ad lib. } \\
\text { Corn meal ad lib. } \\
\text { Barley meal ad lib. } \\
\text { Lentil meal and bran, with sugar ad lib. } \\
\text { Lentil meal and bran, with starch ad lib. } \\
\text { Lentil meal and bran, with sugar and starch. } \\
\text { Lentil meal, bran, sugar, and starch ad lib. }\end{array}$ \\
\hline
\end{tabular}

From the results of the first experiment, the amount of fat contained in the observed increase in live weight in each case was computed, the animals being assumed to have had at the beginning of the fattening the composition of the lean pig analyzed and at its close that of the fat pig. These amounts were then compared with the amounts which could have been produced from the fat and proteids of the food. In order to make the case as unfavorable as possible for the carbohydrates the authors assumed:

First, that all the fat of the food was digested and laid up in the body.

Second, that all the nitrogenous matter of the food was digested, and that it all consisted of true proteids.

Third, that, after deducting the amount of proteids gained by the body, the total carbon of the remainder, minus that required to form urea, was available for fat formation.

The results of the comparison were as follows, calculated per 100 pounds gain in live weight.

*Rep. Brit. Asso. Adv. Sci., 1854. 


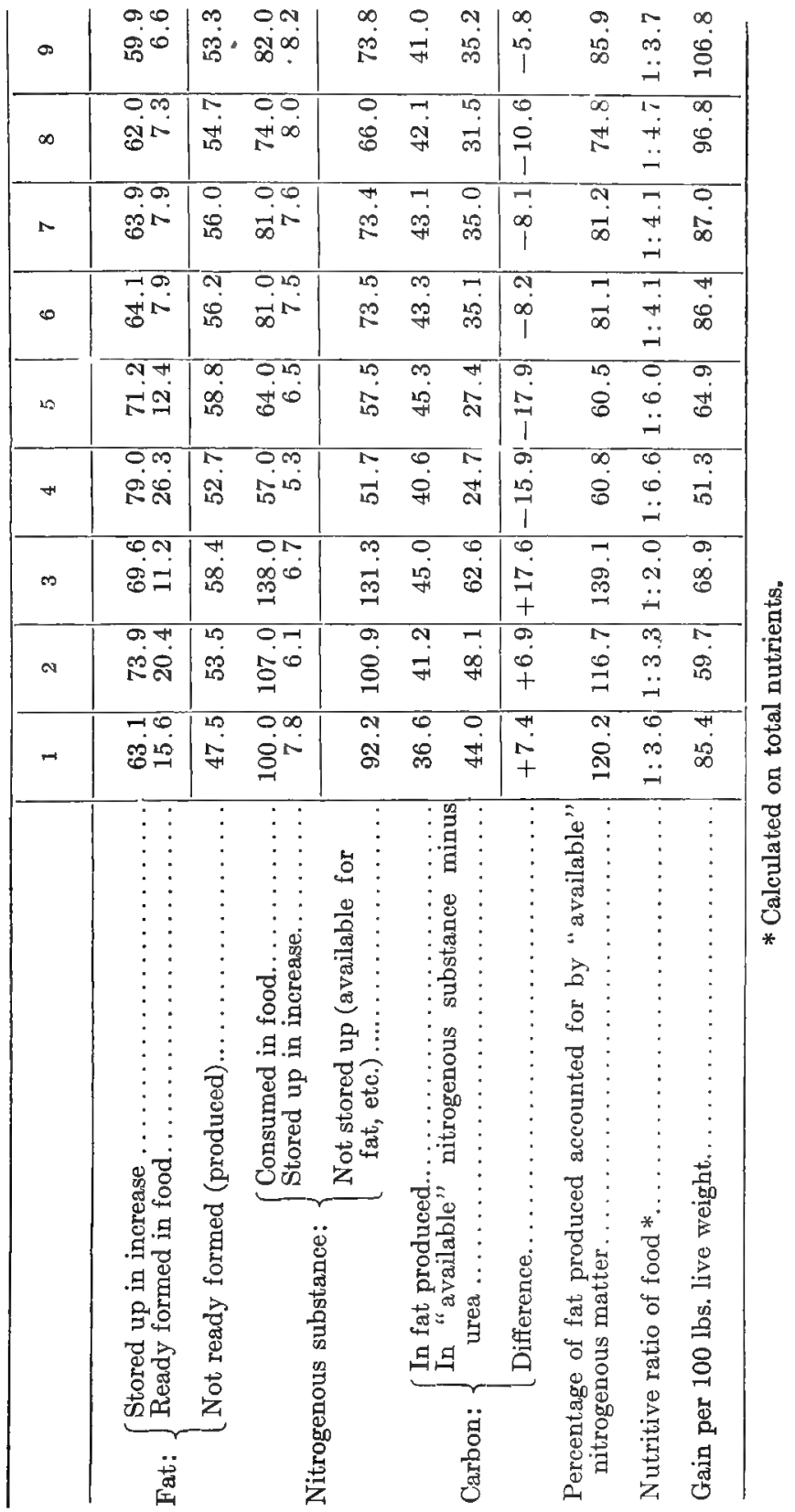


Even on the most extreme assumptions it is only possible to regard the fat produced as derived wholly from the proteids of the food in three cases in which an excessive proportion of the latter was fed. If the probable digestibility of the foods used be considered, and Henneberg's factor (51.4 per cent.) for the possible production of fat from proteids be used, the results show even more decidedly a formation of fat from carbohydrates. In a later paper,* in reply to criticisms, the authors state that they have reviewed and recalculated many of their experiments with the result that, while the experiments with ruminants (sheep and oxen) failed to furnish conclusive evidence of the formation of fat from carbohydrates, a large number of those with pigs unquestionably showed such formation.

In view of their historical interest it has seemed desirable to give the results of Jawes \& Gilbert's experiments in some detail, although at the time they hardly secured the recognition which was due them and Voit's views became the generally accepted theory for the next twenty-five years. Notwithstanding the latter fact, however, results of experiments on herbivorous animals speedily began to accumulate which were difficult to reconcile with Voit's hypothesis.

Experiments on Ruminants.-Experiments on milch cows were made by Voit himself, as already noted. G. Kühn \& Fleischer $\dagger$ a little later discussed the results of two of their extensive feeding experiments on milch cows in their bearing on this point, and $M$. Fleischer $t$ did the same with the results of similar experiments made by Wolff and himself.\& Their results are tabulated on the opposite page.

Neither Voit's nor Fleischer's results are such as to require the assumption of a formation of fat from carbohydrates. Those of Kühn \& Fleischer show a small excess of fat in the milk over that producible from the fat and proteids of the food, but the authors

\footnotetext{
* Jour. Anat. and Physiol., 9, 577; Rothamsted Memoirs, Vol. IV.

† Landw. Vers. Stat., 10, 418; 12, 451.

¥Virchow's Archiv, 51, 30.

\&Jour. f. Landw., 19, 371, and 20, 395.
} 


\begin{tabular}{|c|c|c|c|c|}
\hline & $\begin{array}{l}\text { Fat of } \\
\text { Fodder, } \\
\text { Grms. }\end{array}$ & $\begin{array}{c}\text { Fat from } \\
\text { Protein, } \\
\text { Grms. }\end{array}$ & $\begin{array}{l}\text { Total, } \\
\text { Grms. }\end{array}$ & $\begin{array}{l}\text { Fat of } \\
\text { the Milk, } \\
\text { Grms. }\end{array}$ \\
\hline Voit: $\left\{\begin{array}{c}\text { Experiment } a \ldots \ldots \ldots \ldots \ldots \ldots \\
b \ldots \ldots \ldots \ldots \ldots\end{array}\right.$ & $\begin{array}{l}318.8 \\
276.0\end{array}$ & $\begin{array}{l}401.8 \\
308.5\end{array}$ & $\begin{array}{l}720.6 \\
584.5\end{array}$ & $\begin{array}{l}577.5 \\
337.3\end{array}$ \\
\hline Kühn \& Fleischer: $\{\underset{\text { Experiment I }}{\text { II.. }}$ & $\begin{array}{l}183.5 \\
183.5\end{array}$ & $\begin{array}{l}79.5 \\
69.5\end{array}$ & $\begin{array}{l}263.0 \\
253.0\end{array}$ & $\begin{array}{l}277.5 \\
292.0\end{array}$ \\
\hline Fleischer: $\begin{cases}\text { Experiment } & \text { I. } \ldots \ldots \ldots \ldots\end{cases}$ & $\begin{array}{l}170.5 \\
166.5\end{array}$ & $\begin{array}{l}158.5 \\
170.0\end{array}$ & $\begin{array}{l}329.0 \\
336.5\end{array}$ & $\begin{array}{l}303.5 \\
290.5\end{array}$ \\
\hline
\end{tabular}

regard the differences as within the limits of error in such experiments.

Studies of the results of fattening experiments with ruminants give similar results. On the basis of Lawes \& Gilbert's determinations of the composition of the increase of live weight in fattening, the amount of fat produced in such an experiment may be approximately computed and compared with the amounts of proteids and fat in the food. Such a comparison by the writer * in seventy-seven experiments on sheep showed that, with one or two possible exceptions, the fat and proteids of the food were sufficient to account for the amount of fat formed, although in some of the experiments little margin was left.

Experiments on Swine.-Experiments with swine, on the other hand, as Wolff $\dagger$ has shown, have almost without exception given results which can scarcely be explained except upon the hypothesis of a formation of fat from carbohydrates. These animals, as Lawes \& Gilbert pointed out in their early papers, are especially adapted to experiments of this sort, since they consume a relatively large amount of easily digestible food, have a small proportion of offal to carcass, and are by nature inclined to lay on fat readily. It was therefore to be expected that experiments upon swine would show a production of fat from carbohydrates, if such took place, more decisively than those upon ruminants.

Experiments on pigs by Weiske \& Wildt, $\downarrow$ it is true, on the same plan as those by I.awes \& Gilbert, yielded results consistent with Voit's theory, showing a formation of 5565 grams of fat in the

* Manual of Cattle Feeding, p. 177.

† Ernährung Landw. Nutzth., pp. 354-356

$\ddagger$ Zeitschrift f. Biol., 10, 1. 
body as compared with a possible 6724 grams from the fat and proteids of the food. The feeds used, however, were not well suited to young animals and the gain was abnormally small in proportion to the food consumed, so that the results could not be expected to be decisive. Moreover, the presence of non-proteid nitrogen in the food is not considered in the computation. (See the next paragraph.)

Sources of Uncertainty.-Up to this point the results of experiments on herbivorous and omnivorous animals had been somewhat conflicting. Before taking up the later investigations it is desirable to point out some of the uncertainties attaching to experiments such as those above enumerated. These relate, first, to the amount of fat actually produced, and second, to the possible sources of supply in the food.

The basis for estimating the amount of fat actually produced by a fattening animal was in two cases a comparison with the amount in a supposedly similar animal at the beginning of the fattening, the fattened animal being actually analyzed. In the remainder the increase in live weight was assumed to have the composition found by Lawes \& Gilbert. It need scarcely be pointed out that the results of such comparisons can be only approximate and are subject to a considerable range of error. Only the most decided results one way or the other can be accepted as at all conclusive. In experiments on milch cows the production of milk fat can of course be determined, but the variations in the weight of such an animal often render any conclusions as to gain or loss of body fat. so difficult that the results as a whole are less satisfactory than those on fattening.

The possible sources of fat in the food, aside from the carbohydrates, are the ether extract and the proteids. As regards the first, it is certain that not all the digestible ether extract of stock foods is true fat. With the proteids the case is still worse. In particular we now know that a portion, and in some cases a considerable portion, of the total nitrogenous matter of feeding-stuffs consists of non-proteid material, which so far as we know contributes little if anything directly to fat production. This is a very important source of error. Thus the writer * has shown, as has also Soxhlet, $\dagger$ that if

* Manual of Cattle Feeding, p. 182.

† Compare Soskin, Jour. f. Landw., 42, 203. 
account be taken of this fact the teachings of Weiske \& Wildt's experiment cited above are exactly reversed and show a formation of fat from carbohydrates. A consideration of the same fact, of course, tends to make the results of all similar experiments, including those on milch cows, more favorable to the carbohydrates.

Still further, it is doubtful whether $\mathbf{1 0 0}$ parts of proteids can actually yield 51.4 parts of fat. The latter number was computed by Henneberg from the elementary composition of proteids and of urea to be the maximum amount obtainable. Zuntz,* however, has called attention to the fact that if the proteids actually split up in the manner which Henneberg's calculation supposes, the products must contain all the potential energy of the original material, so that none can be given off during their cleavage. This is a process wholly without analogy in the animal body, and, to say the least, very improbable. It would seem then, that even if we still hold to a formation of fat from proteids, we must considerably reduce our estimate of its amount.

Later Fattening Experiments.-All these considerations tend to strengthen the belief that fat is formed from carbohydrates, and more recent experiments have demonstrated that such is the fact. Henneberg, Kern, \& Wattenberg, $t$ in experiments undertaken to determine the rate of gain and the composition of the increase of fattening sheep, and conducted substantially like those of Lawes $\&$ Gilbert on swine, were the first to furnish proof of the formation of fat from carbohydrates by ruminants. Wolfft having pointed out that their results demonstrated that fact, Henneberg discussed this feature of the experiments in a later pubjication. $\&$ Regarding all the digested ether extract of the food as pure fat, and assuming that all the digested nitrogenous matters were true proteids capable of yielding 51.4 per cent. of fat, he obtained the results given on p. 174 . Forty-two per cent. more fat was produced than could be accounted for by the fat and proteids of the food, even on the extreme assumptions made. Furthermore, not only did some of the nitrogenous substances of the food undoubtedly consist of non-pro-

* Landw. Jahrb., 8, 96.

$\dagger$ Jour. f. Landw., 26, 549.

$\ddagger$ Land w. Jahrb., 8, I. Supp., 269.

\& Zeit f Biol, 17. 345 . 


\begin{tabular}{|c|c|c|}
\hline & $\begin{array}{c}\text { Proteids, } \\
\text { Grms. }\end{array}$ & $\begin{array}{l}\text { Fat, } \\
\text { Grms. }\end{array}$ \\
\hline 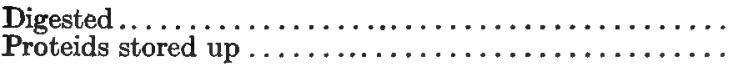 & $\begin{array}{r}10220 \\
936\end{array}$ & 2100 \\
\hline 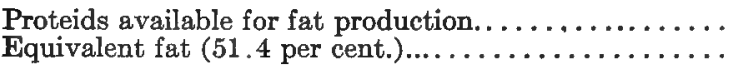 & $\begin{array}{l}9284 \\
\ldots \ldots\end{array}$ & 4772 \\
\hline $\begin{array}{l}\text { Total from fat and proteids. } \ldots \ldots \ldots \ldots \ldots \ldots \ldots \ldots \ldots \\
\text { Actually produced by animal. } \ldots \ldots \ldots \ldots \ldots \ldots\end{array}$ & & $\begin{array}{l}6872 \\
9730\end{array}$ \\
\hline
\end{tabular}

teids, but a high figure was assumed for their digestibility, and in computing the gain of fat by the animal no account was taken of the fat of the wool and of the offal. Henneberg's final conclusion is that no possible errors arising from differences in the animals compared or from irregularities in the consumption of food can explain away the above result.

Soxhlet * made similar experiments with swine fattened on rice, that is, on a feeding-stuff poor in proteids and fat and rich in carbohydrates, with the result that only 17 to 18 per cent. of the fat produced could be accounted for by the digestible protein and fat of the food. In two experiments with the same species of animal by Tschirwinsky $\dagger$ but 43 per cent. and 28 per cent. respectively of the fat production could be thus accounted for. Of six experiments on geese by B. Schulze, $\$$ four, in which a comparatively wide nutritive ratio was used, showed that at least from 5 to 20 per cent. of the fat must have been produced from carbohydrates. Chaniewski \& likewise experimented on geese and obtatined much more decisive results, from 72 to 87 per cent. of the observed fat production being necessarily ascribed to the carbohydrates.

Recent experiments by Jordan $\|$ have shown that the dairy cow may likewise produce fat from carbohydrates. In the first experiment a cow weighing 867 pounds was fed for fifty-nine days with food from which most of the fat had been extracted, the digestible

* Bied. Centr. BI. Ag. Chem., 10, 674.

$\dagger$ Landw. Vers. Stat., 29, 317.

¥ Landw. Jahrb., 11, 57.

§ Zeit. f. Biol., 20, 179.

|l N. Y. State Experiment Station, Bulls. 132 and 197. 
protein of the ration being varied from 184 grams to 841 grams per day. During this time she gained 33 pounds in weight, and her whole appearance was such as to negative the assumption of any considerable loss of body fat. In the second experiment one cow was fed a ration poor in fat, one a normal ration, and one a ration unusually rich in fat, the protein supply being again varied through a considerable range. As in the previous case the gain in weight and the general condition of the cows forbare the assumption that body fat was drawn upon to any material extent. In all instances except the last a considerable formation of fat from carbohydrates was shown.

The following table gives the more important data of the above experiments:

\begin{tabular}{|c|c|c|c|c|c|c|}
\hline Experimenter. & Animal. & $\begin{array}{c}\text { Total } \\
\text { Proteid* } \\
\text { Meta- } \\
\text { bolism, } \\
\text { Grms. }\end{array}$ & $\begin{array}{c}\text { Equiva- } \\
\text { lent Fat, } \\
\text { Grms. }\end{array}$ & $\begin{array}{l}\text { Fat of } \\
\text { Food, } \\
\text { Grms. }\end{array}$ & $\begin{array}{c}\text { Total } \\
\text { from Fat } \\
\text { and } \\
\text { Proteids, } \\
\text { Grms. }\end{array}$ & $\begin{array}{c}\text { Fat } \\
\text { Actually } \\
\text { produced } \\
\text { Grms. }\end{array}$ \\
\hline $\begin{array}{c}\text { Henneberg, Kern, \& } \\
\text { Wattenberg ......... }\end{array}$ & Sheep & 9,284 & 4,772 & 2,100 & 6,872 & 9,730 \\
\hline Soxhlet...$\ldots \ldots \ldots$ & Swine \{ & $\begin{array}{l}3,463 \\
7,169\end{array}$ & & $\begin{array}{l}300 \\
340\end{array}$ & & $\begin{array}{l}10,082 \\
22,180\end{array}$ \\
\hline Tschirwinsky & Swine & $\begin{array}{l}5,934 \\
2,361 \dagger\end{array}$ & $\begin{array}{l}3,050 \\
1,213\end{array}$ & $\begin{array}{l}656 \\
203\end{array}$ & $\begin{array}{l}3,706 \\
1,416\end{array}$ & $\begin{array}{r}8,577 \\
5,429\end{array}$ \\
\hline & & 1,054 & $383 \ddagger$ & 222 & 605 & 387 \\
\hline & & 1,049 & $381+$ & 221 & 602 & 539 \\
\hline Schulze. . & Geese & $\begin{array}{l}785 \\
785\end{array}$ & $\begin{array}{l}286 \pm \\
286 \pm\end{array}$ & $\begin{array}{l}205 \\
205\end{array}$ & $\begin{array}{l}491 \\
491\end{array}$ & $\begin{array}{l}515 \\
612\end{array}$ \\
\hline & & 55 & $194 t$ & 203 & 397 & 492 \\
\hline & & 55 & $194 \ddagger$ & 203 & 397 & 471 \\
\hline & & 11 & & & 75 & \\
\hline Chaniewski..... & Geese & 203 & 105 & 32 & 137 & 640 \\
\hline & & 100 & 51 & & & \\
\hline an: $\left\{\begin{array}{l}59 \text { days } \\
74\end{array}\right\}$ & & 15,109 & 7,766 & 1,490 & 9,256 & 17,585 \\
\hline Jordan: $\left\{\begin{aligned} 74 & \text { "r } \\
4 & \text { " }\end{aligned}\right\}$ & Cows & $\begin{array}{r}34,661 \\
2,209\end{array}$ & $\begin{array}{r}17,816 \\
1,131\end{array}$ & $\begin{array}{l}2,211 \\
1,504\end{array}$ & $\begin{array}{r}20,027 \\
2,635\end{array}$ & $\begin{array}{r}37,037 \\
3,289\end{array}$ \\
\hline
\end{tabular}

In view of the extreme assumptions made in these computations as to the possible contribution by the proteids and fat of the food

* Digested protein of food less gain of protein by the animal.

$\dagger$ In original 2572 grms.

$\ddagger$ Computed on a different basis from the other experiments Compare loc. cit, p. 84 . 
to fat production, and of the very large differences between this amount and the fat computed to have been actually formed, the possible errors of the method are relatively insignificant, and these investigations, together with the earlier ones, must be regarded as establishing the fact of a formation of fat from carbohydrates.

The earliest experiment to be published in full demonstrating the production of fat from carbohydrates in the body of the dog, was by Munk.* The animal was deprived of food long enough to render it certain that but traces of fat remained in the body. It was then fed for twenty-four days on a diet consisting of small amounts of meat, with some gelatine, and large quantities of starch and sugar. In the body of the animal at the close of the experiment 1070 grams of fat were found, of which Munk estimates that at least 960 grams must have been produced during the experiment, while the proteids fed could have produced as a maximum only 415.3 granis and the meat itself contained but 75 grams of fat. Even if a formation of fat from gelatine be admitted, a considerable excess of fat remains unaccounted for except by the carbohydrates of the food.

Respiration Experiments.-There are not wanting, however, for final demonstration, experiments with the respiration apparatus, in which the total income and outgo of nitrogen and carbon has been determined.

Meissl, Strohmer, \& Lorenz, $\uparrow$ in very carefully conducted respiration experiments upon swine, using a wide, a medium, and a narrow nutritive ratio, obtained the following results:

\begin{tabular}{|c|c|c|c|c|c|}
\hline $\begin{array}{l}\text { Food, } \\
\text { Grms. }\end{array}$ & $\begin{array}{l}\text { Proteid } \\
\text { Metabolism, } \\
\text { Grms. }\end{array}$ & $\begin{array}{c}\text { Equivalent } \\
\text { Fat, } \\
\text { Grms. }\end{array}$ & $\begin{array}{l}\text { Fat of } \\
\text { Food, } \\
\text { Grms. }\end{array}$ & $\begin{array}{c}\text { Total } \\
\text { from Fat } \\
\text { and Proteids, } \\
\text { Grms. }\end{array}$ & $\begin{array}{c}\text { Fat } \\
\text { Actually } \\
\text { Produced, } \\
\text { Grms. }\end{array}$ \\
\hline Rice... $^{6}$ & $\begin{array}{l}65.4 \\
64.1\end{array}$ & $\begin{array}{l}33.6 \\
33.0\end{array}$ & $\begin{array}{r}7.9 \\
16.4\end{array}$ & $\begin{array}{l}41.5 \\
49.4\end{array}$ & $\begin{array}{l}353.9 \\
413.2\end{array}$ \\
\hline $\begin{array}{l}\text { Barley ............. } \\
\text { Flesh meal rice and }\end{array}$ & 88.0 & 45.2 & 15.2 & 60.4 & 208.7 \\
\hline whey............ & 381.6 & 196.1 & 48.6 & 244.7 & 256.3 \\
\hline
\end{tabular}

Almost simultaneously C. Yoit $\ddagger$ gave a preliminary account of

* Virchow's Archiv, 101, 91.

$†$ Zeit. f. Biol., 22, 63.

$\ddagger$ Sitzungsber bayr. Acad d. Wiss.; Math. Phys. Classe, 1885, p. 288. 
respiration experiments made in his laboratory by Lehmann \& E. Voit with geese and by Rubner with a dog which demonstrated a production of fat from carbohydrates. Rubner's experiment was shortly afterward published in full.* It was a respiration experiment covering four days immediately following a fortnight's heavy feeding with meat. On the first two days of the experiment the animal fasted and on the second two received only starch and canesugar. The results for the last two days were:

Proteid metabolism............ 15.94 grams.

Equivalent fat, according to Rubner.. 7.65 "

Fat of food. ................ 9.40 "

Maximum from fát and proteids..... 17.05 "

Fat actually produced.......... 117.25 "

Even after making all possible deductions for the fact that some carbon may have been retained in the body in the form of glyrogen instead of fat, and also for a possible residuc of undigested starch in the alimentary canal at the close of the experiments, Rubner still computes that at least 40.7 grams of fat must have had its origin in carbohydrates.

Lehmann \& E. Voit's experiments have only recently appeared. $\dagger$ In their introduction they report also the results of experiments on fattening geese made by C. Voit several years previous to 1883 , which likewise show a production of fat from carbohydrates.

G. Ǩühn and his associates, $\downarrow$ at the Möckern Experiment Station, have demonstrated, by means of respiration experiments in which starch was added to rations but slightly exceeding the maintenance requirement, a formation of fat from carbohydrates by ruminants (oxen). In view of the possibility (see p. 27) that part of the carbon of the urine may be derived from the non-nitrogenous matter of the food, and in order to be on the safe side, the authors assume as possible that all the carbon of the proteids metabolized may have been stored up in the body in the form of fat. On this extreme and improbable assumption their results were as shown on the following page :

* Zeit. f. Biol., 22, 272.

$\dagger$ Ibid., 42, 619 .

† Reported by Kellner; Landw. Vers. Stat., 44, 257. 


\begin{tabular}{|c|c|c|c|c|c|c|}
\hline Animal. & Period. & $\begin{array}{c}\text { Proteid } \\
\text { Metabolism, } \\
\text { Grms. }\end{array}$ & $\begin{array}{c}\text { Equivalent } \\
\text { Fat, } \\
\text { Grms. }\end{array}$ & $\begin{array}{l}\text { Fat of } \\
\text { Food, } \\
\text { Grms. }\end{array}$ & $\begin{array}{c}\text { Maximum } \\
\text { from Fat } \\
\text { and Proteids. } \\
\text { Grms. }\end{array}$ & $\begin{array}{c}\text { Fat } \\
\text { Actually } \\
\text { Produced, } \\
\text { Grms. }\end{array}$ \\
\hline I & $2 a$ & 373.6 & 259 & 86 & 345 & 423 \\
\hline $\overrightarrow{\mathrm{I}}$ & $2 b$ & 382.0 & 265 & 81 & 346 & 332 \\
\hline$\overline{I I}$ & 2 & 297.4 & 206 & 77 & 283 & 434 \\
\hline III & 2 & 104.4 & 72 & 60 & 132 & 281 \\
\hline IV & 2 & 126.9 & 88 & 60 & 148 & 160 \\
\hline III & 3 & 506.9 & 351 & 69 & 420 & 375 \\
\hline IV & 3 & 548.8 & 380 & 74 & 454 & 388 \\
\hline III & 4 & 980 & 679 & 84 & 763 & 526 \\
\hline $\bar{V}$ & $2 a$ & 232 & 161 & 42 & 203 & 396 \\
\hline V & $2 b$ & 268 & 186 & 42 & 228 & 407 \\
\hline $\mathrm{V}$ & 3 & 149 & 103 & 39 & 142 & 703 \\
\hline VI & $2 a$ & 218 & 151 & 40 & 191 & 304 \\
\hline VI & $2 b$ & 232 & 161 & 35 & 196 & 381 \\
\hline VI & 3 & 186 & 129 & 43 & 172 & 507 \\
\hline
\end{tabular}

In most of these experiments the rations were purposely made poor in proteids and fat, and in all such cases, with one exception, a formation of fat from carbohydrates is clearly demonstrated. In three cases in which large amounts of proteids were fed, as well as in some similar experiments not included in the above table, it was possible to account for the fat production otherwise, but such negative results in no degree weaken the positive teaching of the remaining trials.

The more recent investigations of Kellner et al.* at the same Station, in which starch was added to a basal ration, although undertaken primarily for other purposes, likewise show the formation of an amount of fat inconsistent with the hypothesis of its production from the fat and proteids of the ration only.

The failure of Pettenkofer \& Voit to obtain affirmative results in their earlier experiments appears to be largely explicable, in the light of more recent knowledge, from the conditions of the experiments themselves. Pflüger $\dagger$ has recalculated their experiments on the same basis as those upon the formation of fat from proteids (see p. 109), and has pointed out that in the majority of cases the total foor was, according to his computations, scarcely more than sufficient for the maintenance of the organism, thus leaving no excess of any kind for fat production. Moreover, out of those ex-

* Landw. Vers. Stat, 53, 1.

† Arch. ges. Physiol , 52, 239. 
periments in which the conditions were favorable for a production of fat from carbohydrates, some actually do show that result, although they were classed by Voit as "exceptional cases," while its failure to appear in others is explained, according to Pflüger, by the increased metabolism due to maltreatment of the animal and the overloading of its digestive organs with starch.

Whether we admit all of Pflüger's criticism or not, it is now universally conceded that the carbohydrates are an important source of fat. If we are to go further and deny with Pflüger the production of fat from proteids, we are brought back, by a curious reversal of views, substantially to Liebig's classification of the nutrients into "plastic" and "respiratory," but, as already pointed out, it appears altogether probable that the proteids also contribute to fat production. However this may be, it is clear that in the case of herbivorous animals, which ordinarily consume relatively little proteids and fat and large amounts of carbohydrates, the latter are the most important factors in fattening, and the results of Lawes \& Gilbert (p. 167), according to which the gain of fattening animals is largely determined by the supply of non-nitrogenous matters in the food, are seen to be in full accord with the most careful physiological investigation.

Evidence from Respiratory Quotient.--The formation of fat from carbohydrates is a process of reduction. If we suppose all the carbon of 100 parts of dextrose, together with the necessary hydrogen and oxygen, to be united to form fat of the average composition stated on p. 61 , we have the following:

\begin{tabular}{|c|c|c|c|c|c|}
\hline & Dextrose. & $\begin{array}{c}\text { Equivalent, } \\
\text { Fat. }\end{array}$ & Residue. & $\begin{array}{l}\text { Equivalent, } \\
\text { Water. }\end{array}$ & $\begin{array}{c}\text { Excess of } \\
\text { Oxygen. }\end{array}$ \\
\hline 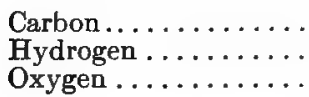 & $\begin{array}{r}40.00 \\
6.67 \\
53.33\end{array}$ & $\begin{array}{r}40.00 \\
6.28 \\
6.01\end{array}$ & $\begin{array}{r}0.39 \\
47.32\end{array}$ & $\begin{array}{l}0.39 \\
3.12\end{array}$ & ${ }^{4} 4.20$ \\
\hline & 100.00 & 52.29 & 47.71 & 3.51 & 44.20 \\
\hline
\end{tabular}

The excess of oxygen we may further suppose to unite with the carbon of 41.44 additional parts of dextrose, producing 60.78 parts of carbon dioxide and 24.86 parts of water. The process would be an intra-molecular combustion analogous to a fermentation, pro- 
ducing carbon dioxide without the intervention of oxygen from out side. The latter fact, of course, is equally true whatever substance combines with the excess of oxygen of the carbohydrate. The tendency, therefore, will be to increase the respiratory quotient and, if large amounts of carbohydrates are thus transformed, to even raise it above unity.

Numerous such instances are on record. Thus Regnault \& Reiset * report a quotient of 1.024 in case of a hen, and Reiset $\dagger$ obtained quotients of 1.004 and 1.054 with a ewe and a boar. Hanriot \& Richet, + in studies on the respiration of man, found that the ingestion of earbohydrates caused the respiratory quotient to rise markedly and sometimes to exceed unity. Later Hanriot § studied the transformations of glucose in the organism of man and obtained similar but more marked results, the quotient reaching as high a value as 1.28 .

Magnus-Levy || has likewise observed quotients greater than unity in the case of a dog fed large quantities of carbohydrates, and Bleibtreu, $\uparrow$ in experiments on fattening geese in a form of Regnaul $t$ respiration apparatus, also verified this fact, as have Kaufmann ** and Laulanie $\dagger \dagger$ in experiments upon dogs with sugar. The extensive respiration experiments of Zuntz \& Hagemann also afford numerous instances of respiratory quotients greater than unity.

The evidence of the respiratory quotient, then, is entirely in accord with the conclusions reached by other methods as to the formation of fat from carbohydrates.

Non-nitrogenous Nutrients of Feeding-stuffs.-It has become customary to regard the digestible non-nitrogenous ingredients of feeding-stuffs, aside from the ether extract, as consisting essentially of carbohydrates. As has several times been urged on

\footnotetext{
* Ann de Chim. et de Phys. [3], 26, 45.

$\dagger$ Ibid. [3], 69, 145.

$\ddagger$ Comptes rend., 106, 419 and 496 .

$\S$ Archives de Physiol., 1893, p. 248.

\| Arch, ges. Physiol., 55, 1.

Tा Ibid., 56, 464; 85, 366.

** Archives de Physiol., 1896, 341.

†† Ibid., 1896, 791.

¥ Landw. Jahrb., 27, Supp III.
} 
preceding pages, however, this is far from being the case as regards the materials actually resorbed from the digestive tract of our common domestic animals, particularly the ruminants. A demonstration of the production of fat from carbohydrates, therefore, does not necessarily show that the chemically diverse materials resorbed from coarse fodders, e.g., are available for fat production.

As a matter of fact, however, what a large proportion of the experiments just cited actually show, under a strict interpretation, is that fat was produced from the non-nitrogenous nutrients of the rations other than fat. In many of the experiments, it is true, notably those with swine and with geese, the ration consisted of concentrated feeding-stuffs whose "nitrogen-free extract" consisted to a large extent of hexose carbohydrates. Similarly, in G. Kühn's experiments the fat production was secured by the addition of starch to rations slightly above the maintenance requirement. In these cases, therefore, at least the larger part of the fat production in excess of that possible from proteids and food fat must be ascribed to the hexose carbohydrates. In experiments like those of Henneberg, Kern \& Wattenberg, and of Jordan, on the other hand, a not inconsiderable proportion of the non-nitrogenous nutrients was necessarily derived from coarse fodders and was, therefore, largely of undetermined nature. In such cases it is obviously impossible to say whether the fat production was at the expense of the hexose carbohydrates only or whether the other non-nitrogenous ingredients participated in it.

Other considerations, however, seem to render a participation of these substances in fat production, directly or indirectly, at least highly probable if not certain.

Crude Fiber.-The experiments of v. Knieriem (p. 161), as we have seen, seem to show that digested cellulose may be as efficient as other carbohydrates in protecting the body fat,- - that is, as part of a maintenance ration. The numerous experiments cited on pp. 117-123 likewise indicate that it has an effect similar to that of other carbohydrates in diminishing the proteid metabolism. Kellner * has also investigated its value in a fattening ration, using for this purpose the material resulting from the treatment of rye straw

* Landw. Vers. Stat., 53, 278. 
with an alkaline solution under pressure and containing $76.78 \mathrm{per}$ cent. of "crude fiber." This material was added to a basal ration somewhat more than sufficient for maintenance. The results as regards the proteid metabolism have already been considered (p. 121); the following table shows the effects also upon the fat production:

\begin{tabular}{|c|c|c|c|c|c|c|c|}
\hline & & \multicolumn{4}{|c|}{ Apparently Digested. } & \multicolumn{2}{|c|}{ Gain. } \\
\hline & & $\begin{array}{c}\text { Crude } \\
\text { Fat, } \\
\text { Grms. }\end{array}$ & $\begin{array}{l}\text { Crude } \\
\text { Fiber, } \\
\text { Grms. }\end{array}$ & $\begin{array}{c}\text { N.-free } \\
\text { Extract, } \\
\text { Grms. }\end{array}$ & $\begin{array}{l}\text { Protein, } \\
\text { Grms. }\end{array}$ & $\begin{array}{l}\text { Protein, } \\
\text { Grms. }\end{array}$ & $\begin{array}{l}\text { Fat. } \\
\text { Grms. }\end{array}$ \\
\hline \multirow[t]{2}{*}{$\begin{array}{l}\mathrm{O} x \mathrm{H:} \\
\underset{6}{\text { Period }} 5 \\
\end{array}$} & $\begin{array}{l}\text { Extracted straw. } \\
\text { Basal ration..... }\end{array}$ & $\begin{array}{l}116 \\
101\end{array}$ & $\begin{array}{l}3129 \\
1083\end{array}$ & $\begin{array}{l}3351 \\
2912\end{array}$ & $\begin{array}{l}654 \\
749\end{array}$ & $\begin{array}{r}157 \\
43\end{array}$ & $\begin{array}{l}735 \\
191\end{array}$ \\
\hline & Difference... & 15 & 2047 & 439 & -95 & 114 & 544 \\
\hline \multirow[t]{2}{*}{$\begin{array}{r}\text { Period } 3 \\
4\end{array}$} & $\begin{array}{l}\text { Starch ............. } \\
\text { Basal ration..... }\end{array}$ & $\begin{array}{r}92 \\
101\end{array}$ & $\begin{array}{l}1057 \\
1083\end{array}$ & $\begin{array}{l}4773 \\
2912\end{array}$ & $\begin{array}{l}629 \\
749\end{array}$ & $\begin{array}{l}78 \\
43\end{array}$ & $\begin{array}{l}565 \\
191\end{array}$ \\
\hline & Difference..... & -9 & -26 & 1861 & -120 & 35 & 374 \\
\hline \multirow[t]{2}{*}{$\begin{array}{l}O x J: \\
\underset{6}{\text { Period }} 5 \\
4\end{array}$} & $\begin{array}{l}\text { Extracted straw... } \\
\text { Basal ration..... }\end{array}$ & $\begin{array}{l}110 \\
107\end{array}$ & $\begin{array}{l}3101 \\
1114\end{array}$ & $\begin{array}{l}3344 \\
2895\end{array}$ & $\begin{array}{l}747 \\
836\end{array}$ & $\begin{array}{l}98 \\
33\end{array}$ & $\begin{array}{l}693 \\
223\end{array}$ \\
\hline & Difference...... & 3 & 1987 & 449 & -89 & 65 & 470 \\
\hline \multirow[t]{2}{*}{$\underset{4}{\operatorname{Period}} \mathbf{3}$} & $\begin{array}{l}\text { Starch........... } \\
\text { Basal ration..... }\end{array}$ & $\begin{array}{r}85 \\
107\end{array}$ & $\begin{array}{l}1105 \\
1114\end{array}$ & $\begin{array}{l}4396 \\
2895\end{array}$ & $\begin{array}{l}764 \\
836\end{array}$ & $\begin{array}{l}91 \\
33\end{array}$ & $\begin{array}{l}472 \\
223\end{array}$ \\
\hline & Difference...... & -22 & -9 & 1501 & -72 & 58 & 249 \\
\hline
\end{tabular}

The varying quantities of nutrients digested stand in the way of a direct comparison of the results. If, however, we reckon 1 gram of digested fat equivalent to 2.25 grams of digested crude fiber or nitrogen-free extract or protein (isodynamic quantities according to the usual method of computation), and if we further convert the gain of proteids into its equivalent amount of fat, on the same principle, by multiplication by 5.7 and division by 9.4 , we have the results shown in the table on the opposite page.

While no great quantitative accuracy attaches to such a computation, it is sufficient to show that the effect produced in this case 


\begin{tabular}{|c|c|c|c|}
\hline & $\begin{array}{l}\text { Total } \\
\text { Carbohydrate } \\
\text { Equivalent } \\
\text { of Nutrients, } \\
\text { Grms. }\end{array}$ & $\begin{array}{l}\text { Total Fat } \\
\text { Equivalent } \\
\text { of Gain, } \\
\text { Groos. }\end{array}$ & $\begin{array}{l}\text { Gain per } \\
\text { Kilogram } \\
\text { Nutrients, } \\
\text { Grms. }\end{array}$ \\
\hline 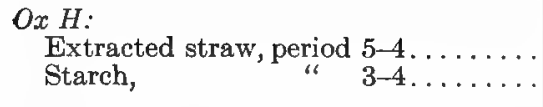 & $\begin{array}{l}2425 \\
1695\end{array}$ & $\begin{array}{l}613 \\
395\end{array}$ & $\begin{array}{l}252.8 \\
233.0\end{array}$ \\
\hline 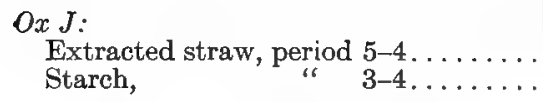 & $\begin{array}{l}2334 \\
1370\end{array}$ & $\begin{array}{l}509 \\
284\end{array}$ & $\begin{array}{l}218.1 \\
207.3\end{array}$ \\
\hline
\end{tabular}

by the addition to the basal ration of digestible matter five sixths of which was derived from crude fiber, was not inferior to that produced by the addition of an equal amount of pure starch.

It would seem that these results may fairly be taken as showing that the products of the digestion of cellulose by ruminants are substantially of equal value with those of the digestion of starch. This, however, by no means warrants the conclusion that starch and cellulose are of equal value in ordinary feeding-stuffs. The material used in these experiments had been so altered mechanically and freed from incrusting materials by the treatment to, which it had been subjected that 88.3 per cent. of its organic matter and 95.8 per cent. of its crude fiber was digested. The same animals digested but 52.5 per cent. of the crude fiber of wheat straw, and the digestible organic matter of the latter proved far less efficient than that of either starch or extracted straw. A full discussion of these facts may be more profitably undertaken in connection with a consideration of the energy relations of feeding-stuffs in Part II; for the present it may suffice to point out that the difference just noted appears to depend on physical rather than chemical causes.

Pentose Carbohydrates.-We have already (p. 156) seen reason to believe that the pentose carbohydrates may serve as a source of energy to the organism and protect other materials from oxidation. This, of course, is equivalent to an indirect production of fat. In the same connection, however, the experiments of Kellner, just mentioned, were referred to as indicating a direct participation by these bodies in fat production. About one third of the digested matter of the extracted rye straw was found to consist of bodies 
yielding furfural, presumably pentosans, as appears from the following modified form of the last table:

\begin{tabular}{|c|c|c|c|}
\hline & \multicolumn{2}{|c|}{$\begin{array}{c}\text { Total Carbohydrate Equivalent } \\
\text { of Nutrients. }\end{array}$} & \multirow{2}{*}{$\begin{array}{l}\text { Total Fat } \\
\text { Equivalent } \\
\text { of Gain, } \\
\text { Grms. }\end{array}$} \\
\hline & $\begin{array}{l}\text { Pentosans, } \\
\text { Grms. }\end{array}$ & Other Substances, & \\
\hline $\begin{array}{l}O x H: \\
\text { Extracted straw, period } 5-4 \ldots \ldots \\
\text { Starch, } \\
3-4 \ldots \ldots\end{array}$ & $\begin{array}{r}809 \\
-34\end{array}$ & $\begin{array}{l}1616 \\
1729\end{array}$ & $\begin{array}{l}613 \\
395\end{array}$ \\
\hline $\begin{array}{l}O x J: \\
\text { Extracted straw, period } 5-4 \ldots \ldots \\
\text { Starch, }\end{array}$ & $\begin{array}{r}834 \\
-89\end{array}$ & $\begin{array}{l}1500 \\
1459\end{array}$ & $\begin{array}{l}509 \\
284\end{array}$ \\
\hline
\end{tabular}

If we regard the furfuroids as not contributing to the fat production, then we must assign to the other nutrients of the extracted straw a value from 66 to 74 per cent. greater than that of the digested matter of the starch, a result which is hardly conceivable. Apparently we must admit that the furfuroids in this case produced approximately the same effect as the other non-nitrogenous nutrients and were at least indirectly if not directly a source of fat. 


\section{CHAPTER VI.}

\section{THE INFLUENCE OF MUSCULAR EXERTION UPON METABOLISM.}

IT is a matter of common experience that muscular exertion results in a very marked increase in the vital activities of the body. The rate of circulation and respiration is greatly quickened and the increased metabolism in the organism is shown by the loss of weight and by the increased demand for food to make good the destruction of tissue. Indeed, no other factor even approaches muscular exertion in the extent to which it increases the metabolic activities of the body.

We have now to.consider in some detail the nature of muscular exertion and the precise character of its effects upon metabolism.

\section{§. General Features of Muscular Activity.}

\section{Muscular Contraction.}

The work of the muscles is accomplished by contracting, and a brief consideration of some of the more prominent general features of muscular contraction will conduce to an intelligent study of the main subject of the chapter. It will be possible here to consider this phase of the subject only in its most general outline, and the reader is referred to works on physiology for details.

When a suitable stimulus, which in the living animal is usually a nerve stimulus, is applied to a muscle it contracts; that is, it tends to grow shorter and thicker. This change is brought about by a shortening and thickening of the individual fibers of which the muscle is built up. A single stimulus, such, for example, as that caused by the making or breaking of an electric circuit, gives rise to what is known as a simple muscular contraction. If such a stimulus is repeated with sufficient frequency it produces a 
series of simple contractions which fuse together, resulting in a state of contraction which continues, subject to the effects of fatigue, as long as the stimulus acts. This form of muscular contraction has received the name of "tetanus." In the living animal the ordinary contractions of the muscles brought about through the nervous system, even those that seem but momentary, are essentially tetanic in their character.

Chemical Changes during Contraction.--Under the influence of a stimulus sufficient to produce a muscular contraction there occurs a sudden and large increase in the chemical changes which are continually going on even in the quiescent muscle. More material is metabolized in the muscle during contraction and energy is thus liberated for the performance of work.

Our knowledge of the nature of these chemical changes in the contracting muscle is comparatively meager, but three main features appear well established:

First, during contraction the neutral or slightly alkaline reaction of the quiescent muscle c anges to an acid reaction, probably through the formation of sarcolactic acid.

Second, there is a large increase in the amount of oxygen taken up by the muscle from the blood and a still greater increase in the amount of carbon dioxide given off by it.*

Third, under normal circumstances, judging from the amount of the urinary nitrogen, there appears to be no considerable increase in the nitrogenous products of metabolism.

From the increase in oxygen consumed and carbon dioxide given off we might be led at first thought to suppose that the increased activity in the muscle during contraction was of the nature of a simple oxidation. Certain other facts, however, seem to show that this view of the matter is inadequate.

Oxidations Incomplete. - That the increased metabolism in the contracting muscle is not a simple oxidation of some material $\mathbf{t}$ carbon dioxide and water is indicated by the fact of the production of lactic or other acid in the muscle. Plainly, if the energy for muscular contraction is produced by oxidation the oxidation is at least incomplete.

* Some good authorities doubt whether the carbon dioxide resulting from muscular exertion actually leaves the muscle in that form. Compare Schüffer, Text-book of Physiology, 1898, Vol. I, p. 911. 
ResPiRatory QUOTIENT.-By analogy with investigations upon respiration we may designate the ratio between the oxygen consumed and the carbon dioxide given off by the muscle as the respiratory quotient of the muscle. Numerous investigations upon this point have shown that during contraction much more carbon dioxide is given off than corresponds to the oxygen consumed, or, in other words, the respiratory quotient of the active muscle is considerably greater than unity.

As early as 1862 Sczelkow * determined the gaseous exchange between the blood and the muscles of the posterior extremities of a dog, tetanus being produced by an electric current. He found that during rest more oxygen disappeared from the blood than corresponded to the carbon dioxide taken up by it, while during tetanus, on the contrary, the carbon dioxide considerably exceeded the oxygen. His results, calculated for the posterior extremities alone, were as follows:

\begin{tabular}{|c|c|c|c|c|}
\hline \multirow[b]{2}{*}{ Experiment. } & & \multicolumn{2}{|c|}{ Per Minute. } & \multirow[b]{2}{*}{$\begin{array}{l}\text { Respiratory } \\
\text { Quotient. }\end{array}$} \\
\hline & & $\begin{array}{l}\text { Carbon } \\
\text { Dioxide } \\
\text { c.c. }\end{array}$ & $\begin{array}{c}\text { Oxygen } \\
\text { c.c. }\end{array}$ & \\
\hline 1. & $\left\{\begin{array}{l}\text { Rest } \ldots \ldots \ldots \ldots \ldots \ldots \ldots \ldots \ldots \ldots \ldots \\
\text { Tetanus . . } \ldots \ldots \ldots \ldots \ldots\end{array}\right.$ & $\begin{array}{r}1.60 \\
10.37\end{array}$ & $\begin{array}{l}4.10 \\
3.92\end{array}$ & $\begin{array}{l}0.41 \\
2.65\end{array}$ \\
\hline 2. & 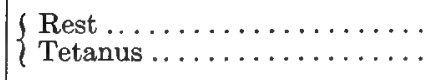 & $\begin{array}{r}2.62 \\
12.38\end{array}$ & $\begin{array}{r}4.25 \\
10.52\end{array}$ & $\begin{array}{l}0.62 \\
1.18\end{array}$ \\
\hline 3... & $\left\{\begin{array}{l}\text { Rest } \ldots \ldots \ldots \ldots \ldots \ldots \ldots \ldots \\
\text { Tetanus . . . . } \ldots \ldots \ldots \ldots \ldots\end{array}\right.$ & $\begin{array}{r}1.73 \\
10.62\end{array}$ & $\begin{array}{l}3.21 \\
7.55\end{array}$ & $\begin{array}{l}0.54 \\
1.41\end{array}$ \\
\hline $4 \ldots \ldots \ldots$ & $\left\{\begin{array}{l}\text { Rest } \ldots \ldots \ldots \ldots \ldots \ldots \ldots \ldots \\
\text { Tetanus ... } \ldots \ldots \ldots \ldots \ldots\end{array}\right.$ & $\begin{array}{r}3.53 \\
12.19\end{array}$ & $\begin{array}{l}4.71 \\
9.38\end{array}$ & $\begin{array}{l}0.75 \\
1.30\end{array}$ \\
\hline $5 \ldots \ldots \ldots$ & 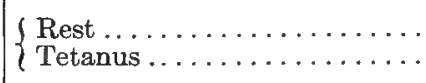 & $\begin{array}{r}2.33 \\
12.95\end{array}$ & $\begin{array}{r}5.82 \\
18.71\end{array}$ & $\begin{array}{l}0.40 \\
0.80\end{array}$ \\
\hline
\end{tabular}

In the above experiments, with a single exception, the quantity of oxygen consumed by the active muscles was more than that taken up in a state of rest, but the increase in the amount of carbon dioxide given off was still greater, so that the respiratory quotient was largely increased, exceeding unity in every instance but one.

* Sitzungsber. Wiener Akad. d. Wiss., Math-Naturwiss. Klasse, 45, II, 171. 
Chauveau \& Kaufmann * have more recently obtained similar results. Their experiments were made upon the Levator labii superioris of the horse, both in a state of rest and in a state of activity consequent upon the consumption of food. From the amount and composition of blood entering and leaving this muscle the following results were obtained for the oxygen consumed and carbon dioxide given off per kilogram of muscle in one minute. On the average of the three experiments, in round numbers, twenty-one times as much oxygen was consumed during work as during rest and twenty-nine times as much carbon dioxide was given off.

\begin{tabular}{c|c|c|c|c|c|c}
\hline \multirow{2}{*}{ Experiment. } & \multicolumn{3}{|c|}{ Oxygen Consumed. } & \multicolumn{2}{c}{ Carbon Dioxide Given Off. } \\
\cline { 2 - 7 } & $\begin{array}{c}\text { Rest, } \\
\text { Grms. }\end{array}$ & $\begin{array}{c}\text { Work. } \\
\text { Grms. }\end{array}$ & $\begin{array}{c}\text { Work } \\
\text { Rest. }\end{array}$ & $\begin{array}{c}\text { Rest. } \\
\text { Grms. }\end{array}$ & $\begin{array}{c}\text { Work. } \\
\text { Grms. }\end{array}$ & $\begin{array}{c}\text { Work }+ \\
\text { Rest }\end{array}$ \\
\hline $2 \ldots \ldots \ldots \ldots \ldots \ldots$ & .00479 & .07148 & 14.9 & .00365 & .12534 & 34.3 \\
$3 \ldots \ldots \ldots \ldots \ldots \ldots$ & .01167 & .20190 & 17.3 & .01168 & .35488 & 30.4 \\
$4 \ldots \ldots \ldots \ldots \ldots$ & .00419 & .14899 & 35.6 & .00518 & .25709 & 49.6 \\
\hline Average....... & .00688 & .14079 & 20.5 & .00864 & .24577 & 28.5 \\
\hline
\end{tabular}

These facts show plainly that the increased metabolism of the active muscle cannot consist wholly of a direct oxidation, since the carbon dioxide given off from the muscle contains more oxygen than direct experiment shows to have been taken up by the muscle during the same time.

OXYGen NOT Essential.-A further and still more striking proof of the above assertion is found in the fact that the living muscle can execute a considerable number of contractions in the entire absence of oxygen.

Setschenow is quoted by Ludwig \& Schmidt $\dagger$ as having found that muscles would contract freely when supplied with oxygen-free blood, while L. Hermann $\ddagger$ has shown that an excised muscle may continue to contract in a vacuum. The well-known investigations of Pfluger $\S$ show that frogs may continue to live and execute more or less extensive motions in an atmosphere of pure nitrogen for

* Comptes rend, 104, 1126, 1352, 1409.

† Verhandl. Sächs Akad. d. Wiss., Math-Phys. Klasse, 20, 12.

$\ddagger$ Unters. u. Stoffw. der Muskeln.

$\S$ Arch. ges. Physiol., 10, 313. 
several hours, giving out considerable amounts of carbon dioxide, and Bunge* has made similar observations upon the movements of certain intestinal worms (Ascaris) in one per cent. salt solution made as nearly oxygen-free as possible.

Weinland $\dagger$ has shown that in the latter case the energy is derived chiefly from the cleavage of glycogen with the production of carbonic and valerianic acids.

Summary.-The three classes of facts just adduced justify the conclusion that the chemical changes by which energy is liberated in a muscular contraction are not simply oxidations, but are of the nature of a cleavage of some complex substance or substances with evolution of carbon dioxide. There is, in other words, a sudden "explosive" decomposition of substances elaborated in the muscle during rest. Of the nature of the material thus broken down we have little definite knowledge. We can say, however, that if it is nitrogenous matter its nitrogen is ordinarily retained in the muscle in some form and that in effect the metabolized material is nonnitrogenous. The increase in the consumption of oxygen during work appears to be to a certain extent a secondary process, accomplishing the further oxidation of the primary products of metabolism. At the same time, the fact that the amount of oxygen consumed responds very promptly to work and also to its cessation shows that these primary products, whatever they may be, are very speedily oxidized, either in the muscle or elsewhere in the organism.

Thermal Changes during Contraction.-A considerable portion of the energy set free during muscular exertion always takes ultimately the form of heat. When the muscle acts without shortening, as when supporting a weight (isometric contraction) - that is, when no external work is done-all the metabolized energy takes the form of heat. If, on the other hand, the weight be lifted (isotonic contraction) - if external work is done-a portion of the energy takes the form of motion. The interesting question of the relation between the external work performed and the total amount of energy metabolized will be considered later. For the present it is sufficient to state that muscular action always produces heat and that a very considerable share of the metabolized energy ultimately takes this form.

* Zeit physiol. Chem . 8, 48.

† Zeit. f. Biol., 42, 55. 
Muscular Tonus.-The chemical and thermal changes just enumerated as characterizing the muscle during contraction are taking place in it to a less extent at all times. Even at rest the muscle respires and produces heat, as is well illustrated by Sczelkow's and Chauveau \& KKaufmann's experiments quoted above.

The living muscles of the body are elastic and may be said to be always slightly on the stretch, as is shown by the fact that when cut they gape open and that they shorten when their attachments to the bones are severed. This slight degree of contraction of the resting muscles has been called muscular tonus, and it is at least a plausible conclusion that the chemical changes taking place in a quiescent muscle furnish the energy to maintain this tonus. According to Chauveau * we may regard the essence of muscular contraction as a sudden increase in the elasticity of the muscle. He holds that all the energy liberated by muscular metabolism is converted first into the elastic force of the muscle and only secondarily into heat. According to this view the slight degree of elasticity of the quiescent muscle is produced by the constant metabolism going on within it. In active muscular contraction this process is greatly exaggerated and the katabolic processes exceed the anabolic, thus giving rise to a great increase in muscular elasticity which in turn may be converted into work. In repose following work, we may assume that the substances broken down during contraction are built up again, while in prolonged repose the two processes must substantially balance each other.

Muscular tonus is most noticeable during the waking hours, under the influence of external stimuli to the central nervous system, and consequently the rate of metabolism and the heat production tend to be greater than during sleep. To this is to be added, as a further cause of greater metabolic activity during the waking hours, those continual slight movements of the body which usually take place even in what is commonly spoken of as a state of rest and which may be designated as incidental movements.

That the total amount of metabolism required for the maintenance of muscular tonus is considerable seems to be indicated by the observations of Röhrig \& Zuntz, $\dagger$ and of Colasanti, + who

* Le Travail Musculaire et l'Energie qu'il Represente. Paris, 1891.

† Arch. ges. Physiol., 4, 57; 12, 522.

$\ddagger I b i d$., 16, 157. 
found that when the motor nerves of the rabbit are paralyzed by curari the rate of metabolism, as measured by the respiratory exchange, falls to about one half the amount during rest and does not react to changes of external temperature. Pflüger * computes from his experiments a similar reduction of about 35 per cent. Under these conditions the heat production of an animal is insufficient to maintain its normal temperature, and unless the loss of heat from the body is hindered by coverings or otherwise it soon perishes. Frank \& F. Voit, $\dagger$ on the contrary, found that curarized dogs excreted no less carbon dioxide than in the normal state, provided the body temperature was kept normal.

\section{Secondary Effects of Muscular Exertion.}

The greater activity of the muscular metabolism during the performance of work gives rise to important secondary effects, particularly upon the circulation and respiration. It is a familiar fact that in active exercise the action of the heart is largely increased and the breathing becomes deeper and more rapid, and that ordinarily the limit of muscular exertion is set, not by the power of the muscles themselves, but by the ability of the heart and lungs to keep pace with the demands upon them.

Circulation.-The circulating blood is the medium by which oxygen is conveyed to the muscles and carbon dioxide and other products of their metabolism removed. The latter function is of special importance, since an accumulation in the muscle of the products of its own metabolism speedily reduces and ultimately - suspends its power to contract. In active muscular exercise, therefore, an increase in the rate of circulation is essential to the continued activity of the muscles. This increase appears to be brought about by the accumulation in the blood of the products of metabolism, which act as a stimulus to the vaso-motor center. The result is a dilation of the peripheral blood-vessels, which is aided by the mechanical effects of muscular contraction. To offset this and prevent a fall of arterial blood pressure, the visceral capillaries are probably constricted, while the rapidity and strength of the heartbeats are largely increased. The rapidity of the circulation as a

* Arch. ges. Physiol., 18, 247.

$\nmid$ Zeit. f. Biol., 42, 349. 
whole is thus greatly augmented, while at the same time a larger percentage of the total blood passes through the muscles. For example, in the experiments of Chauveau \& Kaufmann, cited above, the ratio between the circulation in the resting as compared with the active muscle varied from $1: 3.35$ to $1: 6.60$. Zuntz \& Hagemann,* in their investigations upon the work of the heart, found the average amount of blood passing through the heart of a horse per minute to be during rest 29.16 liters and during work 53.03 liters. By this increase in the rate of circulation through the muscles the carbon dioxide and other injurious products of muscular metabolism are rapidly removed and an abundant supply of oxygen is ensured. In fact it is usually true that during work which is not excessive the venous blood contains less carbon dioxide and more oxygen than during rest.

Since the heart is a muscular organ, it is obvious that this increase in the circulatory activity must add materially to its metabolism. In the performance of work, therefore, there is an expenditure of matter and energy, not only for the work of the skeletal muscles but likewise for the additional work of the heart. Zuntz \& Hagemann in their experiments upon the horse just mentioned compute that during moderate work the metabolism due to the work of the heart amounts to 3.8 per cent. of the total metabolism of the body.

RESPIRATION.-The greater activity of the circulation consequent upon muscular exertion would be futile were not provision made for more efficient aeration of the blood in the lungs through an increased activity of the respiration. The latter appears to be brought about, like the increase in the circulatory activity, by the effect of the greater amount of metabolic products in the blood, acting in this case upon the respiratory center. It has been shown that an accumulation of carbon dioxide in the blood does not have this effect, but that a lack of oxygen, such as occurs, for example, in asphyxiation, provokes powerful movements of the respiratory organs. In ordinary work, however, whatever may be the case in excessive muscular exertion, the effect is not caused by a lack of oxygen, for the blood, as already noted, is usually more arterialized

* Landw. Jahrb., 27, Supp. III, 405. 
than during rest. Apparently the stimulation of the respiratory center is brought about by the other products of muscular metabolism, whatever they may be, which find their way into the blood. Under the influence of this stimulus the respiratory movements increase in frequency or depth or both, thus making possible a more active gaseous exchange between the blood and the air in the lungs. This action is usually so efficient that the expired air during work contains a smaller proportion of carbon dioxide than it does during rest, notwithstanding the fact that the total quantity eliminated is much greater.

Since respiration, like circulation, is maintained by muscular action, it is true in the former case as in the latter that a greater activity of the function necessitates a greater metabolism for that purpose. Zuntz \& Hagemann * have recently investigated the work of respiration in the horse, the augmented respiratory activity being brought about by an admixture of carbon dioxide to the inspired air, this resulting in a marked increase in the depth of the respiratory movements. With the animal upon which most of the experiments were made they found an increment of from 2.02 c.c. to 5.23 c.c. of oxygen consumed for each increment of one liter in the volume of air respired. In general, although with some exceptions, the work of respiration as thus measured increased with the increased depth of the respiratory movements. The results upon other horses were somewhat variable. It was observed, however, that in the performance of ordinary work by the horse the effect was chiefly upon the frequency of respiration rather than its depth. The former effect the authors believe to involve less work than the latter and moreover an amount largely independent of the total volume of air respired.

\section{§. Effects upon Metabolism.}

It is obvious from the foregoing paragraphs that the production of external work is a complex phenomenon. As regards its effects upon the total metabolism, the main features involved seem to be:

1. An explosive decomposition of some unknown "contractile substance". in the muscles.

* Landw. Jahrb., 27, Supp. III, 361. 
2. The oxidation somewhere in the organism of the immediate products of this decomposition to the final excretory products.

3 . Since the state of contraction appears to be only an exaggeration of the muscular condition during rest, we may reasonably suppose that there is a continual re-formation of the "contractile substance" going on.

4. As secondary effects there is a marked increase in the activity of circulation and respiration, thus involving supplementary muscular exertion.

It is plain that however interesting and important to the physiologist may be studies of the changes in the muscle itself, from the point of view of the statistics of nutrition the important thing is the total effect upon the expenditure of matter and energy by the organism under varying conditions of work. The energy relations of the subject will be discussed subsequently in Part II. Here we are concerned more particularly with the nature of the material expended in the production of work, and as a matter of convenience we may, as in the two preceding chapters, take up first the effect upon the proteid metabolism and second that upon the metabolism of the non-nitrogenous substances.

\section{Effects upon Proteid Metabolism.}

Earlier Investigations. Since the muscles, which are the instruments by means of which work is produced, are composed essentially of proteid material, it was natural to regard the proteids as the source of muscular power and to assume that the energy developed during work was supplied by an increased metabolism of these substances. This view was supported by the authority of Liebig, who, however, does not appear to have based it upon any actual experiments, and it was quite generally, although not universally, accepted.

C. Voit* appears to have been the first to subject this idea to investigation. His experiments were made upon a dog weighing about 32 kilograms. The work performed, by running in a treadmill, was considerable, being estimated to average $1.7 \mathrm{kgm}$. per second for the whole twenty-four hours. Experiments were made

* Untersuchungen über den Einfluss des Kochsalzes, des Wassers, und der Muskelbewegungen auf den Stoffwechsel. 1860. Compare the summary by E. v. Wolff in Die Ernährung der landw. Nutzthiere, pp. 386-388. 
both during fasting and with a daily ration of 1500 grams of lean meat. The results obtained were as follows:

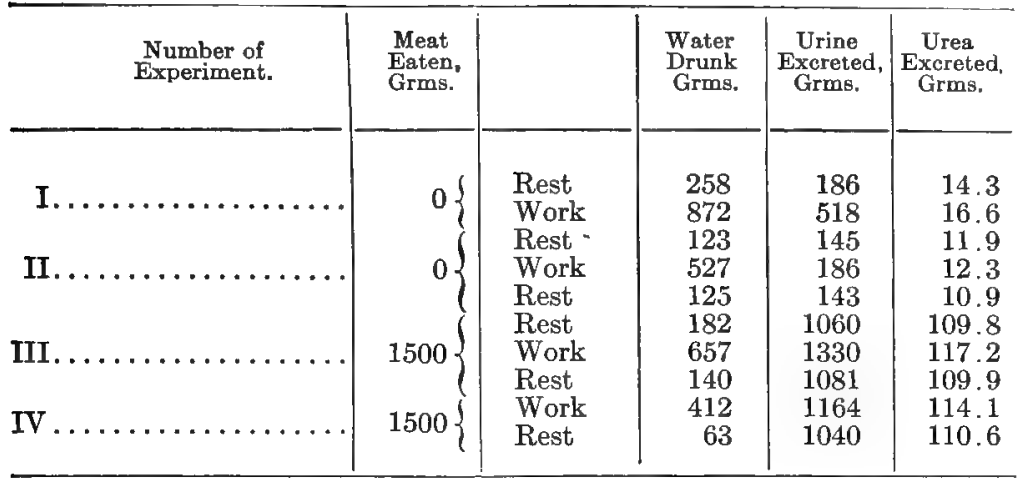

The average increase of the proteid metabolism, as measured by the urea excreted, was in the fasting experiments 11.8 per cent. and in the experiments with food 4.95 per cent. The absolute difference in grams, however, was materially less in the fasting experiments, although approximately the same amount of work was performed in both cases. A similar experiment upon an older and quite fat dog while fasting showed an increase of only 6 per cent. in the proteid metabolism.

Subsequently Pettenkofer \& Voit* made similar experiments upon a man, the work consisting in turning a heavy wheel provided with a brake. The work was performed in the respiration apparatus. The results showed a large increase in the carbon dioxide excreted, but scarcely any effect was noted upon the excretion of nitrogen, as will be seen from the following table:

\begin{tabular}{|c|c|c|c|c|c|c|}
\hline & \multirow{2}{*}{$\begin{array}{l}\text { Nitrogen } \\
\text { of Urine, } \\
\text { Grms. }\end{array}$} & \multirow{2}{*}{$\begin{array}{l}\text { Carbon } \\
\text { Dioxide } \\
\text { Excreted, } \\
\text { Grms. }\end{array}$} & \multicolumn{2}{|c|}{ Water Excreted. } & \multirow{2}{*}{$\begin{array}{l}\text { Oxygen } \\
\text { Taken Up. } \\
\text { Grms. }\end{array}$} & \multirow{2}{*}{$\begin{array}{c}\text { Number } \\
\text { of } \\
\text { Experi- } \\
\text { ments. }\end{array}$} \\
\hline & & & $\begin{array}{l}\text { In } \\
\text { Urine, } \\
\text { Grms. }\end{array}$ & $\begin{array}{l}\text { Evapo- } \\
\text { rated, } \\
\text { Grms. }\end{array}$ & & \\
\hline $\begin{array}{l}\text { Fasting : } \\
\quad \text { Rest } \ldots \ldots \ldots \ldots \\
\quad \text { Work } \ldots \ldots \ldots \ldots\end{array}$ & $\begin{array}{l}12.4 \\
12.3\end{array}$ & $\begin{array}{r}716 \\
1187\end{array}$ & $\begin{array}{r}1006 \\
746\end{array}$ & $\begin{array}{r}821 \\
1777\end{array}$ & $\begin{array}{r}762 \\
1072\end{array}$ & $\begin{array}{l}2 \\
1\end{array}$ \\
\hline $\begin{array}{l}\text { Average diet : } \\
\quad \text { Rest } \ldots \ldots \ldots \ldots \\
\text { Work ........... }\end{array}$ & $\begin{array}{l}17.0 \\
17.3\end{array}$ & $\begin{array}{r}928 \\
1209\end{array}$ & $\begin{array}{l}1218 \\
1155\end{array}$ & $\begin{array}{r}931 \\
1727\end{array}$ & $\begin{array}{l}832 \\
981\end{array}$ & $\begin{array}{l}3 \\
2\end{array}$ \\
\hline
\end{tabular}

* Zeit. f. Biol., 2, 478. 
Pettenkofer \& Voit regard the slight increase in the proteid metabolism which they observed in most cases as a secondary effect of muscular exertion. They have shown, as we have seen, that when the cells of the body are abundantly supplied with non-nitrogenous nutrients, either in the form of food or of body fat, there is a tendency to diminish the proteid metabolism. In work, on the contrary, large amounts of non-nitrogenous material are oxidized, as their respiration experiments show. The supply of these nutrients to the cells is thus diminished, and it is to this that they attribute the increase in proteid metabolism.

Results like those just given can hardly be interpreted otherwise than as showing that the non-nitrogenous constituents of the body or of the food, rather than the proteids, are the source of the energy expended in muscular work, but the first attempt to compare the amount of work performed with the energy available from the proteids metabolized was the famous experiment of Fick \& Wislicenus * in 1866. These observers made an ascent of the Faulhorn and found that the amount of proteids metabolized during and after the ascent, as measured by the urea excreted, was insufficient, according to their computations, to account for more than one third of the energy required to raise their bodies to the height of the mountain, making no allowance for the work of the internal organs, nor for those muscular exertions which did not contribute directly to the work done.

Fick \& Wislicenus found no considerable increase in the urinary nitrogen in their experiment. Subsequent investigators, among whom may be mentioned Parkes, $\dagger$ Noyes, $\ddagger$ Haughton, $\$$ Meissner,\| Schenk, $\uparrow$ and Engelmann,** have reported apparently conflicting results regarding the influence of work on the proteid metabolism. In some cases an increase was observed, while in other cases no material effect was apparent. The increase when observed was never large except in the experiments of Engel-

* Vrtljschr. Naturf. Gesell. Zürich, 10, 317.

$\dagger$ Phil, Mag., 4th ser., 32, 182.

$\ddagger$ Amer. Jour. Med. Sci., Oct., 1867.

\$ Brit. Med. Jour., 15, 22.

|| Virchow's Jahresber., 1868, p. 72.

T Centralb. Med. Wiss., 1874, p. 377.

** Archiv f. (Anat. u.) Physiol., 1871, p. 14. 
mann, and was entirely insufficient to account for the energy expended. Oppenheim made the interesting observation that work pushed to the point of producing dyspnœa caused a marked increase in the proteid metabolism.

Influence of Total Amount of Food-Kellner's InvesTIGATIONS.-- Doubtless the conflicting results of earlier experiments are due in part to defective technique, but they arise in part also, as it would seem, from another cause to which attention was first called by Kellner in 1879-80. Kellner's experiments were made upon the horse. They differed from most earlier experiments, first, in that the comparison was made between different amounts of work instead of between work and rest, and second, that the individual periods instead of covering only a few days were extended over two. or three weeks.

Series I.-Kellner's first series * was made primarily for the purpose of testing the influence of work upon the digestibility of the food, but the total nitrogen of the urine was also determined. The methods employed for this purpose were somewhat imperfect, there being some mechanical loss and probably also a loss of ammonia from the urine, but the author believes the results of the several periods to be fairly comparable. The amount of work performed was measured by a dynamometer. The numerical results of the measurement have since been shown to be too high, but the relative amount in the several periods is not thought to be materially affected by this error. The results of the several periods are briefly summarized in the following table:

\begin{tabular}{|c|c|c|c|c|}
\hline \multirow{2}{*}{ Period. } & \multirow{2}{*}{$\begin{array}{l}\text { Work, } \\
\text { Kgm. }\end{array}$} & \multicolumn{2}{|c|}{ Nitrogen. } & \multirow{2}{*}{$\begin{array}{l}\text { Live Weight } \\
\text { at Close } \\
\text { of Period, } \\
\text { Kgs. }\end{array}$} \\
\hline & & $\begin{array}{c}\text { Digested, } \\
\text { Grms. }\end{array}$ & $\begin{array}{c}\text { In Urine, } \\
\text { Grms. }\end{array}$ & \\
\hline 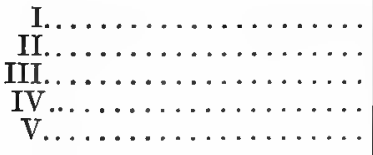 & $\begin{array}{r}625,000 \\
1,250,000 \\
1,875,000 \\
1,100,000 \\
625,000\end{array}$ & $\begin{array}{l}134.41 \\
128.32 \\
132.72 \\
126.40 \\
129.41\end{array}$ & $\begin{array}{r}99.0 \\
109.3 \\
116.8 \\
110.2 \\
98.3\end{array}$ & $\begin{array}{l}534.1 \\
529.5 \\
522.5 \\
508.8 \\
518.0\end{array}$ \\
\hline
\end{tabular}

While the above figures show a considerable nitrogen deficit, the urinary nitrogen increased and decreased with the amount of * Landw. Jahrb., 8, 701. 
work performed in a manner which can scarcely be explained otherwise than as a result of the changes in the latter. The ration consumed was amply sufficient for the light work of the first and fifth periods. When, however, more work was demanded from the animal, the live weight promptly fell off, showing that the total ration was insufficient. This insufficiency of the total ration Kellner believes to be the cause of the increase in the proteid metabolism.

A consideration of the daily results confirms this view. In passing from periods of lighter to those of heavier work the increase followed promptly upon the change. In Period III, with the most severe work, the proteid metabolism continued to increase throughout the period and apparently had not reached its limit at the close. Conversely, when the work was diminished in Periods IV and $\mathrm{V}$ it decreased as promptly as it had increased. Finally, it should be noted that the additional amount of proteids metabolized was entirely insufficient to furnish an amount of energy equivalent to the increase in the work.

In four succeeding series of experiments Kellner * has investigated this phenomenon more fully, some of the sources of error noted above having been avoided in the later researches. The results, as will appear, still show a deficit of nitrogen. Kellner estimates that about 6 grams of nitrogen per day were required for the growth of hoofs, hair, epidermis, etc., and believes that there was some loss of urinary nitrogen mechanically and chemically.

Series II.-In this series of experiments the ration, consisting of 7.5 kilograms of hay and 4 kilograms of beans. was purposely made rich in protein. In spite of this liberal supply of protein. however, the same result as in the first experiment was noted to an even more marked extent. As in the first series, too, the increase in the excretion of nitrogen promptly disappeared when the amount of work was diminished.

Series III.-In this series the animal was brought as nearly as possible into equilibrium with his food upon rather light work. The work was then trebled, while at the same time an addition was made to the non-nitrogenous ingredients of the ration by substituting for a portion of the beans an amount of oats containing the same absolute quantity of protein. In this second period there was a slight increase in the digestibility of the protein and, therefore, a

* Landw. Jahrb., 9, 651. 
corresponding increase in the urinary nitrogen (compare Chapter $\mathrm{V}$ ), but this was small compared with the much greater amount of work performed. Moreover, it did not, as in the first series of experiments, augment from day to day during the period of severe work. The following table shows the principal results of this series, the figures for urinary nitrogen and for live weight being given for the first and second halves of each period:

\begin{tabular}{|c|c|c|c|c|}
\hline \multirow{2}{*}{ Period. } & \multirow{2}{*}{$\begin{array}{l}\text { Work, } \\
\text { Kgm. }\end{array}$} & \multicolumn{2}{|c|}{ Nitrogen. } & \multirow{2}{*}{$\begin{array}{c}\text { Live } \\
\text { Weight, } \\
\text { Kg. }\end{array}$} \\
\hline & & $\begin{array}{c}\text { Digested, } \\
\text { Grms. }\end{array}$ & $\begin{array}{c}\text { In Urine, } \\
\text { Grms. }\end{array}$ & \\
\hline$I \ldots \ldots \ldots \ldots \ldots$ & 810,000 & 173.6 & $\left\{\begin{array}{l}158.9 \\
164.1\end{array}\right.$ & $\begin{array}{l}560.3 \\
556.8\end{array}$ \\
\hline II ...... & $2,430,000$ & 178.8 & $\begin{array}{l}174.0 \\
174.8\end{array}$ & $\begin{array}{l}541.3 \\
539.7\end{array}$ \\
\hline III . & 810,000 & 178.8 & $\begin{array}{l}166.4 \\
171.4\end{array}$ & $\begin{array}{l}542.5 \\
543.3\end{array}$ \\
\hline
\end{tabular}

Series IV.-Upon the basis of the foregoing facts Kellner determined the maximum amount of work which his horse could perform on a fixed medium ration without causing an increase in the proteid metabolism. One kilogram of starch was then added to the ration and the maximum amount of work that could be performed upon this new ration without causing such an increase was determined. In the nature of the case this determination could not be of the highest accuracy, but it is amply sufficient for our present purpose. The principal results are given in the following table, the amount of work being expressed by the number of revolutions of the dynamometer, since relative results are all that are required:

\begin{tabular}{|c|c|c|c|c|c|c|c|}
\hline \multirow{2}{*}{ Period. } & & & & \multirow{2}{*}{$\begin{array}{l}\text { Work, } \\
\text { Rev. }\end{array}$} & \multicolumn{2}{|c|}{ Nitrogen. } & \multirow{2}{*}{$\begin{array}{c}\text { Live } \\
\text { Weight, } \\
\text { Kg. }\end{array}$} \\
\hline & & & & & $\begin{array}{c}\text { Digested, } \\
\text { Grms. }\end{array}$ & $\begin{array}{c}\text { In Urine, } \\
\text { Grms. }\end{array}$ & \\
\hline $\begin{array}{r}\text { I } \ldots \ldots \cdots \\
\text { II } a \ldots \ldots \cdots \cdots \\
\text { III } b \ldots \ldots \cdots \\
\text { IV } \ldots \ldots \cdots \cdots\end{array}$ & 1 & $\begin{array}{l}\text { Without } \\
\text { starch }\end{array}$ & ( & $\begin{array}{l}300 \\
600 \\
600 \\
500 \\
400\end{array}$ & 121.1 & $\begin{array}{l}107.2 \\
110.2 \\
115.6 \\
109.4 \\
109.6\end{array}$ & $\begin{array}{l}540.0 \\
538.3 \\
533.1 \\
532.5 \\
530.7\end{array}$ \\
\hline II $\ldots \ldots$ & \} & $\begin{array}{l}\text { With } \\
\text { starch }\end{array}$ & \} & $\begin{array}{l}800 \\
600\end{array}$ & 120.1 & $\begin{array}{l}115.5 \\
109.6\end{array}$ & $\begin{array}{l}517.1 \\
515.4\end{array}$ \\
\hline
\end{tabular}


Kellner estimates that the maximum amount of work which could be performed on the ration containing starch was 700 revolutions as compared with a maximum of 500 revolutions without starch. Even if this estimate of Kellner's be regarded as high, it is evident from the figures given that the addition of the starch enabled materially more work to be performed without an increase in the proteid metabolism. The results obtained in this and the subsequent series have been made the basis of interesting computations. regarding the utilization of the potential energy of the food which will be considered in Part II.

Series $V$.-This series was precisely similar to the preceding one, except that the addition of non-nitrogenous matter to the ration was. made in the form of oil by substituting flaxseed for linseed meal. The protein of the ration remained unchanged, while the fat was increased by 203 grams. The results were entirely similar to those with starch, as the following table shows:

\begin{tabular}{|c|c|c|c|c|c|}
\hline \multirow{2}{*}{ Period. } & & \multirow{2}{*}{$\begin{array}{l}\text { Work, } \\
\text { Rev. }\end{array}$} & \multicolumn{2}{|c|}{ Nitrogen. } & \multirow{2}{*}{$\begin{array}{c}\text { Live } \\
\text { Weight, } \\
\text { Kg. }\end{array}$} \\
\hline & & & $\begin{array}{l}\text { Digested, } \\
\text { Grrms. }\end{array}$ & $\begin{array}{c}\text { In Urine, } \\
\text { Grms. }\end{array}$ & \\
\hline $\begin{array}{l}\mathrm{I} a \ldots \ldots \\
\mathbf{I} b \ldots \ldots \\
\operatorname{II} a \ldots \ldots \\
\mathbf{I I} b \ldots \ldots\end{array}$ & $\begin{array}{l}\text { Without } \\
\text { addition } \\
\text { of fat }\end{array}$ & $\begin{array}{l}500 \\
500 \\
550 \\
550\end{array}$ & 159.0 & $\begin{array}{l}148.9 \\
149.2 \\
147.5 \\
153.0\end{array}$ & $\begin{array}{l}496.5 \\
493.2 \\
485.8 \\
479.4\end{array}$ \\
\hline $\begin{array}{l}\operatorname{I} a \ldots \ldots \\
\operatorname{I} b \ldots \ldots \\
\operatorname{II} a \ldots \ldots \ldots\end{array}$ & $\begin{array}{l}\text { With } \\
\text { addition } \\
\text { of fat }\end{array}$ & $\begin{array}{l}700 \\
700 \\
650 \\
650\end{array}$ & 153.9 & $\begin{array}{l}148.1 \\
153.9 \\
145.6 \\
145.0\end{array}$ & $\begin{array}{l}476.0 \\
469.0 \\
466.4 \\
460.8\end{array}$ \\
\hline
\end{tabular}

While Kellner's method of investigation may be regarded as somewhat imperfect and necessarily giving but approximate results, yet it suffices to bring out in a very striking manner the intimate relation existing between the supply of non-nitrogenous nutrients in the food of a working animal and the effect of the work upon the proteid metabolism. In conclusion, it should be noted that in all Kellner's experiments there was a fairly abundant supply of protein. Whether the same result would be obtained on a ration containing the minimum amount of proteids required by the organism is not shown. In no case was the increase in the proteid metabolism, when observed, sufficient to supply energy equivalent to the additional work done. 
Later Investigations.-In 1882 North * made experiments upon himself in which a considerable amount of work, mainly walking from 30 to $4 \dot{7}$ miles while carrying a load of about 27 pounds, was performed on one day of each experiment. The account of the experiments does not give sufficient data for computing the total amount of work performed, but it was evidently very considerable and resulted in a marked increase in the excretion of nitrogen. It is not possible, however, to determine whether the total food was adequate for the work days, but it was no greater then than during the periods of rest.

Argutinsky, $\dagger$ in experiments upon himself, observed as a result of rather severe work a very marked increase in urinary nitrogen which continued at least three days after the cessation of the work. Munk $\ddagger$ subsequently criticised Argutinsky's results on the ground that the supply of non-nitrogenous nutrients in his diet was insufficient. Krummacher $\$$ obtained results quite similar to those of Argutinsky, but his experiments are open to the same criticism as those of his predecessor, namely, an insufficient supply of nonnitrogenous nutrients, as he himself points out in a later paper. Hirschfeldt $\|$ failed to observe any material increase in the nitrogen excretion as the result of work upon a diet containing a considerable excess of food over the amount required for maintenance. This was true both upon a diet containing little protein and one abundantly supplied with this nutrient.

Pflüger, like Liebig, regards protein as the sole source of muscular energy. As yet only a preliminary sketch of his investigations has been published. $\mathrm{T}$ Hed a lean dog upon prepared lean meat, that is, upon a nearly pure proteid diet, for seven months. The animal remained apparently in perfect health and was able to perform a large amount of work. Under the influence of the work the excretion of nitrogen was observed to increase somewhat, but not sufficiently to account for the energy expended in the work. This phenomenon Pflüger explains by supposing that during work

* Proc. Roy. Soc., 36, 14.

$\dagger$ Arch. ges. Physiol., 46, 552.

$\ddagger$ Arch. f. (Anat. u.) Physiol., 1890, p. 552.

§ Arch. ges. Physiol., 47, 454.

II Virchow's Arehiv., 121, 501.

If Arch. ges. Physiol., 50, 98. 
the organism economizes in its demands for proteids elsewhere than in the muscles. The further interesting observation was made that with continuous work the proteid metabolism, which at first showed an increase, diminished again and even reached its original value. With a ration containing but little protein and much non-nitrogenous material, a small increase of the proteid metabolism was observed as the result of work. The preliminary account of the experiments affords no adequate data for computing the sufficiency of the total food.

Krummacher,* in his second investigation, made three separate experiments. In the first of these the total food was estimated to be approximately sufficient for maintenance (38 Cals. per kilogram), while in the other two it was much in excess of this. The following table shows the total amount of food per kilogram, expressed as Calories of metabolizable energy, $\dagger$ the amount of work performed, and the percentage increase of the proteid metabolism:

\begin{tabular}{|c|c|c|c|c|}
\hline & \multicolumn{2}{|c|}{ Energy of Food. } & \multirow{2}{*}{$\begin{array}{l}\text { Work } \\
\text { Measured, } \\
\text { Kgm. }\end{array}$} & \multirow{2}{*}{$\begin{array}{c}\text { Tncrease } \\
\text { of Proteid } \\
\text { Metabolism, } \\
\text { Per Cent. }\end{array}$} \\
\hline & $\begin{array}{l}\text { Total. } \\
\text { Cals. }\end{array}$ & 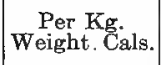 & & \\
\hline 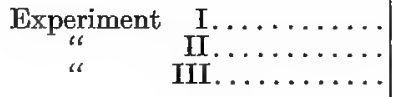 & $\begin{array}{l}2459 \\
5034 \\
5701\end{array}$ & $\begin{array}{l}38 \\
64 \\
72\end{array}$ & $\begin{array}{l}153,070 \\
324,540 \\
401,965\end{array}$ & $\begin{array}{r}21 \\
22 \\
7\end{array}$ \\
\hline
\end{tabular}

The work done consisted in turning an ergostat. It has been shown by subsequent investigators that not over 30 per cent. of the energy of the body material metabolized in the performance of work in this way can be recovered in the work actually done. Assuming this high figure, and further that Krummacher's estimate of the maintenance requirements is accurate, it appears that the food in these experiments was insufficient to supply the energy required for the amount of work actually done.

It was observed, as in other experiments of this nature, that the increased excretion of nitrogen continued for a day or two after the cessation of the work. Only in the first experiment, however, was even the total proteid metabolism during the periods of work, together with the excess above the rest value observed on succeeding

$$
\text { * Zeit. f. Biol., 33, } 108 .
$$$$
\text { † See Chapter X. }
$$ 
days, sufficient to supply an amount of energy equal to that actually measured on the ergostat, so that at least the larger share of the energy must have been derived from non-nitrogenous materials.

Zuntz \& Schumburg,* in investigations upon soldiers, observed an increase of the proteid metabolism as the result of marching, carrying a considerable weight. The increase, however, seemed to bear no direct relation to the amount of work performed, but rather to the conditions under which it was done. Thus excessive heat or sultriness of the atmosphere, resulting in unusual fatigue, was accompanied by an increased excretion of nitrogen. The increase continued during the two days following the work.

Frentzel $\dagger$ experimented upon dogs. In the first series the animals were fed pure fat, while in the second series no food was given. The work, which was done upon a tread power, was considerable. In the first series there was an increase of 9.25 per cent. in the nitrogen excretion in the work experiments, while in the second series a maximum increase of 44.26 per cent. was computed, which, however, is believed by the author to be too high. A method of computation which he considers more nearly correct makes the increase in the second period 13.31 per cent. In the first series of experiments the food consisted of 150 grams of fat per day except upon one of the work days, when only 80 grams were consumed. No data are given regarding the sufficiency of this ration, but according to E. Voit's compilation $\ddagger$ it would appear hardly adequate for the maintenance of a dog of the weight used ( 36 kilograms). The work, therefore, even in the first series, was probably done upon insufficient food. In neither case was the increase in the amount of protein metabolized equivalent in energy content to the actual amount of external work done, and in the first series even the total proteid metabolism was not, while if we allow for the consumption of energy in internal work, heat production, etc., it was not sufficient in either series.

Atwater \& Sherman \& have reported observations upon the

* Arch. f. (Anat. u.) Physiol., 1895, p. 378.

$\dagger$ Arch. ges. Physiol., 68, 212.

$\ddagger$ Zeit. f. Biol., 41, 115

§ U. S. Dept. Agr., Office of Experiment Stations, Bull. 88. 
food consumption, digestion, and metabolism of three bicyclers during a six-day contest. They find that, in spite of an apparently liberal diet containing large amounts of protein, all three riders lost considerable proteid tissue during the race. The conditions of the investigation were not such as to permit of a determination of the sufficiency of the food consumed, but the computations by Carpenter of the actual amount of work done seem to render it very probable that the body fat must have been drawn upon to a considerable extent.

ReCAPItUlation,-The investigations above cited seem to show beyond a doubt that when work is performed upon food less than sufficient to maintain the body and supply the amount of energy required for the work the proteid metabolism is somewhat increased.

Whether the converse of this is true, namely, that when the food is sufficient such an increase in the proteid metabolism does not occur, is not so clear, for the reason that in most, if not all, of the cases we have no adequate data as to the sufficiency of the food. It is plain, however, that the question is not so easily investigated as might appear at first sight, and that the final solution of the relations of work to proteid metabolism can only be reached by means of investigations in which the total metabolism both of matter and energy is determined.

Gain of Proteids during Work. - Caspari and Bornstein have recently made further investigations into the possibility of a gain of protein as a result of work which was mentioned above in connection with Pflüger's experiments.

Caspari * experimented upon a dog which received an amount of food computed to have been fully sufficient for its maintenance and to supply energy for the work done. Furthermore, a considerable portion of the non-nitrogenous nutrients of the ration, consisting largely of carbohydrates, was given shortly before the work was done, while in some cases additional sugar or fat was given at that time. In the first experiment, work was performed upon three successive days. Upon the second of these there was a considerable increase of the urinary nitrogen, but upon the third its amount fell below that of the rest period. The average for the three days . of work was almost exactly equal to the value found for the last

* Arch. ges. Physiol., 83, 509. 
day of the rest period and less than the average for the four previous rest days.

In the second experiment the work was continued for four days, then a rest day intervened, and then the work was continued for five more days. At the outset there was a slight increase of the proteid metabolism, but in the second period of five days it showed a marked decrease resulting in a progressive gain of nitrogen by the body, as is shown in the following tabular statement of the daily average results:

\begin{tabular}{|c|c|c|c|c|c|}
\hline Day. & $\begin{array}{c}\text { External } \\
\text { Work } \\
\text { Done, } \\
\text { Cals. }\end{array}$ & $\begin{array}{l}\text { Nitrogen } \\
\text { of Food, } \\
\text { Grms. }\end{array}$ & $\begin{array}{l}\text { Average } \\
\text { Nitrogen } \\
\text { of Feces, } \\
\text { Grms. }\end{array}$ & $\begin{array}{l}\text { Nitrogen } \\
\text { of Urine, } \\
\text { Grms. }\end{array}$ & $\begin{array}{l}\text { Gain or } \\
\text { Loss of } \\
\text { Nitrogen, } \\
\text { Grms. }\end{array}$ \\
\hline 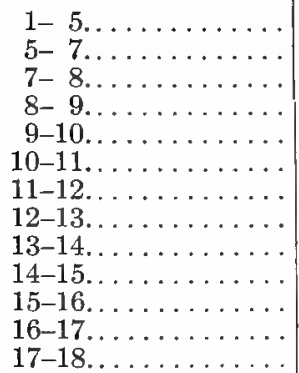 & $\begin{array}{r}0 \\
0 \\
0 \\
597 \\
467 \\
597 \\
596 \\
0 \\
595 \\
590 \\
593 \\
588 \\
586\end{array}$ & $\begin{array}{l}25.11 \\
25.11 \\
25.11 \\
25.11 \\
25.11 \\
25.11 \\
25.11 \\
25.11 \\
25.11 \\
25.11 \\
25.11 \\
25.11 \\
25.11\end{array}$ & $\begin{array}{l}1.89 \\
1.89 \\
1.89 \\
1.78 \\
1.78 \\
1.78 \\
1.78 \\
1.78 \\
1.78 \\
1.78 \\
1.78 \\
1.78 \\
1.78\end{array}$ & $\begin{array}{l}23.68 \\
22.00 \\
21.98 \\
24.72(?) \\
23.32 \\
23.23 \\
21.83 \\
22.06 \\
20.82 \\
19.64 \\
20.39 \\
19.87 \\
19.79\end{array}$ & $\begin{array}{l}-0.46 \\
+1.22 \\
+1.24 \\
-1.39 \\
+0.01 \\
+0.10 \\
+1.50 \\
+1.27 \\
+2.51 \\
+3.69 \\
+2.94 \\
+3.46 \\
+3.54\end{array}$ \\
\hline
\end{tabular}

This gain Caspari ascribes to an actual growth of the muscles as the effect of exercise, this growth according to him taking the form of a hypertrophy of the fibers. No determinations of the gain or loss of carbon were made.

Bornstein,* who had previously investigated the possibility of increasing the store of proteids in the body by the addition of proteids to the food, has also contributed to the investigation of this phase of the question. His experiments were made upon himself. For seven days he consumed a uniform ration containing a moderate amount of protein and sufficient non-nitrogenous nutrients, according to previous experience, to maintain his body. The latter was in equilibrium with the food as regards nitrogen from the first day. Then the proteid supply was increased by approximately 50 per cent. by the ingestion of pure proteids and light work $(17,000$ * Arch. ges. Physiol., 83, 540. 
kgm. per day) done by turning an ergostat. As a result of the increased supply of proteids in the food the proteid metabolism increased promptly, reaching its maximum upon the fifth day, when it very slightly exceeded the supply. From that time, however, it decreased gradually during the remaining thirteen days of the experiment, so that a gain of proteids by the body resulted, which was still in active progress when the experiment was discontinued. Counting from the time when the proteid metabolism reached its maximum the average gain of nitrogen per day was

First five days............... 1.28 grams

Last five days... . ........ . . 2.05 "

Average of all.................. 1.475 "

The author computes that 22 per cent. of the proteids added to the food was stored up in the body. In a previous similar experiment without work it was found that only 16 per cent. was thus stored.

Two respiration experiments with the Zuntz apparatus were made during the work. The difference between their results and those of similar experiments during rest was used as the basis for computing the actual amount of energy metabolized in the body for the performance of work. This was found to be equal to 0.0100875 Cal. per kgm. external work, which is equivalent to 171.5 Cals. for the whole daily work of $17,000 \mathrm{kgm}$. Assuming the original ration to have been a maintenance ration, Bornstein computes that the portion of the added proteids which was actually metabolized was insufficient to supply the energy necessary for the work done and that some of the fat of the body was drawn upon. The loss in live weight was found to agree with this assumption.

The above investigations seem to show, not only that work may be done without increasing the proteid metabolism but that it may actually result in diminishing it, a fact which appears in harmony with the common observation that the tendency of exercise is to build up the muscular tissue.

Summary.-While the results which have been cited are not in all respects conclusive, and while further investigation is required to fully elucidate the relations of muscular exertion to proteid metabolism, the following general conclusions seem to be justified by the evidence now available: 
1. The non-nitrogenous ingredients of the food or of the tissues are the chief source of muscular energy. In by far the greater number, if not all, of the experiments upon this subject the amount of protein metabolized, as measured by the nitrogen excretion, was insufficient to furnish energy equivalent to the work done, the deficiency being in many cases very great. This statement, it will be observed, does not assert that the proteids are not concerned in the production of this energy. We may regard it as very probable that the non-nitrogendus matter metabolized has first entered into the structure of the muscular protoplasm, which, as we know, consists largely of proteids, but in a contraction it is largely, if not wholly, the non-nitrogenous groups contained in the protoplasm which are metabolized rather than the nitrogenous groups.

2. With insufficient food there may be a considerable increase in the proteid metabolism as a result of muscular exertion, especially when pushed to exhaustion.

3. This increase is far from sufficient to supply energy for the work actually done, is not usually proportional to it, and seems dependent to a considerable degree upon accompanying conditions.

4. With sufficient food the increase of the total proteid metabolism consequent upon muscular exertion is at the most slight and possibly equal to zero.

5. In some cases a storage of proteids has been observed to result from the performance of work.

Functions of Proteids.- - If the above conclusions are admitted, it is possible to suppose that in a muscular contraction under favorable conditions-that is, when there is an abundant supply of nonnitrogenous material-there is no increased metabolism of the proteids. This view of the subject would regard the question as being simply one of the relative supply of nutrients, the energy being evolved from non-nitrogenous nutrients when these are in abundance, while in default of them the proteids are drawn upon.

Another view of the subject, however, is possible, and perhaps more probable. It would appear that muscular exertion tends to produce two opposite effects upon the proteid metabolism: first, to break down additional protein, as is shown when work is done upon insufficient food; and second, to build up proteid tissue when 
the food is sufficient, as is illustrated in the experiments of Caspari and Bornstein.

As a basis for a tentative hypothesis, it seems allowable to suppose that both these processes - that of anabolism and katabolism of proteids-are continually taking place in the muscle and that both are exaggerated by exercise. In other words, we may imagine that the performance of work by a normally developed muscle requires an increased proteid katabolism, which is balanced, at least in the course of the twenty-four hours, by a corresponding increase in the proteid anabolism. With a liberal supply of food proteids, then, a part of the latter would, during rest, simply undergo nitrogen cleavage and be used virtually as "fuel," but when work was done they (or part of them) would be used to replace the proteids katabolized in the muscles. Upon this hypothesis, the proteids might play a not unimportant part in the production of muscular work without any evidence of it appearing in an increased nitrogen excretion. It is to be remarked, however, that even on this supposition the proteids could not be regarded as furnishing all, or even, in many cases, a large share, of the energy liberated. On insufficient food, the hypothesis would assume that the energy supply is deficient and that proteids which would otherwise be used for muscular anabolism are diverted to use as "fuel," probably undergoing a preliminary nitrogen cleavage and furnishing their nonnitrogenous residue to the muscles as a source of energy.

The above tentative hypothesis implies that if work were performed upon a ration containing only the minimum amount of proteids required during rest, it would cause an increase of the proteid metabolism, no matter how much non-nitrogenous material was supplied, because there would be no proteids available which could be diverted to repair the waste assumed to be occasioned by muscular activity. Up to the present time, however, we possess no experimental investigation of this phase of the question.

However this may be, we know that the performance of work requires a well-developed muscular system. To produce and develop such a system, a liberal supply of protein is essential, while we may reasonably suppose that to maintain it involves a larger proteid supply in the food than is required to maintain the proteid 
tissue on a lower level. This fact alone would indicate the need of a reasonably liberal supply of protein in the food of working animals. If the hypothesis above outlined be approximately correct, it is necessary that the food also contain protein which during rest may be simply a source of heat, but which during work may be diverted to repair the increased waste of nitrogenous tissues caused by exertion. This accords with the well-established fact that the dietaries selected by athletes and others who undertake severe physical exertion are almost invariably rich in protein.* It is of course difficult to say how far the large amount of proteids in the dietaries of athletes represents a real physiological demand and how far it is a matter of tradition or of taste, but it hardly seems likely that so universal an opinion should be lacking in some considerable basis of fact.

\section{Effects upon the Carbon Metabolism.}

In the foregoing paragraphs we have seen that as a rule the total proteid metabolism is not much affected by muscular exertion. While proteids undoubtedly have important functions in connection with the production of work, it is nevertheless true that normally the energy liberated in muscular contraction is derived chiefly or wholly from the breaking down of non-nitrogenous material. Moreover, even in those cases in which a considerable increase of the proteid metabolism has been observed, its amount has been entirely insufficient to account for the extra evolution of energy. It therefore becomes of especial importance to consider the effects of work upon the carbon balance.

The Gaseous Exchange.-Since the influence of muscular exertion upon the proteid metabolism is at most small, it is possible to compare the carbon metabolism during work and rest without material error upon the basis of the gaseous exchange simply, and as a matter of fact a large share of our knowledge of the subject rests upon determinations of the respiratory exchange.

Is LARGeLY INCREASED. - The fact that muscular work largely increases the evolution of carbon dioxide and water and the consumption of oxygen by the organism is too familiar from ordinary

* For a summary of American experiments bearing upon this point see Atwater \& Benedict, Boston Medical and Surgical Journal, 144, 601 and 629 
experience and too well established scientifically to require more than illustration. The fact of such an increase was shown in the researches of Lavoisier. Scharling, ${ }^{*}$ who as early as 1843 constructed an apparatus somewhat like the Pettenkofer respiration apparatus (see p. 70), states in his account of his experiments that moderate work increases the excretion of carbon dioxide and that it is also greater shortly after a meal. Of other early researches upon this point may be mentioned those of Hirn $\dagger$ in 1857, and especially those of Smith $\ddagger$ in 1859 . The investigations of Pettenkofer \& Voit $\S$ in 1866 appear to have been the first to be executed in accordance with modern methods. Their results have already been cited in their bearing upon the influence of work on proteid metabolism, but may be repeated here:

\begin{tabular}{|c|c|c|c|c|c|c|}
\hline & \multirow{2}{*}{$\begin{array}{c}\text { Nitrogen } \\
\text { of Urine, } \\
\text { Grms. }\end{array}$} & \multirow{2}{*}{$\begin{array}{c}\text { Carbon } \\
\text { Dioxide } \\
\text { Excreted, } \\
\text { Grms. }\end{array}$} & \multicolumn{2}{|c|}{ Water Excreted. } & \multirow{2}{*}{$\begin{array}{l}\text { Oxygen } \\
\text { Taken } \\
\text { Up. } \\
\text { Grms. }\end{array}$} & \multirow{2}{*}{$\begin{array}{l}\text { Number } \\
\text { of Experi- } \\
\text { ments. }\end{array}$} \\
\hline & & & $\begin{array}{l}\text { In } \\
\text { Urine. } \\
\text { Grms. }\end{array}$ & $\begin{array}{l}\text { Evapo- } \\
\text { rated. } \\
\text { Grms. }\end{array}$ & & \\
\hline \multicolumn{7}{|l|}{ Fasting: } \\
\hline Rest & 12.4 & 716 & 1006 & 821 & 762 & 2 \\
\hline \multirow{2}{*}{\multicolumn{7}{|c|}{ Average diet: }} \\
\hline & & & & & & \\
\hline Rest ......... & 17.0 & 928 & 1218 & 931 & 832 & 3 \\
\hline Work ........ & 17.3 & 1209 & 1155 & 1727 & 981 & 2 \\
\hline
\end{tabular}

Subsequent investigators such as Speck, $\|$ Hanriot \& Richet, Katzenstein,** Loewy, $\dagger \dagger$ and many others have fully confirmed the results of the early experimenters. The increase in the oxygen taken up was not actually demonstrated in all of these experiments, but it was in some and may be reasonably inferred in the remainder.

* Ann Chem Pharm, 45, 214

† Comptes rend Soc. de Physique de Colmar, 1857; Revue Scientifique, ler Semestre. 1887.

$\ddagger$ Phil. Trans., 1859, p 681.

$\$$ Zeit f Biol., 2, 478 .

|| Schriften der Gesell. der ges. Naturwiss zu Marburg, 1871; Arch. klin Med., 45, 461.

I Comptes rend., 104, 435 and 1865; 105, 76; Ann. de Chim. et de Phys., (6), 22, 485

** Arch ges Physiol., 49, 330.

†† Ibid., 49, 405. 
Effects are Immediate.--Experiments like those of Pettenkofer \& Voit, extending over twenty-four hours, give simply the total effect of the performance of work upon the carbon balance. By the use of the Zuntz type of apparatus, however, it is possible to follow the gaseous exchange in its details through successive short periods as well as to determine the amount of oxygen consumed. The data thus obtained give a clear picture of the immediate effects of work upon metabolism and have led to the extensive use of this type of apparatus in experiments of this nature. The results of these experiments agree with common experience in showing that these effects appear very promptly and soon reach their maximum, disappearing as promptly after the work ceases. In other words, the increase in the carbon metabolism is very closely confined to the time during which the work is actually performed.

The Respiratory Quotient.-The ratio between carbon dioxide produced and oxygen consumed, commonly known as the respiratory quotient, as has been pointed out in Chapter III, enables us to form a fairly clear idea as to the general nature of the total material metabolized, and hence much study has been bestowed upon the relation between these two quantities.

Is VARIABLE.-We have already seen that the respiratory quotient may vary considerably during repose, being largely determined by the nature of the food. The same thing is true of the respiratory quotient during work.

Zuntz,* in experiments on a fasting dog, obtained the following values for this quotient:

\begin{tabular}{|c|c|c|}
\hline & $\begin{array}{c}\text { Number of } \\
\text { Experi- } \\
\text { ments. }\end{array}$ & $\begin{array}{c}\text { Average } \\
\text { Respiratory } \\
\text { Quotient. }\end{array}$ \\
\hline 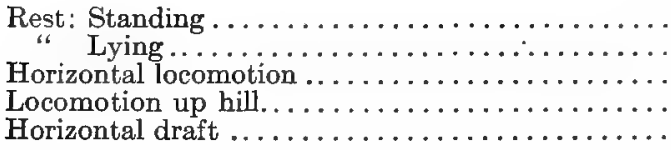 & $\begin{array}{r}2 \\
6 \\
8 \\
5 \\
10\end{array}$ & $\begin{array}{l}0.69 \\
0.71 \\
0.73 \\
0.77 \\
0.77\end{array}$ \\
\hline
\end{tabular}

In Zuntz \& Hagemann's † experiments upon the horse the respiratory quotient in the single work periods ranged from 0.729 upon a

* Arch. ges. Physiol., 68, 191.

† Landw. Jahrb., 27, Supp. III, 296-331. 
ration of green alfalfa to 0.996 upon hay, straw, and oats. The averages obtained for different forms of work were as follows:

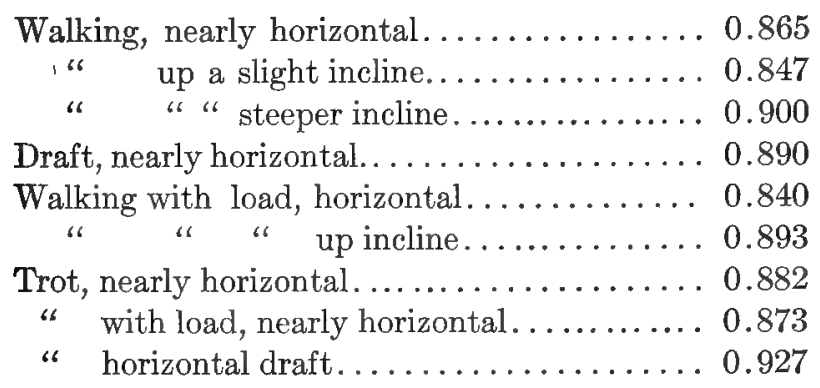

The total range of the respiratory quotient in these experiments was 0.84 to 0.93 . It is thus seen to be higher with herbivorous animals, subsisting largely upon carbohydrates, than with the dog.

Change Caused by Work. - Chauveau states as the result of his investigations upon the origin of muscular power that the performance of work always increases the respiratory quotient.

His first experiments* were made upon a man who had fasted for sixteen hours. The work consisted in the alternate ascent and descent of a stairease, the work of ascending being equal to about $29,000 \mathrm{kgms}$. in the seventy minutes of the experiment. Samples of the expired air were taken by the Tissot apparatus $\dagger$ for five minutes at a time at intervals during the work and the respiratory quotient determined by a comparison of its composition with that of the normal atmosphere. The following were the results for the respiratory quotient:

Immediately before work............ 0.75

First to fifth minute................... 0.84

Tenth to fifteenth minute.............. 0.87

Fortieth to forty-fifth minute............ 0.95

Sixty-fifth to seventieth minute......... 0.74

* Comptes rend., 122, 1163.

† Archives de Physiol., 1896, p. 563. The apparatus is of the Zuntz type. 
A second experiment,* begun after fifteen hours' fasting, was divided into two periods. The first was similar to the previous experiment, but lasted for thirty minutes only, the work of ascent equaling in that time about $30,000 \mathrm{kgms}$. The subject then rested for a time during which he consumed 105 grams of butter. Two hours after the ingestion of the butter the experiment, was repeated, samples of the expired air being taken for three minutes at a time. The results as regards the respiratory quotient were as follows:

\section{Fasting.}

Three minutes before beginning work...... 0.706

Twelfth to fifteenth minute............. 0.804

Twenty-seventh to thirtieth minute........ 0.812

Rest............................... 0.812

\section{Two Hours after Ingestion of Butter.}

Three minutes before beginning work...... 0.666

Twelfth to fifteenth minute.............. 0.783

Twenty-seventh to thirtieth minute....... 0.809

In conjunction with Laulanié $\dagger$ he has also experimented on dogs and rabbits, the muscular contractions being caused by electric shocks. The method of determining the respiratory exchange, as described by Laulanié, consisted in using a Pettenkofer type of apparatus with a small but constant known rate of ventilation. The outgoing air passed through a small gasometer, but the current could be shunted and the sample of air contained in the gasometer analyzed. No details of the experiments or of the methods of calculation are given. The first table on the following page contains Laulanié's summary of the results. $\S$

An even greater increase in the respiratory quotient has been bserved by other investigators. Thus Hanriot \& Richet \|f found

* Comptes rend., 122, 1169

† Ibid., 122, 1244, 1303: Archives de Physiol . 1896, p. 572.

$\ddagger$ Archives de physiol . 1896, pp. 619 and 636 .

\$ Energetique Musculaire, p. 70.

|f Comptes rend., 104, 435 and 1865: 105, 76. 


\begin{tabular}{|c|c|c|c|c|c|}
\hline \multirow{2}{*}{ Animal. } & \multirow{2}{*}{ Food. } & \multirow{2}{*}{$\begin{array}{c}\text { No. of } \\
\text { Expts. }\end{array}$} & \multicolumn{3}{|c|}{ Respiratory Quotient. } \\
\hline & & & $\begin{array}{l}\text { Before } \\
\text { Work. }\end{array}$ & $\begin{array}{l}\text { During } \\
\text { Work. }\end{array}$ & $\begin{array}{l}\text { After } \\
\text { Work. }\end{array}$ \\
\hline $\begin{array}{l}\text { Rabbit } \\
\text { Dog } \ldots \\
\text { Dog } \ldots\end{array}$ & 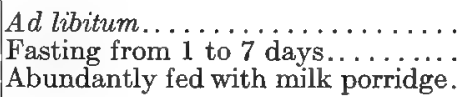 & $\begin{array}{l}7 \\
5 \\
2\end{array}$ & $\begin{array}{l}0.880 \\
0.776 \\
1.016\end{array}$ & $\begin{array}{l}0.970 \\
0.849 \\
1.027\end{array}$ & $\begin{array}{l}0.799 \\
0.733 \\
1.033\end{array}$ \\
\hline
\end{tabular}

in the increments of carbon dioxide and oxygen over the rest values quotients much greater than unity and reaching in one case 3.5 (?). Speck * likewise found an increase in the respiratory quotient as the result of work. Although he observed numerous exceptions, he regards it as the rule that it increases with the severity of the work.

On the other hand, Katzenstein, $\dagger$ in experiments on men, found in some cases no considerable increase in the respiratory quotient during work. He gives the following average results, of which those in the first table do not relate to exactly the same subjects in the three cases:

Turning Ergostat..

Repose.................... 0.754

Light work................... 0.824

Heavy work.................... 0.823

\section{Walking.}

\begin{tabular}{|c|c|c|c|c|}
\hline & $\begin{array}{l}\text { Subject } \\
\text { No. } 1 .\end{array}$ & $\begin{array}{l}\text { Subject } \\
\text { No.2. }\end{array}$ & $\begin{array}{l}\text { Subject } \\
\text { No. } 3 . \neq\end{array}$ & $\begin{array}{l}\text { Subject } \\
\text { No. } 4 .\end{array}$ \\
\hline 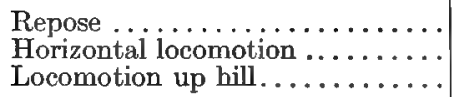 & $\begin{array}{l}0.801 \\
0.805 \\
0.799\end{array}$ & $\begin{array}{l}0.73 \\
0.77 \\
0.79\end{array}$ & $\begin{array}{l}0.77 \\
0.82 \\
0.865\end{array}$ & $\begin{array}{l}0.75 \\
0.895 \\
0.86\end{array}$ \\
\hline
\end{tabular}

In all cases, the determinations of the respiratory exchange covered only a few minutes soon after the work began, and

* Arch. klin. Med., 45, 461.

$\dagger$ Arch. ges. Physiol., 49, 330.

\# very corpulent individual. 
no mention is made of the nature of the diet except in one case (fasting). The individual results were rather variable, but most weight is given to those on Subject No. 1, with whom most of the experiments were made. Katzenstein believes Speck's results to be due in part to a change in the rate of respiration, causing the excretion of carbon dioxide to exceed its actual production (p. 73), and in part to a deficiency of oxygen in the tissue of the contracting muscles.

Loewy, like Katzenstein, found that work pushed to the point of producing a considerable degree of fatigue raised the respiratory quotient, while moderate work did not. Rapid turning of the wheel of the ergostat, preventing full breathing, or compression of the upper arm by means of a rubber band, produced the same effect, which he attributes to a lack of oxygen. The most marked results seem to be those for the first few minutes of work, although in one case work continued for ten to twenty minutes and producing fatigue raised the respiratory quotient.

Probably the most extensive and carefully conducted investigations of this nature are those of Zuntz and his associates upon the dog, and particularly on the horse. Some data from the latter investigations have already been given on p. 212. The following table adds to the averages there quoted those for the corresponding rest periods. In these experiments there was a distinct lowering of the respiratory quotient instead of an increase. In

\begin{tabular}{|c|c|c|c|}
\hline \multirow{2}{*}{ Kind of Work. } & \multirow{2}{*}{ Periods. } & \multicolumn{2}{|c|}{ Respiratory Quotient. } \\
\hline & & Repose. & Work. \\
\hline 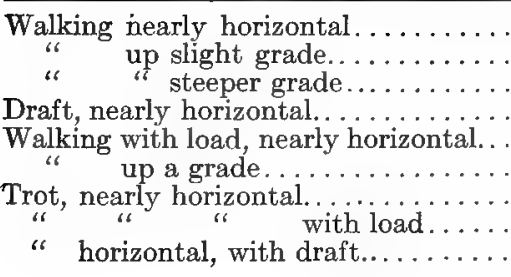 & $\begin{array}{l}a, b, e, f, i, o \\
a, b, e, o \\
a, b, e, f, i, n \\
b, e, f, i \\
\quad e, i, o \\
e, i, o, o \\
a, e, f, o \\
\quad e, i, o \\
g, f, i\end{array}$ & $\begin{array}{l}0.943 \\
0.940 \\
0.953 \\
0.956 \\
0.915 \\
0.915 \\
0.943 \\
0.915 \\
0.943\end{array}$ & $\begin{array}{l}0.865 \\
0.847 \\
0.900 \\
0.890 \\
0.840 \\
0.893 \\
0.882 \\
0.873 \\
0.927\end{array}$ \\
\hline
\end{tabular}

* Arch. ges. Physiol., 49, 405. 
all cases the animal was liberally fed, usually with oats, hay, and cut straw.

VARIATION DURING WORK.-In their experiments cited above, Chauveau \& Laulanie find that the rise of the respiratory quotient which they regard as the invariable result of muscular exertion occurs promptly upon the beginning of the work, and the same thing is shown by the earlier results of Chauveau. As the work is continued, however, the quotient shows a tendency to fall again, sometime even going below its original rest value, while in a period of rest following work a still further decrease is observed. The results of their experiments * are contained in the table on the opposite page.

Zuntz \& Hagemann $\dagger$ also report a number of experiments on the horse in which the respiratory exchange was determined in successive periods of work. The following are their results for the respiratory quotient:

\begin{tabular}{|c|c|c|c|c|}
\hline \multirow{2}{*}{$\begin{array}{c}\text { No. of } \\
\text { Experiment. }\end{array}$} & \multicolumn{3}{|c|}{ Successive Values of Respiratory Quotient. } & \multirow{2}{*}{$\begin{array}{c}\text { Aggregate Length } \\
\text { of Work Periods, } \\
\text { Min. }\end{array}$} \\
\hline & 1 & 2 & $\mathbf{3}$ & \\
\hline 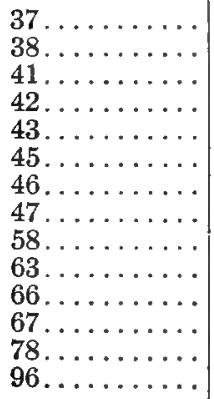 & $\begin{array}{l}.917 \\
.913 \\
.929 \\
.925 \\
.920 \\
.865 \\
.928 \\
.910 \\
.974 \\
.863 \\
.911 \\
.949 \\
.936 \\
.931\end{array}$ & $\begin{array}{l}.865 \\
.806 \\
.889 \\
.948 \\
.931 \\
.868 \\
.921 \\
.926 \\
.905 \\
.820 \\
.922 \\
.934 \\
.909 \\
.904\end{array}$ & $\begin{array}{l}\ldots \\
\ldots \\
.897 \\
.875 \\
.911 \\
\ldots \\
.837 \\
\ldots \ldots \\
.871 \\
.878 \\
.883\end{array}$ & $\begin{array}{c}80 \\
121 \frac{1}{2} \\
102 \\
100 \\
92 \frac{1}{2} \\
54 \\
34 \\
48 \\
65 \\
73 \\
71 \\
124 \\
78 \\
75\end{array}$ \\
\hline
\end{tabular}

The results cited in the foregoing paragraphs would appear to justify the general conclusion that in the case of fasting animals or

* Comptes rend., 122, 1244.

† Loc. cit., pp. 290-292. 
INFLUENCE OF MUSCULAR EXERTION UPON METABOLISM. 217

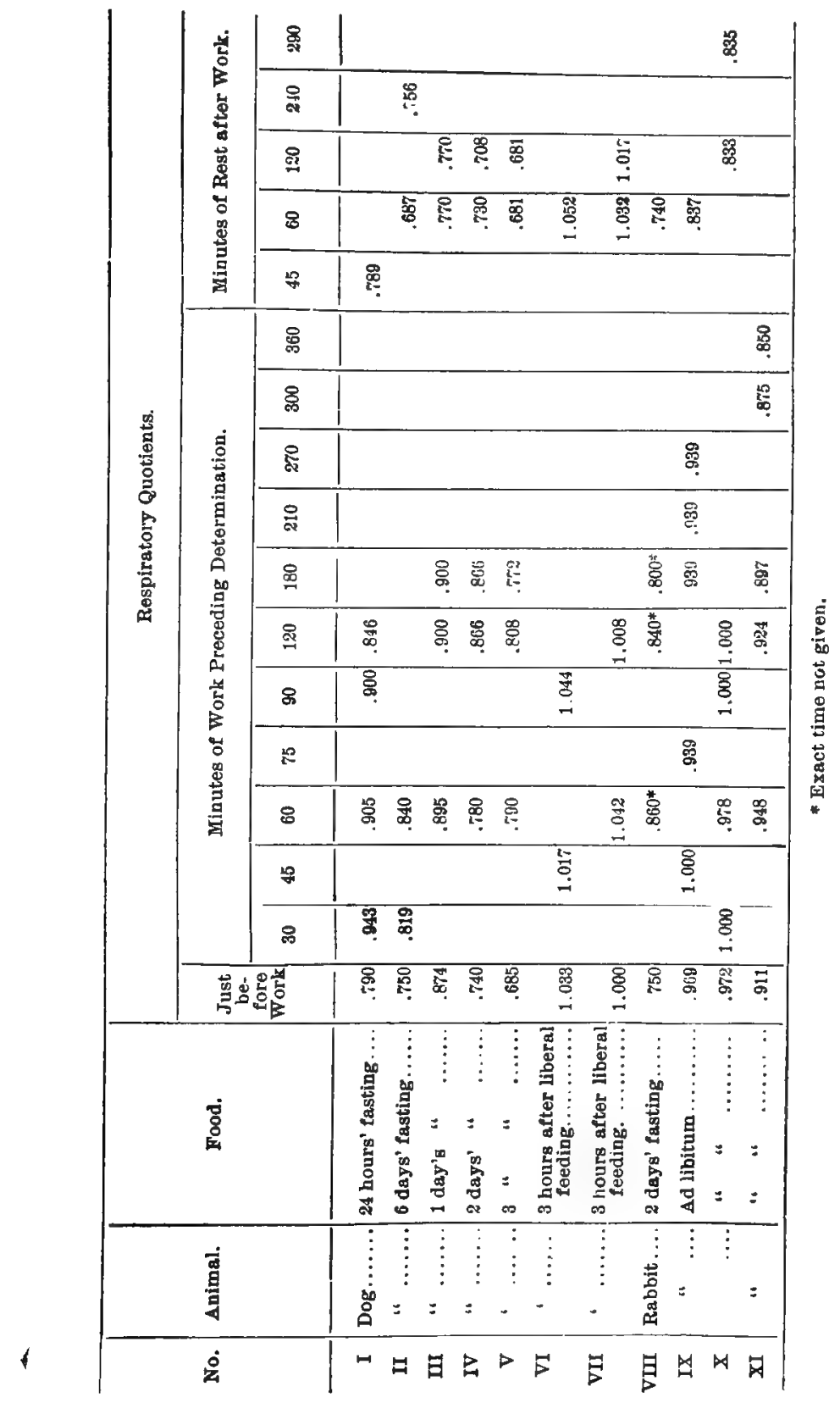


of those insufficiently fed the respiratory quotient is increased by the performance of work, while with well-fed animals, especially those receiving an abundance of carbohydrates, this effect is not apparent. As the work is continued, there appears in many cases to be a tendency toward a diminution of the quotient, while in rest following work a still further decrease may occur.

Nature of Non-nitrogenous Material Metabolized.-As already pointed out, a comparative study of the final products of metabolism during rest and work does not itself afford direct evidence as to the nature of the material actually metabolized in a muscular contraction, but simply shows the total effect of the contraction itself and of the secondary activities resulting from it upon the make-up of the schematic body. When we attempt to go further than this, other methods of investigation are requisite, although experiments like those already cited may afford important confirmatory evidence.

Conclusions From Respiratory Quotient.-The significance of the respiratory quotient in experiments upon work has already been illustrated in Chapter III (p. 76). Neglecting any slight error due to small changes in the proteid metabolism, the variations in the respiratory quotient as outlined in the foregoing paragraphs enable us to trace the corresponding changes in the nature of the carbon metabolism.

The metabolism of a fasting animal at rest is, as was shown in Chapter IV, largely a metabolism of fat. Corresponding to this, the respiratory quotient of such an animal approaches the value 0.7 for pure fat, although never quite reaching it, since some protein is always metabolized. Numerous instances of this fact are seen in the experiments already cited. When such an animal performs work, the respiratory quotient has been found to increase materially, thus showing that, in addition to the fat, carbohydrate material is being metabolized. This is entirely in accord with the well-established fact that muscular exertion causes the glycogen, both of the muscles and of the liver, to decrease and even disappear entirely. With an animal at rest and liberally supplied with carbohydrate food, on the other hand, the respiratory quotient approaches or even reaches unity, showing that the metabolism is essentially carbohydrate in character. When work is required of 
such a subject, little change is noted at first in the respiratory quotient. The cells of the body being richly supplied with carbohydrates apparently utilize these as the most readily available source of energy. In either case, however, continued work makes large demands upon the non-nitrogenous materials available, the store of carbohydrates in the body is rapidly depleted, and the fat of the body is drawn upon to an increasing extent as a source of energy, the necessary result being a diminution in the respiratory quotient.

In the experiments of Chauveau \& Laulanie only the respiratory quotients corresponding to the total metabolism are given, and consequently the changes in the character of the metabolism indicated above can only be traced qualitatively. In Zuntz \& Hagemann's investigations the increments of the carbon dioxide and oxygen over the rest values are given, and from them the proportion of oxygen applied respectively to the oxidation of fat and of carbohydrates is computed. The following average results for the various forms of work show clearly that the ratio of fat to cariohydrates metabolized may vary through a very wide range.

\begin{tabular}{|c|c|c|c|}
\hline \multirow{2}{*}{ Kind of Work. } & \multirow{2}{*}{ Periods. } & \multicolumn{2}{|c|}{$\begin{array}{l}\text { Oxygen per Minute } \\
\text { applied to the Oxida- } \\
\text { tion of }\end{array}$} \\
\hline & & $\begin{array}{l}\text { Fat, } \\
\text { c.c. }\end{array}$ & $\begin{array}{l}\text { Carbohy- } \\
\text { drates, c.c. }\end{array}$ \\
\hline 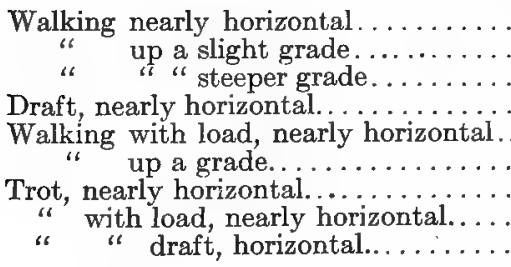 & $\begin{array}{c}a, b, e, f, i, v \\
a, b, e, o \\
a, b, e, f, i, n \\
\quad b, e, f, i \\
e, i, o \\
e, i, o, o \\
a, e, f, o \\
\quad e, i, o \\
g, f, i\end{array}$ & $\begin{array}{r}4.3638 \\
10.433 \\
8.665 \\
8.882 \\
5.962 \\
8.525 \\
7.852 \\
12.718 \\
14.007\end{array}$ & $\begin{array}{l}2.9962 \\
7.465 \\
15.215 \\
12.992 \\
3.317 \\
14.892 \\
14.201 \\
16.023 \\
45.050\end{array}$ \\
\hline
\end{tabular}

The Intermediary Metabolism.-As stated, the conclusions drawn from the respiratory quotient relate, strictly speaking, to the total effect of muscular exertion upon the store of matter in the body. The results of such experiments show that, as a consequence of a given amount of work, a certain quantity of fat and of carbohydrates has been oxidized somewhere in the organism. 
Many eminent physiologists, however, notably Zuntz and his pupils, go further and regard both the fat and the carbohydrates of food or body tissue as immediate sources of muscular energy and as of value for this purpose in proportion to their content of potential energy - that is, to their heats of combustion. In other words, they hold that either fat or carbohydrates may be in effect directly metabolized by the muscular tissue and that each under like conditions yields substantially the same proportion of its potential energy in the form of mechanical work.

On the other hand, Chauveau * and Seegen $\dagger$ and their followers, as has already been indicated, regard the carbohydrates as the immediate source of energy for all the vital activities and hold that fat (or proteids) must first be converted into dextrose by the liver before it can be utilized. It is particularly with regard to muscular exertion that this theory has been elaborated, the conclusions as to other forms of vital activity being to a considerable extent based upon analogy with the former.

Functions of the Liver.-According to this theory the material which is actually metabolized in a muscular contraction is a carbohydrate, viz., either the dextrose carried to the muscle by the blood or the glycogen which is stored up in it. Muscular activity is thus brought into intimate relations with the sugar-forming function of the liver, and a chief office of that organ is considered to be the preparation of the necessary carbohydrate material from the various ingredients of the food. The main facts which have been established may be summarized as follows (compare Chapter II, $\S \S 1$ and 2):

1. Dextrose is being constantly formed by the liver, which not only modifies the carbohydrates of the food but likewise appears to produce dextrose from proteids and particularly, according to this school of physiologists, from fat.

2. Dextrose is as constantly being abstracted from the blood by the tissues, particularly the muscular tissues, as is shown by the constancy of the proportion of dextrose in the blood.

3. The dextrose content of the blood is, according to Chauveau,

* La Vie et l'Energie chez l'Animale.

$\uparrow$ Die Zuckerbildung im Thierkörper. 
maintained during fasting until the very last stages of inanition. When it finally disappears there is a rapid fall in the body temperature and death speedily follows.

4. Both the production of dextrose by the liver and its consumption in the tissues appear to be augmented by muscular exertion.

The latter fact is shown by the well-known experiments of Chauveau \& Kaufmann * upon the masseter muscle of the horse. Comparing the amount of blood passing through the muscle and the decrease in its percentage of dextrose in rest and in work they found that the consumption of dextrose in the two cases was in the proportion of $1: 3.372$. Subsequent experiments $\dagger$ upon the Levator labii superioris of the horse, the results of which as to the gaseous exchange have already been cited (p. 188), gave the following figures for the dextrose abstracted from the blood per kilogram of muscle in one minute:

\begin{tabular}{|c|c|c|c|}
\hline & Rest, Grms. & Work, Grms. & Work $\div$ Rest. \\
\hline $\begin{array}{cc}\text { Experiment } & 2 \ldots \\
\text { " } & 3 \ldots \\
& 4 \ldots\end{array}$ & $\begin{array}{l}0.00598(?) \\
0.06358 \\
0.03976(?)\end{array}$ & $\begin{array}{l}0.07026(?) \\
0.22303 \\
0.12852\end{array}$ & $\begin{array}{r}11.75 \\
3.51 \\
3.23\end{array}$ \\
\hline Average.$\ldots \ldots \ldots \ldots \ldots \ldots$ & 0.03644 & 0.14027 & 3.85 \\
\hline
\end{tabular}

The authors also call attention to the fact that in these two series of experiments the arterial blood supplied to the active muscle contained a higher percentage of dextrose than that supplying the same muscle in a state of repose, notwithstanding the consumption of this substance by the muscle, and conclude that muscular activity stimulates the production of dextrose by the liver. The observation of Külz, $\ddagger$ that prolonged muscular exertion may cause the disappearance of glycogen from the liver, may perhaps be interpreted as sustaining this conclusion.

* Comptes rend., 103, 974, 1057, 1153.

† Ibid., 104, 1126, 1352, 1409.

$\ddagger$ Arch. ges. Physiol., 24, 41. 
Muscular Glycogen.-Especial- interest attaches in this connection to the behavior of the glycogen of the muscles. Nasse* appears to have been the first to show that the muscular glycogen is consumed during contraction. This result has been abundantly confirmed by other investigators, notably by Weiss, $\uparrow$ while, as just stated, Külz has shown that the same thing is true of the glycogen of the liver.

It has also been shown that glycogen accumulates in muscles whose activity has been suspended by section of their nerves or otherwise. An early statement to this effect, unaccompanied by experimental proof, is by MacDonnel. $\$$ Chandelon $\S$ investigated the influence upon the glycogen content of the hind leg of a rabbit of, first, ligature of the arteries, and second, section of the motor nerves. The first treatment caused a large loss and the second a large gain of glycogen. Morat \& Dufourt \| confirmed these results and also found that the formation of muscular glycogen was more rapid in a fatigued quiescent muscle than in a normal one, while Aldehoff 9 has shown that in a fasting animal glycogen persists longer in the muscles than in the liver and reappears first in the former when food is given.

In view of these facts it can hardly be doubted that the muscular glycogen is in some way a source of energy to the muscles, being destroyed during contraction and stored up again during rest.

Chauveau's Interpretation.-By a comparison of their results for dextrose just cited on p. 221 with those for the gaseous exchange of the muscle as given on p. 188, Chauveau \& Kaufmann show that during rest there was a storage of dextrose and of oxygen in the muscle. During work, on the contrary, more carbon dioxide was produced by the muscle than corresponded to the amount of dextrose which was abstracted from the blood, and this carbon dioxide contained more oxygen than was supplied to the muscle by the

* Arch. ges. Physiol., 2, $97 ; 14,482$.

† Sitzungsber. Wiener Akad der Wiss., Math-Nat. Klasse, 64, II, 284.

$\ddagger$ Proc. Roy. Irish Acad., Ser. I, 7, 271.

Arch. ges. Physiol , 13, 626.

II Archives de Physiol , 1892, 327 and 457.

If Zeit. f. Biol., 25, 137. 
blood during the same time. The average results, computed in milligrams per minute, were:

\begin{tabular}{|c|c|c|}
\hline & $\begin{array}{l}\text { During Rest, } \\
\text { Mgrms. }\end{array}$ & $\begin{array}{c}\text { During Work, } \\
\text { Mgrms. }\end{array}$ \\
\hline 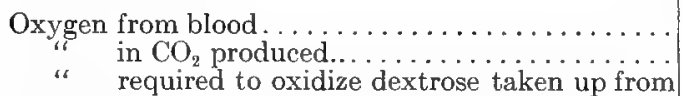 & $\begin{array}{l}0.11803 \\
0.08424\end{array}$ & $\begin{array}{l}2.48490 \\
3.15052\end{array}$ \\
\hline $\begin{array}{l}\text { blood } \\
\text { Carbon of } \mathrm{CO}_{2} \text { produced } \ldots \ldots \ldots \ldots \ldots \ldots \ldots \ldots \\
\text { dextrose taken } \mathrm{up} \ldots \ldots \ldots \ldots \ldots \ldots\end{array}$ & $\begin{array}{l}0.58305 \\
0.03160 \\
0.21862\end{array}$ & $\begin{array}{l}2.35055 \\
1.18128 \\
0.88118\end{array}$ \\
\hline
\end{tabular}

During rest the muscle was storing up both carbohydrate (glycogen) and oxygen, thus supplying itself with a reserve of potential energy. During activity this reserve, as well as the supply brought by the blood, was drawn upon for the performance of work.

The fluctuations of the respiratory quotient resulting from muscular exertion are explained by Chauveau in outline as follows:

At first there is a rapid oxidation of the stored glycogen of the muscles and of the dextrose of the blood, resulting in a respiratory quotient approaching unity. As the work progresses the store of carbohydrate material in the organism becomes relatively exhausted, unless there is a large supply of it in the food, and to meet the demands of the muscles an increased production of dextrose from the fat of the food or of the body takes place in the liver. This. change, however, according to the equation proposed on p. 38, consumes 67 molecules of oxygen for each 18 molecules of carbon dioxide produced. This process, superadded to the combustion of carbohydrates in the muscles, results in the observed lowering of the respiratory quotient. The further lowering of the quotient during a succeeding rest period results from the great diminution. in the amount of carbohydrates oxidized in the muscles, the for mation of carbohydrates from fat in the liver still continuing. for a time in order to replenish the exhausted store of muscular glycogen.

Fat as a Source of Muscular Energy.-According to the above theory, fat is only indirectly a source of muscular energy, in that it serves for the production of dextrose in the liver, and the 
same thing is held to be true of protein so far as it contributes energy for muscular exertion.

As we have seen in Chapter II, however, the formation of dextrose from fat in the liver is by no means universally admitted, and Chauveau's ingenious theory as to the immediate source of muscular energy has not lacked opponents. If it is true, fat has a much lower value for that purpose than corresponds to its potential energy as measured by its heat of combustion. If it be assumed to be converted into dextrose in accordance with the equation on p. 38 , it is easy to compute that about 36 per cent. of its potential energy will be liberated as heat in the process and that consequently only the 64 per cent. remaining in the resulting dextrose will be available to the muscles. Consequently the relative values of fat and dextrose for the production of work will be as 162 to 100 and not as 253 to 100 .

While the evidence of the respiratory quotient is not inconsistent with Chauveau's theory, it is also not inconsistent with the view which supposes fat to be directly metabolized for the production of mechanical work. The difference lies, not in the amounts of carbon dinxide and oxygen evolved but in the place where and the form in which the energy is liberated, and the question can therefore be satisfactorily discussed only on the side of its energy relations.

Postponing that discussion for the present, it may be remarked here that while it appears to be true, as already stated, that the muscular glycogen and the dextrose of the blood are a source of muscular energy, and perhaps the most readily available one, it by no means follows that they are the only source. The muscle contains other non-nitrogenous reserve materials besides glycogen, and notably a not inconsiderable amount of fat and of lecithin. Moreover, recent investigations (see pp. 63 to (5) have shown that the amount of the muscular fat is greater than was formerly supposed, and that some of it cannot be extracted with ether and behaves almost as if in chemical combination. Indeed, it appears not improbable that both fat and carbohydrate molecular groupings, as well as proteids, enter into the structure of living protoplasm. Finally, not only the muscle but the blood which nourishes it contains fat as well as carbohyhrates, the former indeed being more 
abundant than the latter. There would seem to be no inherent difficulty, then, in supposing that the fat of the muscle and of the blood serves directly as a source of energy, although the writer is not aware of any investigations upon the influence of the contraction of a muscle upon its fat-content. 


\section{PART II.}

\section{THE INCOME AND EXPENDITURE OF ENERGY.}

\section{CHAPTER VII.}

\section{FORCE AND ENERGY.}

ForCe is defined as whatever is capable of changing the rate of motion of a mass of matter. When a force acts upon a mass, imparting to it a certain velocity, it does work, the amount of work being measured by the product of the force into the distance through which it acts. Energy may be defined as the capacity to do work. Any mass of matter which can act upon another mass in such a way as to change its rate of motion is said to possess energy.

Kinetic and Potential Energy.-In studying energy we distinguish between kinetic energy, or the energy due to motion, and potential energy, or the energy due to position. The falling weight of a pile-driver at the instant it strikes the pile possesses a certain amount of kinetic energy and does a corresponding amount of work on the pile. When it is raised again a certain amount of work is done on it, and when it comes to rest at the top of the machine a corresponding amount of energy is stored up in it as potential energy. As long as the weight is supported at this point it does no work, but simply possesses the possibility of doing work. When it is allowed to fall again, this potential energy due to its position is converted into the actual or kinetic energy of motion, and when it reaches the point from which it was raised and strikes the pile it does work upon the latter exactly equal to that formerly 
stored up in the weight as potential energy, which again was equal to the energy expended in raising it.

An even simpler example of the conversion of potential into kinetic energy and vice versa is a swinging pendulum. When at rest for an instant at the end of a vibration it possesses a certain amount of potential energy, corresponding to its vertical height above the lowest point of its arc. When it reaches this lowest point, so far as the mechanism of which it forms part is concerned it has no more potential energy because it cannot fall any farther. In place of this, however, neglecting mechanical resistances, it contains an exactly equivalent amount of kinetic energy, due to its motion. During the second half of the swing this kinetic energy is expended in again raising the pendulum, and when it has all been expended the pendulum will (in the absence of external resistance) have been raised to exactly the same height as before above its lowest point. In other words, its kinetic energy will have been reconverted into an equivalent amount of potential energy and so the alternate conversion and re-conversion goes on as long as the pendulum continues to swing.

The same facts which have been illustrated above in the case of the motion of visible masses of matter are likewise true of molecular and atomic motions. When molecules of carbon dioxide and water are converted into starch in the green leaves of the plant, work is done upon them by the energy of the sun's rays. Their constituent atoms are forced apart and compelled to assume new groupings. In this process a certain amount of kinetic energy has disappeared and the resulting system of starch molecules and oxygen molecules contains a corresponding amount of potential energy. Under suitable conditions the reverse process may also take place. The atoms may, so to speak, fall together and resume their old positions, producing the original amounts of carbon dioxide and water and giving off in the process the exact amount of kinetic energy which was originally absorbed. This energy may appear in the form of heat, as in ordinary combustion, or in any other of the various forms of energy, according to circumstances.

The last example is but an illustration of the general fact that in every chemical reaction there occurs a transformation of energy which most commonly takes the form of an evolution or absorption 
of heat. That branch of science which deals with the connection between chemical and thermal processes is known as thermo-chemistry. Since kinetic energy in the animal is derived from chemical processes, and since it largely takes the form of heat, we may regard the study of the transformations of energy in the organism as constituting a branch of thermo-chemistry and proceed to a consideration of the fundamental laws upon which the latter subject is based.

The Conservation of Energy.-In any system of bodies not acted on by external forces the sum of the potential and kinetic energy is constant. In other words, while the ratio of potential to kinetic energy may vary, and while each may take various forms, as mass-motion, heat, electric stress, etc., there is no loss of energy in these conversions. Energy, like matter, is indestructible. This great law of the conservation of energy was first clearly enunciated by Mayer, and forms the foundation of all modern conceptions of physical processes. In the case of the swinging pendulum used above as an illustration the total energy of the system composed of the earth and the pendulum is constant, a portion of it simply alternating between the potential and kinetic states. So, too, in the system of atoms of carbon, hydrogen, and oxygen, the potential energy contained in the system before the starch is burned is simply converted into the kinetic energy of heat, while the total energy of the system remains the same.

Initial and Final States.-An important consequence of the law of the conservation of energy, which was first deduced and demonstrated experimentally by Hess in 1840 , is known as the law of initial and final states. This law is that in any independent system the amount of energy transformed from the potential to the kinetic form, or vice versa, during any change in the system, depends solely upon the initial and final states of the system and not at all upon the rapidity of the transformation or upon the kind or number of the intermediate stages through which it passes. Although this law is true in the general form here stated, it was originally propounded as related to chemical reactions and forms the basis of the science of thermo-chemistry. If we start with starch and oxygen and end with the corresponding quantities of carbon dioxide and water, the amount of kinetic energy evolved is 
the same, no matter whether the starch be burned almost instantaneously in pure oxygen or whether it be subjected to slow oxidation in the tissues of a plant buried in the soil; whether carbon dioxide and water are the immediate products of the action or whether the starch be previously transformed into maltose, glycogen, dextrose, lactic acid, etc., etc., as in the body of the animal. We have simply to determine the potential energy of the system in its initial and in its final state, and the difference is equal to the amount of kinetic energy evolved during the change. The truth of this law, as applied to chemical processes, has been fully demonstrated by the researches of Berthelot and Thomsen. That the same law applies to the processes taking place in the body of the animal is exceedingly probable, a priori, and has been demonstrated experimentally by the researches of Rubner and of Atwater and his associates.

Heats of Combustion.-We have no means of determining the total amount of potential energy contained in a system, but can only measure that portion which is manifested by the change to the kinetic or the potential form during some change in the system. In other words, we may assume the potential energy of the system in some particular state as zero and obtain a numerical expression for its potential energy in some other state as compared with this standard state. For the latter we shall naturally select that one in which no further conversion of potential into kinetic energy can, according to our experience, take place.

In the case of organic substances, such as those entering into the metabolism of the animal, the system consists of the substance itself and oxygen, and the state of complete oxidation is the one in which experience shows that no further evolution of kinetic energy is possible by chemical means. Thus, to recur to the example of starch, if one gram be oxidized in accordance with the equation

$$
\mathrm{C}_{6} \mathrm{H}_{10} \mathrm{O}_{5}+6 \mathrm{O}_{2}=6 \mathrm{CO}_{2}+5 \mathrm{H}_{2} \mathrm{O} \text {, }
$$

the amount of heat evolved will be 4183 cals., ${ }^{*}$ this being the amount of energy converted from the potential to the kinetic form. From the system represented by the second member of the above equation we can get no further evolution of heat. We therefore repre-

* For the units of measurement see the following paragraph. 
sent its potential energy by 0 and accordingly that of the system starch + oxygen by 4183 cals. for each gram of starch. This value is called the heat of combustion of starch, and shows how much energy can be liberated from this substance by its conversion into $\mathrm{CO}_{2}$ and $\mathrm{H}_{2} \mathrm{O}$. It is common to speak of this as the potential energy of the starch, and the expression has the advantage of brevity, but it should not be forgotten that it is really the potential energy of the system $\mathrm{C}_{6} \mathrm{H}_{10} \mathrm{O}_{5}+6 \mathrm{O}_{2}$ as compared with the system $6 \mathrm{CO}_{2}+5 \mathrm{H}_{2} \mathrm{O}$.

In like manner the heat of combustion of any organic compound, or of any mixture of compounds such as a feeding-stuff, represents the amount of energy which a given weight of it evolves in the form of heat when completely oxidized. In the case of nitrogenous badies the final products are $\mathrm{CO}_{2}, \mathrm{H}_{2} \mathrm{O}, \mathrm{N}_{2}$, and in case of proteids $\mathrm{SO}_{3}$.

Heats of combustion may be determined at constant pressure or at constant volume. When the substance is burned under ordinary atmospheric pressure the amount of heat evolved may include, besides that due to the difference in the chemical energy of the substance before and after burning, a mechanical component due to the fact that the volume of the products is not the same as that of the original substances. If it is greater, work is done in overcoming atmosphcric pressure and the heat production is diminished by a corresponding amount. In the contrary case, work is done by the atmosphere upon the products of combustion and heat is evolved. When the substance is burned in a confined volume of oxygen, as in the bomb-calorimeter, the possibility of such mechanical action is eliminated and we obtain a quantity of heat representing solely the difference in chemical energy. The heats of combustion at constant volume are therefore, from a theoretical point of view, the more correct. On the other hand, however, all ordinary processes of combustion, including those occurring in the animal organism, take place under atmospheric pressure, which is practically constant, and therefore the actual heat value of a substance oxidized in the body is measured by its heat of combustion at constant pressure. If there is no change in volume during the combustion, then the two heats of combustion are, of course, identical. This is the case, for example, with the carbohydrates, which 
form so large a part of the food of herbivorous animals. Furthermore, the difference in the case of the other common nutrients is so slight that the heats of combustion as determined with the bombcalorimeter may be used without appreciable error in computing the metabolism of energy in the body. The only substance involved in such computations for which the correction needs to be made is methane, $\mathrm{CH}_{4}$, the heat of combustion of which is at constant volume 13,246 cals. per gram and at constant pressure 13,344 cals.

Units of Measurement.-The unit of force is the dyme, which is defined as the amount of force required to produce in a mass of one gram, in one second, an acceleration of one centimeter per second.

When a orce acts upon a mass, the amount of work done is measured by the product of the force into the distance (measured along the direction of the force) through which it acts. The unit of work is the erg which is defined as the work done by a force of one dyne acting through one centimeter.

Energy has been defined as the power of doing work, and is measured by the amount of work done, that is, in ergs. Since, however, the erg is a very small quantity, it is often more convenient in practice to use a multiple of it. For this purpose the quantity $10^{10} \mathrm{erg}=1$ Kilojoule $(\mathrm{J})$ is a convenient unit. Energy is also frequently expressed in units based on weight instead of mass, the most common being the gram-meter, the kilogram-meter, and the font-pound. The gram-meter is the work done against gravity in raising a weight of 1 gram through 1 meter. Since, however, the force of gravity, and consequently the weight of a given mass, varies at different points on the earth's surface, it is necessary to state also where the weight is.taken. At the level of the sea, in temperate latitudes, the force of gravity equals 980.5 dynes. Under these conditions, then, doing 1 gram-meter of work would be equivalent to exerting a force of 980.5 dynes through $100 \mathrm{~cm}$., which equals 98,050 ergs. The kilogram-meter $(\mathrm{kgm}$.) is the work done against gravity in raising 1 kilogram through 1 meter, and is accordingly 1000 times the gram-meter or 98,050,000 ergs. The foot-pound is the work done against gravity in raising 1 pound through 1 foot and accordingly equals $13,550,000$ ergs.

In addition to mechanical energy the animal produces heat. 
For the measurement of heat various units are in use, but the ones most commonly employed in physiology are the small and the large calorie. The small calorie (cal.) is defined as the amount of heat required to raise the temperature of $1 \mathrm{gram}$ of water through $1^{\circ} \mathrm{C}$. Since, however, the specific heat of water varies somewhat with the temperature, it is necessary to specify the average temperature of the water. The temperature of $18^{\circ} \mathrm{C}$. has been quite commonly used for this purpose, the resulting unit being indicated by the abbreviation cal $_{18}$. Atwater \& Rosa,* however, in their work with the respiration-calorimeter, have employed the temperature of $20^{\circ} \mathrm{C}$., designating their unit by $\mathrm{cal}_{20}$. The difference between the two is very slight, $1 \mathrm{cal}_{20}$ equaling $1.0002 \mathrm{cal}_{18}$. The large calorie (Cal.) is the amount of heat required to raise the temperature of one kilogram of water through $1^{\circ} \mathrm{C}$., or is equal to 1000 small calories. The temperature at which the large calorie is measured may be indicated as in case of the small calorie.

The calorie, however, while commonly used, and while in some respects a convenient unit, is in a sense not a rational one. Since heat is one form of energy, and since, in accordance with the law of the conservation of energy, there is a fixed relation between it and other forms of energy, a rational unit would be one bearing a simple numerical relation to the units employed to measure other forms of energy, or in other words, the erg or some simple multiple of it. As already noted, the Kilojoule $(J)$ is a convenient unit for this purpose. It has two advantages over the Calorie: first, it permits of a direct comparison of heat with other forms of energy (expressed, of course, in units of the same system); and second, it is an "absolute" unit, that is, it is based on the fundamental units of space, mass, and time, and has a perfectly definite magnitude, while the Calorie has not unless the temperature at which it is measured is stated. To this may be added that in discussing physiological relations it avoids the sometimes confusing implication that the quantities of energy dealt with actually exist in all cases as heat.

The relation between the Calorie and the Kilojoule is as follows:

$$
\begin{aligned}
& 1 \mathrm{Cal}_{18}=4.183 \mathrm{~J}=41,830,000,000 \mathrm{ergs} ; \\
& 1 \mathrm{~J}=0.2391 \mathrm{Cal}_{18}=10,000,000,000 \mathrm{ergs} .
\end{aligned}
$$

* U. S. Dept. Agr., office of Expt. Stats., Bull. 63, p. 55. 
Since, however, most of the results of investigations upon the physiological relations of energy are expresserl in calories (often without any statement of temperature) it will be more convenient, in the following pages to employ this unit rather than the more rational Kilojoule.

Finally, since measurements of mechanical energy (as in experiment with working animals) have been commonly made in weight units, it is necessary to know the relation of these to the calorie. These relations are included in the following table, the force of gravity being taken as 980.5 dynes:

EQUTVALENCE OF UNITS OF ENERGY.

\begin{tabular}{|c|c|c|c|c|c|c|}
\hline & \multicolumn{2}{|c|}{ Ergs.* } & \multicolumn{2}{|c|}{ Kilojoules. } & $\begin{array}{l}\text { Gram- } \\
\text { meters. }\end{array}$ & $\begin{array}{c}\text { Kilogram- } \\
\text { meters. }\end{array}$ \\
\hline \multirow[t]{2}{*}{$\begin{array}{l}1 \text { Kilojoule }=\ldots \ldots \ldots \\
1 \text { gram-meter }=\ldots \ldots \\
1 \text { kilogram-meter }=\ldots \\
1 \text { foot-pound }=\ldots \ldots \ldots \\
1 \text { cal }{ }_{1} \ldots \ldots \ldots \ldots \ldots \\
1 \text { Cal }_{1} \ldots \ldots \ldots \ldots \ldots\end{array}$} & \multicolumn{2}{|c|}{$\begin{array}{l}10^{10} \\
980.5 \times 10^{2} \\
980.5 \times 10^{5} \\
135.5 \times 10^{5} \\
4.183 \times 10^{7} \\
4.183 \times 10^{10}\end{array}$} & \multicolumn{2}{|c|}{$\begin{array}{l}980.5 \div 10^{8} \\
980.5 \div 10^{5} \\
135.5 \div 10^{5} \\
\quad 0.004183 \\
\quad 4.183\end{array}$} & $\begin{array}{r}101989 \\
1000 \\
138.2 \\
426.6 \\
426600\end{array}$ & $\begin{array}{l}0.001 \\
\\
0.1382 \\
0.4266 \\
426.6\end{array}$ \\
\hline & \multicolumn{3}{|c|}{$\begin{array}{c}\text { Foot- } \\
\text { pounds. }\end{array}$} & \multicolumn{2}{|c|}{$\mathrm{cal}_{18}$. } & $\mathrm{Cal}_{18 .}$ \\
\hline \multicolumn{2}{|c|}{ 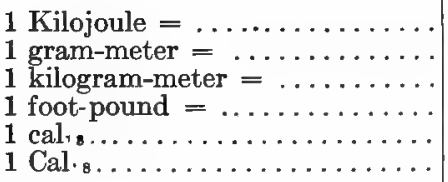 } & \multicolumn{2}{|c|}{$\begin{array}{l}738.1 \\
\quad 0.007236 \\
\quad 7.236 \\
\cdots \\
3.087 \\
3087 .\end{array}$} & \multicolumn{2}{|c|}{$\begin{array}{l}239.1 \\
0.002344 \\
2.344 \\
0.3239 \\
\cdots \\
1000\end{array}$} & $\begin{array}{l}0.2391 \\
0.2344 \div 10^{5} \\
0.002344 \\
0.000324 \\
0.001\end{array}$ \\
\hline
\end{tabular}

* From Ostwald, Grundriss der allgemeinen Chemie. 


\section{CHAPTER VIII.}

\section{METHODS OF INVESTIGATION:}

THE food is the sole known source of energy as well as of matter to the body of the warm-blooded animal, and the total income of potential energy, according to the principles laid down in the preceding chapter, is represented by the heat of combustion of the food.

A portion of this food, as we have seen in Part I, is metabolized in the body, while part of it escapes complete oxidation and is rejected as undigested matter in the feces, as metabolic products in feces, urine, and perspiration, and as combustible intestinal gases. All these substances still contain more or less of their original store of potential energy and collectively constitute one main division of the outgo of energy. Wo may call it, for brevity, the outgo of potential energy. A portion of the food may also be applied to the production and storage of tissue (protein and fat) in the body, and this, from our present point of view, is to be classed with the outgo of potential energy.

The potential energy of the remaining portion of the food, viz., that which is completely oxidized, may take various transitory forms in the organism, but ultimately it leaves it in one of two forms of kinetic energy, viz., as mechanical work or as heat. Here we have the second main division of the outgo of energy, viz., the outgo of kinetic energy. These relations.may be briefly expressed in tabular form, as shown at the head of the opposite page.

As in the corresponding chapter of Part I, it is proposed to consider here simply the general principles of the more important methods available for determining the income and outgo of potential and kinetic energy, without entering into technical details. 


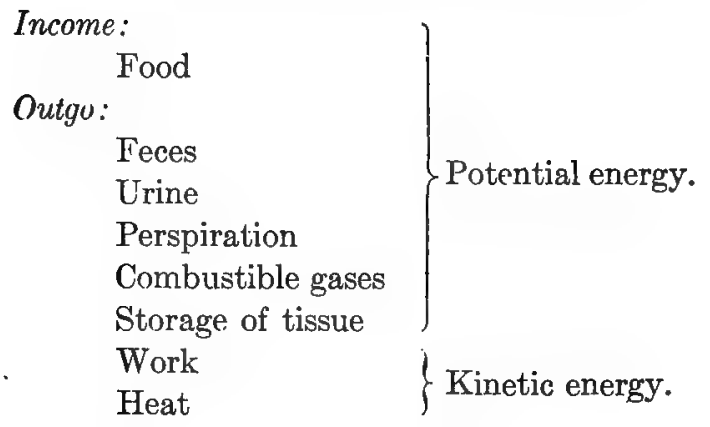

\section{Determination of Potential Energy.}

The Energy of the Food.--The potential energy of the food is conveniently measured by converting it into the kinetic form of heat; that is, by determining its heat of combustion. This determination is effected by means of an instrument known as a calorimeter, in which the heat produced by the complete combustion of a known weight of the substance under examination is absorbed by some calorimetric substance and its amount measured by the change of temperature or of physical state of the latter. The calorimetric substance ordinarily employed is water, the increase in temperature of a known weight of this substance giving directly the amount of heat in calories. It is, of course, essential either that all the heat produced shall be transferred to the calorimetric substance or that it shall be possible to correct the observed results for any heat that may escape absorption.

Another essential is that the oxidation shall be complete, a condition whose fulfillment it is by no means easy to secure. Two general methods have been employed for this purpose. The first was that of Thompson,* as used by Frankland and subsequently modified by Stohmann, $\dagger$ in which the oxidation is effected by mewis of pure potassium chlorate, corrections being made for the heat evolved in the decomposition of the latter substance. The second method, which has almost entirely replaced the first, con-

* Described by Frankland, Proc. Roy. Inst. of Great Britain, June 8, 1866, and Phil. Mag. (4), 32, 182.

† Jour. pr. Chem., 127, 115; Landw. Jahrb., 13, 513. 
sists in burning the substance without any admixture in highly compressed oxygen contained in a lined steel bomb as first devised by Berthelot* and subsequently modified by Mahler, Hempel, and Atwater. With this type of calorimeter very accurate and comparatively rapid work may be done. $\dagger$

Frankland was the first to undertake determinations of the hears of combustion of foods and food ingredients, using the original form of the Thompson calorimeter. Subsequent investigators, of whom may be especially mentioned Stohmann, v. Rechenberg and Danilewski, Berthelot and his associates, Rubner, and Atwater, Gibson \& Woods, have continued these investigations with improved apparatus and more refined methods, ${ }_{+}$and we now possess a considerable mass of data as to the heats of combustion of the more important ingredients of animals and plants and of the products of metabolism. Atwater $\S$ gives the following summary of the results on record up to July, 1894 (see pp. 237-9).

In the course of recent investigations into the energy relations of the food of man and of domestic animals a considerable amount of data has also been secured regarding the heats of combustion of foods and feeding-stuffs. A summary of the results of such determinations on 276 samples of human foods of various kinds has been published by Atwater \& Bryant.\| No similar compilation of heats of combustion of feeding-stuffs is as yet available.

It need hardly be pointed out that, taken by themselves, such results furnish no measure of the relative values of the various feeding-stuffs. Like a chemical analysis, they supply but a single factor, albeit an important one, for such a com-

* Ann. de Chim. et de Phys., (5), 23, 160.

† For the technical details of the method reference may be had to the published descriptions of the apparatus or to Wiley's Principles and Practice of Agricultural Chemical Analysis, Vol. III, p. 569.

$\ddagger$ For a historical sketch of the development of calorimetry, as applied to food substances, compare Atwater, "Chemistry and E.conomy of Food," U. S. Department of Agriculture, Office of Experiment Stations, Bull. 21, pp 116-126. p. 73.

\& Ibid., pp. 127 and 128. Compare also Rep. Storrs Expt. Station, 1899,

|l Rep. Conn. Storrs Fixpt. Station, 1899, p. 97. 
HEATS OF COMBUSTION OF ORGANIC SUBSTANCES.

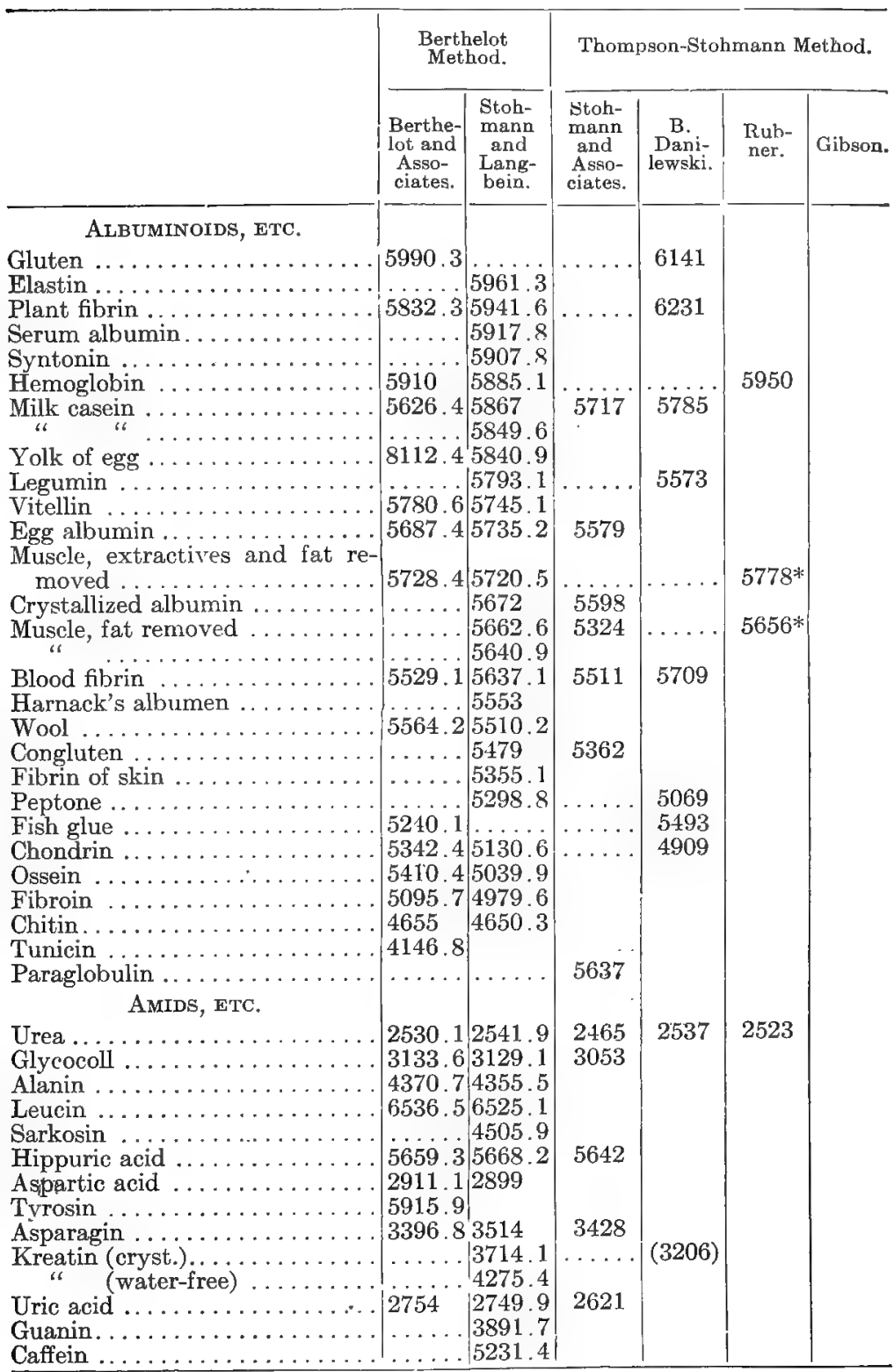

* Calculated ash-free. 


\section{HEATS OF COMBUSTION OF ORGANIC SUBSTANCES (Continued).}




HEATS OF COMBUSTION OF ORGANIC SUBSTANCES (Continued).

\begin{tabular}{|c|c|c|c|c|c|c|}
\hline & \multicolumn{2}{|c|}{$\begin{array}{l}\text { Berthelot } \\
\text { Method. }\end{array}$} & \multicolumn{4}{|c|}{ Thompson-Stohmann Method. } \\
\hline & $\begin{array}{c}\text { Berthe- } \\
\text { lot and } \\
\text { Asso- } \\
\text { ciates. }\end{array}$ & \begin{tabular}{|l} 
Stoh- \\
mann \\
and \\
Lang- \\
bein.
\end{tabular} & $\begin{array}{c}\text { Stoh- } \\
\text { masn } \\
\text { and } \\
\text { Asso- } \\
\text { ciateg. }\end{array}$ & $\begin{array}{c}\text { B. } \\
\text { Dani- } \\
\text { lewski. }\end{array}$ & $\begin{array}{l}\text { Rub- } \\
\text { ner. }\end{array}$ & Gibson. \\
\hline 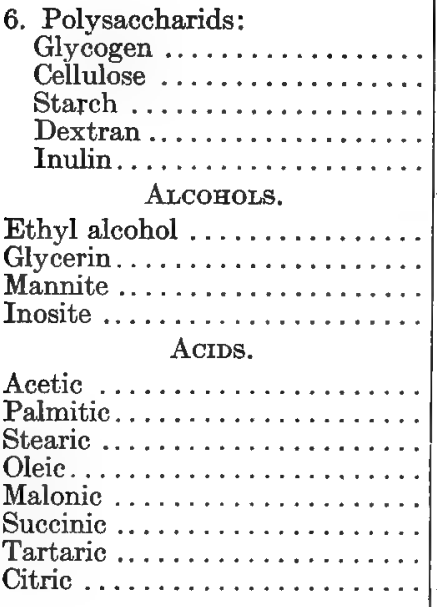 & $\begin{array}{l}3200 \\
4228 \\
4180.4 \\
4187.1 \\
7068 \\
4001.2 \\
3676.8 \\
3490.4 \\
\cdots \ldots . . \\
\cdots \ldots 9.2 \\
1998.2 \\
3006.2 \\
2477.9\end{array}$ & 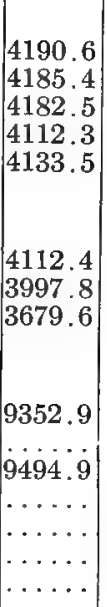 & $\begin{array}{r}4146 \\
4123 \\
4070\end{array}$ & & & 4164 \\
\hline
\end{tabular}

parison. Just as the chemical analysis shows the total amounts of various substances or classes of substances present, so the heat of combustion shows the total amount of potential energy which has been stored up in the feeding-stuff. In both cases the knowledge thus acquired must be combined with data, secured in an entirely different way, as to the availability of these ingredients or this energy before we can form a judgment as to the relative values to the animal.

Computation of Heats of Comisustion.-The heat of combustion of a mixture of various organic substances, such as are contained in ordinary foods and feeding-stuffs, is equal to the sum of the heats of combustion of the single ingredients. If the latter are known we may obtain the heat of combustion of the material in question either by a direct calorimetric determination or by determining chemically the proportions of the several ingredients and multiplying the amount of each into its known heat of combustion. 
The first method, when available, is obviously to be preferred, and is to be regarded as indispensable in all exact investigations into the energy relations of the food of man or of animals. With materials whose proximate composition is fairly well known, however, the agrement between the computed and the actual heat of combustion is very close, as has been shown by Wiley \& Bigelow* and Slosson $\dagger$ for hulled cereals and cereal products. Atwater \& Bryant, in their publication just referred to, have discussed this question very fully in relation to human foods and have proposed a series of factors for the ingredients of the various classes of foods by whose use they obtain a most satisfactory agreement with the calorimetric results.

On the other hand, in case of vegetable products containing much woody and fibrous material the actual heat of combustion is higher than that computed under the ordinary interpretation of the results of chemical analysis. Thus the actual heat of combustion of unhulled oats was found by Wiley \& Bigelow to be about 4.5 per cent. higher than the computed value, and Merrill $\ddagger$ has obtained similar results for wheat middlings and bran and for hay and silage. This obviously arises from the presence among the ill-known bodies constituting the so-called lignin and incrusting substances of compounds having higher heats of combustion than the common carbohydrates. It is not impossible that a series of factors similar to those of Atwater \& Bryant might be worked out for different classes of stock foods, so that their heats of combustion might be computed from their chemical composition. In view; however, of the comparative case and rapidity with which direct calorimetric results can be accumulated it may be doubted whether such an undertaking would repay the labor involved.

Methods have also been proposed and somewhat extensively used for computing the heat of combustion of the digested portion of the food. This phase of the subject, however, can be more profitably considered later.

The Energy of the Excreta.-For the visible excreta (feces and urine) substantially the same method is available as for the food,

* Jour. Am. Chem. Soc., 20, 304.

$\dagger$ Wyoming Expt. Station, Bull. 33.

$\ddagger$ Maine Expt. Station, Bull. 67, p. 169. 
viz., a determination of the heat of combustion. An element of uncertainty, however, which is ordinarily not mut with in the case of the food, arises from the ready decomposability of the excretory products, which is liabie to result in a loss of energy during the drying necessary to preparc them for combustion. The urea of the urine, in particular, is very readily converted into the volatila ammonium carbonate. Comparative determinations of nitrogen in the fresh and in the dried urine will show the amount of nitrogen lost in drying, and on the assumption that oniy urea is decomposed the loss of energy can be readily computed from the known heat of combustion of that substance. Atwater \& Benedict* have found this assumption to be substantially correct for human urine, and the same may be presumed to be the case with the urine of carnivora. It has usually been assumed to be applicable also to the more complex urine of herbivora, although without, so far as the writer is aware, any experimental proof.

A greater or less loss of nitrogen has also been observed in the drying of the feces of domestic animals, particularly of horses and sheep, but the nature of the material decomposed has not yet been investigated, and the same is true of the possible decomposition of non-nitrogenous materials in both urine and feces. Atwater \& Benedict (loc. cit.) found the loss of nitrogen from human feces to be insignificant.

Computation or Energy. - The computation of the energy of the visible excreta is much less satisfactory than in the case of the food on account of our inferior knowledge of the proportions and chemical nature of their ingredients.

The Urine.-Formerly the urine was assumed to be substantially an aqueous solution of urea, and numerous computations of its energy content were made on this basis, particularly in connection with estimates of the metabolizable energy of the proteids, while the same method has been applied also in estimating the metabolizable energy of feeding-stuffs. Rubner $\dagger$ was the first to demonstrate the serious nature of the error involved in this assumption and to show that the energy of the urine is materially greater than the amount thus computed. In the urine of the $\mathrm{dog}$ he found

* U. S. Dept. Agr., Office of Experiment Stations, Bull. 69, p. 22.

$\dagger$ Zeit. f. Biol., 20, 265; 21, 250 and 337; 42, 302. Compare Chapter X. 
the energy content to be from 6.7 to 8.5 Cals. per gram of nitrogen in place of 5.4 Cals. as computed on the assumption that only urea was present, while for human urine he has obtained values ranging from 6.42 Cals. to 8.87 Cals. per gram of nitrogen, and Tangl* has reported even higher figures.

Kellner $\dagger$ has shown that the difference is still greater in the urine of an ox receiving only coarse fodder, the actual energy being about six times that computed on the above assumption and nearly $\mathbf{1 7 5}$ per cent. of that computed after allowing for the hippuric acid present. In subsequent investigations $\ddagger$ he finds that the energy content of the urine of cattle is much more nearly proportional to its carbon than to its nitrogen, being approximately 10 Cals. per gram of carbon.

In six cases reported by Atwater \& Benedict $\S$ in the course of their investigations with the respiration-calorimeter, the amount of energy found in human urine from a mixed diet as compared with that computed from the nitrogen reckoned as urea was:

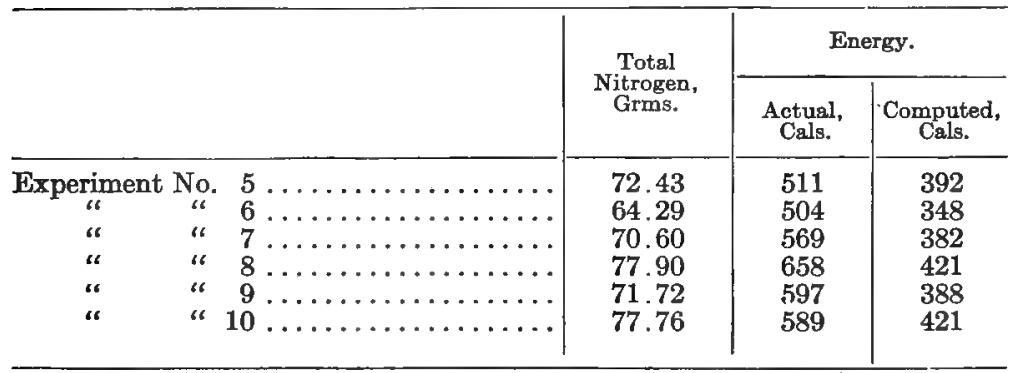

Here, too, it is evident that a computation on the basis of the urea yields results much below the truth, and later experiments by the same authors have fully confirmed this result.

The Feces.-Our knowledge of the proximate principles contained in the feces is so small that no satisfactory computation of their energy content is possible, except perhaps in the case of carnivora on a purely meat diet, where the total amount of feces is

* Arch. f. (Anat. u.) Physiol., 1899, p. 261.

$\dagger$ Landw. Vers. Stat., 47, 275.

$\$$ Ibid., 53, 437 .

$\S$ U. S. Dept. Agr., Office of Experiment Stations, Bull. 63. 
small. On a mixed diet containing any considerable proportion of vegetable matter, and particularly in the case of herbivorous animals consuming large amounts of coarse fodders, only an actual determination of the heat of combustion can be depended upon. Since the feces of these animals contain a larger proportion of the indigestible lignin, etc., than does their food, the heat of combustion of the feces is correspondingly higher, but its actual value must obviously depend to a considerable degree on the character of the food.

Combustible Gases.-Since it is impracticable to collect separately the combustible intestinal gases, we must of necessity compute the amount of potential energy carried off in these substances. This computation has been based on the amount of carbon contained in these gases, determined in the manner indicated on p. 72, upon the assumption that only methane $\left(\mathrm{CH}_{4}\right)$ was present. It has been shown that this gas exists in considerable amounts in the digestive tract of herbivora, and it is probable that the above assumption is substantially accurate, although a small amount of hydrogen has been found by some observers. In experiments by Fries,* at the Pennsylvania Experiment Station, in which both the carbon and hydrogen of the combustible gases excreted by a steer consuming chiefly timothy hay were determined, the following ratios of hydrogen to carbon were obtained:

Period A.

\begin{tabular}{|c|c|}
\hline First day, & $1: 2.900$ \\
\hline Second day, & $1: 2.916$ \\
\hline \multicolumn{2}{|c|}{ Period $B$} \\
\hline First day, & $1: 2.978$ \\
\hline Second day, & $1: 2.947$ \\
\hline \multicolumn{2}{|c|}{ Period C. } \\
\hline First day, & $1: 2.899$ \\
\hline Second day, & $1: 2.951$ \\
\hline \multicolumn{2}{|c|}{ Period D. } \\
\hline First day, & $1: 3.051$ \\
\hline Second day, & $1: 3.096$ \\
\hline Average, & $\overline{1: 2.967}$ \\
\hline Computed for $\mathrm{CH}_{4}$, & $1: 2.976$ \\
\hline
\end{tabular}


These results tend strongly to substantiate the belief that the combustible gases practically consist of methane only.

Perspiration.-In view of the relatively minute amounts of organic matter contained in the perspiration it has generally been regarded as a negligible quantity. The data given on p. 48 for the nitrogen of the sensible perspiration would afford some approximate data for computing the amount of energy contained in it.

The Energy of Tissue Gained.-The amount of potential energy stored up in a gain of tissue, or the amount liberated in the kinetic form in case the gain is negative, cannot, of course, be made the subject of a direct determination. The amounts of protein and of fat gained or lost can, however, be determined by the methods described in Chapter III, and their energy content computed from average figures. The errors involved are those incident to the method of computation from the carbon and nitrogen balance, which have already been considered in the chapter cited, and those arising from uncertainty as to the exact energy content of the material gained by the body.

Protein.-Just as computations of the gain or loss of protein by the body have been based upon the average composition of the proteids, so computations of its energy content have been based upon the average heat of combustion of these substances. The compilation by Atwater on pp. 237-9 contains the available data up to 1894.

For approximate computations the value 5.7 Cals. per gram has been commonly used, while in more exact computations it has been assumed that the gain of protein by the animal has substantially the heat value as well as the chemical composition of fat-free muscular tissue (see p. 63), and the average of Stohmann's two determinations, viz., 5.652 Cals. per gram, has been employed. Köhler's investigation* of the composition of fat and ash-free muscular tissue (p. 64) included determinations of the heats of combustion which are reproduced on the opposite page.

Fat.-Rubner, in his computations, employs the round number 9.4 Cals. per gram for fat, while Kellner uses the value 9.5 Cals. Benedict \& Osterberg, $\dagger$ whose determinations of the composition of

* Zeit. physiol. Chem., 31, 479.

$\dagger$ Amer. Jour. Physiol., 4, 69. 


\begin{tabular}{|c|c|c|}
\hline & $\begin{array}{c}\text { No. of } \\
\text { Samples. }\end{array}$ & $\begin{array}{c}\text { Heat of Combus- } \\
\text { tion per Gram, } \\
\text { Cals. }\end{array}$ \\
\hline 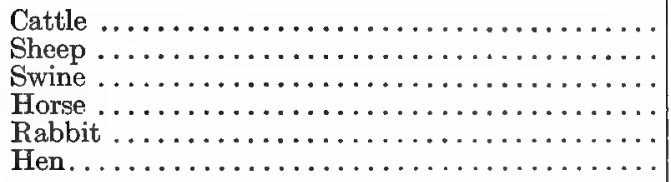 & $\begin{array}{l}4 \\
2 \\
2 \\
3 \\
2 \\
2\end{array}$ & $\begin{array}{l}5.6776 \\
5.6387 \\
5.6758 \\
5.5990^{*} \\
5.6166 \\
5.6173\end{array}$ \\
\hline
\end{tabular}

human fat are given on p. 61, found for the heat of combustion of the same twelve samples values ranging from 9.474 Cals. to 9.561 Cals. per gram, the average being 9.523 Cals. Other results are noted in the table on pp. 237-9.

\section{Determination of Kinetic Energy.}

Mechanical Work. - The energy of the mechanical work done by the animal upon its surroundings is derived, as was seen in Part I, immediately from body materials and mediately from the food, and is one of the two forms in which kinetic energy leaves the body.

The energy of the mechanical work done by the animal may be measured in various ways, the consideration of which belongs to the domain of mechanics and lies outside the scope of the present work. In general two classes of appliances have been used:

First, dynamometers proper, in which the work is expended in overcoming a known resistance, produced either by friction or by a magnetic field, the work done being measured by the tension of a opring or by the amount of electric energy produced.

Second, the tread power, in which the work, aside from that of locomotion, consists in lifting the body vertically and is proportional to the product of the mass of the body into the distance through which it is raised.

Heat.-The second form in which kinetic energy leaves the body is heat. In an animal doing no work all the energy arising from the metabolism in the body ultimately takes this form, and even when mechanical work is done the larger share of the outgo of kinetic energy consists of heat. Part of this heat is imparted to the surroundings of the animal by conduction and radiation and a

* Contained an average of 3.65 per cent.glycogen. 
part is expended in the evaporation of water from skin and lungs and takes the form of the latent heat of water vapor.

Animal Calorimeters.-The direct determination of the heat produced by an animal, especially a large animal, is not an easy task. It requires in the first place a calorimeter large enough to contain the animal and in the second place, for experiments of any length, the maintenance of a sufficient ventilating current of air under such conditions as shall not affect the accuracy of the calorimetric determination, while the latent heat of the water vapor carried out in the air-current must also be taken account of. In other words, such an apparatus must be at once a respiration apparatus and a calorimeter, and hence the name respiration-calorimeter has come to be applied to it. Various forms of animal calorimeters have been devised, some of which may be briefly mentioned.

Lavoisier \& Laplace, ${ }^{*}$ in their investigations upon the origin of animal heat, employed an ice-calorimeter, in which the heat is measured by the amount of ice melted. Crawford $\uparrow$ investigated the same subject using a water-calorimeter, as did, later, Dulong $\ddagger$ and Despretz,§ while more recently Wood,\| and still later Reichert, 9 have also employed the water-calorimeter.

The ice-calorimeter, however, necessarily subjects the animal to an abnormally low temperature, while with the water-calorimeter it has been found very difficult to secure a uniform heating of the different strata of water. These facts led to the employment of air as the calorimetric substance, the heat being measured either by the increase in the volume of a confined body of air at a constant pressure or the increase in the pressure at constant volume, and until quite recently the most exact methods have been based on this principle.

Scharling,** Vogel, $† \dagger$ and Hirn, 㧊 between 1849 and 1864, used

* Hist. Acad. Roy. d. Sc., 1780, 355.

† Experiments and Observations on Animal Heat. London, 1788.

$\$$ Ann. de Chim. et de Phys. (3), 1, 440 .

$\S$ Ibid., (2), 26, 337.

|| Smithsonian Contributions, 1880.

T Univ. Med. Mag., Phila., 2, 173.

*** Jour. pr. Chem., 48, 435.

†† Arch. d. Ver. f. Wiss. Heillk., 1864, p. 442.

施cherches sur l'équivalent méchanique de la chaleur. Paris, 1858. 
crude forms of the air-calorimeter. In 1885 Richet* described an air-calorimeter for small animals, the heat being measured by the increase in the volume of a confined portion of air at constant pressure. His experiments were of short duration (1 to $1 \frac{1}{2}$ hours) and no specific statement is made regarding ventilation and no mention of any determinations of the latent heat of the water vapor.

In 1886 d'Arsonval $\dagger$ described a differential air-calorimeter, and in $1890 \$$ two other forms of animal calorimeter, the first being a water-calorimeter of constant temperature with automatic regulation of the flow of water, for which a high degree of accuracy is claimed, and the second an air-calorimeter, but he reports no experiments with either form. In the same year Laulanie \& (see j). 70) described briefly a Regnault respiration apparatus which was also used as a calorimeter, and has subsequently reported some results obtained by its use.

One of the best known forms of animal calorimeter is that of Rubner.\| This is essentially a Pettenkofer respiration apparatus, the walls of the chamber being double and the whole surrounded by an air space which in its turn is surrounded by a jacket containing water kept at a constant temperature. The amount of heat given off to the calorimeter is measured by the expansion under constant pressure of the confined volume of air between the two walls of the respiration chamber, while from comparative determinations of moisture in the ingoing and outcoming air the heat removed in the latent form is computed.

Rosenthal of has constructed a somewhat similar instrument in which the respiratory portion is a Regnault apparatus, while the heat is measured by the increase in pressure of the air at constant volume, instead of by the increase in its volume as in Rubner's apparatus. Both instruments are therefore air-calorimeters, and the numerical values of their readings must be determined experimentally for each instrument. These two forms of apparatus are of a size sufficient for experiments with small animals (rabbits or small dogs).

* Archives de Physiol , 1885, II, 237.

$\uparrow$ Jour. de l'Anat. et Physiol., 1886.

$\ddagger$ Archives de Physiol., 1890, pp. 610 and 781.

$\S$ Ibid., p. 571.

|| Calormetrische Methodik, Marburg, 1891 ; Zeit. f. Biol., 30, 91.

If Arch. f. (Anat. u.) Physiol., 1894, p. 223. 
In 1894 Haldane, White \& Washbourne* described a form of aircalorimeter in which the expansion caused by the heat produced by the animal in one chamber is balanced by that produced by a flame of hydrogen burning in a second similar chamber. The calorimeter is essentially one of constant volume, but the heat is computed from the amount of hydrogen burned.

Laulanié $\nmid$ in 1895 described a Pettenkofer apparatus with small ventilation (see p. 71) which served also as an air-calorimeter, and still later $\$$ has described a differential water-calorimeter. Kaufmann, $\S$ as mentioned on p. 69 , has determined the respiratory exchange of animals during short periods in a confined volume of air. The apparatus consisted simply of a zinc receptacle which served also as a radiation calorimeter. The internal temperature and that of the surrounding air were measured by recording thermometers and the loss of heat calculated according to Newton's law. The atmosphere in the apparatus was saturated with watervapor at the start, so that the moisture excreted by the animal was condensed and no correction for the heat of vaporization was necessary.

By far the most important form of respiration-calorimeter yet devised, however, not only as regards accuracy but particularly in view of the range of work of which it is capable, is that of Atwater \& Rosa,\| the respiratory part of which has already been mentioned (pp. 72 and 79). In this apparatus water is used as the calorimetric substance, but in the form of a constant current instead of a large stationary mass. As described by the authors the apparatus consists of a Pettenkofer respiration apparatus provided with special devices for the accurate measurement, sampling, and analysis of the air-current. A current of cold water is led through copperabsorbing pipes near the top of the respiration chamber and takes up the heat given off by the subject. The volume of the water used being measured, and its temperature when entering and leaving being taken at frequent intervals, the amount of heat brought out

* Jour. Physiol., 16, 123.

$\dagger$ Archives de Physiol, 1895, p. 619.

$\$$ Ibid., 1898, pp. 538 and 613 .

$\S$ Ibid., 1896, p. 329 .

\|U. S. Dept. Agr., office of Experiment Stations, Bulletins 63 and 69. 
in the water-current is readily calculated. To this is added the latent heat of the water-vapor brought out in the ventilating aircurrent. By means of ingenious electrical devices, a description . of which would occupy too much space here, the temperature of the interior of the apparatus is kept constant, and any loss of heat by radiation through the walls or in the air-current is prevented. In test experiments the apparatus has given extremely accurate results.

An especial advantage of this apparatus is that it is practicable to make it of large size, and also to continue the experiments for an indefinite length of time. The original apparatus was of a size sufficient for experiments on man, while all previous forms were restricted to experiments on small animals. Recently a modified Atwater-Rosa apparatus has been completed under the writer's direction at the Pennsylvania Experiment Station, with the cooperation of the Bureau of Animal Industry of the United States Department of Agriculture, of a size sufficient for investigations with cattle, and still larger ones are in process of construction.

Computation of Heat Production.-The respiration-calorimeter, in its more perfected forms, is a complicated and costly apparatus both in construction and use, and, moreover, is a rather recent development. It was natural, therefore, that attempts should be made to determine the heat production indirectly by computations based on the kind and amount of matter oxidized in the body.

We may conveniently distinguish three distinct although closely related methods of attacking the problem, all of which assume as a fundamental postulate that the oxidation of a given substance in the body liberates the same amount of energy as does its oxidation outside the organism. In the next chapter we shall examine into the correctness of this postulate; for the present we are concerned simply with the methods of computation based on it.

Computation from Gaseous Exchange.-From a knowledge of the ultimate composition and heat of combustion of a substance it is easy to compute the amount of heat which will be produced by the oxidation of an amount of it sufficient to yield a unit of carbon dioxide or to consume a unit of oxygen. Conversely, then, we can compute from the carbon dioxide evolved or the oxygen consumed in a given time the corresponding amount of energy liberated. 
Such computations have been made by different authors for the three principal classes of nutrients, viz., the proteids, carbohydrates, and fats, the results of a few of which are as follows:

\begin{tabular}{|c|c|c|c|c|c|c|c|c|}
\hline & \multicolumn{2}{|c|}{$\begin{array}{l}\text { Magnus- } \\
\text { Levy.* }\end{array}$} & \multicolumn{2}{|c|}{ Zuntz. $\dagger$} & \multicolumn{2}{|c|}{ Kaufmann. $\ddagger$} & \multicolumn{2}{|c|}{ Laulanié.8 } \\
\hline & $\begin{array}{c}\text { Per } \\
\text { Liter } \\
\mathrm{CO}_{2} \\
\text { Cals. }\end{array}$ & $\begin{array}{c}\text { Per } \\
\text { Liter } \\
O_{2} \\
\text { Cals. }\end{array}$ & $\begin{array}{c}\text { Per } \\
\text { Liter } \\
\mathrm{CO}_{2} \\
\text { Cals. }\end{array}$ & $\begin{array}{c}\text { Per } \\
\text { Liter } \\
\mathrm{O}_{2} \\
\text { Cals. }\end{array}$ & $\begin{array}{c}\text { Per } \\
\text { Liter } \\
\text { CO }_{2} \\
\text { Cals. }\end{array}$ & $\mid \begin{array}{c}\text { Per } \\
\text { Liter } \\
\mathrm{O}_{2} \\
\text { Cals. }\end{array}$ & \begin{tabular}{c|}
$\mathrm{Per}$ \\
$\mathrm{Liter}$ \\
$\mathrm{CO}_{2}$ \\
Cals.
\end{tabular} & $\begin{array}{c}\text { Per } \\
\text { Liter } \\
\mathrm{O}_{2} \\
\text { Cals. }\end{array}$ \\
\hline 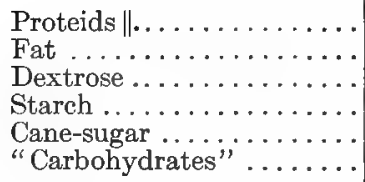 & $\left|\begin{array}{l}5.464 \\
6.586 \\
4.915 \\
4.976 \\
5.090\end{array}\right|$ & $\begin{array}{l}4.289 \\
4.676 \\
4.915 \\
4.976 \\
5.090\end{array}$ & $\mid \begin{array}{l}5.644 \\
6.628 \\
5.047\end{array}$ & $\begin{array}{l}4.476 \\
4.686 \\
5.047\end{array}$ & $\begin{array}{l}5.569 \\
6.648 \\
5.056\end{array}$ & $\begin{array}{l}4.647 \\
4.650 \\
5.056\end{array}$ & 6.571 & $\begin{array}{l}4.6 \\
4.6\end{array}$ \\
\hline
\end{tabular}

Kaufmann also computes from his theoretical equations already given in Part I (pp. 38 and 51) the evolution of heat per liter of oxygen in the various processes of partial oxidation which he believes to take place in the body, with the following results:

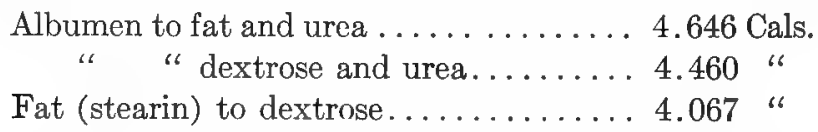

Disregarding the minor differences in the figures of different. authorities, it is evident that the amount of heat produced bears a much more constant relation to the oxygen consumed than to the carbon dioxide produced. For the fats and proteids, especially, the difference is comparatively small. In the case of an animal metab-

* Arch. ges. Physiol., 65, 9.

$\dagger$ Ibid., 68, 191.

$\ddagger$ Archives de Physiol., 1896, pp. 329, 342, 757.

§Ibid., 1898, p. 748.

|| As pointed out on pp.74-75 the determination of the respiratory exchange corresponding to a unit of proteids is not a simple matter In the table Kaufmann's and Laulanié's figures are based upon the theoretical equation (p. 75) for the conversion of albumin into carbon dioxide, water, and urea, while those of Magnus-Levy and Zuntz are derived largely from determinations and estimates by Rubner (Zeit. f. Biol., 21, 363) and others of the proximate composition of the urine of meat-fed animals. As will appear later, these figures are not applicable to the urine of herbivora. 
olizing substantially proteids and fat, then, such as a fasting animal or one consuming only those two nutrients, a determination by any of the methods indicated in Chapter III of the amount of oxygen consumed will afford the basis for at least an approximately correct computation of the energy liberated during the same time, particularly when, as is often the case, the proteid metabolism constitutes but a small proportion of the total metabolism. For the carbohydrates the figures are somewhat higher, and where these bodies constitute a considerable portion of the food the error will be more serious, but even then the results will be of value and especially will afford relatively correct figures for the heat production on the same diet at different times.

The computation from the gaseous exchange of the amount of energy liberated assumes a more exact form in case it is desired to determine the increment arising from some change in the conditions of the experiment, notably from an increase in the muscular work done. In the latter case, as we have seen (Chapter VI), the increased metabolism is largely or wholly that of nonnitrogenous matter. Such being the case, we can compute in the manner indicated on p. 76 from the increments of carbon dioxide and oxygen caused by the work the proportion of each gas corresponding respectively to the oxidation of fat and of carbohydrates, and from this it is easy to compute the corresponding amounts of energy. Thus, to take the example from Zuntz's investigations there given, the increments of oxygen and of carbon dioxide produced by the performance of $1 \mathrm{kgm}$. of work in the case of a dog were computed to be divided as follows:

\begin{tabular}{|c|c|c|}
\hline & $\begin{array}{l}\text { Oxygen } \\
\text { Consumed, } \\
\text { c.c. }\end{array}$ & $\begin{array}{l}\text { Carbon Dioxide } \\
\text { Produced, } \\
\text { c.c. }\end{array}$ \\
\hline $\begin{array}{c}\text { By fat } \\
\text { carbohydrates } \ldots \ldots \ldots \ldots \ldots \ldots \ldots \ldots \ldots \ldots \\
\end{array}$ & $\begin{array}{l}0.6939 \\
0.9765\end{array}$ & $\begin{array}{l}0.4905 \\
0.9765\end{array}$ \\
\hline Total. . & 1.6704 & 1.4670 \\
\hline
\end{tabular}

From this, using Zuntz's factors and assuming that there was no change in the proteid metabolism, the total excess of energy liberated in the body during work over that metabolized during rest is computed as follows: 
Energy from fat...........4.686 cals. $\times 0.6939=3.252$ cals. " " carbohydrates... 5.047 cals. $\times 0.9765=4.927$

Total.

It is obvious that this method of computation affords the means of comparing the total energy metabolized during the performance of a measured amount of work with the quantity recovered in the work itself. It has been extensively used for this purpose by Zuntz and his associates, especially in his investigations in conjunction with Iehmann and Hagemann* upon work production in the horse, which will be considered in a subsequent chapter. The same authors $\dagger$ show that the error introduced by the assumption of unchanged proteid metabolism is too small to be of any significance.

Computation from Total Excreta.-The method just described naturally leads up to a computation based on the gaseous exchange combined with a determination of the urinary products, particularly nitrogen. The latter shows the total amount of proteids metabolized. If we also know, or can compute with sufficient accuracy, the carbon, hydrogen, and oxygen of the urinary solids we have the data from which to compute the portion of the respiratory exchange due to the protein (see p. 75) and the corresponding amount of energy liberated. The residues of carbon dioxide and oxygen can then be distributed between the fats and carbohydrates in the manner already described. This method has been extensively employed by Kaufmann. $\downarrow$ As already stated, he computes the gaseous exchange of the proteids on the assumption of an oxidation to carbon dioxide, water, and urea only, an assumption which, as we have seen, is in some cases considerably wide of the truth.

It is, of course, essential that experiments by this method shall cover a sufficient length of time to ensure that the nitrogen excretion corresponds with the actual proteid metabolism. It is therefore inapplicable to periods of from a few minutes to an hour or so, such as have been generally employed in experiments based on the gaseous exchange only. Kaufmann's experiments extended over five

\footnotetext{
* Landw. Jahrb., 18. 1; 23, 125; 27, Supp. III.

$\dagger$ Ibid., 27, Supp. III., p. 251.

¥ Archives de Physiol., 1896, pp. 329, 342, 757.
} 
hours, but it is open to serious question whether such a period is sufficiently long.

Rubner * has made extensive use of a method substantially the same as that just outlined, but differing in details. The computation is based upon the total nitrogen and carbon (determined or estimated) of urine, feces, and respiration for twenty-four (or twenty-two) hours, the feces being regarded as substantially a metabolic product. The oxygen consumption is not determined. From the results for nitrogen and carbon the proteid and fat metabolism is computed in the manner explained in Chapter III (p. 78). For each gram of carbon in the fat metabolized Rubner reckons 12.31 Cals. of energy, equivalent to 9.4 Cals. per gram of fat, while for each gram of excretory nitrogen (urine and feces) he uses an energy value based on previous experiments $\dagger$ in which the actual heats of combustion of proteids and the products of their metabolism were determined. These results will be considered in another connection (Chapter X). The resulting values for the evolution of energy corresponding to each gram of excretory nitrogen are:

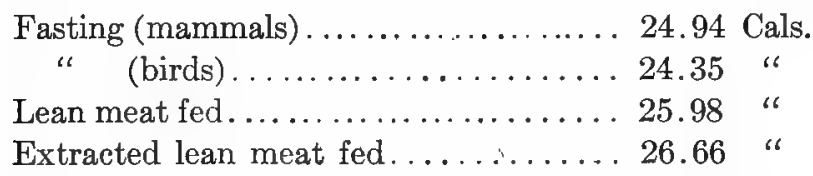

These factors were obtained in experiments on dogs and in strictness apply only to carnivorous animals. By their use, especially if average figures are assumed for some of the minor quantities, such as the carbon of the feces and urine, the determination of the heat production of a quiescent animal in this indirect way becomes a relatively simple matter, while comparisons with direct calorimetric results have shown it to be quite accurate. As was pointed out on p. 78, however, when carbohydrates enter largely into the diet the results are ambiguous, and this fact as well as the marked differences in the character of the excreta forbid its application to herbivorous animals.

Cleavages, Hydrations, etc.-Both the above methods of computing the heat production of an animal assume that the gaseous ex-

* Zeit. f. Biol., 19, 313; 22, 40; 30, 73.

$\dagger I b i d ., 21,250$ and 337. 
change is brought about by what is, in effect, a process of oxidation simply. That many other chemical processes take place in the body is, however, well known, and Berthelot* in particular lays special stress upon the possibility of numerous cleavages, syntheses, hydrations, and dehydrations in which the respiratory quotient may vary between wide limits and in which the heat production is not necessarily proportional to either the oxygen consumed or the carbon dioxide generated. An example of such a process is the formation of fat from carbohydrates, which, as we have seen, may be regarded in the light of an intra-molecular combustion in which no oxygen from outside is consumed, but in which there is an evolution of heat. As an illustration of the opposite possibility-an evolution of heat without production of carbon dioxide-Berthelot instances $\dagger$ the oxidation of a molecule of ethyl alcohol by successive atoms of oxygen to ethyl aldehyde, acetic acid, glycollic acid, oxyglycollic acid, oxalic acid, and finally carbon dioxide and water. Only in the last of these stages is there an evolution of carbon dioxide, yet in each stage there is an evolution of heat varying from 39.9 Cals. to 73.3 Cals. per atom of oxygen.

But while the possibility and even probability of similar reactions in the body of the animal cannot be denied, it certainly seems very questionable, in the light of the results to be considered in the next chapter, whether they have any material bearing upon the determination of the general balance of energy. We know at least approximately the final products of metabolism, and according to the law of initial and final states (p.228) the intermediate reactions can only affect the total amount of energy liberated in case some of the intermediate products are retained in the organism. The only material which we know to be stored up in any considerable quantity in the normal body, however, is fat, and the amount of this we can at least approximately determine. It is of course possible that in an experiment covering a few minutes only, these intermediate reactions may seriously affect the result, but in an experiment covering several hours or a whole day we can hardly conceive such to be the case. Indeed we may probably go still further. It seems to be a general physiological law that the functions of the organism are adjusted to a certain average composition

* Chaleur Animale, Part I.

$\dagger$ Loc. cit., p. 44. 
of its tissues and fluids, and that even a comparatively small variation in the latter calls into action compensatory processes. A striking illustration of this is seen in the promptness with which the respiratory and vascular mechanism reacts to the changes produced in the blood by muscular activity (compare Chapter VI). It seems improbable, therefore, that any sufficient accumulation of the intermediate products of metabolism can take place to seriously in. fluence the results of any but very short experiments. That the methods employed involve other sources of error has already appeared, but with due allowance for these it would appear that the results are worthy of a large degree of confidence.

Computation from Carbon and Nitrogen Balance.-The method of computing the heat production from the total excreta, as employed by Rubner and others for carnivorous animals, we have seen to be inapplicable to herbivora. It, however, shades naturally into a third method, of general applicability, which consists in combining with a determination of the carbon and nitrogen balance by means of the respiration apparatus direct determinations of the potential energy of the food and of the visible excreta by the methods already indicated. Kellner has made extensive use of this method, and the following example, taken from his earliest investigations, ${ }^{*}$ will serve to show clearly the nature of the method. The ox experimented upon was fed daily $8.5 \mathrm{kgs}$ of meadow hay. Respiration experiments showed that on this ration there was a daily gain by the animal of 6.2 grams of nitrogen and 127.2 grams of carbon, equivalent to 37.2 grams of protein and 140.8 grams of fat, the potential energy of which can be computed from the data on p. 244 .

From determinations of the heats of combustion of food, feces, and urine, assuming the combustible gases excreted to consist only of methane, the balance of energy is computed as in the table on p. $256 . \dagger$

Having included under the head of outgo all the known forms in which potential energy as such may be disposed of, the balance of 14,819.5 Cals. is regarded as having been liberated as kinetic energy, and, since no external work was performed, to have taken finally the form of heat. Short of an actual calorimetric experi-

* Landw. Vers. Stat., 47, 275.

† The figures are the corrected ones given in Landw. Vers. Stat., 53, 9. 


\begin{tabular}{|c|c|c|}
\hline & $\begin{array}{l}\text { Income, } \\
\text { Cals. }\end{array}$ & $\begin{array}{l}\text { Outgo, } \\
\text { Cals. }\end{array}$ \\
\hline 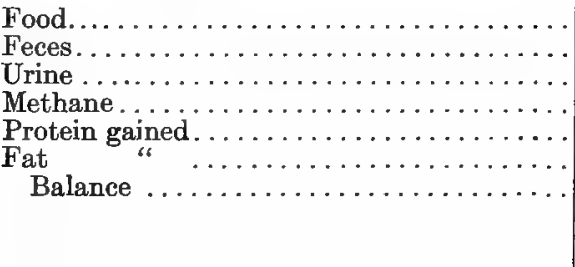 & $\begin{array}{l}32,177.3 \\
\cdots \cdots \cdots \\
\cdots \cdots \cdots \\
\cdots \cdots \cdots \\
\cdots \cdots \cdots \\
\cdots \cdots \cdots \\
32,177.3\end{array}$ & $\begin{array}{r}11,750.3 \\
1,945.0 \\
2,113.7 \\
211.2 \\
1,337.6 \\
14,819.5 \\
32,177.3\end{array}$ \\
\hline
\end{tabular}

ment, this is the most accurate method available for determining the heat production of an animal during a considerable period of time. To short periods it is inapplicable for obvious reasons.

Heat Production and Heat Emission.-In conclusion, it is important to remember that what is determined more or less accurately by all these indirect methods is the amount of energy which takes the kinetic form, and in the absence of mechanical work finally appears as heat. In other words, what is determined is the heat production by the animal. On the other hand, the results obtained with an animal calorimeter show the amount of heat given off by the animal during the experiment, that is, the heat emission. But these two, heat production and heat emission, are by no means necessarily equal. On the one hand, heat produced may be temporarily stored in the body, or, on the other hand, heat retained in the body from a previous period may be given off along with that actually produced during the experiment.

This is sufficiently obvious in case of changes in the body temperature, but even when the latter remains constant the possibility of a temporary storage of the materials of the food, and especially of water, in the body, must be considered. If, for example, the consumption of water in an experiment exceeds the total amount given off in the visible and gaseous excreta, the quantity of heat required to warm the excess of water to the temperature of the body remains in the animal as sensible heat. The heat is produced but not emitted. If, on the other hand, the excretion of water exceeds the consumption, sensible heat is removed from the body in this excess and the emission of heat exceeds the production by a corresponding amount. What is true of water is of course true also, ceteris paribus, of the total income and outgo of matter, although the water, on 
account of its large amount and high specific heat, constitutes the most important factor. The skillful investigator will, of course, seek to plan his experiments so as to avoid these fluctuations so far as possible, but they can rarely be completely eliminated and therefore we cannot expect that the emission of heat will correspond exactly to the production. 
CHAPTER IX.

\section{THE CONSERVATION OF ENERGY IN THE ANIMAL BODY.}

Throughout the preceding chapter, particularly in considering the indirect methods of animal calorimetry, it has been assumed that the law of the conservation of energy applies to the animal body. This is the fundamental postulate upon which all study of nutrition from the standpoint of energy is based, and it is of prime importance, therefore, to examine into the experimental evidence upon which it is based.

The processes of metabolism are essentially chemical processes, and, like other chemical reactions, are accompanied by thermal changes, resulting as a whole in a liberation of kinetic energy. From this point of view, then, the subject may be regarded as a branch of thermo-chemistry.

The applicability of the law of the conservation of energy, and in particular of the law of initial and final states, to the most diverse chemical reactions has been amply demonstrated by the investigations of Hess, Berthelot, Thomsen, and others. It might seem, then. in view of the chemical nature of metabolism, that we were justified in assuming the same law to apply also to the reactions taking place in the body, especially since investigations in other fields of science have led us to regard it as one of the fundamental laws of the universe. On the other hand, however, the reactions occurring in the body are vast in number, are of the most varied character-oxidations, reductions, syntheses, cleavages, hydrations, etc--are infinitely more complex than those which the chemist can produce in his laboratory, and finally, our knowledge of them is as yet but very partial and fragmentary. Moreover, the matter composing the body is living matter, and whatever view we may take as to the nature of life the properties of living matter differ from those of 
dead matter, and we have no scientific right to assume in advance of the evidence that no special forces are operative in the former. In brief, whatever may be the probabilities in the case the applicability of the law to living beings as logically requires experimental demonstration as did its applicability in physics or chemistry, and no little labor has been within the past few years devoted to this problem.

Nature of Evidence.-Before proceeding to a consideration of the experiments bearing upon this question it will be well to make clear the nature of the evidence required.

If the law of the conservation of energy applies to the animal, the following are necessary consequences of it:

1. In an animal doing no work on its surroundings and neither gaining nor losing body substance, the potential energy (heat of combustion) of the food will be equal to the potential energy of the excreta plus the kinetic energy given off in the form of heat plus the energy expended in producing physical and chemical changes in the body.*

2. In an animal doing work on its surroundings, but neither gaining nor losing body substance, the potential energy of the food will be equal to the potential energy of the excreta plus the energy of the heat given off plus the energy of the work done plus the energy expended in producing physical and chemical changes in the body.

3. In an animal doing no work on its surroundings, but gaining or losing body substance, the potential energy of the food will equal the potential energy of the excreta plus the energy of the heat given off plus the potential energy of the gain by the body (a loss by the body being regarded as a negative gain) plus the energy expended in producing physical and chemical changes in the body.

4. In an animal doing work on its surroundings and gaining or losing body substance the potential energy of the food will equal the potential energy of the excreta plus the energy of the heat given off plus the energy of the work done plus the potential energy of the gain by the body (a loss by the body being regarded as a negative

* Surh as changes of temperature or aggregation, cleavages, syntheses, etc. In case these resulted in an evolution of energy, this term of the equation would, of course, have a negative sign. 
gain) plus the energy expended in producing chemical and physical changes in the body.

In actual experimentation it is practically impossible to so adjust the food that there shall be absolutely no gain or loss of body substance, although its amount can be made relatively small. Experiments on this subject, then, necessarily fall under Cases 3 or 4 , and as a matter of fact, in all the experiments hitherto made, the subject has either done no mechanical work or this work has been converted into heat inside the calorimeter and measured along with that directly given off by the body, so that all these experiments fall under Class 3.

The quantities to be determined, then, are

1. Potential energy of food.

2. Potential energy of excreta (feces, urine, hydrocarbons, etc.).

3. The heat produced (including that into which any mechanical work is converted).

4. The potential energy of the gain or loss of body substance.

5 . The energy expended (or evolved) in producing changes in the body.

If we can determine accurately these five factors, and having done so find the equality stated under 3 to exist in a large number of cases, we shall be justified in the conclusion that the law of the conservation of energy applies to the animal organism.

The methods by which the first four of the above factors may be determined formed the subject of the preceding chapter. As regards the fifth, it has commonly been assumed that in an experiment begun and ended at the same hour of the day and under comparable conditions, which has been preceded by a considerable period of uniform feeding and other conditions, and in which the subject was in apparent good health, the initial and final states of the body are substantially the same. While it seems highly probable that this is true, an actual demonstration of its truth is not an easy matter. With respect to the body temperature in particular it is worthy of note that even a slight variation would materially affect the results. Thus in a 1000-pound ox, assuming an average specific heat of 0.8 , a variation of one fifth of a degree Celsius would correspond to 160 Cals. The rectal temperature affords the best available means of control on this point, and a very ingenious 
method for its determination at frequent intervals has been described by Benedict \& Snell.* While it is true that the rectal temperature is not necessarily the average of that of the whole body, we may probably assume with safety that the variations of the two will substantially correspond and therefore that the error introduced by the use of the former will be insignificant.

The question of possible chemical and physical changes in the make-up of the tissues has already been considered in the preceding chapter, where it was pointed out that their effect is in all probability negligible in experiments of any considerable duration.

EARLY Experiments. †-From a slightly different point of view the question under consideration may be stated as that of the source of animal heat. Is the energy given off by the animal in this form (in the absence of external muscular work) equivalent to the heat produced by the oxidation of the same materials outside the body? In this form the question could scarcely fail to attract attention as soon as man began to observe and reflect upon the phenomena of nature.

The ancients regarded the "animal heat" or "vital heat" as "innate" and having its source in the heart. In more recent times it was attributed in a vague way to chemical action, and later was also explained as resulting from mechanical action and in particular from the pulsation of the blood in the blood-vessels. Our real knowledge of the subject, however, dates from the discovery of oxygen and from those researches by Lavoisier and others which established the true nature of combustion and laid the foundations of modern chemistry.

Black $\downarrow$ discovered that carbon dioxide was produced in animals by a process of combustion, and Lavoisier, $\&$ along with his more purely chemical researches, studied the question of animal heat and advanced the hypothesis that respiration consists essentially of a slow oxidation of the carbon and hydrogen of the food by the oxygen of the air, and that this slow combustion is the source of the animal heat.

* Arch. ges Physiol., 90, 33.

$\dagger$ This paragraph follows substantially the historical introduction to Rubner's paper, "Die Quelle der thierischen Wärme." cited below.

$\ddagger$ Lectures on Chemistry, edited by Robison, Edinburgh, 1803

$\S$ Hist. Acad. Roy. d. Sci., Paris, 1780, 355. 
The first part of this hypothesis was readily susceptible of verifieation by a quantitative determination of the oxygen taken up and the carbon dioxide given off, but the second portion was too bold to secure general acceptance. Lavoisier, therefore, with the aid of Laplace, subsequently attempted to secure experimental evidence as to its truth. To this end they determined the amount of heat given off by a guinea pig in an ice-calorimeter, while in a second experiment the animal was placed under a bell-jar and the production of carbon dioxide determined. Having previously determined by means of the ice-calorimeter the heat of combustion of carbon, the results of these two trials gave them data for comparing this amount with that produced by the animal. The computed amount of heat was 25.41 Cals.; that produced by the animal 31.82 Cals.

Several sources of error were inherent in the experimental methods adopted, of some of which Lavoisier was aware, which tended to make the computed amount of heat too small. Taking these into consideration, Lavoisier considered that the experiment substantially confirmed his hypothesis.

At about the same time Crawford * was investigating the same subject, and while his methods were rather primitive and his results less accurate than those of Lavoisier and Laplace, his general conclusions were the same. Of later experiments may be mentioned especially those of Despretz $\dagger$ and of Dulong. $f$ Both investigators employed very similar apparatus, viz., a water-calorimeter through which a current of air was passed, the respiratory products and the heat being determined in the same experiment. The proportion of the oxygen consumed which united with hydrogen was also determined. Both investigators found more heat than they could account for by the oxidation of tissue and concluded that chemical action is the chief but not the only source of animal heat. $\S$

With the advance of physiological knowledge and the recognition of the multiplicity and complexity of the processes taking place in the body, the combustion theory of the origin of animal heat lost rather than gained ground. A few clear-sighted physiologists

* Experiments and Observations on Animal Heat, 1788.

$\dagger$ Ann. de Chim. et de Phys. (2), 26, 337.

$\ddagger$ Ibid. (3), 1, 440.

$\S$ Compare Liebig's discussion of their experiments, Thierchemie, p. 28. 
still adhered to the unity and simplicity of the combustion theory, but in general various subsidiary hypotheses were brought in to account for the observed surplus, such as the motion of the blood, friction, imbibition, etc.

RUBNER'S EXPERIMENTS.-The demonstration of the law of the correlation and conservation of energy in the inorganic world supplied the clue to a rational explanation of the energy manifestations in the living organism, while the subsequent developments of thermochemistry served also to demonstrate a material source of error in the older experiments on animals. In those experiments the computed heat production was based upon the amounts of carbon and hydrogen oxidized and the heats of combustion of those elements, the nitrogenous compounds not being considered. The body, however, does not oxidize free carbon and hydrogen, but various organic compounds, while among its excreta are likewise incompletely oxidized bodies. The computed heat production, therefore, in the early experiments could not fail to be seriously erroneous. From the new point of view, therefore, there appeared no reason to seriously doubt that the animal heat has its sole source in the metabolism of food and tissue, or, in other words, that the law of the conservation of energy applies to the animal body. The first to undertake an experimental demonstration of this fact by modern methods was Rubner.*

His object being primarily to investigate the source of animal heat, his experimental method could be somewhat abbreviated from the general method outlined on p. 260. No external mechanical work having been done by the animals, we have Case 3 of the four possible ones there mentioned. If we let

$$
\begin{aligned}
& F=\text { potential energy of food, } \\
& E=\text { " " " " } \\
& G=\text { " } \quad \text { gain by body, } \\
& H=\text { heat produced, }
\end{aligned}
$$

then, assuming the initial and final states of the body to be the same, we have

$$
F=E+G+H,
$$

* Zeit. f. Biol., 30, 73. 
which may also be given the form

$$
H=(F-G)-E \text {. }
$$

Rubner determined summarily the value of the quantity $F-G$ in the second member of the last equation by the method described in Chapter VIII, p. 253, while the actual heat production was determined by means of his respiration-calorimeter.

The quantities actually determined in these experiments were the weight and nitrogen content of feces and urine, the carbon dioxide of respiration, and the heat produced. The carbon of feces and urine was estimated from their nitrogen and the absence of combustible gases in the respiratory products was assumed. From the total excretion of nitrogen and carbon the amounts of protein and fat metabolized are computed, it being assumed that all the excretory carbon is derived from these two substances. The corresponding amount of potential energy, equivalent to the expression $F-G$ in the equation above, can readily be computed from the heats of combustion of fat and protein. From this the potential energy of the excreta must be subtracted, and this Rubner virtually computes from their total nitrogen on the basis of results obtained in previous experiments with similar food.

A comparison of the heat production as thus computed with that actually measured by means of the calorimeter gave the following results:

\begin{tabular}{|c|c|c|c|c|}
\hline \multirow{2}{*}{ Food. } & \multirow{2}{*}{$\begin{array}{l}\text { Length } \\
\text { of Experi- } \\
\text { ment. } \\
\text { Days. }\end{array}$} & \multicolumn{2}{|c|}{ Total Heat. } & \multirow{2}{*}{$\begin{array}{l}\text { Percentage } \\
\text { Difference. }\end{array}$} \\
\hline & & $\begin{array}{l}\text { Computed, } \\
\text { Cals. }\end{array}$ & $\begin{array}{c}\text { Measured, } \\
\text { Cals. }\end{array}$ & \\
\hline $\begin{array}{l}\text { Fasting } \ldots \ldots \ldots \ldots \ldots \ldots \\
\text { Fat } \ldots \ldots \ldots \ldots \ldots \ldots \\
\text { Meat and fat } \ldots \ldots \ldots \ldots \ldots \\
\text { Meat } \ldots \ldots \ldots \ldots \ldots \ldots\end{array}$ & $\begin{array}{r}5 \\
2 \\
5 \\
8 \\
12 \\
6 \\
7\end{array}$ & $\begin{array}{l}1296.3 \\
1091.2 \\
1510.1 \\
2492.4 \\
3985.4 \\
2249.8 \\
4780.8\end{array}$ & $\begin{array}{l}1305.1 \\
1056.6 \\
1495.3 \\
2488.0 \\
3958.4 \\
2276.9 \\
4769.3\end{array}$ & $\begin{array}{l}+0.69 \\
-3.15 \\
-0.97 \\
-0.17 \\
-0.68 \\
+1.20 \\
-0.24\end{array}$ \\
\hline Total ................ & 45 & 17406.0 & 17349.7 & -0.32 \\
\hline
\end{tabular}

While some of the individual experiments show not inconsiderable discrepancies, the averages of computed and measured heat 
agree very closely and, granting the entire validity of the numerous assumptions involved in this method, would seem to approach a demonstration of the applicability of the law of the conservation of energy to the metabolism of the animal. Aside from errors in the estiniation of the carbon of the excreta from their nitrogen, which are probably small, the chief elements of uncertainty are the assumptions as to the nature of the material metabolized in the body and as to the heat of combustion of the excreta. As regards the former point, Rubner himself points out (loc. cit., pp. 118-121) that a portion of the carbon of the respiration may be derived from glycogen, and even bases upon the calorimetric results in one case a computation of the extent to which this may have occurred. The latter, however, is obviously begging the question, and in his main computations Rubner assumes that only protein and fat were metabolized.

LAULANIE's EXPERIMENTS.-By means of his differential watercalorimeter, Laulanié * has determined the respiratory exchange and the heat production of animals, both fasting and fed. The nitrogen excretion does not appear to have been determined. From the respiratory exchange the heat production is computed, using the data given on p. 250 , and compared with that obtained calorimetrically. In the fasting experiments an evolution of 4.6 Cals. of heat is computed per liter of oxygen consumed. In the experiments in which food was given the author computes from the respiratory quotient the distribution of the oxygen between fat and carbohydrates, neglecting the protein because it yields the same amount of heat per unit of oxygen as does fat, and thence calculates the heat production. Preliminary tests of the calorimeter, by allowing water to cool in it, gave respectively 101.3 per cent., 100.9 per cent., and 99.7 per cent. of the theoretical results. The experiments show a close agreement between the observed and computed amounts of heat, as appears from the table at the top of page 266.

Atwater \& Benedict's Investigations. - By far the most extensive and complete data regarding the conservation of energy in the animal body are those afforded by the investigations of Atwater \& Benedict $\nmid$ upon man. The experiments were made

* Archives de Physiol , 1898, p. 748.

$\dagger$ U. S. Dept. Agr, Office of Experiment Stations, Bull. 109; Memoirs Nat Acad. Sci., 8, 235. 


\begin{tabular}{|c|c|c|c|c|c|c|c|}
\hline 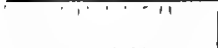 & $1 i$ & Legth & & & Heat & Produ & ction. \\
\hline $\begin{array}{r}\text { Subject. } \\
\\
,\end{array}$ & Food. & $\begin{array}{l}\text { Experi- } \\
\text { ment. } \\
\text { Hours. }\end{array}$ & $\begin{array}{l}\text { Con- } \\
\text { sumed, } \\
\text { Liters. }\end{array}$ & $\begin{array}{l}\text { Quo- } \\
\text { tient. }\end{array}$ & $\begin{array}{c}\text { Ob- } \\
\text { gerv'd, } \\
\text { Cals. }\end{array}$ & $\begin{array}{l}\text { Com- } \\
\text { puted } \\
\text { Cals. }\end{array}$ & $\left\{\begin{array}{c}\text { Comp. } \\
+ \text { Obs. } \\
\%\end{array}\right.$ \\
\hline 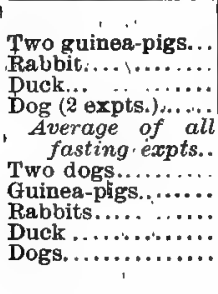 & $\begin{array}{l}\text { Third day of fasting.... } \\
\text { Second day of fasting... } \\
\text { Fasting for } 2 \text { days....... } \\
\text { ". " } 2 \text { and } 4 \text { days. }\end{array}$ & $\begin{array}{c}8 \\
51 \\
4 \\
12 \frac{1}{8} \\
\ldots \ldots \cdots \\
20 \\
\cdots \\
30 \\
8 \\
66\end{array}$ & $\begin{array}{r}8.112 \\
7.650 \\
8.800 \\
30.205 \\
82.812 \\
51.683 \\
31.787 \\
46.445 \\
21.603 \\
180.398\end{array}$ & $\begin{array}{l}0.791 \\
0.752 \\
0.750 \\
0.758 \\
\\
0.766 \\
0.816 \\
0.917 \\
0.893 \\
0.885 \\
0.973\end{array}$ & $\begin{array}{r}37.106 \\
35.254 \\
40.375 \\
141.366 \\
\\
385.403 \\
239.431 \\
155.787 \\
227.086 \\
105.911 \\
882.580\end{array}$ & $\begin{array}{r}37.315 \\
35.190 \\
40.480 \\
138.943 \\
\\
380.935 \\
237.741 \\
154.230 \\
224.050 \\
104.040 \\
887.197\end{array}$ & $\begin{array}{r}100.6 \\
99.8 \\
100.3 \\
98.2 \\
\\
98.8 \\
99.2 \\
99.0 \\
98.6 \\
98.2 \\
100.5\end{array}$ \\
\hline
\end{tabular}

with the aid of the respiration-calorimeter of Atwater \& Rosa (p. 248 ), and in addition to the great pains bestowed to obtain accurate results are especially distinguished by the fact that all the quantities involved were, so far as possible, subjected to direct measurement, estimates being avoided with the necessary exception of the potential energy of the gain or loss by the body. The sublingual or axillary temperature of the subject was also measured in every case. The following results of one of the earliest experiments (No. 5) may serve to illustrate the general features of them all:

\begin{tabular}{|c|c|c|}
\hline & $\begin{array}{l}\text { Income, } \\
\text { Cals. }\end{array}$ & $\begin{array}{l}\text { Outgo, } \\
\text { Cals. }\end{array}$ \\
\hline 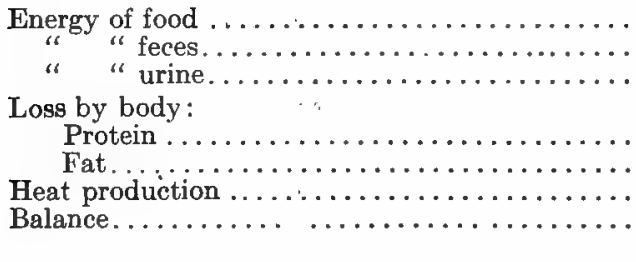 & $\begin{array}{l}2655 \\
\cdots \cdots \\
\cdots \\
\cdots \\
\cdots \\
\cdots\end{array}$ & $\begin{array}{r}143 \\
128 \\
-24 \\
-73 \\
2379 \\
102 \\
\end{array}$ \\
\hline & 2655 & 2655 \\
\hline
\end{tabular}

Aside from the loss of 97 Cals. by the body as computed from the carbon and nitrogen balance, all the quantities in the above statement represent actual determinations of energy and the account balances within $102 \mathrm{Cals}$., which is 3.8 per cent. of the total energy of the food or 4.1 per cent. of the computed heat production. To put the matter in a slightly different way, the heat production as computed by Kellner's method ( p. 255) from the carbon and nitrogen balance and the energy of food and excreta exceeds by 102 Cals. 
the heat production actually measured by the calorimeter. This experiment was one of the two showing the greatest percentage difference between the computed and the observed heat production. In the following statement are tabulated the results of all the experiments thus far reported, arranged without regard to the subject of the experiment or the nature of the diet, but divided into two groups according as active muscular work was or was not performed.

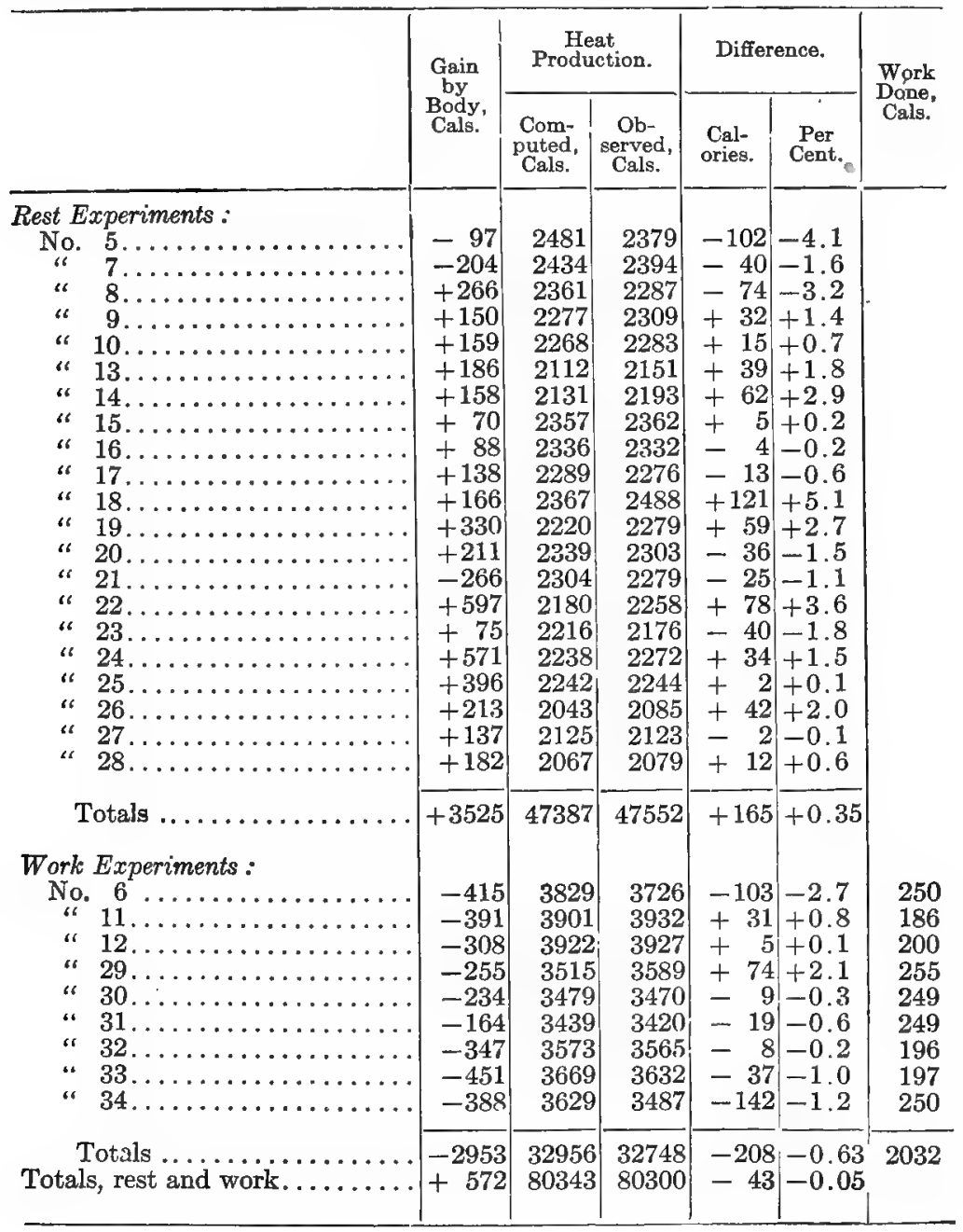


In the former case the observed heat production includes the heat into which the work was converted.

The total of all the experiments shows an almost absolute agreement between the computed and the observed results. To a trifling extent, however, this arises from a compensation between the rest and work experiments, the computed heat tending to be slightly too small in the former and slightly too great in the latter, but the agreement in each series is so close as to amount to a demonstration of the applicability of the law of the conservation of energy to the metabolism of the animal organism. 


\section{CHAPTER X.}

\section{THE FOOD AS A SOURCE OF ENERGY-METABOLIZABLE ENERGY:}

WirH the establishment of the law of the conservation of energy in its application to the animal body, and with the development of the methods of calorimetric research briefly outlined in Chapter VIII, it has become possible to study successfully the problems of animal nutrition from a new standpoint, regarding the food as primarily a source of energy to the body and tracing, to some extent at least, the transformations which that energy undergoes in the organism and particularly the extent to which the latter utilizes it for various purposes.

Some data regarding the total energy of foods and their constituents have already been given in Chapter VIII. It was there pointed out, however, that the total energy, taken by itself, does not furnish a measure of the nutritive value of a substance. It is now necessary to enter upon the question of the availability of this energy to the organism.

Total and Metabolizable Energy.-The heat of combustion of the food represents to us its total store of potential energy. By no means all of this potential energy, however, is accessible to the organism. A part of what the animal eats is not food at all in a physiological sense, but is simply waste matter which passes through the digestive tract unacted upon. Furthermore, that part of it which is digested and resorbed is not completely oxidized in the body, but gives rise to the formation of excretory products which are still capable of liberating energy by oxidation. We have, therefore, at the outset, to distinguish between the total, or gross, energy of the food eaten, represented by its heat of combustion, and the portion of that energy which can be liberated and utilized in 
the organism. It is only this latter portion, of course, of which the body can avail itself, and the term available energy has, therefore, very naturally been proposed for it.

As will appear later, however, the terms available, and availability may also be employed, and have actually been used, in a more restricted sense to designate that part of the energy of the food which can be applied directly by the organism to purposes other than simple heat production. In orcler to avoid the confusion of terms thus arising it has been proposed to modify the term available by the words gross and net. The gross available energy, according to this terminology, signifies all of the total energy of the food which can be utilized by the body for any purpose whatever; that is, it is available energy in the first of the two senses defined above. Similarly, the net available energy signifies the available energy in the second sense, or energy available for other purposes than simple heat production. The term "fuel value" has also been employed by some writers, notably by Atwater, to designate the gross available energy.

It appears to the writer desirable, however, to avoid the double use of the word available, even with the somewhat awkward modifying terms proposed. Strictly speaking, what is meant by gross available energy in the above sense is that portion of the potential energy of the food which the digestive and metabolic processes of the organism can convert into the kinetic form, and its measure, according to the principles enunciated in Chapter VII, is the difference between the potential energy of the food and the potential energy of the various forms of unoxidized matter rejected by the organism. In other words, it is that fraction of the energy of the food which can enter into the metabolism of energy in the body. The writer, therefore, tentatively proposes for it the term metabolizable energy, as expressing the facts without any implication as to the uses made by the body of the energy thus metabolized.

Metabolizable energy, then, may be briefly defined as potential energy of food minus potential energy of excreta, including under excreta, of course, all the wastes of the body. visible and invisible. The methorl is analogous to that of the determination of digestibility. In both cases it is a calculation by difference, and the result shows simply the maximum amount of matter or of energy put at the dis- 
posal of the organism without affording any clue to the use marle of it by the latter, that is, to its availability in the more restricted sense.

In actual investigation, of course, the metabolizable energy of the food is most accurately found by means of direct determination's of the heats of combustion of the food and the waste products: Except in the case of the intestinal gases no serious difficulties stand in the way of these determinations, and with the present improved and simplified methods of calorimetry 'it may' fairly be expected that, in exact experiments, at least the energy of the food, feces, and urine will be directly determined, whilie it is not impossible that more extended investigations than are now available may enable us to make, for different classes of materials, a fairly accurate estimate of the intestinal gases. As results accumulate from such investigations we shall gradually acquire a fund of information regarding the amount of metabolizable energy contained in foods and feeding-stuffs which it is perhaps not chimerical to suppose may one day largely take the place of our present tables of composition and digestibility.

Up to the present time, however, but a comparatively small number of experiments upon domestic animals are'on record in which the metabolizable energy of the food has been actually determined. In a somewhat larger number of cases the loss of energy in feces and urine has been determined, and in others that in the feces only. As regards human food the data are somewhat more abundant, but nevertheless by far the greater part of our scientific knowledge of foods and feeding-stuffs is expressed in terms of (conventional) chemical composition and apparent digestibility. If, therefore, we would not forego the advantages which may be anticipated from a study, from the new point of view, of the accumulated results of the last half-century of experimental work in this domain, it is important that we be able to estimate as accurately as may be the metabolizable energy of the food from its known or estimated composition and digestibility. Not a little labor has been'expended upon both aspects of the subject, particularly by Rubner in relation to the carnivora and man, by Atwater and his associates with relation to human nutrition, by Kellncr as regards ruminants, and by Zuntz and his associates in the case of the horse. 


\section{§ 1. Experiments on Carnivora.}

The comparative simplicity and completeness of the digestive processes of carnivora, together with the great variations which can be made in their diet, have made them favorite subjects for physiological experiments. It is possible to feed a dog or cat on what are close approximations to simple nutrients for a sufficient length of time to permit an accurate determination of the waste products, while with herbivora this is impracticable for obvious reasons.

While earlier experimenters, among whom may be mentioned Frankland,* Traube, $\uparrow$ and Zuntz, $\downarrow$ have concerned themselves with the question of the energy values of foods and nutrients, it is to the fundamental researches of Rubner that we owe not merely more accurate determinations of metabolizable energy, but in particular a clearer conception of its actual significance in nutrition. Rubner's experiments \& were made chiefly with $\operatorname{dogs}$ and were directed toward the determination of what he designates as the physiological heat value of the more important proteid foods, corresponding substantially to what is here called the metabolizable energy.

Proteids. - As regards the non-nitrogenous ingredients of the food, Rubner assumes that, so far as they are digested, their metabolizable energy is the same as their gross energy, or, in other words, that there are no waste products. For example, if a dog is given a certain amount of starch and none appears in the feces it is assumed that the starch has simply undergone hydration and solution in the digestive tract without material loss of energy and that consequently the full amount of energy contained in the starch is available in the resorbed sugar for the metabolism of the body. In herbivora we know that there is a considerable production of gaseous hydrocarbons by fermentation in the digestive tract. The respiration experiments of Pettenkofer \& Voit on dogs, however (compare p. 72), showed but a small excretion of such gases, while Tappeiner \| denies the presence of methane in any part of the dog's alimentary canal. In the case of carnivora, then, the above

* Phil. Mag. (4), 32, 182.

$\dagger$ Virchow's Archiv., 29, 414.

† Landw. Jahrb., 8, 65 .

$\S$ Zeit. f. Biol., 21, 250 and 337.

|| Quoted by Rubner, ibid., 19, 318. 
assumption is at least in harmony with current opinion. Rubner's experiments were therefore directed to the determination of the metabolizable energy of the proteids.

The earlier computations of the metabolizable energy of the proteids by Frankland, Traube, Danilewski, and others * were affected by two sources of error. First, the heats of combustion as determined by the imperfect calorimetric methods then available were seriously in error. Second, the manner of computing the metabolizable energy from these data has been shown by Rubner to be incorrect. Previous to his investigations the metabolizable energy of the proteids had been very generally computed by deducting from their gross energy the energy of the corresponding amount of urea. In other words, it was assumed that all the nitrogen of the proteids was split off in the form of urea and excreted in the urine, which was accordingly regarded as being practically an aqueous solution of urea, and that the non-nitrogenous residue of the proteids was completely oxidized to carbon dioxide and water. Rubner's results show that this assumption is seriously erroneous and gives too high results for the metabolizable energy.

In the first place, it neglects entirely one of the waste products, viz., the feces. The latter are to be regarded in the carnivora, especially on a proteid diet, as a true excretory product. comparable to the organic matter of the urine and containing at most but traces of undigested food. This was early pointed out by Bischoff \& Voit $\dagger$ and is now generally admitted by physiologists. (Compare p. 47.) In Rubner's experiments somewhat over 3 per cent. of the energy of the proteid food was found in the feces.

In the second place, Rubner shows that the urine is far from being a simple solution of urea. $\$$ His previous investigations $\&$ had shown that the extractives of lean meat the form of proteid most commonly used in such experiments, pass through the system unchanged and are excreted in the urine, thus increasing its content of energy. By feeding meat previously treated with water to remove

* Cf. Rubner, loc. cit., p. 341.

$\dagger$ Ernährung des Fleischfressers, p. 291; compare also Müller, Zeit. f. Biol., 20, 327; Rieder, ibid., 20, 378; Tsuboi, ibid., 35, 68.

‡ Compare Chapter VIII, p. 241.

\$ Zeit. f. Biol., 20, 265. 
these extractives, he demonstrates that in this case also the urine is far from being a simple solution of urea. With a daily excretion of 13.22 grams of total urinary nitrogen, there was found in the urine 0.105 gram of kreatinin, 0.656 gram of cyanuric acid, and an undetermined amount of phenol. The proportion of carbon to nitrogen in the urine was also notably higher than in urea, viz., $0.523: 1$ in place of $0.428: 1$, or an excess of about 20 per cent. Rubner concludes that the only sure method of ascertaining the amount of potential energy carried off in the urine is the direct determination of its heat of combustion. Accordingly, in the experiments under consideration, the urine was dried on pumice-stone and burned in the calorimeter, a correction being made for the urea decomposed during the drying. Danilewski,* about the same time, also reported determinations of the heat of combustion of the dry matter of human urine which, like Rubner's, show an excess over that computed from the urea present.

The materials experimented on by Rubner were prepared lean meat, such as has been commonly used in feeding experiments, and meat with the extractives removed by treatment with water, the gross energy of each being determined by burning the dried material in the calorimeter after having removed the fat by extraction with alcohol and ether. $\dagger$ The prepared material (in the moist state) was fed to dogs for from five to eight days, during all or a portion of which time the feces and urine were collected and their content of nitrogen and energy determined. The amounts fed are not stated, but the percentage of the total nitrogen fed which reappeared in the feces is given. A third experiment on a fasting dog was added in which the urine of the second, third, and fourth days was collected and examined.

So far as the proteids are metabolized in the body all their nitrogen which does not reappear in the feces will be found in the urine. On this basis the nitrogen per gram of dry proteids metabolized in these (xperiments was divided as shown in the following table. In the case of the fasting animal, Rubner believes himself justified, on the basis of other experiments, in assuming that the nitrogenous tissue

* Arch. ges. Physiol., 36, 230.

† Subsequent investigations.have shown that the material thus prepared still contains traces of fat. 
metabolized had substantially the same composition and heat-value as the lean meat of the first experiment, and the feces are also assumed to be similar.

\begin{tabular}{|c|c|c|c|}
\hline Food. & $\begin{array}{l}\text { Nitrogen of } \\
\text { Food, } \\
\text { Grms. }\end{array}$ & $\begin{array}{l}\text { Nitrogen of } \\
\text { Feces, } \\
\text { Grms. }\end{array}$ & $\begin{array}{l}\text { Nitrogen of } \\
\text { Urine, } \\
\text { Grms. }\end{array}$ \\
\hline Lean meat $\ldots \ldots$ & 0.1540 & 0.0024 & 0.1516 \\
\hline Fxtracted lean meat. & 0.1659 & 0.0023 & 0.1636 \\
\hline Nothing (body tissue). & 0.1659 & 0.0023 & 0.1636 \\
\hline
\end{tabular}

The energy of the excretory products, calculated per gram of nitrogen, was as follows:

\begin{tabular}{|c|c|c|}
\hline Food. & $\begin{array}{l}\text { Urine, } \\
\text { Cals. }\end{array}$ & $\begin{array}{l}\text { Feces, } \\
\text { Cals. }\end{array}$ \\
\hline $\begin{array}{l}\text { Lean meat } \ldots \ldots \ldots \ldots \\
\text { Extracted lean meat } \ldots \ldots \ldots \\
\text { Nothing......... }\end{array}$ & $\begin{array}{l}7.450 \\
6.695 \\
8.495\end{array}$ & $\begin{array}{l}70.290 \\
81.515\end{array}$ \\
\hline
\end{tabular}

A comparison of the above results for the urine with the energy of urea (5.41 Cals. per gram of nitrogen) fully confirms the conclusions already drawn from its chemical composition.

From the figures of the last two tables, together with the heats of combustion found for the food consumed, viz.,

Lean meat, fat removed...........5.345 Gals. per gram

" " extractives and fat removed...5.754 " " "

we can readily compute the energy of the excreta and by difference the metabolizable energy of the food per gram, as follows:

\begin{tabular}{|c|c|c|c|c|c|c|}
\hline $\begin{array}{l}{ }^{\prime} \\
\text { Energy of food } \ldots \ldots \ldots \ldots \ldots \ldots \\
" \text { " } \text { " feces } \ldots \ldots \ldots \ldots \ldots \ldots \\
\text { Metabolizable energy } \ldots \ldots \ldots \ldots\end{array}$ & \multicolumn{2}{|c|}{ Lean Meat. } & \multicolumn{2}{|c|}{$\begin{array}{l}\text { Extracted } \\
\text { Lean Meat. }\end{array}$} & \multicolumn{2}{|c|}{$\begin{array}{l}\text { Nitrogenous } \\
\text { Body Tissue. }\end{array}$} \\
\hline \multirow{5}{*}{ 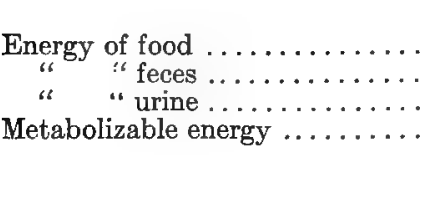 } & Cals. & $\mid \begin{array}{c}\text { Cals. } \\
5.3450\end{array}$ & & $\mid \begin{array}{c}\text { Cals. } \\
5.7540\end{array}$ & & Cals. \\
\hline & 0.1683 & & 0.1854 & & 0.1683 & \\
\hline & 1.1294 & & 1.0945 & & 1.2878 & \\
\hline & 4.0473 & & 4.4741 & & 3.8889 & \\
\hline & 5.3450 & 5.3450 & 5.7540 & 5.7540 & 5.3450 & 5.3450 \\
\hline
\end{tabular}


Rubner makes a slight correction in the above figures for the energy of hydration and solution. The energy of the proteids was determined in the dry state. They were fed, however, moist, and it is known that an evolution of heat takes place when dry proteids are brought in contact with water. Consequently the potential energy of the moist proteids is less than that computed from the calorimetric results. Rubner estimates this loss (loc. cit., p. 307) at 0.5 per cent. The urea leaves the body in solution. Its solution in water, however, causes an absorption of heat equal to 2.4 per cent. of the total energy of the urea, and accordingly (neglecting other organic matter) the heat value of the urine is higher than that calculated from the calorimetric results upon the dried urine. Both these errors tend to make the metabolizable energy appear too great. Rubner's corrections are as follows:

\begin{tabular}{|c|c|c|c|}
\hline & $\begin{array}{c}\text { Lean Meat. } \\
\text { Cals. }\end{array}$ & $\begin{array}{c}\text { Extracted } \\
\text { Lean Meat. } \\
\text { Cals. }\end{array}$ & $\begin{array}{l}\text { Nitrogenous } \\
\text { Body Tissue } \\
\text { Cals. }\end{array}$ \\
\hline Metabolizable energy as above & 4.0473 & 4.4741 & 3.8889 \\
\hline Energy of hydration ......... & 0.0269 & 0.0288 & 0.0269 \\
\hline " "solution $\ldots \ldots \ldots \ldots \ldots$ & 0.0199 & 0.0215 & 0.0199 \\
\hline Corrected metabolizable energy ... & 4.0005 & 4.4238 & 3.8421 \\
\hline
\end{tabular}

The energy lost in hydration is, of course, practically a diminution of the gross energy of the food. The energy absorbed in the solution of the urea can be regarded either as a part of the energy of the excreta or as being a part of the general expenditure of energy by the body in internal work. (See the next chapter.)

Rubner * has also computed the metabolizable energy of a number of proteids for which direct determinations are wanting. For this purpose he uses the results of Stohmann $\dagger$ for the gross energy and assumes. first, that the nitrogen will be divided between feces and urine in the same ratio as in the experiment on extracted lean meat, and second, that the energy of these excretory products per gram of nitrogen will be the same as in that experiment. He thus obtains the following results:

* Loc. cit., p. 351.

$\dagger$ Landw. Jahrb., 13, 513. 


\begin{tabular}{|c|c|c|c|c|}
\hline $\begin{array}{l}\text { Substance. } \\
.\end{array}$ & $\begin{array}{l}\text { Per Cent. } \\
\text { Nitrogen. }\end{array}$ & $\begin{array}{c}\text { Gross } \\
\text { Energy } \\
\text { Por Grm., } \\
\text { Cals. }\end{array}$ & $\begin{array}{c}\text { Loss in } \\
\text { Exereta, } \\
\text { etc..' } \\
\text { Cali. }\end{array}$ & $\begin{array}{l}\text { Metaboliz- } \\
\text { able Energy } \\
\text { Per Grm., } \\
\text { Cals. }\end{array}$ \\
\hline 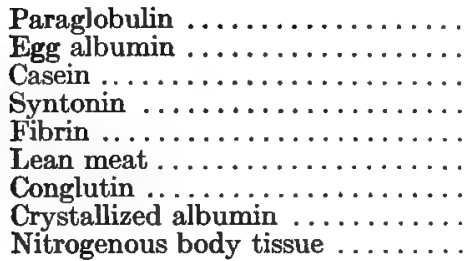 & $\begin{array}{l}15.6 \\
15.7 \\
15.2 \\
16.6 \\
16.6 \\
15.4 \\
17.5 \\
19.2 \\
15.4\end{array}$ & $\begin{array}{l}5.634 \\
5.577 \\
5.715 \\
5.754 \\
5.508 \\
5.345 \\
5.359 \\
5.595 \\
5.345\end{array}$ & $\begin{array}{l}1.263 \\
1.270 \\
1.311 \\
1.329 \\
1.329 \\
1.345 \\
1.390 \\
1.555 \\
1.503\end{array}$ & $\begin{array}{l}4.371 \\
4.307 \\
4.404 \\
4.424 \\
4.179 \\
4.000 \\
3.969 \\
4.090 \\
3.842\end{array}$ \\
\hline
\end{tabular}

\section{§ 2. Experiments on Man.}

Protein.-Rubner * has also reported a single experiment on a man upon a diet of meat with a slight addition of fat. The results, expressed in the same manner as those given in the preceding section, that is, per gram of dry matter of the meat, were-

Energy of food.................. 5.599 Cals.

$$
\begin{aligned}
& \text { " "feces........... 0.434 Cals. } \\
& \text { " " urine........... 1.027 " } \\
& \text { Metabolizable energy........ } 4.138 \text { " } \\
& \overline{5.599} \text { “ } \overline{5.599} \text { “ }
\end{aligned}
$$

Quite a number of determinations are on record of the ratio between the nitrogen and the energy content of human urine. Rubner $\dagger$ reports the following results upon various diets, including the experiment on meat just quoted:

$$
\begin{aligned}
& \text { Energy Per Grm., } \\
& \text { Nitrogen. }
\end{aligned}
$$

Mother's milk......................12.10 Cals.

Cow's milk-infant.................... 6.93

" " -adult...................... 7.71

Mixed diet, poor in fat..................... 8.57 "

" " " " "

" " rich in fat................. 8.87 "

" " " " "

" " " " "-boy............... 6.42 "

Mixed diet-boy....................... 7.50 "

Meat................................ 7.69 "

Potatoes..............................., 7.85 "

*Zeit. f. Biol., 42, $272 . \quad$ † lbid., p. 302. 
With the exception of the mother's milk, the results show but a slightly greater range than those on the dog. The results of Atwater \& Benedict,* cited on p. 242, when computed per gram of nitrogen, give the following results:

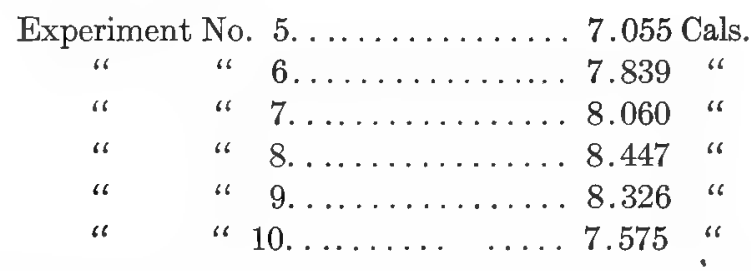

The same authors report $\dagger$ the average of 46 determinations as 7.9 Cals. per gram of nitrogen. Tangl $\ddagger$ has reported materially higher figures, especially for diets containing large amounts of carbohydrates and fat.

In the case of a mixed diet more or less of the potential energy of the feces may be derived from the non-nitrogenous nutrients of the food, and we should hardly be justified in making for these experiments a computation like that made for the meat diet. The rather small range of the figures in most cases, however, would seem to show that the metabolizable energy of the proteids of ordinary mixed dietaries is substantially the same as that found by Rubner for carnivora. Tangl's results perhaps suggest the possibility of the occasional presence in human urine of non-nitrogenous matters similar to those found so abundantly in that of ruminants.

Rubner's Computations.-Rubner's earlier researches did not include experiments upon man, but from the results given in the foregoing section he endeavored to compute approximate factors for the metabolizable energy of the mixed diet of man.\$ For this purpose he estimates that, on the average, 60 per cent. of the protein of the diet is derived from animal sources and 40 per cent. from vegetable. For the animal protein he uses the value found above for lean meat, and for vegetable protein the average of the values for syntonin and fibrin (since these have an ultimate composition

* U. S. Dept. Agr., Office of Experiment Stations, Bull. 69.

$\dagger$ Report Storrs Expt. Station, 1899, p. 100.

$\ddagger$ Arch. f. (Anat. u.) Physiol., 1899, 261.

$\S$ Loc. cit., p. 370 . 
similar to that of the proteids of the grains). Correcting these values for the error involved in the usual computation of protein from nitrogen, he obtains as the average metabolizable energy of the protein $(\mathrm{N} \times 6.25)$ of a mixed diet 4.1 Cals. per gram.

For the fat and carbohydrates it is assumed that all their potential energy is metabolizable, but an allowance is made in the latter case for the error due to the ordinary computation of the carbohydrates by difference and for some minor sources of uncertainty. Rubner's final averages are--

Protein $(\mathrm{N} \times 6.25) \ldots \ldots \ldots 4.1$ Cals. per gram.

Fat.............. 9.3 " " "

Carbohydrates. ........4.1" " " "

The value for protein, by the method of computation, includes an allowance for the metabolic products contained in the feces, but neither it nor the values for the other nutrients include any estimate for the loss through imperfect digestion. In other words, they refer to the digested nutrients.

These figures were designed expressly for computing the metabolizable energy of human dietaries, and even for that purpose are confessedly only approximations. In the absence of more exac figures, however, they have been somewhat extensively used for computing the metabolizable energy of the digested portion of the food of domestic animals. For purposes of approximate estimates such a use of them was perhaps justifiable, but in too many cases their origin seems to have been forgotten and a degree of accuracy ascribed to them which they do not possess. As will be shown presently, later investigations have yielded materially different results for the metabolizable energy of the several classes of nutrients in the fuod of herbivorous animals.

Later Experiments.-Quite recently Rubner* has published the results of some experimental investigations into the validity of the averages or "standard figures" given above. In these experiments the weights and heats of combustion of food, feces, and urine were determined calorimetrically and the metabolizable energy as obtained from these data was compared with that computed by the use of the above factors. In making the latter calculation an allowance

* Zeit. f. Biol., 42, 261. 
was made for the percentage loss in the feces equal to that observed in the actual experiment. The results for the metabolizable energy per day were-

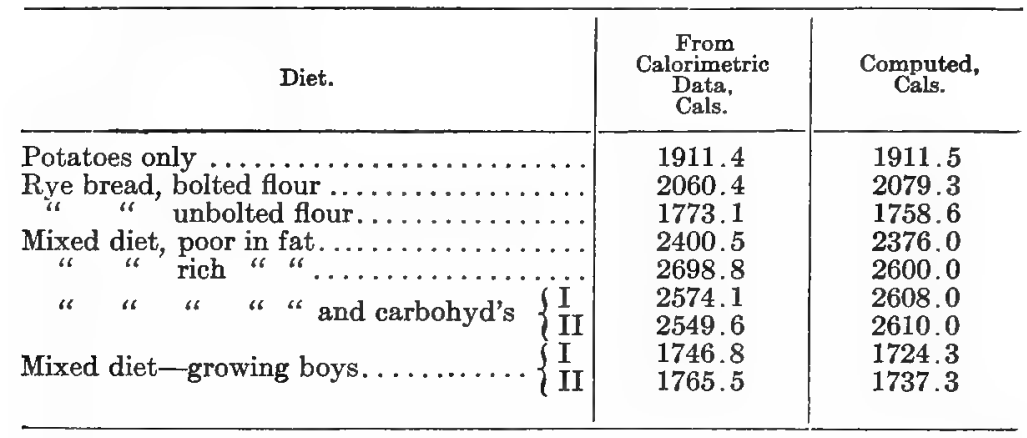

As above noted, the computed results include a deduction for the energy of the undigested matter in the feces. Rubner finds that the heat of combustion of the organic matter of the latter varies but little even on extremes of diet, so that the loss through this channel is approximately proportional to the amount of the excretion. In the experiments on mixed diet the percentage loss of energy in the feces varied from 4.3 per cent. to 7.9 per cent. of the energy of the food.

Atwater's Investigations.-By far the most extensive data as to the metabolizable energy of human foods and dietaries are those derived from the investigations upon human nutrition made under Atwater's direction by the United States Department of Agriculture with the coöperation of Wesleyan University, the Storrs Experiment Station, and various other experiment stations. Atwater \& Bryant* have summarized these results in a preliminary report of which the essential features are given in the following paragraphs.

From the best data available, the heats of combustion of the protein, carbohydrates, and fats of various classes of foods are estimated. In these estimates account is taken as fully as possible of the proportion of nitrogen in proteid and non-proteid forms, and of the varying percentage of nitrogen in different proteids, the nitrogen factors used being those quoted on p. 6. The accuracy of

* Report Storrs Agr. Expt Station, 1899, p. 73. 
these estimates is checked by a comparison of the computed with the actual heats of combustion of 276 different samples of food, the average results showing a close agreement. Assuming the potential energy of the urine to be all derived from the proteids, the average of 7.9 Cals. per gram nitrogen given above (p. 278) corresponds to 1.25 Cals. per gram of protein $(\mathrm{N} \times 6.25)$ metabolized. The loss of energy in the feces is estimated from a number of digestion experiments upon single foods, the results being checked by a comparison of the actual and computed apparent digestibility in 93 digestion experiments on mixed diet. Finally, the proportions of the several nutrients which are derived from different classes of foods in average mixed diets are computed from the results of 185 dietary studies. The final results thus obtained for the metabolizable energy or "fuel value". of the nutrients are shown in the table on page 282 .

The average results for the ordinary mixed diet of man were-

Protein................... 4.0 Cals. per gram

Carbohydrates.............4.0 " " "

Fat.................... 8.9 " "

These factors are smaller than those proposed by Rubner, largely because they relate to the total and not to the digested nutrients. Comparisons of the computed with actual metabolizable energy of mixed dietaries, using the factors of the above table, gave concordant results.

\section{§3. Experiments on Herbivora.}

The MöckeRn Investigations.-The larger share of our present knowledge regarding the metabolizable energy of the food of herbivora is due to the investigations upon mature cattle which have been made by Kellner * since 1894 at the Möckern Experiment Station. In the earlier series of experiments (including those by G. Kühn, reported by Kellner $\dagger$ ) additions of commercial wheat gluten and of starch were made to a basal ration consisting exclusively of coarse fodder (hay or straw). In the later series of experiments additions of the same substances and of oil and beet molasses on the one hand, and of coarse fodders on the other hand, were made to a mixed basal ration.

* Landw. Vers. Stat., 47, 275; 50, 245; 68, $1 . \quad \dagger I b i d ., ~ 44,257$. 


\begin{tabular}{|c|c|c|c|c|c|c|}
\hline \multirow[b]{2}{*}{$\begin{array}{l}\text { Kind of Food } \\
\text { Material. }\end{array}$} & \multirow{2}{*}{$\begin{array}{c}\text { Nutrients } \\
\text { Furnished } \\
\text { by Each } \\
\text { Group per } \\
100 \text { Grms. } \\
\text { Total. }\end{array}$} & \multirow{2}{*}{$\begin{array}{c}\text { Heats of } \\
\text { Combus- } \\
\text { tion per } \\
\text { Grm. }\end{array}$} & \multirow{2}{*}{$\begin{array}{c}\text { Propor- } \\
\text { tion of } \\
\text { Total } \\
\text { Nutrients } \\
\text { Actually } \\
\text { Avail- } \\
\text { able. }\end{array}$} & \multirow{2}{*}{$\begin{array}{c}\text { Total } \\
\text { Energy } \\
\text { per Grm. } \\
\text { in Avail- } \\
\text { able Nu- } \\
\text { trients. }\end{array}$} & \multicolumn{2}{|c|}{ Fuel Value. } \\
\hline & & & & & $\begin{array}{l}\text { Per Grm. } \\
\text { Available } \\
\text { Nutrients. }\end{array}$ & $\begin{array}{l}\text { Per Grm. } \\
\text { Total } \\
\text { Nutri- } \\
\text { ents. }\end{array}$ \\
\hline $\begin{array}{l}\text { Protein: } \\
\quad \text { Meats, fish, etc ... } \\
\text { Eggs ............ } \\
\text { Dairy products ... }\end{array}$ & $\begin{array}{r}\text { Grms. } \\
43.0 \\
6.0 \\
12.0\end{array}$ & $\begin{array}{l}\text { Cals. } \\
5.65 \\
5.75 \\
5.65\end{array}$ & $\begin{array}{c}\text { Рer Cent. } \\
97 \\
97 \\
97\end{array}$ & $\begin{array}{l}\text { Cals. } \\
5.50 \\
5.60 \\
5.50\end{array}$ & $\begin{array}{l}\text { Cals. } \\
4.40 \\
4.50 \\
4.40\end{array}$ & $\begin{array}{l}\text { Cals. } \\
4.25 \\
4.35 \\
4.25\end{array}$ \\
\hline Animal food.... & 61.0 & 5.65 & 97 & 5.50 & 4.40 & 4.25 \\
\hline $\begin{array}{l}\text { Cereals ........... } \\
\text { Legumes.......... } \\
\text { Vegetables ........ } \\
\text { Fruits .......... }\end{array}$ & $\begin{array}{r}31.0 \\
2.0 \\
5.5 \\
0.5\end{array}$ & $\begin{array}{l}5.80 \\
5.70 \\
5.00 \\
5.20\end{array}$ & $\begin{array}{l}85 \\
78 \\
83 \\
85\end{array}$ & $\begin{array}{l}4.95 \\
4.45 \\
4.15 \\
4.40\end{array}$ & $\begin{array}{l}4.55 \\
4.45 \\
3.75 \\
3.95\end{array}$ & $\begin{array}{l}3.70 \\
3.20 \\
2.90 \\
3.15\end{array}$ \\
\hline Vegetable food & 39.0 & 5.65 & 85 & 4.80 & 4.40 & 3.55 \\
\hline Total food & 100.0 & 5.65 & 92 & 5.20 & 4.40 & 4.00 \\
\hline $\begin{array}{l}\text { Fat : } \\
\text { Meat and eggs .... } \\
\text { Dairy produets } . . .\end{array}$ & $\begin{array}{l}60.0 \\
32.0\end{array}$ & $\begin{array}{l}9.50 \\
9.25\end{array}$ & $\begin{array}{l}95 \\
95\end{array}$ & $\begin{array}{l}9.00 \\
8.80\end{array}$ & $\begin{array}{l}9.50 \\
9.25\end{array}$ & $\begin{array}{l}9.00 \\
8.80\end{array}$ \\
\hline Animal food... & 92.0 & 9.40 & 95 & 8.95 & 9.40 & 8.95 \\
\hline Vegetable food & 8.0 & 9.30 & 90 & 8.35 & 9.30 & 8.35 \\
\hline Total food ..... & 100.0 & 9.40 & 95 & 8.90 & 9.40 & $\mathbf{8 . 9 0}$ \\
\hline 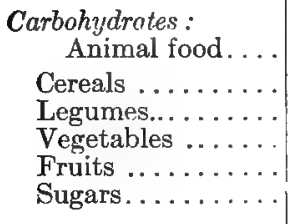 & $\begin{array}{r}5.0 \\
55.0 \\
1.0 \\
13.0 \\
5.0 \\
21.0\end{array}$ & $\begin{array}{l}3.90 \\
4.20 \\
4.20 \\
4.20 \\
4.00 \\
3.95\end{array}$ & $\begin{array}{l}98 \\
98 \\
97 \\
95 \\
90 \\
98\end{array}$ & $\begin{array}{l}3.80 \\
4.10 \\
4.05 \\
4.00 \\
3.60 \\
3.85\end{array}$ & $\begin{array}{l}3.90 \\
4.20 \\
4.20 \\
4.20 \\
4.00 \\
3.95\end{array}$ & $\begin{array}{l}3.80 \\
4.10 \\
4.05 \\
4.00 \\
3.60 \\
3.85\end{array}$ \\
\hline Vegetable food & 95.0 & 4.15 & 97 & 4.00 & 4.15 & 4.00 \\
\hline Total food .. & 100.0 & 4.15 & 97 & 4.00 & 4.15 & 4.00 \\
\hline
\end{tabular}

In each experiment the digestibility of the ration was determined in the usual manner, and also the carbon of food, feces, urine, and respiration (including methane, etc.), and the nitrogen and heats of combustion of food, feces, and urine. The experiments were made with every precaution and extended over a sufficient length of time to ensure normal results. In each experiment the respiratory products were determined in four or five separate periods of twenty-four hours each. No such complete experiments with 
other classes of herbivorous animals have been reported, although partial data are available from experiments on horses and swine.

Method of Stating Results.-The determination of the metabolizable energy of a given ration by experiments like the above is, in principle, very simple, although requiring many appliances and much technical skill. When, however, we attempt to generalize the results much greater difficulties are encountered than in the cases previously considered.

In investigations upon carnivora and upon man the metabolizable energy, as we have just seen, is usually computed upon the total nutrients of the food-that is, upon the total amounts of protein, carbohydrates, and fat-the deduction for the loss of energy in the feces being included in the factors employed. This is possible because the amount of potential energy thus removed is small in itself and subject to relatively small variations on ordinary diet and also because the crude nutrients composing the food are largely chemical compounds which are at least fairly well known.

The food of herbivora, on the contrary, is both more complex and less well known chemically and contains a much larger and very varying proportion of indigestible matter. As a consequence the feces, instead of being chiefly an excretory product, consist mainly of undigested food residues with but a small proportion of metabolic products, and contain a large and variable part of the total potential energy of the food. For all these reasons it seems likely that any attempt to compute general factors for the metabolizable energy of the crude nutrients of feeding-stuffs similar to those of Rubner or Atwater for the nutrients of human foods would be confronted by almost insuperable difficulties.

It was natural, then, to attempt to eliminate these difficulties by computing the results upon the digestible nutrients of the feeding-stuffs, but even here considerable difficulties arise. The digested nutrients, particularly in the case of coarse fodders, are far from being the pure protein, carbohydrates, and fats which our ordinary statements of composition and digestibility assume them to be. Furthermore, a considerable and a variable proportion of the waste of proteid metabolism in the herbivora takes the form of hippuric acid, a body less completely oxidized than urea, and ac- 
cordingly containing more potential energy, while the urine of sheep and cattle also contains not a little non-nitrogenous matter of some sort. Finally, the slow and complicated process of digestion in the herbivora is accompanied by fermentations and the evolution of gaseous hydrocarbons (methane), and perhaps of hydrogen, both of which carry off a more or less variable proportion of the potential energy of the food. By means of experiments with approximately pure nutrients it is possible to secure factors for the metabolizable energy of the digested nutrients of concentrated feeding-stuffs, but in the case of coarse fodders about all that is practicable in this direction is to compute the results of experiments upon the total digestible matter.

There is possible, however, a third method, viz., to compute the metabolizable energy upon the total organic matter of the feedingstuff, expressing it either as Calories per gram or pound of organic matter or as a percentage of the gross energy. In the latter form the result would be analogous to a digestion coefficient and would show what proportion of the total energy of the material, as determined by combustion in the calorimeter, was capable of being metabolized in the body. This method of expressing the results has certain advantages in directness and simplicity, and especially in putting the whole matter on the basis of energy values. In the succeeding paragraphs the available data will be considered from both the standpoints last named.

METABOLIZABLE ENERGY OF ORGANIC MATTER.

For a discussion of the matter from this standpoint we have to rely almost entirely upon the Möckern investigations already mentioned. In the case of those earlier experiments in which the ration consisted exclusively of a single coarse fodder the computation of the metabolizable energy of the latter is, of course, readily made. In the experiments in which the food under investigation was added to a basal ration the computation is somewhat less simple, it being then necessary to compare the gross energy of the added food with the increase in the energy of the excreta in the second period as compared with the first. The details of both methods will be best explained by illustration. 
Total Organic Matter.

Coarse Fodders. Fed Alone.-For Ox H, fed exclusively on meadow hay, Kellner obtained the following results* per day and head:

Ingesta.

$7,263 \dagger$ grams meadow hay....... $\quad 32,177.3$ Cals. Excreta.

$2,547 \dagger$ grams feces..... 11,750.3 Cals.

13,675 " urine...... $1,945.0$ "

158.4 " methane.... 2,113.7 "

Total excreta................ 15,809.0 "

Difference................. 16,368.3 "

Had the ration exactly sufficed for the maintenance of the animal, the difference of $16,368.3$ Cals. would represent exactly its metabolizable energy. In reality, however, the nitrogen and carbon balance indicated a gain by the animal of 37.2 grams of protein $(\mathrm{N} \times 6.00 \ddagger)$ and 140.8 grams of fat, equivalent to 1548.8 Cals., so that the amount of energy actually converted into the kinetic form was $16,368.3-1548.8=14,819.5$ Cals. The potential energy of the 140.8 grams of fat, however, while it was not actually rendered kinetic, might have been had the needs of the organism required it. Its retention in the potential form was, in a sense, temporary and accidental, and its energy should properly be considered as a part of the metabolizable energy of the food.

With the gain of protein, however, the case is different. Its total potential energy equals 211.2 Cals., but not all of this is capable of conversion into kinetic energy. According to Rubner's results (p. 275) each gram of urinary nitrogen derived from the metabolism of the protein of lean meat corresponds to 7.45 Cals. If this result is applicable to the forms of protein consumed by herbivora (and we shall see later that there is good reason to believe that such is approximately the case), then the metabolism of the 37.2 grams of protein gained would have added 46.2 Cals. to the observed potential energy of the urine, while the remaining 165 Cals. would have taken the kinetic form and should, therefore, be regarded as part of the metabolizable energy of the food.
* Loc, cit., 53, 9 .
$\dagger$ Dry matter.
$\ddagger$ Compare pp. 67,68 . 
In other words, to get at the actual metabolizable energy of the ration in this experiment we must add to the observed potential energy of the urine the amount of $46.2 \mathrm{Cals}$. by which it would have been increased had all the protein of the food been metabolized, or, what is the same thing, must subtract this amount from the observed difference between food and excreta. This leaves 16,322.1 Cals. as the metabolizable energy of 7263 grams of dry matter or 6750 grams of organic matter in meadow hay, and the metabolizable energy per gram of organic matter is therefore 2.418 Cals.

Computed in the above manner, the several experiments of this category gave per day and head the following results:

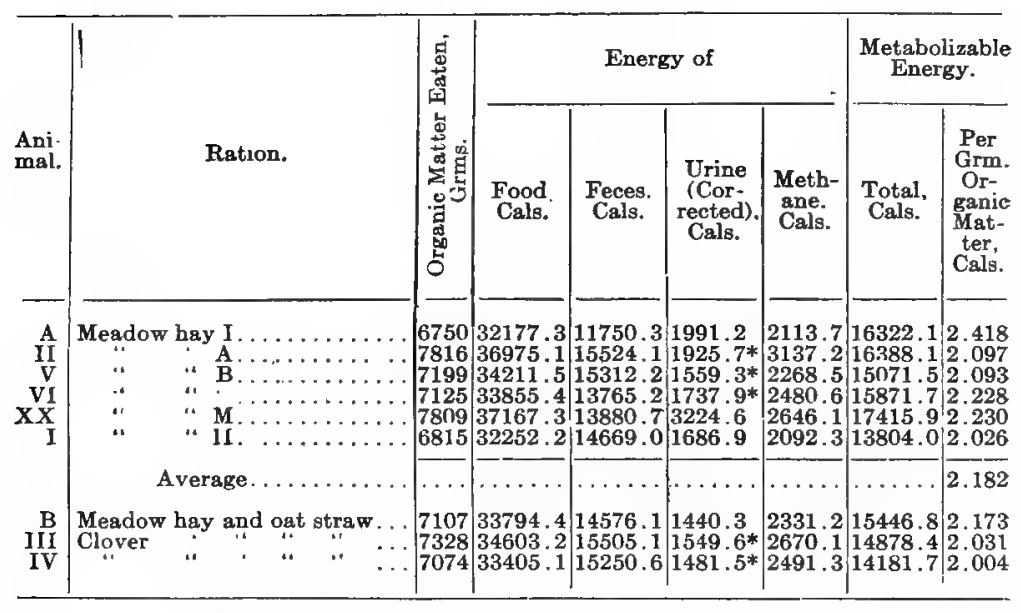

* Energy of urine computed from its carbon content.

It should be noted that the figures for the energy of the feces in these and in all the succeeding experiments include that of the metabolic products contained in them. While the latter are not derived directly from the food they are a part of the expenditure made by the body in the digestion of the food, and there is. therefore, the same reason for including their energy as for including that of the organic matter of the urine.

Both contain a certain amount of potential energy, derived ultimately from the food, which has escaped being metabolized in 
the body and so is to be deducted from the total energy of the food to obtain its metabolizable energy.

Experiments on timothy hay made by the writer,* in which the amount of methane excreted was estimated from the amount of nonnitrogenous nutrients digested, gave the following results, the correction for the gain or loss of nitrogen being computcd in a slightly different way from that explained above:

ENERGY PER GRAM ORGANIC MATTER.

\begin{tabular}{|c|c|c|c|}
\hline & $\underset{\text { Cals }}{\text { Experiment I. }}$ & $\begin{array}{c}\text { Experiment II. } \\
\text { Cals. }\end{array}$ & $\begin{array}{c}\text { Experiment VI. } \\
\text { Cals. }\end{array}$ \\
\hline \multirow{3}{*}{ 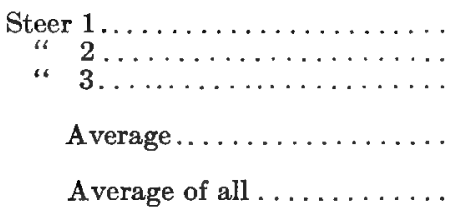 } & $\begin{array}{l}2.104 \\
2.007 \\
1.904\end{array}$ & $\begin{array}{l}1.838 \\
2.164 \\
1.824\end{array}$ & $\begin{array}{l}2.139 \\
2.175 \\
2.176\end{array}$ \\
\hline & 2.005 & 1.942 & 2.163 \\
\hline & \multicolumn{3}{|c|}{2.037} \\
\hline
\end{tabular}

It should be noted that the above figures are, as already stated, approximate only. The energy of the methane was estimated, while the determinations of the energy of the urine were not, in all cases, satisfactory. We are probably justified, however, in regarding the results as a close approximation to the truth.

Coarse Fodders Added to Basal Ration.-As an example of this class of experiments we may take Periods 4 and 7 with Ox H. The rations in the two periods were as follows:

\begin{tabular}{|c|c|c|c|c|c|}
\hline & \multicolumn{2}{|c|}{ Total Weight. } & \multicolumn{3}{|c|}{ Containing Organic Matter. } \\
\hline & $\begin{array}{c}\text { Period } 4 \\
\text { Kgs. }\end{array}$ & $\begin{array}{c}\text { Period 7, } \\
\text { Kgg. }\end{array}$ & $\begin{array}{c}\text { Period 4, } \\
\text { Grms. }\end{array}$ & $\begin{array}{c}\text { Period 7, } \\
\text { Grms. }\end{array}$ & $\begin{array}{l}\text { Difference, } \\
\text { Grms. }\end{array}$ \\
\hline 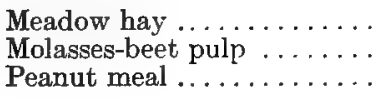 & $\begin{array}{l}4 \\
3 \\
1\end{array}$ & $\begin{array}{l}8 \\
3 \\
1\end{array}$ & $\begin{array}{r}3198 \\
2386 \\
818\end{array}$ & $\begin{array}{r}6495 \\
2413 \\
835\end{array}$ & $\begin{array}{r}3297 \\
27 \\
17\end{array}$ \\
\hline & 8 & 12 & 6402 & 9743 & 3341 \\
\hline
\end{tabular}

* Penna State Experiment Station, Bull 42, p. 153.

$\dagger$ Loc cit., 53, 278-335. 
The potential energy of food and excreta (that of the urine corrected to nitrogen equilibrium) and by difference the amounts of metabolizable energy were:

\begin{tabular}{|c|c|c|c|c|c|}
\hline & $\begin{array}{l}\text { Food, } \\
\text { Cals. }\end{array}$ & $\begin{array}{l}\text { Feces, } \\
\text { Cals. }\end{array}$ & $\begin{array}{c}\text { Urine } \\
\text { (Corrected), } \\
\text { Cals. }\end{array}$ & $\begin{array}{l}\text { Methane, } \\
\text { Cals. }\end{array}$ & $\begin{array}{l}\text { Metaboliz- } \\
\text { able Energy. } \\
\text { Cals. }\end{array}$ \\
\hline $\begin{array}{c}\text { Period } 7 \ldots \ldots \ldots \\
4 \quad 4 \ldots \ldots\end{array}$ & $\begin{array}{l}46,275.0 \\
30,338.1\end{array}$ & $\begin{array}{r}14,104.8 \\
8,574.9\end{array}$ & $\begin{array}{l}2,593.0 \\
1,795.0\end{array}$ & $\begin{array}{l}3,564.2 \\
2,579.4\end{array}$ & $\begin{array}{l}26,013.0 \\
17,388.8\end{array}$ \\
\hline Difference .... & $15,936.9$ & $5,529.9$ & 798.0 & 984.8 & $8,624.2$ \\
\hline
\end{tabular}

The metabolizable energy of the additional 3341 grams of organic matter eaten in Period 7 was therefore 8624.2 Cals. This added food was intended to consist of hay, but the unavoidable variations in the moisture content of the feeding-stuffs resulted in a slightly greater consumption of the other ingredients of the ration also. Of the 3341 grams of additional organic matter, 3297 grams, as the previous table shows, were from the hay and 44 grams from the basal ration. If, then, we would ascertain the metabolizable energy of the added hay only, we must subtract from the difference of 8624.2 Cals. between the two rations the metabolizable energy of this 44 grams of organic matter from the other feeding-stuffs.

But while the gross energy of the latter is known, its metabolizable energy cannot be computed exactly, since it is impossible to determine what part of the energy of the excreta was derived from this particular portion of the ration. By assuming, however, that the same percentage of its gross energy was metabolizable as was the case with the basal ration, and that its non-metabolizable energy was sinilarly distributed between the various excreta, we may compute a correction which, although not strictly accurate, will not, in view of the small quantities involved, introduce any serious error. In this case the gross energy of the 3297 grams of organic matter in the added hay was 15,728.6 Cals., and the table takes the form shown on the opposite page.

As thus computed, the metabolizable energy of the 3297 grams of organic matter added to the basal ration in the form of hay was 8504.8 Cals., equal to 2.580 Cals. per gram. The total correction amounts to 119.4 Cals., and even a considerable relative error in it would not materially change the final results. 


\begin{tabular}{|c|c|c|c|c|c|}
\hline & $\begin{array}{l}\text { Food, } \\
\text { Cals. }\end{array}$ & $\begin{array}{l}\text { Feces, } \\
\text { Cals. }\end{array}$ & $\begin{array}{c}\text { Urine } \\
\text { (Corrected), } \\
\text { Cals. }\end{array}$ & $\begin{array}{l}\text { Methane, } \\
\text { Cals. }\end{array}$ & $\begin{array}{l}\text { Metaboliz- } \\
\text { able Energy, } \\
\text { Cals. }\end{array}$ \\
\hline $\begin{array}{c}\text { Period } 7 \ldots \ldots \ldots \\
4 \ldots \ldots\end{array}$ & $\begin{array}{l}46,275.0 \\
30,338.1\end{array}$ & $\begin{array}{r}14,104.8 \\
8,574.9\end{array}$ & $\begin{array}{l}2,593.0 \\
1,795.0\end{array}$ & $\begin{array}{l}3,564.2 \\
2,579.4\end{array}$ & $\begin{array}{l}26,013.0 \\
17,388.8\end{array}$ \\
\hline $\begin{array}{l}\text { Difference.... } \\
\text { Correction ... . }\end{array}$ & $\begin{array}{r}15,936.9 \\
-208.3\end{array}$ & $\begin{array}{r}5,529.9 \\
-58.9\end{array}$ & $\begin{array}{r}798.0 \\
-12.3\end{array}$ & $\begin{array}{r}984.8 \\
-17.7\end{array}$ & $\begin{array}{l}8,624.2 \\
-119.4\end{array}$ \\
\hline Percentages... & $\begin{array}{r}15,728.6 \\
100.0\end{array}$ & $\begin{array}{r}5,471.0 \\
34.78\end{array}$ & $\begin{array}{r}785.7 \\
5.00\end{array}$ & $\begin{array}{r}967.1 \\
6.15\end{array}$ & $\begin{array}{r}8,504.8 \\
54.07\end{array}$ \\
\hline
\end{tabular}

In these computations it is assumed that the increased metabolizable energy of the ration is derived entirely from the added feeding-stuff, or, in other words, that the latter exerted no influence either upon the digestibility of the basal ration or upon the proportion of its energy lost in urine and in hydrocarbons. That such is the case we have no means of proving, and it is, indeed, unlikely that it is exactly true. The metabolizable energy of the added feeding-stuff as above computed includes any such effects-that is, it represents the net result to the organism of the added coarse fodder.

Table I of the Appendix contains the results of all the experiments of this sort, computed in the manner illustrated above. It will be noted that in all but two cases the correction is less than in the above example. In each case the table shows also the percentage of the gross energy of the feeding-stuff which was found to be metabolizable and the percentage carried off in each of the excreta.

Summary. - The results of the foregoing determinations of the metabolizable energy of the organic matter of coarse fodders are summarized in the table on page 290, which shows the gross and metabolizable energy per gram of organic matter and also the percentage of gross energy found to be metabolizable.

Concentrated Feeding-stuffs.-The metabolizable energy of the organic matter of a concentrated feeding-stuff when added to a basal ration can, of course, be computed by the same method as in the case of added coarse fodders, but, as we shall see, some special difficulties arise in its application.

The only commercial concentrated feeding-stuff upon which such experiments have been reported is beet molasses, although 


\begin{tabular}{|c|c|c|c|}
\hline & \multicolumn{2}{|c|}{$\begin{array}{l}\text { Per Gram Organic } \\
\text { Matter. }\end{array}$} & \multirow[b]{2}{*}{$\begin{array}{l}\text { Per Cent. } \\
\text { Metaboliz } \\
\quad \text { able. }\end{array}$} \\
\hline & $\begin{array}{l}\text { Gross } \\
\text { Energy, } \\
\text { Cals. }\end{array}$ & $\begin{array}{l}\text { Metaboliz- } \\
\text { able } \\
\text { Energy, } \\
\text { Cals. }\end{array}$ & \\
\hline 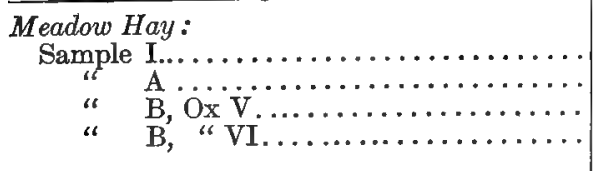 & $\begin{array}{l}4.767 \\
4.731 \\
4.752 \\
\}\end{array}$ & $\begin{array}{l}2.418 \\
2.097 \\
2.093 \\
2.228\end{array}$ & $\begin{array}{l}50.72 \\
44.32 \\
44.06 \\
46.88\end{array}$ \\
\hline 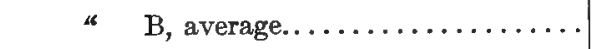 & $\ldots \ldots \ldots$ & 2.161 & 45.47 \\
\hline 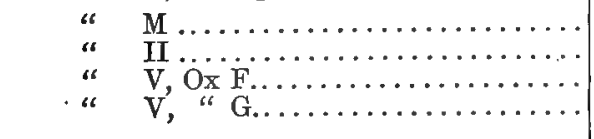 & $\left\{\begin{array}{c}4.760 \\
4.734 \\
4.743\end{array}\right.$ & $\begin{array}{l}2.230 \\
2.026 \\
1.933 \\
2.087\end{array}$ & $\begin{array}{l}46.86 \\
42.80 \\
40.75 \\
44.00\end{array}$ \\
\hline " $\quad \mathrm{v}$, average................. & 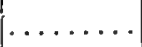 & 2.010 & 42.38 \\
\hline 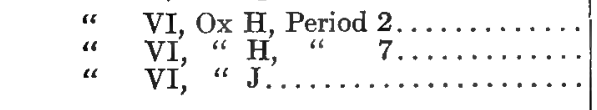 & $\{4.771\}$ & $\begin{array}{l}2.520 \\
2.580 \\
2.540\end{array}$ & $\begin{array}{l}52.82 \\
54.07 \\
53.24\end{array}$ \\
\hline “ VI, average................... & & 2.547 & 53.38 \\
\hline Average of seven samples $\ldots \ldots \ldots \ldots$ & 4.751 & 2.213 & 46.56 \\
\hline Timothy Hay (approximate) .... & 4.670 & 2.037 & 43.62 \\
\hline 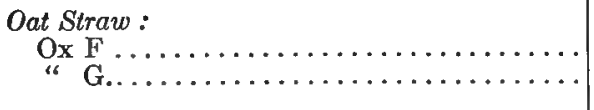 & 4.816\{ & $\begin{array}{l}1.760 \\
1.688\end{array}$ & $\begin{array}{l}36.54 \\
35.05\end{array}$ \\
\hline 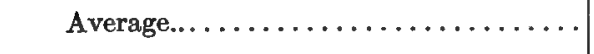 & $\ldots \ldots \ldots$ & 1.724 & 35.80 \\
\hline 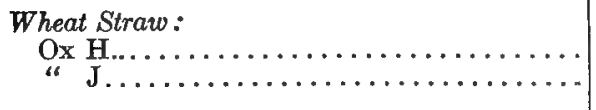 & \} 4.743\{ & $\begin{array}{l}1.411 \\
1.540\end{array}$ & $\begin{array}{l}29.75 \\
32.47\end{array}$ \\
\hline Average............................. & $\ldots \ldots \ldots$ & 1.475 & 31.11 \\
\hline 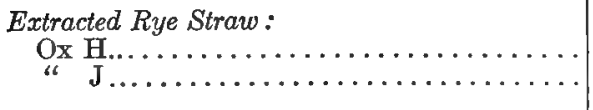 & \} 4.251\{ & $\begin{array}{l}3.261 \\
3.164\end{array}$ & $\begin{array}{l}76.71 \\
74.45\end{array}$ \\
\hline Average...................... & & 3.213 & 75.58 \\
\hline
\end{tabular}

experiments were also made by Kellner with wheat gluten, starch, oil, and extracted straw, the aim of which was to determine the metabolizable energy of the various digestible nutrients.

As an illustration of this class of experiments we may take one upun molasses with $\mathrm{Ox} \mathrm{F}$,* comparing Period 3 , on the basal ration,

* Loc, cit., 53, 172-227. 
with Period 6, on the same ration with the addition of molasses. Comparing, first, the organic matter of the two rations we have the following:

\begin{tabular}{|c|c|c|}
\hline & $\begin{array}{c}\text { Total Organic } \\
\text { Matter Fed, } \\
\text { Grms. }\end{array}$ & $\begin{array}{c}\text { Organic Matter in } \\
\text { Molasses, } \\
\text { Grms. }\end{array}$ \\
\hline 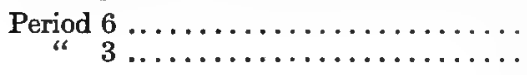 & $\begin{array}{l}8262 \\
6630\end{array}$ & $\begin{array}{r}1702 \\
0\end{array}$ \\
\hline & 1632 & 1702 \\
\hline
\end{tabular}

In the period with molasses 70 grams less of the basal ration was consumed than in the period without, and a correction must accordingly be made for this in the way explained on page 288 . The energy of food and excreta in the two experiments (that of the urine being corrected to nitrogen equilibrium), together with the correction for the 70 grams of organic matter, is shown in the following table:

\begin{tabular}{|c|c|c|c|c|c|}
\hline & $\begin{array}{l}\text { Food, } \\
\text { Cals. }\end{array}$ & $\begin{array}{l}\text { Feces, } \\
\text { Cals. }\end{array}$ & $\begin{array}{l}\text { Urine, } \\
\text { Cals. }\end{array}$ & $\begin{array}{l}\text { Methane, } \\
\text { Cals. }\end{array}$ & $\begin{array}{l}\text { Metaboliz- } \\
\text { able Energy, } \\
\text { Cals. }\end{array}$ \\
\hline \multirow[t]{2}{*}{$\begin{array}{c}\text { Period } 6 \ldots \ldots \ldots \\
3 \ldots \ldots \ldots\end{array}$} & $\begin{array}{l}37,946.2 \\
31,327.8\end{array}$ & $\begin{array}{r}11,365.8 \\
9,599.2\end{array}$ & $\begin{array}{l}1,786.1 \\
1,530.0\end{array}$ & $\begin{array}{l}2,397.9 \\
2,560.7\end{array}$ & $\begin{array}{l}22,396.4 \\
17,637.9\end{array}$ \\
\hline & $\begin{array}{r}6,618.4 \\
+330.8\end{array}$ & $\begin{array}{r}1,766.6 \\
+101.3\end{array}$ & $\begin{array}{r}256.1 \\
+16.2\end{array}$ & $\begin{array}{r}-162.8 \\
+\quad 27.0\end{array}$ & $\begin{array}{r}4,758.5 \\
+186.3\end{array}$ \\
\hline Correction .... & $6,949.2$ & $1,867.9$ & 272.3 & -135.8 & $4,944.8$ \\
\hline
\end{tabular}

Dividing the metabolizable energy of the molasses, 4944.8 Cals., by the number of grams consumed, 1702 , gives the metabolizable energy of 1 gram of organic matter as 2.905 Cals.

Real and Apparent Metabolizable Enfrgy.-The above figures, however, demand more critical discussion. While the addition of molasses to the basal ration increased the amount of potential energy carried off in the feces and urine, it diminished that in the methane; that is, it acted in some way to check the fermentation in the digestive tract to which this gas owes its origin. In other words, under the influence of the molasses the loss of energy by fermentation of the basal ration was diminished by 135.8 Cals., and this amount, by the method of computation, is added to the metabolizable energy of the molasses. 
Moreover, the loss of energy in the feces is a complex of several factors. The amounts of organic matter and of the several nutrients excreted in the feces in the two periods (not corrected for the 70 grams difference in organic matter consumed) were as follows:

\begin{tabular}{|c|c|c|c|c|c|}
\hline & $\begin{array}{l}\text { Organic } \\
\text { Matter, } \\
\text { Grms. }\end{array}$ & $\begin{array}{l}\text { Protein, } \\
\text { Grms. }\end{array}$ & $\begin{array}{l}\text { Crude } \\
\text { Fiber, } \\
\text { Grms. }\end{array}$ & $\begin{array}{c}\text { Nitrogen- } \\
\text { free } \\
\text { Extract, } \\
\text { Grms. }\end{array}$ & $\begin{array}{c}\text { Crude } \\
\text { Fat, } \\
\text { Grms. }\end{array}$ \\
\hline $\begin{array}{c}\text { Period } 6 \ldots \ldots \ldots \ldots \ldots \ldots \\
\longleftarrow \quad 3 \ldots \ldots \ldots \ldots \ldots\end{array}$ & $\begin{array}{l}2132 \\
1797\end{array}$ & $\begin{array}{l}403 \\
284\end{array}$ & $\begin{array}{l}595 \\
527\end{array}$ & $\begin{array}{r}1068 \\
924\end{array}$ & $\begin{array}{l}66 \\
62\end{array}$ \\
\hline Difference............ & 335 & 119 & 68 & 144 & 4 \\
\hline
\end{tabular}

In addition to protein and nitrogen-free extract, which may possibly represent indigestible material in the molasses, the feces contained 68 grams more crude fiber and 4 grams more fat in Period 6 than in Period 3. These cannot have been derived from the molasses, since the latter does not contain these ingredients. This feeding-stuff, in other words, diminished the apparent digestibility of the fiber and fat of the basal ration. As a matter of fact, the ingredients of molasses being practically all soluble in water, it is probable that nearly all the difference in the amount digested is due to the diminished apparent digestibility of the basal ration under the influence of the molasses.

The figure above given for the metabolizable energy includes all these effects; that is, it shows the net result as regards energy obtained from molasses fed under the conditions of these experiments, the nutritive ratio of the basal ration being $1: 5.8$ and that of the molasses ration $1: 6.4$. To get at the actual amount of energy set free from the molasses itself we should need to subtract from the metabolizable energy as calculated above the energy corresponding to the decreased excretion of methane and to add to it the metabolizable encrgy corresponding to the decrease in the amounts of crude fiber and ether extract digested, assuming that all the excess of protein and nitrogen-free extract in the feces was derived from the molasses. Computed in this way* the real metabolizable energy

$*$ One gram of crude fiber $=3.3$ Cals., and one gram of ether extract $=$ 8.3 Cals. See p. 332. 
of the organic matter is 2.977 Cals. per gram. This would be a minimum figure, while if we assume, as suggested above, that the molasses is entirely digestible, this figure is still too low and should be increased to equal the gross energy of the organic matter.

If, however, either one of these latter values were used in computing the metabolizable energy of rations, the results would obviously be too high unless corrections were made for the effect upon the apparent digestibility of the other feeding-stuffs in the ration. The figure first computed, while including several different effects, nevertheless seems better adapted for use in actual computations under average conditions, while the second gives the more accurate idea of the store of metabolizable energy contained in the feedingstuff regarded by itself. The distinction is analogous to that between apparent and real digestibility, and we may accordingly speak of the apparent and the real metabolizable eniergy of feedingstuffs.

The whole of our present discussion of the metabolizable energy of the organic matter (total or digestible) of food materials relates to the apparent metabolizable energy. This is obvious as regards the concentrated feeds from the above example, and logically applies also to those cases in which coarse fodders were added to the basal ration, while in the case of the coarse fodders used alone the distinction vanishes or is reduced to one between apparent and real digestibility. The experiment with beet molasses well illustrates the difficulties in the way of determining the actual metabolizable energy of feeding-stuffs which cannot be used alone.

BeEt Molasses.-In two later experiments the addition of molasses increased instead of diminishing the excretion of methane. The results of the three experiments upon molasses, computed in the same manner as the experiments upon coarse fodders, are contained in Table II of the Appendix.

In the last two experiments 10 to 12 per cent. of the energy of the molasses was lost in the products of intestinal fermentation, but this was more than counterbalanced by its less effect upon the digestibility of the rations, so that the final result is a higher figure for the apparently metabolizable energy than in the first experiment. Summarizing the results per gram as in the case of the coarse fodders we have: 


\begin{tabular}{|c|c|c|c|c|}
\hline ' & $\begin{array}{l}\text { Gross } \\
\text { Energy, } \\
\text { Cals. }\end{array}$ & & $\begin{array}{l}\text { Apparently } \\
\text { Metabolizable } \\
\text { Energy, } \\
\text { Cals. }\end{array}$ & $\begin{array}{c}\text { Per Cent } \\
\text { Metabolizable. }\end{array}$ \\
\hline$\underset{*}{\text { Sample }} \underset{*}{\mathrm{I}} \ldots \ldots \ldots \ldots \ldots$ & $\begin{array}{r}4.084 \\
4.188\end{array}$ & \{ & $\begin{array}{l}2.905 \\
3.308 \\
3.044\end{array}$ & $\begin{array}{l}71.16 \\
79.00 \\
72.70\end{array}$ \\
\hline Average, Sample II ....... & $\ldots \ldots \ldots$ & & 3.176 & 75.85 \\
\hline
\end{tabular}

STARCH.-In a considerable number of the trials commercial starch was added to the basal ration. The earlier experiments by Kühn were intended primarily to throw light on the possible formation of fat from carbohydrates (compare p. 177). In them, starch was added to a ration of coarse fodder only and the nutritive ratio was purposely made very wide, the result being that more or less of the starch escaped digestion. In the later experiments by Kellner the starch was added to a mixed ration. Except in the first two experiments the nutritive ratio was a medium one and but traces of starch escaped digestion. It will be convenient, therefore, to tabulate these two classes of experiments separately, as has been done in Tables III and IV of the Appendix, the computations being made as in the previous cases.

The same remarks which were made on p. 291 concerning the distinction between real and apparent metabolizable energy apply to these results. As computed they represent the net gain to the organism from the consumption of starch and are the algebraic sum of several factors. In particular, there was a considerable loss of energy in the feces, even in the later experiments in which but traces of the starch itself escaped digestion. In other words, the starch either lowered the digestibility of the basal ration or increased the formation of fecal metabolic products or both. The method of computation arlopted virtually looks upon this as part of the necessary expenditure in the digestion of the starch. On the other hand, there are several cases in which there was a decrease in the outgo of potential energy in the urine, even after the results are corrected to nitrogen equilibrium. This, from our present point of view, is credited to the starch and increases its apparent metabolizable energy. 
The results on starch, expressed in Calories per gram of organic matter, may be summarized as follows:

\begin{tabular}{|c|c|c|c|}
\hline & $\begin{array}{l}\text { Gross } \\
\text { Energy, } \\
\text { Cals. }\end{array}$ & $\begin{array}{l}\text { Apparent } \\
\text { Metaboliz- } \\
\text { able } \\
\text { Energy, } \\
\text { Cals. }\end{array}$ & $\begin{array}{l}\text { Per Cent. } \\
\text { Metaboliz- } \\
\text { able. }\end{array}$ \\
\hline $\begin{array}{l}\text { Kühn's Experiments : } \\
\quad \text { Sample I, Ox III. } \ldots \ldots \ldots \ldots \ldots \ldots \ldots \ldots \ldots \ldots \ldots \ldots \ldots \ldots \ldots \ldots \ldots \ldots\end{array}$ & $\begin{array}{l}4.249 \\
4.249\end{array}$ & $\begin{array}{l}3.029 \\
2.705\end{array}$ & $\begin{array}{l}71.21 \\
63.71\end{array}$ \\
\hline Average... . . & 4.249 & 2.867 & 67.46 \\
\hline 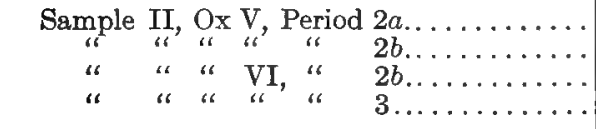 & $\begin{array}{l}4.236 \\
4.236 \\
4.236 \\
4.236\end{array}$ & $\begin{array}{l}3.347 \\
3.161 \\
3.018 \\
2.964\end{array}$ & $\begin{array}{l}78.95 \\
74.68 \\
71.26 \\
69.98\end{array}$ \\
\hline Average.............. & 4.236 & 3.123 & 73.72 \\
\hline Average of I and II...... & 4.243 & 2.995 & 70.59 \\
\hline 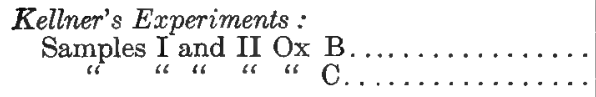 & $\begin{array}{l}4.165 \\
4.165\end{array}$ & $\begin{array}{l}2.027 \\
2.028\end{array}$ & $\begin{array}{l}48.62 \\
48.68\end{array}$ \\
\hline Average... . & 4.165 & 2.028 & 48.65 \\
\hline 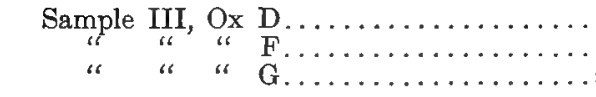 & $\begin{array}{l}4.156 \\
4.156 \\
4.156\end{array}$ & $\begin{array}{l}2.792 \\
2.969 \\
3.214\end{array}$ & $\begin{array}{l}67.20 \\
71.44 \\
77.32\end{array}$ \\
\hline Average........ & 4.151 & 2.992 & 71.99 \\
\hline 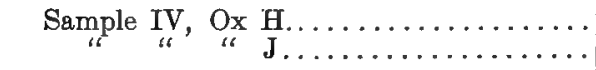 & $\begin{array}{l}4.180 \\
4.180\end{array}$ & $\begin{array}{l}3.313 \\
3.017\end{array}$ & $\begin{array}{l}79.22 \\
72.16\end{array}$ \\
\hline Average.... . & 4.180 & 3.165 & 75.69 \\
\hline Average of III and IV. & 4.168 & 3.079 & 73.84 \\
\hline
\end{tabular}

Wheat GLuten.-Seven experiments upon commercial wheat gluten are reported. three by Kühn and four by Kellner. The chemical composition of the dry matter of the three samples of gluten employed is shown in the first table on the next page.

In Kühn's experiments the gluten caused a marked increase in the apparent digestibility of the basal ration, which by our method of computation augments the apparent metabolizable energy of the gluten, so that in one case it amounts to over 101 per cent. of the gross energy. The correction for organic matter is also rela- 


\begin{tabular}{|c|c|c|c|}
\hline & \multirow{2}{*}{$\begin{array}{l}\text { Kühn's } \\
\text { Experiments, } \\
\text { Per Cent. }\end{array}$} & \multicolumn{2}{|c|}{ Kellner's Experiments. } \\
\hline & & $\begin{array}{c}\text { Oxen B and C, } \\
\text { Per Cent. }\end{array}$ & $\begin{array}{l}\text { Ox D } \\
\text { Per Cent. }\end{array}$ \\
\hline \multirow[t]{2}{*}{ 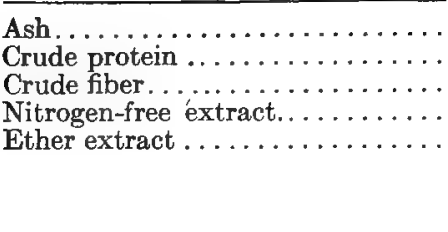 } & $\begin{array}{r}1.36 \\
87.88 \\
0.47 \\
8.07 \\
2.22\end{array}$ & $\begin{array}{r}2.86 \\
83.45 \\
0.08 \\
13.35 \\
0.26\end{array}$ & $\begin{array}{r}2.80 \\
82.67 \\
0.43 \\
13.38 \\
0.72\end{array}$ \\
\hline & 100.00 & 100.00 & 100.00 \\
\hline
\end{tabular}

tively large. In Kellner's experiments the variations are not so great. Computed as before, the results are as shown in Table $\mathrm{V}$ of the Appendix. Summarizing Kellner's figures, as probably the more accurate, we have per gram of organic matter-

\begin{tabular}{|c|c|c|c|}
\hline & $\begin{array}{c}\text { Gross Energy. } \\
\text { Cais. }\end{array}$ & $\begin{array}{l}\text { Apparent } \\
\text { Metabolizable } \\
\text { Energy. } \\
\text { Cals. }\end{array}$ & $\begin{array}{c}\text { Per Cent. } \\
\text { Metabojzable. }\end{array}$ \\
\hline 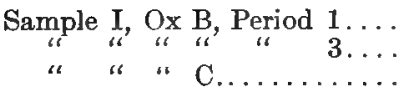 & $\begin{array}{l}5.675 \\
5.675 \\
5.675\end{array}$ & $\begin{array}{l}3.019 \\
3.719 \\
4.062\end{array}$ & $\begin{array}{l}53.18 \\
65.55 \\
71.61\end{array}$ \\
\hline Average.............. & 5.675 & 3.600 & 63.45 \\
\hline Sample II, Ox D.. & 5.808 & 4.061 & 69.90 \\
\hline Average of I and II & 5.742 & 3.831 & 66.68 \\
\hline
\end{tabular}

The wheat gluten was by no means pure protein and the above figures of course apply to the feeding-stuff as a whole, including its fat and carbohydrates as well as its protein. The question of the metabolizable energy of the latter w]ll be considered subsequently.

Peanut Oil.-Three experiments with this substance are reported by Kellner. In the first the oil was given in the form of an emulsion, prepared by saponifying a small portion of the oil with sodium hydrate, and was completely digested In the second and third experiments it was emulsified with lime-water. In this form it was less well digested. and in one case ( $\mathrm{Ox} \mathrm{F}$ ) affected the digestibility of the basal ration unfavorably. The results per gram of organic matter, computed as before, constitute Table VI of the Appendix and are summarized in the following table: 


\begin{tabular}{|c|c|c|c|}
\hline & $\begin{array}{c}\text { Gross Energy, } \\
\text { Cals. }\end{array}$ & $\begin{array}{c}\text { Metabolizable } \\
\text { Energy, } \\
\text { Cals. }\end{array}$ & $\begin{array}{l}\text { Per Cent. } \\
\text { Metabolizable. }\end{array}$ \\
\hline 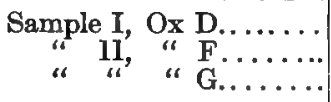 & $\begin{array}{l}9.493 \\
9.464\end{array}$ & $\begin{array}{l}7.382 \\
4.973 \\
5.623\end{array}$ & $\begin{array}{l}77.76 \\
52.52 \\
59.39\end{array}$ \\
\hline Average, II... & $\ldots \ldots \ldots \ldots$ & 5.298 & 55.96 \\
\hline
\end{tabular}

SUMMARY.-The foregoing results may be conveniently summarized in the table below, which shows the average gross energy per gram of organic matter, the percentage of this gross energy carried off unmetabolized in the various excreta, and the apparent metabolizable energy expressed both per gram of total organic matter and as a percentage of the gross energy:

\begin{tabular}{|c|c|c|c|c|c|c|}
\hline & \multirow{2}{*}{$\begin{array}{c}\text { Gross } \\
\text { En'gy } \\
\text { per } \\
\text { Grm. } \\
\text { Or- } \\
\text { ganic } \\
\text { Mat- } \\
\text { ter. } \\
\text { Cals. }\end{array}$} & \multicolumn{3}{|c|}{ Percentage Loss in } & \multicolumn{2}{|c|}{$\begin{array}{l}\text { Apparent } \\
\text { Metabolizable } \\
\text { Energy. }\end{array}$} \\
\hline & & Feces. & Urine. & Methane. & $\begin{array}{c}\text { Per } \\
\text { Grm. } \\
\text { Or- } \\
\text { ganic } \\
\text { Mat- } \\
\text { ter. } \\
\text { Cals. }\end{array}$ & $\begin{array}{c}\text { Per } \\
\text { Cent. } \\
\text { of } \\
\text { Grosg } \\
\text { En'gy. }\end{array}$ \\
\hline Meadow hay........ & 4.751 & 40.96 & 5.71 & 6.77 & 2.213 & 46.56 \\
\hline Timothy hay. & 4.670 & 47.27 & 2.61 & $6.50 *$ & 2.037 & 43.62 \\
\hline Oat straw... & 4.816 & 56.80 & 2.08 & 5.32 & 1.724 & 35.80 \\
\hline Wheat straw... & 4.743 & 58.22 & 2.37 & 8.30 & 1.475 & 31.11 \\
\hline Extracted rye straw..... & 4.251 & 12.75 & -0.79 & 12.46 & 3.213 & 75.58 \\
\hline Beet molasses, Sample II. .... & 4.188 & 9.93 & 2.91 & 11.31 & 3.174 & 75.85 \\
\hline $\begin{array}{l}\text { Starch, Kühn's experiments. . . . } \\
\text { Kellner's experiments: }\end{array}$ & 4.243 & 19.59 & -0.92 & 10.74 & 2.995 & 70.59 \\
\hline Heavy rations............ & 4.165 & 55.91 & -2.07 & -2.49 & 2.028 & 48.65 \\
\hline Medium rations. . . . . . . . . . & 4.168 & 17.61 & -0.66 & 9.21 & 3.079 & 73.84 \\
\hline 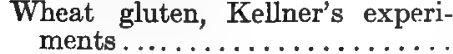 & 5.742 & 20,16 & 1308 & 0.08 & 3.831 & 66.68 \\
\hline 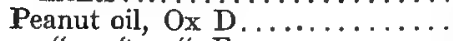 & 9.493 & 24.34 & -1.08 & -1.02 & 7.382 & 77.76 \\
\hline " " $\quad$ F.. & 9.464 & 64.77 & -1.19 & -16.10 & 4.973 & 52.52 \\
\hline " $"$ "G.. & 9.464 & 41.00 & 1.37 & -1.76 & 5.623 & 59.39 \\
\hline
\end{tabular}

* Estimated.

\section{Digestible Organic Matter.}

As appears especially from the figures of the last table, the loss of energy in the feces is the one which is subject to the greatest variation. In other words, the digestibility of a feeding-stuff is the 
most important single factor in determining its content of metabolizable energy. We may eliminate this factor by computing, on the basis of the determinations of digestibility, the energy of the digested organic matter and the proportion of this energy which was lost in urine and methane or was metabolizable. In this way we may secure figures which will be useful as a basis for estimating the energy values of rations in experiments in which it has not been determined, and which will also afford, from some points of view, a better idea of the relative extent of the losses other than those in the feces.

Conrse Fodders Alone. - In the cases in which coarse fodder constituted the exclusive ration the computation from the data given on p. 286 and the amounts of organic matter apparently digested in the several experiments is very simple and yields the following results per gram digested organic matter:

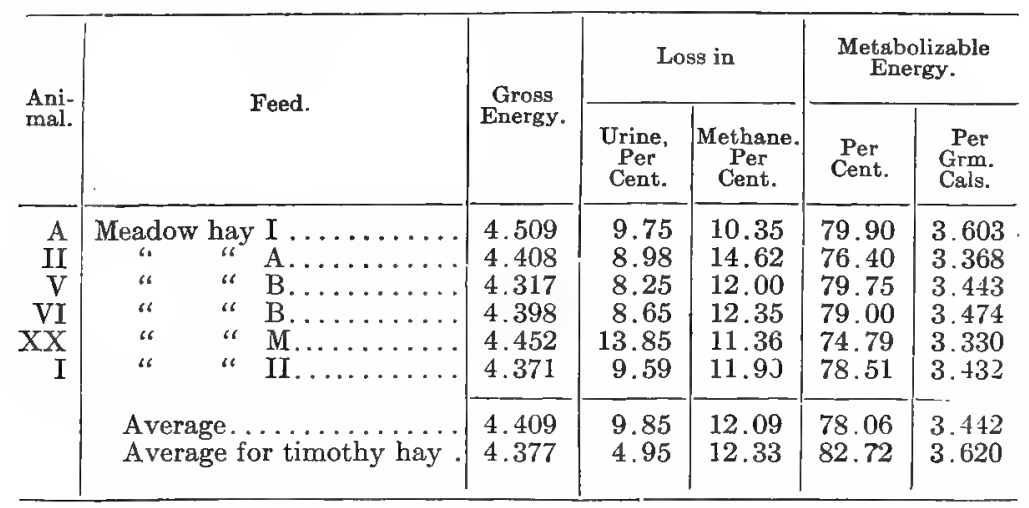

Coarse Fodders Added to Basal Ration.-From the results contained in Table I of the Appendix we may compute in substantially the same manner the total and metabolizable energy of the digestible organic matter of the coarse fodders which were added to the basal rations. In the table referred to, a correction was introduced for the small differences in the amount of the basal rations consumed in the periods compared. In the present computations it has been assumed that the organic matter of these small differences possessed the same digestibility as the total organic matter of the basal ration. For example, in the case of $\mathrm{Ox} \mathrm{H}$, 
Periods 4 and 7 , the amounts of digestible organic matter in the two rations were:

Period $7 \ldots \ldots \ldots \ldots \ldots \ldots \ldots \ldots, 7106$ grams

Period 4.................... 4845 "

Difference............. 2261 "

The table shows, however, that in Period 7 the animal received 44 grams more of total organic matter in the basal ration than in Period 4. In the latter period the digestibility of the organic matter was found to be 75.7 per cent. Consequently, of the excess of 2261 grams of digestible organic matter in Period 7 $44 \times 0.757=33$ grams may be regarded as derived from the basal ration and $2261-33=2228$ grams from the meadow hay added. The corresponding corrected amounts of energy as given in the same table are-

\begin{tabular}{|c|c|c|}
\hline & Total Cals. & $\begin{array}{c}\text { Per Grm. Digested } \\
\text { Organic Matter } \\
\text { Cals. }\end{array}$ \\
\hline $\begin{array}{c}\text { Energy of added hay (corrected)......... } \\
" \text { corresponding feces......... }\end{array}$ & $\begin{array}{r}15728.6 \\
5471.0\end{array}$ & \\
\hline $\begin{array}{c}\text { " " digested matter. . . } \ldots \ldots \ldots \ldots \\
\text { Metabolizable energy } \ldots \ldots \ldots \ldots \ldots \ldots \ldots\end{array}$ & $\begin{array}{r}10257.6 \\
8504.8\end{array}$ & $\begin{array}{l}4.604 \\
3.817\end{array}$ \\
\hline
\end{tabular}

The table on the next page contains the results of these computations expressed per gram of digested organic matter. Kellner* has made the same comparison in a slightly different manner. His results for the gross energy of the digested matter are given subsequently (p. 310). Those for metabolizable energy do not differ materially from those here given.

Concentrated Feeding-Stuffs. - The results of experiments upon concentrated feeding-stuffs may of course be computed in the same manner as those upon coarse fodders just considered. In the case of materials like starch, oil, and gluten, however, which differ widely from ordinary feeding-stuffs and which produce material and readily traceable effects upon the digestibility of the basal ration. relatively little value attaches to computations of the apparent metabolizable energy, and only the average results with these materials have been included in the summary on page 301 for the * Loc. cit, 53, 414 and 447. 


\begin{tabular}{|c|c|c|c|c|c|c|c|}
\hline \multirow{2}{*}{ 离 } & \multirow{2}{*}{ 总 } & & \multirow{2}{*}{$\begin{array}{l}\text { Total } \\
\text { Energy, } \\
\text { Cals. }\end{array}$} & \multicolumn{2}{|c|}{ Loss in } & \multicolumn{2}{|c|}{$\begin{array}{c}\text { Apparent } \\
\text { Metabolizable } \\
\text { Energy. }\end{array}$} \\
\hline & & & & $\begin{array}{c}\text { Urine. } \\
\text { Per Cent. }\end{array}$ & $\begin{array}{l}\text { Methane, } \\
\text { Per Cent. }\end{array}$ & Per Cent. & $\begin{array}{l}\text { Per Grm. } \\
\text { Cals. }\end{array}$ \\
\hline \multirow[t]{2}{*}{$\begin{array}{l}\mathrm{F} \\
\mathrm{G}\end{array}$} & \multirow[t]{2}{*}{$\begin{array}{l}1 \\
2\end{array}$} & $\begin{array}{c}\text { Meadow Hay } \\
\text { Sample V....... } \\
\text { V V...... }\end{array}$ & $\begin{array}{l}4.356 \\
4.496\end{array}$ & $\begin{array}{l}8.61 \\
7.72\end{array}$ & $\begin{array}{l}10.20 \\
12.58\end{array}$ & $\begin{array}{l}81.19 \\
79.70\end{array}$ & $\begin{array}{l}3.537 \\
3.583\end{array}$ \\
\hline & & Average . . . . . & 4.426 & 8.17 & 11.39 & 80.44 & 3.560 \\
\hline \multirow[t]{2}{*}{$\begin{array}{l}\mathrm{H} \\
\mathrm{H} \\
\mathbf{J}\end{array}$} & \multirow[t]{2}{*}{$\begin{array}{l}2 \\
7 \\
2\end{array}$} & 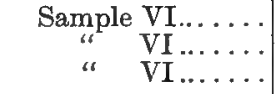 & $\begin{array}{l}4.531 \\
4.604 \\
4.506\end{array}$ & $\begin{array}{l}8.32 \\
7.66 \\
9.64\end{array}$ & $\begin{array}{l}7.74 \\
9.43 \\
9.33\end{array}$ & $\begin{array}{l}83.94 \\
82.91 \\
81.03\end{array}$ & $\begin{array}{l}3.803 \\
3.817 \\
3.651\end{array}$ \\
\hline & & Average . & 4.547 & 8.54 & 8.83 & 82.63 & 3.757 \\
\hline \multirow[t]{2}{*}{$\begin{array}{l}F \\
G\end{array}$} & \multirow[t]{2}{*}{$\begin{array}{l}2 \\
1\end{array}$} & $\begin{array}{c}\text { Oat Straw } \\
\text { Sample II } \\
\text { II ............ }\end{array}$ & $\begin{array}{l}4.441 \\
4.586\end{array}$ & $\begin{array}{l}5.30 \\
4.32\end{array}$ & $\begin{array}{l}10.17 \\
14.42\end{array}$ & $\begin{array}{l}84.53 \\
81.26\end{array}$ & $\begin{array}{l}3.754 \\
3.726\end{array}$ \\
\hline & & Average ...... & 4.514 & 4.81 & 12.30 & 82.89 & 3.740 \\
\hline$\underset{J}{H}$ & $\begin{array}{l}\text { I } \\
\text { I }\end{array}$ & $\begin{array}{l}\text { Wheat Straw : } \\
\text { Sample I....... } \\
\text { I...... }\end{array}$ & $\begin{array}{l}4.488 \\
4.397\end{array}$ & $\begin{array}{l}4.75 \\
6.49\end{array}$ & $\begin{array}{l}20.11 \\
19.67\end{array}$ & $\begin{array}{l}75.14 \\
73.84\end{array}$ & $\begin{array}{l}3.373 \\
3.247\end{array}$ \\
\hline \multirow[t]{2}{*}{$\underset{J}{H}$} & $\begin{array}{l}5 \\
5\end{array}$ & $\begin{array}{l}\text { Average ...... } \\
\text { Extracted } \text { Straw : } \\
\text { Sample I. . . . . } \\
\text { I. I...... }\end{array}$ & $\begin{array}{l}4.240 \\
4.164\end{array}$ & $\begin{array}{l}-0.52 \\
-1.29\end{array}$ & $\begin{array}{l}13.99 \\
14.58\end{array}$ & $\begin{array}{l}86.53 \\
86.71\end{array}$ & $\begin{array}{l}3.668 \\
3.611\end{array}$ \\
\hline & & Average & 4.202 & -0.91 & 14.29 & 86.62 & 3.640 \\
\hline
\end{tabular}

sake of completeness. Those upon peanut oil have been omitted, since the varying effect upon digestibility and upon the methane fermentation makes the results as computed in this way appear of questionable significance.

Summary. - The average results upon the various materials experimented with are summarized on the opposite page.

As appears from the figures of the table, the apparent metabolizable energy of the digestible organic matter of the different coarse fodders is quite uniform. At first sight it appears somewhat surprising that oat straw should show more favorable results than hay, but the reason is readily seen in the smaller loss which takes place in the urine; in wheat straw this loss is somewhat larger, while that 
ENERGY OF DIGESTED ORGANIC MATTER.

\begin{tabular}{|c|c|c|c|c|c|}
\hline & \multirow{2}{*}{$\begin{array}{c}\text { Total } \\
\text { Energy. } \\
\text { Cals. }\end{array}$} & \multicolumn{2}{|c|}{ Loss in } & \multicolumn{2}{|c|}{$\begin{array}{l}\text { Apparent } \\
\text { Metabolizable } \\
\text { Energy. }\end{array}$} \\
\hline & & $\begin{array}{l}\text { Urine, } \\
\text { Per } \\
\text { Cent. }\end{array}$ & $\begin{array}{c}\text { Methane, } \\
\text { Per } \\
\text { Cent. }\end{array}$ & $\begin{array}{l}\text { Per } \\
\text { Cent. }\end{array}$ & $\begin{array}{l}\text { Per } \\
\text { Grm., } \\
\text { Cals. }\end{array}$ \\
\hline Meadow hay (seven samples)........ & 4.439 & 9.62 & 11.52 & 78.86 & 3.501 \\
\hline Timothy hay $\ldots \ldots \ldots \ldots \ldots$ & 4.377 & 4.95 & 12.33 & 82.72 & 3.620 \\
\hline Oat straw............ & 4.514 & 4.81 & 12.30 & 82.89 & 3.740 \\
\hline Wheat straw ......... & 4.443 & 5.62 & 19.89 & 74.49 & 3.310 \\
\hline Extracted straw........ & 4.202 & -0.91 & 14.29 & 86.62 & 3.640 \\
\hline Beet molasses, Sample II... & 4.124 & 3.24 & 12.52 & 84.24 & 3.473 \\
\hline Starch, Kühn's experiments... & 4.192 & -1.19 & 13.42 & 87.77 & 3.679 \\
\hline "Kellner's experiments *...... & 4.012 & -0.92 & 11.12 & 89.80 & 3.603 \\
\hline Wheat gluten, Kellner's experiments.. & 5.749 & 16.59 & 0.02 & 83.39 & 4.792 \\
\hline
\end{tabular}

* Average of Samples III and IV.

in the methane is considerably larger, resulting in a materially lower figure for metabolizable energy.

The results summarized in the two preceding tables, it should be remembered, include, as already pointed out, all the effects produced by the addition of the material under experiment to the basal ration; that is, they give the apparent metabolizable energy. In the case of the coarse fodders no other method of computation is practicable, and the same would be true in most instances of ordinary concentrated commercial feeding-stuffs. In such cases it is rarely possible to distinguish with accuraey between the energy derived from the material experimented with and the subsidiary effects of the latter upon the digestibility of the several ingredients of the ration or upon the losses of energy in urine and methane. We may anticipate, therefore, that the results of future determinations of the metabolizable energy of ordinary feedingstuffs will of necessity be expressed substantially in the summary manner here employed.

With the nearly pure nutrients used in many of Kellner's experiments the case is different. Here it is possible to take account, to a large degree, of the secondary effects, such as those, for example, which in the case of wheat gluten result in figures exceeding 100 per cent. for the apparent metabolizable energy, and to compute results which represent more nearly the actual metabolizable energy contained in the substances themselves. In these cases, therefore, 
the averages of the tables are of less significance than the results given in the following pages, where the digestible nutrients are made the basis of the computation.

\section{ENERGY OF DIGESTIBLE NUTRIENTS.}

The foregoing paragraphs have dealt with the apparent metabolizable energy of feeding-stuffs, and the results have been expressed in terms of total or of digestible organic matter, or as percentages of gross energy. We now turn to a consideration of such data as are available regarding the several conventional groups of nutrients into which the food of herbivorous animals is ordinarily divided and inquire whether it is possible to compute average factors for their metabolizable energy which shall be useful in themselves and be of value particularly for purposes of comparison with earlier experiments. This was the special purpose of Kellner's investigations, and his experiments supply valuable data on these points as regards cattle and presumably other ruminants, which may be supplemented to a certain extent from experiments by other investigators upon horses and swine. In considering the experiments from this standpoint, Kellner's discussion and methods of computation have been closely followed, the attempt being made to compute as accurately as possible the real metabolizable energy of the several nutrients.

\section{Gross Energy.}

If it were possible to add pure nutrients to a basal ration and be sure that they would have no effect upon the utilization of the latter, it would be a comparatively simple matter to determine their real metabolizable energy. As a matter of fact, however, as has been seen, this is not possible. Not only is it impracticable to secure large quantities of pure nutrients, but each such addition to the basal ration is liable to affect especially the digestibility of the latter. Consequently the difference in metabolizable energy between the two rations fails to represent correctly the real metabolizable energy of the nutrient added. In order to compute the latter we must. have a basis for correcting the results for the small variations in the amounts of other nutrients digested, and for this purpose we need to know the total or gross energy of the digested matters. 
Crude Frber.-In four of his experiments on hay fed alone, Kellner* determined the heats of combustion of the crude fiber of the food and of the feces with the following results per gram:

\begin{tabular}{|r|c|c|}
\hline & $\begin{array}{c}\text { Crude Fiber of } \\
\text { Hay, Cals. }\end{array}$ & $\begin{array}{c}\text { Crude Fiber of } \\
\text { Feces, Cals. }\end{array}$ \\
\hline I. . . & 4.4350 & 4.7378 \\
II. . . & 4.3907 & 4.7423 \\
III. . . & 4.4548 & 4.9037 \\
IV.... & 4.4230 & 4.7426 \\
\hline
\end{tabular}

It appears from these figures that the crude fiber of meadow hay has a higher heat value than pure cellulose (4.1854 Cals. according to Stohmann), obviously due to the admixture of compounds richer in carbon, while the indigestible crude fiber of the feces has a still higher heat value. Merrill $\dagger$ has also reported similar results for the crude fiber of oat hay, clover silage, and oat and pea silage, as follows:

\begin{tabular}{|c|c|c|}
\hline & $\begin{array}{c}\text { Crude Fiber of Fodder. } \\
\text { Cals. per Grn. }\end{array}$ & $\begin{array}{l}\text { Crude Fiber of Feces. } \\
\text { Cals. yer Grm. }\end{array}$ \\
\hline $\begin{array}{l}\text { Oat hay ............. } \\
\text { Clover silage ....... } \\
\text { Oat and pea silage... }\end{array}$ & $\begin{array}{l}4.405 \\
4.610 \\
4.667\end{array}$ & $\begin{array}{l}4.662 \\
5.215 \\
4.820\end{array}$ \\
\hline
\end{tabular}

It follows that the digested portions of the crude fiber must contain less potential energy than the crude fiber of the feed, and from the known digestibility of the latter it is easy to calculate what the heat of combustion of the digested portion must be. Kellner's results, after deducting 5.711 Cals. per gram for the slight amounts of nitrogenous matter still contained in the crude fiber, were as shown on the next page.

The average result shows that not only the chemical composition but likewise the heat of combustion of the digested crude fiber varies but little from that of pure cellulose. Merrill's figures, computed in the same manner from the data of the digestion experiments reported by Bartlett, $\ddagger$ but without the correction for

\footnotetext{
* Loc, cit., 47, 299.

† Maine Expt. Station, Bull. 67, p. 170.

$\ddagger$ Ibid., pp. 140 and 150, and Report, 1898, p. 87.
} 


\begin{tabular}{|c|c|c|c|}
\hline & & $\begin{array}{l}\text { Crude } \\
\text { Fiber, } \\
\text { Grms. }\end{array}$ & $\begin{array}{c}\text { Equivalent } \\
\text { Energy, } \\
\text { Cals. }\end{array}$ \\
\hline \multirow{2}{*}{$\mathrm{I}$} & 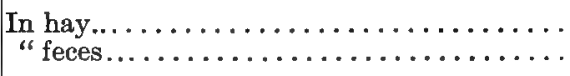 & $\begin{array}{l}2832 \\
1034\end{array}$ & $\begin{array}{r}12532.8 \\
4869.2\end{array}$ \\
\hline & 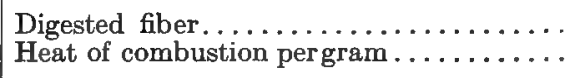 & $\begin{array}{c}1798 \\
\ldots \ldots\end{array}$ & $\begin{array}{r}7663.6 \\
\quad 4.2623\end{array}$ \\
\hline \multirow{2}{*}{ II } & 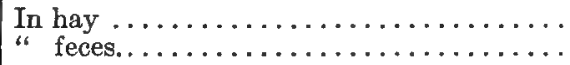 & $\begin{array}{r}2394 \\
822\end{array}$ & $\begin{array}{r}10503.0 \\
3878.1\end{array}$ \\
\hline & 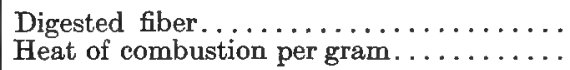 & & $\begin{aligned} 6624.9 \\
4.2143\end{aligned}$ \\
\hline \multirow{2}{*}{ III } & 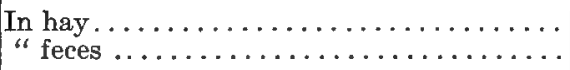 & $\begin{array}{r}2329 \\
769\end{array}$ & $\begin{array}{r}10367.7 \\
3754.0\end{array}$ \\
\hline & $\begin{array}{l}\text { Digested fiber....................... } \\
\text { Heat of combustion per gram. . . . }\end{array}$ & $\begin{array}{c}1560 \\
\ldots \ldots\end{array}$ & $\begin{aligned} 6613.7 \\
4.2396\end{aligned}$ \\
\hline \multirow{3}{*}{ IV } & 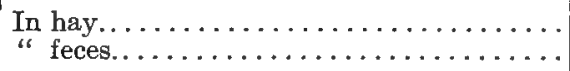 & $\begin{array}{r}1978 \\
716\end{array}$ & $\begin{array}{l}8732.0 \\
3479.2\end{array}$ \\
\hline & 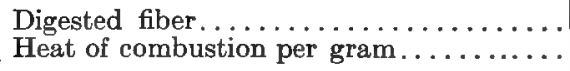 & $\begin{array}{c}1262 \\
\ldots \ldots\end{array}$ & $\begin{array}{l}5252.8 \\
\quad 4.1623\end{array}$ \\
\hline & Average heat of combustion per gram.. & & 4.2196 \\
\hline
\end{tabular}

nitrogenous matter, give the following results per gram for the digested crude fiber:

Oat hay. 4.161 Cals.

Clover silage 4.123 "

Oat and pea silage 4.584 "

Ether Extract.-Similar determinations by Kellner * on the ether extract of hay and feces yielded the following results per gram:

\begin{tabular}{|c|c|c|}
\hline & $\begin{array}{l}\text { Ether Extract } \\
\text { of Hay, Cals. }\end{array}$ & $\begin{array}{l}\text { Ether Extract } \\
\text { of Feces. Cals. }\end{array}$ \\
\hline I. . & 9.1604 & 9.7690 \\
\hline II. . & 93240 & 9.8923 \\
\hline III. & $9.3240\}$ & 9.8646 \\
\hline IV. & 9.0554 & 9.8314 \\
\hline V. & 9.1062 & 9.7640 \\
\hline Average. . & 9.1940 & 9.8243 \\
\hline
\end{tabular}

* Loc. cit., 47, 301. 
A calculation similar to that made for the crude fiber yielded the following figures for the heat of combustion of the digested portion:

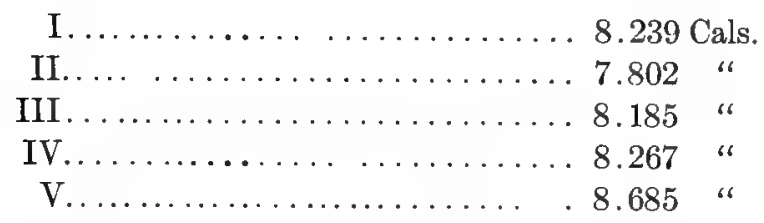

That these results are more or less discordant is not surprising in view of the uncertain elements involved in the determinations. Applying the average figures for the energy per gram of the ether extracts to the total amounts eaten and excreted in the five experiments taken together, we have for the average energy of the apparently digested ether extract 8.322 Cals. per gram, a figure considerably below the results recorded on p. 238 for either animal or vegetable fats. It must be remembered, however, that the ether extract of the feces contains more or less metabolic products, so that the above result does not represent the actual energy of the digested ether extract. It does, however, represent the energy corresponding to the difference between food and feces with which we reckon in computing rations, and from this point of view it is of value.

NItROGEN-FREE EXTRACT.-The nitrogen-free extract cannot be separated and examined like the crude fiber and the ether extract, but it is possible to arrive at an estimate of its heat of combustion indirectly. For this purpose Kellner assumes the average heat of combustion of the proteids (proteid nitrogen $\times 6.25$ ) as 5.711 Cals. per gram and that of the non-proteids as equal to that of asparagin, viz., 3.511 Cals. per gram. By subtracting from the gross energy of food or feces as directly determined the energy of the amounts of proteids, non-proteids, crude fiber, and ether extract shown by analysis to be present, he computes the heat of combustion of the nitrogen-free extract. Furthermore, by comparing the results on food and feces as in the case of the crude fiber the heat of combustion of the digested portion may be computed. The results per gram of such a computation for the same four experiments were:*

* Loc. cit., 47, 303-306. 


\begin{tabular}{|c|c|c|c|}
\hline & $\begin{array}{l}\text { N.-fr. Extract } \\
\text { of Hay, } \\
\text { Cals. per Gram. }\end{array}$ & $\begin{array}{l}\text { N -fr. Extract } \\
\text { of Feces, } \\
\text { Cals. per Gram. }\end{array}$ & $\begin{array}{l}\text { Digested N.-fr. } \\
\text { Extract. } \\
\text { Cals. per Gram. }\end{array}$ \\
\hline 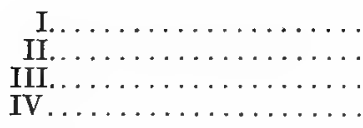 & $\begin{array}{l}4.5713 \\
4.6547 \\
4.5029 \\
4.6081\end{array}$ & $\begin{array}{l}5.2834 \\
5.4212 \\
5.1058 \\
5.2484\end{array}$ & $\begin{array}{l}4.203 \\
4.146 \\
4.246 \\
4.335\end{array}$ \\
\hline Average... . & 4.584 & 5.265 & 4.232 \\
\hline
\end{tabular}

In view of the indirect nature of the computation the results agree as well as could be expected and show that, as might be anticipated from its chemical composition, the heat of combustion of the digested portion of the nitrogen-free extract did not vary widely from that of starch.

Digested Matter of Mixed Rations.-The Möckern experiments afford accurate data as to the energy of the total digested matter of a large number of mixed rations. Kellner * has compared this with the computed energy of the same material. For this computation the factors used were: for fat, 8.322 Cals. per gram; for crude fiber and nitrogen-free extract, the average of Stohmann's figures for starch and cellulose, 4.184 Cals. per gram; for protein provisionally, 5.711 Cals. per gram. Of the fifty-nine experiments, twelve, in which large amounts of wheat gluten or oil were fed, showed sufficient differences to indicate that the figures assumed for protein and fat were too low as applied to these two materials. In the other forty-seven cases the differences were nearly all less than 2 per cent. of the total amount and were in both directions.

The special interest of these results lies in the fact that they show that we may safely use the above figures as indicated on $p$. 302 to correct the results reached from a comparison of two rations.

Nitrogen-free Extract of Starch.-As an example of Kellner's method of computation we may compare the results for $\mathrm{Ox} \mathrm{H}$ in Period 3, with starch, and in Period 4, on the basal ration. The total energy of the apparently digested matter (compare Table IV of the Appendix) was-

Period 3, with starch.......... 28,718 Cals.

Period 4, without starch.......... 21,763 "

Difference.................. 6, 6,955 ،

* Loc. cit., 53, 407. 
A slightly less amount of the basal ration was eaten in Period 3 than in Period 4. The difference in crude nutrients and in estimated digestible nutrients was as follows:

\begin{tabular}{|c|c|c|c|}
\hline & \multirow{2}{*}{$\begin{array}{l}\text { Total, } \\
\text { Grms. }\end{array}$} & \multicolumn{2}{|c|}{ Estimated Digestible. } \\
\hline & & Grms. & $\begin{array}{c}\text { Equivalent } \\
\text { Energy, Cals. }\end{array}$ \\
\hline $\begin{array}{l}\text { Protein } . \ldots \ldots \ldots \ldots \ldots \\
\text { Crude fiber. . . . . . . . . . } \\
\text { Nitrogen-free extract.... }\end{array}$ & $\left.\begin{array}{c}4 \\
13 \\
23\end{array}\right\}$ & $\begin{array}{r}2 \\
24\end{array}$ & $\begin{array}{c}11.4 \\
100.4 \\
111.8\end{array}$ \\
\hline
\end{tabular}

This amount of 112 Cals. should be added to the energy of the digested matter of Period 3 or subtracted from that of Period 4 in order to render them comparable, thus making the real difference due to the starch 7067 Cals. Still further, the starch diminished the digestibility of the other nutrients of the ration by the following amounts:

\begin{tabular}{|l|c|c|}
\hline & Grms. & $\begin{array}{c}\text { Equivalent } \\
\text { Energy, Cals. }\end{array}$ \\
\hline Protein.................... & 118 & 673.8 \\
Crude fiber..... & 9 & 71.1 \\
Ether extract..... & & $\frac{74.9}{819.8}$ \\
\hline
\end{tabular}

Had these amounts been digested in Period 3 as in Period 4, the energy of the digested matter of the ration would have been 820 Cals. greater, and the difference between the two periods would have been $7887 \mathrm{Cals}$. The digestible nitrogen-free extract was 1876 grams more in Period 3 than in Period 4. Assuming all of this to be derived from the starch, we have for the energy of each gram of digested nitrogen-free extract $7887 \div 1876=4.204$ Cals.

The following table* contains the results of all the starch experiments computed in the manner just outlined:

* Loc. cit., 53, 412. 
ENERGY OF DIGESTED NITROGEN-FREE EXTRACT OF STARCE.

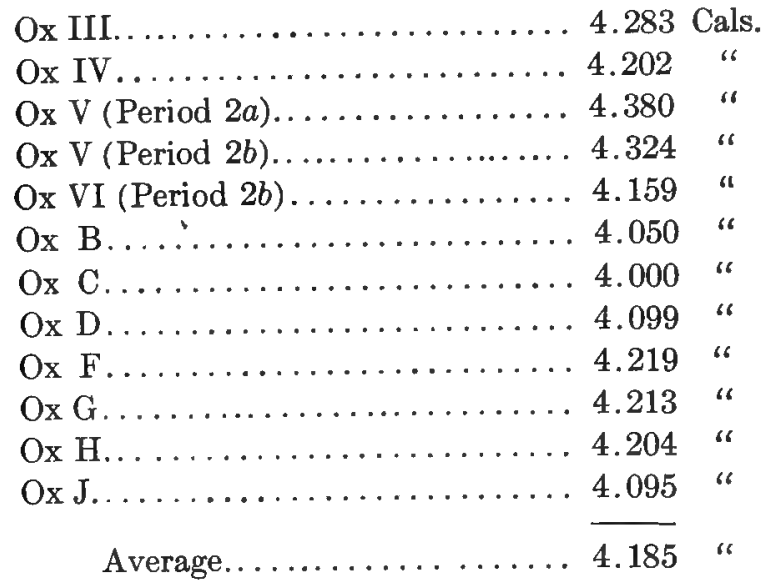

Carbohydrates of Extracted Straw.-Computed in the same manner as the experiments upon starch, the two experiments upon this substance gave the following results:*

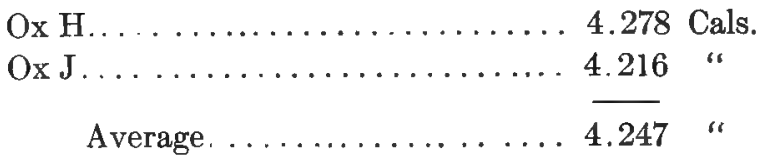

This average is slightly higher than woulu be computed on the assumption that the digested crude fiber and nitrogen-free extract had the heat values respectively of the digested crude fiber of hay and the digested nitrogen-free extract of starch.

Peanut Ora.-Four experiments upon this substance similarly computed give the following results; *

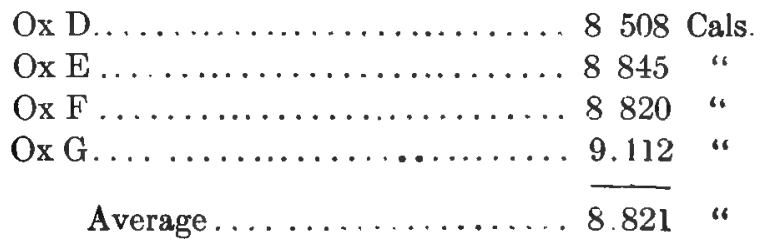

* Loc cit, 63413 and 414 
As in the case of the ether extract of hay, the energy of the digested fat is less than that of the original material, which was 9.478 Cals. per gram.

Protein of Wheat Gluten.-Comparing the experiments with and without this material exactly as in the case of the starch, we have the following results * for the energy of the digested protein:

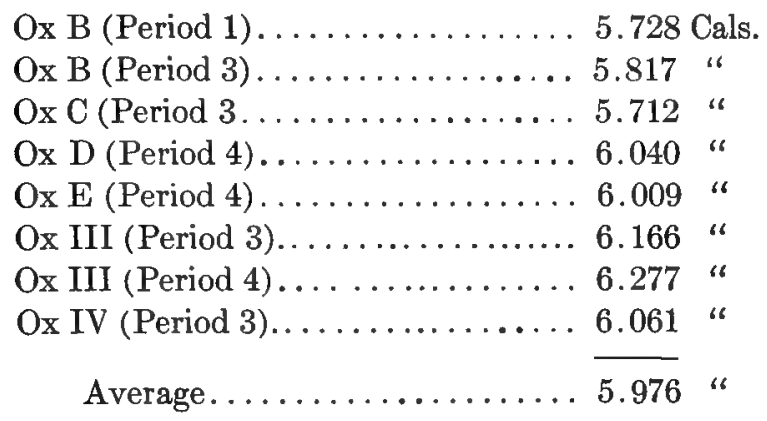

In these trials three different kinds of gluten were used which were prepared by somewhat different processes. The averages for the three sorts separately were as follows:

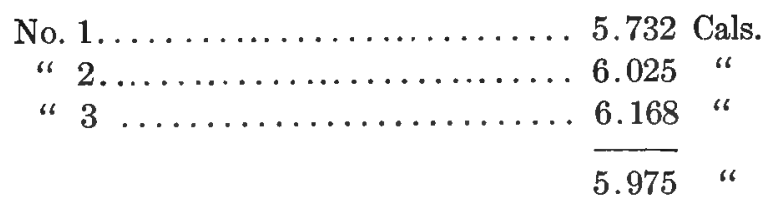

The above figures refer to the so-called crude protein, that is, to nitrogen $\times 6.25$. The proteins of wheat, however, contain considerably over 16 per cent. of nitrogen. Using Ritthausen's factor, namely, 5.7, for the computation of protein from nitrogen reduces the amount of protein in the gluten and increases that of the nitrogen-free extract by the same amount. The energy of the digested protein when computed on this basis equals 6.148 Cals. per gram.

Organic Matter of Coarse Fodders.-For the total digested organic matter of hay and straw the following heat values per gram were computed:*

* Loc. cit., 58, 412 and 414. 


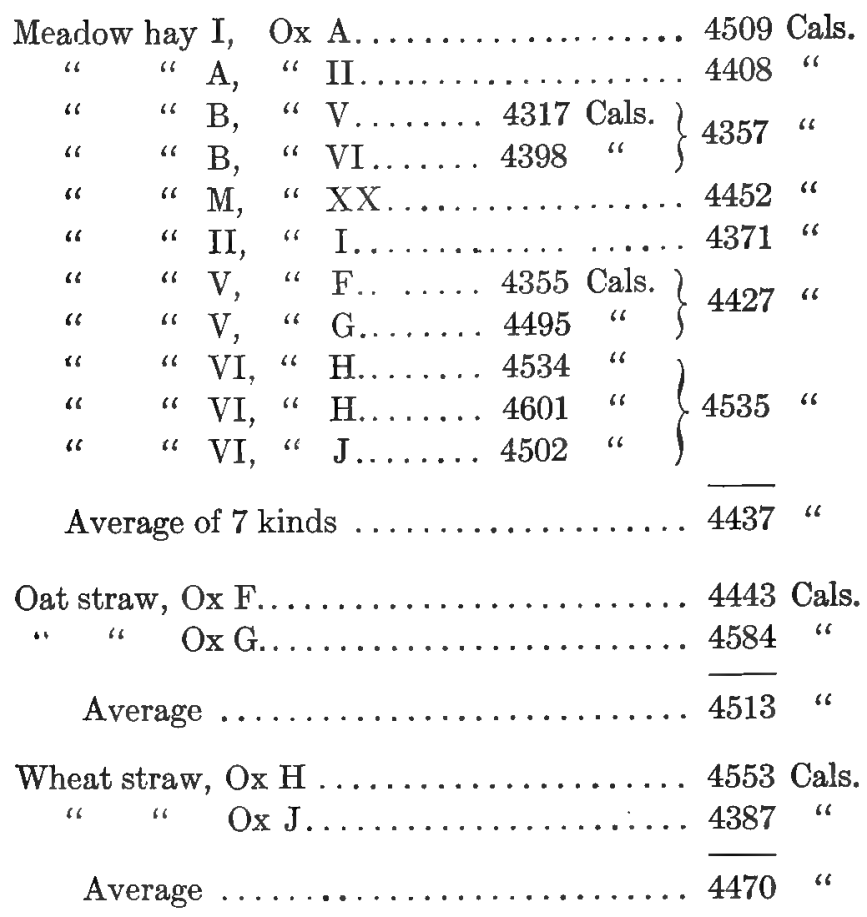

The digestible matter of the straw has apparently about the same heat value as that of hay.

\section{Metabolizable Energy.}

Protein.-A portion of the gross energy of the digested protein is removed in the urea and other nitrogenous products of metabolism, and in addition to this there is to be considered the possibility of a loss of energy by fermentation in the digestive tract.

Losses in Methane.-In nine of the Möckern experiments in which wheat gluten or flesh-meal was added to the basal ration, the amount of carbon excreted in the form of hydrocarbons per day and head was as tabulated on the opposite page.

The differences between the excretion with and without gluten are small in amount and are sometimes positive and sometimes negative, the averages being probably within the limit of experimental error. The percentage losses of energy in methane as 


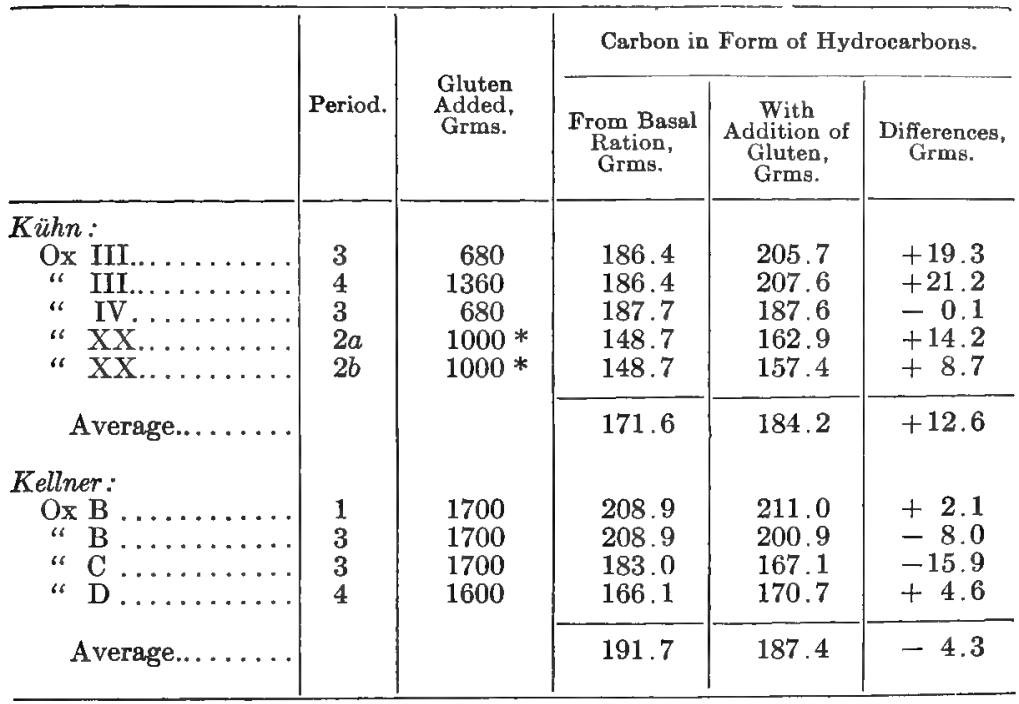

* Flesh-meal.

computed in Table $\mathrm{V}$ of the Appendix, like the figures just given for the carbon of the methane, lead to the conclusion that the protein of the food does not participate in the methane fermentation. Those figures were:

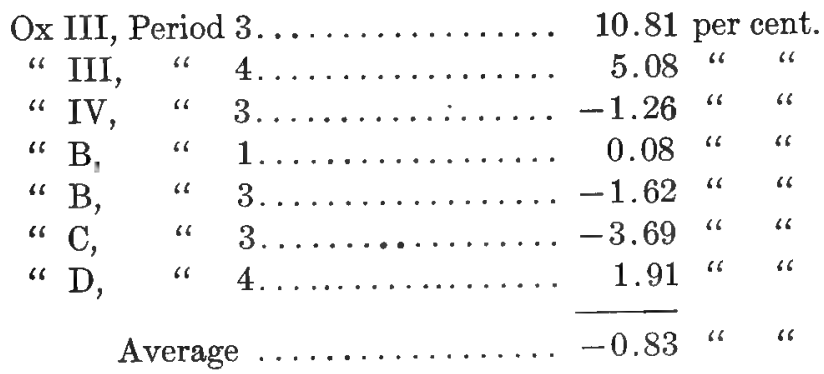

Kellner* reaches the same conclusion by comparing the ratio of the methane carbon to the amount of digested carbohydrates (nitrogen-free extract + crude fiber) in the several periods. The former amounted to the following per cent. of the latter in his experiments:

$$
\text { * Loc. cit., 53, } 420 \text {. }
$$




\begin{tabular}{|c|c|c|}
\hline & $\begin{array}{c}\text { Basal Ration, } \\
\text { Per Cent. }\end{array}$ & $\begin{array}{c}\text { Basal Ration } \\
\text { + Gluten, } \\
\text { Per Cent. }\end{array}$ \\
\hline Ox B $\ldots \ldots \ldots \ldots \ldots \ldots \ldots$ & .2 .94 & 2.96 \\
" $\mathrm{B} \ldots \ldots \ldots \ldots \ldots \ldots \ldots$ & 2.94 & 2.82 \\
" $\mathrm{C} \ldots \ldots \ldots \ldots \ldots \ldots \ldots$ & 2.71 & 2.41 \\
" $\mathrm{D} \ldots \ldots \ldots \ldots \ldots \ldots \ldots$ & 2.75 & 2.71 \\
Average $\ldots \ldots \ldots \ldots \ldots$ & 2.87 & 3.19 \\
\hline
\end{tabular}

Had the large quantities of digestible protein added to the basal rations produced any material amount of methane, that fact must have been reflected in the above percentages. This method of comparison takes into account the probable effect of the carbohydrates of the wheat gluten in increasing the production of methane, and the substantial agreement of the results with and without protein leads to the same conclusion as the preceding data. It seems fair to presume that this conclusion applies to protein in general, although a strict demonstration of it, especially for coarse fodders, would have its difficulties.

Losses IN URINe.-While the assumption that the urine is essentially an aqueous solution of urea leads to grave errors in the case of the carnivora, this is still more emphatically true of the urine of herbivora, particularly of ruminants. The presence in the urine of herbivora of hippuric acid and other nitrogenous compounds less highly oxidized than urea has of course long been known, while, as stated on p. 27, the presence of considerable amounts of nonnitrogenous organic matter was subsequently demonstrated by Henneberg and by G. Kühn in the urine of ruminants.

It follows from these facts that the energy content of the urine of these animals must be higher in proportion to its nitrogen than is the case with carnivora or with man, but the experimental demonstration of this fact and the realization of the extent and importance of the difference are of comparatively recent date.

Cattle.-It is to Kellner * that we owe the first direct determinations of the potential energy of the urine of cattle. The two animals used in the experiment were fed, the one (A) on meadow hay, and the other (B) on meadow hay and oat straw. The results as regards the urine were as follows, per day and head:

* Loc. cit., 47, 275. 


\begin{tabular}{|c|c|c|}
\hline & Ox A. & $\mathrm{O} \times \mathrm{B}$ \\
\hline $\begin{array}{c}\text { Total nitrogen } \ldots \ldots \ldots \ldots \ldots \\
\text { carbon } \ldots \ldots \ldots\end{array}$ & $\begin{aligned} 61.28 & \text { grams. } \\
203.20 & \end{aligned}$ & $\begin{array}{r}46.63 \text { grams. } \\
161.30 \text { “ }\end{array}$ \\
\hline Hippurıc acid $\ldots \ldots \ldots \ldots \ldots$ & $145.00 \quad$ ". & 126.40 \\
\hline Total energy ....... & $1945.00 \mathrm{Cals}$ & 1549.40 Cals. \\
\hline
\end{tabular}

Assuming all the nitrogen not contained in the hippuric acid to have been in the form of urea, we have the following as the distribution of the carbon and of the energy of the urine:

\begin{tabular}{|c|c|c|c|c|}
\hline & \multicolumn{2}{|c|}{ Ox A. } & \multicolumn{2}{|c|}{ Ox B. } \\
\hline & Amount. & Per Cent. & Amount. & Per Cent \\
\hline $\begin{array}{l}\text { Carbon. } \\
\text { In hippuric acid } \ldots \ldots \ldots \\
\text { " urea ............... } \\
\text { " other compounds... }\end{array}$ & $\begin{array}{l}\text { Grms. } \\
87.48 \\
21.40 \\
94.32\end{array}$ & $\begin{array}{l}43.05 \\
10.53 \\
46.42\end{array}$ & $\begin{array}{l}\text { Grms. } \\
76.26 \\
15.75 \\
69.29\end{array}$ & $\begin{array}{r}47.28 \\
9.76 \\
42.96\end{array}$ \\
\hline Total & 203.20 & 100.00 & 161.30 & 100.00 \\
\hline $\begin{array}{l}\text { Energy } \\
\quad \text { In hippuric acid } \ldots \ldots \ldots \\
\text { " urea .............. } \\
\text { " other eompounds... }\end{array}$ & $\begin{array}{l}\text { Cals. } \\
821.30 \\
271.40 \\
852.30\end{array}$ & $\begin{array}{l}42.23 \\
13.95 \\
43.82\end{array}$ & $\begin{array}{l}\text { Cals. } \\
715.90 \\
199.60 \\
633.90\end{array}$ & $\begin{array}{l}46.20 \\
12.88 \\
40.92\end{array}$ \\
\hline Total. & 1945.00 & 100.00 & 1549.40 & 100.00 \\
\hline
\end{tabular}

While the assumption that all the nitrogen was present either as hippuric acid or urea is not strictly correct. still the figures suffice to show, first, that a considerable proportion of the energy of the proteids of the food may be removed in the hippuric acid, and second, that the urine contains relatively considerable amounts of non-nitrogenous organic matter. Had the energy of the urine been computed from its nitrogen reckoned simply as urea the results would have been as follows:

\begin{tabular}{|c|c|c|}
\hline & Ox $A$ & $\mathrm{Ox} B$ \\
\hline 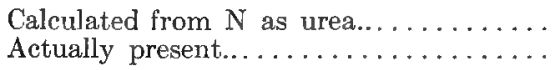 & $\begin{array}{r}331.6 \text { Cals } \\
1945.0\end{array}$ & $\begin{array}{r}252.3 \mathrm{Cals} \\
1549.4 \text { " }\end{array}$ \\
\hline
\end{tabular}

In experiments by the writer on the maintenance ration of cattle,* determinations of the total energy of the urine of steers * Penna. Experiment Station, Bull 42, p. 150. 
were likewise made. Calculated per gram of nitrogen the results were as follows:

\begin{tabular}{|c|c|c|c|}
\hline Feed. & Steer No. 1. & Steer No. 2. & Steer No. 3. \\
\hline 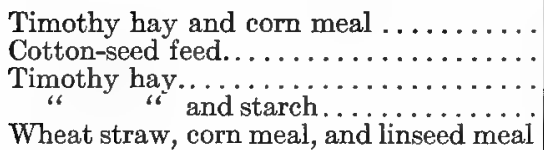 & $\begin{array}{l}37.79 \text { Cals. } \\
40.64 \text { " } \\
19.29 \text { " } \\
25.02 \text { " } \\
11.24 \text { " }\end{array}$ & $\begin{array}{l}28.35 \text { Cals. } \\
34.25 \text { " } \\
18.01 \text { “ } \\
10.77 \text { " }\end{array}$ & $\begin{array}{l}28.82 \text { Cals. } \\
12.47 \text { " } \\
10.95 \text { " }\end{array}$ \\
\hline
\end{tabular}

The methods employed to prepare the urine for combustion were not altogether satisfactory, and the range of possible error is rather large. In but two cases, however, was the encrgy of the urine less than twice that corresponding to its nitrogen calculated as urea (5.434 Cals.), while in one case it reached over seven times that amount. Neither carbon nor hippuric acid having been determined, no computations can be made as to the amount of nonnitrogenous matter present.

Jordan * has reached similar results on the urine of cows, the average energy content per gram of nitrogen being as follows:

\begin{tabular}{c|c|c|c}
\hline & $\begin{array}{c}\text { Total Nitrogen, } \\
\text { Grms. }\end{array}$ & $\begin{array}{c}\text { Potential Energy, } \\
\text { Cals. }\end{array}$ & $\begin{array}{c}\text { Energy per Grm. } \\
\text { Nitrogen, Cals. }\end{array}$ \\
\cline { 1 - 2 } Cow No. 12: & 87.0 & 1658.3 & \\
Period $1 \ldots \ldots \ldots \ldots \ldots \ldots$ & 78.8 & 1547.2 & 19.06 \\
" $2 \ldots \ldots \ldots \ldots \ldots \ldots$ & 42.8 & 1323.5 & 30.63 \\
Cow No. $10 \ldots \ldots \ldots \ldots \ldots \ldots$ & 65.5 & 1452.5 & 22.18 \\
\hline
\end{tabular}

As in the writer's experiments, the energy per gram of nitrogen varies within wide limits, being greatest when the total nitrogen of the urine is least. In other words, it would appear that the non-nitrogenous ingredients of the urine of cattle are subject to less fluctuation than the nitrogenous ingredients.

Kellner's later experiments have fully confirmed his earlier results, as will appear in greater detail in subsequent paragraphs. He finds that the carbon rather than the nitrogen of the urine is the measure of its potential energy, and that an estimate of 10 Cals. per gram of carbon gave for his experiments results closely approximating the truth. $\dagger$

* New York State Experiment Station, Bull. 197, p. 28.

$\dagger$ Loc. cit., 53, 437. 
Other Species.-We may probably assume without serious error that the results obtained with cattle apply in general to sheep and other ruminants. No direct determinations of the energy of the urine of the horse or the hog have yet been reported, but Zuntz \& Hagemann * have made some estimates of it in the case of the horse on a mixed ration of hay, oats, and straw. They determined the total carbon and total nitrogen of the urine and, on the assumption that only urea and hippuric acid are present, compute the proportion of each of these, and thence the energy of the urine. They thus find the potential energy of the latter, per gram of nitrogen, equal to 15.521 Cals. Neither hippuric acid nor energy having been determined directly, it is impossible to check the above computation or to ascertain whether any non-nitrogenous organic matter was present. It is to be noted, however, that the ratio. of carbon to nitrogen in the urine was much lower than in Kellner's experiments on cattle, viz.:

$$
\begin{aligned}
& \text { Zuntz \& Hagemann............ } 1.526: 1 \\
& \text { Kellner, Ox A............. 3.315:1 } \\
& \text { " Ox B............... 3.458:1 }
\end{aligned}
$$

This fact clearly indicates that at least there was very much less non-nitrogenous matter present in the former case.

Meissl, Strohmer \& Lorenz $\dagger$ in their respiration experiments on swine likewise determined carbon and nitrogen in the urine. Computed by the method of Zuntz \& Hagemann the energy of the urine averaged 9.55 Cals. per gram of nitrogen, while the average ratio of carbon to nitrogen was $0.745: 1$. These results would seem to indicate that the loss of energy in the urine of the hog is not very much greater than in that of the carnivora.

Metabolizable Energy of Protein of Concentrated Feeds. -Accepting it as demonstrated that there is no material loss of potential energy in the form of fermentation products of protein, the data regarding the energy of the urine just considered afford the basis for an approximate estimate of the metabolizable energy of the digested protein.

Cattle.-Kellner's experiments upon cattle afford data for computing the metabolizable energy of the digested protein of wheat

* Landw. Jahrb., 27, Supp. III, 239. $\dagger$ Zeit. f. Biol., 22, 63. 
gluten and of beet molasses. The method of computation is precisely similar to that already employed for calculating the metabolizable energy of the total organic matter; that is, the results upon the basal ration are subtracted from those upon the ration containing the material under experiment.

Taking as an example the results upon wheat gluten with $\mathrm{Ox} \mathrm{C}$ in Periods 1 and 3 we have the following comparison:

\begin{tabular}{|c|c|c|c|c|c|c|}
\hline & \multicolumn{4}{|c|}{ Digested. } & \multirow{2}{*}{$\begin{array}{l}\text { Energy } \\
\text { of Urive, } \\
\text { Cals. }\end{array}$} & \multirow{2}{*}{$\begin{array}{c}\text { Gain of } \\
\text { Nitrogen } \\
\text { by } \\
\text { Animal, } \\
\text { Grms. }\end{array}$} \\
\hline & $\begin{array}{l}\text { Protein, } \\
\text { Grms. }\end{array}$ & $\begin{array}{l}\text { Crude } \\
\text { Fiber, } \\
\text { Grms. }\end{array}$ & $\begin{array}{c}\text { Nitrogen- } \\
\text { Free } \\
\text { Extract, } \\
\text { Grms. }\end{array}$ & $\begin{array}{c}\text { Ether } \\
\text { Extract. } \\
\text { Grms. }\end{array}$ & & \\
\hline \multirow{2}{*}{$\begin{array}{c}\underset{6}{\operatorname{Period}} 3 \ldots \ldots \ldots \\
\quad 1 \ldots \ldots \ldots \\
\text { Difference. } \quad 1 \ldots \ldots\end{array}$} & $\begin{array}{r}1694 \\
598\end{array}$ & $\begin{array}{l}1279 \\
1289\end{array}$ & $\begin{array}{l}5648 \\
5464\end{array}$ & $\begin{array}{l}34 \\
40\end{array}$ & $\begin{array}{l}2592.8 \\
1666.4\end{array}$ & $\begin{array}{l}20.31 \\
16.01\end{array}$ \\
\hline & 1096 & -10 & 184 & -6 & 926.4 & 4.30 \\
\hline
\end{tabular}

The difference of 4.3 grams in the amount of nitrogen gained by the animal is equivalent to 32 Cals. which would otherwise have appeared in the urine. This added to the 926.4 Cals. actually found makes a total of 958.4 Cals. for the increase in the potential energy of the urine due to the 1096 grams of protein digested. There are also differences in the amount of non-nitrogenous matters digested, particularly of the nitrogen-free extract. As Tables I, III and IV of the Appendix show, both starch and crude fiber, as represented by the extracted straw, tend to diminish the amount of energy carried off in the urine. These differences were observed when from 2 to 2.5 kilograms of these substances were added to the basal ration. If the differences are proportional to the amount fed, the energy corresponding to the small difference observed in this ex. periment would not exceed 15 or 20 Cals., and may be neglected, while the maximum difference in any experiment of the series would probably not exceed 70 to 75 Cals. Assuming that all the additional protein digested came from the wheat gluten, we have for the corresponding energy of the urine

$958.4 \div 1096=0.874$ Cals. per gram protein digested.

Subtracting this from the total energy of the digested protein as found on p. 309, viz., 5.975 Cals., we have 5.101 Cals. as the metabo- 
lizable energy of one gram of digested protein of wheat gluten in this experiment.

For the four experiments upon this substance, computed as in the above example, the results were as follows:

\begin{tabular}{|c|c|c|c|}
\hline & \multirow{2}{*}{$\begin{array}{l}\text { Protein } \\
\text { digested } \\
\text { from } \\
\text { Gluten, } \\
\text { Grms. }\end{array}$} & \multicolumn{2}{|c|}{$\begin{array}{c}\text { Difference in } \\
\text { Energy of Urine.* }\end{array}$} \\
\hline & & $\begin{array}{l}\text { Total, } \\
\text { Cals. }\end{array}$ & $\begin{array}{l}\text { Per Grm. of } \\
\text { Protein, } \\
\text { Cals. }\end{array}$ \\
\hline 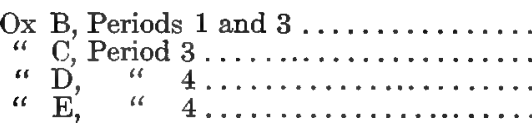 & $\begin{array}{l}2185 \\
1096 \\
1056 \\
1148\end{array}$ & $\begin{array}{r}2547.3 \\
958.4 \\
1061.1 \\
1362.1\end{array}$ & $\begin{array}{l}1.166 \\
0.874 \\
1.005 \\
1.186\end{array}$ \\
\hline Average............... & 1371 & 1482.2 & 1.081 \\
\hline
\end{tabular}

* Corrected to nitrogen equilibrium.

Subtracting from the total energy of the digested protein the potential energy carried off in the urine we have for the metabolizable energy of one gram of protein

$$
\text { 5.975 Cals. }-1.081 \text { Cals. }=4.894 \text { Cals. }
$$

If we use Ritthausen's factor, 5.7, for proteids, the average digested protein becomes 1250 grams and the loss of energy in the urine 1.190 Cals. per gram of protein. Subtracting this from 6.148 Cals., the gross energy of one gram of $N \times 5.7$ (p. 309), we have for the metabolizable energy of the latter 4.958 Cals. per gram.

The average increase in the energy of the urine for each additional gram of nitrogen excreted in these experiments (6.756 Cals.) was almost exactly the same as Rubner found in his experiment on extracted lean meat ( 6.695 Cals.). This may be taken as indicating that the process of proteid metabolism is substantially the same in both classes of animals, while the fact that the result is notably greater than the energy of urea shows that in the herbivora as in the carnivora other waste products than urea result from the proteid metabolism.

In three other experiments beet molasses was added to the basal ration, resulting in the digestion of an increased amount of nitrogenous matter. Computing the results as in the case of the 
wheat gluten, and assuming that the large amounts of soluble carbohydrates digested had no effect on the potential energy of the urine, the results were as follows:

\begin{tabular}{|c|c|c|c|}
\hline & \multirow{2}{*}{$\begin{array}{l}\text { Protein Digested } \\
\text { from Molasses, } \\
\text { Grms. }\end{array}$} & \multicolumn{2}{|c|}{ Difference in Energy of Urine.* } \\
\hline & & Total, Cals. & $\begin{array}{c}\text { Per Grm. Protein, } \\
\text { Cals. }\end{array}$ \\
\hline 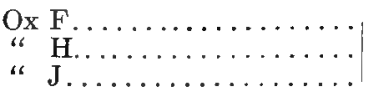 & $\begin{array}{l}117 \\
160 \\
122\end{array}$ & $\begin{array}{l}256.1 \\
240.3 \\
192.6\end{array}$ & $\begin{array}{l}2.189 \\
1.502 \\
1.579\end{array}$ \\
\hline Average $\ldots \ldots \ldots \ldots \ldots$ & 133 & 229.7 & 1.727 \\
\hline
\end{tabular}

* Corrected to nitrogen equilibrium.

It will be seen that the loss of energy in the urine is much greater than in the case of the gluten or than in Rubner's experiments with carnivora. Since it is improbable that the soluble carbohydrates of the molasses escape oxidation, it would appear that some of the nitrogenous material of the latter must have passed through the system unmetabolized. Kellner suspects that it is made up in part at least of xanthin bases.

If we consider the nitrogen of the molasses to represent crude protein $(\mathrm{N} \times 6.25)$ with a heat value of 5.711 Cals. per gram, the metabolizable energy per gram would be 3.984 Cals. In view, however, of the fact that only a very small proportion of the nitrogen of the molasses is in the proteid form, such a calculation seems of doubtful value.

Swine.-In the investigations of Meissl, Strohmer and Lorenz* upon the production of fat from carbohydrates (p. 176) the carbon and nitrogen of the urine were determined in six experiments.

Applying to the results Zuntz \& Hagemann's method of computation (p. 315) we obtain the following estimates for the energy per gram of nitrogen in the urine of the hog in these experiments and for the corresponding metabolizable energy of the digested protein:

* Zeit. f. Biol., 22, 63. 


\begin{tabular}{|c|c|c|c|c|c|c|}
\hline $\begin{array}{c}\text { Experi- } \\
\text { ment } \\
\text { No. }\end{array}$ & Feed. & $\begin{array}{c}\text { Nitrogen } \\
\text { as Urea, } \\
\text { Grms. }\end{array}$ & $\begin{array}{l}\text { Nitrogen } \\
\text { as Hip- } \\
\text { puric } \\
\text { Acıd, } \\
\text { Grms. }\end{array}$ & $\begin{array}{c}\text { Total } \\
\text { Energy } \\
\text { of Urine, } \\
\text { Cals. }\end{array}$ & $\begin{array}{c}\text { Energy } \\
\text { per Grm. } \\
\text { of } \\
\text { Nitrogen, } \\
\text { Cals. }\end{array}$ & $\begin{array}{l}\text { Metabo- } \\
\text { lizable } \\
\text { Energy } \\
\text { per } \\
\text { Grm. } \\
\text { Protein, } \\
\text { Cals. }\end{array}$ \\
\hline $\begin{array}{l}1 \\
2 \\
3 \\
4 \\
5 \\
6\end{array}$ & 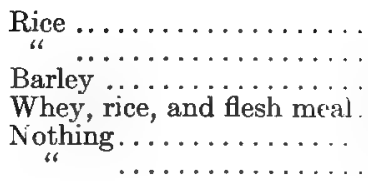 & $\begin{array}{r}9.58 \\
9.22 \\
13.04 \\
59.89 \\
9.35 \\
6.48\end{array}$ & $\begin{array}{l}0.88 \\
1.04 \\
1.04 \\
1.17 \\
0.45 \\
0.29\end{array}$ & $\begin{array}{r}115.7 \\
125.6 \\
146.5 \\
410.0 \\
83.7 \\
56.4\end{array}$ & $\begin{array}{r}11.06 \\
12.24 \\
10.40 \\
6.72 \\
8.54 \\
8.33\end{array}$ & $\begin{array}{l}3.941 \\
3.753 \\
4.048 \\
4.636 \\
4.344 \\
4.379\end{array}$ \\
\hline
\end{tabular}

Kornauth \& Arche * report the following results on the urine of swine fed chiefly upon cockle:

\begin{tabular}{|c|c|c|c|}
\hline $\begin{array}{l}\text { Experiment } \\
\text { No. }\end{array}$ & $\begin{array}{c}\text { Nitrogen, } \\
\text { Grms. }\end{array}$ & $\begin{array}{l}\text { Carbon, } \\
\text { Grms. }\end{array}$ & $\begin{array}{l}\text { Ratio, } \\
\mathrm{C}: \mathbf{N} .\end{array}$ \\
\hline $\begin{array}{l}1 \ldots \ldots \cdots \cdots \\
2 \ldots \ldots \ldots \ldots \\
3 \ldots \ldots \ldots \cdots\end{array}$ & $\begin{array}{l}10.56 \\
10.30 \\
10.41\end{array}$ & $\begin{array}{r}10.30 \\
9.53 \\
9.96\end{array}$ & $\begin{array}{l}0.975: 1 \\
0.926: 1 \\
0.957: 1\end{array}$ \\
\hline Average......... & 10.42 & 9.93 & $0.953: 1$ \\
\hline
\end{tabular}

The results, computed as in the previous case, make the average energy content of the urine 10.27 Cals. per gram of nitrogen, equivalent to a metabolizable energy of 4.067 Cals. per gram of protein.

In the two fasting experiments of Meissl, Strohmer \& Lorenz the ratios of carbon to nitrogen and of computed energy to nitrogen are similar to those obtained with fasting carnivora. The abundant supply of proteids in the diet in the fourth experiment seems to have had the effect of reducing these ratios to values comparable with those obtained by Rubner for extracted meat and by Kellner for the digested protein of wheat gluten. These facts seem to indicate clearly that the nature of the proteid metabolism in all these animals is substantially the same. In the experiments in which ordinary grains were used, the computed energy content of the urine is notably greater relatively to its nitrogen. How far the excess of carbon found in these cases was due to an

* Landw. Vers. Stat., 40, 177. 
increased formation of hippuric acid and what part of it, if any, is to be ascribed to the presence of non-nitrogenous matter in the urine, the experiments afford no means of estimating.

The Horse.-Zuntz \& Hagemann's results on the horse, p. 315, although the result of feeding mixed rations, may be conveniently considered here. The computed energy of the urine was 15.521 Cals. per gram of nitrogen, equivalent to 2.483 Cals. per gram of protein. Assuming for the latter, as before, a value of 5.711 Cals., there remains for the metabolizable energy 3.228 Cals. per gram.

Protein of Coarse Fodders.-Almost the only data on this point are those afforded by Kellner's experiments upon cattle. In those in which coarse fodders were used alone we can of course compute the metabolizable energy of the protein directly from the amount digested and from the energy of the urine. In those experiments in which coarse fodders were added to a basal ration we can compare the two experiments in the same manner as those upon gluten, neglecting, as in that case, the differences in the nonnitrogenous nutrients digested.

Passing over the details of the computation, the final results, including the metabolizable energy of the digested protein computed upon the assumption that its gross energy equals 5.711 Cals. per gram, are as given in the table on the opposite page.*

The writer's experiments on timothy hay, the results of which as regards the energy of the urine have already been given on p. 314, when computed in the same manner as the above experiments give the following results for the metabolizable energy of the digested protein:

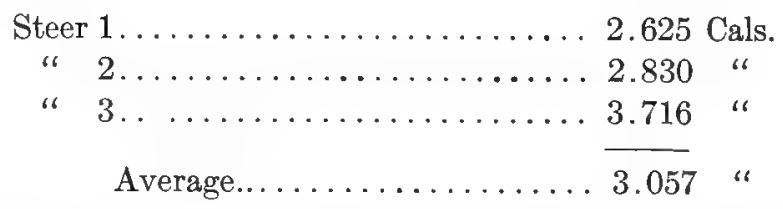

Influence of Non-nitrogenous Matter of Urine.-In the previous paragraphs there appeared reasons for supposing that the processes of proteid metabolism are essentially the same in all domestic

* The figures given in this table for digested protein, energy, etc., refer solely to that derived from the coarse fodder and not to that of the total ration. 


\begin{tabular}{|c|c|c|c|c|}
\hline & Protein & $\begin{array}{c}\text { Diff } \\
\text { Energy }\end{array}$ & $\begin{array}{l}\text { Bnce in } \\
\text { f I rine.* }\end{array}$ & $\begin{array}{l}\text { Metaboliz- } \\
\text { able Energy }\end{array}$ \\
\hline & $\begin{array}{l}\text { Digested, } \\
\text { Grms. }\end{array}$ & $\begin{array}{l}\text { Total, } \\
\text { Cals. }\end{array}$ & $\begin{array}{l}\text { Per Grm. of } \\
\text { Protein } \\
\text { Digested, } \\
\text { Cals. }\end{array}$ & $\begin{array}{l}\text { Digestible } \\
\text { Protein, } \\
\text { Cals. }\end{array}$ \\
\hline Meadow Hay : & & & & \\
\hline No. I, Ox A.. & 440 & 1991.3 & 4.526 & 1.185 \\
\hline " II, " I. . & 342 & 1686.9 & 4.933 & 0.778 \\
\hline “ V, " F. & 137 & 583.2 & 4.257 & 1.454 \\
\hline "V, " $\mathrm{G}_{0} . \ldots \ldots$ & 146 & 556.5 & 3.812 & 1.899 \\
\hline "VI, " H, Period 1.. & 193 & 781.4 & 4.049 & 1. 662 \\
\hline “VI, " $\mathrm{H}$, " $7 \ldots$ & 220 & 798.0 & 3.632 & 2.079 \\
\hline “VI, " J.......... & 213 & 930.5 & 4.368 & 1.343 \\
\hline " $\vec{A}, "$ II.. & 413 & 1925.7 & 4.662 & 1.049 \\
\hline "B, " V. & 451 & 1559.3 & 3.456 & 2.255 \\
\hline " B, " VI.. & 458 & 1737.9 & 3.794 & 1.917 \\
\hline “ $\mathbf{M}^{\prime}, " \mathrm{XX} .$. & 540 & 3224.6 & 5.973 & -0.262 \\
\hline Average ........ & 323 & 1434.1 & 4.439 & 1.272 \\
\hline Oat Straw : & & & & \\
\hline No, II, Ox F.... & 35 & 354.2 & 10.120 & -4.409 \\
\hline " II, " G.... & 48 & 274.0 & 5.710 & -0.001 \\
\hline Average . & 42 & 314.1 & 7.478 & -1.767 \\
\hline Wheat Straw: & & & & \\
\hline No. I, Ox $\mathrm{H}$. & -11 & 289.7 & (?) & (?) \\
\hline " I," & 14 & 413.2 & 29.520 & -23.809 \\
\hline Average .. & 2 & 351.5 & (?) & $(?)$ \\
\hline
\end{tabular}

* Corrected to nitrogen equilibrium.

animals and consequently that the metabolizable energy of the proteids cannot be widely different. In these results upon coarse fodders we meet an apparent contradiction of this conclusion, the metabolizable energy of the digestible protein as above computed being quite variable and much lower than the values found for pure proteids, while in the straw we get large negative values.

These latter results, however, while appearing at first sight paradoxical, furnish the clue to the apparent contradiction. In the case of the straws it is evident that a very considerable part of the potential energy of the urine must have been contained in nonnitrogenous substances, and that the latter must have been derived largely from the non-nitrogenous matter of the food. We have already seen, however, that these non-nitrogenous excretory prod- 
ucts are a normal constituent of the urine of cattle both on hay and on mixed rations. Their effect on the computation becomes more obvious in the case of the straws, simply because of the relatively small amount of protein in the latter feeding-stuffs. In these cases we get impossible results when we assume that all the potential energy of the urine is derived from the proteids metabolized, but it is clear that the results on the hays must be affected by the same error, and there is little question that the low and variable results noted in the table are to be explained in part in this way. We know no essential difference between the real proteids of the different coarse fodders, nor between those of coarse fodders and grain, nor any reason why they should not be metabolized in substantially the same way in the body and possess approximately the same metabolizable energy. It would seem more reasonable, then, to assume that the proteids of coarse fodders are metabolized substantially like those of concentrated fodders, and to take provisionally the results obtained for the protein of wheat gluten as representing approximately the metabolizable energy of the digested protein of the total ration, while we regard the remaining energy of the urine as derived largely from the non-nitrogenous nutrients of the food.

Hippuric Acid.-The statement last made, however, requires some modification. Not a little of the potential energy of the urine of cattle is contained in the hippuric acid which these animals excrete so abundantly. This being a nitrogenous product, it is natural to look upon it as derived from the proteids of the food, but it must not be forgotten that this is only partially true. Its glycocol portion originates in the proteids, but its phenyl radicle appears to be derived in these animals largely, if not wholly, from the non-nitrogenous ingredients of the food (compare p. 45). If the metabolism of one gram of protein is arrested at the glycocol stage by the presence in the organism of benzoic acid, there has already been liberated from it about 3 Cals. of energy, while about 2.7 Cals. remain in the glycocol. The resulting hippuric acid, however, contains about 11.6 Cals. of potential energy, or more than the original protein. In this case, then, the larger share of the energy of the excretory product (8.9 Cals. out of 11.6 Cals.), although contained in a nitrogenous substance, is derived ultimately from the 
non-nitrogenous matter of the food. It is clear, then, that the non-nitrogenous moiety of the hippuric acid and the non-nitrogenous organic matter of the urine together represent a large share of the potential energy of the latter, and that it is quite as incorrect to compute the metabolizable energy of the protein on the assumption that all the energy of the urine is derived from it as it is, on the other hand, to simply deduct from its gross energy the energy of the equivalent amount of urea.

Ether Extract. - Our only data upon this ingredient are furnished by the four experiments upon steers by Kellner in which peanut oil was added to the ration. In the first two experiments this oil was emulsified by means of a small quantity of soap made from the same oil. The result was a milky fluid which was readily digestible and which caused no considerable decrease in the digestibility of the basal ration. In the second two experiments the oil was emulsified with lime-water, giving a thickish mass which was not very well digested and which, in the case of $\mathrm{Ox} F$ particularly, caused a considerable decrease in the digestibility of the crude fiber and nitrogen-free extract of the basal ration. It should be noted that in the experiment with $\mathrm{Ox} \mathrm{E}$ the oil was not added to a basal ration, but was substituted for a part of the bran. From Table VI of the Appendix we obtain the summary tabulated on the next page, showing the effects of the oil upon the loss of energy in the gaseous hydrocarbons and in the urine, the results of the experiment on $\mathrm{Ox} \mathrm{E}$ being included.

Upon the evidence of these four experiments, bearing in mind that the one with Ox E was upon the substitution of oil for bran, we should not be inclined to ascribe to the fat of the food any considerable effect either upon the formation of hydrocarbons or upon the amount of potential energy carried off in the urine. As regards the hydrocarbons, the differences in the cases of Oxen D and G are insignificant. In the case of $\mathrm{Ox} \mathrm{F}$, on the contrary, the production of hydrocarbons was reduced nearly one half; this it may be noted was the case in which there was a considerable effect upon the digestibility of the basal ration. As regards the energy of the urine, the differences, except in the case of $\mathrm{Ox} \mathrm{E}$, are relatively small and are in both directions.

Provisionally, therefore, we are probably justified in assuming 


\begin{tabular}{|c|c|c|c|c|}
\hline $\begin{array}{l}\text { Ani- } \\
\text { mali. }\end{array}$ & Period. & & $\begin{array}{l}\text { Energy of Urine } \\
\text { (Corrected). Cals. }\end{array}$ & $\begin{array}{c}\text { Energy of } \\
\text { Methane, Cals. }\end{array}$ \\
\hline \multirow[t]{2}{*}{$\begin{array}{l}\mathrm{D} \\
\mathrm{D}\end{array}$} & \multirow[t]{2}{*}{$\begin{array}{l}3 \\
1\end{array}$} & \multirow{2}{*}{$\begin{array}{l}\text { With oil................ } \\
\text { Basal ration.......... } \\
\quad \text { Differences........... }\end{array}$} & $\begin{array}{l}2851.2 \\
2407.0\end{array}$ & $\begin{array}{l}2909.0 \\
2957.0\end{array}$ \\
\hline & & & -55.8 & -48.0 \\
\hline \multirow[t]{2}{*}{$\frac{\mathrm{E}}{\mathrm{E}}$} & \multirow[t]{2}{*}{$\begin{array}{l}3 \\
1\end{array}$} & \multirow{2}{*}{$\begin{array}{l}\text { With oil................ } \\
\text { Basal ration.......... } \\
\text { Differences........... }\end{array}$} & $\begin{array}{l}2026.2 \\
2312.9\end{array}$ & $\begin{array}{l}2640.8 \\
2950.4\end{array}$ \\
\hline & & & -286.7 & -309.6 \\
\hline \multirow[t]{2}{*}{$\begin{array}{l}\mathbf{F} \\
\mathbf{F}\end{array}$} & \multirow[t]{2}{*}{$\begin{array}{l}5 \\
3\end{array}$} & \multirow{2}{*}{ 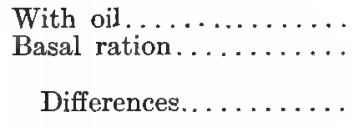 } & $\begin{array}{l}1455.0 \\
1530.0\end{array}$ & $\begin{array}{l}1369.1 \\
2560.7\end{array}$ \\
\hline & & & -75.0 & -1191.6 \\
\hline \multirow[t]{2}{*}{$\begin{array}{l}\mathrm{G} \\
\mathrm{G}\end{array}$} & \multirow[t]{2}{*}{$\begin{array}{l}5 \\
3\end{array}$} & $\begin{array}{l}\text { With oil............... } \\
\text { Basal ration.......... }\end{array}$ & $\begin{array}{l}1452.1 \\
1359.6\end{array}$ & $\begin{array}{l}2371.2 \\
2524.7\end{array}$ \\
\hline & & Differences..... & 92.5 & -153.5 \\
\hline
\end{tabular}

as Kellner does that none of the energy of the fat was lost either in the hydrocarbons or in the urine, and that consequently the metabolizable energy of the digested fat was the same as its gross energy, namely, 8.821 Cals. per gram, as given on p. 308 . If we assume that the ether extract of hay behaves like the peanut oil, taking no part either in the production of methane or in the loss of energy through the urine, its metabolizable energy would likewise be the same as its gross energy, namely,8.322 Cals. per gram, as computed on p. 305 . No results upon the metabolizable energy of the ether extract are available in the case of other species of herbivorous animals.

Carbohydrates. - Those of Kellner's experiments in which starch, as a representative of the readily digestible carbohydrates. and extracted straw, consisting largely of "crude fiber," were added to the basal ration afford data for an approximate computation of the metabolizable energy of this group of nutrients in the ox, and experiments by Lehmann, Hagemann \& Zuntz afford partial data for the horse.

STARCH.-The results of the Möckern experiments, as recorded in Tables III and IV of the Appendix, show that the starch had but a slight effect upon the amount of potential energy carried off in the urine of the ox, although the general tendency was to 
diminish it slightly. On the other hand, the formation of hydrocarbons was markedly increased except in two cases. It has already been shown that the proteids of the food do not take part in the production of these gases, and that the same is probably true of the fat under normal conditions. Neglecting the small effects upon the urine, therefore, we may compare directly the increase in the digested carbohydrates with the increase in the gaseous hydrocarbons, using for this purpose the differences between the two rations uncorrected for the slight variations in the consumption of dry matter.

Taking first the last five of Kellner's experiments; * which seem to represent the most normal conditions, we have the following:

\begin{tabular}{|c|c|c|c|}
\hline & \multicolumn{2}{|c|}{$\begin{array}{c}\text { Difference in Carbohydrates } \\
\text { Digested. }\end{array}$} & \multirow{2}{*}{$\begin{array}{l}\text { Difference in } \\
\text { Energy of } \\
\text { Methane, } \\
\text { Cals. }\end{array}$} \\
\hline & $\begin{array}{c}\text { Crude Fiber, } \\
\text { Grms. }\end{array}$ & $\begin{array}{c}\text { Nitrogen-free } \\
\text { Extract. } \\
\text { Grms. }\end{array}$ & \\
\hline 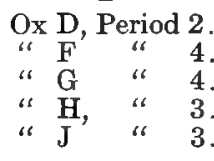 & $\begin{array}{l}-64 \\
-64 \\
-50 \\
-26 \\
-9\end{array}$ & $\begin{array}{l}+1388 \\
+1609 \\
+1598 \\
+1861 \\
+1501\end{array}$ & $\begin{array}{l}+424.4 \\
+822.0 \\
+645.8 \\
+604.5 \\
+769.9\end{array}$ \\
\hline Totals ... & -213 & +7957 & 3266.6 \\
\hline
\end{tabular}

Assuming that the same proportion of hydrocarbons is produced in the fermentation of crude fiber as in that of starch, we may compare the algebraic sum of the two with the energy of the methane as follows:

3266.6 Cals. $\div(7957-213)=0.422$ Cals. per gram.

Subtracting the latter result from the gross energy of the digested nitrogen-free extract of starch, we have for the metabolizable energy of the latter

4.185 Cals. -0.422 Cals. $=3.763$ Cals. per gram.

In the experiments on Oxen $\mathrm{B}$ and $\mathrm{C}$ the basal ration was a heavy one, with a rather wide nutritive ratio, and already contained large amounts of digestible carbohydrates. Under these circumstances the added starch was very imperfectly digested, while

*Loc, cit., 53. 422. 
the production of hydrocarbons was diminished. Kellner suggests that the latter effect may have been due to a partial suppression of the organisms causing the methane fermentation by other species, and suspects that the presence of large amounts of carbohydrates along with little protein favors this result. At any rate, the conditions are evidently unusual if not abnormal.

In Kühn's experiments the starch was added to a ration of coarse fodder. The nutritive ratio was wide, but the absolute amount of carbohydrates was much less than in the two experiments by Kellner just mentioned, less starch appeared to escape digestion, and the production of hydrocarbons was increased in every case. The following are Kühn's* results:

\begin{tabular}{|c|c|c|c|}
\hline & \multicolumn{2}{|c|}{$\begin{array}{l}\text { Difference in Carbohydrates } \\
\text { Digested. }\end{array}$} & \multirow{2}{*}{$\begin{array}{l}\text { Difference in } \\
\text { Energy of } \\
\text { Methane, } \\
\text { Cals. }\end{array}$} \\
\hline & $\begin{array}{c}\text { Crude Fiber, } \\
\text { Grms. }\end{array}$ & $\begin{array}{l}\text { Nitrogen-free } \\
\text { Extract, Grms. }\end{array}$ & \\
\hline $\begin{array}{l}\text { Ox } \\
\text { III, } \\
\text { " }\end{array}$ & $\begin{array}{l}-220 \\
-180 \\
-195 \\
-130 \\
-176 \\
-146 \\
-88 \\
-156\end{array}$ & $\begin{array}{l}1529 \\
1408 \\
1537 \\
1539 \\
2619 \\
1468 \\
1554 \\
2587\end{array}$ & $\begin{array}{r}706.2 \\
856.7 \\
752.6 \\
665.5 \\
1181.0 \\
729.5 \\
649.9 \\
1407.0\end{array}$ \\
\hline Totals .... & -1291 & 14241 & 6948.4 \\
\hline
\end{tabular}

Assuming as before the equivalence of crude fiber and nitrogenfree extract as regards the production of hydrocarbons we have

6948.4 Cals. $\div(14241-1291)=0.537$ Cals. per gram, 4.185 Cals. -0.537 Cals. $=3.648$ Cals, per gram.

Determinations by Lehmann, Hagemann \& Zuntz $\dagger$ of the amount of methane produced by the horse will be considered in connection with the metabolizable energy of crude fiber. Zuntz $\downarrow$ has pointed out that the fermentation of the food in the horse takes place largely in the cœcum and after the more digestible carbohydrates have been resorbed. Accordingly he regards the metabo-

* Loc. sit., 44, 570.

$\dagger$ Landw. Jahrb., 23, 125.

\pm Arch. ges. Physiol., 49, 477 . 
lizable energy of starch and similar bodies in this animal as equal to their gross energy, viz., 4.185 Cals. per gram in the case of starch.

Extracted Straw.-The two experiments in which extracted straw was added to the basal ration, when computed as in the case of the starch experiments, give the following results:

\begin{tabular}{|c|c|c|c|}
\hline & \multicolumn{2}{|c|}{$\begin{array}{c}\text { Difference in Carbohydrates } \\
\text { Digested. }\end{array}$} & \multirow{2}{*}{$\begin{array}{l}\text { Difference in } \\
\text { Energy of } \\
\text { Methane, } \\
\text { Cals. }\end{array}$} \\
\hline & $\begin{array}{l}\text { Crude Fiber, } \\
\text { Grms. }\end{array}$ & $\begin{array}{c}\text { Nitrogen-free } \\
\text { Extract, } \\
\text { Grms. }\end{array}$ & \\
\hline 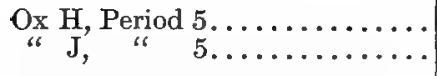 & $\begin{array}{l}2046 \\
1987\end{array}$ & $\begin{array}{l}439 \\
449\end{array}$ & $\begin{array}{l}1425.1 \\
1425.2\end{array}$ \\
\hline Totals ................ & 4033 & 888 & 2850.3 \\
\hline
\end{tabular}

The loss of energy in the hydrocarbons equals 0.579 Cals. per gram of total digestible carbohydrates (of which 82 per cent. was crude fiber), and the corresponding metabolizable energy of the carbohydrates is 3.668 Cals. per gram. This is a materially lower figure than Kellner found for starch and indicates that the loss of energy in the gaseous products of fermentation is greater in the case of crude fiber than in that of the more soluble carbohydrates, an indication which, as we shall see, is confirmed by the results of other experiments.

Carbohydrates of Coarse Fodders. - Upon the same two assumptions, viz., that the carbohydrates are the sole source of the gaseous hydrocarbons, and that the latter represent the entire loss of energy from the digested carbohydrates, we may compute the metabolizable energy of the total digestible carbohydrates of the various coarse fodders exactly as in the case of the extracted straw: the results being tabulated on the next page.

If we average the results for each feeding-stuff and compute them as in the foregoing cases, our findings are as given on p. 329, where the rations are arranged in the order of their crude fiber content. In computing the metabolizable energy, the gross energy of the digested carbohydrates has been assumed to be the average 
COARSE FODDERS ALONE.

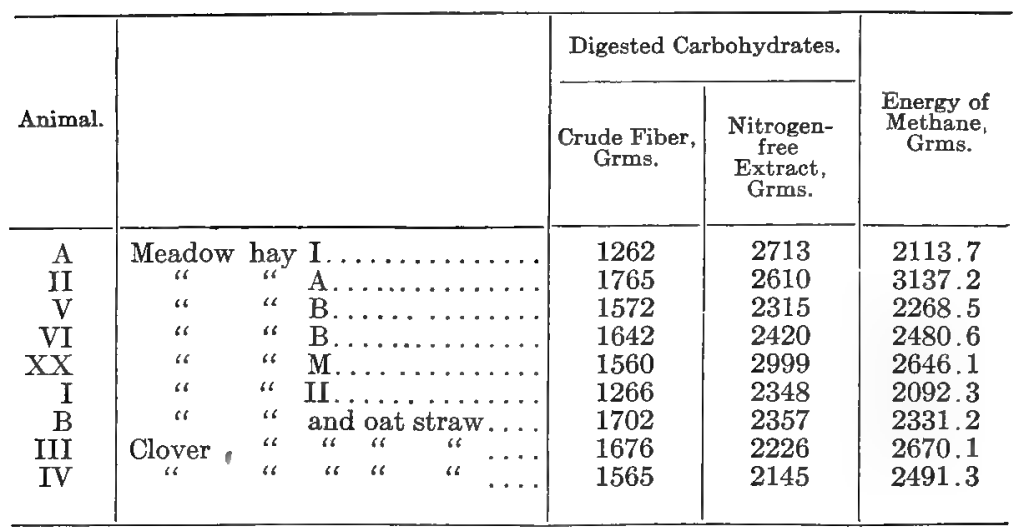

COARSE FODDER ADDED TO BASAL RATION.

\begin{tabular}{|c|c|c|c|c|c|}
\hline \multirow{2}{*}{ Animal. } & \multirow{2}{*}{ Period. } & & \multicolumn{2}{|c|}{$\begin{array}{l}\text { Difference in } \\
\text { Carbohydrates Digested. }\end{array}$} & \multirow{2}{*}{$\begin{array}{c}\text { Energy of } \\
\text { Methane, } \\
\text { Cals. }\end{array}$} \\
\hline & & & $\begin{array}{l}\text { Crude Fiber, } \\
\text { Grras. }\end{array}$ & $\begin{array}{l}\text { Nitrogen- } \\
\text { free } \\
\text { Extract, } \\
\text { Grms. }\end{array}$ & \\
\hline F. & 1 & Meadow hay $V$. & 546 & 836 & 689.9 \\
\hline $\mathrm{G}$ & 2 & " $"$ V. & 538 & 886 & 907.4 \\
\hline $\mathrm{H}$ & 2 & "VI. & 703 & 1129 & 727.2 \\
\hline H & $\overline{7}$ & "VI. & 739 & 1236 & 898.0 \\
\hline$J$ & 2 & " $\quad$ VI. & 683 & 1213 & 984.8 \\
\hline $\mathrm{F}$ & 2 & Oat straw II..... & 694 & 721 & 679.2 \\
\hline $\mathrm{G}$ & 1 & " $"$ II... & 595 & 684 & 923.4 \\
\hline $\mathrm{H}$ & 1 & Wheat straw I.. & 821 & 524 & 1213.0 \\
\hline J & 1 & " $\quad$ I. & 829 & 616 & 1281.0 \\
\hline
\end{tabular}

of the results given on pp. 304 and 306 for the digested crude fiber and nitrogen-free extract of coarse fodders, viz., 4.226 Cals. per gram.

As a whole, the figures given on p. 329 show a tendency toward an increased production of methane with an increase in the proportion of crude fiber, but considerable variations are found in individual cases. It is evident, therefore, from these results, as well as from those already cited in connection with the experiments upon starch and upon molasses, that a variety of factors influence the extent of this fermentation. 


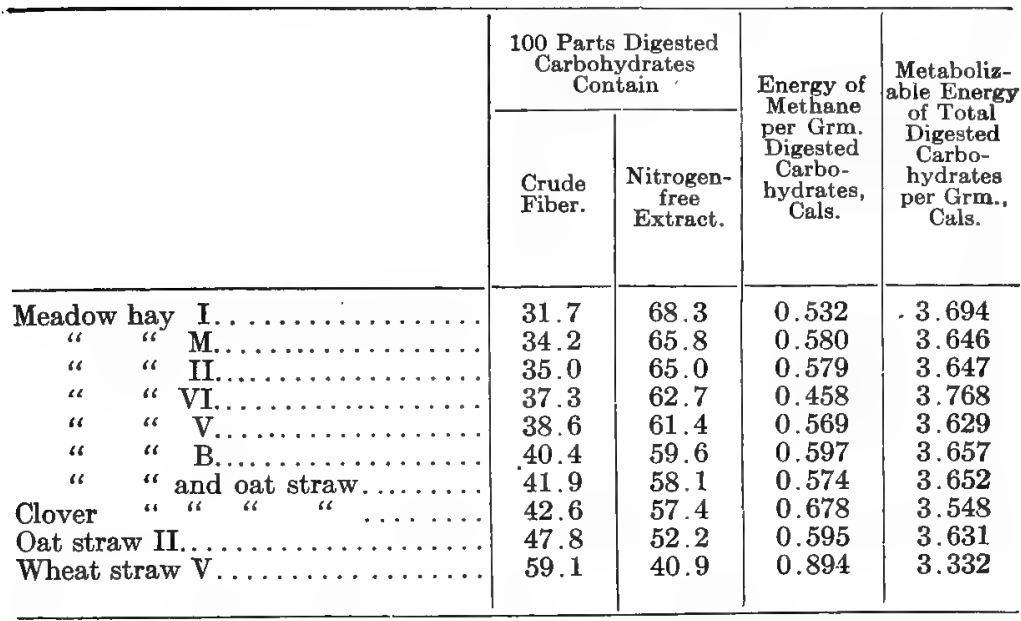

A comparison of the methane production with the digestibility of the feeding-stuffs shows in general that the former is greatest when the latter is least, that is, with the feeding-stuffs which tend to remain longest in the digestive tract. Here too, however, exceptions occur, and it would appear that the physical condition of the feeding-stuff is not without its influence. The exceedingly complicated nature of digestion in ruminants, and the fact that it is a chemical rather than a physiological process, and is therefore subject to considerable variations according to the nature and amount of the food, render it difficult, if not impossible, with our present knowledge to compute very trustworthy averages for the amount of energy carried off in this way.

CRUde FIBer. Ruminants.-Both the ultimate composition and the heat of combustion of the digested nitrogen-free extract have been shown to agree quite closely with those of starch, and the nutritive value of the former has commonly been assumed to be the same as that of the latter. If we are justified in somewhat extending this, and assuming that the nitrogen-free extract of coarse fodders suffers the same loss by the methane fermentation as does starch, the figures of the preceding paragraphs supply data for computing the corresponding loss suffered by the crude fiber. 
In the case of the extracted straw, for example, there was digested in the total of the two experiments :

Crude fiber................ 4033 grams

Nitrogen-free extract........... 888 "

Assuming the loss of energy in the methane to have been 0.422 Cal. per gram of nitrogen-free extract digested (the same as that found by Kellner for starch, p. 325), the 888 grams of these substances correspond to a loss of 374.7 Cals. Subtracting this from the total loss of 2850.2 Cals., we have 2475.5 Cals. as the energy of the methane produced from 4033 grams of crude fiber, which is equal to $0.614 \mathrm{Cal}$. per gram. The total energy of the digested crude fiber was shown on p. 304 to be approximately 4.220 Cals. per gram. Subtracting the loss in the methane, 0.614 Cal., leaves 3.606 Cals. as the metabolizable energy of one gram of digested crude fiber. A similar computation of the average results upon the other coarse fodders affords the figures of the following table for the metabolizable energy of one gram of digestible crude fiber:

\begin{tabular}{|c|c|c|}
\hline Digestible Crude Fiber of & $\begin{array}{l}\text { Loss in Methane, } \\
\text { Cals. }\end{array}$ & $\begin{array}{c}\text { Metabolizable } \\
\text { Energy, } \\
\text { Cals. }\end{array}$ \\
\hline 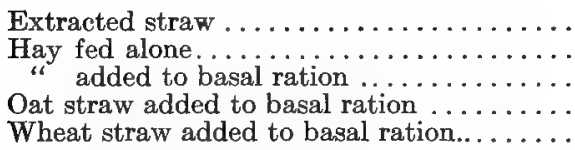 & $\begin{array}{l}0.614 \\
0.909 \\
0.614 \\
0.783 \\
1.219\end{array}$ & $\begin{array}{l}3.606 \\
3.311 \\
3.606 \\
3.437 \\
3.001\end{array}$ \\
\hline
\end{tabular}

The loss of energy in methane, as thus computed, is in all instances greater than in the case of starch. Owing, however, to the slightly higher value obtained for the gross energy of the digested crude fiber, the difference in metabolizable energy between starch and crude fiber is somewhat less marked, and is hardly sufficient of itself to justify assigning a materially lower nutritive value to the latter.

It is worthy of note also that the loss in the methane appears to be a very variable one, justifying the conclusion already reached that other factors than the proximate composition of the food materially affect the extent of the methane fermentation.

The Horse.-The production of methane by the horse appears to be much less copious than that by ruminants. Lehmann, Hage- 
mann \& Zuntz * in eight respiration experiments obtained the following results, the hydrocarbons being computed as methane:

Crude Fiber Digested.

698.5 grams

$\begin{array}{cc}538.9 & \text { “ } \\ 451.7 & \text { “ } \\ \text { “ } & \text { “ } \\ \text { “ } & \text { “ } \\ \text { ، } & \text { “ }\end{array}$

Methane Excreted.

26.8 grams

33.4 "

13.0 "

$20.0 " 1$

16.4 "

$31.0 "$ "

22.1 "

23.0 "

As already noted on p. 326, Zuntz $\dagger$ has pointed out that the fermentation of the food in the horse takes place largely in the cœcum and after the more digestible carbohydrates have been resorbed. The authors consequently compute the excretion of methane entirely upon the crude fiber of the food. On the average of the eight somewhat discordant experiments, in which the food consisted of oats, hay, and cut straw, 100 grams of digested crude fiber yielded 4.7 grams of methane, which corresponds exactly with the results reported by Tappeiner $\ddagger$ for the artificial fermentation of cellulose. In the same experiments an excretion of approximately 0.203 gram of hydrogen per 100 grams digested crude fiber was observed. Deducting the corresponding amounts of energy from the energy of the apparently digested cellulose we have-

Total energy of 1 gram..... ... 4.220 Cals.

Energy of $\mathrm{CH}_{4}(0.047$ gram). 0.627 Cal.

Energy of $\mathrm{H}(0.002$ gram $) \ldots 0.070$ ". $0.697 \mathrm{Cal}$.

Metabolizable energy of 1 gram.......... 3.523 Cals. $\$$

While less methane is apparently produced by the horse than by the ox, the assumption that it all arises from the fermentation of the crude fiber gives the latter a metabolizable energy not greatly different from that found in the case of the ox. It is of course

* Landw. Jahrb., 23, 125.

† Arch. ges. Physiol., 49, 477.

$\ddagger$ Zeit. f. Biol., 20, 88 .

\& As computed by the authors, 3.487 Cals. on the basis of 4.185 Cals. total energy per gram of crude fiber. 
implied in this that the metabolizable energy of the digested nitrogen-free extract is the same as its gross energy.

Summary.-The results recorded in the preceding paragraphs regarding the metabolizable energy of the several classes of digestible nutrients are summarized in the following table:

METABOLIZABLE ENERGY OF DIGESTIBLE NUTRIENTS.

\begin{tabular}{|c|c|c|c|}
\hline & $\begin{array}{l}\text { Cattle, } \\
\text { Cals. per } \\
\text { Grm. }\end{array}$ & $\begin{array}{c}\text { Horse, } \\
\text { Cals. per } \\
\text { Grm. }\end{array}$ & $\begin{array}{c}\text { Swine, } \\
\text { Cals. per } \\
\text { Grm. }\end{array}$ \\
\hline 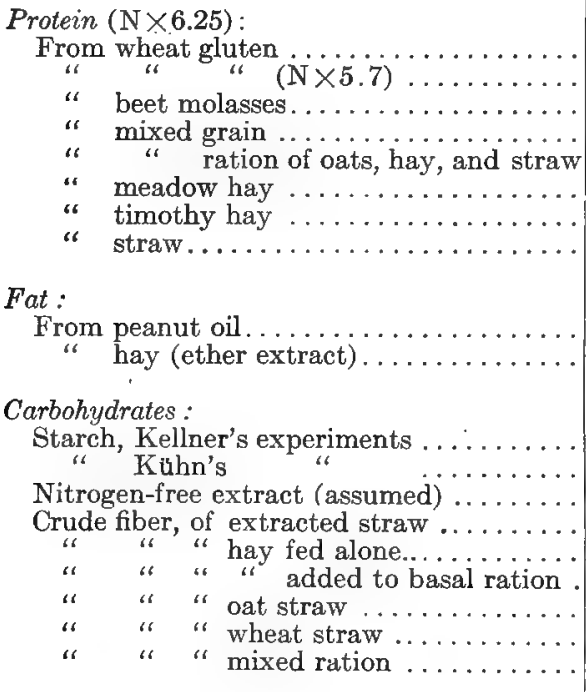 & $\begin{array}{c}8.821 \\
8.322 \\
\\
3.763 \\
3.648 \\
\cdots . . \% \\
3.606 \\
3.311 \\
3.606 \\
3.437 \\
3.001\end{array}$ & 3.228 & 4.083 \\
\hline
\end{tabular}

Perhaps the most striking thing about these figures is the wide range of the results upon the same class of nutrients. For reasons already stated, this is most noticeable with the protein, but it is sufficiently marked also with the other two groups. Moreover, such meager data as we possess regarding other animals than the ox indicate that the results vary with the species of animal, a fact which should not surprise us, but which, nevertheless, adds materially to the complexity of the subject and greatly widens the range of necessary investigation. It is obvious, therefore, that at present our knowledge is too imperfect to allow of the assignment of average values for the metabolizable energy of the different classes of 
nutrients (as ordinarily determined) even for a single species of animal.

The results tabulated above, however, are amply sufficient to justify the statement on p. 279 that Rubner's averages are not applicable to herbivorous animals, and that the metabolizable energy as computed with their aid is likely to vary widely from the truth. Indeed, since Rubner's factor for fat (9.3 Cals. per gram) is 2.27 times that for carbohydrates and protein (4.1 Cals. per gram) a computation of the metabolizable energy of feeding-stuffs or rations as it has not uncommonly been made simply gives a series of figures about 4.1 times as great as that obtained for total digestible matter when the digestible fat is reduced to its starch equivalent by multiplication by $2 \frac{1}{4}$. So far, then, as a comparison of one feeding-stuff or ration with another is concerned, this process adds no whit to our knowledege. It does, it is true, give some idea, albeit an inadequate one, of the total amount of metabolizable energy present. As yet, however, our accurate knowledge of the energy requirements of domestic animals for various purposes is comparatively meager. If we base our computations on the feeding standards now current, we simply repeat with them the useless multiplication performed on the feeding-stuffs. On the other hand, if we take the results of such exact experiments on the metabolism of energy as are available, then, as the above results show, we shall be computing the energy requirements upon one basis and the energy supply upon a materially different one.

Significance of the Results.-A much more fundamental problem than that raised in the foregoing paragraph confronts us when we come to reflect upon the general method by which it has been attempted to compute the metabolizable energy of nutrients, and to consider the real significance of the results. In so doing we may properly confine ourselves to the results upon cattle, those for horses and for swine being more or less fragmentary and uncertain. By far the larger proportion of the results above tabulated, as well - as the most important of them, are based on experiments in which additions were made to a basal ration, the computation being by difference. As was pointed out in discussing the apparent metabolizable energy of the organic matter on previous pages, and as was specifically illustrated in the case of one experiment on 
molasses (p.291), the difference in the metabolizable energy of the excreta is the algebraic sum of the differences in the energy of methane, urine, and the several proximate ingredients of the feces, and some of these differences may be positive and others negative. The computations of the metabolizable energy of the organic matter as made in the earlier paragraphs give the net result to the animal under the condition of the experiment and include all the secondary effects upon digestion, etc.

In the computations here considered Kellner's methods have been followed. In the first place the influence of the added feed upon the digestibility of the basal ration has been eliminated by basing the computation upon the digested matter. Still further, such effects as the decrease of the methane excretion in certain of the experiments with molasses, oil, and starch, and the diminished export of energy in the urine under the influence of starch and extracted straw, have not entered into the computation. In other words, the endeavor has been to determine the actual amount of energy liberated by the breaking down of the molecules of the digested starch or protein or fat in the organism without regard to these various incidental effects; that is, to determine the real and not the apparent metabolizable energy.

Either method of computation would seem to be entirely defensible, and our choice between them will be largely determined by the point of view. For the purposes of the physiologist, desirous of tracing the details of the chemistry and physics of metabolism, the results obtained by the latter method will be of more interest. On the other hand, the student of nutrition who is especially interested in the problems of feeding will not fail to note that the results thus reached represent, from his standpoint, only a part of the truth. They show (barring errors of detail) how much energy is liberated in the body from the several nutrients, but the loss or saving of energy in the incidental processes constitutes just as real a part of the balance of energy which he wishes to determine as the energy liberated from the nutrients themselves, and must be taken . account of in his calculations. Whether this can best be done by using some such factors as those just tabulated and then making a correction for these incidental gains and losses, or whether the method followed in the earlier paragraphs is to be preferred, it 
would probably be premature to attempt to decide at present. Pending further investigation and experience, however, it should be remembered that the figures on p. 332 will give, in most cases, too high results for the metabolizable energy of mixed rations, while the same thing is still more emphatically true of Rubner's factors.

One additional point requires mention. In discussing the metabolizable energy of protein it was pointed out (p. 320) that it is at least a plausible hypothesis that the proteids are metabolized in the herbivora substantially as in carnivora, and that the excess of energy in the urine is derived from the non-nitrogenous ingredients of the food. If we accept this hypothesis, however, and assume the metabolizable energy of protein $(\mathrm{N} \times 6.25)$ to be in the neighborhood of 4.9 Cals. per gram, then the figures for the non-nitrogenous nutrients are subject to a still further deduction, especially in the case of coarse fodders. If we were to assign to the fat its full value as given, it would not be difficult to compute the metabolizable energy of the carbohydrates on this basis, and probably a set of factors could be worked out which would correspond to the actual results obtained with mixed rations. These, however, if successfully obtained, would be substantially identical with the results given on previous pages for the apparent metabolizable energy of total or of digestible organic matter, and it does not appear that the former would offer sufficient advantages over the latter to justify the labor involved in their computation. 


\section{CHAPTER XI.}

\section{INTERNAL WORK.}

\section{$\S$ I. The Expenditure of Energy by the Body.}

Having considered the food in the light of a supply of energy to the animal, it now becomes desirable to take a more general view of the subject and inquire into the uses to which the energy of the food is applied in the organism.

We have already distinguished between that portion of the potential energy of the food which is convertible into kinetic energy in the body, and which we have here called metabolizable energy, and that portion of it which is rejected for one reason or another in the potential form in the various excreta. This latter portion we may dismiss from consideration for the present. The former portion-the metabolizable energy - as common experience informs us, is applied to two main purposes. First, it supplies the energy for carrying on the various activities of the body. Second, if the supply is in excess of the requirements of the body a portion of it may temporarily escape conversion into the kinetic form and be stored up as gain of tissue, notably of fat. We may say briefly, then, that the metabolizable energy of the food is used, first, for the production of "physiological work" and second, for the storage of energy.

Physiological Work.-The term "physiological work" in the previous sentence is employed in a somewhat loose and general sense to designate all those activities in the body which are sustained by the metabolizable energy of the food and whose ultimate result is the production of heat or motion. A more definite idea of what the term includes may be gained by a consideration of the chief factors which go to make up the physiological work of the body. 
Work of The Voluntary Muscles.-The most obvious form of physiological work is that performed by the contraction of the voluntary muscles, either in the performance of useful work or in the various incidental movements made during the waking hours. In a sense the production of muscular work may be said to be the chief end of the metabolizable energy of the food, inasmuch as all the other activities of the body (apart from the reproductive functions and, of course, from mental activities) are accessory to this. In amount, however, the energy of muscular work is much less than the energy expended in other forms of physiological work and consumes a comparatively small percentage of the metabolizable energy of the food.

INTERNAI WORK.- The body of an animal in what we commonly speak of as a state of rest is still performing a large amount of work. The most evident forms of this are the work of circulation and respiration. In addition to these, however, there are less obvious kinds of work whose total is probably very considerable. The body is an aggregate of living cells. The living cell, however, is constantly carrying on activities of various sorts, and these activities require a supply of energy, although how much of the energy of the food is consumed in the various processes of secretion, osmosis, karyokinesis, etc., it is difficult to say.

In the numerous varieties of internal work the energy involved passes through various forms. Ultimately, however, since it accomplishes no work upon the surroundings of the animal, it is converted into heat and leaves the body either by radiation and conduction, as the latent heat of water vapor or as the sensible heat of the excreta.

Work of Digestion and Assimilation.-Logically the work of digestion and assimilation would be classed as part of the internal work of the body, but motives of convenience make it desirable to consider it separately.

In a fasting animal, with the digestive tract empty, the various forms of internal work indicated above go on with a considerable degree of constancy, and the resulting heat production is quite uniform under like conditions. If food be given to such an animal there results very promptly an increase in the excretion of carbon 
dioxide and the absorption of oxygen and in the amount of heat produced. In general terms this is brought about in four ways:

First, the muscular work required for masticating and swallowing the food and moving it through the digestive apparatus involves an expenditure of energy which finally gives rise to the evolution of heat.

Second, the activity of the various secreting glands of the digestive tract is stimulated, again making a demand for energy and giving rise to an increased heat production.

Third, the work of the resorbing cells likewise makes demand for energy.

Fourth, the various fermentations, cleavages, hydrations, and syntheses which the food ingredients undergo in the course of digestion, resorption, and assimilation may occasion in individual cases either an evolution or an absorption of energy, but taken as a whole result in the production of a greater or less amount of heat and consume a corresponding amount of the metabolizable energy of the food.

Production of Heat. - The body temperature of the healthy warm-blooded animal is practically constant, any considerable variation from the average indicating some serious disturbance of the animal economy. Since this temperature is ordinarily higher than that of the environment, a continual production of heat is necessary to maintain it.

As stated above, the various forms of internal work, including the work of digestion and assimilation, give rise in the aggregate to the evolution of a large amount of heat, and this heat is of course. available for the maintenance of the body temperature.

Whether its amount is sufficient for this purpose, or whether under any or all circumstances there is a production of heat for its own sake, simply to keep the animal warm, is still a debatable question. Many eminent physiologists, notably Chauveau and his associates, hold that the primary function of metabolism is tofurnish energy for the physiological processes going on in the body. They hold that the potential energy of the food is converted immediately into some form of physiological energy, which in its turn, in fulfilling its functions, is converted into heat which serves incidentally to maintain the body temperature. In other words, they regard heat as substantially an excretion and would consider that 
in the course of organic evolution those forms have survived in which the incidental heat production was sufficient to meet the demand of the environment.

Other physiologists no less eminent hold that at least an exceptional demand for heat (low external temperature) may be met by a direct combustion of food or body material for that purpose. We shall have occasion later to give further consideration to these divergent views.

Summary.-The following scheme may serve to summarize what has been said above regarding the uses to which the energy of the food is put in the body, the possible direct heat production being considered, for convenience, as part of the physiological work of the body in order to include it among the other forms of the expenditure of energy:

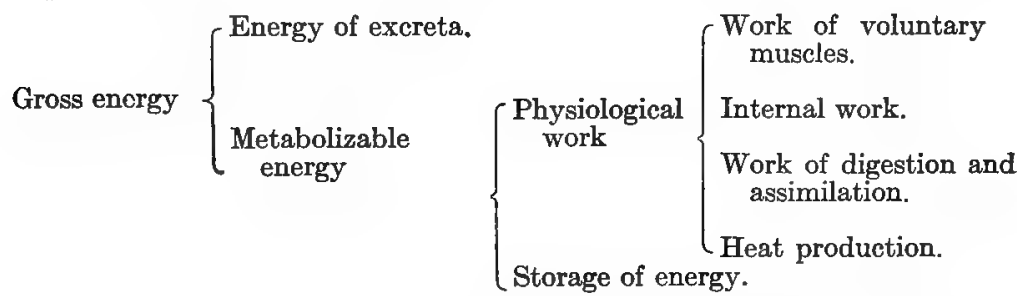

For the sake of directness of statement, language has been used above which seems to imply that the food is directly oxidized somewhat like the fuel in a locomotive. While statistically the effect is the same as if this were the case, it must not be forgotten that the body itself constitutes a reservoir of potential energy and that the energy liberated in its various activities comes primarily from the potential energy stored up in its various tissues, while the function of the food is to make good the loss this occasioned.

The metabolism of matter and energy in the body might be compared to the exchange of water in a mill-pond. The water in the pond may represent the materials of the body itself, while the water running in at the upper end represents the supply of matter and energy in the food, and that going down the flume to the millwheel the metabolism required for the production of physiological work as above defined. The water flowing into the pond does not immediately turn the wheel, but becomes part of the pond and loses its identity. Part of it may be drawn into the main current 
and enter the flume comparatively soon, while another part may remain in the pond for a long time. Pursuing the comparison still further, as but a small proportion of the energy liberated in the descent of the water in the flume takes the form of mechanical energy, most of it being converted into heat, so in the body but a small proportion of the energy expended in physiological work takes ultimately the form of mechanical energy. Finally, if we compare the flow of water in the stream below the dam to the heat production of the body, that flow miay be increased, in case of need, in two ways, viz., by opening the gate wider and letting more water pass through the flume (increase of physiological work) or by lowering the dam and allowing more water to flow over, corresponding to a heat production for its own sake, if such takes place.

The succeeding sections of this chapter will be devoted to a consideration of the expenditure of energy in the various forms of internal work, including that of digestion and assimilation, while the subjects of the production of external work and of] the storage of energy may be more appropriately considered in subsequent chapters.

\section{$\S 2$. The Fasting Metabolism.}

If an animal be deprived of food,for a sufficient length of time to empty the digestive tract, and kept in a state of rest as regards muscular exertion, the expenditure of energy in external work and in the work of digestion and assimilation are both eliminated, while there can be, of course, no storage of energy. Under these conditions the metabolism of energy in the organism is confined to the maintenance of those essential vital activities which were grouped above under the term "internal work" in the narrower sense, together with any direct production of heat for its own sake. The fasting animal, then, affords the most favorable opportunity to study the laws governing the expenditure of energy for the internal work of the body. The fasting metabolism has already been considered in Part I from the side of the matter involved; here we are concerned with its energy relations.

Nature of Demands for Energy.

Without attempting to enter into details, it may be said that the internal work of the fasting organism may be roughly classified 
as muscular, glandular, and cellular. To the demand for energy for these purposes we have probably to add, at least in some cases, a direct demand for heat production.

Muscular Work.-The more obvious forms of muscular work in the quiescent animal are circulation and respiration. To these are to be added as minor factors any movements of other internal organs, and especially the general tonus of the muscular system, while finally, the various incidental movements made by such an animal, although not logically belonging in the category of internal work, practically have to be classed there in actual experimentation. It would be aside from the purpose of this volume to enter into any detailed consideration of these forms of internal work, but a few general statements regarding their amount may be of interest.

Circulation.-The work performed by the heart is determined by two factors, viz., the weight of the blood moved and the mean arterial pressure overcome. Quite divergent results have been obtained by various investigators for the former factor, while the latter is more readily determinable. Zuntz \& Hagemann * estimate the output of blood by the heart of the horse from a comparison between the blood gases and the respiratory exchange, and compute the expenditure of energy in circulation to be 5.01 per cent. of the total metabolism of the horse in a state of rest and 3.77 per cent. during moderate work. Hill $\dagger$ estimates the average work of the heart in man at about 24,000 kilogram-meters in twenty-four hours. As the velocity of the circulation increases, the friction in the peripheral blood-vessels, and consequently the arterial pressure, rapidly augments, so that in case of severe muscular exertion, for example, the work of the heart may readily become excessive.

Respiration.-The work of respiration consists essentially of an expansion of the thorax against the resistance caused by the atmospheric pressure and the elasticity of the lungs and the rib cartilages. Zuntz \& Hagemann $f$ estimate its amount in the horse at about 4.7 per cent. of the total metabolism.

Muscular Tonus.-As was pointed out in Chapter VI, the living

* Landw. Jahrb., 27, Supp. III, 371.

$\dagger$ Schäffer's Text-book of Physiology, II, 43.

\$oc. cit. 
muscle is in a constant state of slight tension or tonus, and is constantly the seat of metabolic activities which we may presume serve, in part at least, to maintain that tonus. This is, of course, cquivalent to saying that there is a continual liberation of kinetic encrgy in the resting muscle, which temporarily takes the form of muscular elasticity but ultimately appears as heat. As to the amount of energy thus liberated exact information seems to be lacking, but in view of the relatively large mass of the muscles as compared with that of the other active tissues we may assume that it is not inconsiderable. The same thing would seem to be indicated also, as noted in Chapter VI (p. 191), by the great decrease in the metabolism and heat production ordinarily observed as the result of paralysis of the motor nerves by curari.

Incidental Muscular Work.-It is rare that an animal, even when at rest in the ordinary sense, does not execute more or less motions of various parts of the body, all of which involve a conversion of potential energy into the kinetic form. Even apparently insignificant movements may materially increase the amount of metabolism. Zuntz \& Hagemann, * for example, report a respiration experiment upon a horse in which the uneasiness caused by the presence of a few flies in the chamber of the apparatus caused an increase of over 10 per cent. in the metabolism. Johanson, Landergren, Sonden \& Tigerstedt, $\dagger$ in two-hour periods, found the following average and minimum values per day and kilogram weight for the excretion of carbon dioxide by a fasting man during sleep, the results plainly showing the increased metabolism due to restlessness:

\begin{tabular}{|c|c|c|}
\hline & $\begin{array}{c}\text { Average, } \\
\text { Grms. }\end{array}$ & $\underset{\text { Grms. }}{\text { Minimum, }}$ \\
\hline 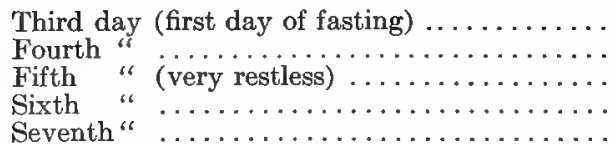 & $\begin{array}{l}7.296 \\
7.704 \\
8.136 \\
7.488 \\
7.212\end{array}$ & $\begin{array}{l}6.744 \\
6.768 \\
7.524 \\
6.684 \\
6.564\end{array}$ \\
\hline
\end{tabular}

Subsequently Johanson $\$$ compared the excretion of carbon dioxide by a fasting man when simply lying in bed (awake) with

* Landw. Jahrb., 23, 161.

† Skand. Arch. f. Physiol., 7, 29.

† Ibid., 8, 85. 
that obtained when all the muscles were as perfectly relaxed as possible. The results per hour were:

$$
\begin{array}{lll}
\text { Lying in bed . . . . . . . . . . } & 24.94 \text { grams } \\
\text { Complete muscular relaxation. . . } & 20.72 \text { " }
\end{array}
$$

- Furthermore, there is more or less muscular exertion involved during the waking hours in maintaining the relative position of the different members of the body. This is notably true of the effort of standing. In experiments with the respiration-calorimeter under the writer's direction* the heat production of a steer per minute while standing and lying was found to be approxinuately

\begin{tabular}{|c|c|c|c|}
\hline & $\begin{array}{l}\text { Lying, } \\
\text { Cals. }\end{array}$ & $\begin{array}{l}\text { Standing: } \\
\text { Cals. }\end{array}$ & $\begin{array}{l}\text { Ratio, Lying to } \\
\text { Standing. }\end{array}$ \\
\hline 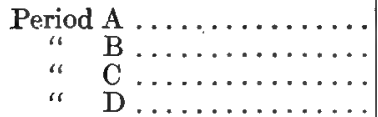 & $\begin{array}{l}5.322 \\
5.781 \\
6.310 \\
6.605\end{array}$ & $\begin{array}{l}7.031 \\
7.700 \\
8.177 \\
8.495\end{array}$ & $\begin{array}{l:}1: 1.321 \\
1: 1.332 \\
1: 1.296 \\
1: 1.286\end{array}$ \\
\hline
\end{tabular}
as follows:

Zuntz $\uparrow$ found an even greater difference in the case of the dog, the average oxygen consumption per minute being-

Lying .................... 174.3 c.c.

Standing.................

In experiments of any considerable duration on normal animals it is impossible to avoid more or less expenditure of energy in this incidental muscular work, while it is often a matter of difficulty to make the different periods of an experiment comparable in this respect.

GLANDUlar Work.-The activity of the various secretory, absorptive, and excretory organs may be conveniently summarized under this head. While the purpose of the glandular metabolism is, in the majority of cases, primarily a chemical one, the accom. plishment of this purpose involves an expenditure of energy which,

* Proc. Soc Prom. Agr. Sei, 1902.

† Arch. ges. Physiol , 68, 191. 
so far as it is not removed from the body in the potential form in the secretions or excretions, ultimately takes the form of heat.

Moreover, the fundamental features of glandular metabolism appear to be indentical with those of muscular metabolism. Thus Henderson * has shown that the active submaxillary gland of the dog does not lose nitrogen as compared with the inactive gland, but does lose weight, evidently from the metabolism of nonnitrogenous matter. Similarly, Bancroft $\dagger$ found the respiratory exchange of the same gland during activity to be three or four times that during rest. If we may accept these results as typical, we must conclude that glandular, like muscular metabolism is largely at the expense of non-nitrogenous matter, and shall not hesitate to summarize the two together as parts of the internal work of the body.

Cenlular Work.-While both muscular and glandular work are forms of cell activity, a passing mention may be made for the sake of completeness of such processes as imbibition, filtration, osmosis, protoplasmic motion, karyokinesis, etc., which, while taking place in the various organs, are so general in their nature and form so essential a part of our conception of cell life that it seems proper to speak of them collectively as cellular work. As to the quantitative importance of these activities, so far as they can be differentiated from the special functions of the various organs, we lack the data for forming any definite conception, although it would appear that it must be small.

\section{Heat Production.}

As we have just seen, the forms of internal work are numerous and some of them are not readily accessible to measurement. All of them, however, have this in common, that the energy used in their performance ultimately assumes the form of heat.

This being the case, while the single factors making up the internal work are not readily determined, a determination of the total heat produced by a fasting animal in a state of rest (either directly or by computation from the amount and kind of matter metabolized) will show the total amount of energy consumed in the

* Am. Jour. Physiol., 3, 19.

† Journal of Physiol , 27, 31. 
performance of the internal work and how it varies under varying conditions. Carnivorous animals, with their short and relatively simply digestive canal, lend themselves most readily to experiments of this sort although rabbits and guinea-pigs have been employed to some extent, as well as, for short periods, men.

Constancy Under Uniform Conditions. - Attention has already been called in Chapter IV to the relative constancy of the total metabolism of the fasting animal, particularly as compared with the total mass of active tissue in the body. This constancy has been especially emphasized by Rubner,* and forms the basis of his determinations of the replacement values of the several nutrients which will be considered in the following chapter. With a rabbit the following daily averages, computed per 100 parts of nitrogen in the body, were obtained:

\begin{tabular}{|c|c|c|}
\hline Day of Experiment. & $\begin{array}{l}\text { Nitrogen in } \\
\text { Urine. }\end{array}$ & $\begin{array}{l}\text { Fat } \\
\text { Metabolized. }\end{array}$ \\
\hline $\begin{array}{l}\text { Third to eighth........ } \\
\text { Ninth to fifteenth...... }\end{array}$ & $\begin{array}{l}2.16 \\
2.19\end{array}$ & $\begin{array}{l}16.2 \\
13.8\end{array}$ \\
\hline
\end{tabular}

Since the ratio of proteids to fat metabolized did not vary greatly in these trials, the total amount of carbon dioxide excreted may be taken as an approximately accurate measure of the total metabolism. For the several days of the experiment, this was as follows:

\begin{tabular}{|c|c|c|c|}
\hline \multirow[b]{2}{*}{ Day. } & \multirow{2}{*}{$\begin{array}{c}\text { Average Live } \\
\text { Weight, } \\
\text { Grms. }\end{array}$} & \multicolumn{2}{|c|}{ Carbon Dioxide Excreted. } \\
\hline & & $\begin{array}{c}\text { Per Head, } \\
\text { Grms. }\end{array}$ & $\begin{array}{l}\text { Per Kg. } \\
\text { Live Weight, } \\
\text { Grms. }\end{array}$ \\
\hline 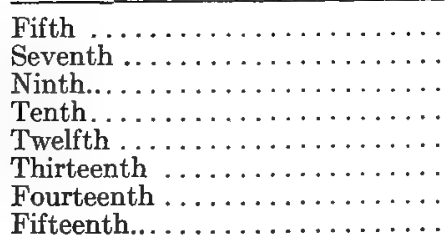 & $\begin{array}{l}2091 \\
2002 \\
1907 \\
1864 \\
1764 \\
1731 \\
1716 \\
1697\end{array}$ & $\begin{array}{l}36.1 \\
31.8 \\
30.3 \\
29.2 \\
30.2 \\
27.4 \\
27.4 \\
25.5\end{array}$ & $\begin{array}{l}17.26 \\
15.90 \\
15.90 \\
15.65 \\
17.18 \\
15.81 \\
15.95 \\
15.90\end{array}$ \\
\hline
\end{tabular}

* Zeit. f. Biol., 17, 214 ; 19, 312. 
With a dog the following results were obtained:

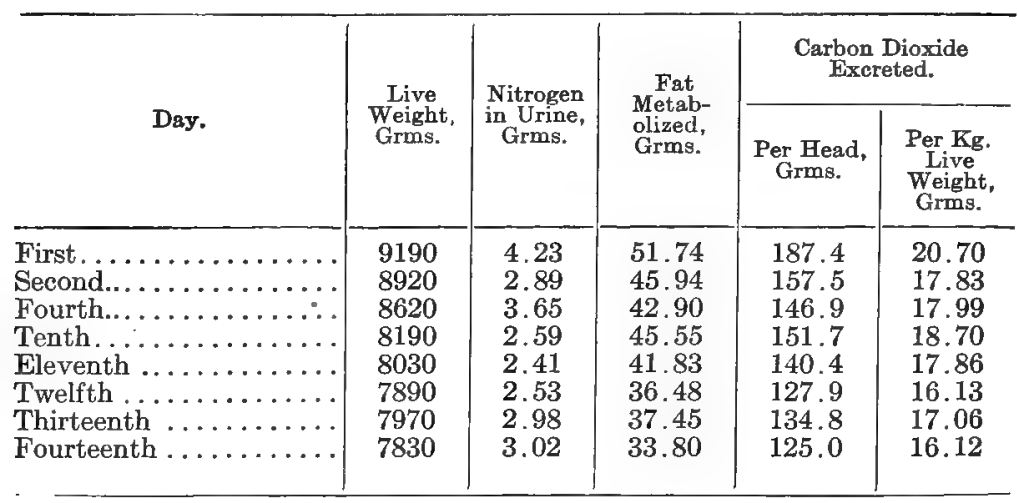

Rubner also quotes the following results by Kuckein on a cock:

$$
\begin{aligned}
& \text { Carbon Dioxide per } \\
& \text { Kg. Live Weight. }
\end{aligned}
$$

Third .............21.73 grams.

Fifth............... 21.47 "

Seventh............21.43" "

Rubner's experiments on a guinea-pig* show a similar constancy, the heat production being computed from the total metabolism;

\begin{tabular}{|c|c|c|}
\hline Day. & $\begin{array}{l}\text { Heat Prod } \\
\text { per Kilog }\end{array}$ & ram. \\
\hline First. & . 149.9 & \\
\hline Second. & $\ldots 162.6$ & " \\
\hline Third. . & . 156.5 & \\
\hline Fourth. & 140.5 & \\
\hline Fifth... & 137.3 & \\
\hline Sixth. . & 150.6 & \\
\hline Seventh & 157.4 & " \\
\hline Eighth. & 155.6 & “ \\
\hline Ninth. & 162.6 & \\
\hline
\end{tabular}

Concerning this point Rubner says: $\nmid$ "The uniformity of the fasting metabolism proves that, in spite of the undoubted limitatation of all the voluntary functions which can cause a consumption of matter, no further reduction of the metabolism is possible,

* Biologische Gesetze, p. 15.

$\dagger$ Loc. cit., 19, 326. 
and we recognize from this that we have to do here with a constant metabolism which is indissolubly connected with life itself. The animal in the fasting state adjusts itself to the minimum metabolism."

In other words, the metabolism and consequent heat production of the fasting, quiescent animal speedily reaches a minimum which represents the aggregate demands of the vital activities of the organism for energy; that is, which represents the internal work of the body in the sense in which the words are here used, plus the metabolism required for any direct production of heat which may be necessary to maintain the normal temperature of the animal.

The relative importance of the internal work in the narrower sense and of the direct heat production as regards their demands for a supply of energy will appear more clearly when we consider, in the following paragraphs, the effects of varying conditions, and particularly of the thermal environment, upon the heat production of the fasting animal.

Influence of Thermal Environment on Heat Production.*-An animal, particularly in the temperate zones, is subject to considerable variations of external conditions, particularly of temperature, which, in the first place, tend to affect the rate at which it emits heat, and secondarily, within certain limits to modify the amount of heat produced in the body.

Body Temperature.-As regards their body temperature, animals have been divided into two great classes: the cold-blooded (poikilothermic), whose temperature as a rule differs but slightly from that of their surroundings, and the warm-blooded (homoiothermic), whose temperature remains approximately constant during health whatever be that of their surroundings. Since all our domestic animals, as well as man himself, belong to the second group, it alone will be considered in the following paragraphs.

Since the animal is constantly producing heat in the various ways already indicated, it is obvious that in order to maintain a constant body temperature it nust be able to give off this heat at the same average rate at which it is produced. Ranke illustrates this necessity in a striking manner by computing that if the

* The discussion of this subject follows to a considerable extent that of Ranke in the introduction to his "Einwirkung des Tropenklimas auf die Ernährung des Menschen," Berlin, 1900. 
body of a man were unable to give off the heat which it produces, a single day would suffice to raise it to a pasteurizing temperature, while in the course of a year, at the same rate, a temperature of over $17,000^{\circ} \mathrm{C}$. would be reached.

Furthermore, since the external conditions of temperature are subject to frequent and sudden changes, it is obvious that the balance between heat production and emission must be capable of prompt adjustment to varying circumstances.

Thermic RaNGe.-The ahility of the animal body to adapt itself to changes of temperature has, however, often been exaggerated. As a matter of fact this adaptation is possible only within a comparatively narrow range, and unless we hold fast to this fundamental idea we are in danger of reaching fallacious and absurd conclusions. Man has considerably extended the range of climate within which he can exist by means of clothing, shelter, artificial heat, and even to a slight extent artificial refrigeration, and this fact often leads unconsciously to an overestimate of the possible thermic range. These means of artificial protection result essentially in modifying the temperature to which the body is actually exposed, and the same is true in a less degree of the differences in the summer and winter coats of animals. The fact still remains that the actual thermic range of any species is and must be strictly limited. All life implies a certain amount of metabclism, and consequently of heat production. With rising temperature a point must sooner or later be reached at which the animal is unable to impart this heat to its surroundings as fast as it is produced, and in which the rise in temperature necessarily resulting will prove fatal. With falling temperature a point will be reached at which the greatest possible amount of metabolism in the body will be unable to equal the rate at which heat is lost to the surroundings and the animal will perish from cold. Both the maximum and minimum points and the extent of the thermic range will vary for different species and varieties of animals, but at best the range is relatively small.

Means of Regulation.-Within the thermic range of a given animal the adjustment to its thermal environment may be effected in one or both of two ways, viz., by a regulation of the rate of emission of heat or by a variation in the heat production. 
Regulation of Rate of Emission.-Heat is given off by the body in four principal ways: (1) by conduction; (2) by radiation; (3) by evaporation of water; (4) as the sensible heat of the excreta.

By conduction, heat is transferred directly from the body to its surroundings, including such solid objects as it may be in contact with and particularly the air. The rate of loss in this way will depend upon the relative temperature and conductivity of the surface of the body and of the substances with which it is in contact, and in case of the air will be also influenced by the rate of motion of the latter relatively to that of the body.

By radiation, a constant exchange of heat goes on between the body and objects not in immediate contact with it. Since the body is usually warmer than its surroundings, the net result of this exchange is a loss of heat by the body, the amount of which depends upon the specific radiating power of the surface of the body and upon the difference in temperature between the latter and surrounding objects.

By evaporation of water from the skin, and to a less degree from the mucous membrane of the air-passages, a large amount of heat may be removed as latent heat of vaporization. The amount of water evaporated from the skin, and consequently the rate at which heat is carried off, will depend in part on the amount transpired by the skin, but when this is abundant, chiefly upon the relative humidity of the air and upon its rate of movement.

Finally, the heat removed in the excreta is relatively small, and in the case of the fasting animal in particular is insignificant as compared with the losses through the other three channels.

In general we may say that the rate of emission of heat in all of the first three ways named is determined by two sets of conditions, viz., those relating to the environment of the animal (temperature, relative humidity, movement of air) and those relating to the animal itself and particularly to its surface.

The conditions of the first set, of course, are beyond the control of the organism. Their tendency is to produce the same effect upon the rate of emission of heat that they would upon that of a lifeless body, viz., to increase it as the temperature of the surroundings is lowered and their conducting power increased. In the case of the 
living animal this tendency is offset by the regulative mechanism acting upon the second set of conditions, so that, e.g., a fall in the temperature of its surroundings within certain limits instead of increasing the rate of emission, as in the case of a lifeless body, has no effect upon it. This regulation of the rate of emission is effected chiefly by means of changes in the temperature and state of moisture of the skin, brought about on the one hand through the vaso-motor mechanism and on the other through the special nerves of perspiration.

Variations of external temperature acting upon the peripheral nerves influence by reflex action the activity of the vaso-motor nerves which regulate the caliber of the minute blood-vessels. Exposure to cold causes a contraction of the capillaries of the skin and a relaxation of those of the viscera. As a result more blood passes through the latter, while the flow through the skin is diminished, the latter becomes paler, and since the heat given off is not fully replaced by the blood current, its temperature falls. Exposure to heat has the contrary effect. The capillaries of the skin relax, more blood flows through them, the skin becomes flushed and its temperature rises, while the flow of blood to the viscera is checked. A fall in the temperature of the skin, however, tends to diminish the rate of emission of heat both by conduction and radiation, while a rise in its temperature has the opposite effect, thus counteracting the tendency of changes of external temperature. In other words, the "emission constant" of the skin changes to meet changes in external conditions. So exactly are these mechanisms adjusted in health that within certain rather narrow limits they maintain the rate of emission of heat, and consequently the average temperature of the body, very nearly constant.

Obviously, however, there must be a limit above which the temperature and radiating power of the skin cannot be increased to compensate for a rise in external temperature. The second method of regulation then comes more markedly into play through the familiar act of perspiration,.or sweating. At high temperatures the activity of the sweat-glands is greatly stimulated, in part doubtless by the more abundant supply of blood to the skin, but chiefly by reflex stimulation of the special nerves which control the secretion of sweat. The evaporation of the relatively large amount 
of water thus supplied to the surface of the skin is a powerful means of refrigeration, as we know no less from common experience than from scientific determinations, the evaporation of a single gram of water requiring approximately $0.592 \mathrm{Cal}$. of heat. With very high temperatures, especially in a humid atmosphere, however, even this method of disposing of the heat becomes insufficient and the extreme upper limit of the thermal range is passed.

These two methods of regulation of the body temperature are often spoken of collectively as "physical" regulation.

Variations in Amount of Heat Produced.- Just as there is a superior limit beyond which the regulation of the body temperature by the means above described cannot be carried, so it is obvious that there must be a lower limit of regulation. However much the cutaneous circulation may be reduced, the skin will always lose hcat to a sufficiently cold environment faster than it is being generated by the internal work of the body. Under these circumstances the only method by which the temperature of the animal can be maintained is an increase in the rate of generation of heat.

That changes of external temperature affect the amount of heat generated was shown by the experiments of Lavoisier and the observations of Liebig, but Liebermeister* appears to have been the first to clearly enunciate the theory of regulation by variations in the rate of production. The fact of such regulation has been fully demonstrated by numerous subsequent investigators. As a typical example we may take the well-known experiments of Theodor $\dagger$ on a cat, some of the results of which are as follows:

\begin{tabular}{c|c|c||c|c|c}
\hline $\begin{array}{c}\text { Temperature, } \\
\text { Deg. } \\
\text { Cent. }\end{array}$ & $\begin{array}{c}\text { CarbonDioxide } \\
\text { Excreted, } \\
\text { Grms. }\end{array}$ & $\begin{array}{c}\text { Oxygen } \\
\text { Taken Up, } \\
\text { Grms. }\end{array}$ & $\begin{array}{c}\text { Temperature. } \\
\text { Deg. } \\
\text { Cent. }\end{array}$ & $\begin{array}{c}\text { CarbonDioxıde } \\
\text { Excreted, } \\
\text { Grms. }\end{array}$ & $\begin{array}{c}\text { Oxygen } \\
\text { Taken Up, } \\
\text { Grms. }\end{array}$ \\
\hline-5.5 & 19.83 & 17.48 & 12.3 & 17.63 & 17.71 \\
$-\mathbf{- 3 . 0}$ & 18.42 & 18.26 & 16.3 & 15.73 & 14.74 \\
0.2 & 18.24 & 19.95 & 20.1 & 14.34 & 12.78 \\
5.0 & 17.90 & 14.82 & 29.6 & 13.12 & 10.87 \\
\hline
\end{tabular}

Numerous other investigators have obtained similar results, but the effect of low temperature in stimulating the heat production of warm-blooded animals is too well established to require an

* Arch f. (Anat. u.) Physiol., 1860, pp. 520 and 589; 1861, p. 661.

$\uparrow$ Zeit. f. Biol., 14, 51. 
extended citation of authorities here. Some of Rubner's* more recent results, however, are of interest as showing the delicacy of the reaction. The experiments were made on fasting dogs in a state of complete rest, the heat production being computed from the total metabolism of carbon and nitrogen:

\begin{tabular}{|c|c|c|c|}
\hline \multirow{2}{*}{\multicolumn{2}{|c|}{$\begin{array}{l}\text { Tempera- } \\
\text { ture, Deg. C. } \\
13.8 .\end{array}$}} & \multirow{2}{*}{\multicolumn{2}{|c|}{$\begin{array}{l}\text { Heat Production per } \\
\text { Kg. in } 24 \text { Hours. }\end{array}$}} \\
\hline & & & \\
\hline \multirow{3}{*}{ I } & 14.9. & $\ldots 74.74$ & “ \\
\hline & 17.3. & $\ldots 69.78$ & " \\
\hline & 18.0. & 67.06 & " \\
\hline \multirow{4}{*}{ II } & 11.8. & $40.60^{\circ}$ & " \\
\hline & 12.9.. & 39.13 & “ \\
\hline & $15.9 .$. & 35.99 & 6 \\
\hline & 17.5. & 35.22 & " \\
\hline \multirow{3}{*}{ III } & & 39.65 & "6 \\
\hline & 19.5. & .. 35.10 & " \\
\hline & 27.4. & .... 30.82 & 6 \\
\hline
\end{tabular}

This method of regulation of the body temperature is often briefly designated as "chemical" regulation.

Just how the additional generation of heat is effected is not so clear. From the fact that the muscles are the seat of a very large part of the heat production of the body we should naturally be inclined to look to them as the source of the increase. In quite a number of experiments on man, of which those of A. Loewy $\dagger$ and of Johansson $\ddagger$ may be especially mentioned, a stimulation of the heat production with falling temperature was only observed when there was visible muscular action, such as shivering, while in the other cases only the "physical" regulation occurred. Any contraction of the muscles would of course be a source of heat, but the increase with falling external temperature has been repeatedly observed with animals in the absence of this obvious cause. Whether in such cases there is an increase in the tonus of the muscles, involving an increase in their metabolism, or whether, through some form of reflex stimulation, the rate of oxidation is accelerated

* Biologische Gesetze, p. 10.

$\dagger$ Arch. ges. Physiol., 46, 189.

\$ Skand. Arch. f. Physiol., 7, 123. 
simply for the sake of the heat produced is still an unsettled question and one which, for our present purpose, we need not pause to consider. As to the fact of the increase there is no question.

Critical Temperature.-In early writings upon this subject the influence of external temperature in increasing or diminishing the heat production of the body was frequently spoken of as if it were of unlimited application, and the same idea has passed more or less fully into the popular literature of the subject. But little reflection is necessary, however, to show that this cannot be the case. Common observation teaches us that neither our own metabolism nor that of our domestic animals, as roughly measured by the consumption of food, is affected, for example, by the difference between winter and summer to any such extent as would correspond to the difference in average temperature. Moreover, if every rise in external temperature diminished the heat production, there would be a temperature at which no heat production at all would occur and at which, therefore, life could exist without metabolism, which is a contradiction in terms. This extreme case renders clear the fundamental error of this view, viz., that of regarding the heat production as an end in itself and not as, substantially, an incident of the general metabolism.

Carl Voit* was the first to demonstrate by exact scientific experiments the limits within which the influence of temperature upon metabolism (the so-called chemical regulation) is confined. His experiments were a continuation of those of Theodor (p. 351), and were made upon a man weighing about $70 \mathrm{kgs}$. and wearing ordinary clothing. After exposure for some time to the temperature to be tested he passed six hours in the chamber of the respiration apparatus, fasting and in complete rest. During the six hours the excretion of carbon dioxide and nitrogen was as follows:

\begin{tabular}{r|c|c||c|c|c}
\hline $\begin{array}{c}\text { Temperature. } \\
\text { Deg. C. }\end{array}$ & $\begin{array}{c}\text { Carbon } \\
\text { Dioxide. } \\
\text { Grms. }\end{array}$ & $\begin{array}{c}\text { Urinary } \\
\text { Nitrogen } \\
\text { Grms. }\end{array}$ & $\begin{array}{c}\text { Temperature. } \\
\text { Deg. C. }\end{array}$ & $\begin{array}{c}\text { Carbon } \\
\text { Dioxide. } \\
\text { Grms. }\end{array}$ & $\begin{array}{c}\text { Urinary } \\
\text { Nitrogen. } \\
\text { Grms. }\end{array}$ \\
\cline { 2 - 5 } & 210.7 & 4.23 & 23.7 & 164.8 & 3.40 \\
$\mathbf{4 . 4}$ & 206.0 & 4.05 & 24.2 & 166.5 & 3.34 \\
$\mathbf{9 . 0}$ & 192.0 & 4.20 & 26.7 & 160.0 & 3.97 \\
$\mathbf{1 4 . 3}$ & 155.1 & 3.81 & 30.0 & 170.6 & $\ldots .$. \\
$\mathbf{1 6 . 2}$ & $\mathbf{1 5 8 . 3}$ & $\mathbf{4 . 0 0}$ & & & \\
\hline
\end{tabular}

* Zeit. f. Biol., 14, 57. 
Later and more comprehensive experiments with animals by Rubner have given corresponding results. Thus with two guineapigs the following figures were obtained in 24-hour experiments:*

\begin{tabular}{c|c|c||c|c|c}
\hline \multicolumn{3}{c||}{ Mature Animal. } & \multicolumn{3}{c}{ Young Animal. } \\
$\begin{array}{c}\text { Temperature } \\
\text { of Air. } \\
\text { Deg. C. }\end{array}$ & $\begin{array}{c}\text { Temperature } \\
\text { of Animal, } \\
\text { Deg. C. }\end{array}$ & $\begin{array}{c}\mathrm{CO}_{2} \text { per Kg. } \\
\text { and Hour, } \\
\text { Grms. }\end{array}$ & $\begin{array}{c}\text { Temperature } \\
\text { of Air, } \\
\text { Deg. C. }\end{array}$ & $\begin{array}{c}\text { Temperature } \\
\text { of Animal, } \\
\text { Deg. C. }\end{array}$ & $\begin{array}{c}\mathrm{CO}_{2} \text { per Kg. } \\
\text { and Hour, } \\
\text { Grms. }\end{array}$ \\
\hline 0 & 37.0 & 2.905 & 0 & 38.7 & 4.500 \\
11.1 & 37.2 & 2.151 & 10 & 38.6 & 3.433 \\
20.8 & 37.4 & 1.766 & 20 & 38.6 & 2.283 \\
25.7 & 37.0 & 1.540 & 30 & 38.7 & 1.778 \\
30.3 & 37.7 & 1.317 & 35 & 39.2 & 2.266 \\
34.9 & 38.2 & 1.273 & & & \\
40.0 & 39.5 & 1.454 & & & \\
\hline
\end{tabular}

A later experiment by Rubner $\dagger$ upon a dog, in which the heat production was measured by a calorimeter, gave the following results:

Temperature of Air.

Heat Production per $\mathrm{Kg}$.

$7.6^{\circ} \mathrm{C} \ldots \ldots \ldots \ldots . \ldots . . .63 .5$ Cals

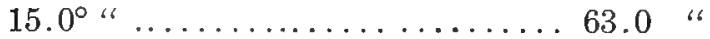

$20.0^{\circ} " \ldots \ldots \ldots \ldots \ldots \ldots \ldots \ldots \ldots .53 .5$ "

$25.0^{\circ} " \ldots \ldots \ldots \ldots \ldots \ldots \ldots \ldots \ldots \ldots .2 .2$

$30.0^{\circ}$ «................ 56.2 "

The uniform testimony of these various experiments is that for each species there is a certain external temperature at which the metabolism and consequent heat production reach a minimum. With man in ordinary clothing it would appear to lie at about $15^{\circ} \mathrm{C}$. $\$$ with the $\operatorname{dog}$ at about $20^{\circ} \mathrm{C}$, and with the guinea-pig at about $30^{\circ}-35^{\circ} \mathrm{C}$. Below this point the heat production rises or falls with changes of external temperature; or, in other words, the constancy of the body temperature is secured, in part at least, by

* Biologische Gesetze, p. 13.

$\uparrow$ Archiv f. Hygiene, 11, 285.

$\ddagger$ Rubner (Biol. Gesetze, p. 30 ) says that for naked man it is about $37^{\circ} \mathrm{C}$. 
means of the so-called "chemical" regulation, that is, by variations in the production of heat.

Above this point the heat production, instead of a further decrease, shows an increase, which, however, is slight as compared with the differences observed as a result of the "chemical" regulation. Here we are obviously in the domain of the "physical" regulation-the regulation by changes in the emission constant of the skin. This temperature at which the chemical regulation ceases, and which presumably varies for different species of animals, Ranke calls the critical temperature. Below it the regulation is chiefly "chemical," above it chiefly "physical." The slight increase in the metabolism above the critical point is plausibly explained as due to the greater activity of the organs of circulation, respiration, and perspiration required for the "physical" regulation.

Rubner's experiments also show that the portion of the thermic range lying above the critical temperature falls into two distinct subdivisions. For a certain distance above that point, the factors chiefly concerned in the regulation of the body temperature are conduction and radiation, which keep pace with the rising temperature in the manner already explained. At the same time, there is a small increase in the rate of evaporation of water, approximately equivalent to the slight increase in the metabolism above the critical temperature to which attention has just been called. Matters go on in this way through a certain range of temperature until the regulative capacity of the vaso-motor mechanism is utilized to its maximum. If the external temperature still rises, the emission of heat by conduction and radiation begins to decrease as it would in a lifeless object, and the deficit thus occasioned is made up by a sudden increase in the exhalation of water vapor, coinciding, in man, with the production of visible perspiration. This sudden increase in the activity of the sweat-glands is accompanied, as we should expect, by an increase in the total metabolism and consequent heat production.

These phenomena are well illustrated by Rubner's experiments with a fasting dog, already partially cited on the opposite page. The following table shows the amount of heat carried off by conduction and radiation and as latent heat of water-vapor at the 
several temperatures, and the same facts are also shown graphically in the accompanying diagram.

\begin{tabular}{|c|c|c|c|}
\hline \multirow[b]{2}{*}{$\begin{array}{l}\text { Temperature } \\
\text { of Air. } \\
\text { Deg. C. }\end{array}$} & \multirow[b]{2}{*}{$\begin{array}{l}\text { Total Heat } \\
\text { Production, } \\
\text { Cals. }\end{array}$} & \multicolumn{2}{|c|}{ Disposed of by } \\
\hline & & $\begin{array}{l}\text { Conduction } \\
\text { and } \\
\text { Radiation. } \\
\text { Cals. }\end{array}$ & $\begin{array}{c}\text { As Latent } \\
\text { Heat of Water } \\
\text { Vapor, } \\
\text { Cals. }\end{array}$ \\
\hline $\begin{array}{r}7.6 \\
15.0 \\
20.0 \\
25.0 \\
30.0\end{array}$ & $\begin{array}{l}83.5 \\
63.0 \\
53.5 \\
54.2 \\
56.2\end{array}$ & $\begin{array}{l}71.7 \\
49.0 \\
37.3 \\
37.3 \\
30.0\end{array}$ & $\begin{array}{l}11.8 \\
14.0 \\
16.2 \\
16.9 \\
26.2\end{array}$ \\
\hline
\end{tabular}

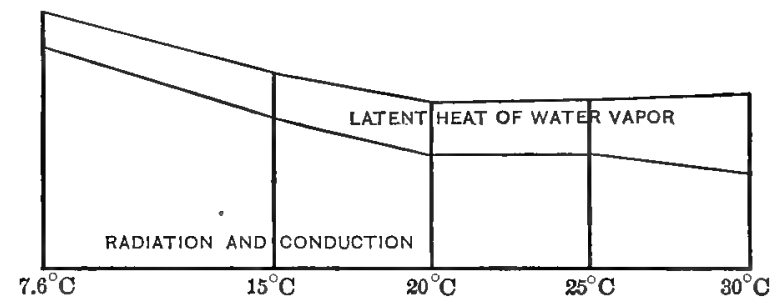

It appears, then, that a certain minimum heat production, corresponding to the metabolism at the critical temperature, is inseparably connected with the life of the animal. The very fact that the heat production at this temperature is a minimum shows that its amount is not determined by the needs of the organism for heat. If the latter were the controlling condition, a rise of external temperature should still further reduce the generation of heat, while as a matter of fact it is accompanied by a slight increase up to the point where the amount of heat produced overpasses the ability of the organism to dispose of it and death results. The natural conclusion is that the metabolism at the critical temperature is that which is necessary for the performance of the various functions of the organism, and that the heat production at this temperature, therefore, represents the amount of energy necessarily consumed in the internal work of the body. This is, of course, Rubner's conclusion (p. 346) in a slightly altered form.

The case is not unlike that of a room in which a fire must be kept burning for some purpose-a kitchen, for example. In winter, changes in external temperature may be met by burning more or 
less fuel. As spring advances, the fire is reduced until it is just sufficient for the necessary work. If the weather still continues to grow warmer, since the fire cannot be further reduced the excess of heat is gotten rid of by opening the windows more or less, while, to carry out the analngy, in very hot weather we may sprinkle the floor or wet the walls to secure relief from heat through the evaporation of water.

Modification of Conception of Critical Temperature.In our discussion thus far we have considered chiefly the influence of external temperature on metabolism and heat production. This is, however, by no means the only condition affecting the heat balance of the body. Of the other meteorological factors, three call for special mention, viz., wind, insolation, and in particular relative humidity.

Wind.-In a perfectly still atmosphere, the layer of air next to the skin becomes warmed and loaded with water vapor and constitutes to a certain degree a protective envelope which is removed with comparative slowness by gaseous diffusion. A current of air, by removing this protecting layer and bringing fresh portions of air in contact with the body, increases the emission of heat both by conduction and by evaporation of water. This is in accord with the common experience that a degree of cold which can readily be borne when the air is still becomes intolerable in a brisk wind, while, on the other hand, the oppressiveness of a very hot day is sensibly relieved by even a slight breeze. The effect of wind, then, is to transpose the thermic range of the animal to a higher place in the thermometric scale, and to correspondingly raise the critical temperature.

Insolation.-The direct rays of the sun impart a considerable amount of heat to the body. The effect of insolation, therefore, is the reverse of that of wind, viz., to transpose the thermal range and the critical temperature downward. A similar effect is produced, of course, by the sun's heat when reflected from surrounding objects, or by the radiant heat from hot objects, the earth, for example. On the other hand, the radiation from the body into space during the night, especially at high altitudes and through a dry, clear atmosphere, may have a very considerable effect in the contrary direction. 
Relative Humidity. - The relative humidity of the air affects the emission of heat in two principal ways. At low temperatures, where the evaporation of water plays a subordinate rôle, it increases the rate of emission by increasing the conductivity and specific heat of the air, and also the conductivity of the skin and the body covering (hair, fleece, clothing), these effects outweighing its influence in diminishing the relatively small amount of evaporation Moist cold is, therefore, more trying than dry cold.

At high temperatures, on the other hand, where a large proportion of the heat is removed by evaporation, a high relative humidity, by checking this evaporation, hinders the emission of heat, this effect overbalancing any slight increase in conduetivity. Moist heat is accordingly more oppressive than dry heat.

An increase in the relative humidity, then, abbreviates the thermal range at both ends, while at moderate temperatures it appears to have but little effect, a diminution of the loss by evaporation being compensated for by an increase in radiation and conduction.

Critical Thermal Environment.-From the above it is obvious that the so-called critical temperature is not a constant, even for the same species or the same individual, but that other. factors than the temperature of the air materially affect it.

What is constant (relatively at least) is the rate at which heat is produced in the body by the metabolism necessary to sustain its various physiological activities, that is, by its internal work. In order to maintain the normal body temperature, the total outflow of heat through its various channels must, at its minimum, be equal to the amount thus liberated in the organism. The outflow of heat, as we have seen, is affected directly or indirectly by the external conditions, and largely by the three just mentioned. Innumerable combinations of these conditions are possible, and any one of them whose combined effect upon the animal is to make the outflow of heat equal to the rate of evolution due to the internal work will constitute a critical point in the above sense. Any change in such a set of conditions which tends to increase the outflow of heat will, like a fall in temperature, be met chiefly by an increased heat production. Any change tending in the opposite direction will be compensated for by the effects upon the organ- 
ism whch have already been described and which result in maintaining the rate of emission of heat at a point enough higher than before to provide for carrying off the extra heat arising from the physiological work of the regulative mechanism itself. In other words, instead of a critical temperature, we get the conception of a critical thermal environment, which may be reached under a variety of conditions, and below which we have the domain of "chemical" regulation, while above it is the region of "physical" regulation.

Influence of Size of Animal on Heat Production. - The total metabolism of a large animal is necessarily greater than that of a small one of the same species, but it is not proportional to the weight, being relatively greater in the smaller animal under comparable conditions.

Relation of Heat Production to Surface.-Bergmann* appears to have been the first to connect the fact just stated with the relatively greater surface of the smaller animal, but we are indebted to Rubner $\uparrow$ for the first quantitative investigation of this phase of the subject. His experiments were made on six dogs whose weights varied from 3 to 24 kilograms each. The total metabolism (proteids and fat) of each of these animals in the fasting state was determined in from two to thirteen experiments, and from their results the average heat production of each animal was computed. The table on page $360 \ddagger$ shows the air temperature and the computed heat production per kilogram live weight in each experiment, and also the same corrected to the uniform temperature of $15^{\circ} \mathrm{C}$. This correction is made on the basis of Theodor's experiments (see p. 351), according to which a difference of $1^{\circ}$ Centigrade caused the amount of oxygen taken up by the cat to vary 1.11 per cent. The first series consists of a selection from Pettenkofer \& Voit's experiments.

Whether we consider the observed or the corrected heat production we find that with the single exception of the corrected result for No. VI the amount per unit of live weight increases as the weight itself decreases.

* Cited by Rubner.

$\dagger$ Zeit. f. Biol , 19, 535 .

$\ddagger$ The figures of the table are computed from those given by Rubner in loc. cit., $\mathrm{p} 540$, and differ in some cases from the summary given in loc. cit., p 542 . 


\begin{tabular}{|c|c|c|c|c|c|}
\hline \multirow{2}{*}{$\begin{array}{l}\text { No. of } \\
\text { Ani- } \\
\text { mal. }\end{array}$} & \multirow{2}{*}{ Date. } & \multirow{2}{*}{$\begin{array}{l}\text { Live } \\
\text { Weight, } \\
\text { Kgs. }\end{array}$} & \multirow{2}{*}{$\begin{array}{l}\text { Air Tem- } \\
\text { perature, } \\
\text { Deg. C. }\end{array}$} & \multicolumn{2}{|c|}{$\begin{array}{l}\text { Heat Production } \\
\text { per } \mathrm{Kg} \text {. }\end{array}$} \\
\hline & & & & $\begin{array}{l}\text { Observed, } \\
\text { Cals. }\end{array}$ & $\begin{array}{l}\text { Corrected } \\
\text { to } 15^{\circ} \\
\text { Cals. }\end{array}$ \\
\hline \multirow[t]{2}{*}{ I } & $\begin{array}{c}\text { Pettenkofer \& Voit's } \\
\text { experiments }\end{array}$ & $\begin{array}{l}30.96 \\
29.87 \\
31.44 \\
30.38\end{array}$ & $\begin{array}{l}17.1 \\
17.7 \\
16.2 \\
13.9\end{array}$ & $\begin{array}{l}38.99 \\
31.82 \\
37.39 \\
36.54\end{array}$ & $\begin{array}{l}39.90 \\
32.77 \\
37.89 \\
36.09\end{array}$ \\
\hline & Average . . . . . . . . & 30.66 & 16.2 & 36.18 & 36.66 \\
\hline \multirow[t]{2}{*}{ II } & 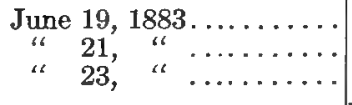 & $\begin{array}{l}24.11 \\
23.75 \\
23.27\end{array}$ & $\begin{array}{l}15.0 \\
15.0 \\
15.0\end{array}$ & $\begin{array}{l}41.40 \\
40.22 \\
41.10\end{array}$ & $\begin{array}{l}41.40 \\
40.22 \\
41.10\end{array}$ \\
\hline & Average...$\ldots \ldots \ldots$ & 23.71 & 15.0 & 40.91 & 40.91 \\
\hline \multirow[t]{2}{*}{ III } & $\begin{array}{c}\text { Feb. } 24,1882 \ldots \ldots \ldots \ldots \\
\text { " } 28, \quad \text { " } \quad \ldots \ldots \ldots \ldots \ldots \\
\text { Mch. } 1, \quad \text { " } \ldots \ldots \ldots \ldots\end{array}$ & $\begin{array}{l}19.80 \\
19.01 \\
18.79\end{array}$ & $\begin{array}{l}16.9 \\
14.5 \\
16.0\end{array}$ & $\begin{array}{l}47.95 \\
45.71 \\
42.79\end{array}$ & $\begin{array}{l}48.91 \\
45.48 \\
43.22\end{array}$ \\
\hline & Average . . . . . . . . . & 19.20 & 15.8 & 45.48 & 45.87 \\
\hline \multirow[t]{2}{*}{ IV } & $\operatorname{Jan}_{"} 12,1880 \ldots \ldots \ldots \ldots$ & $\begin{array}{l}18.20 \\
17.20\end{array}$ & $\begin{array}{l}13.9 \\
16.6\end{array}$ & $\begin{array}{l}50.72 \\
41.54\end{array}$ & $\begin{array}{l}50.11 \\
42.29\end{array}$ \\
\hline & Average ........... & 17.70 & 15.3 & 46.13 & 46.20 \\
\hline \multirow[t]{2}{*}{ V } & 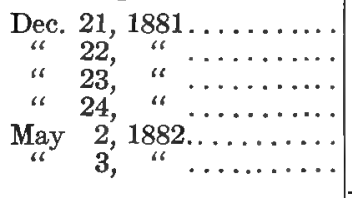 & $\begin{array}{r}9.05 \\
8.83 \\
8.68 \\
8.53 \\
11.11 \\
10.87\end{array}$ & $\begin{array}{l}19.2 \\
20.9 \\
20.2 \\
21.0 \\
18.4 \\
20.0\end{array}$ & $\begin{array}{l}66.32 \\
60.28 \\
64.88 \\
60.66 \\
61.16 \\
57.86\end{array}$ & $\begin{array}{l}69.10 \\
64.19 \\
68.58 \\
64.66 \\
63.42 \\
61.04\end{array}$ \\
\hline & Average ............ & 9.51 & 19.95 & 61.86 & 65.16 \\
\hline \multirow[t]{4}{*}{ VI } & 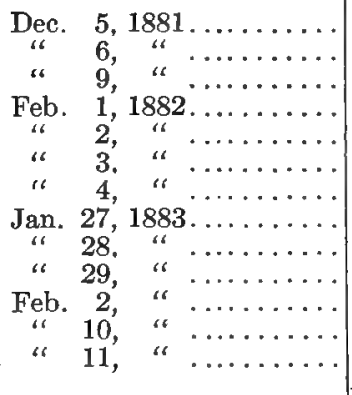 & $\begin{array}{l}6.84 \\
6.36 \\
6.14 \\
6.83 \\
6.69 \\
6.56 \\
6.40 \\
6.66 \\
6.50 \\
6.36 \\
6.21 \\
6.15 \\
5.98\end{array}$ & $\begin{array}{l}15.8 \\
23.6 \\
20.7 \\
18.2 \\
18.0 \\
15.0 \\
16.5 \\
14.6 \\
16.4 \\
16.3 \\
15.9 \\
18.4 \\
19.2\end{array}$ & $\begin{array}{l}65.01 \\
63.65 \\
58.13 \\
71.07 \\
76.85 \\
71.60 \\
75.03 \\
61.55 \\
54.91 \\
53.64 \\
52.57 \\
61.06 \\
54.24\end{array}$ & $\begin{array}{l}65.77 \\
69.70 \\
61.79 \\
73.56 \\
79.39 \\
71.60 \\
76.23 \\
61.11 \\
55.73 \\
54.39 \\
53.09 \\
63.22 \\
56.73\end{array}$ \\
\hline & Average ............. & 6.44 & 17.6 & 63.02 & 64.79 \\
\hline & 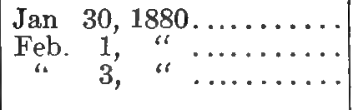 & $\begin{array}{l}3.34 \\
3.05 \\
2.91\end{array}$ & $\begin{array}{l}15.0 \\
12.7 \\
20.6\end{array}$ & $\begin{array}{l}84.45 \\
97.86 \\
80.00\end{array}$ & $\begin{array}{l}84.45 \\
95.41 \\
84.88\end{array}$ \\
\hline & Average...$\ldots \ldots \ldots$ & 3.10 & 16.1 & 87.44 & 88.25 \\
\hline
\end{tabular}


INTERNAL WORK.

SUMMARY.

\begin{tabular}{|c|c|c|c|c|}
\hline \multirow{2}{*}{$\begin{array}{c}\text { No. of } \\
\text { Animal. }\end{array}$} & \multirow{2}{*}{$\begin{array}{c}\text { Average Live } \\
\text { Weight, } \\
\text { Kgs. }\end{array}$} & \multicolumn{2}{|c|}{ Heat Production per $\mathrm{Kg}$. } & \multirow{2}{*}{$\begin{array}{c}\text { Relative Hea } \\
\text { Production } \\
\text { (Corrected). } \\
\text { Cals. }\end{array}$} \\
\hline & & $\begin{array}{l}\text { Observed, } \\
\text { Cals. }\end{array}$ & $\begin{array}{l}\text { Corrected } \\
\text { to } 15^{\circ}, \\
\text { Cals. }\end{array}$ & \\
\hline 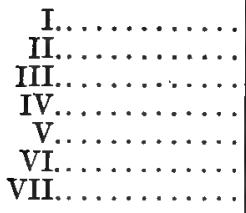 & $\begin{array}{r}30.66 \\
23.71 \\
19.20 \\
17.70 \\
9.51 \\
6.44 \\
3.10\end{array}$ & $\begin{array}{l}36.18 \\
40.91 \\
45.48 \\
46.13 \\
61.86 \\
63.02 \\
87.44\end{array}$ & $\begin{array}{l}36.66 \\
40.91 \\
45.87 \\
46.20 \\
65.16 \\
64.79 \\
88.25\end{array}$ & $\begin{array}{l}100 \\
112 \\
125 \\
126 \\
178 \\
177 \\
241\end{array}$ \\
\hline
\end{tabular}

Rubner also determined approximately the surface exposed by his animals, in part by direct measurement and in part by calculation, and computed the heat production per square meter of surface, with the following results:

\begin{tabular}{|c|c|c|}
\hline No. of Animal. & $\begin{array}{l}\text { Surface, } \\
\text { Sq. Cm. }\end{array}$ & $\begin{array}{l}\text { Heat } \\
\text { Production per } \\
\text { Square Meter, } \\
\text { Cals. }\end{array}$ \\
\hline 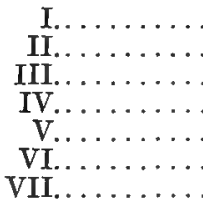 & $\begin{array}{r}10750 \\
8805 \\
7500 \\
7662 \\
5286 \\
3724 \\
2423\end{array}$ & $\begin{array}{l}1046 \\
1112 \\
1207 \\
1097 \\
1183 \\
1120 \\
1214\end{array}$ \\
\hline
\end{tabular}

He also cites * the results of experiments by Senator on the heat production of fasting dogs, and a respiration experiment by Regnault \& Reiset, as follows:

\begin{tabular}{|c|c|c|c|c|}
\hline \multirow[b]{2}{*}{ No. } & \multirow{2}{*}{$\begin{array}{c}\text { Live Weight, } \\
\text { Kgs. }\end{array}$} & \multirow{2}{*}{$\begin{array}{l}\text { Calculated } \\
\text { Surface, } \\
\text { Sq. Cm. }\end{array}$} & \multicolumn{2}{|c|}{ Heat Production. } \\
\hline & & & $\begin{array}{c}\text { Per Kg. } \\
\text { Live Weight, } \\
\text { Cals. }\end{array}$ & $\begin{array}{c}\text { Per Square } \\
\text { Meter of Sur- } \\
\text { face, Cals. }\end{array}$ \\
\hline 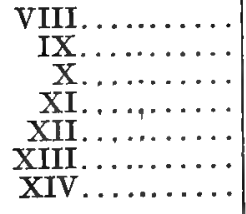 & $\begin{array}{r}10.80 \\
7.52 \\
6.09 \\
5.68 \\
5.40 \\
4.24 \\
5.59\end{array}$ & $\begin{array}{l}5423 \\
4285 \\
3722 \\
3534 \\
3462 \\
2924 \\
3508\end{array}$ & $\begin{array}{l}52.31 \\
53.76 \\
63.04 \\
68.40 \\
74.16 \\
69.12 \\
72.82\end{array}$ & $\begin{array}{r}1035 \\
944 \\
1031 \\
1101 \\
1157 \\
1003 \\
1154\end{array}$ \\
\hline
\end{tabular}

* Loc. cit., p. 551. 
With one exception, the results per square meter agree very well with those of Rubner, both absolutely and relatively.

Rubner has also shown in later experiments * that the same thing is substantially true of guinea-pigs, both at zero and at the temperature of about 30 degrees, at which the heat production is at its mimimum (critical temperature). He likewise points out $\nmid$ that the well-known rapid metabolism of children as compared with adults is, so far as the available data show, quite closely proportional to their relative surface, and observations on the diet of a dwarf $\$$ gave a like result.

Richet, $\S$ working with an air-calorimeter of constant pressure, in which the heat production was measured by the amount of water displaced by the expansion of the air, obtained the following results on rabbits, and similar results upon guinea-pigs are also reported:

\begin{tabular}{|c|c|c|c|c|}
\hline $\begin{array}{c}\text { Number of } \\
\text { Experiments. }\end{array}$ & $\begin{array}{c}\text { Live Weight, } \\
\text { Kgs. }\end{array}$ & $\begin{array}{l}\text { Heat } \\
\text { per Kg.. } \\
\text { Cals. }\end{array}$ & $\begin{array}{l}\text { Total Heat } \\
\text { Expressed in } \\
\text { e.c. of Water } \\
\text { Displaced. }\end{array}$ & $\begin{array}{l}\text { The Same } \\
\text { per Urit of } \\
\text { Surface. }\end{array}$ \\
\hline 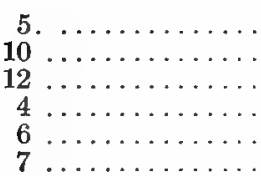 & $\begin{array}{l}2.0-2.2 \\
2.2-2.4 \\
2.4-2.6 \\
2.6-2.8 \\
2.8-3.0 \\
3.0-3.2\end{array}$ & $\begin{array}{l}4.730 \\
3.985 \\
3.820 \\
3.650 \\
3.570 \\
3.320\end{array}$ & $\begin{array}{r}119 \\
110 \\
115 \\
119 \\
125 \\
-130\end{array}$ & $\begin{array}{l}130 \\
129 \\
127 \\
128 \\
127\end{array}$ \\
\hline
\end{tabular}

It would appear from the description of the experiments that only the heat given off by radiation and conduction was measured, no specific statements being made as to ventilation or as to the loss of heat as latent heat of water-vapor. The experiments were also of short duration, ranging from sixty to ninety minutes.

The same author in later experiments $\|$ determined the respiratory exchange 9 of rabbits of different weights. Computing the

* Biologische Gesetze, pp. 17-18.

$\dagger$ Zeit. f. Biol., 21, 390.

$\ddagger$ Biologische Gesetze, p. 9.

$\S$ Archives de Physiol, 1885, II, 237.

|| Ibid., 1890, pp. 17 and 483; 1891, p. 74; Comptes rend, 109, 190.

T By means of an apparatus described briefly in Comptes rend., 104, 435 
results per square centimeter of surface by the use of Meeh's formula (p. 364) he obtained the following figures, while similar results are also reported on guinea-pigs, rats, and birds.

\begin{tabular}{|c|c|c|}
\hline $\begin{array}{c}\text { Number of } \\
\text { Experiments. }\end{array}$ & $\begin{array}{c}\text { Average Live } \\
\text { Weight, Kgs. }\end{array}$ & $\begin{array}{c}\text { Carbon Dioxide } \\
\text { yer Square Cm. } \\
\text { of Surface, } \\
\text { Mgrs. }\end{array}$ \\
\hline $4 \ldots \ldots \ldots \ldots \ldots$ & 24.0 & 2.65 \\
$5 \ldots \ldots \ldots \ldots$ & 13.5 & 2.60 \\
$7 \ldots \ldots \ldots \ldots$ & 11.5 & 2.81 \\
$4 \ldots \ldots \ldots \ldots$ & 9.0 & 2.81 \\
$3 \ldots \ldots \ldots \ldots$ & 6.5 & 2.69 \\
$3 \ldots \ldots \ldots \ldots$ & 5.0 & 2.57 \\
$4 \ldots \ldots \ldots \ldots$ & 3.1 & 2.71 \\
\hline
\end{tabular}

E. Voit * has recently published an extended compilation of results bearing upon this point, including experiments on man, dogs, rabbits, swine, geese, and hens, the heat production being in most cases computed from the metabolism of carbon and nitrogen. The results when computed per square meter of surface, while they show not inconsiderable variations in some individual cases, nevertheless as a whole substantially confirm the conclusion that the fasting metabolism is in general proportional to the surface. Still more recently Oppenheimer $\dagger$ has shown that the law also holds good for infants.

Causes of Variations.-In comparing experiments made upon different animals by different observers at different times some variation in the results would naturally be expected. The experiments compiled by Voit were not all made at the same temperature, but the range in most cases is relatively small and can hardly have exerted any considerable influence. Differences between the different animals as to their normal rate of emission of heat (thickness of coat, quality of skin) may perhaps have also had an effect, although probably a small one.

A more important source of error seems to lie, as Voit points out, in the computation of the results to unit surface, what is actually measured, of course. being the total heat production of the animal. In solids which are of the same shape, that is, which

* Zeit. f. Biol., 41, 113. †Ibid., 42, 147. 
are geometrically similar figures, the surface is proportional to the two-thirds power of the volume. If we let $S=$ surface and $V=$ volume, then $S=k V^{2}$, in which $k$ is a constant for any given form. Putting $W=$ weight, if the bodies have the same specific gravity we may substitute $W$ for $V$ in the above equation, and we then have

$$
S=k W^{3}, \quad k=\frac{S}{W^{\frac{2}{3}}} .
$$

On the assumption that the bodies of animals of the same species constitute similar figures and have the same specific gravity, the value of $k$ has been determined for several species, as follows (the weight being expressed in kilograms and the surface in square centimeters) :

\begin{tabular}{|c|c|c|}
\hline Man......... & 12.9 & Meeh (Zeit. f. Biol., 15, 425). \\
\hline Dog. & 11.2 & Rubner (Ibid., 19, 548). \\
\hline Rabbit. & 12.9 & Rubner (Ibid., 19, 553). \\
\hline Horse . & 9.02 & Hecker (Zeit. f. Veterinark., 1894). \\
\hline Hen. . & 10.45 & Rubner (Zeit. f. Biol., 19, 553). \\
\hline Guinea-pig ... . & 8.89 & Rubner (Biol. Gesetze, p. 17). \\
\hline Rat ......... & $9.13\}$ & \\
\hline Frog . . . . . & $4.62\}$ & \\
\hline
\end{tabular}

The heat production per unit of surface in most of the foregoing experiments is computed by the use of these factors. The results of such computations, however, are necessarily approximations only. While animals of the same species are of the same general shape, we can by no means regard them as being exactly similar figures in the geometrical sense, nor can we safely assume them to be of exactly the same specific gravity, since changes in the amount of contents of stomach and intestines, and particularly in the quantity of fat in the body, would cause greater or less variations. Moreover, the state of fatness has, as Voit points out, still another effect. As an animal grows fat, the increase in size is mainly transverse and not longitudinal, the effect being like that of increasing the diameter of a cylinder of fixed length.* In such a case, however, the increase in the surface is not proportional to the two-thirds power of the volume, nor to the square root of the vol-

* In the case of an animal, of course, we have the additional fact that the deposit of fat is not of uniform thickness over the whole surface of the body. ' 
ume, as Voit states. The curved surface of the cylinder will be proportional to the square root of its volume, while the surface of the two ends will be proportional to the volume, and the ratio of total surface to volume will depend upon the ratio of length to diameter, being greater as the latter becomes less.

Obviously, the calculation of the surface of an animal from its weight is a more or less uncertain one, and it is not surprising that the results should be somewhat fluctuating. It seems very doubtful, however, whether the larger differences found in Voit's compilation can be explained in this way, and Voit shows that there is another factor to be considered, viz., the mass of active cells in the body, which has a material bearing on the results. Before proceeding to a discussion of this point, however, it is desirable to consider briefly the significance of the general fact of the close relation between heat production and surface.

Significance of Results.-Let us imagine an animal exposed to its "critical thermal environment" ( $p .358$ ) to gradually shrink in size while the external conditions remain the same. Under such circumstances the loss of heat to its surroundings will tend to increase relatively to its mass - that is, the body, like an inanimate object, will tend to cool more rapidly. This tendency can be met and the body temperature maintained in only two ways, viz., either by some modification of its surface-e.g., thicker hair-which will lower what we may call its emission constant, or by a relative increase in its rate of heat production.

The results which we have been considering show that in general the emission constant, i.e. the rate of heat emission per unit of surface, is substantially the same in small and large animals, and that the greater loss of heat in the former case is met by an increased production. In this aspect the effect is simply an extension of the influence of falling temperature, the increased demand for heat being met by an increased supply, so that the extent of surface appears as the determining factor of the amount of metabolism.

In the case of an animal exposed to a temperature below the critical point, however, the increased demand for heat appears to be met largely by a stimulation of those processes of metabolism which do not result in any visible form of work, while the internal work, 
in the more restricted sense of the ordinary functions of the internal organs, does not seem to be materially affected. Are we justified in assuming the same thing to be true in our imagined shrinkage of an animal? In other words, is the work of the internal organs proportional to the mass of the body and is the increased heat production in the smaller animal due to the same cause as that observed when an animal is exposed to a falling temperature?

It appears quite clear that this question must be answered in the negative. It is a well-known fact that the circulation, respiration, and other functions are as a rule more active in small than in large animals, and this greater activity must necessarily result in the evolution of relatively more heat. If we raise the temperature of the surroundings to a point corresponding to the critical thermal environment, we may, as we have seen, regard the heat production as representing the internal work in the narrower sense. Rubner * reports experiments of this sort upon four guinea.pigs at $0^{\circ} \mathrm{C}$. and at $30^{\circ} \mathrm{C}$., which gave the following results for the production of carbon dioxide:

\begin{tabular}{c|c|c|c|c}
\hline \multirow{2}{*}{$\begin{array}{c}\text { Weight of } \\
\text { Animal, } \\
\text { Grms. }\end{array}$} & \multicolumn{2}{|c|}{$\mathrm{CO}_{2}$ per Hour at $0^{\circ} \mathrm{C}}$. & \multicolumn{2}{c}{$\mathrm{CO}_{2}$ per Hour at $30^{\circ} \mathrm{C}}$. \\
\cline { 2 - 3 } & $\begin{array}{c}\text { Per Kg. } \\
\text { Weight, } \\
\text { Grms. }\end{array}$ & $\begin{array}{c}\text { Per Square } \\
\text { Meter Surface, } \\
\text { Grms. }\end{array}$ & $\begin{array}{c}\text { Per Kg. } \\
\text { Weight, } \\
\text { Grms. }\end{array}$ & $\begin{array}{c}\text { Per Square } \\
\text { Meter Surface, } \\
\text { Grms. }\end{array}$ \\
\hline 617 & 2.905 & 27.85 & 1.289 & 12.35 \\
568 & 3.249 & 30.30 & 1.129 & 10.53 \\
223 & 4.462 & 30.47 & 1.778 & 12.14 \\
206 & 4.738 & 31.56 & 1.961 & 13.16 \\
\hline
\end{tabular}

With the first and third of these animals direct experiment showed that the minimum production of carbon dioxide (critical point) was reached at about $30^{\circ}-35^{\circ}$, and we may fairly assume this to be true of the other two. At $30^{\circ} \mathrm{C}$., then, we may assume that the "chemical" regulation was practically eliminated and that the observed metabolism was that due to the work of the internal organs. Under these conditions, as the figures show, the metabolism was still approximately proportional to the surface of the animal, and consequently greater per unit of weight in the smaller than in the larger animals.

* Biologische Gesetze, pp. 12-18. 
Strong confirmation of this conclusion is afforded by the experiments previously cited. In many of them, notably in Rubner's, the range of size is so great that to regard the differences in heat production as arising from a direct stimulation of the metabolism, as in the case of a fall in the external temperature, leads to improbable consequences. Thus a comparison of the largest with the smallest dog in Rubner's experiments (p. 361) shows that if we regard the heat production of the former as representing simply the work of the internal organs, over 56 per cent. of the heat production of the smaller animal must, on the supposition that the internal work is proportional to the mass of the body, have arisen from some other source. Such an enormous increase in the metabolism of the body simply for the sake of heat production can hardly be regarded as probable. Still further, if we assume (compare p. 354) a temperature of about $20^{\circ} \mathrm{C}$. to represent the critical point for the dog, then, on the hypothesis that the necessary internal work per unit of weight is the same, we find that a fall of one degree in temperature must have produced about six times the effect upon the metabolism of the smallest dog that it did on that of the largest one, while if we take the other alternative and seek to explain the results on the assumption of a higher critical temperature for the smallest dog, we find for the latter about $36 \frac{1}{2}^{\circ} \mathrm{C}$.

Taking these considerations along with the results of Rubner's trials with the four guinea-pigs, it seems most reasonable to assume, in default of more extensive investigations directed to this specific point, that the critical temperature is substantially the same for large and small animals of the same species and that the work of the internal organs is approximately proportional to the surface of the animal.

Substantially the same conclusion has been reached by v. Hösslin * from a quite different point of view. He points out that the increased production of heat below the critical temperature is not proportional to the difference in temperature between the body and its surroundings, as it should be, according to Newton's law, if the emission constant of the surface remained the same. Taking as an example Theodor's experiments (p. 351) he makes the following comparisons:

* Arch. f. (Anat. u.) Physiol., 1888, p. 323. 


\begin{tabular}{c|c|c|c|c}
\hline $\begin{array}{c}\text { External } \\
\text { Temperature, } \\
\text { Degrees. }\end{array}$ & \multicolumn{2}{|c|}{$\begin{array}{c}\text { Difference Between Body and } \\
\text { External Temperature. }\end{array}$} & \multicolumn{2}{c}{ Carbon Dioxide in 12 Hours. } \\
\cline { 2 - 4 } & $\begin{array}{c}\text { Total, } \\
\text { Degrees. }\end{array}$ & Relative. & $\begin{array}{c}\text { Total, } \\
\text { Grms. }\end{array}$ & Relative. \\
\hline 30.8 & 7.2 & 1.0 & 12.03 & 1.00 \\
20.1 & 17.9 & 2.5 & 14.34 & 1.19 \\
12.3 & 25.7 & 3.6 & 17.76 & 1.48 \\
0.2 & 37.8 & 5.25 & 18.24 & 1.52 \\
-5.5 & 43.5 & 6.0 & 19.83 & 1.65 \\
\hline
\end{tabular}

It would appear from these figures that even below the critical temperature the "physical" regulation plays a large part in the regulation of the body temperature, being simply supplemented by the "chemical" regulation, and that therefore the demand for heat has not the determining influence upon the heat production which Rubner supposes. According to v. Hösslin the apparent dependence of the total metabolism upon the surface is only a particular case of a general morphological law and he points out:

First, that since, according to him, the velocity of the circulation does not vary greatly in large and small animals, the average amount of blood passing through the organs, and consequently their supply of oxygen, will be proportional to the total crosssection of the blood-vessels, which again, similar form being assumed, will be proportional to the two-thirds power of the volume (or weight) of the body.

Second, that the capacity of the alimentary canal to digest and resorb food and thus to supply material for metabolism is limited in the same proportion.

Third, that the work of locomotion-substantially the only form of external work in the wild state-at a given speed is proportional to the two-thirds power of the weight.

In short, v. Hösslin claims that all the important physiological activities of the body, including, of course, its internal work and the consequent heat production, are substantially proportional to the two-thirds power of its volume, and that since the external surface bears the same ratio to the volume, a proportionality necessarily exists between heat production and surface. According to this view, then, the heat production of the fasting animal at the critical temperature represents the internal work, which is proportional 
to the two-thirds power of the volume of the body, while below this point there is superadded a stimulating effect upon the heat production, which, since it acts through the surface, we may assume to be proportional to the latter.

Comparison of Species.-In the foregoing discussion comparisons have been made between large and small animals of the same species, with the result that both their internal work and their total fasting metabolism appear to be closely proportional to their surface. Going a step further and comparing the average results of the several species with each other, $\mathrm{E}$. Voit * reaches the interesting and striking result that the same relation of total fasting metabolism to surface is substantially true as between different species. The following table contains the averages, with the addition of the fasting metabolism of the horse as computed by Zuntz \& Hagemann, which Voit believes with good reason to be too low:

\begin{tabular}{|c|c|c|c|c|}
\hline & \multirow{2}{*}{$\begin{array}{l}\text { Average Tem- } \\
\text { perature, } \\
\text { Deg. C. }\end{array}$} & \multirow{2}{*}{$\begin{array}{c}\text { Average } \\
\text { Weight, Kgs. }\end{array}$} & \multicolumn{2}{|c|}{ Fasting Metabolism. } \\
\hline & & & $\begin{array}{l}\text { Per Kg., } \\
\text { Cals. }\end{array}$ & $\begin{array}{l}\text { Per Square } \\
\text { Meter, Cals. }\end{array}$ \\
\hline 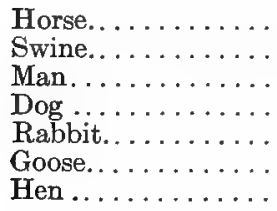 & $\begin{array}{l}9.1(?) \\
20.1 \\
14.3 \\
18.0 \\
18.2 \\
15.0 \\
18.5\end{array}$ & $\begin{array}{r}441 \\
128 \\
64.3 \\
15.2 \\
2.3 \\
3.5 \\
2.0\end{array}$ & $\begin{array}{l}11.3 \\
19.1 \\
32.1 \\
51.5 \\
75.1 \\
66.7 \\
71.0\end{array}$ & $\begin{array}{r}>948 \\
1078 \\
1042 \\
1039 \\
776 \\
967 \\
943\end{array}$ \\
\hline
\end{tabular}

With the exception of the rabbit, the average heat production of these various animals per unit of surface does not show any greater variations than have been observed between different animals of the same species, more or less of which, as we have seen, can probably be accounted for by errors in the estimate of the surface of the body.

Accepting the fact of the general proportionality of heat production to surface, and passing over for the moment the exceptional case of the rabbit, it is plain that the considerations which have been adduced in discussing the results upon the same * Loc. cit., p. 120. 
species will in the main apply to a comparison of different species. It is true that what data we have indicate that there may be more or less difference between the critical temperatures for different species, but in view of the enormous range in the size of the animals experimented on this cannot largely modify the results. Any reasonable assumptions as to critical temperatures and as to rates of variation per degree in heat production would still leave the corrected results substantially proportional to the surface. Apparently we must conclude that in all these different species, as well . as in larger and smaller animals of the same species, the internal work, as measured by the total metabolism at the critical temperature, is substantially proportional to the surface.

How generally this may be true we have at present no means of judging. It is clear, however, that in the process of organic evolution one of the very important factors has been the demand for heat exerted by the environment upon the animal. This has been met to some extent by modifications in the coat of the animal, but to a very large degree by changes in the rate of heat production, with the result that, other things being equal, those forms have survived whose normal heat production, resulting from internal work alone, was sufficient to maintain their temperature under the average conditions surrounding them without, on the one hand, calling largely into play the processes of "chemical" regulation, nor, on the other hand, producing so much heat as to render it difficult for the body to get rid of it.

Relation of Heat Production to Mass of Tissue.-As already indicated, E. Voit. in his article cited above, has shown that while the heat production is in general proportional to the surface, there is also another determining factor, viz., the mass of the active cells in the organism, a rough measure of which is the total nitrogen of the body exclusive of that of the bones and the skin. This conclusion is based chiefly on experiments with fasting animals. As the weight of such an animal decreases, its relative surface must increase, and, as was shown on p. 364, probably more rapidly than in proportion to the two-thirds power of the weight. Under these circumstances we should naturally expect that the relative heat production would increase, but as a matter of fact it rather shows a tendency to decrease. E. Voit, in discussing the results of Rubner 
and others, computes the heat production per unit of surface, and also compares it with the amount of nitrogen computed to be present in the organs of the animal on the several days of the experiment. The following results of one of Rubner's experiments with rabbits are typical of those obtained in this way:

\begin{tabular}{|c|c|c|c|c|c|}
\hline \multirow[b]{2}{*}{ Day of Fasting. } & \multirow[b]{2}{*}{$\begin{array}{l}\text { Average } \\
\text { Live } \\
\text { Weight, } \\
\text { Grms. }\end{array}$} & \multicolumn{4}{|c|}{ Heat Production per Day. } \\
\hline & & $\begin{array}{c}\text { Total, } \\
\text { Cals. } \\
\text {. }\end{array}$ & $\begin{array}{l}\text { Per Kg. } \\
\text { Cals. }\end{array}$ & $\begin{array}{c}\text { Per } \\
\text { Square } \\
\text { Meter of } \\
\text { Surface, } \\
\text { Cals. }\end{array}$ & $\begin{array}{c}\text { Per } 100 \\
\text { Nitrogen, } \\
\text { Cals. }\end{array}$ \\
\hline Third & 2185 & 155 & 71.0 & 730 & 310 \\
\hline Fifth & 2093 & 117 & 55.9 & 556 & 243 \\
\hline Seventh & 2007 & 102 & 50.8 & 499 & 220 \\
\hline Ninth ... . . . . . . . & 1923 & 97 & 50.5 & 488 & 221 \\
\hline Tenth and twelfth $\ldots \ldots \ldots$ & 1841 & 95 & 51.6 & 494 & 227 \\
\hline Thirteenth and fourteenth.. & 1735 & 88 & 50.7 & 463 & 222 \\
\hline Fifteenth and sixteenth $\ldots .$. & 1646 & 81 & 49.2 & 452 & 218 \\
\hline Seventeenth and eighteenth & 1507 & 72 & 47.8 & 428 & 219 \\
\hline
\end{tabular}

The heat production per unit of surface is seen to decrease at first rapidly and later more slowly, while the heat production per unit of weight shows but a slight decrease and that per unit of nitrogen scarcely any. From these and other similar results, Voit concludes that the law of the proportionality of heat production to surface as enunciated by Rubner and as extended by himself must be limited in its application to animals in like bodily condition, and that an animal with a low stock of nitrogenous tissue will, under the same conditions, show a lower heat production per unit of surface than a well-nourished animal. The exceptionally low average for the rabbit noted on p. 369 he explains on this hypothesis as resulting from the frequent use for such experiments of animals in a poorly nourished and "degenerate" condition resulting from long confinement.

The result has an interesting bearing in another direction. Most of the experiments cited by Voit were probably made at temperatures below the critical points for the several animals. In our previous discussion we have assumed that under these circumstances the heat regulation is accomplished largely by "chemical" means-by variations in the rate of production. In these experi- 
ments, on the contrary, since the heat production decreased along with the decrease of nitrogenous tissue, we see the regulation of body temperature effected by a diminution in the rate of emission of heat, which, however, was in most cases less marked than in the instance just cited. Either we must conclude that the abnormal condition arising from fasting enables the animal to diminish the rate of emission of heat to an extent not possible to the wellnourished one, or we may suppose that in the latter case the stimulation of the metabolism by the abstraction of heat begins before the possibilities of "physical" regulation have been exhausted; that, in other words, the domains of "chemical" and "physical" regulation overlap. Obviously the latter conclusion is entirely in harmony with v. Hösslin's views as stated on pp. 367-8.

\section{§3. The Expenditure of Energy in Digestion and Assimilation.}

\section{General Conception.}

Food Increases Metabolism.-That the consumption of food increases the metabolism and consequent heat production in the body has been known since the time of Lavoisier, who observed the oxygen consumption of man to increase materially (about 37 per cent.) after a meal. Regnault \& Reiset* also, among their respiration experiments on animals, report the following results for the oxygen consumption of two rabbits while fasting and after eating:

\begin{tabular}{|c|c|c|}
\hline Animal. & $\begin{array}{c}\text { Fasting, } \\
\text { Grms. }\end{array}$ & $\begin{array}{c}\text { After Eating, } \\
\text { Grms. }\end{array}$ \\
\hline A......... & 2.518 & 3.124 \\
B........ & 2.731 & 3.590 \\
\hline
\end{tabular}

Subsequent investigations by Vierodt, Smith, Speck, Fredericq, v. Mehring \& Zuntz, Wolfers, Potthast, Hanriot \& Richet, † MagnusLevy, Zuntz \& Hagemann, Laulanié, and others, some of which will be considered more specifically in subsequent paragraphs, have fully confirmed these earlier results, so that the fact of an increased metabolism consequent upon the ingestion of food is undisputed.

* Ann. de Chim. et de Phys. (3), 26, 414.

$\dagger$ Ibid. (6), 22, 520 . 
Cause of the InCrease.-Two possible explanations of the above fact naturally suggest themselves, viz., that, on the one hand, the more abundant supply of food material to the cells of the body may act as a direct stimulus to the metabolic processes, or, on the other hand, that the increased metabolism may arise from the greater activity of the organs of digestion, or finally, that both causes may act simultaneously.

The results obtained by Speck,* who found that the increase began very promptly (within thirty minutes) after a meal, would indicate that it can hardly be due to a stimulating action of the resorbed food upon the general metabolism, but must arise, in large part at least, from the activity of the digestive organs. Specific investigations upon this point were undertaken by Zuntz \& v. Mehring. $\dagger$ They found that glycerin, sugar, egg-albumin, purified peptones, and the sodium salts of lactic and butyric acids $\ddagger$ when injected into the circulation caused no material increase in the amount of oxygen consumed as determined in successive short periods by the.Zuntz form of respiration apparatus. It is well established that some of these substances do increase the metabolism when given by the mouth, and the authors verified this fact for sugar and for sodium lactate and likewise showed that substances like sodium sulphate, which are not metabolized in the body, caused a similar rise in the metabolism when introduced into the digestive tract. They therefore conclude that the effect of the ingestion of food upon the metabolism is due chiefly to the expenditure of energy required in its digestion. Wolfers $\S$ and Potthast, $\|$ in experiments supplementary to those just mentioned, obtained confirmatory results.

On the other hand, Laulanié, $\uparrow$ in the experiments mentioned on p. 180 in their bearings upon the formation of fat from carbohydrates, obtained almost as marked an increase in the oxygen consumption subsequent to the injection of sugar into the circulation as after its administration by the mouth.

* Arch. exper. Pathol. and Pharm., II, 1874, p. 405.

$\dagger$ Arch. ges. Physiol., 15, 634; 32, 173.

$\ddagger$ The results of their experiments upon organic acids have already been cited in Chapter V, p. 157, in another connection.

$\S$ Arch. ges. Physiol., 32, 222.

|l Ibid., 32, 280.

I Archives de Physiol., 1896, p. 791. 
On the whole, however, and in view of the patent fact that the activity of the digestive apparatus consequent upon the consumption of food must lead to an expenditure of energy, the results of Zuntz \& v. Mehring appear to have been generally accepted as proof that it is this influence rather than any direct effect of the resorbed food upon the metabolism to which the increase of the latter after a meal is to be ascribed. This increased expenditure is often, although rather loosely, spoken of as the "work of digestion."

FACTORS OF WORK OF Digestion.-In the process of digestion we are probably safe in assuming that the muscular work of prehension, mastication, deglutition, rumination, peristalsis, etc., constitutes an important source of heat production. A secondary source of heat production, which we may designate as glandular metabolism, is the activity of the various secretory glands which provide the digestive juices, to which may be added also the work of the resorptive mechanisms. Furthermore, the various processes of solution, hydration, cleavage, etc., which the nutrients undergo during digestion contribute their share to the general thermic effect.

Fermentations.-To the above general sources of heat production during the digestive process, there is to be added as a very important one in the case of ruminating animals the extensive fermentation which the carbohydrates of the food undergo. We have already seen that a considerable fraction of the gross energy of these bodies is carried off in the potential form in the combustible gases produced. A further portion is liberated as heat of fermentation. This latter portion forms a part of the metabolizable energy of the food as defined in the preceding chapter, since it assumes the kinetic form in the body. Since, however, it appears immediately as heat, it can be of use to the body only indirectly, as an aid in maintaining its temperature. While, therefore, it does not constitute work in the strict sense of the term, the heat produced by fermentation constitutes a part of the expenditure of metabolizable energy in digestion, and therefore is included under the term "work of digestion" in the general sense in which the term is frequently used.

Warming Ingesta.--The food, and particularly the water, consumed by an animal have to be warmed to the temperature of the body. To the extent that this warming of the ingesta is accomplished at the expense of the heat generated by the muscular, gland- 
ular, and fermentative actions indicated above, it does not call for any additional expenditure of energy and so does not, from the statistical point of view, constitute part of the "work of digestion." If, however, at any time the warming of the ingesta requires more heat than is produced by these processes-if, for example, a large amount of very cold water is consumed-it is evident that the surplus energy required will be withdrawn from the stock otherwise available for other purposes, and to this extent will increase the expenditure of energy consequent upon digestion.

The Expenditure of Extergy in Assimilation.-While our knowledge of the changes which the nutrients undergo after resorption is very meager, we may regard it as highly probable that they undergo important transformations before they are fitted to serve directly as sources of energy for those general vital activities of the body represented in gross by the fasting metabolism.

Thus the proteoses and peptones produced in the course of digestive proteolysis are synthesized again to proteids, while the proteids, when the supply is large, undergo, as was shown in Chapter $\mathrm{V}$, rapid nitrogen cleavage, leaving a non-nitrogenous residue as a source of energy. According to some authorities, as we have seen, the resorbed fat undergoes conversion into dextrose in the liver before entering into the general metabolism of the body. Even the carbohydrates, at least so far as they are not directly resorbed as dextrose, seem to undergo more or less transformation before entering into the general circulation.

In brief, there seems good reason to believe that the crude materials resulting from the digestion of the food undergo more or less extensive chemical transformations before they are ready to serve as what Chauveau calls the "potential" of the body-that is, as the immediate source of energy for the vital functions. Of the nature and extent of these transformations we are largely ignorant. So far as they are katabolic in their nature, a liberation of energy is necessarily involved. Any anabolic processes of course would absorb energy, but the energy so absorbed must come ultimately. from the katabolism of other matter, and in all probability there would be more or less escape of kinetic energy in the process.

Moreover, as was pointed out in the opening paragraphs of Chapter II in discussing the general nature of metabolism, as well 
as in the Introduction, the vital activities are intimately connected with the katabolic processes going on in the protoplasm of the cells. As was there stated, it is highly probable that the molecules of the protoplasm are much more complex than those of the proteids, fat and carbohydrates of the food (compare pp. 17 and 224). To what extent it is necessary that the resorbed nutrients shall be synthesized to these more complex compounds before they can serve the purposes of the organism we are hardly in position to say, but so far as it is required it can be accomplished only by an expenditure of energy derived ultimately from the food and constituting a part, and not impossibly a large part, of the work of assimilation.

Summary. - The considerations of the foregoing paragraphs make it plain that the exercise of the function of nutrition, as is the case with the other functions of the body, involves the expenditure of energy. In general, we may say that this energy is expended for the two purposes indicated in the title of this section, viz., for digestion, or the transformation of the crude materials of the food and their transference to the fluids of the body, and for assimilation, or the conversion of these resorbed materials into the "potential" of the organism. Each of these two general purposes is served by a variety of processes, and the attempt to assign to each its exact share in the increased metabolism brought about by the ingestion of food is a physiological problem at once interesting and complicated.

For our present purpose, however, viz., a consideration from the statistical point of view of the income and expenditure of energy by the organism, we are concerned primarily with the total expenditure caused by the ingestion of food rather than with the single factors composing it. As a matter of convenience it may be permissible to retain the designation above given, viz., the work of digestion and assimilation, but it should not be forgotten that other processes may conceivably be concerned in the matter. In particular, any increased heat production resulting from a direct stimulation of the metabolic processes or of the incidental muscular activity of the animal by the resorbed food, such for example, as Zuntz \& Hagemann * have observed with the horse as a result of abundant feeding, particularly with Indian corn, would be included under the term as here used.

* Landw. Jahrb., 27, Supp. III, 234 and 259. 


\section{Experimental Results.}

General Methods. - It follows from what has been said above that two general methods, or more properly two modifications of one general method, may be employed to determine the total expenditure of energy due to the ingestion of food.

First, since the energy expended in the various processes outlined above is ultimately converted into heat, we may determine the heat production of the animal while fasting and compare with it the heat production during the digestion and assimilation of a known amount of food. The excess of heat produced in the latter case as compared with the former will represent the increased expenditure of energy in the work of digestion and assimilation.

Second, we may determine the total income and outgo of energy in the fasting and in the fed animal by one of the methods indicated in Chapter VIII. In this case the extent to which the net loss of energy by the body has been diminished by means of the food will show how much of the metabolizable energy of the latter has been utilized by the organism in place of that previously drawn from the metabolism of tissue. The part of the metabolizable energy not thus utilized has obviously been expended in some of the various operations of digestion, assimilation, etc. The two methods are complementary, in the one case the expenditure for digestion, assimilation, etc., being determined directly and in the other by difference.

A point of some importance, at least logically, is that the determinations should be made below the point of maintenance. The term assimilation as above defined includes all those processes by which the resorbed nutrients are prepared for their final metabolism in the performance of the vital functions. When we give food in excess of the maintenance requirement, however, there is added to this the set of processes by which the excess food is converted inta suitable forms for more or less temporary storage in the body. These may be presumed to consume energy, and as it would seem, to a more or less variable extent. At any rate, we have no right to assume in advance that the relative expenditure of energy above the maintenance point in the storage of excess material is the same as that below the maintenance point for the processes of assimilation as above defined. In other words, it is not necessarily nor even, it would seem, probably the case that the resorbed 
portion of a maintenance ration is first converted into the same materials (particularly fat) that are deposited in the body when excess food is given, and that these materials are then metabolized in the performance of the bodily functions. It is at least conceivable, if not likely, that a much less profound transformation, and one involving a smaller loss of energy, suffices to prepare the resorbed nutrients for their functions as "potential" than is required for their storage as gain of tissue.

Finally, the comparison need not necessarily be made, and indeed in case of most agricultural animals cannot well be made, with the fasting state. While this method is the simpler when practicable, a comparison of the total heat production or of the balance of energy on two different rations (both being less than the maintenance requirement) will afford the data for a computation by difference (exactly similar to that employed in the determination of metabolizable energy in Chapter $\mathrm{X}$ ) of the expenditure of energy in the digestion and assimilation of the food added to the basal ration.

The most important quantitative investigations upon the work of digestion are those of Magnus-Levy* on the dog and on man, and those of Zuntz \& Hagemann $†$ upon the horse.

Experiments on the Dog. - In Magnus-Levy's experiments the respiratory exchange of the animal was determined by means of the Zuntz apparatus at intervals of one or two hours during fasting and after feeding. The single periods were twenty-five to thirty minutes long, and the external conditions were maintained as uniform as possible.

FAT.-Fat (in the form of bacon free from visible lean meat), when given in quantities not materially exceeding in heat value the fasting metabolism, resulted in a slight increase of the latter, beginning about one to three hours after eating, reaching its maximum between the fifth and ninth hours, and disappearing about the twelfth hour. The maximum increase observed was 12 per cent., seven hours after eating. In amounts largely exceeding the equivalent of the fasting metabolism the effect of fat was somewhat more marked and longer continued, a maximum increase of $\mathbf{1 9 . 5}$ per cent. being observed in one case seven hours after eating, while

* Arch. ges. Physiol., 55, 1.

$\dagger$ Landw. Jahrb., 27, Supp. III. 
the metabolism was still slightly above its fasting value after eighteen hours. The respiratory quotient in every case sank to a value closely corresponding to that for the oxidation of pure fat.

The experiments do not permit an exact estimate of the total increase of the metabolism during the twenty-four hours, since the observations were not always made at hourly intervals and but few of the trials extended over a full day. By selecting, however, the two in which the data are most complete and computing as accurately as may be the average rate of consumption of oxygen per minute, it is possible to obtain an approximate expression for the total heat production. For this purpose the average oxygen per minute is multiplied by 1440 and this product by the calorific equivalent of the oxygen, viz., 3.27 Cals. per gram in this case, and the following results obtained, the heat production during fasting being in each instance that found in the particular experiment under consideration:

\begin{tabular}{|c|c|c|c|c|c|c|}
\hline \multirow{3}{*}{$\begin{array}{c}\text { No. of } \\
\text { Experiment. }\end{array}$} & \multirow{3}{*}{$\begin{array}{l}\text { Fat } \\
\text { Eaten, } \\
\text { Grms. }\end{array}$} & \multirow{3}{*}{$\begin{array}{c}\text { Energy } \\
\text { of Food, } \\
\text { Cals. }\end{array}$} & \multicolumn{4}{|c|}{ Heat Production in 24 Hours. } \\
\hline & & & \multirow{2}{*}{$\begin{array}{l}\text { Fasting, } \\
\text { Cals. }\end{array}$} & \multirow{2}{*}{$\begin{array}{l}\text { With } \\
\text { Food, } \\
\text { Cals. }\end{array}$} & \multicolumn{2}{|c|}{ Increase. } \\
\hline & & & & & Cals. & $\begin{array}{l}\text { Per Cent. } \\
\text { of Food. }\end{array}$ \\
\hline $100 \ldots$ & $\begin{array}{l}131.6 \\
305.5\end{array}$ & $\begin{array}{l}1250 \\
2902\end{array}$ & $\begin{array}{r}972 \\
1055\end{array}$ & $\begin{array}{r}991 \\
1142\end{array}$ & $\begin{array}{l}19 \\
87\end{array}$ & $\begin{array}{l}1.53 \\
2.99\end{array}$ \\
\hline
\end{tabular}

Carbohydrates.-Carbohydrates produccd a more marked effect upon the metabolism than did fat, and one which showed itself more promptly. In the experiments on the dog the food consisted of rice, either alone or with the addition of small amounts of fat, sugar, or meat; in other words, the animal was on a mixed diet in which carbohydrates predominated.

On the average of a series of six experiments in which the food consisted of 500 grams of rice, 200 grams of meat, and 25 grams of fat, the metabolism increased by fully 30 per cent. within the first hour and continued to increase more slowly until the maximum of 39 per cent. was reached at the sixth to eighth hour. From that time it decreased to 25 per cent. in the twelfth hour and then rather suddenly dropped nearly to the fasting value. The respiratory quotient rose from 0.78 during fasting to 0.90 in the first hour, and 
reached very nearly 1.00 by the third hour, remaining at substantially this value for sixteen to eighteen hours and not falling to the fasting value in twenty-four hours. Two parallel experiments in which 400 grams of meat were fed showed that a part, but by no means all, of the above increase was to be ascribed to the 200 grams of meat. The small amount of fat given can hardly have affected the result. The author estimates that of the total calculated increase of 22 per cent. over the fasting metabolism about 5 per cent. may have been due to the proteids of the food and the remainder to the carbohydrates. This conclusion is confirmed by the results of two experiments in which rice, sugar, and fat were given. The increase in the metabolism was of precisely the same character as in the other experiments, but less in amount.

In all these experiments the food was in excess of the fasting metabolism. In another series in which the food, consisting of rice, either alone or with a small amount of sugar, was about equivalent to the fasting metabolism, the increase in the metabolism was slightly less, although otherwise the results were similar to those of the other trials.

Computing the results per twenty-four hours, as in the case of the fat, we have the following approximate figures for the three series:

\begin{tabular}{|c|c|c|c|c|c|c|}
\hline \multirow{3}{*}{$\begin{array}{c}\text { No. of } \\
\text { Experiment. }\end{array}$} & \multirow{3}{*}{$\begin{array}{l}\text { Food,* } \\
\text { Grms. }\end{array}$} & \multirow{3}{*}{$\begin{array}{c}\text { Metab- } \\
\text { oliza- } \\
\text { ble } \\
\text { Energy } \\
\text { of } \\
\text { Food, } \dagger \\
\text { Cals. }\end{array}$} & \multicolumn{4}{|c|}{ Heat Production in 24 Hours. } \\
\hline & & & \multirow{2}{*}{$\begin{array}{l}\text { Fast- } \\
\text { ing, } \\
\text { Cals. }\end{array}$} & \multirow{2}{*}{$\begin{array}{l}\text { With } \\
\text { Food, } \\
\text { Cals. }\end{array}$} & \multicolumn{2}{|c|}{ Increase. } \\
\hline & & & & & Cals. & $\begin{array}{l}\text { Per } \\
\text { Cent. of } \\
\text { Food. }\end{array}$ \\
\hline $\begin{array}{r}68,70 \\
71,73, \\
74, \text { and } 75\end{array}$ & $\left.\begin{array}{lr}\text { Proteids....... } & 71.3 \\
\text { Carbohydrates.. } & 375.0 \\
\text { Fat........... } & 31.0\end{array}\right\}$ & 2121 & 1040 & 1271 & 231 & 10.89 \\
\hline 84 and 87\{ & $\left.\begin{array}{lr}\text { Proteids....... } & 28.1 \\
\text { Carbohydrates.. } & 457.5 \\
\text { Fat ......... 25.0 }\end{array}\right\}$ & 2226 & 1132 & 1292 & 160 & 7.19 \\
\hline $107 \ldots \ldots\{$ & 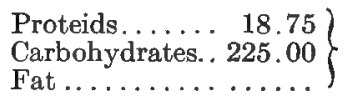 & 999 & 991 & 1080 & 89 & 8.91 \\
\hline
\end{tabular}

* Rice estimated to contain 75 per cent. carbohydrates and 1 per cent. nitrogen.

$\uparrow$ Computed by the writer, using Rubner's factors. 
Proteids.-Proteids in the form of meat or a mixture of meat and flesh-meal, with in some cases small amounts of fat, caused a very marked and prompt increase in the metabolism of the dog. The maximum effect was usually reached about the third or fourth hour and continued with but slight diminution up to the seventh or eighth hour with small rations and as long as to the twelfth or fifteenth hour with large rations. As in the case of fat and carbohydrates, the increase was greater with large rations, but its amount largely exceeded that caused by either of the two former groups of nutrients, reaching in some cases 90 or more per cent. of the fasting value.

The results were more irregular than in the preceding experiments, and were apparently influenced by a peculiar effect of the food upon the type of respiration. The author, however,* computes from three selected series of experiments the following approximate averages for the twenty-four hours:

\begin{tabular}{|c|c|c|c|c|c|c|c|}
\hline & \multirow{3}{*}{$\begin{array}{c}\text { No. of } \\
\text { Experiment. }\end{array}$} & \multirow{3}{*}{$\begin{array}{c}\text { Proteids } \\
\text { Eaten, } \\
\text { Grms. }\end{array}$} & \multirow{3}{*}{$\begin{array}{c}\text { Metabo- } \\
\text { lizable } \\
\text { Energy } \\
\text { of Food, } \\
\text { Cals. }\end{array}$} & \multicolumn{4}{|c|}{ [Heat Production in 24 Hours. } \\
\hline & & & & \multirow{2}{*}{$\begin{array}{c}\text { Fasting, } \\
\text { Cals. }\end{array}$} & \multirow{2}{*}{$\begin{array}{c}\text { With } \\
\text { Food, } \\
\text { Cals. } \\
\end{array}$} & \multicolumn{2}{|c|}{ Increase. } \\
\hline & & & & & & Cals. & $\begin{array}{l}\text { Per Cent } \\
\text { of Food. }\end{array}$ \\
\hline $\begin{array}{r}83 \\
102 \\
95\end{array}$ & $\begin{array}{cr}\text { and } & 89 . \\
\text { “ } & 106 . \\
\text { “" } & 96 .\end{array}$ & $\begin{array}{r}82.5 \\
230.0 \\
370.6\end{array}$ & $\begin{array}{r}338 \\
943 \\
1520\end{array}$ & $\begin{array}{r}1030 \\
963 \\
1059\end{array}$ & $\begin{array}{l}1086 \\
1079 \\
1303\end{array}$ & $\begin{array}{r}56 \\
116 \\
244\end{array}$ & $\begin{array}{l}16.57 \\
12.30 \\
16.05\end{array}$ \\
\hline
\end{tabular}

The amount of the proteid metabolism was not determined in these experiments, but the author points out that they were made on the first day of the feeding, and that it is probable that the proteid metabolism, and consequently the heat production, would have increased more or less had the feeding, particularly with excess of food, been continued longer.

Bone, when fed in large quantities to the dog, was found to cause a greater increase in the metabolism than corresponded to the nitrogenous matter estimated to have been resorbed from it, and the difference is ascribed to the mechanical effect upon the digestive tract. 
Experiments on Man.-Magnus-Levy's experiments upon man were made substantially like those upon the dog, the subject lying upon a sofa, as completely at rest as possible, and breathing through a mouth-piece.

FAT.-Two experiments with fat, computed in the same way as those upon the dog, gave the following results:

\begin{tabular}{|c|c|c|c|c|c|c|}
\hline \multirow{3}{*}{ No. of Experiment. } & \multirow{3}{*}{$\begin{array}{l}\text { Fat } \\
\text { Eaten, } \\
\text { Grms. }\end{array}$} & \multirow{3}{*}{$\begin{array}{l}\text { Energy } \\
\text { of Food, } \\
\text { Cals. }\end{array}$} & \multicolumn{4}{|c|}{ Heat Production in 24 Hours. } \\
\hline & & & \multirow{2}{*}{$\begin{array}{l}\text { Fasting, } \\
\text { Cals. }\end{array}$} & \multirow{2}{*}{$\begin{array}{c}\text { With } \\
\text { Food, } \\
\text { Cals. }\end{array}$} & \multicolumn{2}{|c|}{ Increase. } \\
\hline & & & & & Cals. & $\begin{array}{l}\text { Per Cent. } \\
\text { of Food. }\end{array}$ \\
\hline $\begin{array}{l}81 \ldots \ldots \ldots \ldots \ldots \\
21 \ldots \ldots \ldots \ldots \ldots\end{array}$ & $\begin{array}{r}94.0 \\
195.6\end{array}$ & $\begin{array}{r}893 \\
1855\end{array}$ & $\begin{array}{l}1537 \\
1524\end{array}$ & $\begin{array}{l}1547 \\
1582\end{array}$ & $\begin{array}{l}10 \\
58\end{array}$ & $\begin{array}{l}1.12 \\
3.13\end{array}$ \\
\hline
\end{tabular}

CARBohydrates.-Numerous experiments on a man were made in which the diet consisted chiefly of bread, and a smaller number in which the effect of sugar was studied. With bread the increase in the metabolism was more prompt than in the experiments on the dog, but smaller in amount, varying from about 12 to as high as 33 . per cent., according to the amount eaten. By the end of the third hour the effect had nearly disappeared, but it was then followed by a second increase, less in amount but continuing longer, which the author suggests may have been due to the commencement of intestinal digestion. With sugar (both cane and grape) the increase was equally prompt, although rather less in amount, but disappeared entirely after two or three hours. None of the experiments extended over more than ten hours and usually over less, and the data given are insufficient for a satisfactory computation of the total increase for the twenty-four hours. The respiratory quotient was considerably raised, but did not reach 1.00 in any case.

Proterds.-Experiments upon the effect of proteids on the respiratory exchange yielded results similar to those obtained with the dog, but do not permit of a satisfactory computation of averages for the twenty-four hours.

Mixed Diet.-Results with a mixed diet the ingredients of which are not specified have been reported by Johansson, Lander- 
gren, Sondèn \& Tigerstedt.* The experiments were made in a large Pettenkofer respiration apparatus and extended over twentytwo hours, the results being computed to twenty-four hours. The total heat production, as computed from the carbon and nitrogen balance, and the computed metabolizable energy of the food were:

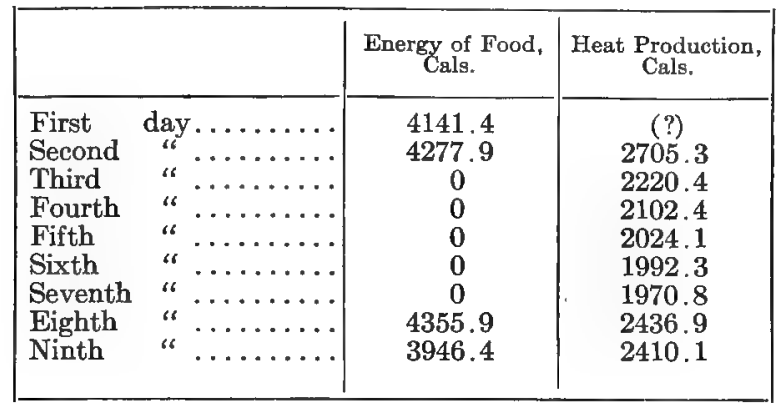

The above figures furnish a striking example of the constancy of the fasting metabolism, and of the marked increase brought about by the consumption of food. Omitting the results for the first day of fasting and for the first day of the experiment we obtain the following averages:

Average energy of food ......... 4193.4 Cals.

Metabolism:

With food................. 2517.4 "

Fasting................ 2022.4 "

Increase.

Total.................... 495.0 "

Per cent. of food............ 11.76 Per cent.

It is to be noted, however, that the food in this experiment was considerably in excess of the fasting requirements, so that there was a notable storage of material and energy in the body.

SUMMARY.-The results of the foregoing approximate computations of the increased expenditure of energy for twenty-four hours are summarized in the following table, which also includes a comparison of the metabolizable energy of the food with the fasting metabolism:

* Skand. Arch. Physiol., 7, 29. 


\begin{tabular}{|c|c|c|c|}
\hline Food. & $\begin{array}{c}\text { Metabolizable } \\
\text { Energy of } \\
\text { Food, Cals. }\end{array}$ & $\begin{array}{l}\text { Excess Above } \\
\text { Fasting } \\
\text { Metabolism, } \\
\text { Cals. }\end{array}$ & $\begin{array}{l}\text { Digestive Work } \\
\text { in Per Cent. } \\
\text { of Metaboliz- } \\
\text { able Energy. }\end{array}$ \\
\hline $\begin{array}{l}\text { Fas: } \\
\qquad \begin{aligned} \text { Experiments on } \operatorname{man} \ldots \ldots \\
\text { " } \quad \text { " } \operatorname{dog} . \ldots \ldots\end{aligned}\end{array}$ & $\begin{array}{r}893 \\
1855 \\
1250 \\
2902\end{array}$ & $\begin{array}{r}-644 \\
+331 \\
+278 \\
+1847\end{array}$ & $\begin{array}{l}1.12 \\
3.13 \\
1.53 \\
2.99\end{array}$ \\
\hline Average ............... & $\ldots \ldots \ldots$ & $\ldots \ldots$ & 2.19 \\
\hline $\begin{array}{l}\text { Chiefly Carbohydrates: } \\
\quad \text { Experiments on dog ........ }\end{array}$ & $\begin{array}{r}2121 \\
2226 \\
999\end{array}$ & $\begin{array}{r}+1081 \\
+1094 \\
+8\end{array}$ & $\begin{array}{r}10.89 \\
7.19 \\
8.91\end{array}$ \\
\hline Average .... & $\ldots \ldots$ & $\ldots \ldots \ldots$ & 8.99 \\
\hline $\begin{array}{l}\text { Proteids : } \\
\qquad \text { Experiments on } \operatorname{dog} . . . \ldots \ldots\end{array}$ & $\begin{array}{r}338 \\
943 \\
1520\end{array}$ & $\begin{array}{r}-692 \\
-20 \\
+461\end{array}$ & $\begin{array}{l}16.57 \\
12.30 \\
16.05\end{array}$ \\
\hline Average...$\ldots \ldots \ldots \ldots$ & $\ldots \ldots \ldots \ldots$ & $\ldots \ldots \ldots \ldots$ & 14.97 \\
\hline $\begin{array}{l}\text { Mixed Diet : } \\
\quad \text { Experiments on man .... }\end{array}$ & 4193 & +2171 & 11.76 \\
\hline
\end{tabular}

It is clear that proteids caused the greatest increase in the metabolism and fat the least, while the carbohydrates occupied an intermediate position. In the case of fat the increase in the heat production seems to show a slight tendency to become greater with amounts of food largely in excess of the fasting metabolism, but with the carbohydrates and proteids no distinct effect of this sort is apparent.

These results, particularly those on proteids, afford a good illustration of the fact that the increase in the heat production caused by the ingestion of food is not due solely to the increased muscular work involved, since if we were to suppose the latter to be the case it is not apparent why the proteids, which are digested pretty promptly and with comparative ease, should cause seven times as much work as the fats. The results certainly suggest strongly that a large part of the heat production in the former case arises from the considerable chemical cleavage which the proteids undergo in digestion and still more from the stimulative effect of food proteids 
on the nitrogen cleavage; in other words, that what was called on p. 375 the work of assimilation is an important factor.

Results on FAT.-The relatively small increase in the metabolism resulting from the ingestion of fat is worthy of notice as bearing upon the hypothesis, already several times referred to, that it undergoes a cleavage into dextrose, carbon dioxide, and water in the liver, and that the resulting dextrose is the material which serves as the source of potential energy for the general metabolism. As was pointed out in Chapter V (p. 153), however, the dextrose derived from one gram of fat according to the commonly accepted equation would contain about 6.1 Cals. of potential energy out of the 9.5 Cals. contained in the original fat. In other words, over one third of the energy of the fat would be liberated as heat in the intermediary metabolism supposed to take place in the liver. While the heat production was not directly measured in MagnusLevy's experiments, and while the method of computation employed may be open to criticism in details, his results certainly fail to indicate any such large increase in the metabolism as this hypothesis would require.

It should be noted, in conclusion, that the above experiments did not include a determination of the work of mastication and ingestion of the food, and also that, according to the author, there was little if any production of fat in the experiments in which carbohydrates were fed.

Experiments on the Horse.-Zuntz, Lehmann \& Hagemann* have investigated the effect of digestive work and also of the mastication of the food on the metabolism of the horse, the respiratory exchange being determined by the Zuntz method and a correction made for the cutaneous and intestinal respiration. In addition to this, however, other data were secured which serve the authors as the basis for computations of the energy metabolism of the animal and of the available energy of the digested food. Since their most important conclusions as to digestive work are based in large part on the results of these computations it is necessary to consider their method in some detail.

Method of Computation.-At six different times between the

* Landw. Jahrb., 27, Supp., III, pp. 271-285. 
years 1888 and 1891 digestion experiments were made * in whichr the total nitrogen metabolism $\dagger$ and the carbon of the food and of the visible excreta were determined. The ration in every case consisted of hay and a mixture of six parts of oats with one of cut straw; the chemical composition of these feeds was quite similar in the several experiments, the greatest variation being in the last experiment (October 16-22, 1891).

From the results of these experiments the metabolism of energy in the respiration experiments is computed in the following. manner:

First, the results of the several digestion experiments are combined in such a way as to give an average corresponding to the ration during the respiration experiment. E.g., in Period 1 (loc. cit., p. 256) the ration consisted of $6 \mathrm{kgs}$. of oats, $1 \mathrm{~kg}$. of straw, and $6 \mathrm{kgs}$. of hay. As no single digestion experiment was made on just this ration, the results of the first one are taken four times, those of the second three times, and those of the third once, and the sums divided by eight. These averages are taken as representing the digestibility and the urinary carbon and nitrogen during the respiration experiment.

Second, from the average carbon and nitrogen of the urine as thus obtained its content of urea and hippuric acid is computed, and from these data, on the assumption of average composition for the metabolized proteids, the portion of the elements of the latter completely oxidized in the body, from which again the amount of oxygen required and of carbon dioxide produced is computed.

Third, from the computed amount of crude fiber digested, assuming it to have the composition $\mathrm{C}_{6} \mathrm{H}_{10} \mathrm{O}_{5}$ and that 100 grams yield 4.7 grams of methane, is computed the oxygen required for its oxidation and the carbon dioxide resulting.

Fourth, after subtracting the amounts of oxygen and carbon dioxide, as above computed, corresponding to the proteids and crude fiber oxidized, from the totals found in the respiration experiment, the remainders are divided between fat and carbohydrates

* Loc. cit., pp. 211-236.

$\uparrow$ The nitrogen of the feces was determined in the air-dried material. Subsequent experience has shown that there is some loss of nitrogen in airdrying. 
in the manner described on page 76 on the assumption that the fat has the composition $\mathrm{C} 76.54$ per cent., $\mathrm{H} 12.01$ per cent., O 11.45 per cent., and the carbohydrates that of starch.

Fifth, on the basis of the chemical processes thus computed the amount of energy set free is estimated from the known (average) heats of combustion of the materials oxidized.

While the calculation involves numerous assumptions, and while, therefore, the result is of the nature of an approximation, most of the assumptions are so nearly correct as not to contain the possibility of serious error. The two which seem most questionable are the peculiar method of computing the digestibility of the food and the proteid metabolism, and the computation of the proximate composition of the urine.

Influence of Food Consumption on Metabolism.-The influence of the ingestion of food in increasing the oxygen consumption and the energy metabolism of the animal is illustrated by the following tabulation of the results obtained in Period b (loc. cit., p. 282). (The animal was standing quietly, but otherwise was in a state of rest.)

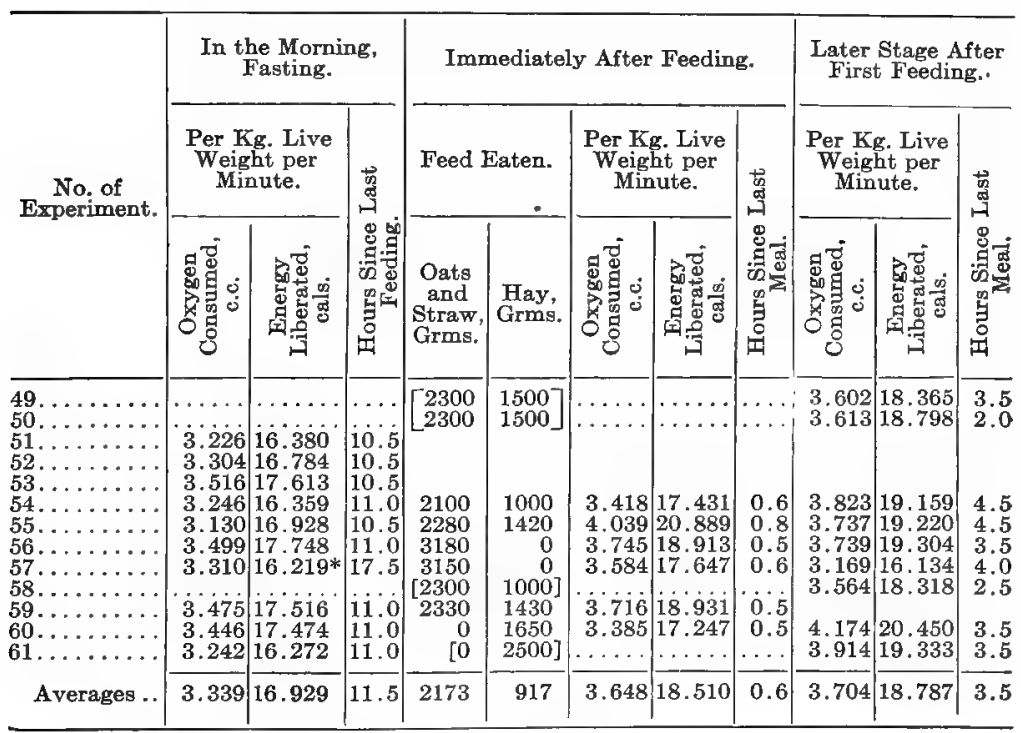

* Animal was uneasy. 
The average energy metabolism thirty-six minutes after eating, computed as previously described, is somewhat more than 9 per cent. greater than that shortly before eating, and a still further increase was observed at the end of three hours. The effect is precisely similar to that observed in Magnus-Levy's experiments. It was not, however, followed through the twenty-four hours, as in some of those experiments.

Comparison of Hay and Grain.-It was found further that coarse fodder (hay) produced a much more marked effect than did grain. The following comparison of the average of the experiments of Period $c$ on an exclusive hay diet with that of Period $f$ on a mixed ration illustrates this fact:

\begin{tabular}{|c|c|c|}
\hline & Period $c$. & Period $f$. \\
\hline $\begin{array}{l}\text { Time since last fed } \ldots \ldots \ldots \ldots \ldots \ldots \\
\text { Ration: }\end{array}$ & $2.6 \mathrm{hrs}$. & $2.8 \mathrm{hrs}$. \\
\hline Hay. & About $10.5 \mathrm{kgs} * *$ & $4.75 \mathrm{kgs}$. \\
\hline Oats ... & $\ldots \ldots \ldots \ldots \ldots \ldots$ & $6.00 " 6$ \\
\hline $\begin{array}{l}\text { Straw. . } \\
\text { Total digested nutrients (fat } \times \text {. }\end{array}$ & & $1.00 " 4$ \\
\hline $\begin{array}{c}2.5) \\
\text { Per kilogram and minute: }\end{array}$ & 4125 grms. $\dagger$ & 5697. grms.† \\
\hline $\begin{array}{l}\text { Oxygen consumed } \ldots \ldots \ldots \ldots \\
\text { Carbon dioxide given off .............. } \\
\text { Energy set free (computed).... }\end{array}$ & $\begin{array}{l}3.9837 \text { c.c. } \\
3.6586 \text { " } \\
19.552 \text { cals. }\end{array}$ & $\begin{array}{l}3.6986 \text { c.c. } \\
3.6695 \text { " } \\
18.339 \text { cals }\end{array}$ \\
\hline
\end{tabular}

Notwithstanding the greater total weight of food consumed in Period $f$, and the much larger amount of digestible matter contained in it, the oxygen consumption and the computed amount of energy liberated are notably greater in Period $c$, on the hay ration. The average time which had elapsed since the last feeding, as well as the external conditions, having been substantially the same in both periods, $t$ and the animal having been in a state of rest, the effect is ascribed to an increase in the expenditure of energy in digestion due to the difference in the physical properties of the two rations. This difference is chemically characterized by the greater

* The exact amount of hay eaten is not stated. The digestible matter is computed from the composition of the hay by the use of Wolff's coeffcients.

† Computed in the manner described above, p. 386.

$\ddagger$ It varied considerably in the individual experiments composing Period $f$. 
proportion of crude fiber in the hay ration. Ascribing the difference in digestive work entirely to the crude fiber, the authors endeavor to estimate the expenditure of energy on this ingredient as follows:

Digestrve Work for Crude Fiber.-The hay ration contained 1572 grams less of (estimated) digestible matter and 648 grams more of total crude fiber than the mixed ration. The computed evolution of energy per head for the twenty-four hours was greater by 772 Cals. in the hay period. On the basis of MagnusLevy's résults the authors assume that the expenditure of energy in the digestion of the nutrients exclusive of crude fiber equals 9 per cent. of the total energy of the digested matter. For 1572 grams (fat being reduced to its starch equivalent) this amounts to $4.1 \times 1572 \times 0.09=580$ Cals. Accordingly, the energy metabolism should have been 580 Cals. less in Period $c$ than in Period $f$. It. was actually 772 Cals. greater, a difference of 1352 Cals. This difference is ascribed to the presence of the 648 grams more of total crude fiber, and corresponds to 2.086 Cals. per gram. With an average digestibility of 55 per cent. this would equal 3.793 Cals. per gram of digested crude fiber, an amount slightly exceeding its metabolizable energy as computed on p. 331. In other words, it would appear that all the metabolizable energy of the crude fiber (or even more, should the digestibility fall below the percentage assumed) is consumed in the work of digestion and converted into heat, leaving none available for external work, and this result seems to coincide strikingly with the results obtained by Wolff * by an entirely different method. (Compare Chapter XIII, §2.)

It is to be observed, however, that the basis of Zuntz \& Hagemann's computation is the difference between the energy required for the digestion of the 648 grams of crude fiber and that required for the digestion of an equal amount of fiber-free nutrients. To get at the total expenditure upon the digestion of the crude fiber we should make the following computation:

The nutrients other than crude fiber digested were in Period $f$ 5124 grams and in Period $c 2608$ grams, a difference of 2516 grams. The corresponding difference in the work of digestion would, on the

* Grundlagen, etc., Neue Beiträge, 1887, p. 94 
above assumptions, be $4.1 \times 2516 \times 0.09=928$ Cals. Adding this, as before, to the observed difference of 772 Cals. gives a total of 1700 Cals. as the effect of the 648 grams of crude fiber, which equals 2.623 Cals. per gram. With a digestibility of 55 per cent., this corresponds to 4.768 Cals. per gram of digested crude fiber, or materially more than its metabolizable energy.

UnCertainties of the COMputation.-The whole method of computation, however, is open to serious criticism on at least two points, aside from the rather indefinite statements as to the amount of hay consumed in Period $c$ and as to the distribution of the ration between the three feedings in Period $f$.

First, the estimate for the work of digestion of the nutrients other than crude fiber which forms the basis of the computation is derived chiefly from the experiments of Magnus-Levy on dogs and man. Those experiments were not only made with highly digestible food, but the digestive work is computed as a percentage of the total (gross) energy of the food. The food of the horse contained in the dry matter 40.94 per cent. of indigestible substances in Period $f$ and 54.37 per cent. in Period $c$, or if we leave out of account the crude fiber the corresponding figures are 31.99 per cent. and 58.45 per cent. A considerable part of the work of digestion undoubtedly consists of muscular work, which must be performed on the indigestible as well as the digestible matter of the food. Moreover these indigestible matters, by their mechanical stimulus and by acting in a certain sense as diluents, may perhaps cause a more abundant secretion of the digestive juices. These facts are entirely ignored when the figures for digestive work derived from experiments on dogs and man are applied simply to the digested food of the horse.

Second, the method of computation assumes that the difference between the metabolism on the two rations which was observed 2.7 hours after eating would have retained the same absolute (not relative) value during the twenty-four hours. The justification for this assumption is found in a comparison * of the results of a single respiration experiment, made one half hour after feeding, with the average of two experiments in which the excretion of carbon

$$
\text { * Loc. cit., p. } 218 .
$$


dioxide was determined for twenty-four hours in a Pettenkofer respiration apparatus. After allowing for the work of mastication in the latter experiment the results were found to agree within 8.8 per cent. The authors, therefore, conclude that with regular feeding the respiratory exchange cluring the forenoon hours, when their experiments were made, corresponds substantially to the average metabolism for the twenty-four hours, exclusive of the work of mastication. It is to be remarked, however, that this conclusion is not fully in harmony with the results quoted on p. 387, which plainly show a marked decrease in the metabolism during the night. Moreover, numerous other determinations of the respiratory exchange at the same hours and on similar food show quite wide variations. In view of this discrepancy, as well as of the somewhat narrow basis of comparison, it certainly appears questionable whether a computation of Periods $c$ and $f$ for twenty-four hours can be safely made.

Zuntz \& Hagemann's results unquestionably show that the work of digestion is greater with coarse fodder than with grain. That this difference is due, at least in large part, to the greater amount of crude fiber in the former is extremely probable. In view, however, of the two sources of uncertainty just pointed out, as well as of the numerous minor assumptions involved in the calculations, we must conclude that the data available are insufficient for an accurate quantitative estimate of the digestive work required by crude fiber.

Work of Mastication.-The foregoing computations relate to the expenditure of energy in the digestion of the food after it has entered the stomach. The same authors have also determined the increase in the gaseous exchange caused by mastication, deglutition, etc. For this purpose they compare * the excretion of carbon dioxide and the consumption of oxygen during the time actually occupied in eating with the corresponding amounts during rest as found from the average of a number of experiments made under identical conditions. On the assumption that the proteid metabolism is unaltered, the proportion of carbohydrates and fat metabolized and the corresponding amounts of energy are computed by

* Loc. cit., p. 271. 
the method described on pp. 76 and 252. The following is a summary of the results computed per kilogram of feed:

\begin{tabular}{|c|c|c|c|c|}
\hline Fodder. & $\begin{array}{c}\text { No. of } \\
\text { Experi- } \\
\text { ments. }\end{array}$ & $\begin{array}{c}\text { Oxygen } \\
\text { Consumed, } \\
\text { Liters. }\end{array}$ & $\begin{array}{c}\mathrm{CO}_{2} \\
\text { Excreted, } \\
\text { Liters. }\end{array}$ & $\begin{array}{c}\text { Equivalent } \\
\text { Energy, } \\
\text { Cals. }\end{array}$ \\
\hline 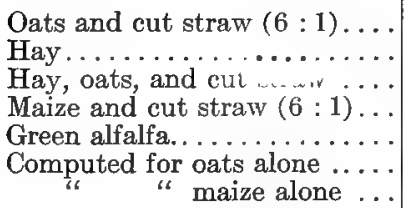 & $\begin{array}{l}8 \\
8 \\
8 \\
2 \\
7\end{array}$ & $\begin{array}{r}12.964 \\
33.840 \\
20.072 \\
7.133 \\
6.171 \\
\cdots\end{array}$ & $\begin{array}{r}10.679 \\
27.813 \\
17.677 \\
6.205 \\
4.980 \\
\ldots\end{array}$ & $\begin{array}{r}64.17 \\
167.44 \\
100.79 \\
35.72 \\
30.42 \\
47.00 \\
13.80\end{array}$ \\
\hline
\end{tabular}

As was to have been expected, the work of mastication proves to be much greater in the case of hay than in that of grain. Maize gave a remarkably low result, while the lowest was obtained with green fodder. Even when the results on the latter are computed per kilogram of dry matter, they are still about 40 per cent. lower than those on hay. A few experiments on old horses with defective teeth gave somewhat higher results for the mixture of oats and cut straw.

The absolute amount of energy expended in mastication, etc., is very considerable. On the average of three periods, on a ration consisting of $5.6 \mathrm{kgs}$. of oats, $0.93 \mathrm{kgs}$. of cut straw, and $5.18 \mathrm{kgs}$. of hay, it is computed at 1287.1 Cals., an amount equal to 11.2 per cent. of the total metabolism during rest.

Conclusions.-The researches of Zuntz \& Hagemann are of great value in that they demonstrate the large proportion of the energy of the food which is consumed in its prehension, mastication, digestion, and assimilation in the case of herbivorous animals, and that this proportion is largely influenced by the physical character of the food. Thus the hard but brittle maize required much less energy for its mastication than the softer but tougher and more woody oats, and the dry matter of the green alfalfa decidedly less than that of the hay. These results indicate quite clearly that no accurate estimates of the work of mastication can (at least in the present state of our knowledge) be based on the chemical composition of feeding-stuffs. As noted above, Zuntz \& Hagemann attempt to compute the work of digestion upon that basis. It 
certainly seems open to question, however, whether in this case also other properties than those expressed by the percentage of crude fiber may not materially affect the result, ${ }^{*}$ and it will be wise, until the subject receives further investigation, to accept their computations as tentative and approximate. $\dagger$

* Compare Kellner's results on cattle, Chapter XIII, §1.

$\dagger$ A somewhat extended critique by Pfeiffer of these researches, together with replies by Zuntz \& Hagemann, will be found in Landw. Vers. Stat., 54, $101 ; 55,117 ; 56,283$ and 289. 


\section{CHAPTER XII.}

\section{NET AVAILABLE ENERGY-MAINTENANCE.}

THe organic matter contrained in the body of an animal we have learned to regard in the light of a certain capital of stored-up energy, at the expense of which the vital activities of the organism are carried on. The function of the food is to make good the losses thus occasioned. The food is frequently spoken of as "the fuel of the body." In a certain limited sense the comparison is admissible, but it may easily be pushed too far, and a closer analogy is that with a stream of water supplying a reservoir and serving to replenish the drafts made upon it for water.

The food in the form in which it is consumed, however, is by no means ready to enter directly into the composition of the tissues of the body and add to its store of potential energy, but on the contrary, as we have seen, a very considerable amount of energy must be expended in the separation of the indigestible matters from the digestible and in the conversion of the latter into such forms as are suitable for the uses of the living cells of the body.

When, therefore, we give food to a quiescent fasting animal we do two things: we supply it with metabolizable energy, depending in amount upon the quantity and nature of the food, to take the place of the energy expended in its internal work, but we at the same time increase its expenditure of energy by the amount necessary to separate the metabolizable from the non-metabolizable energy of the food.

The case is analogous to that of a steam-boiler which is fired by means of a mechanical stoker driven by steam from the same boiler. Each pound of coal fed into the fire-box is capable of evolving a certain amount of heat, representing its metabolizable energy in the above sense, and that heat is capable of producing a 
certain quantity of steam. A definite fraction of the latter, however, is required to introduce the next pound of coal into the furnace and therefore is not available for driving the main engine. To recur to the illustration of the reservoir, it is as if the water, instead of simply flowing into the reservoir, actuated a pump or a hydraulic ram which lifted part of it to the required level.

Gross and Net Availability.-As stated in Chapter X, the difference between the potential energy of the food and that of the excreta represents the maximum amount of energy which is available to the organism for all purposes. This quantity has sometimes been designated as gross available energy, but has here been called metabolizable energy.

A portion of this metabolizable energy, however, as just pointed out, has to be expended in the various processes which have been grouped together under the term work of digestion and assimilation. This portion ultimately takes the form of heat, thus tending to increase the heat production of the animal by a corresponding amount, and becomes unavailable for other purposes in the body, since, so far as we know, the organism has no power to convert heat into other forms of energy. The remainder of the metabolizable energy of the food represents the amount which that food contributes directly towards the maintenance of the capital of potential energy in the body. It is the measure of the net advantage derived by the body from the introduction into it of the food.* From this point of view the energy remaining after deducting the expenditure caused by the ingestion of the food from its metabolizable or gross available energy has been called the net available energy. There are obvious objections to the use of the words available and availability in two senses, but no better term for net available has yet been suggested, while the use of available energy in the sense of metabolizable energy has become quite general. It appears necessary, therefore, to retain for the present the modifying words gross and net to avoid ambiguity.

Distinction between Availability and Utilization.-The net available energy of the food in the above sense represents the

* As will appear later, this somewhat broad statement appears to be subject to modification in certain cases in which there is an indirect utilization of the heat resulting from the work of digestion and assimilation. 
net contribution which it makes to the demands of the vital functions for energy or, in other words, its value as part of a maintenance ration. This must be clearly distinguished from its value for the storage of additional energy in the body-that is, its value for productive purposes. In the latter case it is quite possible that the conversion of the digested nutrients into suitable forms for storage (fat of adipose tissue, ingredients of milk solids, proteids of new growth, etc.) involves a greater expenditure of energy than is required to convert them into forms fitted to serve as sources of cnergy to the body cells (work of assimilation). The consideration of this question belongs in the succeeding chapter, but meanwhile it is important to bear in mind that the net available energy, in the sense in which the term is here employed, is a distinct conception from that of the utilization of energy in fattening, milk production, etc., and has reference to the availability of the energy of the food for maintenance.

It is evident from the above paragraphs that the value of a feeding-stuff to the animal is not measured solely by its metabolizable energy, since materials containing the same proportion of the latter may require the expenditure of very unequal amounts of energy for their digestion and assimilation and, therefore, may contain very unequal amounts of net available energy. Plainly, then, it is a matter of much importance to know the net availability of the metabolizable energy of the various nutrients and feeding-stuffs, and thus to learn the proportions in which they may replace each other.

\section{§. Replacement Values.}

We have already seen (Chapter V, p. 148) that, aside from a certain minimum of proteids, the several nutrients can mutually replace each other to a very large if not to an unlimited extent, either one or all serving, according to circumstances, to supply the demand for energy.

In 1882 v. Hösslin * published an extended discussion of Pettenkofer \& Voit's respiration experiments from this point of view, using such data regarding the potential energy of the nutrients as were then available. He calls attention to the wide range of re-

* Virchow's Archiv, 89, 333. 
placement possible, quoting also Lawes \& Gilbert's conclusions * on the same point drawn from their experiments on fattening swine, and asserts that the nutrients replace each other according to their content of available energy. Danilewsky $\dagger$ also advanced similar views, but Rubner $\ddagger$ appears to have been the first to investigate the subject experimentally.

Isodynamic VALUES.-We have already seen that the total metabolism of a fasting animal is approximately constant, representing the rate at which the store of matter and energy in the body is drawn upon to support the necessary internal work. If we determine the total metabolism of such an animal and then give it a known quantity of some nutrient, as fat, e.g., the loss of tissue will be diminished by a certain amount, which will represent the net available energy of the nutrient and which may be compared with the amount fed. Similarly, a second and third nutrient may be fed and thus their relative values for the prevention of loss of tissue be determined. For example, a dog after fasting for six days was given on the seventh and eighth days 720 and 760 grams respectively of fresh lean meat. The average nitrogen and fat metab. olism for the fifth and sixth days (fasting) and the seventh and eighth days was as follows: §

\begin{tabular}{|c|c|c|c|}
\hline Food. & $\begin{array}{l}\text { Total Nitrogen } \\
\text { Excretion } \\
\text { Grms. }\end{array}$ & $\begin{array}{c}\text { Fat } \\
\text { Metabolized } \\
\text { Grms. }\end{array}$ & $\begin{array}{c}\text { Temperature } \\
\text { Deg C. }\end{array}$ \\
\hline $\begin{array}{l}\text { Nothing (fifth and sixth days).... } \\
\text { Meat (seventh and eighth days). }\end{array}$ & $\begin{array}{r}3.16 \\
20.63\end{array}$ & $\begin{array}{l}75.92 \\
30.72\end{array}$ & $\begin{array}{l}18.0 \\
19.2\end{array}$ \\
\hline Difference . & +17.47 & -45.20 & +1.2 \\
\hline
\end{tabular}

The result of the feeding with meat 'was, of course, a great increase in the proteid metabolism. The increase of 17.47 grams in the nitrogen excreted was equivalent to 113.38 grams of dry matter of the meat. The metabolism of this amount of proteid matter, therefore, enabled the organism to diminish the metabolism of fat

* Phil Trans, 160, 541

$\dagger$ Die Kraftvorräte der Nahrungsstoffe; Arch. ges. Physiol., 1885, p. 230.

\$Zeit. f. Biol , 19, 313

\$ The original account of the experiments is contained in Zeit. f. Biol., 19, 313; these figures are the corrected values given in ibid., 22, 45. 
by 45.20 grams. For the prevention of loss of tissue in this experiment, then, 250 parts of the dry matter of the meat were apparently equivalent to 100 parts of fat. The food, however, was given at the temperature of the room. To warm it and the 100 c.c. of water consumed to the temperature of the body would require an amount of heat equal to that produced by the oxidation of 1.4 grams of fat. Adding this to the 45.2 grams above gives 46.6 grams of fat as the equivalent of 113.38 grams of dry matter of the meat, or a ratio of 100:243.

Another similar experiment gave as a final result a ratio of 100:253, or after correction for the warming of the food 100:243, and a third longer experiment with extracted lean meat (syntonin) yielded the ratio 100:227, or corrected as before 100:225.

If now, from the results of Rubner's determinations of the metabolizable energy of the proteids ( $p .276$ ), we compute the amount of each which contains the same quantity of metabolizable energy as 100 grams of fat and compare it with the above ratios we have the following as the amounts equivalent to 100 grams of fat:

\begin{tabular}{|c|c|c|}
\hline Dry Matter of- & $\begin{array}{c}\text { Computed } \\
\text { from Met- } \\
\text { abolizable } \\
\text { Energy, } \\
\text { Grms." }\end{array}$ & $\begin{array}{c}\text { Found in } \\
\text { Experiments } \\
\text { on Animals, } \\
\text { Grms. }\end{array}$ \\
\hline Lean meat: & & \\
First experiment...... & 235 & 243 \\
Second ". ...... & 235 & 243 \\
Extracted meat...... & 213 & 225 \\
\hline
\end{tabular}

The computed and observed equivalents differ by only 4.3 per cent. and 5.6 per cent. respectively, and hence Rubner concludes that protein replaces fat in metabolism substantially in inverse proportion to its "physiological heat value," or, in other words, to its metabolizable energy.

Rubner has also made similar experiments with cane-sugar and starch, comparing them in each case with the body fat, as in the above experiments, and has also made trials in which grape-sugar was substituted for the fat of the food. In computing the results of these experiments any change in the proteid metabolism was reduced to its equivalent in fat as computed from its metabolizable 
energy. The following table contains the final results, including those on proteids just given:

EQUIVALENT TO 100 GRMS. OF FAT.

\begin{tabular}{|c|c|c|}
\hline Dry Matter of- & $\begin{array}{c}\text { Computed } \\
\text { from } \\
\text { Metabolizable } \\
\text { Energy, } \\
\text { Grms. }\end{array}$ & $\begin{array}{l}\text { Found in } \\
\text { Experiments } \\
\text { on Animals, } \\
\text { Grms. }\end{array}$ \\
\hline Lean meat . . . . . . . & 235 & 243 \\
\hline Extracted meat .... & 213 & $\begin{array}{l}243 \\
225\end{array}$ \\
\hline Cane-sugar . . & 235 & $\begin{array}{l}234 \\
235\end{array}$ \\
\hline Starch ... & 229 & $\begin{array}{l}234 \\
232\end{array}$ \\
\hline Grape-sugar . . . . . . & 255 & $\begin{array}{l}258 \\
254\end{array}$ \\
\hline
\end{tabular}

The equivalents found by experiment correspond quite closely with those computed from the metabolizable energy, and on these facts Rubner bases the law of isodynamic replacement, which may be briefly stated as follows: In amounts less than a maintenance ration the nutrients replace each other in inverse proportion to their metabolizable energy. The quantities which thus replace each other are accordingly said to be isodynamic. It need scarcely be pointed out that the minimum of proteids required for the maintenance of the nitrogenous tissues is not included under this law.

Rubner is careful to limit this law to small amounts of food. In his earlier publications he states that it holds only below the maintenance ration; later * he asserts that it obtains up to an excess of about 50 per cent. over the maintenance ration.

Isoglycosic VALUES.-Mention has already been made of the theory of isoglycosic values maintained by Chauveau and his school, according to which the net available energy of the digested nutrients is measured by the amount of sugar they are considered to be capable of producing in the organism according to the equations given in Chapter II. Chauveau $\uparrow$ computes that the metabolism of 100 parts of proteids according to Gautier's scheme (p. 51), together with the partial oxidation of the resulting fat (p. 38), would yield

* Biologische Gesetze, p. 20. $\dagger$ Comptes rend., 126, 1073. 
81.5 parts of dextrose. Laulanie * computes that 100 parts of fat, carbohydrates, and albumin would produce the following amounts of dextrose:

100 parts of fat produce.......... 161 parts of dextrose

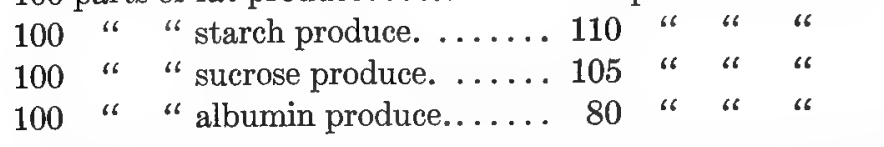

The corresponding isoglycosic values would be as follows, Rubner's isodynamic values being added for comparison:

\begin{tabular}{|c|c|c|}
\hline & $\begin{array}{l}\text { Isodynamic } \\
\text { Weights. }\end{array}$ & $\begin{array}{l}\text { Isoglycosic } \\
\text { Weights. }\end{array}$ \\
\hline Fat.... & 100 & 100 \\
\hline Starch & 229 & 146 \\
\hline Cane-sugar . . . . . . . . . . & 235 & 153 \\
\hline Dextrose .............. & 255 & 161 \\
\hline Lean meat . . . . . . . . . . & 235 & \\
\hline Extracted meat ......... & 213 & \\
\hline Albumin ............... & $\ldots$ & 201 \\
\hline
\end{tabular}

It is evident that the chief point of difference is the relative value of fat and carbohydrates.

Experiments on Maintenance.-As regards the relative values of the several nutrients in a maintenance ration the above conclusions are in part based on theoretical considerations and in part are deductions from the experiments upon the influence of work on the respiratory quotient and upon the nature of the non-nitrogenous material metabolized which were considered in Chapter VI, pp. 211 to 225 . Contejean,$\dagger$ however, has made direct experiments upon the replacement values of fat and carbohydrates.

His experiments were made with dogs. In the first series the animal, weighing about $20 \mathrm{kgs}$., received a basal ration of $500 \mathrm{grams}$ of meat (1000 in the first period), estimated to be ample to maintain nitrogen equilibrium. To this were added in the several periods varying amounts of lard, sugar, and gelatin. The live weight of the animal was taken daily at the same hour and under uniform conditions, and the urinary nitrogen was determined. No mention is made of the fecal nitrogen. The total heat produc-

* Energetique musculaire, p. 101.

† Archives de Physiol., 1896, p. 803. 
tion for the four days of each experiment (excluding preliminary feeding) is computed from the proteid metabolism as measured by the urinary nitrogen, on the assumption that all the fat contained in the meat and all the non-nitrogenous nutrients added were metabolized. An exception is made in the fourth period, however, in which the author computes from a comparison of the gains of nitrogen and of live weight that there was a gain of about 50 grams (?) of fat by the animal. The results are contained in the first six columns of the following table:

\begin{tabular}{|c|c|c|c|c|c|c|c|c|}
\hline \multirow{2}{*}{$\begin{array}{l}\overrightarrow{0} \\
\text { 怘 } \\
\text { ه }\end{array}$} & \multirow{2}{*}{\multicolumn{3}{|c|}{ Food. }} & \multirow{2}{*}{$\begin{array}{c}\text { Gain or } \\
\text { Loss of } \\
\text { Weight, } \\
\text { Grms. }\end{array}$} & \multicolumn{2}{|c|}{ Gain or Loss of } & \multirow{2}{*}{$\begin{array}{c}\text { Esti- } \\
\text { mated } \\
\text { Heat } \\
\text { Pro- } \\
\text { duction, } \\
\text { Cals. }\end{array}$} & \multirow{2}{*}{$\begin{array}{c}\text { Cor- } \\
\text { rected } \\
\text { Heat } \\
\text { Pro- } \\
\text { duction. } \\
\text { Cals. }\end{array}$} \\
\hline & & & & & $\begin{array}{c}\text { Nitrogen, } \\
\text { Grms. }\end{array}$ & $\begin{array}{l}\text { Equivalent } \\
\text { Flesh, } \\
\text { Grmas. }\end{array}$ & & \\
\hline $\mathrm{I}$ & $1000 \mathrm{~g}$ & rms. & meat .... & -395 & +19.39 & +570 & 4548 & 6190 \\
\hline II \{ & $\begin{array}{r}500 \\
40\end{array}$ & & $\left.\begin{array}{c}\text { " } \ldots . . . \\
\text { lard } \ldots . .\}\end{array}\right\}$ & -170 & -1.86 & -55 & 3903 & 4981 \\
\hline III \{ & $\begin{array}{r}500 \\
80\end{array}$ & & $\left.\begin{array}{l}\text { meat.... } \\
\text { lard.... }\end{array}\right\}$ & +50 & +1.81 & +53 & 5326 & 5354 \\
\hline IV \{ & $\begin{array}{l}500 \\
100\end{array}$ & & $\left.\begin{array}{l}\operatorname{meat} . . . \\
\operatorname{lard} . . .\end{array}\right\}$ & $+335(?)$ & +6.36 & +187 (?) & 5486 & 4566 \\
\hline $\mathrm{V}\{$ & $\begin{array}{l}500 \\
100\end{array}$ & $" 6$ & $\left.\begin{array}{l}\text { meat. . . . } \\
\text { sugar ... }\end{array}\right\}$ & +152 & +6.04 & +170 & 3811 & 3980 \\
\hline VI \{ & $\begin{array}{l}500 \\
100\end{array}$ & $" 6$ & $\left.\begin{array}{l}\text { meat.... } \\
\text { gelatin .. }\end{array}\right\}$ & -105 & -1.10 & -32 & 4088 & 4773 \\
\hline
\end{tabular}

Making the comparison of fat and carbohydrates, as the essential point, it would appear from Contejean's results that 100 grams of sugar was fully as efficient as 80 grams of fat, while according to Rubner's figures about 180 grams of sugar would be required. Corresponding to this is the lower computed heat production in the sugar period, the excess in the fat periods being ascribed to the cleavage of fat believed to occur in the liver.

If, however, there is justification for computing the gain of fat by the body in the fourth period by subtracting the gain of flesh from the total gain in weight, the same method is equally applicable to the other periods. By its use the figures of the last column of the table have been computed by the writer. While the heat production in the sugar period as thus estimated is still below that of the fat periods, the rather wide range in the results of the latter serves to illustrate the uncertainties of such computation. 
In a second series of experiments a ration of 150 grams of meat and 100 grams of lard appeared to be equivalent to one of 300 grams of meat and 50 of lard. In a third series, fat, sugar, and gelatin were each given for two days to a fasting dog, the live weight * and urinary nitrogen being determined daily. The results were as follows:

\begin{tabular}{|c|c|c|c|}
\hline Date. & Live Weight, Kgs. & Food. & $\begin{array}{c}\text { Urinary } \\
\text { Nitrogen, } \\
\text { Grms. }\end{array}$ \\
\hline 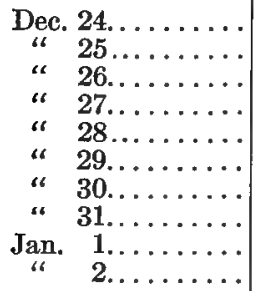 & $\begin{array}{l}25.780 \\
25.125 \\
24.765 \\
24.780+.095 \text { feces } \\
24.616+.064 \text { " } \\
24.215 \\
23.920 \\
23.870+.038 \text { " } \\
23.500 \\
23.200\end{array}$ & $\begin{array}{l}\text { Nothing } \\
\text { " } \\
200 \text { grms. sugar } \\
200 \text { " } \\
\text { Nothing } \\
200 \text { grms. fat } \\
200 \text { " } \\
\text { Nothing } \\
200 \text { grms. gelatin }\end{array}$ & $\begin{array}{r}5.56 \\
6.05 \\
5.59 \\
4.13 \\
4.59 \\
6.56 \\
6.85 \\
4.97 \\
28.77\end{array}$ \\
\hline
\end{tabular}

Neglecting the variations in the urinary nitrogen, Contejean makes the following comparison of the daily loss of live weight, from which he draws the conclusion that 200 grams of sugar, equivalent to 792 Cals., was more efficient in maintaining the animal than 200 grams of fat, equivalent to 1876 Cals.

\section{Gain or Loss of Live Weight per Day.}

Average for fasting........

-377 grams

Sugar :

First day.......... +110 grams

Second day......... -100 "

$$
\text { Average ......... }+5 \text { " }
$$

Fat:

First day......... -295 grams

Second day.........- 12 "

Average -154 “

* In taking the live weight any feces voided during the previous twentyfour hours were added to the weight of the animal, so that the computed gain or loss of weight does not include the feces. 
Experiments in which Work was Done.-Somewhat earlier in point of time than the above experiments by Contejean were similar ones by Chauveau* in which the animal performed a uniform (unmeasured) amount of work per day. No attempt was made to determine the equivalence between food or body metabolism and the work performed, but the latter was simply used as a means of increasing the metabolism, while the relative value of the several nutrients in maintaining the store of energy in the body was estimated from the effect upon the live weight. The experiments, therefore, are not, properly speaking, work experiments, but belong in the same category as those of Contejean-that is, they aim to show in what proportions the nutrients may replace each other in a maintenance ration.

In the first series the basal ration consisted of 400 grams of lean meat, to which was added in alternate six- or five-day periods either 51 grams of lard or an isodynamic quantity (121 grams) of cane-sugar. In one period 128.5 grams of dextrose was used instead of the cane-sugar. The animal (bitch) averaged about 16.8 kgs. in weight. The gain or loss of weight in each period (difference between first and last weighings) was as follows:

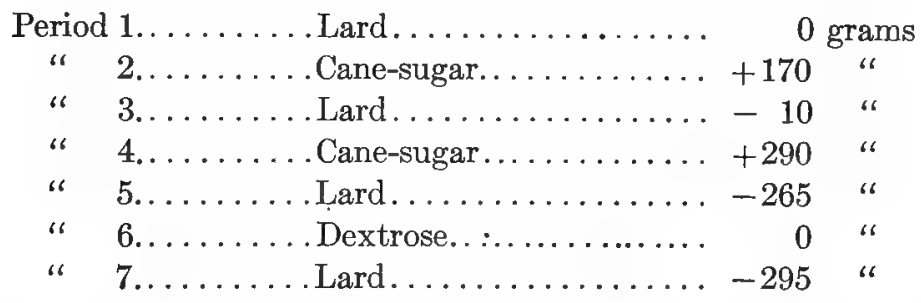

In the first four periods the cane-sugar seems to have caused a gain in weight as compared with practical maintenance on the lard. During the last three periods the animal was in heat and a loss of weight upon the lard ration resulted, which was arrested on the dextrose ration. Water was given ad libitum for several hours after the work, but withdrawn at least twelve hours before weighing. No record is given of the amount of it consumed or of the water content of the materials fed.

In another experiment, in which twice as much work was done, * Comptes rend., 125, 1070; 126, 795, 930, 1072. 
fat and cane-sugar replaced each other in isoglycosic proportions, viz., 110 grams of fat and 168 of cane-sugar. In this case the amount of water consumed was uniform, viz., 400 grams. The gain or loss of live weight in five-day periods was:

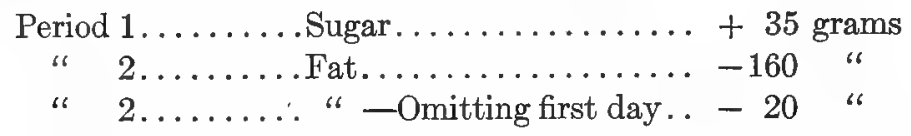

A third experiment, in which amounts of sugar intermediate between the isoglycosic and isodynamic equivalents of the fat were fed, showed a gain on the former as compared with practically no change on the fat.

In a second series of experiments isoglycosic amounts of lard (110 grams) and cane-sugar (168 grams) were alternated every five or three days for eighty-five days, the basal ration consisting of 500 grams of lean meat, and 400 grams of water being consumed per day. The estimated heat values of these rations were respectively 1513 Cals. and 1145 Cals., but notwithstanding this difference they appeared to be equally efficient in maintaining the live weight.

Whatever weight may attach to the deductions from the experiments upon work production, it is hardly necessary to urge that such a method of investigation as that employed in the above trials, while it may afford useful indications, is altogether too crude to disprove the theory of isodynamic values based upon Rubner's more elaborate experiments.

Respiration Experiments.-Kaufmann * has also reported respiration experiments in support of the views regarding the intermediary metabolism promulgated by Chauveau. In his experiments the nitrogen excretion, respiratory exchange, and heat production of dogs variously fed were determined, in five-hour periods, by means of a radiation calorimeter in which the products of respiration were allowed to accumulate. (See pp.69 and 248.) From the theoretical equations given in Chapter II he computes the figures given on the opposite page for the consumption of oxygen, production of carbon dioxide, and heat evolution in the various reactions.

Besides determinations of the fasting metabolism the experiments included feeding exclusively with meat and also with rations rich in carbohydrates and in fat. For each diet, on the basis of the * Archives de Physiol., 1896, pp. 329, 342, and 757. 


\begin{tabular}{|c|c|c|c|c|}
\hline & \multicolumn{3}{|c|}{ Per Grm. of Substance. } & \multirow{2}{*}{$\begin{array}{c}\text { Heat } \\
\text { per Liter } \\
\text { of Oxygen } \\
\text { Con- } \\
\text { sumed, } \\
\text { Cals. }\end{array}$} \\
\hline & $\begin{array}{l}\text { Oxygen } \\
\text { Con- } \\
\text { sumed, } \\
\text { Liters. }\end{array}$ & $\begin{array}{c}\text { Carbon } \\
\text { Dioxide } \\
\text { Produced, } \\
\text { Liters. }\end{array}$ & $\begin{array}{c}\text { Heat } \\
\text { Evolved, } \\
\text { Cals. }\end{array}$ & \\
\hline Albumin to fat and urea..... & 0.481 & 0.4777 & 2.234 & 4.646 \\
\hline " "dextrose and urea. & 0.713 & 0.5480 & 3.180 & 4.460 \\
\hline " $" \mathrm{CO}_{2}, \mathrm{H}_{2} \mathrm{O}$ " $" \quad \ldots \ldots$ & 1.045 & 0.8720 & 4.857 & 4.647 \\
\hline Stearin " " " " dextrose .. & 0.840 & 0.2257 & 3.417 & 4.067 \\
\hline$" \quad "$ " and $\mathrm{H}_{2} \mathrm{O} \ldots \ldots \ldots \ldots$ & 2.043 & 1.4290 & 9.500 & 4.650 \\
\hline Dextrose " " " " $" \ldots \ldots \ldots .$. & 0.744 & 0.7440 & 3.762 & 5.056 \\
\hline
\end{tabular}

determination of the respiratory products, the author assumes a scheme of metabolism in accordance with the theory, and finds that the heat production as computed on this assumption agrees quite closely with that actually determined.

Aside from questions of method, particularly whether a fivehour period is sufficiently long, it is to be remarked that the results of Kaufmann's experiments are ambiguous. They show that it is possible to interpret the facts in accordance with his theory, but they do not exclude the possibility of other explanations. For this reason it seems unnecessary to cite the experiments in detail, and for the same reason they are at best but confirmatory evidence in favor of the theory of isoglycosic values.

\section{$\S$ 2. Modified Conception of Replacement Values.}

The theory of isodynamic replacement as announced by Rubner constituted the first systematic application of the general laws of energy to the problems of animal nutrition. As such it has exerted a profound influence upon subsequent study of the subject in that it has been chiefly instrumental in leading to a practical application of the long-known fact that the food is primarily a supply of energy. It was based, of course, upon the conception that the law of the conservation of energy obtains in the animal body, and in subsequent experiments, which have been described in Chapter IX, Rubner gave at least a partial demonstration of the truth of this conception.

Rubner's general ideas still form the basis of our views regard- 
ing the metabolism of energy in the body, but, as was natural, his first conclusions have undergone more or less modification, in part at his own hands.

Digestive WorK.-The law of isodynamic replacement as stated above is equivalent to saying either that all the metabolizable energy of the food below a maintenance ration is net available energy or that the percentage availability of all the nutrients experimented with is the same. The latter supposition, however, appears to be negatived by the results of Magnus-Levy and others on digestive work.

If, however, a fraction of the metabolizable energy of the food is applied to the work of digestion and assimilation, it is plain that this fraction cannot serve directly for tissue building. In his first paper, Rubner, while not denying the fact of the consumption of energy in digestive work, appears to regard its amount as insignificant, although what he specifically claims is that the total metabolism below the maintenance ration is not increased by the ingestion of food. In support of this view he gives the results of three experiments in which fat was fed; that is, the nutrient which, according to Magnus-Levy's later results, causes the least digestive work. Of these, one on a dog, in which approximately a maintenance ration was given, showed no increase of the metabolism over the fasting state. In the other two experiments, one on a dog and one on a rabbit, more fat was consumed than corresponded to the fasting metabolism, and an increase of the latter was observed amounting to approximately 3 per cent. and 12 per cent. respectively. Feeding with bone also caused an increase of about 12 per cent.

In a later publication,* however, he recognizes the apparent inconsistency between the effects of small and large amounts of food, and propounds a hypothesis to explain it which, in its general features at least, seems in harmony with the observed facts. This hypothesis is outlined in the following paragraphs, although in a slightly different manner than by Rubner.

Indirect Utilization of Heat Resulting from Digestive Work.-In Chapter XI we acquired the conception of the critical thermal environment. According to the ideas there advanced, * Biologische Gesetze, Marburg, 1887, p. 20. 
the heat production of a quiescent, fasting animal below the critical point is made up of-

1. The heat produced by the internal work.

2. The heat produced by the processes of "chemical" regulation.

The first of these we may regard as substantially constant, while the latter varies to meet varying conditions and thus maintain the constancy of body temperature. When we give food to such an animal we introduce a third source of heat, viz., the work of digestion and assimilation. Other conditions remaining the same, the tendency would be to raise the temperature of the body, and this tendency can be overcome either by means of "chemical " or "physical" regulation. Recurring to the illustration of the room on p. 356, it is as if a second fire were kindled in it. To maintain constant temperature, either the first fire must be lowered or the windows must be opened.

The fact, however, that below the critical point the heat regulation of the body appears to be largely "chemical" renders it probable that the regulation is effected by the former method; that is, that the heat produced by the work of digestion is utilized to warm the body and that correspondingly less energy is withdrawn from that stored in the tissues of the body.* Under these circumstances the total heat production of the animal would not be increased by the ingestion of food, and all the metabolizable energy of the food would be apparently available; that is, we should have the phenomenon of isodynamic replacement.

Digestive Work Above Critical Pornt.-The statements of the last paragraph refer to conditions below the critical point. Above this point no such indirect utilization of the heat resulting from digestive work is possible, since the heat production has already been reduced to the minimum due, as was concluded on p. 356, to internal work. The excess of heat arising from the work of digestion is then disposed of by "physical" means.

Thus Rubner $\dagger$ obtained the following results for the carbon

* Loewy (Arch. ges. Physiol., 46, 189; quoted by Magnus-Levy, ibid., p. 116) claims to have shown that such a substitution or compensation does not take place in man.

$\dagger$ Biologische Gesetze, pp. 17-25. 
dioxide produced per square meter by guinea-pigs at $0^{\circ} \mathrm{C}$. and at $30^{\circ} \mathrm{C}$. (critical temperature), when fasting and after the consumption of food ad libitum.

PER SQUARE METER OF SURFACE.

\begin{tabular}{|c|c|c|c|c|c|}
\hline \multicolumn{3}{|c|}{ Fasting.* } & \multicolumn{3}{|c|}{ Fed. } \\
\hline $\begin{array}{c}\text { Live Weight, } \\
\text { Grms. }\end{array}$ & $\begin{array}{l}\text { At } 0^{\circ} \mathrm{C} . \\
\mathrm{CO}_{2}, \text { Grms. }\end{array}$ & $\begin{array}{l}\text { At } 30^{\circ} \mathrm{C} \text {. } \\
\mathrm{CO}_{2} \text {, Grms. }\end{array}$ & $\begin{array}{l}\text { Live Weight, } \\
\text { Grms. }\end{array}$ & $\begin{array}{l}\mathrm{At} 0^{\circ} \mathrm{C} . \\
\mathrm{CO}_{2}, \mathrm{Grms}^{2}\end{array}$ & $\begin{array}{l}\text { At } 30^{\circ} \mathrm{C} \\
\mathrm{CO}_{2} \text {, Grms. }\end{array}$ \\
\hline $\begin{array}{l}617 \\
568 \\
223 \\
206\end{array}$ & $\begin{array}{l}27.85 \\
30.30 \\
30.47 \\
31.56\end{array}$ & $\begin{array}{l}12.35 \\
10.53 \\
12.14 \\
13.16\end{array}$ & $\begin{array}{l}670 \\
520 \\
240 \\
220\end{array}$ & $\begin{array}{l}29.49 \\
29.08 \\
34.07 \\
30.59\end{array}$ & $\begin{array}{l}14.10 \\
16.19 \\
17.69 \\
18.94\end{array}$ \\
\hline Average.: & 30.05 & 12.05 & Average. & 30.81 & 16.73 \\
\hline
\end{tabular}

* Already cited on p. 366.

Comparing the averages we see that at $0^{\circ} \mathrm{C}$., considerably below the critical point, the consumption of food did not materially increase the total metabolism per unit of surface. On the other hand, at a temperature close to the critical point the average heat production was increased nearly 39 per cent. by the consumption of food.

It appears also that at this higher temperature the heat production of the fed animals was no longer proportional to their surface, but was relatively greater in the smaller animals. Rubner explains this by the supposition that (the animals being fed ad libitum) the consumption of food by the animals was in proportion to their fasting metabolism; that is, to their surface. Under these circumstances the factor of surface enters twice, and the heat production is approximately proportional to the square of the surface.

Rubner * has also made calorimetric determinations of the heat production of a dog at different temperatures with the results shown on the opposite page. Not only did the feeding increase the heat production, but it eliminated the effect of rising temperature in diminishing it; that is, it lowered the critical temperature.

Critical Amount of Food.-The very probable hypothesis of a substitution of the heat produced by the work of digestion for that

* Sitzungsber. der k. bayer. Akad. d. Wiss., Math.-phys. Classe, 15, 452. 


\begin{tabular}{|c|c|c|c|}
\hline \multicolumn{2}{|c|}{ Fasting. } & \multicolumn{2}{c|}{ Fed Small Amount of Meat. } \\
\cline { 1 - 2 } $\begin{array}{c}\text { Temperature, } \\
\text { Deg. C. }\end{array}$ & $\begin{array}{c}\text { Heat } \\
\text { Production, } \\
\text { Cals. }\end{array}$ & $\begin{array}{c}\text { Temperature, } \\
\text { Deg. C. }\end{array}$ & $\begin{array}{c}\text { Heat } \\
\text { Production, } \\
\text { Cals. }\end{array}$ \\
\hline 13.2 & 39.65 & 19.5 & 42.64 \\
19.5 & 35.10 & 18.2 & 41.13 \\
27.4 & 30.82 & 23.7 & 41.83 \\
& & 24.8 & 41.10 \\
\hline
\end{tabular}

arising, below the critical point, from the "chemical" regulation of the body temperature affords a very reasonable explanation of the apparent discrepancy between the law of isodynamic replacement as propounded by Rubner and the no less certain fact that the work of digestion and assimilation makes a demand on the body for energy, which energy finally takes the form of heat and is not available for other purposes.

A consequence of this hypothesis, however, which is sufficiently obvious has indeed been pointed out, but hardly seems to have received the attention which it deserves in view of its important bearing on the theoretical aspects of metabolism.

If we give increasing amounts of food to a fasting animal we progressively increase the evolution of heat due to digestive work, and this heat, according to the hypothesis, if the thermal environment is below the critical point, is substituted for the heat previously produced by the metabolism of tissue. There must be a limit to the possibility of this substitution, however, just as there must be to the "chemical" regulation of body temperature (p. 353), since otherwise there would be a ration on which all the heat of the body was derived from the work of digestion and the internal work was performed without evolution of heat. The limit is indeed the same in both cases and is reached when all the heat previously evolved by the processes of "chemical" regulation has been replaced by the heat arising from digestive work. Beyond that point the conditions are the same as in the fasting animal above the critical point, and the excess of heat is gotten rid of by "physical" regulation. We may call the amount of food whose ingestion produces the quantity of heat necessary to just reach this limit the critical amount of food. Below that amount the apparent 
availability of the metabolizable energy of the food will be 100 per cent. or we shall have isodynamic replacement. Above that amount we shall have an availability depending upon the relation of the work of digestion and assimilation to the total metabolizable energy.

Graphic Representation.-The critical amount of food will 'depend chiefly upon two things, viz., the distance below the critical thermal environment at which the experiment is made and the amount of energy that has to be expended in the digestion and assimilation of the food: The greater the former quantity, the more of the total metabolism of the animal will be due to the "chemical" regulation and therefore capable of being replaced, while the greater the work of digestion the less food must be consumed to furnish by its digestive work the heat necessary to a complete substitution.

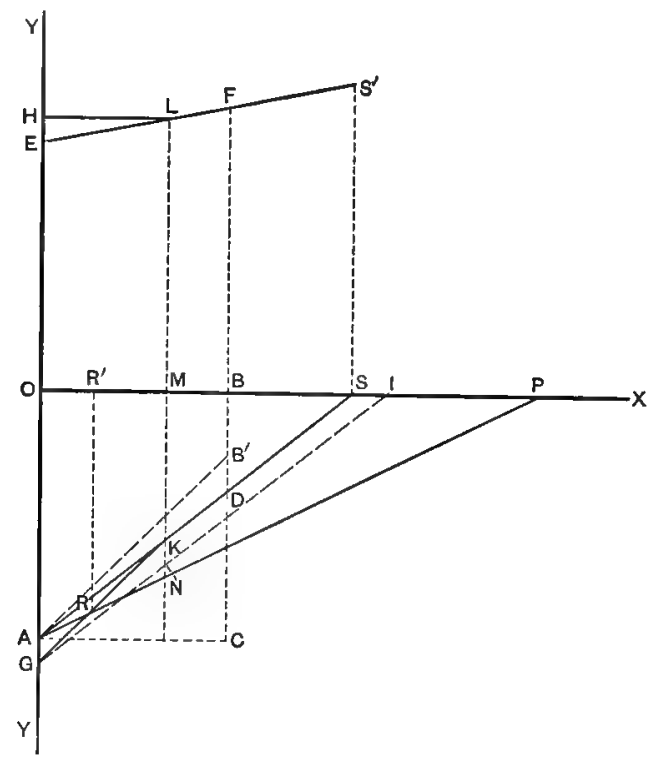

On the two coördinate axes $O X$ and $O Y$ let distances along $O X$ represent the metabolizable energy of the food consumed and distances along $O Y$ the effect of this food upon the store of potential energy in the body. In the first instance, let us take the case of a 
fasting animal and suppose the thermal environment to be at the critical point. The distance $O A$ may then represent the loss of potential energy (tissue) from the body caused by the internal work. If now we supply the animal with food 80 per cent. of whose metabolizable energy is available, with any given amount of energy thus supplied, as $O B=A C, 80$ per cent. of that energy, represented by $C D$, will serve to maintain the store of potential energy in the body, while 20 per cent., or $D B^{\prime}$, will be absorbed by the work of digestion, etc., and converted into heat. Accordingly if we assume that the work of digestion is proportional to the amount of food eaten, the line $A D$ will indicate the availability of the particular food and may be represented algebraically by the equation

$$
y=a x,
$$

in which $a=\tan D A C=$ the percentage availability.

We may also represent the heat production on the same axes. With no food it will be $O E$ equal to $O A$. With an amount of food equal to $O B$ it will be equal to $O E+D B^{\prime}=B F$, and the line $E F$, expressed algebraically by

$$
y=(1-a) x
$$

will represent the law of heat production.

let us next suppose that, the animal being again deprived of food, the external demand for heat is increased, by a fall of temperature, e.g., and that to meet this demand the metabolism is increased by an amount $A G$, and the heat production consequently by the equal amount $E H$. If we now give the same food as before, its real availability will be unchanged and will be represented by the line GI, parallel to $A D$. Up to the critical amount of food, however, the heat resulting from the digestive work will, as we believe, be substituted progressively for that represented by $E H$ and resulting from the metabolism $A G$. The apparent availability, therefore, will be represented by the line $G K$, making an angle of $45^{\circ}$ with the axes, and the heat production by the line $H L$, parallel to $O X$. When the food consumed reaches an amount $O M$ at which the line $G K$ intersects $A D$, the limit of this substitution is reached, since the amount of digestive work, $K N$, equals the amount of additional metabolism $A G$ caused by the fall in temperature. In other words, 
$O M$ is the critical amount of food. Beyond this amount the energy expended in the work of digestion will become waste energy, serving simply to increase the outflow of heat, and the apparent and real availability of the food will coincide.

Plainly, the critical amount of food will vary with circumstances. If the experiment is made at or above the critical thermal environment for the fasting animal the smallest quantity must cause an increase in the heat production and the critical amount will be 0 (or, mathematically, a negative quantity). As the external conditions fall below the critical thermal environment, the point $K$ will be further and further removed from $A$ until finally the point of intersection might even lie above $O X$, that is, above the maintenance ration. The relative availability of the food, too, will be a factor in determining the critical amount. Thus if the true availability of the food were expressed by the line $A P$ instead of $A D$, the point of intersection would lie at $R$ and $O R^{\prime}$ would be the critical amount of food.

\section{§ 3. Net Availability.}

The modified conception of replacement values discussed in the preceding section and in the introductory paragraphs of this chapter renders it evident that both the theory of isodynamic replacement, as first announced and later modified by Rubner, and the rival theory of isoglycosic replacement are but aspects of the more general question of the availability of the metabolizable energy of the food. That the several nutrients are of use to the body and can replace each other in the food in inverse ratio to their available energy is simply a necessary consequence of the law of the conservation of energy. The important question is how much of their energy is really available. Rubner's theory regards all the metabolizable energy of the food as virtually available, directly or indirectly, for maintenance, and this view has been quite generally accepted. Chauveau's theory of isoglycosic replacement has the merit of distinctly recognizing the fact of a possible expenditure of energy in the assimilation of the digested food, but, on the other hand, it takes no account of the digestive work, and moreover, so far as maintenance values are concerned, rests, as we have seen, upon a rather insecure foundation. Plainly, the real question at 
issue can only be settled by experiments in which the actual availability of the energy of the food or of its various ingredients is determined.

\section{Determinations of Net Availability.}

Since the net available energy of the food is equal to its metabolizable energy minus the energy expended in digestion and assimilation, the two general methods for the determination of the latter quantity which were outlined in the preceding chapter (p. 377) are also, from the converse point of view, methods for the determination of net availability. In our study of digestive work we considered chiefly the results of direct determinations of the increase in the heat production due to the ingestion of food; for our present purpose the results of any accurate determinations of the metabolism upon varying known amounts of the same food may be used.

The experimental evidence available is far from being as full as could be wished, but in the following paragraphs the attempt has been made to summarize such data as are accessible. In considering these results it should be remembered that, as explained on p. 396, the net available energy means the energy available for maintenance. In a considerable number of the experiments to be considered, more or less gain was made by the animals, but it seems better to give the results of each series of experiments in full, reserving a discussion of the results with productive rations for a subsequent chapter.

Experiments on Carnivora.-The most extensive data regarding the metabolism of the carnivora in its relations to the food supply are those afforded by the investigations of Pettenkofer \& Voit and of Rubner. These have already been considered in Chapter $\mathrm{V}$ from the standpoint of matter and chiefly in a qualitative way; we have now to study them quantitatively in their bearing upon the income and expenditure of energy by the body.

In Pettenkofer \& Voit's experiments; and in the earlier ones by Rubner, the quantities of energy involved must be computed from the chemical data. In Rubier's experiments upon the source of animal heat, cited in Chapter IX, the actual heat production of the animals was determined, but in no case was there a direct determination of the total income and expenditure of energy, and in particular the data as to the energy of the food are incomplete. For 
the study of replacement values by Rubner's method the latter factor was not necessary, but for a determination of the percentage availability of the energy of the food it is indispensable. In the following paragraphs the necessary computations of energy have been made by the writer, using Rubner's factors so far as possible.*

In the case of Pettenkofer \& Voit's experiments the average results given in Chapter $\mathrm{V}$ have been made the basis of the computation.

Proteids.-From the average results obtained by Pettenkofer $\&$ Voit $\uparrow$ with different amounts of lean meat (see p. 104), the metabolizable energy of the food and of the resulting gain (or loss) by the body may be computed as follows:

\begin{tabular}{|c|c|c|c|c|c|}
\hline \multirow{2}{*}{$\begin{array}{l}\text { Food, } \\
\text { Grms. }\end{array}$} & \multirow{2}{*}{$\begin{array}{c}\text { Metabolizable } \\
\text { Energy of } \\
\text { Food, } \\
\text { Cals. }\end{array}$} & \multicolumn{3}{|c|}{ Computed Heat Production. } & \multirow{2}{*}{$\begin{array}{c}\text { Gain by } \\
\text { Body, } \\
\text { Cals. }\end{array}$} \\
\hline & & $\begin{array}{l}\text { From } \\
\text { Proteids, } \\
\text { Cals. }\end{array}$ & $\begin{array}{l}\text { From Fat, } \\
\text { Cals. }\end{array}$ & $\begin{array}{l}\text { Total, } \\
\text { Cals. }\end{array}$ & \\
\hline $\begin{array}{r}0 \\
500 \\
1000 \\
1500\end{array}$ & $\begin{array}{r}0 \\
442 \\
883 \\
1325\end{array}$ & $\begin{array}{r}146 \\
530 \\
954 \\
1325\end{array}$ & $\begin{array}{r}895 \\
443 \\
179 \\
-38\end{array}$ & $\begin{array}{r}1041 \\
973 \\
1133 \\
1287\end{array}$ & $\begin{array}{r}-1041 \\
-531 \\
-250 \\
+38\end{array}$ \\
\hline
\end{tabular}

* The following factors were used in computing these experiments:

Metabolizable Energy of Food:

Bacon (Speck), 92.2 per cent. fat (Zeit. f. Biol., 30, 138).

$1 \mathrm{grm}$. pork fat, 9.423 Cals. (itid., 21, 333).

$1 \mathrm{grm}$. butter fat, 9.216 Cals. (U.S. Dept. Agr., Office of Expt. Stations, Bull. 21, p. 127).

1 grm. cane-sugar, 4.001 Cals. (Zeit. f. Biol., 21, 266).

1 grm. grape-sugar, 3.692 Cals. (Stohmann, Zeit. f. Biol., 22, 40).

1 grm. starch, 4.123 Cals. (Stohmann, ibid., 19, 376).

Fresh lean meat, 3.4 per cent. nitrogen.

$1 \mathrm{grm}$. nitrogen in meat, 25.98 Cals. (Zeit. f. Biol., 21, 321).

$1 \mathrm{grm}$. nitrogen in syntonin, 26.66 Cals. (ibid., 21, 309).

Energy of Metabolism :

$1 \mathrm{grm}$. excretory nitrogen (urine and feces).

(a) No proteids fed:

Birds, 24.35 Cals. (Zeit. f. Biol., 19, 367).

Mammals, 24.94 Cals. (ibid., 22, 43).

(b) Meat fed, 25.98 Cals. (Ibid.).

(c) Syntonin fed, 26.66 Cals. (ibid.).

$1 \mathrm{grm}$. carbon in fat, 12.31 Cals. (ibid.).

† Zeit. f. Biol., 7, 489. 
As compared with the fasting state, the 883 Cals. of metabolizable energy supplied, for example, in 1000 grams of meat diminished the loss of energy by the body by $1041-250=791$ Cals. The latter quantity, then, represents the extent to which the 883 Cals. supplied in the food aided in maintaining the stock of potential energy in the body, while the remaining 92 Cals. was consumed in the work of digestion and assimilation as defined on previous pages; that is, it increased by this amount the heat production of the animal. Accordingly we compute that in this case 89.6 per cent. of the metabolizable energy of the meat was available, while the digestive work consumed 10.4 per cent. Computing the other experiments in the same way we have-

\begin{tabular}{|c|c|c|}
\hline $\begin{array}{c}\text { Metabolizable } \\
\text { Energy of } \\
\text { Food, Cals. }\end{array}$ & $\begin{array}{c}\text { Gain Over } \\
\text { Fasting } \\
\text { Metabolism, } \\
\text { Cals. }\end{array}$ & $\begin{array}{c}\text { Net Avail- } \\
\text { ability, } \\
\text { Per Cent. }\end{array}$ \\
\hline 442 & 510 & 115.4 \\
883 & 791 & 89.6 \\
1325 & 1079 & 81.5 \\
\hline
\end{tabular}

From Rubner's experiments * with proteids (see p. 106) the following figures are computed in the same manner as those above:

\begin{tabular}{|c|c|c|c|c|c|c|c|}
\hline & \multirow[b]{2}{*}{$\begin{array}{l}\text { Food, } \\
\text { Grms. }\end{array}$} & \multirow{2}{*}{$\begin{array}{l}\text { Metab- } \\
\text { olizable } \\
\text { Energy } \\
\text { of Food, } \\
\text { Cals. }\end{array}$} & \multirow{2}{*}{$\begin{array}{l}\text { Heat } \\
\text { Produe- } \\
\text { tion, } \\
\text { Cals. }\end{array}$} & \multicolumn{2}{|c|}{ Gain. } & \multirow{2}{*}{$\begin{array}{c}\text { Net } \\
\text { Avail- } \\
\text { ability. } \\
\text { Per } \\
\text { Cent. }\end{array}$} & \multirow{2}{*}{$\begin{array}{l}\text { Tem- } \\
\text { perature } \\
\text { Deg. C. }\end{array}$} \\
\hline & & & & $\begin{array}{l}\text { Total, } \\
\text { Cals. }\end{array}$ & $\begin{array}{c}\text { Over } \\
\text { Fasting } \\
\text { Metab- } \\
\text { olism, } \\
\text { Cals. }\end{array}$ & & \\
\hline \multirow[t]{3}{*}{ Meat....... } & $\begin{array}{r}0 \\
415\end{array}$ & $\begin{array}{r}0 \\
367\end{array}$ & $\begin{array}{l}573^{*} \\
596^{*}\end{array}$ & $\begin{array}{l}-573 \\
-229\end{array}$ & 344 & 93.74 & 19.2 \\
\hline & 0 & 0 & $793^{*}$ & -793 & & & 18.0 \\
\hline & 740 & 654 & $825^{*}$ & -171 & 622 & 95.15 & 19.2 \\
\hline \multirow{2}{*}{ Extracted meat } & 0 & 0 & $931^{*}$ & -931 & & & 14.9 \\
\hline & $\begin{array}{r}740 \\
0\end{array}$ & $\begin{array}{r}939 \\
0\end{array}$ & $\begin{array}{l}959^{*} \\
261 \dagger\end{array}$ & $\begin{array}{l}-20 \\
-261\end{array}$ & 911 & 97.03 & 15.6 \\
\hline \multirow[t]{2}{*}{ Meat. } & 390 & 347 & $334 \dagger$ & +13 & 274 & 78.98 & \\
\hline & 350 & 309 & $379 \dagger$ & -70 & 191 & 61.80 & \\
\hline \multirow[t]{2}{*}{ " } & 0 & 0 & $528 \dagger$ & -528 & & & \\
\hline & 580 & 512 & $681 \dagger$ & -169 & 359 & 70.12 & \\
\hline
\end{tabular}

* Computed.

$\dagger$ Calorimetric determinaulon.

* Zeit. f. Biol., 22, 43-48; 30, 117-135. 
To the above results we may add those of Magnus-Levy's determinations (p. 381) of the work of digestion and assimilation in the dog on a meat diet as follows:

\begin{tabular}{c|c|c|c|c}
\hline $\begin{array}{c}\text { Proteids Eaten, } \\
\text { Grms. }\end{array}$ & $\begin{array}{c}\text { Metabolizable } \\
\text { Energy of } \\
\text { Food, } \\
\text { Cals. }\end{array}$ & $\begin{array}{c}\text { Expended in } \\
\text { Digestion and } \\
\text { Assimilation, } \\
\text { Cals. }\end{array}$ & \multicolumn{2}{|c}{ Net Available. } \\
\cline { 2 - 4 } & 33.5 & 56 & 282 & Total, \\
Cals. & Per Cent. \\
\hline 230.0 & 943 & 116 & 827 & 83.43 \\
$370.6 *$ & 1520 & 244 & 1276 & 83.95 \\
\hline
\end{tabular}

* In excess of maintenance requirements.

The wide range of the results obtained by Rubner would seem to indicate either that the net availability of the energy of the proteids may vary with different animals and under different conditions or that the experimental methods were not sufficiently sharp for the purpose now in view. The value of an average drawn from such results is questionable, but for the sake of comparison it is included below along with those derived from Voit's and Magnus-Levy's experiments, Voit's first result being omitted because impossible. The figures express the average net availability as a percentage of the metabolizable energy.

Voit's experiments ........... 85.60 per cent.
Rubner's experiments ......... 82.80 " "
Magnus-Levy's experiments...... 85.03 " “

FAT.-Computing the results obtained by Pettenkofer \& Voit* and by Rubner $\dagger$ upon the effects of fat on the total metabolism (see pp. 144-146) in the same manner as those upon the proteids, and adding Magnus-Levy's results (p. 379), we have the table opposite.

Rubner's and Magnus-Levy's results do not differ widely, and their average, 96.4 per cent., indicates a relatively small expenditure of energy in the digestion and assimilation of fat, which does not appear to materially increase above the maintenance requirement. Most of Pettenkofer \& Voit's experiments give materially lower results above that point, and the one case in which the food

$$
\begin{aligned}
& \text { * Zeit. f. Biol., 5, 370; 7, 440-443; 9, 3-13. } \\
& \text { † Ibid., 19, 328-334; 30, } 123 .
\end{aligned}
$$




\begin{tabular}{|c|c|c|c|c|}
\hline \multirow[b]{2}{*}{ Food. } & \multirow[b]{2}{*}{$\begin{array}{c}\text { Metab- } \\
\text { olizable } \\
\text { Energy } \\
\text { of Food, } \\
\text { Cals. } \\
\text {. }\end{array}$} & \multicolumn{2}{|c|}{ Gain. } & \multirow[b]{2}{*}{$\begin{array}{c}\text { Net } \\
\text { Avail- } \\
\text { ability, } \\
\text { Per Cent. }\end{array}$} \\
\hline & & $\begin{array}{l}\text { Total, } \\
\text { Cals. }\end{array}$ & $\begin{array}{c}\text { Over } \\
\text { Fasting } \\
\text { Metab- } \\
\text { olism or } \\
\text { Basal } \\
\text { Ration, } \\
\text { Cals. }\end{array}$ & \\
\hline 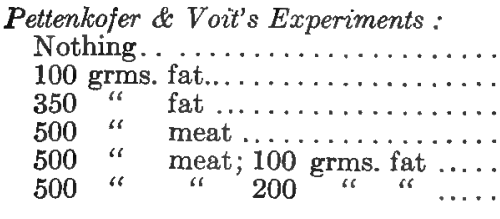 & $\begin{array}{r}0 \\
942 \\
3298 \\
442 \\
1384 \\
2326\end{array}$ & $\begin{array}{r}-1086 \\
-275 \\
+878 \\
-554 \\
+329 \\
+837\end{array}$ & $\begin{array}{r}811 \\
1964 \\
883 \\
1391\end{array}$ & $\begin{array}{l}86.1 \\
59.6 \\
93.7 \\
73.8\end{array}$ \\
\hline 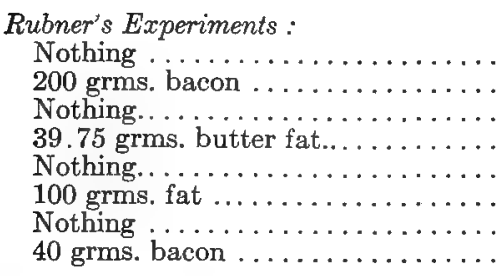 & $\begin{array}{r}0 \\
1738 \\
0 \\
356 \\
0 \\
942 \\
0 \\
348\end{array}$ & $\begin{array}{r}-658 \\
+1016 \\
-373 \\
-17 \\
-466 \\
+428 \\
-261 \\
+49\end{array}$ & $\begin{array}{r}1674 \\
356\end{array}$ & $\begin{array}{r}98.6 \\
100.0 \\
94.9\end{array}$ \\
\hline 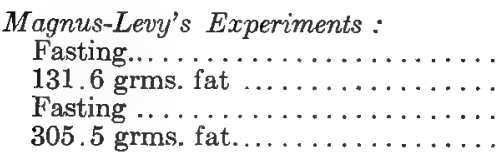 & $\begin{array}{r}0 \\
1250 \\
0 \\
2902\end{array}$ & $\begin{array}{r}-972 \\
+259 \\
-1055 \\
+1760\end{array}$ & 1231 & 98.5 \\
\hline
\end{tabular}

supply was below the amount required for maintenance also gives a rather low availability as compared $\mathrm{w}$ th that obtained by the other experimenters.

CARbohydrates.- Tabulating as in the previous cases the results of Pettenkofer \& Voit* and of Rubner $\dagger$ (see pp. 146-152), and adding those of Magnus-Levy (p. 380), we have the figures shown on the next page.

As was the case with fat, most of Pettenkofer \& Voit's experiments give figures notably lower than those obtained by the other two investigators. The averages of the latter, omitting the figures which exceed 100 per cent., are:

Rubner's experiments ........ 88.9 per cent.

Magnus-Levy's experiments ....... 91.0 " "

* Zeit. f. Biol., 9, $485 . \quad$ † Tbid., 19, 357-379; 22, 273. 


\begin{tabular}{|c|c|c|c|c|}
\hline \multirow[b]{2}{*}{ Food. } & \multirow[b]{2}{*}{$\begin{array}{l}\text { Metab- } \\
\text { olizable } \\
\text { Energy } \\
\text { of Food, } \\
\text { Cals. }\end{array}$} & \multicolumn{2}{|c|}{ Gain. } & \multirow[b]{2}{*}{$\begin{array}{l}\text { Net } \\
\text { Avail- } \\
\text { ability, } \\
\text { Per Cent. }\end{array}$} \\
\hline & & $\begin{array}{l}\text { Total, } \\
\text { Cals. }\end{array}$ & $\begin{array}{l}\text { Over } \\
\text { Fasting } \\
\text { Metab- } \\
\text { olism or } \\
\text { Basal } \\
\text { Ration, } \\
\text { Cals. }\end{array}$ & \\
\hline 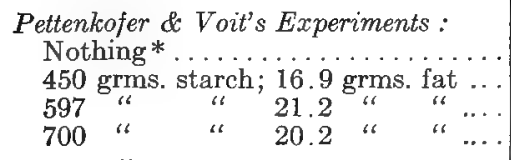 & $\begin{array}{r}0 \\
2015 \\
2661 \\
3076\end{array}$ & $\begin{array}{r}-1098 \\
+353 \\
-198 \\
+853\end{array}$ & $\begin{array}{r}1451 \\
900 \\
1951\end{array}$ & $\begin{array}{l}72.0 \\
33.8 \\
63.4\end{array}$ \\
\hline $\begin{array}{l}500 \text { " meat } \ldots \ldots \ldots \ldots \ldots \ldots \\
500 \text { " meat; } 200 \text { grms. starch } \\
500 \text { grms. meat; } 200 \text { grms. dextrose }\end{array}$ & $\begin{array}{r}442 \\
1316 \\
1180\end{array}$ & $\begin{array}{r}-554 \\
+137 \\
+108\end{array}$ & $\begin{array}{l}691 \\
662\end{array}$ & $\begin{array}{l}79.1 \\
89.7\end{array}$ \\
\hline 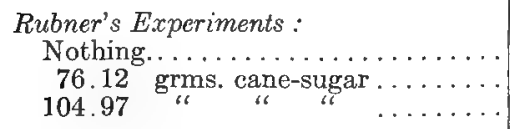 & $\begin{array}{r}0 \\
305 \\
420\end{array}$ & $\begin{array}{r}-436 \\
-116 \\
-22\end{array}$ & $\begin{array}{l}320 \\
414\end{array}$ & $\begin{array}{r}104.9 \\
98.6\end{array}$ \\
\hline 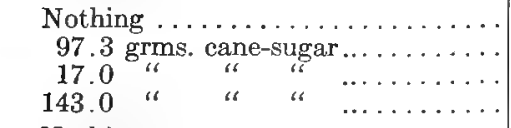 & $\begin{array}{r}0 \\
389 \\
68 \\
572\end{array}$ & $\begin{array}{l}-451 \\
-87 \\
-374 \\
+190 \dagger\end{array}$ & $\begin{array}{r}364 \\
77 \\
641\end{array}$ & $\begin{array}{r}93.6 \\
113.2 \\
112.0\end{array}$ \\
\hline $\begin{array}{l}\text { Nothing } \ldots \ldots \ldots \ldots \\
42.96 \text { grms. starch (digested) . . . . . }\end{array}$ & $\begin{array}{r}0 \\
177\end{array}$ & $\begin{array}{l}-302 \\
-138\end{array}$ & 164 & 92.6 \\
\hline 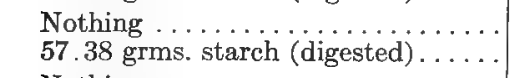 & $\begin{array}{r}0 \\
244\end{array}$ & $\begin{array}{l}-354 \\
-140\end{array}$ & 214 & 87.8 \\
\hline 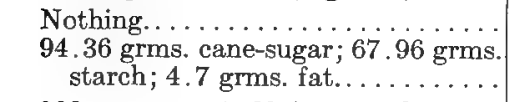 & $\begin{array}{r}0 \\
702\end{array}$ & $\begin{array}{l}-302 \\
+365 \dagger\end{array}$ & 667 & 95.0 \\
\hline 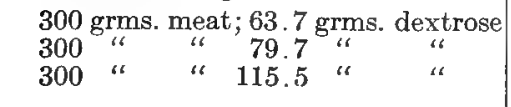 & $\begin{array}{l}500 \\
559 \\
691\end{array}$ & $\begin{array}{r}-126 \\
-84 \\
+34\end{array}$ & $\begin{array}{r}42 \\
160\end{array}$ & $\begin{array}{l}71.1 \ddagger \\
83.7 \ddagger\end{array}$ \\
\hline $\begin{array}{l}\text { Magnus-Levy's Experiments: } \\
\text { Chiefly rice............ }\end{array}$ & $\begin{array}{r}2121 \\
2226 \\
999\end{array}$ & $\begin{array}{r}+850 \\
+934 \\
-81\end{array}$ & $\begin{array}{r}1890 \\
2066 \\
910\end{array}$ & $\begin{array}{l}89.1 \\
92.8 \\
91.1\end{array}$ \\
\hline
\end{tabular}

* Fasting metabolism estimated from previous experiments.

$\uparrow$ Gain of carbon assumed to be all in the form of fat.

$\ddagger$ Of dextrose added.

Experiments on Herbivora.-Comparatively few experiments have been reported from which the net availability of the food of herbivorous animals can be computed, and as regards the common farm animals in particular there is an almost entire lack of data, although numerous experiments upon the relative value of various 
materials for productive feeding have been reported and will be considered in the following chapter.

FAT.-Rubner's experiments include one * in which fat was fed to a rabbit with the following results:

\begin{tabular}{|c|c|c|}
\hline & Fasting. & $\begin{array}{c}\text { Fed } 26.1 \text { Grms. } \\
\text { Bacon. }\end{array}$ \\
\hline 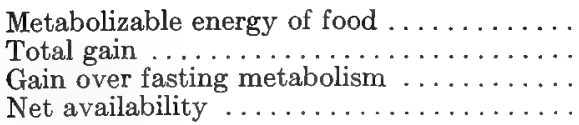 & $\begin{array}{l}0 \quad \text { Cals. } \\
-101 \\
\ldots \ldots \ldots \\
\ldots \ldots \ldots\end{array}$ & $\begin{array}{r}227 \text { Cals. } \\
+122 \text { “ } \\
223 \text { “ } \\
98.2 \%\end{array}$ \\
\hline
\end{tabular}

In connection with his investigations upon cellulose, v. Knieriem $\dagger$ also experimented upon the influence of fat on the metabolism of the rabbit. The basal ration consisted of milk, to which was added in the second period 3.94 grams of dry butter fat per day. Computing the amounts of energy by the use of Rubner's factors the results were:

\begin{tabular}{|c|c|c|c|}
\hline & $\begin{array}{l}\text { Metabolizable } \\
\text { Energy, Cals. }\end{array}$ & Gain, Cals. & $\begin{array}{l}\text { Net Availability, } \\
\text { Per Cent. }\end{array}$ \\
\hline 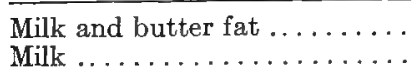 & $\begin{array}{l}207.3 \\
169.8\end{array}$ & $\begin{array}{l}-19.5 \\
-55.2\end{array}$ & \\
\hline Difference... & 37.5 & 35.7 & 95.2 \\
\hline
\end{tabular}

Carbohydrates.-Rubner $\neq$ reports three experiments with cane-sugar on a cock from which the following results are computed:

\begin{tabular}{|c|c|c|c|c|}
\hline \multirow{2}{*}{ Food. } & \multirow{2}{*}{$\begin{array}{l}\text { Metaboliz- } \\
\text { able Energy } \\
\text { of Food, } \\
\text { Cals. }\end{array}$} & \multicolumn{2}{|c|}{ Gain. } & \multirow{2}{*}{$\begin{array}{c}\text { Net } \\
\text { Availability } \\
\text { Per Cent. }\end{array}$} \\
\hline & & $\begin{array}{l}\text { Total, } \\
\text { Cals. }\end{array}$ & $\begin{array}{l}\text { Over Fast- } \\
\text { ing Metab- } \\
\text { olism, Cals. }\end{array}$ & \\
\hline $\begin{array}{l}\text { Nothing } \ldots \ldots \ldots \ldots \ldots \ldots \\
34 \text { grms. cane-sugar } \ldots \ldots \ldots \\
\text { Nothing } \ldots \ldots \ldots \ldots \ldots \\
45 \text { grms. cane-sugar } \ldots \ldots \ldots \\
50 \text { " }{ }^{\prime \prime}{ }^{\prime} \ldots \ldots\end{array}$ & $\begin{array}{r}0 \\
136 \\
0 \\
180 \\
200\end{array}$ & $\begin{array}{l}-239 \\
-121 \\
-258 \\
-101 \\
-\quad 53\end{array}$ & $\begin{array}{l}157 \\
205\end{array}$ & $\begin{array}{r}86.8 \\
87.2 \\
102.5\end{array}$ \\
\hline
\end{tabular}

* Zeit. f. Biol., 19, $333 . \quad$ †Ibid., 21, $119 . \quad \ddagger$ Ibid., 19, 366. 
From the comparisons of cellulose and cane-sugar made by $\mathrm{v}$. Knieriem (loc. cit.) and cited on p. 161, the following figures for the net availability of the energy of the latter substance may be computed:

\begin{tabular}{|c|c|c|c|c|c|c|}
\hline \multirow{2}{*}{$\begin{array}{l}\text { 䓪 } \\
\text { 貟 } \\
\text { 畜 }\end{array}$} & \multirow{2}{*}{ 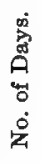 } & \multirow[b]{2}{*}{ Food per Day. } & \multirow{2}{*}{$\begin{array}{c}\text { Metab- } \\
\text { olizable } \\
\text { Energy } \\
\text { of Food, } \\
\text { Cals. }\end{array}$} & \multicolumn{2}{|c|}{ Gain. } & \multirow{2}{*}{$\begin{array}{l}\text { Net } \\
\text { Avail- } \\
\text { ability, } \\
\text { Per Cent. }\end{array}$} \\
\hline & & & & $\begin{array}{l}\text { Total, } \\
\text { Cals. }\end{array}$ & $\begin{array}{l}\text { Over } \\
\text { Basal } \\
\text { Ration, } \\
\text { Cals. }\end{array}$ & \\
\hline $\begin{array}{l}\text { III } \\
\text { IV } \\
\text { V }\end{array}$ & $\begin{array}{l}5 \\
4 \\
3\end{array}$ & 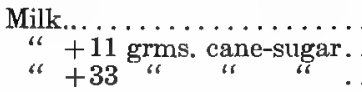 & $\begin{array}{l}350.1 \\
393.7 \\
480.7\end{array}$ & $\begin{array}{l}-37.9 \\
-15.9 \\
+69.9\end{array}$ & $\begin{array}{r}22.0 \\
107.8\end{array}$ & $\begin{array}{l}50.5 \\
82.5\end{array}$ \\
\hline
\end{tabular}

A series of experiments by May* upon the effect of fever on metabolism affords incidentally a few data bearing on the availability of the energy of dextrose. In his experiment No. 5 (loc. cit., p. 23) the ingestion of 30 grams of grape-sugar, an amount approximately equivalent to the fasting metabolism, caused no increase in the computed heat production as compared with that during fasting. In this experiment there was no fever. In Experiment No. 6 (p. 25), with fever, the ingestion of the same amount of grape-sugar produced a computed gain of 2.88 grams carbon as fat, but caused no increase in the computed heat production. Experiment No. 7 (p. 26) was similar to No.6, but showed a decrease in the computed heat production, which, however, coincided with a decrease in the fever. On the whole, May's results appear in accord with Rubner's hypothesis of a substitution of the heat resulting from digestive work for that arising from the metabolism of tissue.

Pentoses.-Cremer's experiments $\dagger$ with rhamnose upon rabbits, cited in Part I, p. 157, afford data for computing the net availability of this representative of the pentoses. For this purpose Cremer computes from the excretion of nitrogen and carbon (neglecting the feces), in the manner described in Chapter VIII, p. 253, the amount of energy liberated by the metabolism of protein and fat in the body, assuming that the rhamnose, after deducting the small amounts in feces and urine, was completely oxidized. The following are the results for each day of the four experiments:

$$
\text { *Zeit. f. Biol., 30, } 1 . \quad \dagger I b i d ., 42,451 .
$$




\begin{tabular}{|c|c|c|}
\hline Food, Grms. & $\begin{array}{l}\text { Metabolizable } \\
\text { Energy of Food, } \\
\text { Cals. }\end{array}$ & $\begin{array}{c}\text { Loss by Body, } \\
\text { Cals. }\end{array}$ \\
\hline $\begin{array}{l}\text { Experiment } 1 . \\
\quad \text { Nothing } \ldots \ldots . \ldots \ldots \ldots \\
\quad \text { Rhamnose, } 11.584 \text { grms.... } \\
\quad \text { Nothing } \ldots \ldots \ldots \ldots \ldots \ldots\end{array}$ & $\begin{array}{c}0 \\
45.3 \\
0\end{array}$ & $\begin{array}{l}147.4 \\
114.0 \\
113.3\end{array}$ \\
\hline 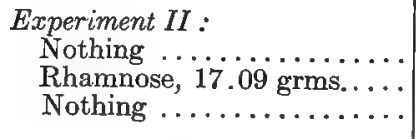 & $\begin{array}{c}0 \\
66.8 \\
0\end{array}$ & $\begin{array}{l}180.7 \\
111.6 \\
184.8\end{array}$ \\
\hline 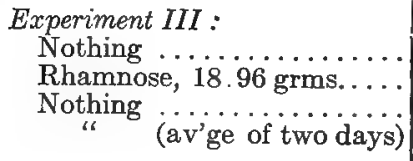 & $\begin{array}{c}0 \\
74.1 \\
0 \\
0\end{array}$ & $\begin{array}{r}129.1 \\
54.3 \\
113.1 \\
113.4\end{array}$ \\
\hline 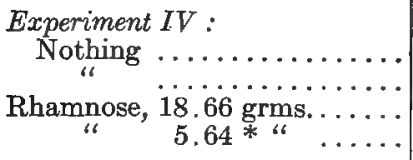 & $\begin{array}{c}0 \\
0 \\
72.9 \\
22.0\end{array}$ & $\begin{array}{r}146.0 \\
141.4 \\
53.3 \\
98.1\end{array}$ \\
\hline
\end{tabular}

* The total amount of rhamnose (24.3 grms.) was given on the first day, but it is estimated from the results for the carbon excretion that this amount of it was not metabolized until the second day.

The results as to net availability obtained by comparison with the several fasting days vary considerably, as the following statement shows, several of them exceeding 100 per cent.:

\section{Experiment $I$.}

Compared with first day............ 74 per cent. " " third day............. Negative

Experiment $I I$.

Compared with first day ........... 103 per cent. " " third day............. 110 " "

Experiment III.

Eompared with first day............ 101 per cent. " " third day ............. 79 " "

Second and third with fourth and fifth days. 80 " "

\section{Experiment IV.}

Third compared with second day. ....... 121 per cent. " " " fourth day ........ 88 " " 
The great variations in the results, as well as the large proportion of cases in which the availability appears to exceed 100 per cent., show that little value attaches to them as quantitative determinations, although they undoubtedly show that rhamnose possesses a comparatively high nutritive value.

Crude Fiber.-The experiments of $\mathrm{v}$. Knieriem have already been cited in Chapter $\mathrm{V}$ in their general bearings upon the metabolism of matter. As was there noted, certain corrections were necessary on account of the residue of undigested cellulose remaining in the digestive canal at the close of the experiment. The results given below are based on those computed by the author, as summarized on p. 161, on the assumption that the resorption of the remaining digestible crude fiber.was complete after two days.

\begin{tabular}{|c|c|c|c|c|c|c|}
\hline \multirow[b]{2}{*}{ 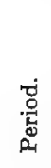 } & \multirow{2}{*}{ 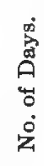 } & \multirow{2}{*}{ Food per Day. } & \multirow{2}{*}{$\begin{array}{c}\text { Metab- } \\
\text { olizable } \\
\text { Energy } \\
\text { of Food, } \\
\text { Cals. }\end{array}$} & \multicolumn{2}{|c|}{ Gain. } & \multirow{2}{*}{$\begin{array}{c}\text { Net } \\
\text { Avail- } \\
\text { ability, } \\
\text { Per Cent }\end{array}$} \\
\hline & & & & $\begin{array}{l}\text { Total, } \\
\text { Cals. }\end{array}$ & $\begin{array}{c}\text { Over } \\
\text { Basal } \\
\text { Ration, } \\
\text { Cals. }\end{array}$ & \\
\hline I & 9 & Milk $\ldots \ldots \ldots \ldots \ldots \ldots \ldots$ & 341.7 & -46.8 & & \\
\hline II & 10 & $\left.\begin{array}{c}+22 \text { grms. crude fiber } \\
\text { for eight days.... }\end{array}\right\}$ & 374.6 & -6.9\{ & $\begin{array}{l}39.9 \\
31.0\end{array}$ & $\begin{array}{l}121.3 * \\
126.5 \dagger\end{array}$ \\
\hline III & 5 & Milk................... & 350.1 & -37.9 & $\ldots$ & $\ldots$ \\
\hline
\end{tabular}

* Compared with Period I.

$\dagger$ Compared with Period III.

It is evident from the above figures that while the experiments show qualitatively a nutritive value for the cellulose, they are insufficient for a quantitative determination of its amount.

In striking contrast with these results are the conclusions drawn by Zuntz \& Hagemann from their experiments upon the horse which have already been considered in the previous chapter ( $\mathrm{pp}$. 389-391). As was there explained in detail, these investigators have estimated the expenditure of energy in the digestion of crude fiber from a comparison of the computed heat production in two sets of experiments in which the proportion of coarse fodder eaten differed considerably, it being assumed that 9 per cent. of the metabolizable energy of the nutrients other than crude fiber was consumed in their digestion. On this basis the digestive work caused by the crude fiber is computed at 2.086 Cals. per gram, or rather more than 
its average metabolizable energy. In other words, it is computed that under the conditions of these experiments, with a ration more than sufficient for maintenance, the net availability of the energy of the crude fiber was practically zero. The authors report no experiments upon rations below the maintenance requirement, but appear to regard the metabolizable energy of the crude fiber as being indirectly available, under such conditions, substantially in the manner assumed by Rubner and already explained.

As has been noted, Zuntz \& Hagemann's conclusions as to the value of crude fiber for work production are in apparent harmony with those of Wolff, which will be discussed in the next chapter, but on the other hand they contrast sharply with the results of Kellner (see Chapter XIII, \&1), who observed a high percentage utilization of the energy of one form of crude fiber in the ration of fattening cattle. On previous pages some reasons were presented for questioning the quantitative accuracy of Zuntz \& Hagemann's computations, but even aside from these their conclusions as regards the value of crude fiber are difficult to reconcile with obvious facts. Thus they compute (loc. cit., p. 280) that the expenditure of energy in the mastication and digestion of average straw is greater than its metabolizable energy, so that for the horse this material has a negative value. When forming part of a maintenance ration we miy probably assume that below the critical amount of food (p. 408) the heat generated during the digestion of the straw would be of use to maintain the body temperature, but this could not possibly suspend the expenditure of energy in the various forms of internal work, such as respiration and circulation. Since, however, by hypothesis, the straw can contribute no energy directly for these purposes, it follows that the consumption of this material alone cannot rerluce the loss of tissue below the amount requisite to supply energy for the internal work, while on an exclusive straw ration above the critical amount of food the more straw the animal consumed the sooner it would starve.

ORGANIC AcIDs.-The results of a considerable number of experiments in which salts of organic acids were injected into the blood have already been presented in Chapter V (p. 157). The general result was that lactic and butyric acids caused little or no increase in the heat production of the animal-in other words 
that practically all their potential energy was available to prevent loss of tissue. In such experiments, of course, there is no digestive work in the proper sense. What they indicate is that what we have called rather loosely the work of assimilation for these substances is practically zero. Acetic acid, on the other hand, was found by Mallèvre to increase the consumption of oxygen by from 10 to 17 per cent., indicating a considerable waste of energy directly or indirectly. The general nature of these experiments is not such, however, as to afford data of much direct value in relation to the question of the availability of the energy of ordinary foods.

Tiмотну Hay.-The experiments described in the foregoing paragraphs relate to pure or nearly pure nutrients. Experiments upon a steer have been made by the writer in conjunction with Fries in which the availability of the apparent metabolizable energy of timothy hay has been determined. To a basal ration considerably below the maintenance requirement, consisting of 3250 grams of hay and 400 grams of linseed meal, three different additions of timothy hay were made, the digestibility of the ration in each period being determined, and likewise the total balance of nitrogen, carbon, and energy by means of the respiration-calorimeter. The results as to energy, uncorrected for the very small differences in the organic matter of the basal ration consumed and for the changes in the live weight of the animal, were as follows:*

\begin{tabular}{|c|c|c|c|c|c|c|c|c|}
\hline & \multicolumn{2}{|c|}{ Period A. } & \multicolumn{2}{|c|}{ Period B. } & \multicolumn{2}{|c|}{ Period C. } & \multicolumn{2}{|c|}{ Period D. } \\
\hline & $\begin{array}{l}\text { Outgo, } \\
\text { Cals. }\end{array}$ & $\begin{array}{c}\text { Income, } \\
\text { Cals. }\end{array}$ & $\begin{array}{l}\text { Outgo, } \\
\text { Cals. }\end{array}$ & $\begin{array}{c}\text { Income, } \\
\text { Cals. }\end{array}$ & $\begin{array}{l}\text { Outgo, } \\
\text { Cals. }\end{array}$ & $\begin{array}{c}\text { Income, } \\
\text { Cals. }\end{array}$ & $\begin{array}{l}\text { Outgo, } \\
\text { Cals. }\end{array}$ & $\begin{array}{l}\text { Income, } \\
\text { Cals. }\end{array}$ \\
\hline $\begin{array}{l}\text { Feed ........... } \\
\text { Excreta: } \\
\text { Feces........... } \\
\text { Urine........ } \\
\text { Methane..... } \\
\text { Metabolizable ... }\end{array}$ & $\begin{array}{r}6,446 \\
863 \\
996 \\
6,618\end{array}$ & $\mid \begin{array}{l}\ldots \ldots \\
\cdots \cdots \\
\cdots \cdots \\
\cdots \cdots \\
\end{array}$ & $\begin{array}{r}8,590 \\
974 \\
1,251 \\
9,482\end{array}$ & $20,297 \mid$ & $\mid \begin{array}{r}\cdots \\
11,477 \\
1,125 \\
1,374 \\
11,222\end{array}$ & 25,198 & $\begin{array}{r}14,276 \\
1,220 \\
1,896 \\
12,255\end{array}$ & 29,647 \\
\hline $\begin{array}{l}\text { Metabolizable ... } \\
\text { Heat produced ... } \\
\text { Gain or loss..... }\end{array}$ & $\begin{array}{c}14,923 \\
\ldots \ldots \\
9,067 \\
\ldots \ldots\end{array}$ & $\begin{array}{r}14,923 \\
6,618 \\
\cdots 2,449\end{array}$ & $\begin{array}{l}20,297 \\
10,206 \\
\ldots \ldots\end{array}$ & $\mid \begin{array}{r}20,297 \\
9,482 \\
\cdots \\
724\end{array}$ & $\begin{array}{r}10,606 \\
616\end{array}$ & \begin{tabular}{|c|}
25,198 \\
11,222 \\
$\ldots \ldots$ \\
$\ldots \ldots$
\end{tabular} & $\begin{array}{r}11,183 \\
1,072\end{array}$ & $\begin{array}{l}29,647 \\
12,255\end{array}$ \\
\hline & 9,067 & 9,067 & 10,206 & 10,206 & 11,222 & 11,222 & 12,255 & 12,255 \\
\hline
\end{tabular}

* Proc. Soc. Prom. Agr. Sci., 1902. 
Subtracting the results on the basal ration of Pcriod A from those of the other periods, as in previous cases, we have the following:

\begin{tabular}{|c|c|c|c|}
\hline & $\begin{array}{c}\text { Metabolizable } \\
\text { Energy, } \\
\text { Cals. }\end{array}$ & $\begin{array}{l}\text { Gain of Tissue, } \\
\text { Cals. }\end{array}$ & $\begin{array}{l}\text { Net Availability, } \\
\text { Per Cent. }\end{array}$ \\
\hline \multirow[t]{2}{*}{ 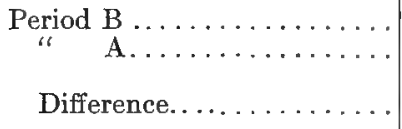 } & $\begin{array}{l}9,482 \\
6,618\end{array}$ & $\begin{array}{r}-724 \\
-2, \pm 49\end{array}$ & \multirow{3}{*}{60.24} \\
\hline & 2,864 & 1,725 & \\
\hline \multirow[t]{2}{*}{ Period $\begin{array}{c}\mathrm{C} \\
\mathrm{A} \ldots \ldots \ldots \ldots \ldots \ldots \ldots \ldots\end{array}$} & $\begin{array}{r}11,222 \\
6,618\end{array}$ & $\begin{array}{r}616 \\
-2,449\end{array}$ & \\
\hline & 4,604 & 3,065 & \multirow[t]{2}{*}{66.57} \\
\hline \multirow[t]{3}{*}{$\underset{*}{\operatorname{Period}} \mathrm{D} \ldots \ldots \ldots \ldots \ldots \ldots \ldots \ldots$} & $\begin{array}{r}12,255 \\
6,618\end{array}$ & $\begin{array}{r}1,072 \\
-2,249\end{array}$ & \\
\hline & 5,637 & 3,521 & 62.46 \\
\hline & & & 63.09 \\
\hline
\end{tabular}

Strictly speaking, only the first of the above percentages represents the net availability for maintenance, since the other two include some gain. From the difference observed between the metabolism of the animal standing and lying, however, it was computed approximately what the gains would have been had the same position been maintained for the whole twenty-four hours, with the following results:

\begin{tabular}{|c|c|c|c|}
\hline & $\begin{array}{l}\text { Metabolizable } \\
\text { Energy, Cals. }\end{array}$ & $\begin{array}{l}\text { Gain, Standing, } \\
\text { Cals. }\end{array}$ & $\begin{array}{l}\text { Net Availability, } \\
\text { Per Cent. }\end{array}$ \\
\hline \multirow{2}{*}{ 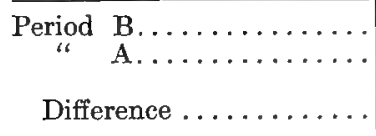 } & $\begin{array}{l}9,482 \\
6,618\end{array}$ & $\begin{array}{l}-1,606 \\
-3,507\end{array}$ & \multirow{3}{*}{66.37} \\
\hline & 2,864 & 1,901 & \\
\hline \multirow{2}{*}{ 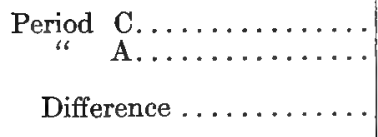 } & $\begin{array}{r}11,222 \\
6,618\end{array}$ & $\begin{array}{r}-550 \\
-3,507\end{array}$ & \\
\hline & 4,604 & 2,957 & \multirow[t]{2}{*}{64.23} \\
\hline \multirow{3}{*}{ 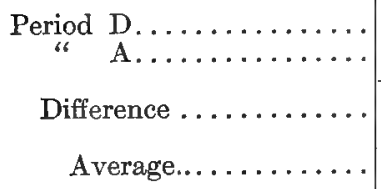 } & $\begin{array}{r}12,255 \\
6,618\end{array}$ & $\begin{array}{r}23 \\
-3,507\end{array}$ & \\
\hline & 5,637 & 3,530 & 62.62 \\
\hline & & $\ldots \ldots \ldots \ldots \ldots$ & 64.41 \\
\hline
\end{tabular}




\begin{tabular}{|c|c|c|c|}
\hline & $\begin{array}{l}\text { Metabolizable } \\
\text { Energy, Cals. }\end{array}$ & $\begin{array}{l}\text { Gain, Lying, } \\
\text { Cals. }\end{array}$ & $\begin{array}{l}\text { Net Availability. } \\
\text { Per Cent. }\end{array}$ \\
\hline Period $\underset{*}{\mathrm{~A}} \mathrm{~A} \ldots \ldots \ldots \ldots \ldots \ldots$ & $\begin{array}{l}9,482 \\
6,618\end{array}$ & $\begin{array}{r}1,157 \\
-1,046\end{array}$ & \multirow{3}{*}{76.92} \\
\hline Difference ........... & 2,864 & 2,203 & \\
\hline 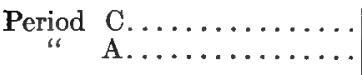 & $\begin{array}{r}11,222 \\
6,618\end{array}$ & $\begin{array}{r}2,136 \\
-1,046\end{array}$ & \\
\hline Difference........ & 4,604 & 3,182 & \multirow[t]{2}{*}{69.12} \\
\hline $\begin{array}{c}\text { Period } \\
\text { " } \\
\mathrm{A}\end{array} \ldots \ldots \ldots \ldots \ldots \ldots \ldots$ & $\begin{array}{r}12,255 \\
6,618\end{array}$ & $\begin{array}{r}2,743 \\
-1,046\end{array}$ & \\
\hline Difference....... & \multirow[t]{2}{*}{5,637} & \multirow[t]{2}{*}{3,789} & 67.22 \\
\hline Average.... & & & 71.08 \\
\hline
\end{tabular}

The results are likewise shown graphically on the accompanying diagram, in which the full line represents the average availability

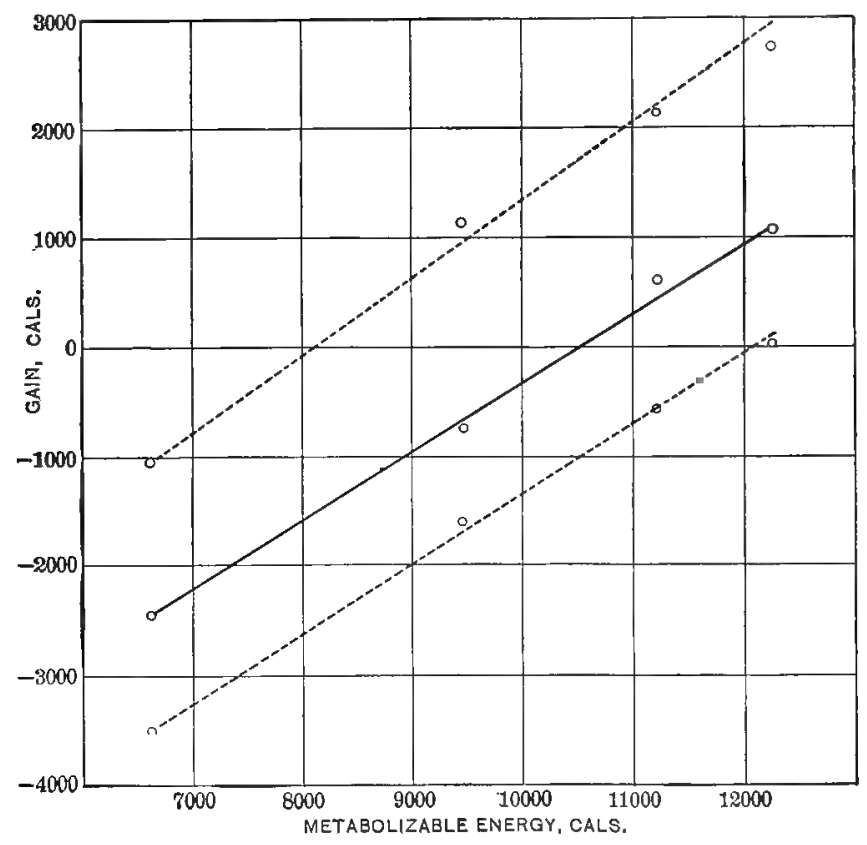


observed and the broken lines that computed respectively for standing and lying, while the points indicate the results for each period. As computed standing, the results are all practically at or below the maintenance point, and their fairly close agreement with each other and with those actually observed indicates that the net availability of the metabolizable energy of this sample of timothy hay was between 63 and 65 per cent.

Summary.-The foregoing data as to availability are summarized in the following table, those experiments in which the total ration was less than the maintenance requirement being separated from those in which more or less gain by the body took place:

Experiments on Carnivora. Below Maintenance.

\section{Proteids :}

Pettenkofer \& Voit . . . $\ldots\left\{\begin{array}{r}\text { Per Cent. } \\ 115.4 \\ 89.6 \\ 93.7 \\ 95.2 \\ 97.0 \\ 61.8 \\ 70.1\end{array}\right.$

Magnus-Levy . . . . . . . $\begin{aligned} & 83.4 \\ & 87.7\end{aligned}$

Fat :

Pettenkofer \& Voit ..... 86.1

Rubner............... 100.0

Starch :

Pettenkofer \& Voit....... 33.8

Rubner............. $\left\{\begin{array}{l}92.6 \\ 87.8\end{array}\right.$

Magnus-Levy (rice)...... 91.1

Dextrose :

Rubner............. 71.3

Cane-sugar:

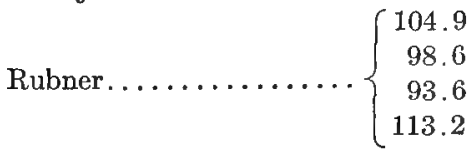

Starch and Cane-sugar :

Rubner.

95.0
Experiments on Herrivora. Below Maintenance.
Fat : $\quad$ Per Cent.

v. Knieriem............ 95.2

Cane-sugar:

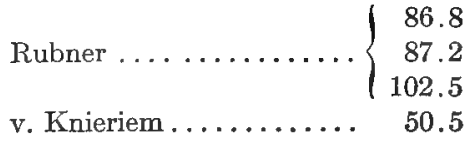




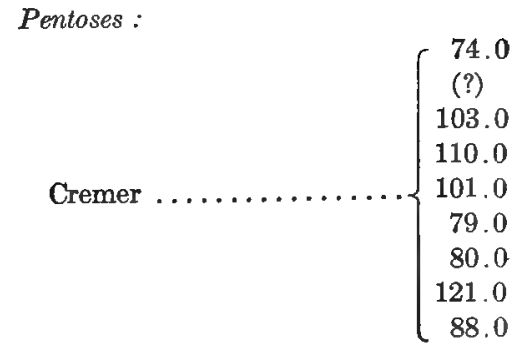

Crude Fiber:

v. Knieriem.......... $\left\{\begin{array}{l}121.3 \\ 126.5\end{array}\right.$

Timothy Hay:

Armsby \& Fries........ 63-65

Above Maintenance.

Proteids:

Pettenkofer \& Voit ...... 81.5

Rubner............... 79.0

Magnus-Levy ......... 84.0

Fat:

Pettenkofer \& Voit...... $\left\{\begin{array}{l}59.6 \\ 93.7 \\ 73.8\end{array}\right.$

Fat:

Per Cent.

Rubner............. 98.2

\section{Above Maintenance.}

Rubner.............. $\left\{\begin{array}{l}98.6 \\ 94.9 \\ 89.1\end{array}\right.$

Magnus-Levy ........ $\left\{\begin{array}{l}98.5 \\ 97.0\end{array}\right.$

Starch:

Pettenkofer \& Voit....... $\left\{\begin{array}{l}72.0 \\ 63.4 \\ 79.1\end{array}\right.$

Magnus-Levy (rice) ...... $\left\{\begin{array}{l}89.1 \\ 92.8\end{array}\right.$

Dextrose :

Pettenkofer \& Voit....... 89.7

Rubner............. 83.7

Cane-sugar :

Cane-sugar:

Rubner.............. 112.0

v. Knieriem........... 82.5

Cane-sugar and Starch:

Rubner............ 95.0 
It scarcely seems possible to draw any well-founded conclusions regarding the net availability of the several nutrients from such widely divergent results as those tabulated above, even if the extreme and obviously incorrect figures be discarded. Two things, however, seem worthy of remark.

First, in but few cases does the net availability of the food reach 100 per cent., and most of those results relate to cane-sugar or rhamnose; that is, to cases in which some of the gain of carbon which is computed as fat may have been in the form of a carbohydrate. It would seem fairly safe to conclude, therefore, that no such complete substitution of the heat resulting from digestive work for that resulting from the general metabolism took place as Rubner's hypothesis supposes. Apparently, under the conditions of these experiments, there was, in most cases at least, a material loss of energy in digestive work.

Second, there is no clear indication of a smaller loss of energy below than above the maintenance ration, although the wide range of the results renders a definite conclusion upon this point hazardous. This question, however, may be more properly considered in connection with a study of the utilization of the net available energy of the food.

Finally, it is to be said that if the validity of the conception of a critical amount of food, as developed on p. 409, be admittedthat is, of an amount of food below which the heat resulting from the work of digestion and assimilation is substituted for that produced by the general metabolism, while above it no such substtution takes place-a very important element is lacking for the interpretation of the above experiments, except, perhaps, those on timothy hay, in which the uniformity of the results with varying amounts seems to show clearly that all the rations supplied more than the critical amount of food. If that conception is correct, to determine the real availability of the energy of a food it is necessary to compare the effects of two quantities both of which are greater than the critical amount. On the other hand, the complete substitution of energy supposed by Rubner could only be demonstrated by comparing quantities less than the critical amount, while a comparison of quantities below the latter amount (including, of course, fasting) with those exceeding it 
can give only mixed results varying with the quantities compared.

It seems tolerably clear, then, that the whole subject of the net availability of foods and nutrients needs reinvestigation by more rigorous methods and with due regard to the amounts of the food materials compared and to the thermal environment of the animals experimented upon.

\section{Discussion of Results.}

For the reasons just stated, any strict quantitative discussion of the above results seems impossible. At the same time, certain general conclusions may be at least tentatively deduced from them which, even though to a considerable extent speculative, may at least serve provisionally as a connecting thread between the known facts.

Influence of Amount of Food on Availability.-In the foregoing paragraphs it has been tacitly assumed that the amount of food eaten has no influence on its availability, or, to state it in another way, that the expenditure of energy in digestion and assimilation is proportional to the quantity of food. To express the same thing in mathematical terms, we have assumed, in constructing the diagram on p. 410 , that the net available energy is a linear function of the metabolizable energy.

While it seems highly probable that such is the case the only experiments bearing specifically upon this question of which the writer is aware are those upon timothy hay just cited. An examination of the graphic representation of the results strongly supports the hypothesis that the net availability of the food is independent of its amount, but the evidence of so few experiments must naturally be accepted with some reserve. The other recorded results, as computed above, apart from the possible source of uncertainty pointed out on p. 429, show such considerable variations in individual cases that it scarcely seems possible to reach any definite conclusions from them regarding the influence of quantity of food. As will appear in the next chapter, the extensive respiration experiments made in recent years at the Möckern Experiment Station by G. Kühn and $O$. Kellner upon fattening cattle indicate that the 
actual gain obtained (expressed in terms of energy), at least within certain limits, is proportional to the amount of metabolizable energy supplied in excess of maintenance. This would mean that above the maintenance ration the energy required for digestion and assimilation plus that consumed in the chemical changes incident to the formation of new tissue (compare p. 396) is proportional to the amount of food. If this be true it seems more reasonable to conclude that each of these forms of work separately is proportional to the amount of food than to assume a compensation between the two, and granting this, we should have every reason to suppose that the same proportionality would hold good for the work of digestion and assimilation below the maintenance requirement.

Character of Food.-The investigations of Zuntz \& Hagemanm (pp. 385-393) have shown that, in the case of the horse at least and doubtless with other animals also, the work of digestion and assimilation varies with the kind of food, a result which is entirely in accordance with what we should expect. For reasons stated in describing their experiments, their results are to be regarded as qualitative rather than quantitative, but they suffice to demonstrate the very marked difference as regards availability which exists between the relatively pure nutrients employed in the experiments of Pettenkofer \& Voit, Magnus-Levy, Rubner, and others and the feeding-stuffs consumed by our herbivorous domestic animals, and to show the fallacy involved in applying the results of the former experiments directly to the latter case. The same conclusion is also indicated by the few results upon timothy hay on p. 424.

Unfortunately no other direct determinations of the availability of the food of herbivorous animals in amounts below the maintenance ration are on record, so that we are unable to compare either different feeding-stuffs or different species of animals in this respect. The extensive investigations of the Möckern Experiment Station mentioned in the previous paragraph show how large a proportion of the metabolizable energy of the food of fattening animals becomes economically waste energy, thus fully confirming the conclusions drawn from Zuntz \& Hagemann's experiments upon the horse, but they afford no means of distinguishing between the work of diges- 
tion and assimilation and the energy expended in converting the resorbed material into permanent tissue.

- The Maintenance Ration.-As already defined, net available energy is that portion of the metabolizable energy of the food which serves to make good the losses of potential energy arising from the internal work plus the work of digestion and assimilation, or, in other words, which contributes towards the maintenance of the stored-up capital of energy. We may, therefore, appropriately consider the bearings of the known facts regarding availability upon the amount of food required for maintenance.

Relations to Availability.-Not a little effort has been expended in determining the maintenance requirements of farm animals on the more or less tacit assumption that this quantity is a constant for the same animal, and the same assumption has even more largely controlled in computations based on the experimental data obtained.

By the maintenance ration, of course, we understand a ration just sufficient to prevent any loss of tissue-that is, of potential energy - by the animal. To accomplish this we must give a ration containing net available energy equal in amount to the potential energy lost when no food is given. Expressed thus in terms of net available energy, the maintenance requirement under given conditions is a constant and is equal to the energy of the fasting metabolism.

The maintenance requirement, however, particularly in the case of farm animals, has not usually been expressed thus, since the necessary data are lacking, but in terms of total digestible matter or of real or supposed metabolizable energy. When thus expressed, however, it is apparent in the light of the foregoing discussion that the maintenance requirement must be a variable, depending upon the availability of the metabolizable energy of the food. Referring again to the graphic representation on p. 410 , it is evident that, under the conditions there represented, with an availability expressed by $\tan D A C$, the amount of metabolizable energy required for maintenance will be equal to $O S$. Furthermore, it is equally evident that as the availability decreases and the angle $D A C$ consequently becomes more acute $O S$ will increase. Only when the critical amount of food, $O M$, is greater than the fasting metabolism 
and the point $K$ falls above the axis $O X$ will there be an apparent exception to this law. In that case, since the energy expended in digestion and assimilation seems to be indirectly utilized, the apparent availability will be 100 per cent. and the metabolizable energy required for maintenance will be constant and equal to the energy of the fasting metabolism.

This case might and perhaps does occur with animals whose food consumes little energy in digestion, such as the carnivora. As was pointed out on p. 412, however, an increase in the work of digestion tends to reduce the critical amount of food and there would appear to be good reason for believing that, in ruminants at least if not in the horse, it lies considerably below the point of maintenance.

Relative Value of Grain and Coarse Fodder.-We know from the investigations of Zuntz \& Hagemann (pp. 385-393) that the work expended in the digestion of coarse fodders is, in the horse and presumably therefore in other animals, materially greater than that caused by grain. It follows, then, that a unit of digestible matter or of metabolizable energy should have more value for maintenance in the latter than in the former.

That such is the case with cattle is rendered probable by experiments by the writer.* In the absence of a respiration apparatus the nutritive effect of the rations was judged of from the live weight and the proteid metabolism during relatively long periods and the methane production was computed from the carbohydrates digested. A ration in which only about 24 per cent. of the digested organic matter was derived from coarse fodder, as compared with rations consisting exclusively of coarse fodder, gave the following results for the metabolizable energy of the maintenance ration per day and $500 \mathrm{kgs}$. live weight:

Exclusive coarse fodder, 12 experiments... 12,771 Cals. Largely grain, 3 experiments. . . ....... 11,023 “

Such determinations of the maintenance requirements of the horse as have been made tend to confirm the results obtained with ruminants. Wolff, in his investigations upon work production described in the following chapter, has computed the maintenance requirements of the horse in the manner there explained both from

* Penna. Expt. Station, Bull. 42, p. 159. 
his own experiments and from those of Grandeau and LeClerc, with the following results per $500 \mathrm{kgs}$. live weight:

Total Digestible Nutrients, * Grms.

On hay alone .................. 4586

About equal parts hay and grain ......... 4190

About $\frac{3}{4}$ grain and $\frac{1}{4}$ hay (Grandeau) ....... 3626

Zuntz \& Hagemann, $\uparrow$ from the results of a respiration experiment with the horse, make a still lower estimate of the maintenance requirement, viz., 3265 grams total nutrients per $500 \mathrm{kgs}$. live weight on a ration of which about four sevenths was grain, but after allowing for the differences in crude fiber content compute a satisfactory agreement between their results and Wolff's. Since their estimate for the work of digestion of crude fiber is really based on the difference in digestive work required by coarse fodder and by grain this is equivalent to showing that the latter is more valuable for maintenance than the former.

On the other hand, Grandeau and LeClerc $\ddagger$ in later experiments on exclusive hay feeding found that the live weight was almost exactly maintained for a month on $8 \mathrm{kgs}$. of hay per day, the total digested nutrients being as follows:

\begin{tabular}{|c|c|c|c|c|}
\hline \multirow{2}{*}{\multicolumn{2}{|c|}{ Animal. }} & \multirow{2}{*}{$\begin{array}{l}\text { Live Weight, } \\
\text { Kgs. }\end{array}$} & \multicolumn{2}{|c|}{ Total Digestible Nutrients. } \\
\hline & & & $\begin{array}{l}\text { Per Head, } \\
\text { Grms. }\end{array}$ & $\begin{array}{c}\text { Per } 500 \text { Kgs., } \\
\text { Grms. }\end{array}$ \\
\hline $\begin{array}{c}\text { No. } \\
\text { " }\end{array}$ & $\begin{array}{l}1 \ldots \ldots \\
2 \ldots \ldots \\
3 \ldots \ldots\end{array}$ & $\begin{array}{l}395 \\
419 \\
413\end{array}$ & $\begin{array}{l}2892 \\
3036 \\
3058\end{array}$ & $\begin{array}{l}3660 \\
3622 \\
3701\end{array}$ \\
\hline
\end{tabular}

These figures do not materially exceed the average computed by Wolff from their previous experiments on heavy grain rations. The horses had a half-hour's walking exercise daily, so that the ration seems to have been amply sufficient for maintenance, and no reason for the divergent result is obvious.

While none of these comparisons have the conclusiveness of

* Including fat $\times 2.4$.

$\dagger$ Loc. cit., pp. 422-4.

$\$$ L'alimentation du Cheval du Trait, 3d memoir, pp. 23-31. 
complete metabolism experiments, their results as a whole indicate clearly that the metabolizable energy of the grains is more valuable for maintenance than that of the coarse fodders, a fact undoubtedly due to the greater expenditure of energy in the digestion and assimilation of the latter.

The maintenance ration of horses, cattle, and sheep, then, as ordinarily expressed (i.e., in units of digestible matter or of metabolizable energy) is not a constant but a variable, depending on the availability of the metabolizable energy, and such a statement of it, to be definite, must be accompanied by a statement of the kind of feed used.

No similar experiments upon swine appear to have been made. The ordinary feed of this animal, however, probably varies less in availability than that of ruminants, and it may be presumed that no such striking differences would be found.

Value of Crude Fiber.-As a result of Wolff's conclusions concerning the apparent worthlessness of crude fiber for work production, as discussed in the succeeding chapter, and of Zuntz \& Hagemann's estimates regarding its digestive work (p. 389), there has been a tendency to ascribe the difference between grain and coarse fodders to the greater amount of crude fiber in the latter, forgetting that what these investigators have actually shown is simply the lower value of the digestible matter from coarse fodders, and that their conclusions regarding crude fiber are deductions from the observed facts. Kellner's more recent experiments (see p. 182 and Chapter XIII, §1) have demonstrated that at least one form of crude fiber is nearly as efficient in producing a gain of fat by cattle as is starch. A fortiori, therefore, it should be equally valuable for maintenance. We have as yet no sufficient evidence to justify us in ascribing the difference between grain and coarse fodder to the crude fiber as such aside from its influence on the mechanical structure of the material.

Influence of Thermal Environment.-It has been not uncommonly assumed that the maintenance requirement of an animal is affected by changes in the temperature and other external factors which combine to determine the refrigerating effect of the environment; in other words, the heat production of the animal has been looked upon more or less distinctly as an end in itself. 
We have already seen reason to believe that this is the case to a very limited extent only, even in the fasting animal, and to a still less degree in one consuming food. If we are justified in thinking that the critical amount of food for herbivorous animals is ordinarity less than the maintenance requirement, it follows that the heat production on a maintenance ration is in excess of the actual needs of the organism for heat by an amount depending upon the availability of the metabolizable energy of the food, and that this excess of heat is disposed of by "physical" regulation. That such is the case appears to be clearly indicated by the writer's experiments upon timothy hay (p. 424), since there was obviously no such indirect utilization of the heat resulting from the work of digestion and assimilation as takes place, according to Rubner's theory, below the critical amount of food. If, now, the temperature to which such an animal is exposed falls, it is in accord with all that we know regarding the regulative processes in the body to suppose that the additional draft on it for heat will be compensated for by a fall in the emission constant rather than by an increased production of heat, or, to put it in another way, that some of the heat resulting from digestive work will be utilized to maintain the temperature of the animal instead of being at once dissipated.

No exact experiments upon the influence of external temperature on the maintenance requirement appear to have been made, but Kern, Wattenberg \& Pfeiffer * have investigated the influence of the greater exposure to cold caused by shearing upon the metabolism of sheep consuming a maintenance ration. A slight decrease in the proteid metabolism was found to result, due, as Pfeiffer conjectures, to a more rapid growth of wool after shearing, but the corresponding difference in the metabolism of energy is insignificant. The removal of a nine-months fleece appears to have caused at first an increased excretion of carbon dioxide, but this practically disappeared within four or five days and is probably to be attributed to greater muscular activity on the part of the shorn animals. Comparing the results before shearing with those obtained from five to sixteen days after, we have the following averages, the amount of water-vapor given off being only an approximate estimate:

\footnotetext{
* Jour. f. Landw., 39, 1.
} 


\begin{tabular}{|c|c|c|}
\hline & $\begin{array}{c}\text { Carbon dioxide } \\
\text { per Day, } \\
\text { Grms. }\end{array}$ & $\begin{array}{c}\text { Estimated } \\
\text { Water-Vapor } \\
\text { per Day, } \\
\text { Grms. }\end{array}$ \\
\hline Before shearing ( 4 experiments) & 719.6 & 1939 \\
\hline After " (4 ") & 725.1 & 434 \\
\hline
\end{tabular}

The total metabolism, as indicated by the excretion of carbon dioxide, shows scarcely any increase as a result of the shearing; and if we accept Pfeiffer's suggestion that the result for the first of the four days ( 736 grams) may have been slightly affected by the stimulation of movement above noted, the difference becomes still less. On the other hand, the difference in the amount of water-vapor given off is very striking and apparently admits of but one conclusion, viz., that drawn by Pfeiffer, that the unshorn animals upon a maintenance ration produced an excess of heat which was gotten rid of by evaporation of water, while the shorn animals, instead of meeting the greater refrigerating effect of their surroundings by an increased metabolism, simply evaporated less water and thus compensated for the increased loss of heat by radiation and conduction.

Even in the case of man, where the digestive work is much less than in the herbivora, the heat production on a maintenance ration may be in excess, and even largely in excess, of the minimum requirement, it being simply a question of clothing, temperature, etc. This has been most strikingly demonstrated by Ranke,* who shows that with relatively high temperature and humidity the heat production on a maintenance ration may be so great as to even produce pathological effects and that under such circumstances the consumption of food is instinctively reduced below the maintenance requirement.

Sanborn, $\dagger$ in experiments upon the maintenance ration of swine, found the amount of middlings required, per hundred pounds of live weight, to be as tabulated on the next page. The second summer experiment is not comparable with the others, since the smaller animal would require a relatively greater maintenance ration. The remaining experiments seem to show a lower requirement for maintenance in winter than in summer.

* Einfluss des Tropenklimas auf die Ernährung des Menschen, and Zeit. f. Biol., 40, 288.

$\dagger$ Mo. State Agr. Coll., Bull. 28, pp. 5 and 6. 


\begin{tabular}{|c|c|c|}
\hline & $\begin{array}{l}\text { Live Weight, } \\
\text { Lbs. }\end{array}$ & $\begin{array}{c}\text { Maintenance } \\
\text { Requirement, } \\
\text { per } 100 \text { Pounds } \\
\text { Lbs. }\end{array}$ \\
\hline Winter (temp. about $40^{\circ} \mathrm{F}$. ) ... \{ & 173.5 & $\begin{array}{l}1.65 \\
1.89\end{array}$ \\
\hline Summer (" " $" 80^{\circ}$ F.) $\left.\ldots\right\}$ & $\begin{array}{r}173.6 \\
48.3\end{array}$ & $\begin{array}{l}2.02 \\
2.07\end{array}$ \\
\hline
\end{tabular}

On the other hand, Cooke,* in a series of experiments on swine at the Colorado Station, found the following amounts of computed digestible matter required for maintenance per hundred pounds live weight of animals weighing from 85 to 182 pounds per head:

In hot weather................. 0.93 lbs.

In moderate weather............... 1.25 "

In cold weather.................. 1.41 "

Consumption of Water.-A not inconsiderable amount of energy is usually required to raise the ingesta to the temperature of the body. This is particularly true of the water consumed, especially in case of the herbivora, both by reason of its relatively large amount as compared with the dry matter of the food and on account of its high specific heat. At first thought it might seem that the warming of the ingesta is part of the work of digestion, since it is an expenditure of energy in preparing the food for assimilation. This same matter or its equivalent, however, finally leaves the body, in the form of various excreta, at body temperature, thus carrying off as sensible heat substantially the same amount of energy which was imparted to it when its temperature was raised, and this heat it imparts in cooling to the environment of the animal. It would seem, then, that the warming of the ingesta may be more logically regarded as a part of the general draft for heat which the surroundings make upon the animal, the process being simply a little less direct than the loss of heat by radiation and conduction through the skin.

From this point of view the influence of the consumption of cold food and particularly of cold water will be subject to the same general laws as the other forms of the demand for heat. On a ration supplying less than the critical amount of metabolizable

*Private communication. 
energy any increase in the consumption of water (taking this as the typical case) will increase the metabolism by an amount sufficient to warm the water to the body temperature. Above the critical amount of food the excess of heat arising from the digestive work will, we may reasonably suppose, be applied to the warming of the additional water consumed, and only when this is insufficient will an increased metabolism be required to make up the deficit. In case of farm animals, however, it would appear that the waste heat even on a maintenance ration is ordinarily sufficient, and more than sufficient, to supply all the energy needed for warming the ingesta.

The Time Element.-One important factor in modifying the results of the demand for heat, particularly with relation to the water consumption, is what we may call the time element. Hitherto it has been tacitly assumed that all the factors making up the demand for heat act at a uniform rate. As a matter of fact this is at best only partially true. Ordinarily a farm animal is watered but once or twice per day and then consumes a relatively large amount in a few minutes. A sudden demand for heat is thus set up, since this water must be raised to body temperature within a comparatively short time. It is quite conceivable, therefore, that the demand for heat may temporarily exceed the supply, requiring the deficit to be made up by an increased metabolism, while if the same water consumption were distributed uniformly over the twelve or twentyfour hours no such effect would be produced. Such a temporary increase in the heat production, however, cannot be made up for later when the heat production is in excess, but is a permanent loss. Once converted into heat, the energy of food or tissue has, so to speak, escaped from the grasp of the organism, which appears to have no power to reconvert it into any other form of energy. We may plausibly suppose that these considerations constitute a partial explanation of the advantages observed in practice from the warming of drinking-water and the installation of self-watering devices in the stable.

What is true in regard to the consumption of water is of course equally true of other forms of the demand for heat. The time element is an important factor. Thus an exposure of an hour or two in a cold yard or to a cold rain may cause an increased metabolism 
and heat production although the average conditions for the twentyfour hours may be such that the necessary production of heat by the internal work and the work of digestion and assimilation would be more than sufficient for the needs of the animal.

Influence of Size of Animal.-The discussion of the heat production of the fasting animal in Chapter XI led us to the conclusion that under comparable conditions, at least for the same species of animal, the internal work is probably approximately proportional to the surface of the body. This, however, is equivalent to saying that the quantity of net available energy required for maintenance is proportional to the body surface. Furthermore, if we are right in supposing that the available energy is a linear function of the metabolizable energy, the amount of the latter required for maintenance will also be proportional to the surface of the body. Referring once more to the diagram on p. 410 , if $O A$ is proportional to the body surface, then $O S$, which for a given food bears a fixed ratio to $O A$, must also be proportional to the surface. If the critical point, $K$, lies above the maintenance requirement, then the metabolizable energy required for maintenance will equal the fasting metabolism, and this, as shown on pp. $359-363$, is proportional to the surface.

Apparently, then, we are justified in concluding that the maintenance requirements of different normal animals of the same species are proportional to their body surface, or, for approximate computations, to the two-thirds power of their live weights. It must not be overlooked, however, that the results upon which this conclusion is based were obtained largely with the dog, an animal which when at rest lies down, and which, therefore, in these experiments was in a state of almost complete muscular relaxation. Our common farm animals, on the contrary, pass a considerable portion of their time standing, which involves an expenditure of energy in muscular work. This expenditure we should naturally assume to be proportional to the mass to be sustained rather than to its surface, and if this be true we have here a second determining factor in the maintenance requirement. How important this factor is it is diffcult to say, although the writer's results with a steer (p. 343) indicate that it is a large one. Its tendency would be to make the maintenance requirement increase more rapidly than the surface. 
Moreover, so far as we can judge from the accounts of Rubner's experiments, it would seem likely that what were designated on p. 342 as incidental muscular movements are a more important factor in determining the maintenance requirements of farm animals than they are in fixing that of the dog.

While, therefore, we are probably justified in retaining provisionally the computation of the maintenance requirement in proportion to the real or estimated surface, it should be with a clear understanding that it is at present a deduction from experiments on other species and under more or less different conditions.

Effect of Fattening on Maintenance Requirement.-An interesting question, and one of practical importance, is what effect the progressive change in weight of the same animal as it is fattened has upon its maintenance requirement. We can hardly suppose that the internal work of the body will be materially increased by such a gain. The increased mass of tissue must involve, of course, some increase in metabolism, but all that we know of metabolism of adipose tissue indicates that it is very sluggish. The most important effect might be anticipated to be an increase in the muscular exertion required in standing, perhaps counterbalanced to a greater or less extent by the tendency of the fat animal to pass more of its time in a recumbent position.

Zuntz \& Hagemann * have investigated the effect of a load carried on the back upon the metabolism of the horse, and have found the latter to be proportional to the total mass (horse plus load), but the applicability of this result to another species of animal and to an increase of weight caused by fattening may perhaps be questioned. The only experiments upon cattle bearing on this point are those of Kellner, $\nmid$ who has compared the maintenance requirements of fattened and unfattened cattle. It being impossible to hit upon exactly the maintenance ration, it is computed from the actual results. In case there was a loss of tissue the maintenance requirement of the animal is computed by subtracting the potential energy of the excreta from the potential energy of food plus tissue lost; in other words, the replacement of energy claimed by Rubner is assumed to occur. When there was a gain of tissue, on

* Landw. Jahrb., 27, Supp. III, 269.

$\dagger$ Landw. Vers. Stat., 50, 245; 53, 14. 
the other hand, the amount of metabolizable energy required to produce it is computed on the basis of the results upon utilization obtained in other experiments, this larger amount being added to the energy of the excreta and the sum of the two subtracted from the potential energy of the food; that is the energy of digestion and assimilation above the maintenance ration is assumed to be waste energy.

Computed in this way, and assuming further that the maintenance requirements of different animals are substantially proportional to the two-thirds powers of their live weights, the results are as follows:

\begin{tabular}{|c|c|c|c|c|}
\hline & $\begin{array}{c}\text { No. of } \\
\text { Animals. }\end{array}$ & $\begin{array}{l}\text { Live } \\
\text { Weight, } \\
\text { Kgg. }\end{array}$ & $\begin{array}{l}\text { Stable } \\
\text { Tem- } \\
\text { perature, } \\
\text { Deg, C. }\end{array}$ & $\begin{array}{c}\text { Main- } \\
\text { tenance } \\
\text { Require- } \\
\text { ment, } \\
\text { Cals. }\end{array}$ \\
\hline $\begin{array}{l}\text { Observed: } \\
\quad \text { Unfattened } \ldots \ldots \ldots \ldots \ldots \ldots \ldots \ldots \ldots \ldots \ldots \ldots \\
\text { Fattened } \ldots \ldots \ldots \ldots \ldots \ldots\end{array}$ & $\begin{array}{l}7 \\
3\end{array}$ & $\begin{array}{l}632 \\
785\end{array}$ & $\begin{array}{l}15.2 \\
15.7\end{array}$ & $\begin{array}{l}13,470 \\
19,671\end{array}$ \\
\hline $\begin{array}{l}\text { Computed to same live weight: } \\
\text { Unfattened } \ldots \ldots \ldots \ldots \ldots \ldots \\
\text { Fattened } \ldots \ldots \ldots \ldots \ldots \ldots\end{array}$ & $\begin{array}{l}7 \\
3\end{array}$ & $\begin{array}{l}800 \\
800\end{array}$ & $\begin{array}{l}15.2 \\
15.7\end{array}$ & $\begin{array}{l}15,760 \\
19,920\end{array}$ \\
\hline
\end{tabular}

Kellner concludes from these figures that the maintenance requirements of fattened animals are greater per unit of surface than those of unfattened ones.

These experiments, it is true, were on different animals and the individuality of the animal is an important factor in determining the maintenance requirement. The results on the seven unfattened animals, when computed to $600 \mathrm{kgs}$. live weight, show a range of 1760 Cals., or 13.54 per cent. of the average, while the three results on fattened animals, computed to $800 \mathrm{kgs}$. live weight, show a range of 2420 Cals., or 12.16 per cent. of the average. Moreover, in making up the average of the unfattened animals, one animal was excluded on the ground that the results were probably abnormally high, but the same animal is subsequently included among the three fattened animals the results on which are averaged.

Even after making all allowances for these facts, however, the results for the fattened animals are decidedly higher relatively 
than for the unfattened, but how much higher can hardly be determined from such averages.

Comparing the results on the one animal common to the two series of experiments we have-

\begin{tabular}{|c|c|c|}
\hline & $\begin{array}{c}\text { Live Weight, } \\
\text { Kgs. }\end{array}$ & $\begin{array}{c}\text { Maintenance, } \\
\text { Cals. }\end{array}$ \\
\hline Observed: & & \\
\hline Unfattened... . & 611.5 & $16,835.6$ \\
\hline Fattened.............. & 750 & $18,959.6$ \\
\hline Computed to $800 \mathrm{kgs.:}$ & & \\
\hline Unfattened......... & 800 & 20,140 \\
\hline Fattened........... & 800 & 19,800 \\
\hline
\end{tabular}

According to the above figures the maintenance ration of this animal was practically proportional to the two-thirds power of its live weight. On the other hand, however, its maintenance requirement in the unfattened state was much higher than the average for the seven unfattened animals, while after fattening it did not differ materially from the average for the three fattened animals. If, then, we are to regard the above result as correct we must assume that by chance all three of the fattened animals had a higher normal rate of metabolism than the seven unfattened ones, which is not exactly probable. Although this leaves the question in a rather unsatisfactory state, it would seem that we must be content to let it rest there pending further comparative experiments on identical animals in different stages of fattening. 


\section{CHAPTER XIII.}

\section{THE UTILIZATION OF ENERGY.}

ACCORDING to the conceptions discussed in the preceding chapter a certain portion of the metabolizable energy of the food is consumed in what has been called in a broad sense the work of digestion and assimilation, while the remainder constitutes net available energy and contributes to the maintenance of the store of potential energy in the body. If the food is sufficient to supply net available energy equal to that dispensed by the internal work of the body, the balance between income and expenditure of energy is just maintained. If we increase the food beyond this maintenance requirement we supply the body with an excess of net available energy. In general terms we can say that this excess may be disposed of in two ways: it may be utilized for the peformance of external work, or it may give rise to a storage of potential energy in the body in the form of new tissue,* particularly of fat tissue. It appears probable, however, that neither of these processes takes place without more or less loss of energy in the form of heat. This is certainly true of the performance of muscular work, as has already been mentioned ( $p .189$ ) and as will be shown in detail on subsequent pages. Out of the total potential energy of the material metabolized rather more than one third, in the most favorable case, is actually recovered in the form of external work, the remainder taking the form of heat. In this case, then, we might speak of the coefficient of utilization of the energy as being about one third.

In the utilization of surplus energy by storage of tissue it appears likely that there must be also a loss of energy, although,

* From this point of view the production of milk is to be regarded as the formation of new tissue. 
as will appear later, we are not yet in a position to make any such definite statements regarding its amount as in the case of muscular work, and although the writer's few results on timothy hay cited on p. 424 afford no indication of such a loss, the utilization of the metabolizable energy for the production of gain seeming to have been practically equal to its net availability. It is obvious, however, that the conversion of the resorbed nutrients of the food into the ingredients of tissue involves profound chemical changes, and we can hardly suppose that these take place without some evolution of heat. As a good illustration we may take the case of a carbohydrate. As resorbed into the blood it appears to be in the form of a sugar, and it would seem that this sugar can serve, without any very extensive chemical changes, to sustain the metabolism incident to the internal work of the body; that is, that it is oxidized more or less directly in the various tissues to supply energy for their physiological work. When, however, a surplus of a carbohydrate is to be utilized for the storage of energy in the form of fat, the case is different. The formation of fat from a carbohydrate is chemically a process of reduction, and the oxygen which is removed from the carbohydrate must unite with the carbon and hydrogen either of other molecules of the carbohydrate or of other ingredients of food or tissue, in either case giving rise to an evolution of heat. If we suppose the transformation to take place according to the equation given in Chapter II (p. 24), the resulting fat would contain about 87 per cent. of the energy of the dextrose. Whether this percentage expresses the actual facts of the case or not, it is very improbable that this or any similar synthetic process takes place in the body without the evolution of some heat.

Provisionally, then, we seem justified in assuming that only a part of the net available energy supplied to the organism above the maintenance requirement can be utilized to increase the store of potential energy in the body, and we may speak in this case, as in that of muscular work, of the coefficient of utilization. Reproducing here the essential parts of the graphic representation on p. 410 , we may now complete it so as to represent in a general and qualitative way the relations indicated above, assuming provisionally that the effects are linear functions of the food. As 
before, $O G$ represents the fasting metabolism at a temperature below the critical point and $O M$ the critical amount of food at this temperature. Then the line GKS represents the availability of the food, $H L S^{\prime}$ the heat production, and $O S$ the maintenance requirement. Beyond the point $S$ we may assume that the net availability of the food remains the same, represented by the line ST. But a fraction of this net available energy, however, can be

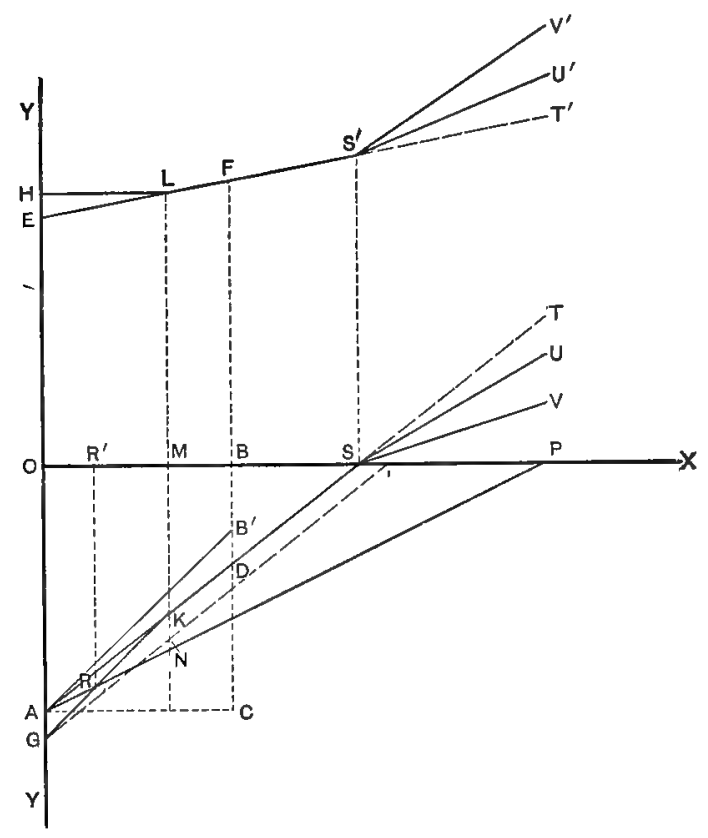

recovered as mechanical work, and its utilization will therefore be represented by some such line as $S V$, while the heat production will be correspondingly increased as represented by $S^{\prime} V^{\prime}$. Similarly the proportion of the net available energy which in the quiescent animal is stored up in the form of new tissue may be expressed by a line $S U$ and the corresponding heat production by $S^{\prime} U^{\prime}$. What the relation between the proportions utilized in the two cases is we do not know, and the diagram is intended to be simply schematic; 
but we do know that the proportion is materially greater in the latter case, since the heat production of a fattening animal is obviously much less than that of a working animal utilizing the same amount of food.

In the following pages the attempt has been made to bring together the more important experimental evidence bearing upon the utilization of food energy for the production of tissue and of work. Before, however, proceeding to a consideration of our present knowledge upon the subject, attention should be called once more to the fact that we are here dealing with it from the statistical point of view of the balance between income and expenditure of energy of the body.

In an animal performing work, each muscular contraction metabolizes a certain quantity of energy, part of which finally appears as heat and part as mechanical work. Besides this, however, a secondary result is an increase in the activity of the organs of circulation and respiration which requires the expenditure of a certain amount of energy, this energy ultimately taking the form of heat and being added to that resulting directly from the activity of the skeletal muscles. When we compare the actual external work done with the total energy metabolized for its performance, and so compute the coefficient of utilization, we group all these sources of heat production and regard them as, from the economic standpoint, a waste of energy, just as in a heat engine the energy which escapes conversion into work is regarded as waste energy notwithstanding the fact that the loss is inevitable. So, too, in the production of new tissue we look upon total gain of potential energy by the body as constituting the net useful result of the feeding, and the coefficient of utilization in this case, as in that of muscular work, would express the relation which this bears to the net available energy supplied in the food. That the effect of abundant food may be in some cases to stimulate the metabolism of tissue or the "incidental" muscular work (p. 342) is rendered probable by Zuntz \& Hagemann's results with the horse (see p. 376). All these effects are part of the necessary expenditure of energy by the body, and however interesting physiologically are statistically sources of loss. 


\section{$\S$ I. Utilization for Tissue Building.}

Under this head we have to consider almost exclusively experiments upon the fattening of mature animals. While the growth of young animals and the production of milk are both forms of tissue building, the experimental data available seem too scanty to justify including them in the scope of the present work. For convenience we may first bring together the recorded results and later discuss them in their more general bearings.

One difficulty, however, is encountered at the outset in our inadequate knowledge of the net availability of nutrients and feeding-stuffs, as pointed out in the foregoing chapter. Until this gap is filled. it is of course impossible to compare the gain of energy by the body with the supply of net available energy. Accordingly the results of the experiments upon productive feeding can at present be utilized only to determine what proportion of the metabolizable energy of the food is recovered in the gain of tissue, and the experiments cited in the following paragraphs will be considered from this point of view.

\section{Experimental Results.}

Experiments on Carnivora.-In connection with the discussion of net availability in the preceding chapter a number of experiments were cited (p. 428) in which more or less gain was made by the animals. In addition to these Rubner* has made a preliminary report of investigations upon the effect of abundant feeding on the heat production A dog weighing $25 \mathrm{kgs}$. received successively isodynamic amounts of lean meat. fat, and carbohydrates (kind not stated) equivalent to 155 per cent. of its fasting metabolism, a two-days' fast intervening in each case between the different rations. Few details are given, but presumably the methods were those of Rubner's other experiments already described (compare p. 253). In a second experiment the effects of two different amounts of meat were also compared. In the following table the results of these experiments have been put into the same form as those on net availability in the preceding chapter, the data given being per day and head:

* Sitzungsber. k. bayer. Akad. der Wiss., Math -phys. Classe, 15, 452 


\begin{tabular}{|c|c|c|c|c|}
\hline & \multirow{2}{*}{$\begin{array}{c}\text { Metabolizable } \\
\text { Energy of } \\
\text { Food. } \\
\text { Cals. }\end{array}$} & \multirow{2}{*}{$\begin{array}{l}\text { Total Gain. } \\
\text { Cals. }\end{array}$} & \multicolumn{2}{|c|}{$\begin{array}{l}\text { Gain Over Fasting } \\
\text { Metabolism. }\end{array}$} \\
\hline & & & $\begin{array}{l}\text { Total, } \\
\text { Cals. }\end{array}$ & $\begin{array}{c}\text { Per Cent. of } \\
\text { Energy of } \\
\text { Food. }\end{array}$ \\
\hline $\begin{array}{l}\text { Nothing . . . . . . . } \\
\text { Fat ......... } \\
\text { Carbohydrates.... } \\
\text { Meat ........ }\end{array}$ & $\begin{array}{r}0 \\
1549 \\
1549 \\
1549 \\
1463 \\
2181\end{array}$ & $\begin{array}{l}-944 \\
+540 \\
+509 \\
+418 \\
+332 \\
+805\end{array}$ & $\begin{array}{l}1484 \\
1453 \\
1362 \\
1276 \\
1749\end{array}$ & $\begin{array}{l}95.8 \\
93.8 \\
87.9 \\
87.2 \\
80.2\end{array}$ \\
\hline
\end{tabular}

Experiments by Gruber * upon the formation of fat from proteids (see p. 112) afforded the following results, computed $\dagger$ by the use of the factors given on p. 414:

\begin{tabular}{|c|c|c|c|c|}
\hline & \multirow{2}{*}{$\begin{array}{l}\text { Metabolizable } \\
\text { Energy of } \\
\text { Food } \\
\text { Cals. }\end{array}$} & \multirow{2}{*}{$\begin{array}{c}\text { Total Gain } \\
\text { Cals. }\end{array}$} & \multicolumn{2}{|c|}{$\begin{array}{c}\text { Gain Over Fasting } \\
\text { Metabolism. }\end{array}$} \\
\hline & & & $\begin{array}{l}\text { Total } \\
\text { Cals. }\end{array}$ & $\begin{array}{l}\text { Per Ceat. of } \\
\text { Energy of } \\
\text { Food. }\end{array}$ \\
\hline $\begin{array}{l}\text { Nothing . . . . . . . } \\
1500 \text { grms meat: } \\
\text { 1st series. . . . } \\
2 \mathrm{~d} \text { series.... }\end{array}$ & $\begin{array}{r}0 \\
1325 \\
1325\end{array}$ & $\begin{array}{r}-743 \\
\\
250 \\
296\end{array}$ & $\begin{array}{r}993 \\
1039\end{array}$ & $\begin{array}{l}74.9 \\
78.4\end{array}$ \\
\hline Average ........ & 1325 & 273 & 1016 & 76.7 \\
\hline
\end{tabular}

The difficulty in interpreting these results, as well as those tabulated on p. 428 , as already stated, lies in our imperfect data regarding the net availability of the materials below the point of mainte. nance. Rubner, in discussing his results, assumes an availability of 100 per cent., or in other words that the fasting metabolism is the measure of the amount of metabolizable energy required for maintenance. He accordingly subtracts this amount from the total metabolizable energy of the food and regards the remainder as excess food, which may be utilized for the storage of energy. The percentage utilization of this excess was as shown in the following table, to which Gruber's results, computed by the writer in the same way, have been added:

* Zeit f Biol , 42, 409 .

† From the last two complete days of each series 


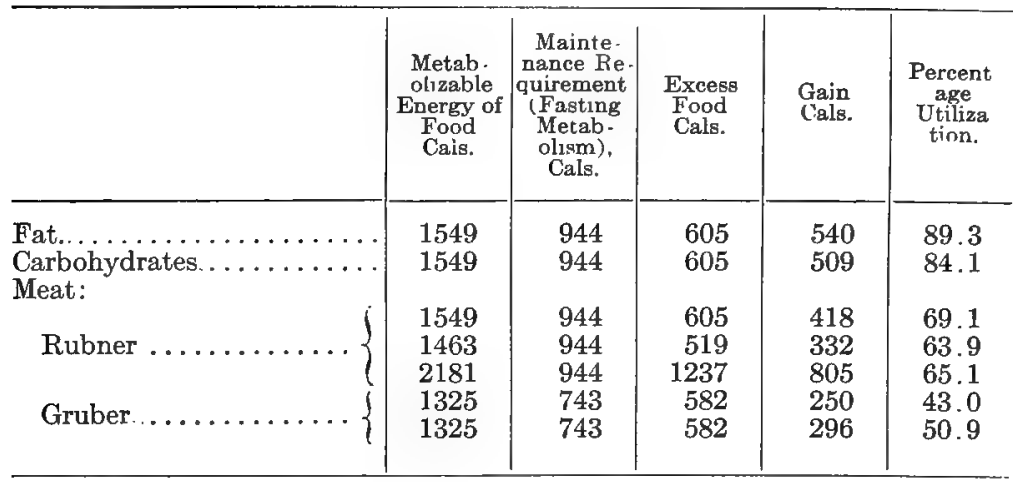

As was shown in the preceding chapter, however, while the recorded determinations of net availability are far from satisfactory they show with a considerable degree of probability that there is some loss of energy below the maintenance point and that 100 per cent. of net availability is at least not ordinarily reached. A lower net availabilty, however, means a larger maintenance requirement, and this in turn results in a larger computed percentage utilization of the excess food.

In the following table the latter percentage has been computed by the writer for most of the experiments tabulated on p. 428 , as well as for those of Rubner and Gruber just cited, on the assumption that the net availability below the maintenance requirement was:

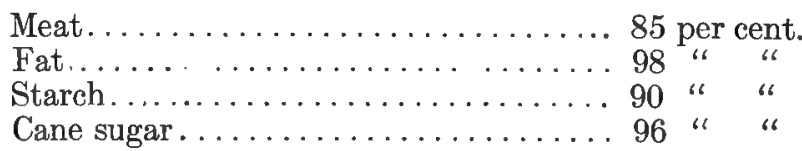

The factor for meat is the average of all the results on p. 427; that for fat is based on Magnus-Levy's results upon digestive work; those for starch and cane-sugar are the averages of Rubner's results, omitting those which exceed 100 per cent. By dividing the fasting metabolism by the above percentages we may compute the amount of metabolizable energy required for maintenance on the above assumption, while subtracting this from the metabolizable energy of the food leaves the amount of excess food, which can be compared with the observed gain. 


\begin{tabular}{|c|c|c|c|c|c|c|}
\hline - & $\begin{array}{l}\text { Fasting } \\
\text { Metab- } \\
\text { olism, } \\
\text { Cals. }\end{array}$ & $\begin{array}{l}\text { Metab- } \\
\text { olizable } \\
\text { Energy } \\
\text { of } \\
\text { Food, } \\
\text { Cals. }\end{array}$ & \begin{tabular}{|} 
Com- \\
puted \\
Main- \\
tenance \\
Cals.
\end{tabular} & $\begin{array}{l}\text { Excess } \\
\text { Food, } \\
\text { Cals. }\end{array}$ & $\begin{array}{l}\text { Gain, } \\
\text { Cals. }\end{array}$ & $\begin{array}{l}\text { Per- } \\
\text { centage } \\
\text { Utiliza- } \\
\text { tion. }\end{array}$ \\
\hline \multicolumn{7}{|l|}{ Proteids (meat): } \\
\hline \multirow{2}{*}{ Pettenkofer \& Voit.......... } & 1041 & 1325 & 1225 & 100 & 38 & 38.0 \\
\hline & $\begin{array}{l}201 \\
944\end{array}$ & $\begin{array}{r}548 \\
1549\end{array}$ & 1169 & $\begin{array}{r}400 \\
380\end{array}$ & $\begin{array}{r}10 \\
418\end{array}$ & 110.0 \\
\hline \multirow{2}{*}{ Rubner ............. } & 944 & 1463 & 1169 & 294 & 332 & 112.9 \\
\hline & 944 & 2181 & 1169 & 1012 & 805 & 79.6 \\
\hline \multirow{2}{*}{ Gruber $\ldots \ldots \ldots \ldots \ldots \ldots\{$} & 743 & 1325 & 920 & 405 & 250 & 61.7 \\
\hline & 743 & 1325 & 920 & 405 & 296 & 73.1 \\
\hline \multicolumn{7}{|l|}{ Fat: } \\
\hline \multirow{2}{*}{ Pettenhofer \& Voit....... } & 1086 & 3298 & 1108 & 2190 & 878 & 40.1 \\
\hline & $\begin{array}{l}554^{*} \\
554^{*}\end{array}$ & $\begin{array}{r}942 \\
1884\end{array}$ & $\begin{array}{l}565 \\
565\end{array}$ & $\begin{array}{r}377 \\
1319\end{array}$ & $\begin{array}{l}329 \\
837\end{array}$ & $\begin{array}{l}87.3 \\
63.5\end{array}$ \\
\hline & 658 & 1738 & 671 & 1067 & 1016 & 95.2 \\
\hline \multirow{3}{*}{ Rubner } & 466 & 942 & 476 & 466 & 428 & 91.8 \\
\hline & 261 & 348 & 266 & 82 & 49 & 59.8 \\
\hline & 944 & 1549 & 963 & 586 & 540 & 92.1 \\
\hline \multicolumn{7}{|l|}{ Starch: } \\
\hline \multirow{2}{*}{ Pettenkofer \& Voit....... } & 1098 & 2015 & 1220 & 795 & 353 & 44.4 \\
\hline & 1098 & 3076 & 1220 & 1856 & 853 & 46.0 \\
\hline \multirow{2}{*}{$\begin{array}{l}\text { Rubner ("carbohydrates", as- } \\
\text { sumed to be starch)........ }\end{array}$} & $554 *$ & 874 & 616 & 258 & 137 & 53.1 \\
\hline & 944 & 1549 & 1049 & 500 & 509 & 101.8 \\
\hline \multicolumn{7}{|l|}{$\begin{array}{l}\text { Cane-sugar: } \\
\text { Rubner .. }\end{array}$} \\
\hline Rubner .. & 451 & 572 & 470 & 102 & 190 & 186.3 \\
\hline \multicolumn{7}{|l|}{$\begin{array}{l}\text { Cane-sugar and Starch (93 per } \\
\text { cent. availability): }\end{array}$} \\
\hline Rubner ................. & 302 & 702 & 325 & 377 & 365 & 96.8 \\
\hline
\end{tabular}

While as a whole the results of the computation would seem to indicate that the percentage utilization for tissue building is less than the percentage availability, the remarkable range of the figures and the uncertain basis upon which they are computed do not encourage any attempt at a critical discussion.

Experiments on Man.-The only respiration experiments upon man which the writer has been able to find in which any large amount of excess food was given are those of Johansson, Landergren, Sondén \& Tigerstedt $\uparrow$ already cited on p. 383 in their bearing on the subject of digestive work. If we assume, on the basis of

* Loss on basal ration.

† Skand. Arch. f. Physiol., 7, 29. 
Magnus-Levy's results, that the work of digestion in man equals about 9 per cent. of the metabolizable energy of the food, the average results of the experiments are as follows:

Fasting metabolism. . . . . . . . . . 2022.4 Cals.

Metabolizable energy of food........... 4193.4 "

Computed maintenance requirement..... 2222.5 "

Excess food........................ 1970.9 “

Gain ......................... 1676.0 "

Percentage utilization $\ldots \ldots \ldots \ldots \ldots \ldots .85 .0$ per cent.

The computation gives a somewhat lower percentage for the utilization of the excess food than that assumed for the availability of the maintenance food.

Experiments on Swine.-Meissl, Strohmer \& Lorenz * in their investigation upon the sources of animal fat made six respiration experiments with swine, the results of which afford some data as to the utilization of their food by these animals. In Experiments $V$ and VI, made on two different animals, no food was given, and the following results were obtained, the energy equivalent to the loss of tissue being computed as in Rubner's experiments in the previous chapter:

\begin{tabular}{c|c|c|c|c|c|c|c}
\hline $\begin{array}{c}\text { Experi- } \\
\text { ment. }\end{array}$ & $\begin{array}{c}\text { Tempera- } \\
\text { ture, } \\
\text { Deg. C. }\end{array}$ & $\begin{array}{c}\text { Hours } \\
\begin{array}{c}\text { Since } \\
\text { Last } \\
\text { Feeding. }\end{array}\end{array}$ & $\begin{array}{c}\text { Live } \\
\text { Weight, } \\
\text { Kgs. }\end{array}$ & $\begin{array}{c}\text { Loss of } \\
\text { Nitrogen, } \\
\text { Grms. }\end{array}$ & $\begin{array}{c}\text { Loss of } \\
\text { Carbon, } \\
\text { Grms. }\end{array}$ & $\begin{array}{c}\text { Metab- } \\
\text { Metal } \\
\text { olism, } \\
\text { Cals. }\end{array}$ & $\begin{array}{c}\text { Metism per } \\
\text { oliso Kgg. } \\
\text { Live } \\
\text { Weight, } \\
\text { Cals. }\end{array}$ \\
\hline V..... & 20 & 24 & 140 & 9.80 & 224.51 & 2607 & 2083 \\
VI.... & 20 & 12 & 120 & 9.55 & 375.78 & 229 & 2029 \\
\hline
\end{tabular}

The experiment begun only twelve hours after the last feeding obviously gave too high results, owing to the presence of food in the digestive canal. That this source of error was substantially eliminated after twenty-four hours appears probable from the close agreement of the results with those obtained after seventy-two hours. The average fasting metabolism per $100 \mathrm{kgs}$. live weight is 2056 Cals. and this average has been made the basis of the computations which follow, except in Experiment I. This experiment

* Zeit. f. Biol., 22, 63.

† Assumed to be proportional to the two-thirds power of the weight. 
having been made on the same individual as Experiment $V$, the result of the latter is used directly.

In Experiments I and II the ration consisted of rice, in Experiment III of barley, and in Experiment IV of rice, flesh-meal, and whey. In all cases large amounts of food were consumed and a rapid production of fat was observed. The digestibility of the food was determined. Its metabolizable energy has been computed by the writer from the results of the digestion experiments by the use of the following factors:*

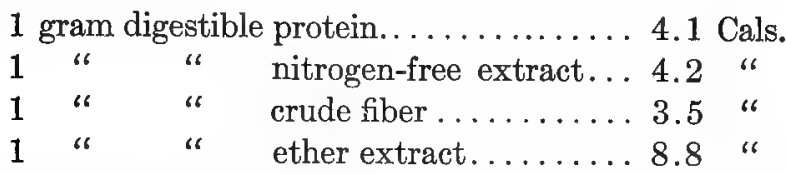

No attempt was made in these experiments to determine the methane, if any existed, in the respiratory products. The results per day and head were as follows:

\begin{tabular}{|c|c|c|c|c|c|c|}
\hline Experiment. & $\begin{array}{l}\text { Tem- } \\
\text { perature, } \\
\text { Deg. C. }\end{array}$ & $\begin{array}{c}\text { Live } \\
\text { Weight, } \\
\text { Kgs. }\end{array}$ & $\begin{array}{c}\text { Computed } \\
\text { Fasting } \\
\text { Metabolism, } \\
\text { Cals. }\end{array}$ & $\begin{array}{c}\text { Metab- } \\
\text { olizable } \\
\text { Energy. } \\
\text { Cals. }\end{array}$ & $\begin{array}{c}\text { Energy of } \\
\text { Gain, Cals. }\end{array}$ & $\begin{array}{c}\text { Nutritive } \\
\text { Ratio. }\end{array}$ \\
\hline $\begin{array}{r}\mathrm{I} \ldots \ldots \ldots \\
\mathrm{II} \ldots \ldots \ldots \\
\mathrm{III} \ldots \ldots \\
\mathrm{IV} \ldots \ldots \\
\ldots\end{array}$ & $\begin{array}{l}18.0 \\
18.5 \\
19.3 \\
16.7\end{array}$ & $\begin{array}{r}140 \\
70 \\
125 \\
104\end{array}$ & $\begin{array}{l}2607 \\
1621 \\
2386 \\
2111\end{array}$ & $\begin{array}{l}7157 \\
7167 \\
5125 \\
6129\end{array}$ & $\begin{array}{l}3464 \\
4048 \\
1774 \\
2556\end{array}$ & $\begin{array}{l}1: 15.4 \\
1: 14.1 \\
1: 9.3 \\
1: 2.4\end{array}$ \\
\hline
\end{tabular}

No determinations were made of the actual requirements for maintenance as distinguished from the fasting metabolism, and hence the data are lacking for a computation of the net availability of the metabolizable energy of the food on the one hand and the percentage utilization of the excess food on the other. Cooke's results mentioned on p. 438, however, seem to give some indication that the maintenance demand of swine may not be greatly in excess of the fasting metabolism. If in these experiments we assume the same net availability as that just assumed in the case of man, viz., 91 per cent., we obtain the following figures:

* Compare p. 332.

$\dagger$ In this experiment the ether extract of the feces exceeded that of the food by $23.95 \mathrm{grms}$. This excess has been assumed to have a heat value of 4.2 Cals. per grm. and a corresponding amount deducted from the computed energy of the other digested nutrients. 


\begin{tabular}{|c|c|c|c|c|c|c|c|c|}
\hline 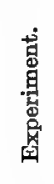 & Food. & $\begin{array}{c}\text { Nutri- } \\
\text { tive } \\
\text { Ratio. } \\
1 .\end{array}$ & $\begin{array}{c}\text { Com- } \\
\text { puted } \\
\text { Fasting } \\
\text { Metab- } \\
\text { olism. } \\
\text { Cals. }\end{array}$ & $\begin{array}{l}\text { Metab- } \\
\text { olizable } \\
\text { Energy } \\
\text { of } \\
\text { Food, } \\
\text { Cals. }\end{array}$ & \begin{tabular}{|c} 
Com- \\
puted \\
Main- \\
tenance \\
Re- \\
quire- \\
ment, \\
Cals.
\end{tabular} & $\begin{array}{l}\text { Excess } \\
\text { Food, } \\
\text { Cals. }\end{array}$ & $\begin{array}{l}\text { Gain, } \\
\text { Cals. }\end{array}$ & $\begin{array}{c}\text { Per- } \\
\text { cent- } \\
\text { age } \\
\text { Utiliza- } \\
\text { tion. }\end{array}$ \\
\hline \multirow{4}{*}{$\begin{array}{l}\text { II } \\
\text { III } \\
\text { IV }\end{array}$} & Rice & 15.4 & 2607 & 7157 & 2865 & 4292 & 3464 & 80.7 \\
\hline & & 14.1 & 1621 & 7167 & 1781 & 5386 & 4048 & 75.2 \\
\hline & Barley... & 9.3 & 2386 & 5125 & 2622 & 2503 & 1774 & 70.9 \\
\hline & $\begin{array}{l}\text { Rice, flesh-meal, } \\
\text { and whey....... }\end{array}$ & 2.4 & 2111 & 6129 & 2320 & 3809 & 2556 & 67.1 \\
\hline
\end{tabular}

It is interesting to note that the utilization as thus computed diminishes as the proportion of protein in the ration increases, a result which the low average figures obtained on pp. 427 and 450 for the availability and the percentage utilization of the proteids would lead us to expect. Obviously, however, too much value should not be attached to such computations as the above.

Kornauth \& Arche* in an investigation on the feeding value of sockle have also made respiration experiments with a swine. The food consisted in Period II of cockle, barley, and maize, and in Period. III of rape-cake, barley, and maize, the amounts of the several nutrients actually digested being nearly the same in the two periods. In each period two respiration experiments were made which gave concordant results. The following table contains the average results for each period computed on the same basis as in the experiments of Meissl, Strohmer \& Lorenz. No fasting experiments having been made, the average results of the experiments by the last-named authors have been used, the average live weight of $50 \mathrm{kgs}$. being taken as the basis.

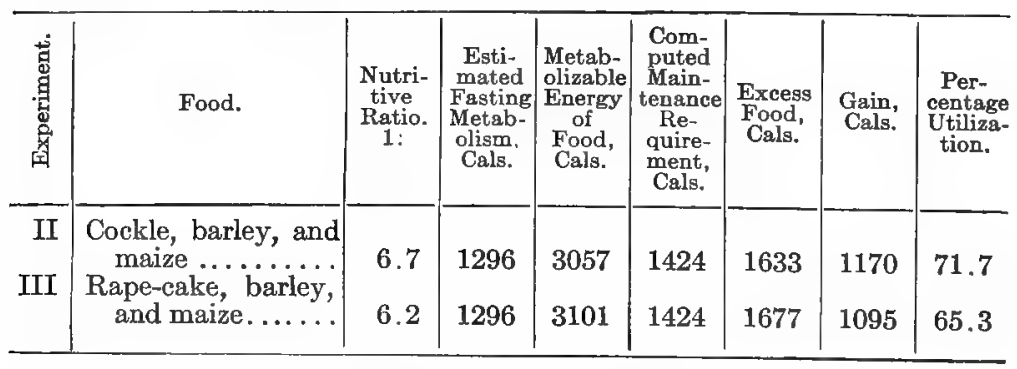

* Landw. Vers. Stat., 40, 177. 
The percentages as thus computed are seen to agree fairly well with the ones computed for those of Meissl, Strohmer \& Lorenz's experiments in which the proportion of protein in the food was similar.

Experiments on Ruminants. - Experiments upon ruminants necessarily differ in one important respect from those hitherto considered. With carnivora and with swine it is possible to determine the fasting metabolism, or, in other words, to trace the line representing the net availability or the utilization throughout its entire extent. With herbivora, and particularly with ruminants, this is practically impossible, for obvious reasons, and the course of the lower portion of the line is imaginary. This, however, is no obstacle to a determination of the net availability or percentage utilization of the food within the limits as to amount prescribed by the nature of the animals. As is clear from the graphic discussion of the problem on pp. 410 and 446, all that is necessary is to determine the gain or loss of energy by the body corresponding to two different amounts of food above or below maintenance. A simple comparison of differences then gives in the one case the percentage utilization and in the other the net availability of the energy of the food added. The method is the same principle as that already employed in computing the metabolizable energy of the added food.

The Möckern Experiments.-The very extensive and elaborate investigations upon cattle at the Möckern Experiment Station by G. Kühn and Kellner,* which have already been discussed in relation to the metabolizable energy of the food, are also our chief source of knowledge regarding the utilization of this energy by ruminants and will necessarily constitute the principal basis of the present discussion.

These experiments were chiefly upon the fattening of mature cattle, various additions being made to basal rations which were themselves in almost every case more than sufficient for maintenance. The actual gain of carbon and nitrogen by the animals, both on the basal and the augmented rations, was accurately determined, and from the data thus obtained the gain of proteids and fat and of energy was computed in the usual way. By a comparison of the * Landw. Vers. Stat., 44, 257; 47, 275; 50, 245; 53, 1. 
gains on the basal and on the augmented ration, then, we may determine what proportion of the metabolizable energy of the added food was stored in the gain of tissue. In other words, we may determine two points on the line $S U$ in the figure on p. 446, thereby determining the line if it is a straight line.

If the added metabolizable energy of the larger ration were derived solely from the material added, the result would show the utilization of the energy of that material. As we have seen, however, in connection with the discussion of the metabolizable energy of the food in Chapter X, this is rarely if ever the case with herbivorous animals. The difference in metabolizable energy between two rations usually includes, in addition to the real metabolizable energy of the added food, differences in the digestibility of the original ration and in the losses in urine and methane. Accordingly, we are here confronted with the same alternative as before, viz., whether to attempt to eliminate these secondary effects and base our computations on the real metabolizable energy of the feeding-stuff under experiment or to take the apparent metabolizable energy as representing the actual amount of energy contributed to the metabolism of the body. In the one case, if successful, we shall obtain a result which will be physiologically correct but which when applied in practice will require modification for the secondary effects just mentioned. In the other case we shall have a summary expression including all these results, but with the disadvantage of being more or less empirical in its nature. Either method has its advantages and disadvantages. In the present case we shall use the apparent metabolizable energy of feeding-stuffs as computed on pp. 285-297 and in Tables I-VI of the Appendix as the basis of computation. This does not, of course, affect the absolute amount of energy utilized from a unit weight of the material, but only the percentage calculated upon the metabolizable energy.

Sources of Uncertainty in Computation.-While the computation of the energy utilized from feeding-stuffs in the manner just indicated is in principle very simple, certain complications arise in its execution from the impossibility of securing exactly comparable conditions of experiment. Two of these in particular require consideration here.

Differences in Organic Matter Consumed.-As was noted in the 
discussion of the metabolizable energy of feeding-stuffs, the unavoidable slight variations in the moisture-content of the latter in the Möckern experiments resulted in slight differences in the amounts of organic matter of the basal ration consumed in the several periods. A comparison, then, between two periods, as regards metabolizable energy and resulting gain, shows the effect of the added feeding-stuff plus the effect of this small difference. For the metabolizable energy an approximate correction was computed. In order to make a similar correction in the resulting gain of tissue, however, it is necessary to know to what extent this difference in metabolizable energy contributed to the observed gain; that is, to know the percentage utilization of the basal ration. No direct determinations of this factor, however-that is, no comparisons of the results of feeding different amounts of the basal ration-were made. In his discussion of the results Kellner virtually assumes a percentage of utilization by subtracting from the total metabolizable energy of the food the average amount required for maintenance as determined by his own experiments and then comparing the energy in excess of the maintenance requirement with the resulting gain.

Differences in Live Weight.-The live weights of the animals in the Möckern experiments differed considerably in the different periods. This would probably result in differences in the requirements for maintenance, although the data at hand seem insufficient. to satisfactorily determine the relation between live weight and maintenance (see p. 441). Kellner assumes that the maintenance ration is in proportion to the two-third power of the live weight, a result which has already been shown to correspond fairly well with the results upon $\mathrm{Ox} B$, although in apparent conflict with the average results obtained on other animals.

Utilization of Basal Ration.-In order to be able to correct the results for differences in organic matter consumed and differences in live weight, it is necessary, as has just been pointed out, to know the percentage utilization of the basal ration. This Kellner assumes in assuming a maintenance ration. There are, however, serious objections to this method of procedure. First, the maintenance ration used by Kellner is an average, based on results which were obtained with a number of animals, not including all those used in the fatten- 
ing experiments, and which show a range of 13.5 per cent. of the average. Second, the computed maintenance ration is based upon experiments with coarse fodder only. We have seen reason to believe, however (pp. 388-391) that the net availability of the metabolizable energy in coarse fodders is decidedly less than in case of concentrated feeds, and that consequently more metabolizable energy would be required for maintenance on a ration composed of coarse fodder than on one containing concentrated feeds, as did Kellner's basal rations. In other words, Kellner's assumed maintenance ration is probably somewhat too large and his computed utilization of the basal ration, therefore, also somewhat too high. Third, it is by no means demonstrated that the maintenance ration of fattened as compared with unfattened animals is, as Kellner assumes, in proportion to the two-third power of the live weight.

In the absence of any direct determinations of the utilization of the basal rations, however, there seems to be no course open but to follow substantially Kellner's method of computation and assume a maintenance ration for each of the animals in proportion to the two-thirds power of its live weight during the period under consideration.

Computation of Results.-The method of computing the corrections for the differences in live weight and in the amount of the basal ration consumed may be illustrated by the same two periods which were used on pages $288-9$ to exemplify the computation of metabolizable energy, viz., Periods 4 and 7 with $\mathrm{Ox} \mathrm{H}$, on meadow hay. In Period 4, on the basal ration, the live weight was 668.9 kgs., the computed maintenance requirement 13,989.1 Cals., and the gain by the animal 2003.2 Cals. The percentage utilization therefore was as follows:

Metabolizable energy of ration...... 17,388.8 Cals.

Computed maintenance requirement . 13,989.1 "

Excess food............... 3399.7 Cals.

Gain ................... 2003.2 "

Percentage utilization........... $58.9 \%$

In Period 7 the total metabolizable energy of the ration was 26,013.0 Cals. and the gain 5643.2 Cals. Of the excess of 8624.2 
Cals. over Period 4, however, it was computed that 119.4 Cals. were due to an increased consumption of the ingredients of the basal ration, leaving 8504.8 Cals. as the metabolizable energy of the added hay. This 119.4 Cals., however, contributed to the increased gain of 3640.0 Cals. made by the animal. If we assume the percentage 58.9 just computed to apply to it, the corresponding gain would be $119.4 \times 0.589$ or 70.3 Cals., leaving 3569.7 Cals. as the gain produced by the 8504.8 Cals. of metabolizable energy derived from the meadow hay.

In Period 7, however, the animal weighed $736.0 \mathrm{kgs}$., and his computed maintenance requirement was therefore 14,909.6 Cals. of metabolizable energy, or 920.5 Cals. more than in Period 4. In other words, if he had weighed no more in Period 7 than in Period 4 , there would have been 920.5 Cals. more metabolizable energy which could have served to produce a gain of tissue. Assuming, as before, that $58.9 \%$ of this would be stored in the body, the resulting gain would have been $920.5 \times 0.589$ or 542.2 Cals. Adding this to the gain of 3569.7 Cals. just computed makes a total of 4111.9 Cals. as the computed gain to be credited to 8504.8 Cals. of metabolizable energy in the hay added, which is equivalent to a percentage utilization of 48.4 per cent. Expressed in tabular form, the results of these comparisons are as follows:-

\begin{tabular}{|c|c|c|c|c|c|c|c|c|}
\hline & 家 & 密 & 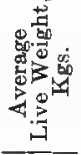 & $\begin{array}{c}\text { Metabo- } \\
\text { lizable } \\
\text { Energy, } \\
\text { Cals. }\end{array}$ & $\begin{array}{c}\text { Com- } \\
\text { puted } \\
\text { Mainte- } \\
\text { nance. } \\
\text { Cals. }\end{array}$ & $\begin{array}{c}\text { Excess } \\
\text { over } \\
\text { Mainte- } \\
\text { nance, } \\
\text { Cals. }\end{array}$ & $\begin{array}{c}\text { Energy } \\
\text { of Gain } \\
\text { (Cor- } \\
\text { rected). } \\
\text { Cals. }\end{array}$ & 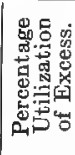 \\
\hline $\begin{array}{l}\text { Meadow Hay, VI: } \\
\text { Basal ration + hay ........ } \\
\text { Correction for organic matter }\end{array}$ & \multirow[t]{2}{*}{$\mathrm{H}$} & \multirow[t]{2}{*}{7} & \multirow[t]{2}{*}{736.0} & $\begin{array}{r}26.013 .0 \\
-119.4\end{array}$ & \multirow[t]{3}{*}{$14,909.6$} & $\begin{array}{r}11,103.4 \\
-119.4 \\
\end{array}$ & $\begin{array}{r}5,643.2 \\
-70.3\end{array} \mid$ & \\
\hline Correction for live weight... & & & & $25,893.6$ & & $\begin{array}{r}10.984 .0 \\
+920.5 \\
\end{array}$ & $\begin{array}{r}5.572 .9 \\
+542.2\end{array}$ & \\
\hline Basal ration... & \multirow[t]{2}{*}{$\mathrm{H}$} & 4 & \multirow[t]{2}{*}{668.9} & $17,388.8$ & & $\begin{array}{r}11,904.5 \\
3,399.7\end{array}$ & $\left|\begin{array}{l}6,115.1 \\
2,003.2\end{array}\right|$ & 58.9 \\
\hline Difference...... & & 4 & & $8,504.8$ & $13,989.1$ & $8,504.8$ & $4,111,9$ & 48.4 \\
\hline
\end{tabular}

Table VII of the Appendix contains the details of the computations of percentage utilization according to the above method. The results differ somewhat from those reported by Kellner,*

* Loc. cit., pp. 63, 133, 226, and 334 . 
first. because they include a correction for the differences in organic matter consumed, and second, because the energy of the gain has been corrected for the amount of nitrogen retained in the body in the same manner as the energy of the urine (compare p. 285), viz., by deducting 7.45 Cals. per gram of nitrogen. In most cases the metabolizable energy is that already computed in Tables I to VI of the Appendix, being based on actual calorimetric determinations in food and feces. In two instances (distinguished by being bracketed) the metabolizable energy has been computed by the writer from such data as are available.*

In Table VII the final results are expressed as percentages of the metabolizable energy utilized. By combining them with the results contained in the six preceding tables of the Appendix they may likewise be expressed as percentages of the gross energy of the several materials and also as energy utilized per gram of total organic matter. The summary on pp. 461-2 contains the results expressed in all of these ways.

Earlier Experiments.-The earlier respiration experiments of Henneberg \& Stohmann $\dagger$ on oxen, in 1865, while made in accordance with the experience then available, are now known to be defective in several respects. The respiratory products were determined for twelve hours only, while the same authors subsequently showed that twenty-four hours was the minimum time necessary. The food consumed on the respiration day was less than the average for the whole experiment, but how much less does not appear, and finally the methods used for the determination of the hydrocarbon gases excreted have subsequently been shown to give too low results. It seems useless therefore to enter into an elaborate computation of the results. In the later experiments of the same authors $\$$ with sheep, these sources of inaccuracy were largely re-

* The data used in these computations are as follows:

For Ox IV the average results for Periods $1 a$ and $1 b$ have been computed on the assumption that the heat values of food and excreta per gram in Period $1 b$ were the same as those determined in Period $1 a$.

For $\mathrm{Ox} \mathrm{V}$ the metabolizable energy in Period 3 has been computed by adding to that in Period $2 a 3345$ Cals. for each gram of organic matter in the starch added, this being the metabolizable energy computed for the starch in Period $2 a$.
$\dagger$ Neue Beiträge, p. 287.
$\ddagger$ Loc. cit , p 68 . 
ENERGY UTILIZED.

\begin{tabular}{|c|c|c|c|}
\hline & $\begin{array}{l}\text { Per Cent. of } \\
\text { Metabo- } \\
\text { lizable } \\
\text { Energy. }\end{array}$ & $\begin{array}{l}\text { Per Cent. of } \\
\text { Grosg } \\
\text { Energy. }\end{array}$ & $\begin{array}{l}\text { Per Grm. } \\
\text { Total } \\
\text { Organic } \\
\text { Matter. }\end{array}$ \\
\hline 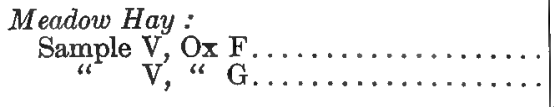 & $\begin{array}{l}40.4 \\
36.2\end{array}$ & $\begin{array}{l}16.5 \\
15.9\end{array}$ & $\begin{array}{l}0.780 \\
0.756\end{array}$ \\
\hline 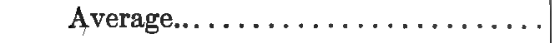 & 38.3 & 16.2 & 0.768 \\
\hline 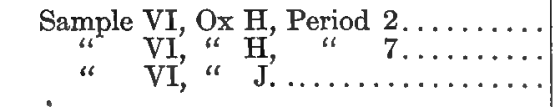 & $\begin{array}{l}50.4 \\
48.4 \\
34.8\end{array}$ & $\begin{array}{l}26.5 \\
26.1 \\
18.5\end{array}$ & $\begin{array}{l}1.266 \\
1.247 \\
0.883\end{array}$ \\
\hline Average. ${ }_{\mathrm{V}} \ldots \ldots \ldots \ldots \ldots \ldots \ldots$ & $\begin{array}{l}44.5 \\
41.4\end{array}$ & $\begin{array}{l}23.7 \\
20.0\end{array}$ & $\begin{array}{l}1.132 \\
0.950\end{array}$ \\
\hline 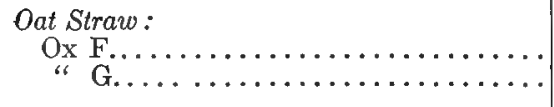 & $\begin{array}{l}38.8 \\
33.4\end{array}$ & $\begin{array}{l}14.2 \\
11.7\end{array}$ & $\begin{array}{l}0.682 \\
0.564\end{array}$ \\
\hline 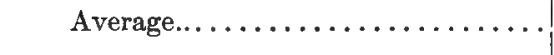 & 36.1 & 12.9 & 0.623 \\
\hline 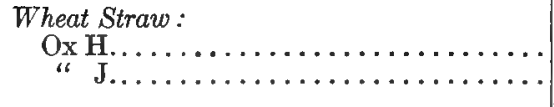 & $\begin{array}{l}10.8 \\
24.0\end{array}$ & $\begin{array}{l}3.2 \\
7.8\end{array}$ & $\begin{array}{l}0.153 \\
0.373 \\
\end{array}$ \\
\hline 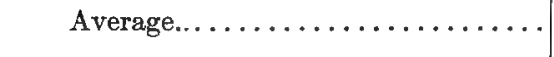 & 17.4 & 5.5 & 0.263 \\
\hline 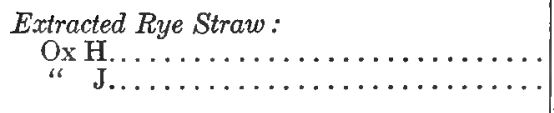 & $\begin{array}{l}67.3 \\
58.6\end{array}$ & $\begin{array}{l}51.6 \\
43.6\end{array}$ & $\begin{array}{l}2.194 \\
1.854\end{array}$ \\
\hline 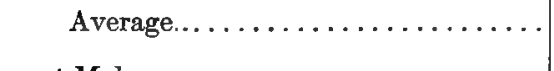 & 63.0 & 47.6 & 2.024 \\
\hline $\begin{array}{c}\text { eet Molasses : } \\
\text { Sample } \\
\text { "I, } \\
\text { " } \\
\text { II } \\
\text { II, " }\end{array}$ & $\begin{array}{l}58.5 \\
83.4 \\
50.2\end{array}$ & $\begin{array}{l}41.6 \\
65.9 \\
36.5\end{array}$ & $\begin{array}{l}1.700 \\
2.760 \\
1.529\end{array}$ \\
\hline Average $\ldots \ldots \ldots \ldots \ldots \ldots \ldots$ & 66.8 & 51.2 & 2.145 \\
\hline 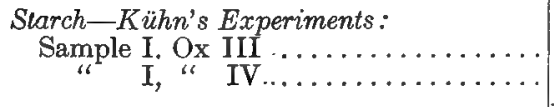 & $\begin{array}{l}50.0 \\
49.2\end{array}$ & $\begin{array}{l}35.6 \\
31.3\end{array}$ & $\begin{array}{l}1.514 \\
1.331\end{array}$ \\
\hline Average. . . . . . . . . . . . . & 49.6 & 33.5 & 1.423 \\
\hline 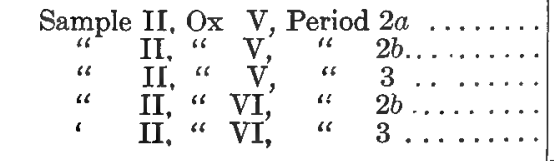 & $\begin{array}{l}53.2 \\
53.7 \\
59.7 \\
48.1 \\
46.6\end{array}$ & $\begin{array}{l}42.0 \\
40.1 \\
34.3 \\
32.6\end{array}$ & $\begin{array}{l}1.779 \\
1.699 \\
1.452 \\
1.380\end{array}$ \\
\hline $\begin{array}{l}\text { Average } \ldots \ldots \ldots \ldots \ldots \ldots \ldots \\
\text { Average } \mathrm{I} \text { and } \mathrm{i} \mathrm{I} \ldots \ldots \ldots \ldots \ldots \ldots\end{array}$ & $\begin{array}{l}50.4 \\
50.0\end{array}$ & $\begin{array}{l}37.3 \\
35.4\end{array}$ & $\begin{array}{l}1.578 \\
1.501\end{array}$ \\
\hline
\end{tabular}


ENERGY UTILIZED (Continued).

\begin{tabular}{|c|c|c|c|}
\hline & $\begin{array}{l}\text { Per Cent. of } \\
\text { Metabo- } \\
\text { lizable } \\
\text { Energy. }\end{array}$ & $\begin{array}{c}\text { Per Cent. of } \\
\text { Gross } \\
\text { Energy. }\end{array}$ & $\begin{array}{l}\text { Per Grm } \\
\text { Total } \\
\text { Organic } \\
\text { Matter }\end{array}$ \\
\hline 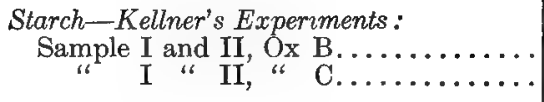 & $\begin{array}{l}65.4 \\
57.6\end{array}$ & $\begin{array}{l}31.8 \\
28.0\end{array}$ & $\begin{array}{l}1.325 \\
1.168\end{array}$ \\
\hline 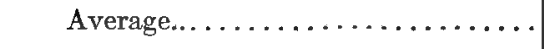 & 61.5 & 29.9 & 1.247 \\
\hline 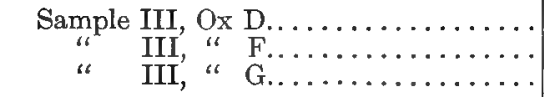 & $\begin{array}{l}53.7 \\
64.8 \\
65.8\end{array}$ & $\begin{array}{l}36.1 \\
46.2 \\
50.9\end{array}$ & $\begin{array}{l}1.500 \\
1.922 \\
2.116\end{array}$ \\
\hline 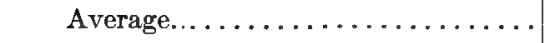 & 61.4 & 44.4 & 1.846 \\
\hline Sample IV, Ox $\underset{\text { IV }}{\mathrm{H}} \ldots \ldots \ldots \ldots \ldots \ldots \ldots \ldots \ldots \ldots \ldots \ldots \ldots \ldots$ & $\begin{array}{l}56.0 \\
54.8\end{array}$ & $\begin{array}{l}44.4 \\
39.5\end{array}$ & $\begin{array}{l}1.855 \\
1.652\end{array}$ \\
\hline $\begin{array}{l}\text { Average } \ldots \ldots \ldots \ldots \ldots \ldots \ldots \\
\text { Average III and } \mathrm{IV} \ldots \ldots \ldots \ldots \ldots \ldots\end{array}$ & $\begin{array}{l}55.4 \\
58.4\end{array}$ & $\begin{array}{l}42.0 \\
43.2\end{array}$ & $\begin{array}{l}1.754 \\
1.800\end{array}$ \\
\hline 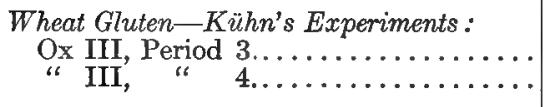 & $\begin{array}{l}45.3 \\
48.0\end{array}$ & $\begin{array}{l}37.0 \\
35.8\end{array}$ & $\begin{array}{l}2.289 \\
2.213\end{array}$ \\
\hline 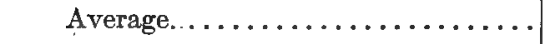 & 46.7 & 36.4 & 2.251 \\
\hline Ox IV $\ldots \ldots \ldots \ldots \ldots \ldots \ldots \ldots \ldots \ldots \ldots \ldots$ & 58.2 & 58.9 & 3.645 \\
\hline 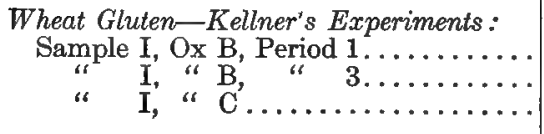 & $\begin{array}{l}36.9 \\
49.7 \\
43.2\end{array}$ & $\begin{array}{l}19.6 \\
32.6 \\
30.9\end{array}$ & $\begin{array}{l}1.115 \\
1.849 \\
1.850\end{array}$ \\
\hline Average $\ldots \ldots \ldots \ldots \ldots \ldots \ldots$ & 43.3 & 27.7 & 1.605 \\
\hline 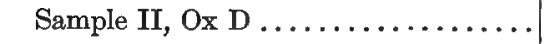 & 37.3 & 26.1 & 1.516 \\
\hline Average of $I$ and II. . . . . . . . & 40.3 & 26.9 & 1.561 \\
\hline 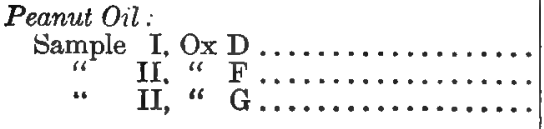 & $\begin{array}{l}51.6 \\
65.1 \\
69.4\end{array}$ & $\begin{array}{l}40.1 \\
34.2 \\
41.2\end{array}$ & $\begin{array}{l}3.811 \\
3.238 \\
3.903\end{array}$ \\
\hline Average. ..... & 67.3 & 37.7 . & 3.571 \\
\hline
\end{tabular}


moved, but the experiments were upon maintenance feeding only and afford no data for a computation of utilization.

A series of respiration experiments on sheep was made by Kern \& Wattenberg at the Göttingen-Weende Experiment Station in 1879, the results of which were reported after Kern's death by Henneberg \& Pfeiffer.* Varying quantities of nearly pure proteids (conglutin or flesh-meal) were added to a basal ration of hay and barley meal, the amount of proteids in the ration being regularly increased by about 50 grams in each of four successive periods and then similarly diminished through three more periods.

The experiments suffered from some defects in technique which later experience has remedied, the results most strikingly affected being those for the amount of methane excreted. For the first two periods no results are reported; for the remaining periods they are quite variable, and those on different days of the same period differ widely. The authors consider that their figures represent the minimum amount present, and in their final computations use the average of all the five periods as the basis for estimating the quantity of carbon excreted in this form. The amounts as actually determined showed a considerable diminution in the periods in which most proteids were fed, contrary to Kühn's results, but it is worthy of note that the average proportion of carbon dioxide to methane was not much different from that found by the latter. The determinations of carbon dioxide in the respiratory products likewise showed considerable fluctuations from day to day, but as the results are mostly the average of three or four trials of twentyfour hours each it may be assumed that these variations are more or less compensated for. The respiratory products were determined for both animals together, although ail the other data were secured for each individual. The results given on the following pages, therefore, are the totals for both animals.

It is stated that addition of proteids to the ration resulted in the diminution, and final disappearance in the middle period, of the hippuric acid of the urine, but the actual amounts present are reported only for the first and last periods. It is not possible, therefore, to make any satisfactory computation of the energy of the urine or of the proper factor for the metabolizable energy of the digested proteids of the total ration. By another method of com-

* Jour. f. Landw., 38, 215. 
putation, however, it seems possible to secure an approximate idea of the relation of added food to gain.

By subtracting from the food digested in Periods II-VI the average amount digested in Periods $\mathrm{I}$ and VII, on the basal ration, we find the amounts of added food, consisting chiefly of proteids. Reckoning the metabolizable energy of the added proteids at 4.958 Cals. per gram (compare p. 317), that of the crude fiber and nitrogen-free extract at 3.674 Cals., and that of the ether extract at 8.322 Cals., we get the approximate metabolizable energy of the added food, and can compare it with the energy of the corresponding gain. Thus for Period II we have the following:

DIGESTED.

\begin{tabular}{|c|c|c|c|c|}
\hline & $\begin{array}{l}\text { Protein,* } \\
\text { Grms. }\end{array}$ & $\begin{array}{l}\text { Crude } \\
\text { Fiber, } \\
\text { Grms. }\end{array}$ & $\begin{array}{c}\text { Nitrogen- } \\
\text { free } \\
\text { Extract, } \\
\text { Grms. }\end{array}$ & $\begin{array}{l}\text { Ether } \\
\text { Extract } \\
\text { Grms. }\end{array}$ \\
\hline $\begin{array}{l}\text { Period II } \ldots \ldots \ldots \ldots \\
\text { Periods I and VII . . . . . } \ldots\end{array}$ & $\begin{array}{l}211.33 \\
101.05\end{array}$ & $\begin{array}{l}280.77 \\
277.91\end{array}$ & $\begin{array}{l}643.22 \\
633.12\end{array}$ & $\begin{array}{l}20.88 \\
21.60\end{array}$ \\
\hline Difference............. & 110.28 & 2.86 & 10.10 & -0.72 \\
\hline $\begin{array}{c}\text { Equivalent metabolizable } \\
\text { energy................ }\end{array}$ & $\begin{array}{l}\text { Cals. } \\
546.8\end{array}$ & $\begin{array}{l}{ }^{1} \\
\text { Cals. } \\
47.6\end{array}$ & & $\begin{array}{l}\text { Cals. } \\
-6\end{array}$ \\
\hline
\end{tabular}

* Protein of basal ration and of feces equals $N \times 6.25$; that of conglutin or flesh-meal equals its total organic matter.

GAIN.

\begin{tabular}{|r|c|c|}
\hline & Protein Grms. & Fat. Grms. \\
\hline Period II ......... & $\mathbf{1 5 . 0 0}$ & 69.27 \\
Periods I and ViI. .. & $\mathbf{6 . 8 5}$ & 19.66 \\
\cline { 2 - 2 } & $\mathbf{8 . 1 5}$ & 49.61 \\
Difference ........ & Cals. & Cals. \\
Equivalent energy & 46.3 & 471.5 \\
\hline
\end{tabular}

The figures for the gain are those given by the authors, based on the assumption of a uniform excretion of methane throughout the experiments; the gain of protein includes that contained in the wool produced. The animals gained slightly in weight, in addition to the growth of wool. Computed on this basis, the percentage of the energy of the added food which was utilized was as follows: 


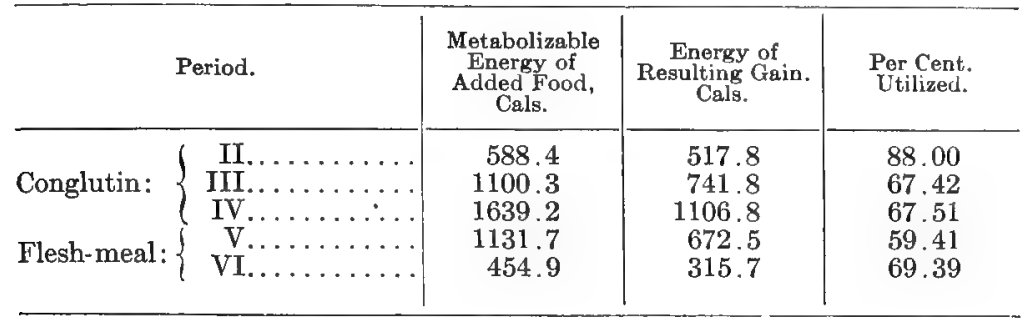

A computation based on the observed amounts of methane would affect the above figures in two ways. First, if the added proteids diminished the production of methane, this was equivalent to an increase in the apparent metabolizable energy of the food, and the figures for the latter must be correspondingly increased. Second, the gain of fat will also appear relatively greater in the intermediate periods, II-VI, and the figures for the energy of the gain must also be increased. Computed on this basis the results are:

\begin{tabular}{|c|c|c|c|c|}
\hline & Period. & $\begin{array}{c}\text { Energy of } \\
\text { Added Food, } \\
\text { Cals. }\end{array}$ & $\begin{array}{c}\text { Energy of } \\
\text { Resulting Gain, } \\
\text { Cals. }\end{array}$ & $\begin{array}{l}\text { Per Cent. } \\
\text { Utilized. }\end{array}$ \\
\hline Conglutin: \{ & $\left\{\begin{array}{l}\text { II... } \\
\text { III.. } \\
\text { IV.. } \\
\text { V... } \\
\text { VI.. }\end{array}\right.$ & $\begin{array}{r}715.4 \\
1245.8 \\
1902.3 \\
1288.2 \\
582.1\end{array}$ & $\begin{array}{r}605.7 \\
842.4 \\
1288.8 \\
780.7 \\
403.6\end{array}$ & $\begin{array}{l}84.68 \\
67.63 \\
67.76 \\
60.59 \\
69.33\end{array}$ \\
\hline
\end{tabular}

No obvious explanation of the exceptionally high results obtained in Period II presents itself. Those of the remaining periods do not seem to indicate any considerable differences in the utilization of different quantities. The figures are notably higher than those computed from the Möckern experiments, but in view of the uncertainties attaching to them too much stress should not be laid on this fact.

\section{Discussion of Results.}

As was pointed out at the beginning of this section, and as was further apparent in considering the results of experiments upon carnivora, our knowledge of the net availability of the energy of feeding-stuffs and nutrients is too imperfect to permit the experi- 
mental results above detailed to be discussed from the standpoint of the percentage utilization of the net available energy.

Furthermore, even confining ourselves to a consideration of the utilization of the metabolizable energy of the food, we have already seen that the recorded results upon carnivorous animals show such wide divergencies as to render it difficult if not impossible to draw any quantitative conclusions from them.

For the present, accordingly, our discussion of the utilization of energy must be confined chiefly to the results which have been reached with herbivora, and in the main to the Möckern experiments, and we must content ourselves with an attempt to trace the relations between metabolizable energy and energy utilized, or, to look at the subject from the other point of view, with determining the proportion of the metabolizable energy of the food which is expended in the combined work of digestion, assimilation, and tissue building. From the practical standpoint this is of course the important thing, since either form of expenditure of energy constitutes, in the economic aspect of the matter, a waste, but it is nevertheless to be regretted that it is at present impossible to further analyze this waste.

Influence of Amount of Food.-As in the discussion of net availability in Chapter XII, we have thus far assumed the energy utilized to be a linear function of the net available or of the metabolizable energy of the food. Before proceeding further it becomes important to consider how far this assumption is justified by the facts on record.

CARnivora.- Of the experiments upon carnivora recorded on preceding pages, those of Rubner with different amounts of meat, when computed by his method (that is, assuming an availability of 100 per cent. below the maintenance point, as on p. 450), appear to indicate that the utilization above that point is constant. If, however, a lower percentage of availability is assumed, as on p. 451, this constancy disappears. None of the other results there summarized seem suitable for discussion from this point of view.

Swine.-If in the experiments of Meissl, Strohmer \& Lorenz, as computed on p. 454 , we express the estimated metabolizable energy of the excess food as a percentage of the fasting metabolism, we have the following comparison of the percentage utilization with 
the relative amount of excess food, to which may be added Kornauth \& Arche's results similarly computed:

\begin{tabular}{|c|c|c|}
\hline & $\begin{array}{c}\text { Excess Over } \\
\text { Fasting } \\
\text { Metabolism, } \\
\text { Per Cent. }\end{array}$ & $\begin{array}{l}\text { Percentage } \\
\text { Utilization. }\end{array}$ \\
\hline \multicolumn{3}{|l|}{ Meissl: } \\
\hline Experiment I. & 133 & 80.7 \\
\hline "6 & 250 & 75.2 \\
\hline III. & 74 & 70.9 \\
\hline "IV. & 180 & 67.1 \\
\hline \multicolumn{3}{|l|}{ Kornauth \& Arche: } \\
\hline Experiment III. & 126 & 71.7 \\
\hline " IV.. & 129 & 65.3 \\
\hline
\end{tabular}

While there is some variation in the percentage utilization, as would naturally be expected in experiments with different animals, the range in the relative amount of excess food is much greater and there is no indication of a connection between the two.

Rominants. - The earlier Möckern experiments by G. Kühn include one upon wheat gluten and two upon starch in which two different quantities were added to the basal ration of the same animal. The final results were as follows:

\begin{tabular}{c|c|c|c|c}
\hline Wheat gluten.... & Animal. & Period & $\begin{array}{c}\text { Added to } \\
\text { Aasal Ration, } \\
\text { Kggs }\end{array}$ & $\begin{array}{c}\text { Percentage } \\
\text { Utilization } \\
\text { of Metaboliz- } \\
\text { able Energy. }\end{array}$ \\
\hline Starch.......... & III & 3 & 0.68 & 45.3 \\
III & 4 & 1.36 & 48.0 \\
V & $2 a$ & 2.0 & 53.2 \\
V & $2 b$ & 2.0 & 53.7 \\
V & 3 & 3.5 & 59.7 \\
VI & $2 b$ & 2.0 & 48.1 \\
VI & 3 & 3.5 & 46.6 \\
& & &
\end{tabular}

These results do not indicate that any material effect is exerted upon the utilization of the metabolizable energy of the food by the amount consumed, since the differences are small in themselves and in both directions.

The results, reported by Pfeiffer, of experiments upon the addition of varying amounts of proteids to a basal ration, as computed by the writer (p. 465), likewise show a fairly constant percentage 
utilization of the energy of the proteids used, with the exception of the strikingly higher result of the first period.

A similar conclusion may be drawn from a study of the Möckern results as a whole, as recorded in Table VII of the Appendix. While the computed percentages in each series vary more or less in the different experiments, the differences are in most cases not large and appear to bear no relation either to the total quantity of food given or to the amount of the particular food under experiment which was added to the basal ration, but to be due rather to individual differences in the animals. This is strikingly shown in the following table, in which the results upon hay, wheat gluten, and starch are arranged in the order of the percentage utilization:

\begin{tabular}{c|c|c|c|c|c}
\hline Feeding-stuff. & Animal. & Period. & $\begin{array}{c}\text { Metaboliz- } \\
\text { able Energy } \\
\text { of Added } \\
\text { Food, } \\
\text { Cals. }\end{array}$ & $\begin{array}{c}\text { Total Excess } \\
\text { Over Com- } \\
\text { puted Main- } \\
\text { tenance, } \\
\text { Cals. }\end{array}$ & $\begin{array}{c}\text { Percentage } \\
\text { Utilization } \\
\text { of Metabo- } \\
\text { lizable } \\
\text { Energy. }\end{array}$ \\
\hline Meadow hay ......... & J & 2 & 7875 & 12,192 & 34.8 \\
Wheat gluten ......... & G & 2 & 5726 & 9,780 & 36.2 \\
F & 1 & 5506 & 10,184 & 40.4 \\
H & 7 & 8505 & 11,905 & 48.4 \\
H & 2 & 7875 & 11,275 & 50.4 \\
B & 1 & 4483 & 15,129 & 36.9 \\
D & 4 & 5713 & 17,373 & 37.3 \\
C & 3 & 6033 & 19,635 & 43.2 \\
III & 3 & 2913 & 8,982 & 45.3 \\
III & 4 & 5332 & 11,401 & 48.0 \\
B & 3 & 5507 & 16,153 & 49.7 \\
IV & 3 & 3645 & 7,132 & 58.2 \\
VI & 3 & 8264 & 12,364 & 46.6 \\
VI & $2 b$ & 5038 & 9,138 & 48.1 \\
IV & 2 & 4350 & 3,411 & 49.2 \\
III & 2 & 4998 & 6,592 & 50.0 \\
V & $2 a$ & 5425 & 8,821 & 53.2 \\
V & 3 & 9658 & 13,054 & 59.7 \\
D & 2 & 4420 & 16,080 & 53.7 \\
J & 3 & 4826 & 9,142 & 54.8 \\
H & 3 & 6668 & 10,068 & 56.0 \\
C & 2 & 3027 & 16,829 & 57.6 \\
F & 4 & 5009 & 9,686 & 64.8 \\
B & 2 & 3291 & 13,937 & 65.4 \\
G & 4 & 5387 & 9,441 & 65.8 \\
\hline
\end{tabular}

But while this is true of each series by itself, a comparison of the two series upon starch leads to a different conclusion. In Kühn's experiments the basal rations consisted largely or exclusively of coarse fodder. In Kellner's experiments the starch was 
added to a materially heavier basal ration containing considerable grain and therefore already tolerably rich in starch and other carbohydrates. In spite of the smaller average amounts of starch added, then, Kellner's results in a sense represent the percentage utilization of larger quantities of starch than do Kühn's; that is, they represent the utilization of starch at a greater distance above the maintenance ration. The average utilization (pp. 461-2) was-

Kühn's experiments . ........... 50.0 per cent.

Kellner's experiments, moderate rations . . 5 58.4 " " " "

" " heavy rations.....61.5 " "

It would appear, then, from these figures that the metabolizable energy of starch was more fully utilized in rations containing a relatively large quantity of it. At least a partial explanation of this seems to be afforded by the variations in the production of hydrocarbons (methane). As was mentioned in discussing the metabolizable energy of starch, the conditions in Kühn's experiments were such as to permit a considerable proportion of the starch to undergo the methane fermentation, while the more abundant supply of it in Kellner's experiments resulted in reducing, or in some cases wholly suppressing. this fermentation of the starch. The effect of this, as there pointed out. was to make the metabolizable energy per gram greater in Kellner's than in Kühn's experiments, but it has also another result. As we have seen, the methane fermentation constitutes part of the work of digestion, in the general sense in which that term is here employed, the amount of the latter being measured by the heat evolved. This amount being less in Kellner's than in Kühn's experiments, the net availability of the metabolizable energy of the starch should be greater, and, other things being equal, the storage of energy (gain of tissue) should also be greater.

Kellner * computes that for each 100 grams of starch digested there was produced, on the average, methane corresponding to the following amounts of carbon:

In Kühn's experiments............. 3.0 grams

In Kellner's experiments. . ......... 2.3 "

* Loc. cit., p. 423. 
An approximate computation of the probable differences in the heat evolved by the fermentation, based on such data as are available, gives as a result 0.159 Cal. per gram of starch, or somewhat more than one-half the difference in average utilized energy, viz., 0.265 Cal. per gram. The data on which the computation is based, however, are too uncertain to allow us to attach very much value to the results, except perhaps as an indication that the supposed cause of the difference in the utilization of the energy is insufficient to fully account for the effect.

Conclusions.- It cannot be claimed that the above results are sufficiently extensive or exact to permit final conclusions to be drawn, but their general tendency seems to be in favor of the hypothesis that the proportion of energy utilized is substantially independent of the quantity of food, provided that the changes in the latter are not so great as to modify the course of the fermentations in the digestive tract. The results upon starch just considered seem to indicate that if the variations in quantity or make-up of the ration are pushed beyond that point, a difference in the proportion of the energy utilized may be caused by a difference in the digestive work; in other words, that it is the availability that is modified rather than the proportion of the available energy which is recovered as gain. While not denying that the latter function may be also modified, either directly as the effect of varying amounts of food, or indirectly by changes in the chemical nature of the substances resorbed from the digestive tract under varying conditions of fermentation, it seems probable that the main effect is that upon availability.

It is to be observed that the rations used in these experiments, while not heavy fattening rations, still produced very fair gains. The experimental periods were comparatively short and hence the testimony of the live weight itself is liable to be misleading. Taking the actual gains of fat and proteids as shown by the respiration experiments, however, and comparing them with the composition of the increase of live weight in fattening as determined by Lawes \& Gilbert, it appears that the total gain per day was equivalent to from 0.9 to 2.5 pounds gain in live weight per day in the experiments on coarse fodder, while in those upon concentrated feeds the corresponding range is from 1 to 3 pounds. 
It may be remarked further that the rations in Kühn's experiments differed materially from those ordinarily used in practice, both as to their make-up and their very wide nutritive ratio, so that the conditions may fairly be regarded as in a sense abnormal. Kellner's rations represent more nearly normal conditions, and they fail, as we have seen, to give any clear indications of an influence of amount of food upon the proportion of energy utilized. Whether other feeding materials show a behavior analogous to that of starch, future investigations must decide. In the meantime we are apparently justified in discussing such results as are now on record upon the provisional hypothesis that, within reasonable limits, the utilization of energy is independent of the amount of food, or, in other words, is a linear function.

Influence of Thermal Environment. - The influence of the thermal environment of the animal upon its heat production and upon the net availability of the energy of the food has already been fully discussed in previous pages and needs only a brief consideration here.

Ruminants. - We have already found reason to think that in ruminants the heat production on the ordinary maintenance ration is in excess of the needs of the body. Kühn's and Kellner's results show us that from 25 to 72 per cent. of the metabolizable energy of the food supplied in excess of the maintenance requirement was converted into heat, so that the heat production was frequently increased 40 or 50 per cent. above that which was observed on the maintenance ration. Under these circumstances we can hardly suppose that any moderate changes in the thermal environment would sensibly affect either the availability of the food energy or its percentage utilization.

The writer is not aware of any exact determinations of the influence of the thermal environment upon the heat production of fattening ruminants, but the above conclusion is in harmony with the practical experience of many feeders that moderate exposure to cold is no disadvantage, but rather an advantage in maintaining the health and appetite of the animals, and it appears also to have the support of not a few practical feeding trials.*

* Compare Henry, "Feeds and Feeding," second edition, p. 364, and Waters, Bulletin Mo. Bd. Agr., September, 1901, p. 23. 
Naturally this can be true only within limits, and exposure to very low temperatures, especially in a damp climate, and particularly to cold rains, causing a large expenditure of heat in the evaporation of water from the surface of the body, may very well pass the limit and cause an increase in the metabolism simply to maintain the temperature of the body. Finally, the time element, as pointed out on p. 439 , is one to be taken into consideration.

SwINE.-As was remarked on p. 435, the work of digestion is doubtless less with the swine than in ruminants, on account of the more concentrated nature of his food, and as was shown on p. 438, the maintenance requirement appears to be affected by the thermal environment. The same reason would tend to make fattening swine more susceptible to this influence than fattening ruminants. This conclusion is borne out by the experiments of Shelton * at the Kansas Agricultural College, who found that swine kept in an open yard during rather severe weather required 25 per cent. more corn to make a given gain than those sheltered from extreme cold.

Influence of Character of Food.-Attention was called in the previous chapter to the fact that the expenditure of energy in the digestion and assimilation of the food is largely dependent upon the nature of that food, but as was there pointed out, we have few quantitative determinations of the differences. Experiments of the class now under consideration show marked variations in the proportion of the metabolizable energy of different foods which is utilized, and we should naturally be inclined to ascribe these variations to differences in the work of digestion and assimilation rather than to differences in the physiological processes involved in tissue production.

The data recorded in the foregoing pages constitute only a beginning of the study of the utilization of the energy of feedingstuffs, but a brief consideration of the main results will prove at least suggestive.

Concentrated Feeding-stuffs.-As we saw in connection with the discussion of the metabolizable energy of feeding-stuffs in Chapter X, the Möckern experiments, to which we owe the larger share of our present knowledge regarding the metabolism of energy in farm animals, were made for the purpose of comparing the * Rep. Prof. of Agriculture, 1883. 
principal classes of nutrients rather than commercial feeding-stuffs. Accordingly such representative materials as starch, oil, and gluten were largely used, and we have as yet but few determinations either of the metabolizable energy of ordinary concentrated feedingstuffs or of its percentage utilization. We have already considered to some extent the advantages and disadvantages resulting from making the pure nutrients, on the one hand, or actual feeding-stuffs, on the other, the starting-point for investigations. Passing over this question for the present, we may conveniently group together here such results as are on record for materials other than coarse fodders.

Starch.-Starch, as a representative of the readily digested carbohydrates, has, as we have seen, received a large share of attention. The results obtained are tabulated in the Appendix, and have already been partially considered in their bearings upon the influence of amount of food. It was there noted that the earlier series of experiments by $K u ̈ h n$, in which the starch was added to a ration of coarse fodder only, gave results differing decidedly from those obtained later by Kellner from the addition of starch to a mixed fattening ration. Among the latter experiments, moreover, were two (animals $\mathrm{B}$ and $\mathrm{C}$ ) which were exceptional in that very large total amounts of starch were contained in the ration, relatively large amounts escaping digestion, while none of the added starch underwent the methane fermentation.

A clear image of the fate of the total potential energy supplied to the organism in the starch is best obtained by a study of its percentage distribution among the several excreta, the work of digestion, assimilation, and tissue building, and the gain secured, as in the table on page 474 , in which each of the three sets of experiments indicated above is given separately. The figures for the work of digestion, etc., are, of course, obtained by difference.

As pointed out in the discussion of metabolizable energy, the percentage of the gross energy carried off in the feces includes, as here computed, not only the energy of the undigested portion of the starch itself, but also that of the portion of the basal ration which escaped digestion under the influence of the starch. This is especially true of Kellner's experiments with moderate rations, in which little or no starch could be detected in the feces. Similarly, the 
PERCENTAGL DISTRIBUTION OF GROSS ENERGY OF STARCH.

\begin{tabular}{|c|c|c|c|c|c|c|c|}
\hline - & 营 & 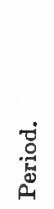 & $\underset{\text { Feces. }}{\text { In }}$ & $\underset{\text { Urine. }}{\text { In }}$ & 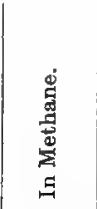 & $\begin{array}{c}\text { Work of } \\
\text { Diges- } \\
\text { tion, } \\
\text { Assimi- } \\
\text { lation, } \\
\text { and } \\
\text { Tissue } \\
\text { Build- } \\
\text { ing. }\end{array}$ & $\underset{\text { Gain. }}{\text { In }}$ \\
\hline \multirow{6}{*}{ Kühn's experiments. } & III & 2 & 20.02 & -1.29 & 10.06 & 35.61 & 35.60 \\
\hline & IV & 2 & 25.29 & -1.01 & 12.01 & 32.41 & 31.30 \\
\hline & V & $2 a$ & 8.82 & 1.03 & 11.20 & 36.95 & 42.00 \\
\hline & $\mathrm{V}$ & $2 b$ & 15.73 & -0.27 & 9.86 & 34.58 & 40.10 \\
\hline & VI & $2 b$ & 22.49 & -2.61 & 8.86 & 36.96 & 34.30 \\
\hline & VI & 3 & 19.03 & -0.88 & 11.87 & 37.38 & 32.60 \\
\hline \multirow{3}{*}{$\begin{array}{l}\text { Kellner's experiments: } \\
\text { Moderate rations... }\end{array}$} & $\mathrm{D}$ & 2 & 29.99 & -3.27 & 6.08 & 31.10 & 36.10 \\
\hline & $\mathbf{F}$ & 4 & 16.42 & 0.73 & 11.41 & 25.24 & 46.20 \\
\hline & $\mathrm{G}$ & 4 & 13.35 & 0.35 & 8.98 & 26.42 & 50.90 \\
\hline & $\mathrm{H}$ & 3 & 15.72 & -2.32 & 7.38 & 34.82 & 44.40 \\
\hline \multirow{2}{*}{\multicolumn{8}{|c|}{ Kellner's experiments: }} \\
\hline & & & & & & & \\
\hline Heavy rations. . & B & 2 & 59.60 & -3.25 & -4.96 & 16.82 & 31.80 \\
\hline Averages: & $\mathrm{C}$ & 2 & 52.22 & -0.89 & -0.01 & 26.68 & 28.0 \\
\hline Kühn's experiments .. & & & 19.59 & -0.92 & 10.74 & 35.19 & 35.40 \\
\hline Kellner's experiments: & & & & & & & \\
\hline Moderate rations.... & & & 17.61 & -0.66 & 9.21 & 30.64 & 43.20 \\
\hline Heavy rations. . & & & 55.91 & -2.07 & -2.49 & 18.75 & 29.90 \\
\hline
\end{tabular}

negative losses in the urine and, in two cases, in the methane mean, of course, that under the influence of starch the metabolic or other processes were so modified that less of the potential energy of the basal ration was lost through these channels. The starch, so to speak, borrowed energy from the basal ration. In brief, the figures of the table give us a picture of the aggregate net results of supplying 100 units of additional potential energy in the form of starch, or in other words, of the "apparent" utilization.

As between Kühn's results and those of Kellner upon moderate rations, the chief difference, as already noted, is the less evolution of methane in the latter and, apparently as, in part, a consequence of this, the smaller expenditure of energy in the work of digestion, etc. Combined with the slightly smaller loss in the feces, this results in making the energy utilized a much larger percentage of the gross energy. Apparently Kellner's figures correspond most nearly to normal conditions of feeding and may be taken to represent the average utilization of starch under these circumstances. 
In Kellner's two experiments on heavy rations the enormous losses in the feces cut down the percentage utilization to a very low figure and thus render difficult a direct comparison with the other averages.

While the above form of stating the results appears the simplest and most direct, it is of interest also to eliminate the influence of varying digestibility by computing the percentage distribution of the gross energy of the apparently digested portion of the starch. This is particularly the case since Kellner's computations of his experiments are made in a somewhat similar way. Combining the data given on p. 461, regarding the percentages of metabolizable energy utilized, with those on p. 301 for the energy of the apparently digested matter, we have the following:

DISTRXBUTION OF ENERGY OF APPARENTTLY DIGESTED STARCH.

\begin{tabular}{|c|c|c|c|c|}
\hline & $\begin{array}{l}\text { In Urine. } \\
\text { Per Cent. }\end{array}$ & $\begin{array}{l}\text { In Methane. } \\
\text { Per Cent. }\end{array}$ & $\begin{array}{c}\text { Work of } \\
\text { Digestion, } \\
\text { Assimilation, } \\
\text { and Tissue } \\
\text { Building. } \\
\text { Per Cent. }\end{array}$ & $\begin{array}{l}\text { In Gain. } \\
\text { Per Cent. }\end{array}$ \\
\hline $\begin{array}{l}\text { Kühn's experiments ........ } \\
\text { Kellner's experiments: }\end{array}$ & -1.19 & 13.42 & 43.89 & 43.88 \\
\hline $\begin{array}{l}\text { Moderate rations. . . . . . . . } \\
\text { Heavy rations ....... }\end{array}$ & $\begin{array}{l}-0.92 \\
-4.95\end{array}$ & $\begin{array}{r}11.12 \\
-6.15\end{array}$ & $\begin{array}{l}37.36 \\
42.77\end{array}$ & $\begin{array}{l}52.44 \\
68.33\end{array}$ \\
\hline
\end{tabular}

Kellner's computations are made in a different manner.* Omitting in the computation of metabolizable energy the correction for nitrogen gained or lost, he compares the period in which starch was fed with that on the basal ration substantially as has been done above. He then, however, introduces a correction for the influence of the starch upon the digestibility of the basal ration. For example, comparing Periods 3 and 4 on $\mathrm{Ox} \mathrm{H}$, he finds in the manner shown on p. 307 , Chapter $X$, that the equivalent of 820 Cals. less of the basal ration was digested in the period in which starch was added to it, while there is a further correction of 112 Cals. to be made for the less amount of organic matter of the basal ration consumed in Period 3, making a total difference of 932 Cals. Of the gross energy of the basal ration, 79.9 per cent. was found to be met-

* Compare Landw. Vers. Stat., 53, 450. 
abolizable, so that the above difference in gross energy would correspond to 745 Cals. of metabolizable energy. Of the metabolizable energy of the basal ration in excess of maintenance, 59.6 per cent. was recovered in the gain. If, then, the differences in organic matter consumed and in the digestibility of the basal ration had not offset some of the effect of the starch in Period 3, there would have been 745 Cals. more of metabolizable energy disposable from the basal ration, and presumably the gain resulting from this would have been 59.6 per cent. of 745 Cals., or 444 Cals. We have, then, by this method the following:

\begin{tabular}{|c|c|c|}
\hline & $\begin{array}{c}\text { Metabolizable } \\
\text { Energy Above } \\
\text { Maintenance, } \\
\text { Cals. }\end{array}$ & $\begin{array}{c}\text { Energy of } \\
\text { Gain, } \\
\text { Cals. }\end{array}$ \\
\hline 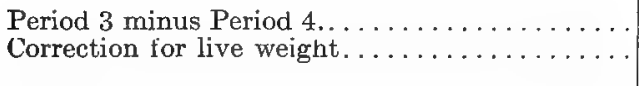 & $\begin{array}{r}6667 \\
67 \\
\end{array}$ & $\begin{array}{r}3752 \\
40\end{array}$ \\
\hline $\begin{array}{l}\text { Correction for organic matter and for decreased } \\
\text { digestibility } \ldots \ldots \ldots \ldots \ldots \ldots \ldots \ldots \ldots \ldots \ldots \ldots \ldots\end{array}$ & $\begin{array}{r}6600 \\
745\end{array}$ & $\begin{array}{r}3712 \\
444\end{array}$ \\
\hline Percentage utilization...... & $\begin{array}{r}7345 \\
\ldots \ldots\end{array}$ & $\begin{array}{l}4156 \\
56.6 \%\end{array}$ \\
\hline
\end{tabular}

Kellner's results, then, assuming that the corrections are accurate, represent respectively the metabolizable and the utilizable energy of the digested matter of the starch itself, while the results as computed on the preceding pages represent, as was there pointed out, a balance between the various negative and positive effects of the addition of starch. In other words, Kellner attempts to compute the real as distinguished from the apparent utilization of the energy of the starch. The comparison on the opposite page of the percentages obtained in this way with those computed on p. 461 will therefore be of interest.

Kellner also computes by his method the distribution of the gross energy of the digested starch in Kühn's experiments and in his own experiments on moderate rations. As calculated in Chapter X, pp. 325-6, the average loss of potential energy in methane was 12.7 per cent. in Kühn's experiments, and 10.11 per cent. in Kellner's, while none of the potential energy of the digested starch passed 
UTILIZATION OF METABOLIZABLE ENERGY OF STARCH.

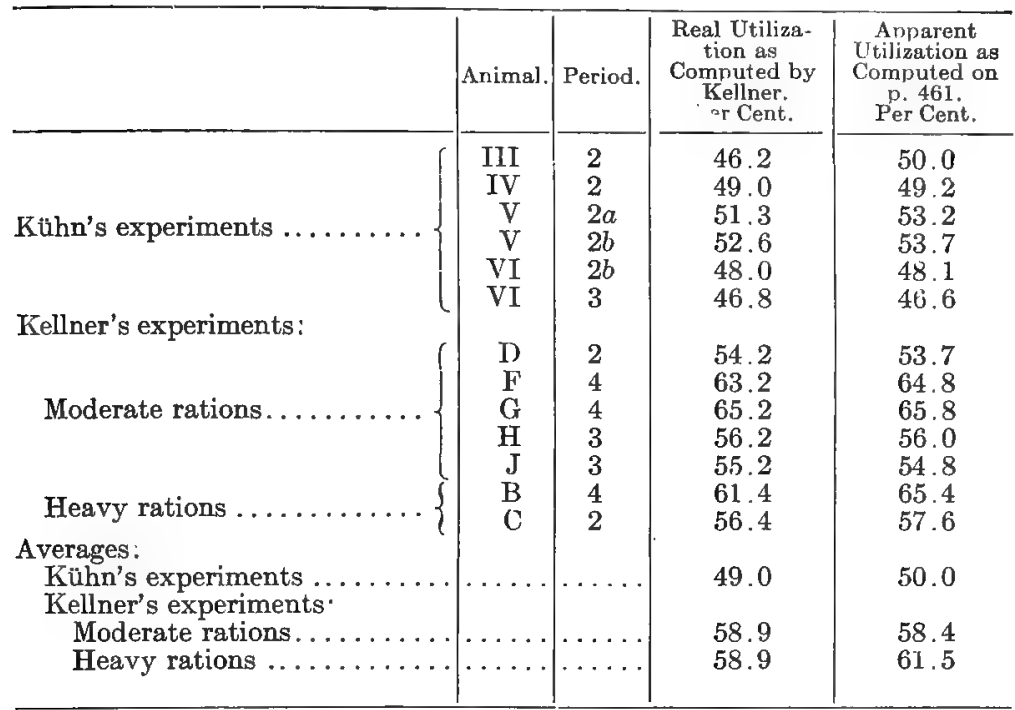

into the urine. In the two cases, then, 87.30 per cent. and 89.89 per cent. respectively of the potential energy of the digested starch was metabolizable. Of this metabolizable energy 49.0 per cent. and 58.9 per cent. respectively was recovered in the gain. Combining these figures we have-

DISTRIBUTION OF ENERGY OF DIGESTED STARCH.

\begin{tabular}{|c|c|c|c|c|}
\hline & $\begin{array}{l}\text { In Urine. } \\
\text { Per Cent. }\end{array}$ & $\begin{array}{l}\text { In Methane } \\
\text { Per Cent }\end{array}$ & $\begin{array}{l}\text { Work of } \\
\text { Digestion. } \\
\text { Assimilation. } \\
\text { and Tissue } \\
\text { Building } \\
\text { Per Cent. }\end{array}$ & $\begin{array}{l}\text { In Gain } \\
\text { Per Cent. }\end{array}$ \\
\hline $\begin{array}{l}\text { Kühn's experiments........... } \\
\text { Kellner's experiments; } \\
\quad \text { Moderate rations........... }\end{array}$ & $\begin{array}{l}0 \\
0\end{array}$ & 12.70 & 44.52 & $\begin{array}{l}42.78 \\
52.94\end{array}$ \\
\hline
\end{tabular}

The final results for the energy recovercd in the gain of tissue, whether. expressed as a percentage of metabolizable energy or of energy of digested matter, are substantially the same numerically as those reached by the former method of computation, but this agreement is purely accidental, and the significance of the 
figures is essentially different, as already explained. From the results last given, assuming the gain of energy to have been entirely in the form of fat, Kellner * computes that the conversion of starch into fat in cattle takes place according to the following scheme:

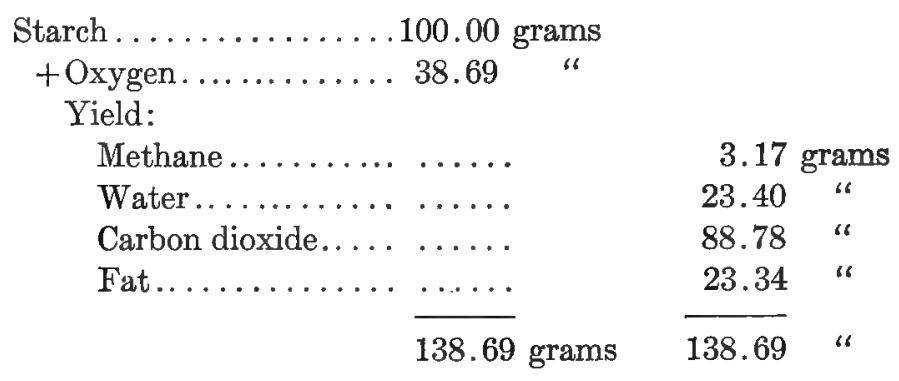

Oil.--Applying to Kellner's three experiments upon the addition of oil to a basal ration the same method of computation which was used for the starch-that is, computing the apparent utilizationwe have the results shown in the two following tables:

DISTRIBUTION OF GROSS ENERGY OF OIL.

\begin{tabular}{|c|c|c|c|c|c|c|c|}
\hline & 离 & 苞 & $\begin{array}{l}\text { In Feces. } \\
\text { Per Cent. }\end{array}$ & $\begin{array}{l}\text { In Urine. } \\
\text { Per Cent. }\end{array}$ & $\begin{array}{c}\text { In } \\
\text { Methane. } \\
\text { Per Cent. }\end{array}$ & $\begin{array}{l}\text { Work of } \\
\text { Digestion. } \\
\text { Assimila: } \\
\text { tion and } \\
\text { Tissue } \\
\text { Building. } \\
\text { Per Cent. }\end{array}$ & $\begin{array}{l}\text { In Gain. } \\
\text { Per Cent. }\end{array}$ \\
\hline 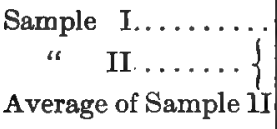 & \begin{tabular}{|c|}
$\mathrm{D}$ \\
$\mathrm{F}$ \\
$\mathrm{G}$ \\
$\cdots$
\end{tabular} & $\begin{array}{c}\mathbf{3} \\
\mathbf{5} \\
\mathbf{5} \\
\cdots\end{array}$ & $\begin{array}{l}24.34 \\
64.77 \\
41.00 \\
52.89\end{array}$ & $\begin{array}{r}-1.08 \\
-1.19 \\
1.37 \\
0.09\end{array}$ & $\begin{array}{l}-1.02 \\
-16.10 \\
-\quad 1.76 \\
-8.93\end{array}$ & $\begin{array}{l}37.66 \\
18.32 \\
18.19 \\
18.25\end{array}$ & $\begin{array}{l}40.10 \\
34.20 \\
41.20 \\
37.70\end{array}$ \\
\hline
\end{tabular}

DISTRIBUTION OF ENERGY OF APPARENTLY DIGESTED OIL.

\begin{tabular}{|c|c|c|c|c|c|c|}
\hline & Animal. & Period. & $\begin{array}{l}\text { In Urine. } \\
\text { Per Cent. }\end{array}$ & $\begin{array}{c}\text { In } \\
\text { Methane } \\
\text { Per Cent. }\end{array}$ & $\begin{array}{l}\text { Work of } \\
\text { Digestion } \\
\text { Assimila- } \\
\text { tion and } \\
\text { Tissue } \\
\text { Building } \\
\text { Per Cent. }\end{array}$ & $\begin{array}{l}\text { In Gain. } \\
\text { Per Cent. }\end{array}$ \\
\hline $\begin{array}{c}\text { Sample I. . . . . . . . . . . . . . . } \\
\text { II } \\
\text { Average for Sample II }\end{array}$ & $\begin{array}{c}\mathrm{O} \\
\mathrm{F} \\
\mathrm{G} \\
\ldots \ldots\end{array}$ & $\begin{array}{r}3 \\
5 \\
5 \\
\ldots \\
\ldots\end{array}$ & $\begin{array}{r}-1.42 \\
-3.38 \\
2.32 \\
-0.53\end{array}$ & $\begin{array}{r}1.34 \\
-45.69 \\
-3.01 \\
-24.35\end{array}$ & $\begin{array}{l}49.76 \\
52.01 \\
30.83 \\
41.42\end{array}$ & $\begin{array}{l}53.00 \\
97.06 \\
69.86 \\
83.46\end{array}$ \\
\hline
\end{tabular}


As was noted in the discussion of metabolizable energy in Chapter $\mathrm{X}$, the results on $\mathrm{Ox} \mathrm{F}$ appear to be exceptional, but those upon the other two show considerable differences, and it is evident that further investigation will be necessary to obtain satisfactory data upon the effect of oil fed in this way.

Kellner's method of computation, hased upon the provisional conclusion on p. 323 , Chapter $\mathrm{X}$, that oil has substantially no effect upon the loss of energy in urine and methane under normal conditions, gives the following results:

PERCENTAGE OF METABOLIZABLE ENERGY UTILIZED.

\begin{tabular}{|c|c|c|}
\hline & $\begin{array}{l}\text { As Computed } \\
\text { by Kellner. }\end{array}$ & $\begin{array}{l}\text { As Computed } \\
\text { on p. } 462 .\end{array}$ \\
\hline 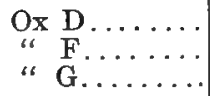 & $\begin{array}{c}52.2 \\
\cdots \cdots \\
59.4\end{array}$ & $\begin{array}{l}51.6 \\
65.1 \\
69.4\end{array}$ \\
\hline
\end{tabular}

DISTRIBUTION OF ENERGY OF DIGESTED OIL.

\begin{tabular}{|c|c|c|c|c|c|c|}
\hline & $\begin{array}{l}\text { Ani- } \\
\text { mal. }\end{array}$ & Period. & $\begin{array}{l}\text { In Urine, } \\
\text { Per Cent: }\end{array}$ & $\begin{array}{c}\text { In Methane, } \\
\text { Per Cent. }\end{array}$ & $\begin{array}{c}\text { Work of } \\
\text { Digestion, } \\
\text { Assimilation } \\
\text { and Tissue } \\
\text { Building, } \\
\text { Per Cent. }\end{array}$ & $\begin{array}{l}\text { In Gain, } \\
\text { Per Cent. }\end{array}$ \\
\hline Sample I........ & $\stackrel{\mathrm{D}}{\mathrm{G}}$ & $\begin{array}{l}3 \\
5\end{array}$ & $\begin{array}{l}0 \\
0\end{array}$ & $\begin{array}{l}0 \\
0\end{array}$ & $\begin{array}{l}47.8 \\
40.6\end{array}$ & $\begin{array}{l}52.2 \\
59.4\end{array}$ \\
\hline $\begin{array}{l}\text { A verage....... } \\
\text { Average computed } \\
\text { as on p. } 478 . . .\end{array}$ & $\begin{array}{l}\cdots \cdots \\
\cdots \cdots\end{array}$ & $\ldots \ldots$ & $\begin{array}{l}0 \\
0.5\end{array}$ & $\begin{array}{c}0 \\
-2.2\end{array}$ & 40.3 & $\begin{array}{l}55.8 \\
61.4\end{array}$ \\
\hline
\end{tabular}

The numerical results of these experiments show more clearly than was the case with the starch the difference in the two methods of computation. Both methods agree, however in showing that the combined expenditure of energy in the digestion and assimilation of the oil and in tissue building is very considerable. We have already seen that the expenditure of energy in the digestion of fat by carnivora and by man is comparatively small. If we are justified in assuming that the same thing is true of ruminants, the result just reached signifies that the digested fat undergoes extensive transformations before being finally deposited in the adipose tissue. 
Until, however, we have satisfactory determinations of the percentage utilization of fat by carnivora, or of its net availability in ruminants, or both, no final conclusion on this point is possible.

Wheat Gluten.-The three samples of this feeding-stuff experimented upon contained respectively $87.88,83.45$, and 82.67 per cent. of crude protein in the dry matter, the remainder being chiefly starch, with the exception of 2.22 per cent. of ether extract in the first lot. A reference to the results obtained for the metabolizable energy will show that they were variable and also that, especially in the earlier experiments, the incidental effects were large. Tabulating the results as in case of starch and oil we have the results contained in the tables on this and the opposite pages.

DISTRIBUTION OF GROSS ENERGY OF WHEAT GLUTEN.

\begin{tabular}{|c|c|c|c|c|c|c|c|}
\hline & 戛 & . & $\begin{array}{l}\text { In Feces. } \\
\text { Per Cent. }\end{array}$ & 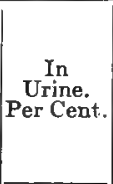 & $\begin{array}{c}\text { In } \\
\text { Methane. } \\
\text { Per Cent. }\end{array}$ & $\begin{array}{c}\text { Work of } \\
\text { Diges- } \\
\text { tion, } \\
\text { Assimila- } \\
\text { tion, and } \\
\text { Tissue } \\
\text { Building. } \\
\text { Per Cent. }\end{array}$ & $\begin{array}{l}\text { In Gain. } \\
\text { Per Cent. }\end{array}$ \\
\hline Ka & $\begin{array}{l}\text { III } \\
\text { III }\end{array}$ & $\begin{array}{l}3 \\
4\end{array}$ & $\begin{array}{r}-10.38 \\
-\quad 1.28\end{array}$ & $\begin{array}{l}17.85 \\
21.71\end{array}$ & $\begin{array}{r}10.81 \\
5.08\end{array}$ & $\begin{array}{l}44.72 \\
38.69\end{array}$ & $\begin{array}{l}37.00 \\
35.80\end{array}$ \\
\hline & $\begin{array}{l}\text { Av. } \\
\text { IV }\end{array}$ & 3 & $\begin{array}{r}-5.83 \\
-16.17\end{array}$ & $\begin{array}{l}19.78 \\
16.18\end{array}$ & $\begin{array}{r}7.95 \\
-1.26\end{array}$ & $\begin{array}{l}41.70 \\
42.35\end{array}$ & $\begin{array}{l}36.40 \\
58.90\end{array}$ \\
\hline Kellner's experiments: & $\begin{array}{l}\mathrm{B} \\
\mathrm{B} \\
\mathrm{C}\end{array}$ & $\begin{array}{l}1 \\
3 \\
3\end{array}$ & $\begin{array}{l}30.16 \\
22.55 \\
20.89\end{array}$ & $\begin{array}{l}16.58 \\
13.52 \\
11.19\end{array}$ & $\begin{array}{r}0.08 \\
-1.62 \\
-3.69\end{array}$ & $\begin{array}{l}33.58 \\
32.95 \\
40.71\end{array}$ & $\begin{array}{l}19.60 \\
32.60 \\
30.90\end{array}$ \\
\hline 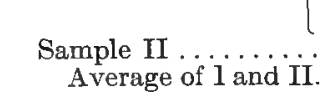 & Av. & 4 & $\begin{array}{l}24.53 \\
15.80 \\
20.16\end{array}$ & $\begin{array}{l}13.76 \\
12.39 \\
13.08\end{array}$ & $\begin{array}{r}-1.74 \\
1.91 \\
0.08\end{array}$ & $\begin{array}{l}35.75 \\
43.80 \\
39.78\end{array}$ & $\begin{array}{l}27.70 \\
26.10 \\
26.90\end{array}$ \\
\hline
\end{tabular}

The exceptionally small loss of energy in the urine in the case of Ox IV, Period 3, and the total suppression of the methane fermentation, as well as the fact that the metabolizable energy was apparently greater than the gross energy, seem to justify excluding this experiment from the average, although there was apparently nothing abnormal in the ration fed. In the experiment with Ox D, Period 4, the nutritive ratio was very narrow $(1: 3.3)$, and Kellner considers this a probable explanation of the 
DISTRIBUTION OF ENERGY OF APPARENTLY DIGESTED MATTER.

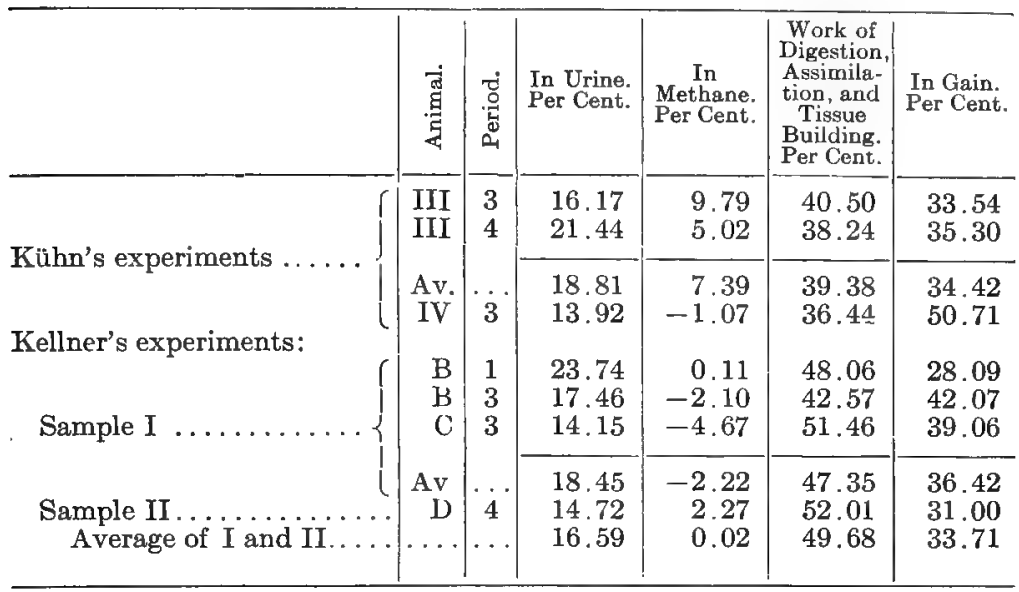

relatively small utilization of the protein as computed by his method. (See below.) An unexpected result is that while the earlier sample of gluten seems to have increased the methane fermentation, the later samples, although containing more starch, caused a decrease in the methane production except in case of Ox D.

Digestible Protein.-Kellner does not attempt to compute the energy utilized from the wheat gluten as a whole by his method, but uses the results as a basis for computing the utilization of the energy of the digested protein. He finds that of the metabolizable energy of the latter. computed in the manner described in Chapter X (p. 316), the following percentages were recovered in the gain:

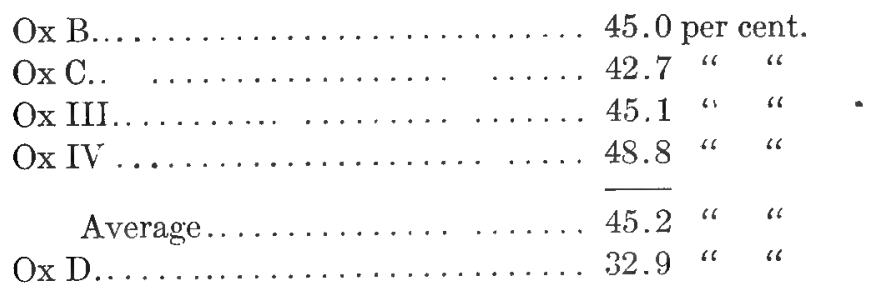

The average loss of energy in the urine was found (p. 317) to be 19.3 per cent. of the gross energy of the digested protein. Applying 
this average to the above figures, and assuming with Kellner that the protein does not take part in the methane fermentation, we have the following:

DISTRIBUTION OF ENERGY OF DIGESTED PROTEIN,

\begin{tabular}{|c|c|c|c|c|}
\hline Animal. & $\begin{array}{l}\text { In Urine. } \\
\text { Per Cent. }\end{array}$ & $\begin{array}{l}\text { In Methane. } \\
\text { Per Cent. }\end{array}$ & $\begin{array}{c}\text { Work of } \\
\text { Dugestion. } \\
\text { Assimilation } \\
\text { and Tissue } \\
\text { Building. } \\
\text { Per Cent }\end{array}$ & $\begin{array}{l}\text { In Gain. } \\
\text { Fer Cent. }\end{array}$ \\
\hline 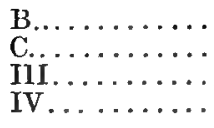 & 19.30 & 0 & $\begin{array}{l}44.38 \\
46.24 \\
44.30 \\
41.32\end{array}$ & $\begin{array}{l}36.32 \\
34.46 \\
36.40 \\
39.28\end{array}$ \\
\hline $\begin{array}{r}\text { Average ..... } \\
\mathrm{D} . . . . . . . .\end{array}$ & & & $\begin{array}{l}44.07 \\
54.15\end{array}$ & $\begin{array}{l}36.63 \\
26.55\end{array}$ \\
\hline
\end{tabular}

There is a wide discrepancy between these results and those computed on p. 465 from the experiments of Kern \& Wattenberg upon sheep with conglutin and flesh-meal. Omitting the apparently exceptional result of Period II, we have the following as the percentages of the (computed) metabolizable energy of the digested proteids which was utilized in those experiments:

\begin{tabular}{|c|c|c|}
\hline & Period. & Fer Cent \\
\hline Conglutin........ & III & $\begin{array}{l}67.63 \\
67.76\end{array}$ \\
\hline Average........... & $\ldots \ldots$ & 67.70 \\
\hline Flesh-meal. ....... & $\begin{array}{l}\mathrm{V} \\
\mathrm{VI}\end{array}$ & $\begin{array}{l}60.59 \\
69.33\end{array}$ \\
\hline Average....... & $\ldots \ldots$ & 64.96 \\
\hline
\end{tabular}

While the gain in these cases includes a considerable growth of wool, it seems difficult to suppose that this alone can have made the conditions so much more favorable for the storing up of the added protein as to account for the great difference between these results and Kellner's, and it must apparently be left to further investigation to clear up the matter. 
It need hardly be added that none of these results are directly comparable with those computed above, after another method, for the wheat gluten as a whole.

Beet Molasses.-The results of the three experiments upon beet molasses show such great differences, as was noted in Chapter $X$ and as is further apparent from the following table, that any discussion of them would evidently be premature:

DISTRIBUTION OF GROSS ENERGY OF BEET MOLASSES.

\begin{tabular}{|c|c|c|c|c|c|c|c|}
\hline & 兽 & 总 & $\begin{array}{l}\text { In Feces. } \\
\text { Per Cent. }\end{array}$ & $\begin{array}{l}\text { In Urine. } \\
\text { Per Cent. }\end{array}$ & $\begin{array}{c}\text { In } \\
\text { Methane. } \\
\text { Per Cent. }\end{array}$ & \begin{tabular}{|c} 
Work of \\
Digestion, \\
Assimilation, \\
and Tissue \\
Building. \\
Per Cent. \\
\end{tabular} & $\begin{array}{l}\text { In Gain. } \\
\text { Per Cent. }\end{array}$ \\
\hline $\begin{array}{cc}\text { Sample } & \text { I........ } \\
\text { " } & \text { II...... }\end{array}$ & $\underset{\mathrm{J}}{\mathrm{F}}$ & $\begin{array}{l}6 \\
6 \\
6\end{array}$ & $\begin{array}{r}26.87 \\
5.40 \\
14.45\end{array}$ & $\begin{array}{l}3.92 \\
3.16 \\
2.67\end{array}$ & $\begin{array}{l}-1.95 \\
12.44 \\
10.18\end{array}$ & $\begin{array}{l}29.56 \\
13.10 \\
36.20\end{array}$ & $\begin{array}{l}41.60 \\
65.90 \\
36.50\end{array}$ \\
\hline Average... & $\ldots$ & $\ldots$ & 9.92 & 2.92 & 11.31 & 24.65 & 51.20 \\
\hline
\end{tabular}

Rice.-The two experiments upon swine by Meissl, Strohmer \& Lorenz, when computed as on p. 454, show that of the (estimated) metabolizable energy of the food approximately the following percentages were recovered in the gain:

Period I................. 80.7 per cent.

"II . . 75.2 " "

Average.............. 78.0 " "

These results are notably higher than any obtained in experiments on ruminants. Like the results on barley and cockle below they are the expression in another form of the well-known superiority of the swine as an economical producer of meat.

Barley.-For the utilization of the energy of this grain the single experiment by Meissl, Strohmer \& Lorenz gives 70.9 per cent. of the (estimated) metabolizable energy.

Mixed Grains.-For mixed grains Kornauth \& Arche's results on swine give figures which do not differ materially from the result just computed for barley, viz.:

Experiment II............... 71.7 per cent. 
CoArse Fodders.--Kellner's results upon hay, straw, and extracted straw are the only data regarding the utilization of the energy of this class of feeding-stuffs which we as yet possess. Only those experiments in which coarse fodder was added to a mixed basal ration are available for a computation of this sort.

Meadow Hay.-The two kinds of meadow hay (V and VI) used in Kellner's experiments gave the following results for the distribution of their energy, computed as in previous instances:

DISTRIBUTION OF GROSS ENERGY OF MEADOW HAY.

\begin{tabular}{|c|c|c|c|c|c|c|c|}
\hline & 兽 & 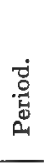 & $\begin{array}{c}\text { In } \\
\text { Feces. } \\
\text { Per Cent. }\end{array}$ & $\begin{array}{c}\text { In } \\
\text { Urine. } \\
\text { Per Cent }\end{array}$ & $\begin{array}{c}\text { In } \\
\text { Methane. } \\
\text { Per Cent. }\end{array}$ & $\begin{array}{l}\text { Work of } \\
\text { Digestion, } \\
\text { Assimila- } \\
\text { tion. and } \\
\text { Tissue } \\
\text { Building. } \\
\text { Per Cent. }\end{array}$ & $\begin{array}{l}\text { In Gain. } \\
\text { Per Cent. }\end{array}$ \\
\hline \multirow[t]{2}{*}{ Sample V.......... } & \multirow{2}{*}{$\begin{array}{c}\mathbf{F} \\
\mathrm{G} \\
\mathrm{Av} .\end{array}$} & \multirow{2}{*}{$\begin{array}{l}1 \\
2 \\
\ldots\end{array}$} & $\begin{array}{l}49.81 \\
44.80\end{array}$ & $\begin{array}{l}4.32 \\
4.26\end{array}$ & $\begin{array}{l}5.12 \\
6.94\end{array}$ & $\begin{array}{l}24.25 \\
28.10\end{array}$ & $\begin{array}{l}16.50 \\
15.90\end{array}$ \\
\hline & & & 47.30 & 4.29 & 6.03 & 26.18 & 16.20 \\
\hline \multirow[t]{2}{*}{ Sample VI......... } & \multirow[t]{2}{*}{$\begin{array}{r}\mathrm{H} \\
\mathrm{J}\end{array}$} & \multirow[t]{2}{*}{$\begin{array}{l}2 \\
7 \\
2\end{array}$} & $\begin{array}{l}37.07 \\
34.78 \\
34.30\end{array}$ & $\begin{array}{l}5.24 \\
5.00 \\
6.33\end{array}$ & $\begin{array}{l}4.87 \\
6.15 \\
6.13\end{array}$ & $\begin{array}{l}26.32 \\
27.97 \\
34.74\end{array}$ & $\begin{array}{l}26.50 \\
26.10 \\
18.50\end{array}$ \\
\hline & & & $\begin{array}{l}35.38 \\
41.34\end{array}$ & $\begin{array}{l}5.52 \\
4.91\end{array}$ & $\begin{array}{l}5.72 \\
5.87\end{array}$ & $\begin{array}{l}29.68 \\
27.93\end{array}$ & $\begin{array}{l}23.70 \\
19.95\end{array}$ \\
\hline
\end{tabular}

DISTRIBUTION OF FNERGY OF APPARENTLY DIGESTED MATTER.

\begin{tabular}{|c|c|c|c|c|c|c|}
\hline & 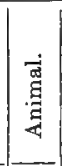 & 离 & $\begin{array}{l}\text { In Urine. } \\
\text { Per Cent. }\end{array}$ & $\begin{array}{c}\text { In } \\
\text { Methane. } \\
\text { Per Cent. }\end{array}$ & $\begin{array}{c}\text { Work of } \\
\text { Digestion, } \\
\text { Assimila- } \\
\text { tion, and } \\
\text { Tissue } \\
\text { Building. } \\
\text { Per Cent. }\end{array}$ & $\begin{array}{l}\text { In Gain. } \\
\text { Per Cent. }\end{array}$ \\
\hline \multirow{2}{*}{ Sample V.......... } & \multirow{2}{*}{$\begin{array}{c}\mathrm{F} \\
\mathrm{G} \\
\mathrm{Av}\end{array}$} & \multirow{2}{*}{$\begin{array}{c}1 \\
2 \\
\ldots\end{array}$} & $\begin{array}{l}8.61 \\
7.72\end{array}$ & $\begin{array}{l}10.20 \\
12.58\end{array}$ & $\begin{array}{l}48.39 \\
50.85\end{array}$ & $\begin{array}{l}32.80 \\
28.85\end{array}$ \\
\hline & & & 8.17 & 11.39 & 49.62 & 30.82 \\
\hline Sample VI . & $\underset{\mathrm{J}}{\mathrm{H}}$ & $\begin{array}{l}2 \\
7 \\
2\end{array}$ & $\begin{array}{l}8.32 \\
7.66 \\
9.64\end{array}$ & $\begin{array}{l}7.74 \\
9.43 \\
9.33\end{array}$ & $\begin{array}{l}41.63 \\
42.77 \\
52.83\end{array}$ & $\begin{array}{l}42.31 \\
40.14 \\
28.20\end{array}$ \\
\hline Average of $\mathrm{V}$ and VI. & $\begin{array}{l}\text { Av. } \\
\ldots\end{array}$ & $\ldots$ & $\begin{array}{l}8.54 \\
8.34\end{array}$ & $\begin{array}{r}8.83 \\
10.78\end{array}$ & $\begin{array}{l}45.75 \\
49.08\end{array}$ & $\begin{array}{l}36.88 \\
31.80\end{array}$ \\
\hline
\end{tabular}


Computed by Kellner's method, the percentage of the metabolizable energy of the hay which was recovered as gain of tissue was as follows, as compared with the results obtained by the writer's method:

PERCENTAGE OF METABOLIZABLE ENERGY RECOVERED.

\begin{tabular}{|c|c|c|c|}
\hline & & $\begin{array}{l}\text { Computed by } \\
\text { Kellner's } \\
\text { Method. }\end{array}$ & $\begin{array}{l}\text { Computed by } \\
\text { the Writer's } \\
\text { Method. }\end{array}$ \\
\hline Hay V.. & $\underset{*}{\mathrm{Ox}} \underset{\mathrm{G}}{\mathrm{F}} \ldots \ldots \ldots \ldots \ldots$ & $\begin{array}{l}42.8 \\
37.7\end{array}$ & $\begin{array}{l}40.4 \\
36.2\end{array}$ \\
\hline Hay VI .......... & 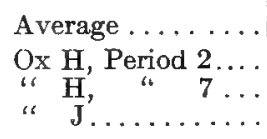 & $\left\{\begin{array}{l}40.2 \\
49.9 \\
35.8\end{array}\right.$ & $\begin{array}{l}38.3 \\
50.4 \\
48.4 \\
34.8\end{array}$ \\
\hline Average of $\mathrm{V}$ and VI... & $\begin{array}{c}\text { Average } . . . \ldots \ldots \\
\ldots \ldots \ldots \ldots \\
\ldots \ldots \ldots\end{array}$ & $\begin{array}{l}42.8 \\
41.5\end{array}$ & $\begin{array}{l}44.5 \\
41.4\end{array}$ \\
\hline
\end{tabular}

Computing the results upon the gross energy of the digested matter of the hay, Kellner obtains the following:

DISTRIBUTION OF ENERGY OF DIGESTED MATTER.

\begin{tabular}{|c|c|c|c|c|}
\hline$\therefore$ & $\begin{array}{l}\text { In Urine. } \\
\text { Per Cent. }\end{array}$ & $\begin{array}{l}\text { In Methane. } \\
\text { Per Cent }\end{array}$ & $\begin{array}{l}\text { Work of } \\
\text { Digestion. } \\
\text { Assimilation, } \\
\text { and Tissue } \\
\text { Building.- } \\
\text { Per Cent. }\end{array}$ & $\begin{array}{l}\text { In Gain. } \\
\text { Per Cent. }\end{array}$ \\
\hline $\mathrm{Hay}_{\text {" } \mathrm{VI}} \ldots \ldots \ldots \ldots \ldots$ & $\begin{array}{l}8.2 \\
8.8\end{array}$ & $\begin{array}{r}11.5 \\
9.0\end{array}$ & $\begin{array}{l}48.00 \\
48.10\end{array}$ & $\begin{array}{l}32.3 \\
34.1\end{array}$ \\
\hline Average........ & 8.5 & 10.3 & 48.00 & 33.2 \\
\hline
\end{tabular}

As in some previous cases, the numerical results of the two methods of computation do not vary greatly, but their essentially different significance should not be forgotten.

Oat Straw.-For the single sample of this feeding-stuff experimented on, the results, arranged in the same order as before, were as follows: 
DISTIBUTION OF GROSS ENIRGY OF OAT STRAW.

\begin{tabular}{|c|c|c|c|c|c|c|}
\hline Animal. & Period. & $\begin{array}{l}\text { In Feces. } \\
\text { Per Cent. }\end{array}$ & $\begin{array}{l}\text { In Urine. } \\
\text { Per Cent. }\end{array}$ & $\begin{array}{c}\text { In } \\
\text { Methane. } \\
\text { Per Cent. }\end{array}$ & $\begin{array}{c}\text { Work of } \\
\text { Digestion, } \\
\text { Assimula- } \\
\text { tion, and } \\
\text { Tissue } \\
\text { Building. } \\
\text { Per Cent. } \\
\end{array}$ & $\begin{array}{l}\text { In Gain, } \\
\text { Per Cent. }\end{array}$ \\
\hline $\begin{array}{l}\mathrm{F} \\
\mathrm{G}\end{array} \ldots \ldots \ldots \ldots \ldots \ldots$ & $\begin{array}{l}2 \\
1\end{array}$ & $\begin{array}{l}56.77 \\
56.86\end{array}$ & $\begin{array}{l}2.29 \\
1.86\end{array}$ & $\begin{array}{l}4.40 \\
6.23\end{array}$ & $\begin{array}{l}22.34 \\
23.35\end{array}$ & $\begin{array}{l}14.20 \\
11.70\end{array}$ \\
\hline Average. . & & 56.81 & 2.08 & 5.31 & 22.85 & 12.95 \\
\hline
\end{tabular}

\section{DISTRIBUTION OF ENERGY OF APPARENTLY DIGESTED MATTER.}

\begin{tabular}{|c|c|c|c|c|c|}
\hline Animal. & Period. & $\begin{array}{l}\text { In Urine. } \\
\text { Per Cent. }\end{array}$ & $\begin{array}{l}\text { In Methane. } \\
\text { Per Cent. }\end{array}$ & $\begin{array}{c}\text { Work of } \\
\text { Digestion, } \\
\text { Assimilation, } \\
\text { and Tissue } \\
\text { Building. } \\
\text { Per Cent. }\end{array}$ & $\begin{array}{l}\text { In Gain. } \\
\text { Per Cent. }\end{array}$ \\
\hline $\begin{array}{l}F \ldots \ldots \ldots \ldots \\
G \ldots \ldots \ldots \ldots\end{array}$ & $\begin{array}{l}2 \\
1\end{array}$ & $\begin{array}{l}5.30 \\
4.32\end{array}$ & $\begin{array}{l}10.17 \\
14.42\end{array}$ & $\begin{array}{l}51.73 \\
54.12\end{array}$ & $\begin{array}{l}32.80 \\
27.14\end{array}$ \\
\hline Average....... & $\ldots \ldots$ & 4.81 & 12.30 & 52.92 & 29.97 \\
\hline
\end{tabular}

PERCENTAGE OF METABOLIZABLE ENERGY RECOVERED.

\begin{tabular}{|c|c|c|}
\hline & $\begin{array}{c}\text { Computed by } \\
\text { Keliner's Method. }\end{array}$ & $\begin{array}{l}\text { Computed by the } \\
\text { Writer's Method. }\end{array}$ \\
\hline${ }^{\mathrm{Ox}} \mathrm{F} \ldots \ldots \ldots \ldots \ldots \ldots \ldots$ & $\begin{array}{l}39.9 \\
35.3\end{array}$ & $\begin{array}{l}38.8 \\
33.4\end{array}$ \\
\hline Average . . & 37.6 & 36.1 \\
\hline
\end{tabular}

DISTRIBUTION OF ENERGY OF DIGESTED MATTER (KELLNER).

\begin{tabular}{c|c|c|c|c}
\hline & $\begin{array}{c}\text { In Urine. } \\
\text { Per Cent. }\end{array}$ & $\begin{array}{c}\text { Work of } \\
\text { In Methane. } \\
\text { Per Cent. }\end{array}$ & $\begin{array}{c}\text { Assimilion, } \\
\text { and Tissue, } \\
\text { Building. } \\
\text { Per Cent. }\end{array}$ & $\begin{array}{c}\text { In Gain. } \\
\text { Per Cent. }\end{array}$ \\
\hline Average F and G... & 4.7 & 12.2 & 51.9 & 31.2 \\
\hline
\end{tabular}


Wheat Straw.-Tabulating the results upon wheat straw in the same manner as those for oat straw we have-

DISTRIBUTION OF GROSS ENERGY OF WHEAT STRAW.

\begin{tabular}{|c|c|c|c|c|c|c|}
\hline Animal. & Period. & $\begin{array}{l}\text { In Feces. } \\
\text { Per Cent. }\end{array}$ & $\begin{array}{l}\text { In Urine. } \\
\text { Per Cent. }\end{array}$ & $\begin{array}{c}\text { In } \\
\text { Methane. } \\
\text { Per Cent. }\end{array}$ & $\begin{array}{l}\text { Work of } \\
\text { Digestion, } \\
\text { Assimila- } \\
\text { tion, and } \\
\text { Tissue } \\
\text { Buildng. } \\
\text { Per Cent. }\end{array}$ & $\begin{array}{l}\text { In Gain. } \\
\text { Per Cont. }\end{array}$ \\
\hline $\begin{array}{l}\mathrm{H} \\
\mathbf{J}\end{array} \ldots \ldots \ldots \ldots \ldots$ & $\begin{array}{l}1 \\
1\end{array}$ & $\begin{array}{l}60.41 \\
56.03\end{array}$ & $\begin{array}{l}1.88 \\
2.85\end{array}$ & $\begin{array}{l}7.96 \\
8.65\end{array}$ & $\begin{array}{l}26.55 \\
24.67\end{array}$ & $\begin{array}{l}3.20 \\
7.80\end{array}$ \\
\hline Average & & 58.21 & 2.37 & 8.31 & 25.61 & 5.50 \\
\hline
\end{tabular}

DISTRIBUTION OF ENERGY OF APPARENTLY DIGESTED MATTER.

\begin{tabular}{|c|c|c|c|c|c|}
\hline Animal. & Period. & $\begin{array}{l}\text { In Urine. } \\
\text { Per Cent. }\end{array}$ & $\begin{array}{l}\text { In Methane. } \\
\text { Per Cent. }\end{array}$ & $\begin{array}{l}\text { Work of } \\
\text { Digestion, } \\
\text { Assimilation, } \\
\text { and Tissue } \\
\text { Building. } \\
\text { Per Cent. }\end{array}$ & $\begin{array}{l}\text { In Gain. } \\
\text { Per Cent. }\end{array}$ \\
\hline $\begin{array}{l}\mathbf{H} \\
\mathbf{J}\end{array} \ldots \ldots \ldots$ & $\begin{array}{l}1 \\
1\end{array}$ & $\begin{array}{l}4.75 \\
6.49\end{array}$ & $\begin{array}{l}20.11 \\
19.67\end{array}$ & $\begin{array}{l}67.03 \\
56.12\end{array}$ & $\begin{array}{r}8.11 \\
17.72\end{array}$ \\
\hline Average.. & & 5.62 & 19.89 & 61.57 & 12.92 \\
\hline
\end{tabular}

PERCENTAGE OF METABOLIZABLE ENERGY RECOVERED.

\begin{tabular}{|c|c|c|}
\hline & $\begin{array}{l}\text { Computed by } \\
\text { Kellner's Method. }\end{array}$ & $\begin{array}{l}\text { Computed by the } \\
\text { Writer's Method. }\end{array}$ \\
\hline \multirow{2}{*}{$\begin{array}{r}\mathrm{Ox} \underset{\mathrm{J}}{\mathrm{H}} \ldots \ldots \ldots \ldots \ldots \\
\quad \text { Average } \ldots \ldots \ldots\end{array}$} & $\begin{array}{l}11.2 \\
24.3\end{array}$ & $\begin{array}{l}10.8 \\
24.0\end{array}$ \\
\hline & 17.8 & 17.4 \\
\hline
\end{tabular}

DISTRIBUTION OF ENERGY OF DIGESTED MATTER (KELLNER).

Average of $\mathrm{H}$ and $\mathbf{J}$.

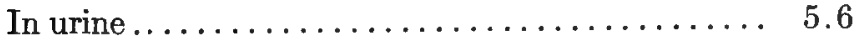

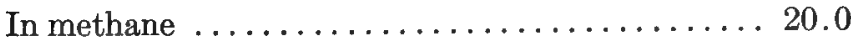

Work of digestion, assimilation, and tissue building. 61.2

In gain........................ 13.2

100.0 
Extracted Straw.-As previously noted in another connection, this material consisted of rye straw which had been treated with an alkaline liquid under pressure, substantially as in the manufacture of straw paper. It contained in the water-free state 76.78 per cent. of crude fiber and $\mathbf{1 9 . 9 6}$ per cent. of nitrogen-free extract. Considerable interest attaches to the results obtained upon this substance as representing to a degree the crude fiber of the food of herbivorous animals. Computed as before, these results were:

DISTRIBUTION OF GROSS ENERGY OF EXTRACTED STRAW.

\begin{tabular}{|c|c|c|c|c|c|c|}
\hline Animal. & Period. & $\begin{array}{l}\text { In Feces. } \\
\text { Per Cent. }\end{array}$ & $\begin{array}{l}\text { In Urine. } \\
\text { Per Cent. }\end{array}$ & $\begin{array}{c}\text { In } \\
\text { Methane. } \\
\text { Per Cent. }\end{array}$ & $\begin{array}{l}\text { Work of } \\
\text { Digestion, } \\
\text { Assimila- } \\
\text { tion, and } \\
\text { Tissue } \\
\text { Building. } \\
\text { Per Cent. }\end{array}$ & $\begin{array}{l}\text { In Gain. } \\
\text { Per Cent. }\end{array}$ \\
\hline $\begin{array}{l}\mathbf{H} \ldots \ldots \\
\mathbf{J} \ldots \ldots\end{array}$ & $\begin{array}{l}5 \\
5\end{array}$ & $\begin{array}{l}11.35 \\
14.14\end{array}$ & $\begin{array}{l}-0.46 \\
-1.11\end{array}$ & $\begin{array}{l}12.40 \\
12.52\end{array}$ & $\begin{array}{l}25.11 \\
30.85\end{array}$ & $\begin{array}{l}51.60 \\
43.60\end{array}$ \\
\hline Average & $\ldots$ & 12.75 & -0.79 & 12.46 & 27.98 & 47.60 \\
\hline
\end{tabular}

DISTRIBUTION OF ENERGY OF APPARENTLY DIGESTED MATTER.

\begin{tabular}{|c|c|c|c|c|c|}
\hline Animal. & Period. & $\begin{array}{l}\text { In Urine. } \\
\text { Per Cent. }\end{array}$ & $\begin{array}{l}\text { In Methane. } \\
\text { Per Cent. }\end{array}$ & $\begin{array}{l}\text { Work of } \\
\text { Digestion, } \\
\text { Assimilation, } \\
\text { and Tissue } \\
\text { Building. } \\
\text { Per Cent. }\end{array}$ & $\begin{array}{l}\text { In Gain. } \\
\text { Per Cent. }\end{array}$ \\
\hline$\underset{\mathbf{J}}{\mathbf{H}} \ldots \ldots \ldots \ldots \ldots \ldots$ & $\begin{array}{l}5 \\
5\end{array}$ & $\begin{array}{l}-0.52 \\
-1.29\end{array}$ & $\begin{array}{l}13.99 \\
14.58\end{array}$ & $\begin{array}{l}28.29 \\
35.89\end{array}$ & $\begin{array}{l}58.24 \\
50.82\end{array}$ \\
\hline Average... & $\ldots$ & -0.91 & 14.29 & 32.09 & 54.53 \\
\hline
\end{tabular}

PERCENTAGE OF METABOLIZABLE ENERGY RECOVERED.

\begin{tabular}{|c|c|c|}
\hline & $\begin{array}{c}\text { Computed by } \\
\text { Kellner's Method. }\end{array}$ & $\begin{array}{l}\text { Computed by the } \\
\text { Writer's Method. }\end{array}$ \\
\hline$\underset{" \mathrm{Ox}}{\mathrm{O}} \mathbf{\mathrm { H }} \ldots \ldots \ldots \ldots \ldots \ldots$ & $\begin{array}{l}67.5 \\
58.7\end{array}$ & $\begin{array}{l}67.3 \\
58.6\end{array}$ \\
\hline Average........ & 63.1 & 63.0 \\
\hline
\end{tabular}


DISTRIBUTION OF ENERGY OF DIGESTED MATTER (KELLNER).

Average of

$\mathrm{H}$ and $\mathrm{J}$.

In urine........................ 0.0

In methane...................... 14.0

Work of digestion, assimilation, and tissue building. 31.7

In gain....................... 54.3

100.0

As was noted in discussing the results upon metabolizable energy, the treatment to which the straw was submitted left it in a condition in which its digestibility, and consequently its percentage of metabolizable energy, compared favorably with that of starch. As we now see, this analogy extends also to its effect in producing gain, the figures showing in this respect a slight superiority on the part of the extracted straw, as appears from the following summary:

RECOVERED IN GAIN.

\begin{tabular}{|c|c|c|}
\hline & $\begin{array}{l}\text { Starch (Kellner's } \\
\text { Experiments on } \\
\text { Moderate } \\
\text { Rations). }\end{array}$ & Extracted Straw. \\
\hline 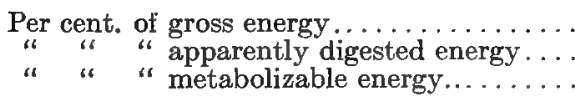 & $\begin{array}{l}43.4 \\
53.1 \\
59.0\end{array}$ & $\begin{array}{l}47.6 \\
54.5 \\
63.0\end{array}$ \\
\hline
\end{tabular}

The reason for this strikingly high value of the extracted straw as compared with the low value indicated for crude fiber by the results of Zuntz and Wolff will be considered in a subsequent paragraph.

Summary.-For convenience of reference the foregoing results may be summarized in the tables on pages 490 and 491, showing respectively the percentage distribution of the gross energy of the feeding-stuffs, that of the energy of the apparently digested organic matter, and the percentage utilization of 
the metabolizable energy according to the two methods of computation adopted:

DISTRIBUTION OF GROSS ENERGY.

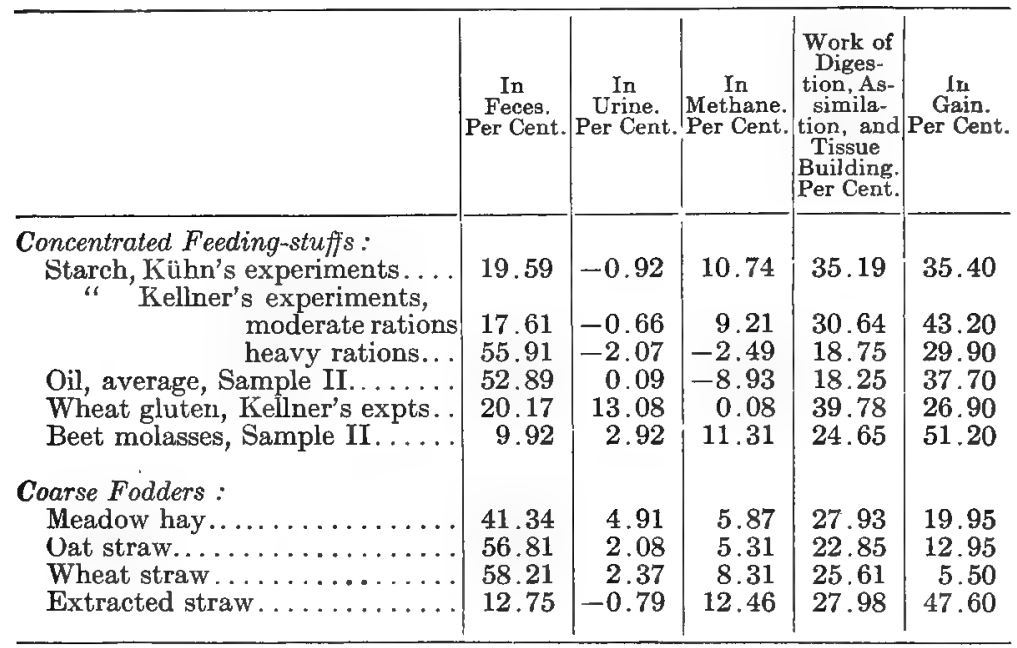

DISTRIBUTION OF ENERGY OF APPARENTLY DIGESTED MATTER.

\begin{tabular}{|c|c|c|c|c|}
\hline & $\begin{array}{c}\text { In } \\
\text { Urine. } \\
\text { Per Cent. }\end{array}$ & $\begin{array}{c}\text { In } \\
\text { Methane. } \\
\text { Per Cent. }\end{array}$ & \begin{tabular}{|c|} 
Work of \\
Digestion, \\
Assimila. \\
tion, and \\
Tissue \\
Building. \\
Per Cent.
\end{tabular} & $\begin{array}{c}\text { In } \\
\text { Gain. } \\
\text { Per } \\
\text { Cent. }\end{array}$ \\
\hline \multicolumn{5}{|l|}{ Concentrated Feeding-stuffs: } \\
\hline $\begin{array}{l}\text { Starch, Kühn's experiments ............. } \\
\text { Kellner's experiments, moder- }\end{array}$ & & & & \\
\hline $\begin{array}{c}\text { ate rations .................. } \\
\text { “ Kellner's experiments, heavy }\end{array}$ & -0.92 & 11.12 & 37.36 & 52.44 \\
\hline rations. . . . . . . . . . & -4.95 & -6.15 & 42.77 & 68.33 \\
\hline 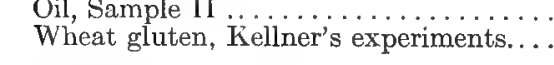 & $\begin{array}{r}-0.53 \\
16.59\end{array}$ & $\begin{array}{r}-24.35 \\
0.02\end{array}$ & $\begin{array}{l}41.42 \\
49.62\end{array}$ & $\begin{array}{l}83.46 \\
33.71\end{array}$ \\
\hline \multicolumn{5}{|l|}{ Coarse Fodders: } \\
\hline Meadow hay.. & 8.34 & 10.78 & 49.08 & 31.80 \\
\hline Oat straw..... & 4.81 & 12.30 & 52.92 & 29.97 \\
\hline Wheat straw ............ & 5.62 & 19.89 & 61.57 & 12.92 \\
\hline Extracted straw............ & -0.91 & 14.29 & 32.09 & 54.53 \\
\hline
\end{tabular}


PERCENTAGE UTILIZATION OF METABOLIZABLE ENERGY.

\begin{tabular}{|c|c|c|}
\hline & $\begin{array}{c}\text { Real } \\
\text { Utilization } \\
\text { as Computed } \\
\text { by Kellner. }\end{array}$ & $\begin{array}{l}\text { Apparent } \\
\text { Utilization. }\end{array}$ \\
\hline 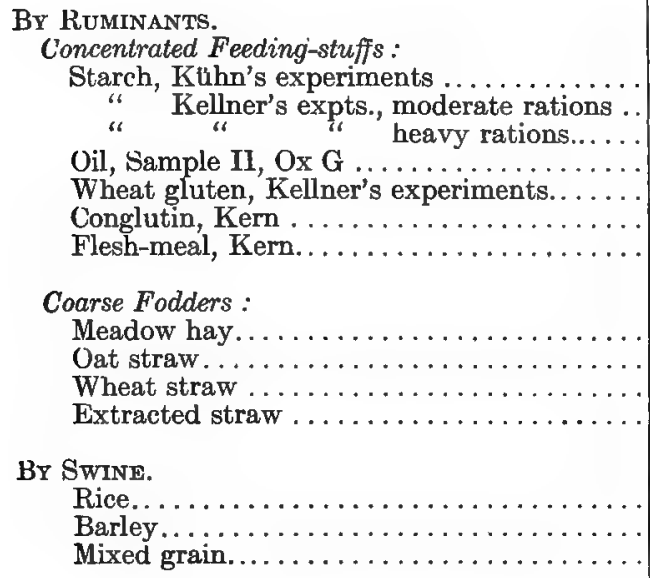 & $\begin{array}{l}49.0 \\
58.9 \\
58.9 \\
59.4 \\
45.2 * \\
67.7 * \\
65.0 *\end{array}$ & $\begin{array}{l}50.0 \\
58.4 \\
61.5 \\
69.4 \\
40.3\end{array}$ \\
\hline
\end{tabular}

The Expenditure of Energy in Digestion, Assimilation, and Tissue Building.-As was shown in the introductory paragraphs on p. 466. the recorded data do not permit us to distinguish between the energy expended in the digestion, resorption, and assimilation of the various feeding-stuffs experimented upon and the energy which we have reason to believe is required for the conversion of the assimilated material into tissue. Accordingly these two factors have been grouped together in the foregoing summaries of results. Some interesting facts are revealed, however, by a comparison of the total expenditure of energy for these two purposes in the several cases. Kellner's results, as the latest and apparently most accurate and representative, have been made the chief basis of the comparison, the figures being those computed by the writer and therefore showing the aggregate net effect upon the balance of energy, that is, the "apparent" utilization.

CoArse Fodders.-A comparison of the coarse fodders with each other brings out the interesting fact that while the percentage of the gross energy recovered in the gain varied from 5.5 to 47.6 . 
the percentage expended in digestion, assimilation, and tissue building varied only from 22.85 to 27.98. Expressing the same thing in absolute figures, we have the following:

ENERGY PER GRAM OF ORGANIC MATTER.

\begin{tabular}{|c|c|c|}
\hline & $\begin{array}{c}\text { Gross, } \\
\text { Cals. }\end{array}$ & $\begin{array}{l}\text { Expended in Diges- } \\
\text { tion, Assimilation, } \\
\text { and Tissue Building, } \\
\text { Cals. }\end{array}$ \\
\hline Meadow hay........ & 4.751 & 1.327 \\
\hline Oat straw ........... & 4.816 & 1.100 \\
\hline Wheat straw....... & 4.743 & 1.214 \\
\hline Extracted straw.... & 4.251 & 1.190 \\
\hline Average.. & 4.640 & 1.208 \\
\hline
\end{tabular}

In other words, the combined energy required to separate the digestible from the indigestible portion of one gram of organic matter, resorb it, and convert the resorbed portion into tissue was not greatly different for these four materials. They differed widely in their nutritive effect, not because of a greater or less expenditure of energy for these purposes, but chiefly because the same expenditure of energy resulted in making a much larger amount of material digestible in some cases than in others.

Concentrated Feeding-STUFrs.-A still more striking result is reached when we compare the results on coarse fodders with those on concentrated feeding-stuffs. Taking the figures of Kellner's experiments for the latter, and omitting his results on heavy rations of starch, we have the following data for starch, oil, and wheat gluten:

ENERGY PER GRAM OF ORGANIC MATTER.

\begin{tabular}{|c|c|c|}
\hline . & $\begin{array}{l}\text { Gross, } \\
\text { Cals. }\end{array}$ & $\begin{array}{l}\text { Expended in } \\
\text { Digestion, } \\
\text { Assimilation } \\
\text { and Tissue } \\
\text { Building, } \\
\text { Cals. }\end{array}$ \\
\hline $\begin{array}{l}\text { Starch (Kellner). } \\
\text { Oil ... (K̈.llnèr) } \\
\text { Gluten (Ko. }\end{array}$ & $\begin{array}{l}4.168 \\
9.464 \\
5.742\end{array}$ & $\begin{array}{l}1.277 \\
1.728 \\
2.281\end{array}$ \\
\hline
\end{tabular}

We thus reach the seemingly paradoxical result that the total expenditure of energy in the production of new tissue is decidedly 
greater in the case of these three materials, and notably the last two, than in the four coarse fodders previously tabulated.

The paradox largely disappears, however, when we remember that while the larger share of the work of digestion has to do with the total dry matter of the food, the work of assimilation and tissue. building has to be performed only upon the digested matter, and that the proportion of the latter is much larger in the starch, oil, and gluten than in the coarse fodders. We have already (pp. 375 and 445) seen reason to suppose that the processes of assimilation and tissue building consume a considerable share of the metabolizable energy of the food, although we are still ignorant as to how much and as to how the proportion differs with different materials, and the above results serve to confirm this conclusion.

If, simply as an illustration; we assume that the uniform proportion of 30 per cent. of the metabolizable energy of the several feeding-stuffs is thus consumed, then if we deduct this amount from the totals above computed we shall have the work of digestion alone as follows:

ENERGY PER GRAM OF ORGANIC MATTER.

\begin{tabular}{|c|c|c|c|c|}
\hline & $\begin{array}{c}\text { Metaboliz- } \\
\text { able } \\
\text { Energy } \\
\text { (p. 297). } \\
\text { Cals. }\end{array}$ & $\begin{array}{l}\text { Assumed } \\
\text { Work of } \\
\text { Assirnilation } \\
\text { and Tissue } \\
\text { Building } \\
\text { (30 Per Cent. } \\
\text { of Metaboliz- } \\
\text { able), Cals. }\end{array}$ & $\begin{array}{l}\text { Total Ex- } \\
\text { penditure } \\
\text { as Above. } \\
\text { Cals. }\end{array}$ & $\begin{array}{c}\text { Work of } \\
\text { Digestion Alone. } \\
\text { Cals. }\end{array}$ \\
\hline 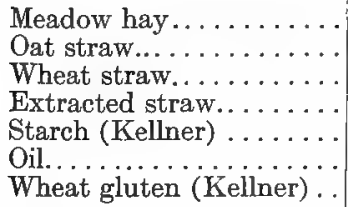 & $\begin{array}{l}2.213 \\
1.724 \\
1.475 \\
3.213 \\
3.079 \\
5.298 \\
3.831\end{array}$ & $\begin{array}{l}0.664 \\
0.517 \\
0.443 \\
0.964 \\
0.923 \\
1.589 \\
1.149\end{array}$ & $\begin{array}{l}\text { 1. } 327 \\
1.100 \\
1.214 \\
1.190 \\
1.277 \\
1.728 \\
2.284\end{array}$ & 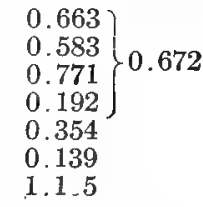 \\
\hline
\end{tabular}

This arbitrary assumption reduces the work of digestion of the starch to about one half that expended upon a like amount of material in the form of coarse fodders which yield chiefly carbohydrates to the organism. Moreover, we must remember that in the case of starch there is a considerably greater loss of energy in the methane fermentation than with the same amount of total organic matter in coarse fodders, and that this loss is included in the work of digestion. The high figure found for the wheat gluten we might be 
inclined to explain by its well-known effect in stimulating the metabolism in the body-that is, by supposing that for this substance our assumption of 30 per cent. for the work of assimilation and tissue building is too low.

The computed work of digestion is small in the case of the oil, as the results obtained in other experiments would lead us to expect. At the same time it should be remembered that the figures given are derived from two experiments only, while a third gave quite different results, showing in particular a decidedly higher figure for the combined work of digestion, assimilation, and tissue building. It is obvious, therefore, that further investigation is necessary to fix the value of oil in this respect.

Crude Fiber.-Finally, it will be observed that our arbitrary assumption results in making the work of digestion of the extracted straw less than two thirds that of starch. We should naturally suppose that the mechanical work involved in digestion would be fully as great in the case of the former as in that of the latter, while, as the figures for methane show, the extracted straw underwent a more extensive fermentation than the starch. Obviously, the mechanical and chemical treatment to which the straw was subjected so modified the cellulose and removed incrusting matters as to produce a material which behaved substantially like starch in the alimentary canal, both as regards its digestibility and its relation to ferments.* Correspondingly, the total work of digestion, assimilation, and tissue building is not widely different in the two cases. It is only when we arbitrarily assume a high percentage for the work of assimilation and tissue building, as was done above for the sake of illustrating the general question, that this difference and that in the amount of metabolizable energy combine to give the relatively low figure for digestive work noted above.

\section{§ 2. Utilization for Muscular Work.}

When a muscle is subjected to a suitable stimulus (normally a nerve stimulus) there occurs, as we have seen, a sudden and rapid increase in its metabolism. This increased metabolism appears to

* Lehmann (Landw. Jahrb., 24, Supp. I, 118) had previously shown that the apparent digestibility of the crude fiber and nitrogen-free extract of straw and chaff thus treated was increased by from 79 to 133 per cent. 
consist largely in a breaking down or cleavage of some substance or substances contained in the muscle, resulting in a rapid increase in the excretion of carbon dioxide and the consumption of oxygen by the animal. In this process of breaking down or cleavage there is a corresponding transformation of energy, a portion of the potential energy of the metabolized material appearing finally as heat, while a part may take the form of mechanical energy. The inquiry naturally arises what proportion of the total energy liberated during the increased metabolism is recovered as mechanical work and what proportion takes the form of the (for this purpose) waste energy of heat. The question is not only one of great theoretical interest to the physiologist, but the efficiency of the working animal regarded. as a machine for the conversion of the potential energy of feedingstuffs into mechanical work is also of the highest practical importance.

EFficiency of Singie Muscle.-A large amount of experimental work has been devoted to the study of the single muscle as a machine. The subject is a complicated one, and unanimity of views upon it has by no means been attained, especially as to the mechanism of muscular contraction. As regards the efficiency of the muscle as a converter of energy, however, one fact is perfectly well established, viz., that it varies within quite wide limits.

If the two ends of a muscle be attached to fixed points, so that it cannot shorten, a suitable stimulus will still cause it to contract in the technical sense of the word; that is, a state of tension will be set up in the muscle tending to pull the two supports nearer together (isometric contraction). In such a contraction there is an expenditure of potential energy and a corresponding increase of muscular metabolism. but no external work is done. In other words, all the potential energy finally takes the form of heat and the mechanical efficiency is zero. This is the case, for example, in the standing animal. A not inconsiderable muscular effort is required to maintain the members of the body in certain fixed positions, and a corresponding generation of heat takes place, but no mechanical work is done.

But even when the muscle is free to shorten and thus do mechanical work, its efficiency is found to be variable, the chief determining factors being the load and the degree of contraction. The 
maximum efficiency of the muscle is reached when the load is such that the muscle can just raise it, while this maximum load diminishes as the muscle contracts until when the latter reaches the limit of shortening it of course becomes zero. Conversely, if the muscle be stretched beyond what may be called its normal length, as is the case in the living body, the weight which it can lift, and consequently its efficiency, is increased. In these respects the muscle behaves like an elastic cord, and some authorities, notably Chauveau,* regard the essence of muscular contraction as consisting of a direct conversion of the potential energy of the "contractile material" of the muscle into muscular elasticity.

Efficiency of the Living Anrmal.-According to the above principles the maximum efficiency of a muscle would be obtained when it was loaded to its maximum at each point in the contraction; that is, when the load diminished uniformly from the maximum corresponding to the initial length of the muscle to zero at the point of greatest contraction. Such conditions, however, rarely if ever obtain in the animal. Of its many muscles some serve largely or wholly to maintain the relative positions of the different parts of the body, and consequently have an efficiency approaching zero. Others contract to a varying extent and under loads less than the maximum. Some muscles, owing to their anatomical relations, work at a less mechanical advantage than others, while the extent to which a given group of muscles is called into action will vary with the nature of the work.

If, then, the efficiency of the single muscle is variable, that of the body as a whole would seem likely to be even more so, thus rendering it difficult to draw any trustworthy direct conclusions as to the efficiency of the bodily machine from studies of the efficiency of the single muscle. Moreover, the performance of labor by an animal sets up various secondary activities, notably of the circulatory and respiratory organs, which consume their share of potential energy and yet do not contribute directly to the performance of the work, and the extent of these secondary activities varies with the nature and the severity of the work. When, therefore, as is here the case, we consider the whole animal in the light of a machine for converting the potential energy of the food into

* Le Travail Musculaire. Paris, 1891. 
mechanical work, we are perforce, by the very complexity of the problem, driven to the statistical method of comparing the total income and outgo of energy in the various forms of work.

THE UTILIZATION OF NET AVAILABLE ENERGY.

Both the activity of the skeletal muscles in the performance of work and the supplementary activity of the muscles concerned in circulation, respiration, etc., is carried on at the expense of energy stored in the muscles themselves or perhaps in the blood which circulates through them. The body thus suffers a loss of energy which is replaced from the energy of the food. If, then, we supply a working animal, in addition to its maintenance ration, with an amount of food exactly sufficient to make good the loss, the total energy metabolized in the performance of the work will represent the net available energy of the excess food, since this by definition is that portion of the gross energy which contributes to the maintenance of the store of potential energy in the body.

It is true that in our discussion of the net available energy of the food we regarded it as making good the losses that occur below the maintenance requirement, and the question may arise whether the availability as thus measured is the same as the availability for the production of muscular work. In reality, however, the two cases are not radically different. Even below the point of maintenance the internal work of the body consists very largely of muscular work, and it is the energy metabolized in the performance of this work which appears to constitute the chief demand for available food energy. It would appear highly probable, therefore, that the net availability of the metabolizable energy of the food will be found to be substantially the same whether that energy be employed to prevent a loss from the body as a consequence of its internal work below maintenance or on account of the performance of external work above maintenance.

If, then, we cause an animal to perform a known amount of external work and measure the increase in the amoun't of energy metabolized in the body, we may regard the latter as representing net available energy derived from previous food, and a comparison 
of this quantity with the work done will give the coefficient of utilization for the particular animal and kind of work experimented on.

\section{The Efficiency of the Animal as a Motor.}

The relation just indicated between the work performed and the total energy metabolized in its performance is not infrequently regarded as expressing the efficiency of the animal as a motor, but it should be clearly understood that this is true only in a limited sense. A coefficient computed in the manner outlined above takes account only of the loss which occurs in the conversion of the stored energy of the body into external mechanical work. It neither includes the expenditure of energy required for the digestion and assimilation of the food, nor does it take account of the large amount of energy continually consumed in the internal work of the animal machine. It does not, therefore, furnish a direct measure of the economy with which the animal machine uses the energy supplied to it, but is comparable rather to the theoretical thermo-dynamic efficiency of a steam-engine. With this limitation, however, the phrase may be used as a matter of convenience.

Quite extensive investigations upon this point are already on record. They have generally taken the form of what may be called respiration experiments. The respiratory exchange of carbon dioxide and oxygen has been determined, first, in a state of rest, and, second, during the performance of a measured amount of work. From the difference between these two values the quantity of material metabolized and the amount of energy consequently liberated have been computed and compared with the energy recovered in the form of mechanical work.

This method of experimentation has been largely developed and employed by Zuntz and his associates * in experiments upon man, the dog, and especially the horse. Since the present work relates especially to the nutrition of domestic animals, the results upon the latter animal are of peculiar interest, but their study may be advantageously preceded by a somewhat brief consideration of the results upon the dog and upon man.

* Compare Chapter VIII, pp. 251-2 
Experiments on the Dog.-The following experıments by Zuntz, ${ }^{*}$ while not the earliest upon record, may serve to illustrate the general methods employed and as introductory to the more elaborate experiments upon the horse.

The following table shows the average oxygen consumption and carbon dioxide excretion, determined by the Zuntz apparatus, of a dog when lying, standing, and performing work upon a treadpower, and also the amount of work done, all computed per minute:

\begin{tabular}{|c|c|c|c|c|c|c|c|c|}
\hline \multirow{2}{*}{$\begin{array}{l}\text { Weight } \\
\text { of Agi- } \\
\text { mal } \\
\text { and } \\
\text { Load } \\
\text { Kgs. }\end{array}$} & \multirow[b]{2}{*}{$\begin{array}{c}\text { No of } \\
\text { Ex- } \\
\text { peri- } \\
\text { ments. }\end{array}$} & \multirow[t]{2}{*}{ s } & \multicolumn{3}{|c|}{$\begin{array}{l}\text { Respiration per } \\
\text { Minute. }\end{array}$} & \multicolumn{3}{|c|}{ Work per Minute. } \\
\hline & & & $\begin{array}{l}\text { Oxy - } \\
\text { gen } \\
\text { c.c. }\end{array}$ & $\begin{array}{l}\mathrm{CO}_{2} \\
\text { c.c. }\end{array}$ & $\begin{array}{l}\text { Respir- } \\
\text { atory } \\
\text { Quo- } \\
\text { tient. }\end{array}$ & $\begin{array}{c}\text { Work } \\
\text { of } \\
\text { Ascent } \\
\mathrm{Kgm} \text {. }\end{array}$ & $\begin{array}{c}\text { Work } \\
\text { of } \\
\text { Draft. } \\
\text { Kgm. }\end{array}$ & $\begin{array}{l}\text { Dis- } \\
\text { tance. } \\
\text { travel- } \\
\text { led. } \\
\text { Meters. }\end{array}$ \\
\hline $\begin{array}{l}26.932 \\
26.674 \\
27.175\end{array}$ & $\begin{array}{r}6 \\
2 \\
8 \\
5 \\
10\end{array}$ & $\begin{array}{l}\text { Lying i } \text { (Magnus-Levy) } \\
\text { Standing } \\
\text { Ascending slight incline. } \\
\text { Draft nearly horizontal. }\end{array}$ & $\begin{array}{r}174.3 \\
172 . \\
245.6 \\
725.3 \\
1285.3 \\
1028.8\end{array}$ & $\begin{array}{l}124.7 \\
123.8 \\
170.2 \\
525.2 \\
990.6 \\
798.9\end{array}$ & $\begin{array}{l}0.71 \\
0.72 \\
0.69 \\
0.73 \\
0.77 \\
0.77\end{array}$ & $\begin{array}{r}\cdots \cdots \\
\cdots \cdots \\
13.23 \\
365.82 \\
22.83\end{array}$ & $\begin{array}{l}\ldots \cdots \\
\cdots \cdots \\
\cdots \cdots \\
\cdots \cdots \\
202.91\end{array}$ & $\begin{array}{l}78.566 \\
79.497 \\
70.420\end{array}$ \\
\hline
\end{tabular}

The work per minute as given in the above table does not include the energy expended in horizontal locomotion. The work of draft is the product of the distance traversed into the draft; the work of ascent equals the same distance multiplied by the sine of the angle of ascent. A remarkable increase ( 41 per cent.) in the metabolism when standing over that when lying was observed (compare p. 343) but does not enter into the subsequent computations.

The two experiments on ascending a grade afford data for computi ig the increased metabolism corresponding, on the one hand, to one gram-meter of work done against gravity, and, on the other, to the transportation of one kilogram through one meter horizontally. The latter, of course, is not work in the mechanical sense, but it requires the consumption of a certain amount of material, the liberated energy being employed in successive liftings of the body and in overcoming internal resistances and ultimately appearing as heat. It includes, of course, the increased metabolism required for the maintenance of the erect position.

* Arch. ges. Physiol., 68, 191. 
If from the totals given in the table we subtract the figures for rest, 'we have the following as the increments of the respiration due to the work, including the work of standing:

\begin{tabular}{|c|c|c|}
\hline & $\begin{array}{c}\text { Oxygen. } \\
\text { c.c. }\end{array}$ & $\begin{array}{c}\text { Carbon Dioxide, } \\
\text { c.c. }\end{array}$ \\
\hline $\begin{array}{c}\text { Ascending slight incline. } \\
\text { steeper " }\end{array}$ & $\begin{array}{c}551.0 \\
1111.0\end{array}$ & $\begin{array}{c}500.5 \\
865.9\end{array}$ \\
\hline
\end{tabular}

The weight of the animal and the distance traversed having differed somewhat, the results may be rendered comparable by computing them per kilogram of weight and per meter of distance traversed-that is, by dividing in each case by the product of weight into distance. Expressing the results in gram-meters and cubic millimeters for convenience we have-

\begin{tabular}{|c|c|c|c|}
\hline & $\underset{\text { c.mm }}{\text { Oxygen }}$ & $\begin{array}{l}\text { Carbon Dioxide } \\
\text { c mm. }\end{array}$ & $\begin{array}{c}\text { Work of Ascent, } \\
\text { gr.-m. }\end{array}$ \\
\hline$\underset{" \text { Ascending slight incline.... }}{\text { steeper }}$ & $\begin{array}{l}260.40 \\
523.93\end{array}$ & $\begin{array}{l}189.27 \\
408.35\end{array}$ & $\begin{array}{r}6.252 \\
172.512\end{array}$ \\
\hline
\end{tabular}

If we let $x$ equal the oxygen consumption required for the transportation of $1 \mathrm{~kg}$. through 1 meter and $y$ that required per grammeter of work of ascent we have

$$
\begin{aligned}
& x+6.252 y=260.40 \text { c.mm. } \\
& x+172.512 y=523.93 \text { c.mm. }
\end{aligned}
$$

whence we have

$$
\begin{aligned}
& x=250.49 \text { c.mm. } \\
& y=1.585 \text { c.mm. }
\end{aligned}
$$

A similar computation for the carbon dioxide gives

Locomotion, per kg. and meter...... 181.033 c.mm.

Per gram-meter of work of ascent... 1.317 c.mm.

and the corresponding respiratory quotient is 0.723 .

With these data in hand it is easy to compute the increased respiratory exchange corresponding to one gram-meter of work of draft as follows: 


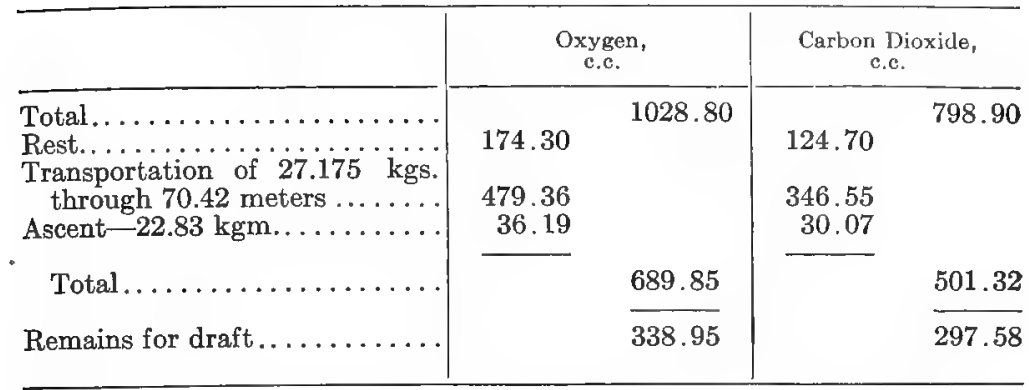

For one gram-meter of work of draft we have, therefore,

Oxygen.................... 1.6704 c.mm

Carbon dioxide.............. 1.467 c.mm

Respiratory quotient........... 0.878

It appears from the above that the work of draft required somewhat more metabolism than the same amount of work of ascent. The individual experiments of this and other series likewise show that variations in the speed and in the angle of ascent affect the result. For the present, however, we may confine ourselves to a consideration of the average figures.

It remains to compute from the results for oxygen and carbon dioxide the corresponding amounts of energy liberated. The data are insufficient for an exact computation. It having been shown, however (compare Chapter VI), that even severe work causes but a slight increase in the proteid metabolism, the author assumes that the additional metabolism in these experiments was entirely at the expense of carbohydrates and fat and computes the proportion of each from the respiratory quotient. The results are admittedly not exact. Besides the uncertainty just mentioned, there is the possibility that irregularities in the excretion of carbon dioxide may affect the respiratory quotient in short trials and, moreover, we must bear in mind the possibility of various cleavages and hydrations as affecting the evolution of energy in such experiments (compare Berthelot's criticism on p. 254). The author does not, however, regard these possible errors as very serious. Computed on this basis the results are as follows, expressed both in terms of heat (calories) and in gram-meters ( 1 cal. equals 425 gram-meters): 
For 1 gram-meter, ascent....... 0.0076681 cal. $=3.259$ gr.-m.

" 1 " " $\operatorname{draft} . \ldots \ldots \ldots . . .0 .008180 "=3.476$ " "

" locomotion per $\mathrm{kg}$. and meter. . 1.1787 cals. $=500.95$ "

According to the above figures the performance of one grammeter of work required the metabolizing of material whose potential energy was equal to 3.259 gr.-m. in the one case and 3.476 gr.-m. in the other. In other words, these amounts of net available energy were liberated in the kinetic form in the body, one gram-meter in each case being recovered as external work while the remainder ultimately took. the form of heat.

This is equivalent to a utilization of 30.7 per cent. of the net available energy in ascent and of 28.77 per cent. in draft. It is to be noted that these figures refer only to that portion of the increased metabolism which is applied to the production of external work and do not include that necessary for the transportation of the animal's weight. The corresponding ratio for this portion could only be obtained on the basis of complicated and uncertain computations of the mechanical work of locomotion. If, however, instead of this we assume that this most common form of muscular activity is performed with the same economy as the work of ascent, we can conversely compute the mechanical work of locomotion for $1 \mathrm{~kg}$. through 1 meter as

$$
500.95 \text { gr.-m. } \times .307=153.8 \text { gr.-m. }
$$

Experiments on Man.-In connection with his experiments on the dog already described, Zuntz * cites the results of a number of experiments with man upon the work of locomotion and of ascent, the average results of which are summarized in the table opposite, to which have been added the results of later experiments by Frentzel \& Reach. $\dagger$

Experiments on the Horse.-Very extensive investigations on the production of work by the horse have been made by Zuntz in conjunction with Lehmann and Hagemann. $\$$ Some of the results of these investigations have already been discussed in their bearing on the question of digestive work (pp. 385-393), and the method

* Loc cit., p. 208.

$\dagger$ Arch. ges Physiol., 83, 494.

‡Landw. Jahr., 18, 1; 23, 125; 27, Supp III 


\begin{tabular}{|c|c|c|c|c|c|}
\hline \multirow[b]{2}{*}{ Experimenter. } & \multirow{2}{*}{$\begin{array}{l}\text { Weight } \\
\text { (with } \\
\text { Appara- } \\
\text { tus), } \\
\text { Kgs. }\end{array}$} & \multicolumn{2}{|c|}{ Energy Expended in } & \multirow{2}{*}{$\begin{array}{c}\text { Horizontal } \\
\text { Velocity } \\
\text { per } \\
\text { Minute, } \\
\text { Meters. }\end{array}$} & \multirow[b]{2}{*}{$\begin{array}{l}\text { Grade, } \\
\text { Per Cent. }\end{array}$} \\
\hline & & $\begin{array}{c}\text { Loco- } \\
\text { motion } \\
\text { per Kg. } \\
\text { and Meter, } \\
\text { Kgm. }\end{array}$ & $\begin{array}{c}\text { Per Kgm. } \\
\text { Work of } \\
\text { Ascent. } \\
\text { Kgm. }\end{array}$ & & \\
\hline \multirow[t]{2}{*}{ Katzenstein ........ } & 55.5 & 0.334 & & 74.48 & $9.6-13.3$ \\
\hline & 72.9 & 0.217 & 3.190 & $71.32\}$ & 6.5 \\
\hline \multirow[t]{3}{*}{ Schumburg \& Zuntz } & 67.9 & 0.211 & 3.140 & $71.46\}$ & \\
\hline & $\begin{array}{l}80.0 \\
88.2\end{array}$ & $\begin{array}{l}0.288 \\
0.263\end{array}$ & $\begin{array}{l}3.563 \\
3.555\end{array}$ & $\begin{array}{l}51.23 \\
43.34\end{array}$ & $30.7-62.0$ \\
\hline & 72.6 & 0.284 & 2.913 & $62 \quad 04\}$ & \\
\hline \multirow[t]{2}{*}{ Loewy ............ } & 81.1 & 0.231 & 2.921 & $60.90\}$ & $23.0-30.5$ \\
\hline & 80.0 & 0.244 & 2.729 & 56.54 & \\
\hline \multirow{5}{*}{$\begin{array}{l}\text { Frentzel: } \\
\text { Normal gait. } \ldots \ldots \ldots \\
\text { Slow } \quad \ldots \ldots \ldots \\
\text { Reach: } \\
\text { Normal gait. } \ldots \ldots \ldots \\
\text { Slow } \quad \ldots \ldots \ldots \ldots\end{array}$} & 86.5 & & & & \\
\hline & 86.5 & 0.233 & 2.746 & $\begin{array}{l}00.94 \\
3, .92\end{array}$ & \\
\hline & & & & & 23.3 \\
\hline & 65.8 & 0.230 & 2.846 & 63.95 & \\
\hline & 55.8 & 0.251 & & $34.58 J$ & \\
\hline
\end{tabular}

of computing the total metabolism in the rest experiments has been explained; it remains to consider the results of the work experiments. The larger proportion of the experiments were upon the same horse (No. III), and the summaries and averages on subsequent pages represent chiefly the results with this animal.

The work was done upon a special tread-power located in the open air, and during the rest experiments the animal likewise stood in the tread-power. The inclination of the platform of the power could be varied, and it could also be driven by a steam-engine, so that by setting it horizontal the work performed by the animal was reduced to that of locomotion alone. The distance traversed was measured by a revolution-counter, and in the experiments on draft the animal pulled against a dynamometer.

The large number of experiments (several hundred) are grouped by the authors into fourteen periods according to the season (winter or summer) and the kind and amount of food consumed, each of these periods including a considerable number of experiments both on rest and on different forms of work. On each day from two to eight experiments were usually made, some on rest and some on work of various sorts. The average of all the rest experiments in each period is then compared with similar averages for the various 
kinds of work in order to eliminate so far as possible the influence of variations in external temperature and in the feeding, as well as to reduce the probable error of experiment.

WORK AT A WALK.-The experiments may be grouped into those in which the work was performed respectively at a walk and a trot. Those of the former category, being the more numerous, may be considered first.

Work of Locomotion.-The following detailed comparison of the experiments of Period $a$ upon rest and upon walking without load or draft will serve to further explain the method:

REST EXPERIMENTS. PERIOD $a$.

Ration, $6 \mathrm{Kg}$. Oats, $1 \mathrm{Kg}$. Straw, 6-7 Kg Hay.

\begin{tabular}{|c|c|c|c|c|c|c|}
\hline \multirow{2}{*}{ No. of Experiment. } & \multicolumn{2}{|c|}{$\begin{array}{l}\text { Per Kg Live Weight } \\
\text { and Minute. }\end{array}$} & \multirow{2}{*}{$\begin{array}{c}\text { Respira- } \\
\text { tory } \\
\text { Quotient. }\end{array}$} & \multirow{2}{*}{$\begin{array}{l}\text { Air Tem- } \\
\text { perature } \\
\text { Deg. C. }\end{array}$} & \multirow{2}{*}{$\begin{array}{l}\text { Relative } \\
\text { Velocity } \\
\text { of Wind. }\end{array}$} & \multirow{2}{*}{$\begin{array}{l}\text { Hours } \\
\text { Since Last } \\
\text { Feeding. }\end{array}$} \\
\hline & $\begin{array}{l}\text { Oxygen } \\
\text { c.c. }\end{array}$ & $\begin{array}{l}\text { Carbon } \\
\text { Dioxide } \\
\text { c.c. }\end{array}$ & & & & \\
\hline $\begin{array}{l}37 a \ldots \ldots \ldots \ldots \\
38 b \ldots \ldots \ldots \\
38 f \ldots \ldots \\
39 a \ldots \ldots \\
44 a \ldots \ldots \\
45 g \ldots \ldots \ldots \\
46 a \ldots \ldots \ldots\end{array}$ & $\begin{array}{l}3.94 \\
3.92 \\
3.98 \\
4.06 \\
4.11 \\
3.89 \\
3.71\end{array}$ & $\begin{array}{l}3.81 \\
4.02 \\
3.42 \\
4.04 \\
3.86 \\
3.63 \\
3.44\end{array}$ & $\begin{array}{l}0.968 \\
1.025 \\
0.861 \\
0.997 \\
0.940 \\
0.933 \\
0.929\end{array}$ & $\begin{array}{r}-5.0 \\
-0.5 \\
2.0 \\
5.3 \\
4.7 \\
2.0 \\
9.0\end{array}$ & $\begin{array}{l}0 \\
1 \\
1 \\
3 \\
1 \\
1 \\
3\end{array}$ & $\begin{array}{l}3.0 \\
2.5 \\
5.6 \\
2.0 \\
1.5 \\
3.5 \\
1.5\end{array}$ \\
\hline $\begin{array}{c}\text { Average } \ldots . . . \\
\text { Corrected } * . . .\end{array}$ & $\begin{array}{l}3.94 \\
4.04\end{array}$ & $\begin{array}{l}3.75 \\
3.86\end{array}$ & 0.950 & 2.5 & 1.4 & 2.8 \\
\hline
\end{tabular}

In the same period eight experiments were made in which the $\therefore$ ad-power was set as nearly horizontal as possible and driven by the steam-engine, the animal being simply required to maintain his place on the power. The results for oxygen were as shown in the first portion of the following table:

* A comparison of Zuntz's method with the results obtained in the Pettenkofer respiration apparatus showed that the gazeous exchange through the skin and intestines amounted to about $2 \frac{1}{2}$ per cent. of the pulmonary respiration in case of the oxygen and 3 per cent in case of the carbon dioxide. These additions are accordingly made to the figures of the respiration experiments and the results designated as "corrected." 
WALKING WITHOUT LOAD OR DRAFT. PERIOD $a$.

Per Kg. Live Weight.

\begin{tabular}{|c|c|c|c|c|c|c|c|}
\hline \multirow{3}{*}{$\begin{array}{l}\text { No. of } \\
\text { Experiment. }\end{array}$} & \multirow{3}{*}{$\begin{array}{c}\text { Live } \\
\text { Weight } \\
\text { Kgs. }\end{array}$} & \multicolumn{4}{|c|}{ Observed. } & \multirow{2}{*}{\multicolumn{2}{|c|}{$\begin{array}{c}\text { Oxygen Equivalent } \\
\text { to Work. }\end{array}$}} \\
\hline & & \multicolumn{3}{|c|}{ Per Minute. } & \multirow{2}{*}{$\begin{array}{c}\text { Work of } \\
\text { Ascent, } \\
\text { Per Meter } \\
\text { Traveled. } \\
\text { Gr.-m. }\end{array}$} & & \\
\hline & & $\begin{array}{c}\text { Oxygen } \\
\text { c.c. }\end{array}$ & $\begin{array}{c}\text { Distance } \\
\text { Traveled } \\
\text { Meters. }\end{array}$ & $\begin{array}{c}\text { Work of } \\
\text { Ascent, } \\
\text { Kgm. }\end{array}$ & & $\begin{array}{c}\text { Per } \\
\text { Minute, } \\
\text { c.c. }\end{array}$ & $\begin{array}{l}\text { Per Meter } \\
\text { Traveled. } \\
\text { c.mm. }\end{array}$ \\
\hline $\begin{array}{l}40 d \ldots \ldots \\
44 b \ldots \ldots \\
45 b \ldots \ldots \\
45 d \ldots \ldots \\
46 b \ldots \ldots \\
46 c \ldots \ldots \\
47 b \ldots \ldots \\
47 c \ldots \ldots\end{array}$ & $\begin{array}{l}429 \\
434 \\
428 \\
428 \\
430 \\
430 \\
434 \\
434\end{array}$ & $\begin{array}{r}9.0 \\
11.3 \\
12.2 \\
12.7 \\
10.8 \\
11.7 \\
12.3 \\
11.2\end{array}$ & $\begin{array}{l}57 \\
87 \\
94 \\
95 \\
92 \\
99 \\
98 \\
93\end{array}$ & $\begin{array}{l}0.57 \\
0.84 \\
0.89 \\
0.87 \\
0.70 \\
0.74 \\
0.79 \\
0.76\end{array}$ & $\begin{array}{r}10 \\
10 \\
9 \\
9 \\
8 \\
8 \\
8 \\
8\end{array}$ & $\begin{array}{l}5.1 \\
7.3 \\
8.2 \\
8.7 \\
6.9 \\
7.8 \\
8.4 \\
7.3\end{array}$ & $\begin{array}{l}89 \\
84 \\
88 \\
92 \\
74 \\
79 \\
86 \\
78\end{array}$ \\
\hline $\begin{array}{l}\text { Average ... } \\
\text { Corrected. }\end{array}$ & 430.9 & 11.405 & 89.338 & 0.764 & 8.643 & $\begin{array}{l}7.463 \\
\ldots \ldots\end{array}$ & $\begin{array}{l}83.793 \\
85.888\end{array}$ \\
\hline
\end{tabular}

If from the oxygen consumption in each of the above experiments we subtract the average rest value for the same period ( 3.94 c.c.) the remainder will represent the increase due to the work, as shown in the seventh column, and this divided by the distance traveled gives the figures of the eighth column.

The average respiratory quotient of that part of the respiration due to the work in these eight experiments was 0.894 . On the very probable assumption that the work caused no material change in the metabolism of either proteids * or crude fiber, or in other words, that the energy for work was derived substantially from soluble carbohydrates and fat, the calorific equivalent of 1 c.c. of oxygen is computed and the following calculation of energy made for the average of the eight experiments (compare pp. 76 and 251). These results are not corrected for cutaneous and intestinal respiration.

Per K̆g. Live Weight per Minute.

Oxygen combined with fat ........... 3.4415 c.c.

Oxygen combined with starch......... 4.0215 "

Total. ..................... 7.4630"

Equivalent energy ............ 36.420 cals.

* The authors show that even a considerably increased proteid metabolism would not materially affect the computation of energy. 
Energy per Meter Traveled (Including Work of Ascent).

Per kg. total mass *............... 0.3948 cal.

Per kg. live weight ............. $\left\{\begin{array}{l}0.4077 \text { “ } \\ 0.1733 \mathrm{kgm} .\end{array}\right.$

Work of ascent............... 8.643 gr.-m.

Determinations of the work of locomotion were made in six different periods, or thirty-five experiments in all. The average for each period, computed in terms of energy as in the above example, is given in Table VIII of the Appendix. It is to be noted that these results still include the small amount of work expended in ascending the slight incline. This factor is determined in the manner shown in the following paragraph.

Work of Ascent.--In four periods experiments were made (thirteen in all) upon the work of ascending a moderate grade at a walk. The average results, computed on the same basis as before, are contained in Table IX of the Appendix.

By comparing the average results of these two series of experiments in the manner explained on $p$. 500, letting $x$ equal the oxygen or energy required per kilogram live weight for locomotion through 1 meter horizontally and $y$ the corresponding quantities for the performance of 1 gram-meter of work of ascent we have the following equations:

$$
\begin{gathered}
\text { For Oxygen. } \\
x+4.395 y=83.480 \text { c.mm. } \\
x+107.041 y=222.941 \text { c.mm. } \\
\text { For Energy } . \\
x+4.395 y=0.4035 \text { cal. } \\
x+107.041 y=1.0795 \text { cals. }
\end{gathered}
$$

\begin{tabular}{|c|c|c|c|}
\hline & \multirow{2}{*}{$\begin{array}{l}\text { Oxygen, } \\
\text { c mm. }\end{array}$} & \multicolumn{2}{|c|}{ Energy. } \\
\hline & & cals. & Kgm. \\
\hline $\begin{array}{l}\text { Locomotion per meter: } \\
\text { Per kg. live weight............. } \\
\text { "" total mass. . . . . . . . . } \\
\text { Ascent, per kilogram-meter . . }\end{array}$ & $\begin{array}{r}77.509 \\
75.048 \\
1359.00\end{array}$ & $\begin{array}{l}0.3746 \\
0.3618 \\
6.5858\end{array}$ & $\begin{array}{l}0.1592 \\
0.1538 \\
2.7990\end{array}$ \\
\hline
\end{tabular}

Solving these we obtain the following values respectively for the work of locomotion per meter and for the energy expended in

* Weight of animal plus weight of apparatus carried. 
doing $1 \mathrm{kgm}$. of work of ascent, and the utilization of the available energy in the latter case is 35.73 per cent.

Work of Draft.-For the work of draft at a walk, up a slight incline, the results tabulated in Table $\mathrm{X}$ of the Appendix were obtained.

Giving $x$ and $y$ the same significance as before, and letting $z$ represent the oxygen or energy corresponding to one gram-meter of work of draft, we have the following equation, based on the results per kilogram live weight and meter traveled:

$$
x+5.115 y+153.127 z=306.561 \text { c.mm. }=1.5021 \text { cals. }
$$

Substituting in this the average values of $x$ and $y$ obtained as indicated in the previous paragraph, but from a larger number of experiments, we have

$$
z=1.4504 \mathrm{c.mm} .=.007143 \mathrm{cal} \text {. per gram-meter. }
$$

The above details of a few of the experiments may serve to illustrate the methods of computation employed. Similar determinations were made upon various forms of work under differing conditions, the results of which will be given later.

Correction for Speed.-Before final data could be obtained, however, it was found necessary to take account of the speed of the animal, since comparisons of the various periods showed that the metabolism due to the work of locomotion at a walk increased materially as the velocity increased.

To compute the necessary correction, the authors divide the thirty-five experiments of Table VIII into three groups according to the speed. For each group the oxygen and energy corresponding to the work of ascent are computed, using the values of $y$ given on the previous page (1359 c.mm.; 6.5858 cals.), and subtracted from the total, leaving the following as the amounts per kilogram

\begin{tabular}{|c|c|c|c|c|c|c|}
\hline $\begin{array}{c}\text { No. of } \\
\text { Experi- } \\
\text { ments. }\end{array}$ & $\begin{array}{l}\text { Velocity } \\
\text { per Minute, } \\
\text { Meters. }\end{array}$ & $\begin{array}{l}\text { Oxygen } \\
\text { Consumed } \\
\text { per Kg. } \\
\text { and Meter, } \\
\text { c.mm. }\end{array}$ & $\begin{array}{c}\text { Respira- } \\
\text { tory } \\
\text { Quotient: }\end{array}$ & $\begin{array}{l}\text { Oxygen Re- } \\
\text { calculated to } \\
\text { Respiratory } \\
\text { Quotient of } \\
0.86 \text {, c.mm }\end{array}$ & $\begin{array}{l}\text { Increase of } \\
\text { Oxygen per } \\
\text { Meter } \\
\text { Velocity, } \\
\text { c.mm. }\end{array}$ & $\begin{array}{c}\text { Heat Value } \\
\text { of Oxygen } \\
\text { per Meter } \\
\text { (Corrected). } \\
\text { cals. }\end{array}$ \\
\hline $\begin{array}{r}6 \\
20 \\
9\end{array}$ & $\begin{array}{l}78.00 \\
90.16 \\
98.11\end{array}$ & $\begin{array}{l}66.69 \\
76.04 \\
80.97\end{array}$ & $\begin{array}{l}0.896 \\
0.848 \\
0.873\end{array}$ & $\begin{array}{l}67.32 \\
75.80 \\
81.23\end{array}$ & $\begin{array}{l}0.697 \\
0.683\end{array}$ & $\begin{array}{l}0.3363 \\
0.3787 \\
0.4058\end{array}$ \\
\hline
\end{tabular}
live weight due to horizontal locomotion: 
On the average, an increase of 1 meter per minute in the speed was found to cause an increased metabolism corresponding to-

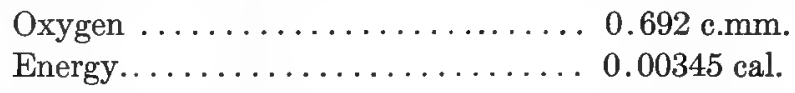

A similar computation for the experiments on ascending a considerable grade without load or draft showed a similar difference, which, however, seemed to be chiefly or entirely due to variations in the work of locomotion. When the amount of the latter was computed with the correction for speed just given, the metabolism due to the actual work of ascent seemed to be independent of the speed, the only exception being two experiments at a rapid walk in which over exertion of the animal was suspected.

In the thirteen experiments on the work of ascending a moderate grade contained in Table IX, the average speed was 81.95 meters per minute, while in the thirty-five experiments with which they are compared (Table VIII) the average speed was 90.16 meters. From the table on p. 506 we compute that the consumption of oxygen $(R . Q .=0.86)$ and the corresponding energy values per kilogram and meter at these speeds would be-

\begin{tabular}{|c|c|c|}
\hline & $\begin{array}{c}\text { Oxygen } \\
\text { c.mm. }\end{array}$ & $\begin{array}{l}\text { Energy. } \\
\text { cals. }\end{array}$ \\
\hline $\begin{array}{l}\text { At } 90.16 \mathrm{M} . \text { velocity } \\
\text { “ } 81.95 \mathrm{M} .\end{array}$ & $\begin{array}{l}75.80 \\
70.05\end{array}$ & $\begin{array}{l}0.3746 \\
0.3462\end{array}$ \\
\hline
\end{tabular}

Substituting this corrected value of $x$ in the equations on p. 506, we have as the corrected value of $y$ per kilogram-meter for ascending a moderate grade

$$
6.851 \text { cals. }=2.912 \mathrm{kgm} .=34.3 \text { per cent. }
$$

In brief, a correction for the value of $x$ is computed. using the first value of $y$, and then this corrected value of $x$ is used to compute the corrected value of $y$. In other words, the method is one of approximation, but the errors of the corrected values are presumably less than the unavoidable errors of experiment.

Effect of Load.--In a number of experiments the horse carried on the saddle a load, consisting of lead plates, corresponding to that of a rider. The mere sustaining of such a weight at rest was found 
to increase the gaseous exchange, the total metabolism being substantially proportional to the total mass (horse + load), but in computing the work experiments the same rest values are used as for the preceding experiments; that is, the results include the work required to simply sustain the weight as well as that required to move it. Computing the results in the same manner as before the authors obtain for an average speed of 90.18 meters per minute the following results:

\section{Locomotion per Meter.}

Per kg. live weight ....... $0.5004 \mathrm{cal} .=0.2126 \mathrm{kgm}$. Ascent.

" " total mass........ $0.3914 "=0.1663$ "

Per kilogram-meter $\ldots \ldots \ldots 6.502$ cals. $=2.7640 "=36.19 \%$

A comparison of these figures with those on p. 506 shows that for this animal a load of $127 \mathrm{kgs}$. caused about 8 per cent. increase in the energy expended, per $\mathrm{kg}$. of total mass, in horizontal locomotion, but no increase in that expended per kilogrammeter in ascent.

Work of Descent.-In descending a grade the force of gravity acts with instead of against the animal and tends therefore to diminish the metabolism. On the other hand, however, as the steepness of the grade increases the animal is obliged to put forth muscular exertions to prevent too rapid a descent, and this tends to increase the metabolism. It was found that an inclination of $2^{\circ} 52^{\prime}$ caused the maximum decrease in the metabolism. At $5^{\circ} 45^{\prime}$ the metabolism was the same as at $0^{\circ}$, while on steeper grades it was greater than on a level surface.

WoRK AT A TROT.-A smaller number of experiments were made upon work at a trot under varying conditions. In trotting, the up and down motion of the body is much greater than in walking, while but a small part of the muscular energy thus expended is available for propulsion. It was therefore to be expected that the energy required for horizontal locomotion would be greater at a trot than at a walk, and the results of the experiments corresponded fully with this expectation, the computed energy per meter being found to be

Per kg. live weight.............. 0.5660 cal.

" " mass (horse +load) .......... 0.5478 " 
at a speed of 195 meters per minute. The fact of such an increased expenditure of energy in trotting as compared with walking has also been confirmed by the results of Grandeau, which will be considered in another connection. It was also found that in trotting, unlike walking, the work of locomotion was independent of the speed within the limits experimented upon (up to a speed of 206 meters per minute, or about $7 \frac{1}{2}$ miles per hour). A load of $127.2 \mathrm{kgs}$. increased the work of locomotion per $\mathrm{kg}$. of mass by about 10 per cent. as compared with the increase of 8 per cent. at a walk. One experiment on work of ascent and one on horizontal draft, both without load, showed a utilization of, respectively, 31.96 per cent. and 31.70 per cent., but two other experiments on horizontal draft, in which the work was thought to have been excessive, gave an average of only 23.35 per cent.

Summary.-The final results of the experiments upon the horse may be summarized as follows:

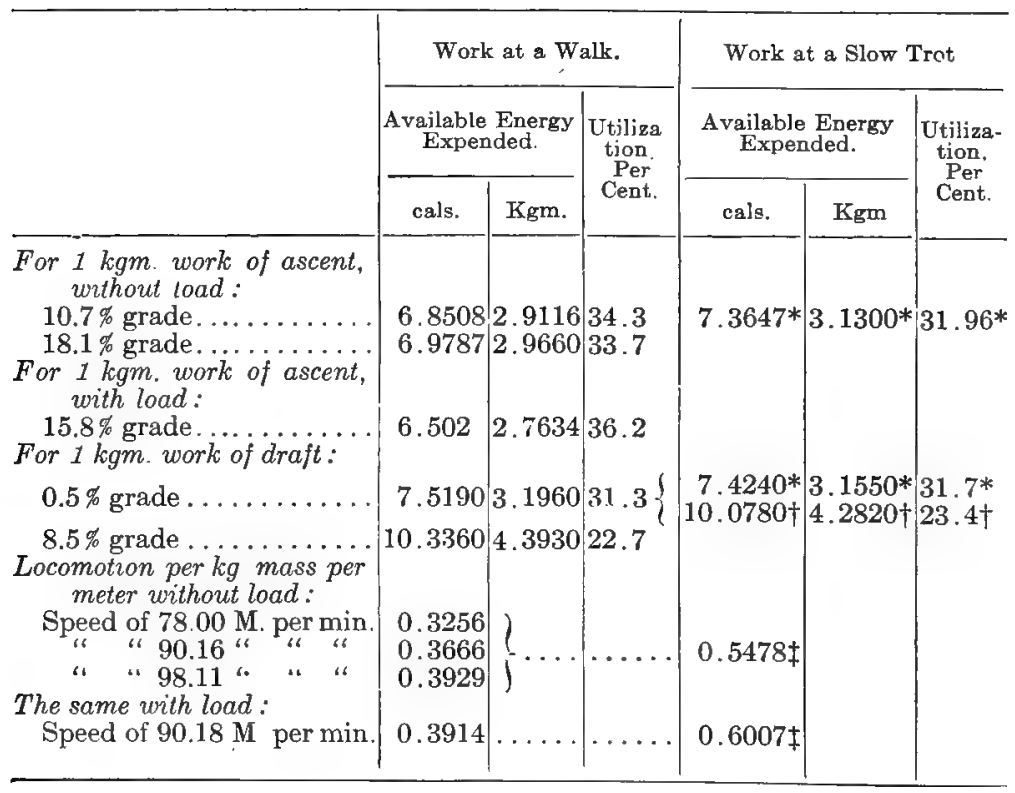

* Single experiment.

† Two experiments. Work probably excessive.

$\ddagger$ Independent of speed. 


\section{Conditions Determining Efficiency.}

From the results recorded in the preceding paragraphs it appears that, as we were led to expect from a consideration of the efficiency of the single muscle, the efficiency of the animal as a converter of potential energy into mechanical work varies with the nature of the work and the conditions under which it is performed, although the variations are perhaps hardly as great as might have been expected. In general, we may say that in the neighborhood of one third of the potential energy directly consumed in muscular exertion is recovered as mechanical work. This appears to be a high degree of efficiency as compared with that of any artificial transformer of potential energy yet constructed. The steam-engine, the chief example of such transformers, even in its most highly perfected forms, rarely utilizes over 15 per cent. of the potential energy of the fuel, while in ordinary practice one half of this efficiency is considered a good result.

The comparison is misleading. however, for three reasons: First, the figures given in the preceding pages relate to the utilization of the net available energy of the food. As we have seen, however, a certain expenditure of energy in digestion and assimilation is required to render the food energy available, while still another portion of the latter is lost in the potential energy of the excreta. In the case of herbivorous animals. these two sources of loss very materially reduce the percentage utilization when computed upon the gross energy of the food. Second, the comparison takes no account of the large amount of energy consumed continually throughout the twenty-four hours for the internal work of the body of the animal, and which continues irrespective of whether the animal is used as a motor or not. Third, the expenditure of energy in locomotion is not considered in computing the efficiency of one third. When these three points are allowed for but little remains of the apparent superiority of the animal as a prime motor, even omitting from consideration the greater cost of his fuel (food).

It remains now to consider somewhat more specifically the in. fluence upon the efficiency of the animal machine of some of the more important conditions. 
KIND OF WorK.-Of the forms of work investigated, that of ascent, that is, of raising the weight of the body (with or without load), appears to be the one which is performed most economically. The horse in ascending a moderate grade without load showed an efficiency of 34.3 per cent., while with a load of $127 \mathrm{kgs}$. a slightly higher efficiency was obtained, viz., 36.2 per cent. (The latter figure, however, includes some estimated corrections for speed.) For the dog (p. 502) the average result was 30.7 per cent. For man the figures of the table on p. 503 correspond to from 28.1 to 36.6 per cent.

The efficiency, however, was found to decrease with the steepness of the grade. Thus with the horse it fell from 34.3 to 33.7 per cent., with an increase of the grade from 10.7 to 18.1 per cent. The experiments of Loewy on man, averaged on p. 503, show the same result in a more striking manner. Taking separately the experiments on each subject we have the following:

\begin{tabular}{|c|c|c|c|}
\hline \multirow{2}{*}{$\begin{array}{c}\text { Grade } \\
\text { Per Cent. }\end{array}$} & \multicolumn{3}{|c|}{ Efficiency. } \\
\cline { 2 - 4 } & $\begin{array}{c}\text { A. L. } \\
\text { Per Cent. }\end{array}$ & $\begin{array}{c}\text { J. L } \\
\text { Per Cent. }\end{array}$ & $\begin{array}{c}\text { L } \mathbf{Z} \\
\text { Per Cent. }\end{array}$ \\
\hline 23 & 34.3 & 36.1 & 36.6 \\
30.5 & 34.3 & 32.6 & 36 \\
36.6 & 29.0 & 32.3 & $\mathbf{3 2 . 2}$ \\
\hline
\end{tabular}

The work of horizontal locomotion consists largely of successive liftings of the weight of the body. It might therefore be expected from the above results that this work would be performed even more economically than that of ascent, since it is obviously the form of muscular activity for which animals like the horse and dog are specially adapted. In the case of the walking horse, Kellner * has proposed a formula based on mechanical considerations, for computing the work of locomotion. Zuntz $\dagger$ has applied this formula to the animal used in his experiments and computed the mechanical work of locomotion at the three speeds for which the total metabolism was also determined (p. 507).

Landw. Jahrb., 9658.

$\dagger$ Ibid, 27. Supp III, p 314. 
A comparison of these figures, expressing the total metabolism in its mechanical equivalent, is as follows:

\begin{tabular}{|c|c|c|c|}
\hline & \multicolumn{3}{|c|}{ Per Kg. Mass and Meter. } \\
$\begin{array}{c}\text { Speed } \\
\text { Meters per } \\
\text { Minute. }\end{array}$ & $\begin{array}{c}\text { Total } \\
\text { Metabolism } \\
\text { Gram-meters. }\end{array}$ & $\begin{array}{c}\text { Computed } \\
\text { Work } \\
\text { Gram-meters. }\end{array}$ & $\begin{array}{c}\text { Percentage } \\
\text { Efficiency. }\end{array}$ \\
\cline { 2 - 4 } & 138.4 & 49.14 & 35.5 \\
78.00 & 155.8 & 54.54 & 35.00 \\
90.16 & 167.0 & 58.40 & 34.97 \\
98.11 & & & \\
\hline
\end{tabular}

This computation gives an efficiency slightly greater than that obtained for the ascent of a grade without load, and in so far tends to confirm our conjecture, but the basis on which the work of locomotion is computed can hardly be regarded as sufficiently accurate to give this result the force of a demonstration.

The work of draft appears to be performed considerably less economically than that of ascent or locomotion. Thus, for the horse, the efficiency for nearly horizontal draft was found to be 31.3 per cent. at a walk, and in one experiment at a trot 31.7 per cent., as against 34-36 per cent. for ascent. In two other experiments at a trot, in which the work may have been excessive, a much lower efficiency was found, viz., 23.4 per cent. For draft up a grade of 8.5 per cent. at a walk the efficiency was greatly reduced, viz., to 22.7 per cent. The above figures refer to the work of draft only, after deducting the energy required for locomotion and ascent. A similar difference was likewise observed with the dog (p. 502), the efficiency in nearly horizontal draft being 28.8 per cent. as compared with 30.7 per cent. for work of ascent.

Experiments on man, not cited in the above pages, in which the work was performed by turning a crank, have shown decidedly lower figures for the percentage utilization.

SPEED AND Gait.- The energy expended by the horse in locomotion at a walk was found to increase with the speed at the rate of 0.00334 cal. per meter and kilogram mass for each increase of 1 meter in the speed per minute. Kellner's mechanical analysis of the work of locomotion mentioned above divides it into two parts, viz., that expended in lifting the body of the 
animal and that expended in imparting motion to the legs. The former portion is regarded as constant, while the latter portion would increase with the speed. The very close proportionality between the work thus computed and the total metabolism, as shown by the table on the preceding page, is a strong confirmation of the correctness of both methods and places the conclusion as to the influence of speed upon metabolism beyond reasonable doubt. It is to be remembered, however, that it is the total metabolism per kilogram and meter which increases with the speed. The percentage utilization of the energy, so far as the data at our command enable us to determine, apparently remains constant. Practically, however, it is the former fact which interests us, since the expenditure of energy in locomotion is comparable to that in internal work and has only an indirect economic value. A similar effect of speed on the metabolism in horizontal locomotion was observed by Zuntz * in experiments on man. In those with the dog; on the other hand, the variations in speed were between 64.2 and 85.9 meters per minute, but no material difference in the metabolism due to locomotion was observed.

In trotting, a horse expends much more energy per unit of horizontal distance than in walking. Thus, trotting at an average speed of 195 meters per minute (a little over 7 miles per hour), as compared with walking at an average speed of 90.16 meters per minute, gave the following results for the metabolism per kilogram mass and meter distance.

$$
\begin{aligned}
& \text { Trotting................... } 0.5478 \text { cal. } \\
& \text { Walking...................... 0.3666 " }
\end{aligned}
$$

On the other hand, speed is, so to speak, obtained more economically at the trot than at the walk. In the averages just given the speed was increased by 116 per cent., while the metabolism was increased by only 49 per cent. The same result is reached in another way by computing, by means of the factor given at the beginning of this paragraph $(0.00334$ cal. $)$, the theoretical walking speed which would give a metabolism equal to the average metabolism in trotting. We find this to be 147 meters per second, as compared with 195 meters at a trot. Moreover, it was found that at the trot the metabolism did not increase with the speed, within the limits of the ex-

* Arch. ges. Physiol., 68, 198. 
periments. These, however, did not include speeds above 206 meters per minute (about $7 \frac{1}{2}$ miles per hour), and the work was done on a tread-power, so that there was no air resistance. At this moderate speed it is not probable that the latter factor would be a large one, but it is one which increases as the square of the velocity, so that at high speeds it constitutes the larger portion of the resistance. At high speeds, too, the muscles contract to a greater degree, thus decreasing their efficiency, and additional auxiliary muscles are called into play, both directly and to aid the increased respiration. It is a matter of common experience that while a horse is able to travel for a number of miles consecutively at 6 to 7 miles per hour, drawing a considerable load, he can maintain his highest speed for only a short time even without load, and does this only at the cost of largely increased metabolism. It is evident then that there is a limit beyond which an increase of trotting speed must increase the metabolism with comparative rapidity.

LOAD.- Supporting a load on the back while standing was found to increase the metabolism of the horse No. III approximately in proportion to the load-that is, the metabolism computed per unit of mass (horse + load) increased but very slightly. In locomotion with a load the metabolism is, of course, increased, since the load as well as the body of the animal must be lifted at each step. The increase over the metabolism at rest and without load, both walking and trotting, was found in the case of Horse III to be somewhat greater ( $8-10$ per cent.) than the increase in the mass moved (horse+load).

After making allowance for this increase in the work of locomotion, the efficiency in ascent with a load was found to be unaffected by the latter; that is, the energy expended in lifting a unit of mass (horse+load) through a unit of distance remained substantially the same. Indeed the figure obtained (36.2 per cent.) is slightly higher than that without load (34.3 per cent). Interesting individual differences in the above particulars were, however, observed between Horse No. III and some of the other animals experimented upon, particularly Nos. II and XIII, which form the subject of a succeeding paragraph.

Species and Size of Animal.-In ascending a moderate grade, the efficiency seems to be about the same in the horse and in 
man, while in the dog it is apparently somewhat less, as is seen from the following comparison:

\begin{tabular}{|c|c|c|}
\hline & $\begin{array}{c}\text { Grade, } \\
\text { Per Cent. }\end{array}$ & $\begin{array}{l}\text { Efficiency, } \\
\text { Per Cent. }\end{array}$ \\
\hline Man........... & 23 & 35.7 \\
\hline Horse......... & 10.7 & 34.3 \\
\hline " $\quad \ldots$ & 18.1 & 33.7 \\
\hline $\operatorname{Dog} \ldots \ldots \ldots \ldots \ldots$ & 17.2 & 30.7 \\
\hline
\end{tabular}

The energy expended in horizontal locomotion, on the other hand, showed more marked differences, viz.:

\begin{tabular}{|c|c|c|}
\hline & $\begin{array}{l}\text { Speed, Meters } \\
\text { per Minute. }\end{array}$ & $\begin{array}{c}\text { Energy Expended } \\
\text { per Kg. Mass } \\
\text { per Meter, Kgm. }\end{array}$ \\
\hline $\begin{array}{l}\operatorname{Dog} \ldots \ldots \ldots \ldots \ldots \ldots \\
\text { Man } \ldots \ldots \ldots \ldots \ldots \ldots \\
\text { Horse } \ldots \ldots \ldots \ldots \ldots\end{array}$ & $\begin{array}{l}78.57 \\
42.32-74.48 \\
78.00\end{array}$ & $\begin{array}{c}0.501 \\
0.211-0.334 \\
0.138\end{array}$ \\
\hline
\end{tabular}

The relatively high figure for the dog is perhaps due in part to the considerable muscular effort apparently required (p.499) to maintain the erect posture. It has been shown by v. Hösslin,* however, by a mechanical analysis of the work of locomotion, that the latter does not increase as rapidly as the weight of the animal, but in proportion to its two-thirds power, or, in other words, approximately in proportion to the surface. If we compare the experiments upon different species of animals on this basis-that is, if we divide the total energy expended by the animal for locomotion by the product of the distance traversed into the two-thirds power of the weight -we obtain the following figures:

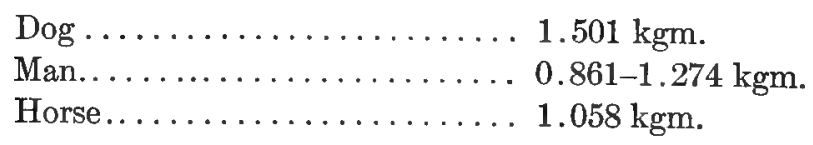

Computed in this way, the figures for the horse and those for man at a comparable speed (74.48 M. per min.) do not differ greatly, and v. Hösslin's conclusions are to this extent confirmed. The figures for the dog still remain higher than the others. If, in the case of * Archiv f. (Anatomie u.) Physiol., 1888, p. 340. 
this animal, we compare the total metabolism in locomotion with that during standing instead of lying, as was done in the case of the horse, the figure is reduced to $1.303 \mathrm{kgm}$., or not much higher than in the case of man. It must be remembered, however, that the figures above given for man include the metabolism due to standing.

INDIVIDUALITY.-Zuntz \& Hagemann's investigations show that the efficiency of the horse is affected to a considerable degree by the individual differences in animals. The experiments whose results are summarized on p. 510 were upon a single animal (No. III). In addition to these a small number of experiments were made with several other animals, mostly old and more or less worthless ones, besides the considerable.number upon Horse No. II previously reported by Lehmann \& Zuntz.* The results are computed by the authors in terms of energy and corrected for speed upon the basis of the results obtained with Horse No. III.

In a single case the work of ascent required slightly less expenditure of energy than with Horse No. III, and in another case the work of horizontal locomotion, computed to the same live weight in proportion to the two-thirds power of the latter (see the opposite page) was also less than for Horse No. III, but as a rule these old, defective horses gave higher results. For ascent, omitting one exceptional case, the range was as follows:

Per Kgm. of Work.

Minimum..... 5.906 cals. $=39.84$ per cent. efficiency

Maximum..... 9.027 " $=26.07$ " " "

Horse No. III . . 6.851 " $=34.30$ " " "

With one very lame horse (string-halt) the figures reached the maximum of 12.343 cals., or an efficiency of only 16.6 per cent.

A similar range was observed in the results on horizontal locomotion. Reduced to a speed of $78 \mathrm{M}$. per minute and to the live weight of No. III, the range was as follows:

$$
\text { Per Meter and Kilogram Live Weiqht. }
$$

Minimum.................... $9.284 \mathrm{cal}$.

Maximum.................... 0.441 "

Horse No. III................. 0.336 "

* Landw. Jahrb., 18, 1. 
The very lame horse mentioned above gave a still higher figure, viz., 0.566 cal.

A somewhat larger number of experiments with Horse No. XIII brought out the interesting fact that the increase in the metabolism caused by carrying a load on the back was markedly less than in the case of No. III, both at rest and in motion.

PER KILOGRAM MASS (HORSE+LOAD).

\begin{tabular}{|c|c|c|}
\hline & $\begin{array}{l}\text { Without Load, } \\
\text { cals. per Minute. }\end{array}$ & $\begin{array}{l}\text { With Load, } \\
\text { cals. per Minute. }\end{array}$ \\
\hline Standing: & & \\
\hline Horse XIII. . . . . . . . & 15.990 & 14.670 \\
\hline $\begin{array}{l}\text { Walking horizontally : } \\
\text { Horse XIII. } \\
\text { "، III } \ldots \ldots \ldots\end{array}$ & $\begin{array}{l}\text { cals. per Meter. } \\
0.389 \\
0.367\end{array}$ & $\begin{array}{l}\text { cals. per Meter. } \\
0.388 \\
0.391\end{array}$ \\
\hline $\begin{array}{c}\text { Trotting Horizontally: } \\
\text { Horse XII. X..... } \\
\text { III........ }\end{array}$ & $\begin{array}{l}0.553 \\
0.548\end{array}$ & $\begin{array}{l}0.488 \\
0.601\end{array}$ \\
\hline
\end{tabular}

While, without load, Horse No. XIII showed a greater metabolism, both while walking and trotting than did Horse No. III, the additional effort required for carrying a load was relatively less, so that in every case the metabolism per unit of mass, instead of increasing, remained unchanged or even diminished. The percentage efficiency of the animal in ascending a grade was also not materially affected by the load, while with Horse No. III it appeared to increase slightly.

The experiments with Horse No. II previously reported,* when recalculated $\dagger$ in the same manner as the later ones, likewise show interesting individual differences. For horizontal locomotion, after correcting for varying speeds, we have per kilogram mass (horse + load) the following:

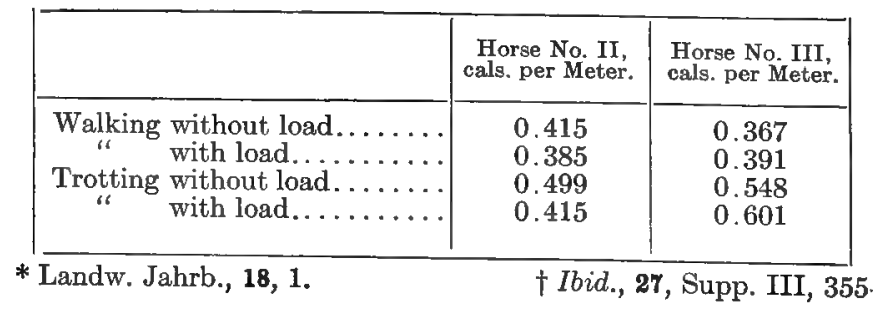


As these figures show, No. II was decidedly inferior to No. III in walking without load. In trotting, on the other hand, he was somewhat the superior of No. III, or in other words the change from walking to trotting caused much less increase in his metabolism. Like No. XIII, he carried a load with decidedly less expenditure of energy than did No. III. For the forms of work in which the percentage efficiency could be measured the results were as follows, the grades, however being not exactly the same for No. II as for No. III:

\begin{tabular}{|c|c|c|}
\hline & $\begin{array}{c}\text { Horse No. II, } \\
\text { Per Cent. }\end{array}$ & $\begin{array}{c}\text { Horse No. III, } \\
\text { Per Cent. }\end{array}$ \\
\cline { 1 - 2 } Ascending, moderate grade... & 33.2 & 34.3 \\
"، heavier grade.... & 31.7 & 33.7 \\
Draft, nearly horizontal...... & 29.0 & 31.3 \\
up a grade........... & 22.4 & 22.7 \\
\hline
\end{tabular}

It seems a fair presumption that such individual differences as those above instanced are caused, in large part at least, by differences in the conformation of the animals resulting from heredity or "spontaneous" variation. A strain of horses which has been bred and trained especially for the saddle through a number of generations might very naturally be expected to be more efficient in carrying a load than a strain which has been bred for speed in harness or strength in draft, while the latter might as naturally excel the former in efficiency at the trot or in draft. Similarly, a race of horses developed in a hilly country might be expected to be more efficient in ascending a grade than one inhabiting a flat region. It would seem, too, that these differences may be not inconsiderable. The results cited suggest an interesting line of thought and investigation for the student of breeding.

Training and Fatigue.- It is a familiar experience that any unaccustomed form of work is much more fatiguing at first than it is later. This is due in part to the fact that in making unfamiliar motions more accessory groups of muscles are called into activity than are necessary later when more skill has been acquired. The experience of a learner on the bicycle is an excellent example of this. In the second place, however, simple exercise of a group of 
muscles in a particular way seems to increase their average mechanical efficiency.

Gruber,* in two series of experiments upon himself, obtained the following figures for the excretion of carbon dioxide during rest, horizontal locomotion, and hill climbing, all the trials being made about the same length of time (four to five hours) after the last meal:

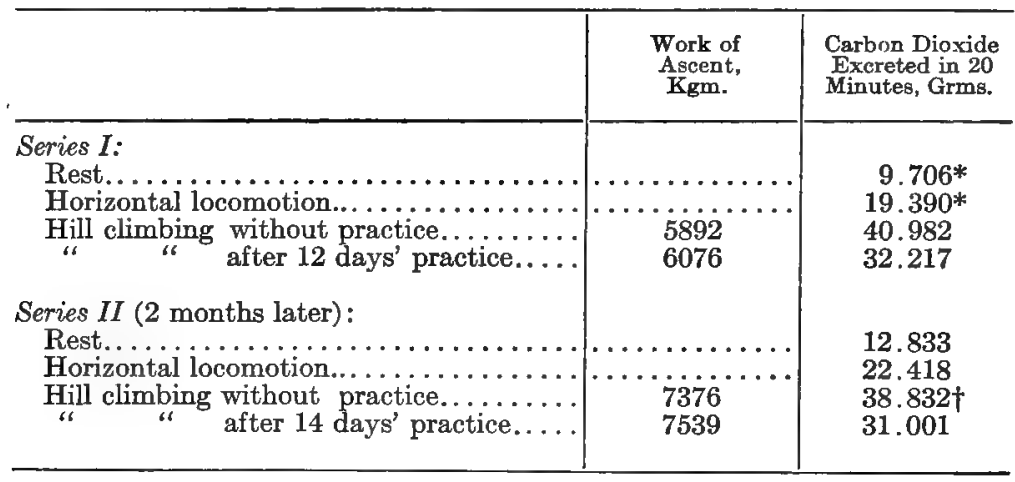

* Some carbon dioxide may have escaped absorption.

† Some carbon dioxide lost.

Schnyder $\dagger$ has confirmed and extended Gruber's results. In experiments in a treadmill upon two different subjects he obtained the following figures for the work performed per gram of carbon dioxide excreted in excess of that given off during rest:

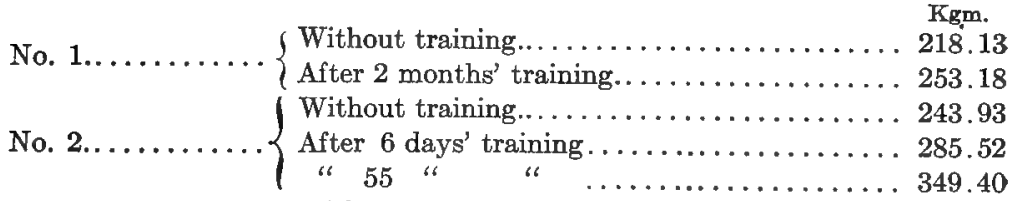

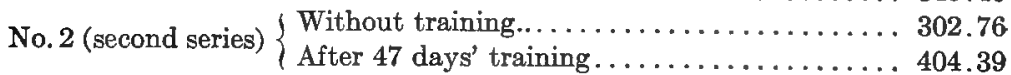

That the greater efficiency after training is not due solely to a diminished use of accessory muscles is shown by Schnyder's experiments on convalescents. His results were as follows:

* Zeit. f. Biol., 28, 466

† Ibid., 33, 289. 


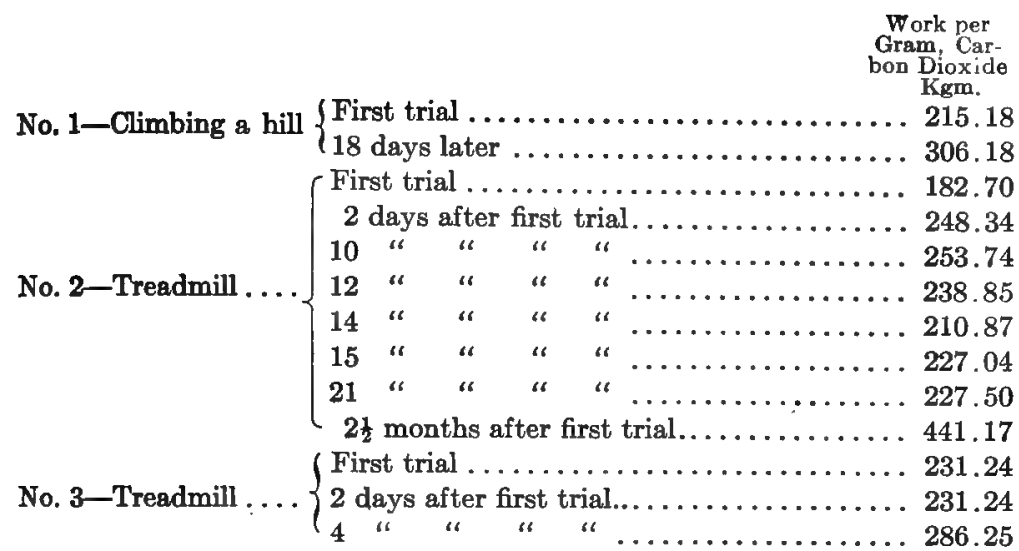

In walking the same distance ( 468 M.) No. 1 excreted the following excess of carbon dioxide over the rest value:

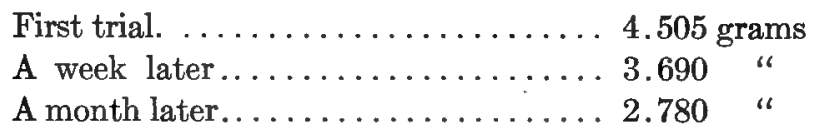

It appears from these results that the gradual strengthening of the muscles during convalescence results in a more economical performance of their work, largely independent of any special training for a particular kind of work. It seems a justifiable conclusion, therefore, that a part of the gain due to training arises from its direct effect in strengthening the muscles, as well as from the increased skill acquired in their use. Conversely, the effect of fatigue in increasing the relative metabolism, as shown by Loewy,* would seem to be in part a direct effect. Schnyder summarizes the matter in the statement that it is not the work itself, but the muscular effort required, which determines the amount of metabolism.

In the case of domestic animals kept chiefly for work, however, we may safely assume that they are constantly in a state of training, and that the results obtained by Zuntz and his associates on the horse are applicable to work done by normal animals within the limits of the experimental conditions.

* Arch. ges. Physiol., 49, 405. 
Relative Values of Nutrients. - In the foregoing discussion it has been tacitly assumed that the stored-up energy of the proteids, fats, and carbohydrates of the body is all net available energy, ready to be utilized directly for the production of mechanical work. As we have seen, however, on previous pages, a school of physiologists, of which Chauveau may stand as the representative, denies this, and holds that the fait in particular must be converted into a carbohydrate before it can become directly available.

In discussing the source of muscular energy in Chapter VI it was shown that the recorded results as regards the nature of the material metabolized were insufficient to decide the question, since the final excretory products are qualitatively and quantitatively the same whether the fat is directly metabolized in the muscle or undergoes a preliminary cleavage in the liver or elsewhere in the body. The results as to energy, however, would be materially different in the two cases. The dextrose resulting from the cleavage of fat, according to Chauveau's schematic equation (p. 38), would contain but about 64 per cent. of the potential energy of the fat, the remainder being liberated as heat. We cannot, however, suppose that the energy of this dextrose can be utilized by the muscle any more completely than that of dextrose derived directly from the food. It follows, then, that the percentage utilization of the total energy metabolized during muscular work should be materially greater when the metabolized material consists largely or wholly of carbohydrates than when it consists chiefly of fat. By supplying food consisting largely of one or the other of these materials, it is possible to bring about these conditions, and a determination of the respiratory exchange and the nitrogen excretion will then afford a check upon the nature of the material metabolized and the means of computing the utilization of its potential energy.

Investigations of this sort have been reported from Zuntz's laboratory. The earliest of these were by Zuntz \& Loeb* upon a $\mathrm{dog}$, the method being substantially the same as that with which the preceding pages have made us familiar. Their final results for the energy metabolized per kilogram and meter traveled (including the work of ascent) were:

* Arch. f. (Anat. u.) Physiol., 1894, p. 541. 


\begin{tabular}{|c|c|c|}
\hline Diet. & $\begin{array}{l}\text { Respiratory } \\
\text { Quotient. }\end{array}$ & Energy, cals. \\
\hline 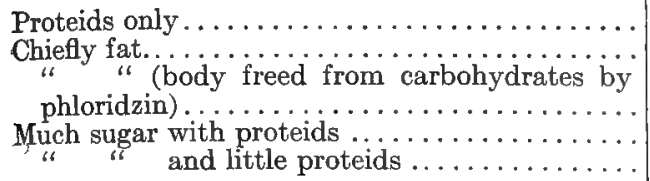 & $\begin{array}{l}0.78 \\
0.74 \\
0.71 \\
0.83 \\
0.88\end{array}$ & $\begin{array}{l}2.58 \\
2.43 \\
2.71 \\
2.58 \\
2.63\end{array}$ \\
\hline
\end{tabular}

The differences are quite small, while, as Zuntz points out, if 2.6 cals. represent the demand for energy per unit of work when carbohydrates are the source it should, according to Chauveau's theory, rise to about 3.68 cals. when the energy is derived exclusively from fat.

Altogether similar results have been recently reported from Zuntz's laboratory by Heineman,* and by Frentzel \& Reach, $\dagger$ in experiments on man.

In Heineman's experiments the work, which was never excessive, consisted in turning an ergostat, the respiratory exchange being determined by means of the Zuntz apparatus and the total urinary nitrogen being also determined. From these data, reckoning 1 gram of urinary nitrogen equivalent to 6.064 liters of oxygen, $\ddagger$ the average amount of energy metabolized on the various diets, and the proportion derived respectively from proteids, fats, and carbohydrates, is computed. By comparison with rest experiments the increments of oxygen and carbon dioxide due to the work were determined, and from these the energy consumed per kilogram-meter of work was calculated upon three different assumptions: first, that the proteid metabolism was not increased by the work; second, that it increased proportionally to the oxygen consumption; third, that as large a proportion of the energy for the work was furnished by the proteids as is consistent with the observed respiratory exchange. The results are summarized in the following table:

\footnotetext{
* Arch. ges. Physiol., 83, 441.

† Ibid., 83, 477.

$\ddagger$ Zuntz, Arch. ges. Physiol., 68, 204.
} 


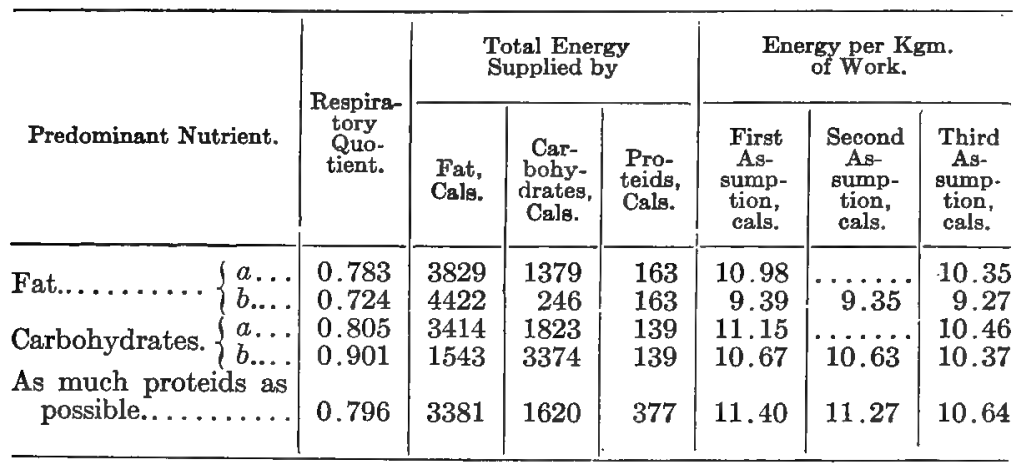

The subject was not able to consume even approximately enough proteids to supply the demands for energy, so that the experiments are virtually a comparison of the utilization of fat and carbohydrates in different proportions. With the exception of the third group, the results seem to show that the energy of the fat metabolized was utilized, if anything, rather more fully than that of the carbohydrates.

Frentzel \& Reach experimented upon themselves, the work being done by walking in a tread-power; otherwise the methods were similar to those of Heineman. In computing the results of the experiments on a carbohydrate and a fat diet they assume that there was no increase in the proteid metabolism as a consequence of the work. For the experiments on a proteid diet they compute the results both on this assumption and also on the assumption of a maximum participation of the proteids in work production. Calculated in this way the total evolution of energy per kilogram weight and meter traveled was as given in the table on p. 525 . The results show a slight advantage on the side of the carbohydrates, which in the case of Frentzel is regarded by the authors as exceeding the errors of experiment. They compute, however, that it is far too small to afford any support to Chauveau's theory.

Zuntz* has recalculated Heineman's results, using slightly different data but reaching substantially the same result. $\mathrm{He}$ shows, however, that they are affected by the influence of training already discussed on p. 519. Arranging the experiments in chronological order, it becomes evident that the work was done * Arch. ges. Physiol., 83, 557. 


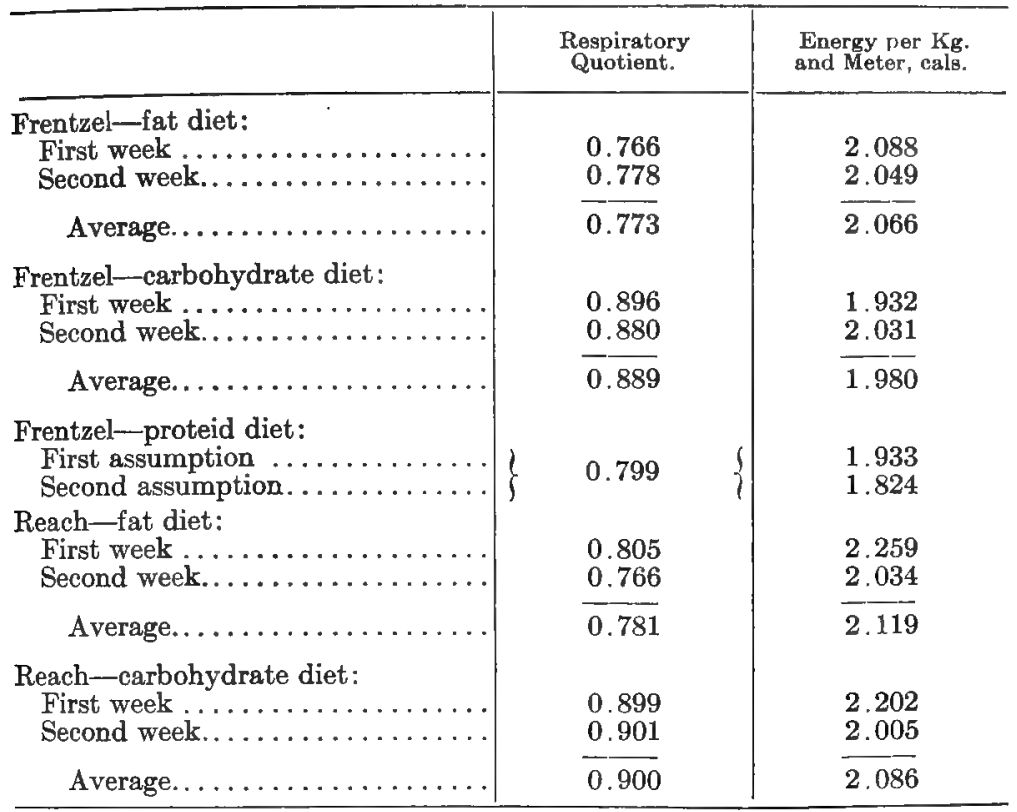

with increasing efficiency, largely independent of the food, and the fact that most of the experiments with fat came later in the series than those with carbohydrates largely, although perhaps not entirely, accounts for the observed difference in efficiency, while the low figure for proteids is accounted for by the fact that these were among the earliest experiments. A similar effect appears in the experiments of Frentzel \& Reach, although it is less marked, since walking is a more accustomed form of work than turning a crank. On the whole, Zuntz concludes that these experiments warrant the conclusion that in work production the materials metabolized in the body replace each other in proportion to their heats of combustion-that is, in isodynamic and not isoglycosic proportions.

\section{THE UTILIZATION OF METABOLIZABLE ENERGY.}

เ he investigations just discussed give us fairly full data as to the utilization of the stored-up energy of the body in the production of external work, and this, as we have seen (p. 497), is substantially equivalent to a knowledge of the utilization of the net 
available energy of the food. These determinations by Zuntz and his co-workers, however, do not bring the energy recovered as mechanical work into direct relation with the energy of the food; that is to say (aside from such computations of available energy as those made by Zuntz \& Hagemann* for the food of the horse), they do not tell us how much of the energy contained in a given feeding-stuff we may expect to recover in the form of mechanical work, but only what proportion of the stored-up energy resulting from the use of this feeding-stuff is so recoverable.

It is the former question rather than the latter, however, which is of direct and immediate interest to the feeder of working animals. The feeding-stuffs which he employs are comparable to the fuel of an engine, and the practical question is how much of the energy which he pays for in this form he can get back as useful work.

Methods of Determination.-Two general methods are open for the determination of the percentage utilization of the energy of the food.

It is obvious that if we know the net availability of the energy (gross or metabolizable) of a given food material we can compute its percentage utilization in work production from the data of the foregoing paragraphs with a degree of accuracy depending upon that of the factors used. For example, if we know that the net available energy of a sample of oats is 60 per cent. of its gross energy, then if the oats are fed to a draft horse utilizing, according to Zuntz \& Hagemann, 31.3 per cent. of the net available energy, it is obvious that the utilization of the gross energy of the oats is $60 \times 0.313=$ 18.78 per cent. An entirely similar computation could of course be made of the percentage utilization of the metabolizable energy of the oats.

Unfortunately, however, as we have already seen, our present knowledge of the net availability of the energy of feeding-stuffs and nutrients for different classes of animals is extremely defective, and extensive investigations in this direction are an essential first step in the determination of the percentage utilization of the energy of feeding-stuffs in work production by this method. Until trustworthy data of this sort are supplied, results like those of Zuntz \& Hagemann can be applied to practical conditions only on the basis

* Landw. Jahrb., 27, Supp. III, 279 and 429. 
of more or less uncertain estimates and assumptions regarding the expenditure of energy in digestion and assimilation such as those discussed in Chapter XI, \& 3.

The second possible general method for the determination of the percentage utilization of the energy of the food in work production is that employed in the determination of the utilization in tissue production. Having brought the animal into equilibrium as regards gain or loss of tissue and amount of work done with a suitable basal ration, the material to be tested is added and the work increased until equilibrium is again reached. The increase in the work performed compared with the energy of the material added would then give the percentage utilization of the latter.

The accurate execution of this method would require the employment of a respiration apparatus or a respiration-calorimeter for the exact determination of the equilibrium between food and work, while the skill of the experimenter would doubtless be taxed in the endeavor to so adjust food and work as to secure either no gain or loss of tissue or equality of gain or loss in the two periods to be compared. Indeed, it may safely be said that exact equality would, as a matter of fact, be reached rarely and by accident, and that as a rule it would be necessary to correct the observed results for small differences in this respect. To make such corrections accurately, however, requires, as we have seen in $\$ 1$ of this chapter, a knowledge of the net availability and percentage utilization of the food, and we are thus brought back to the necessity for more accurate knowledge upon fundamental points.

The extensive investigations of Atwater \& Benedict * upon man appear to be the only ones yet upon record in which the actual balance of matter and energy during rest has been quantitatively compared with that during the performance of a measured amount of work. Unfortunately, however, the gains and losses of energy by the bodies of the subjects in these experiments were relatively considerable, while the experiments thus far reported seem to afford no sufficient data for computing the net availability of the food for maintenance or its percentage utilization for the production of gain. Moreover, the authors appear to regard the measurements

* U. S. Department Agr., Office of Experiment Stations, Bull. 109; Memoirs Nat. Acad. Sci., 8, 231. 
of the work done as not altogether satisfactory. In a preliminary paper* Atwater \& Rosa compute a utilization of 21 per cent. Inasmuch as they have not further discussed the question of the utilization of the food energy for work production it would seem premature to attempt to do so here. It may be remarked, however, that the figures given seem to indicate a rather low degree of efficiency for the particular form of work investigated (riding a stationary bicycle).

\section{Wolff's Investigations.}

The horse, being par excellence the working animal, has naturally been the subject of experiments upon the relation of food to work. While as yet the respiration apparatus or calorimeter has not been applied to the study of this phase of the subject, two extensive and important series of investigations have been made upon the work horse, viz., by Wolff and his associates in Hohenheim and by Grandeau, LeClerc, and others $\dagger$ in Paris, in which the attempt has been made to judge approximately of the equilibrium between food and work from the live weight and the urinary nitrogen.

Grandeau's experiments were made for the Compagnie générale des Voitures in Paris, and were directed specifically toward a scientific investigation of the rations already in use by the company and to a study of the most suitable rations for the different kinds of service required of the horses. They were, therefore, while executed with the greatest care and exactness, largely "practical" in their aim.

Wolf's experiments were made at the Experiment Station at Hohenheim and were broader in their scope, being directed largely to a determination of the ratio of (digested) food to work. The following paragraphs are devoted chiefly to an outline of Wolff's experiments, but with more or less reference also to Grandeau's results.

Methods.-In discussing the effects of muscular exertion on metabolism in Chapter VI, mention was made of the interesting

* Phys. Rev., 9, 248; U. S. Dept. Agr., Office of Experiment Station, Bull. 98, p. 17.

$\dagger$ L'alimentation du Cheval de Trait, Vols. I, II, III, and IV, and Annales de la Science Agronomique, 1892, I, p. 1; 1893, I, p. 1; and 1896, II, p. 113 
results obtained by Kellner regarding the influence of excessive work upon the proteid metabolism of the horse. It was there shown that when the work was increased beyond a certain amount there resulted a prompt increase of the urinary nitrogen and at the same time a steady falling off in the live weight. The method employed in Wolff's experiments, and which originated with Kellner, is based upon this fact. It may perhaps be best illustrated by one of Kellner's earliest experiments, ${ }^{*}$ in which starch was added to a basal ration, the results of which have already been referred to in Chapter VI (p. 199).

In the first period the daily ration consisted of $6 \mathrm{kgs}$. of oats and $6 \mathrm{kgs}$. of hay, while in the second period $1 \mathrm{~kg}$. of rice starch was added. Digestion trials showed that there was digested from these rations the following:

\begin{tabular}{|c|c|c|c|}
\hline & $\begin{array}{c}\text { Period I, } \\
\text { Grms. }\end{array}$ & $\begin{array}{c}\text { Period II, } \\
\text { Grms. }\end{array}$ & $\begin{array}{c}\text { Increase. } \\
\text { Grms. }\end{array}$ \\
\hline $\begin{array}{l}\text { Crude protein } \ldots \ldots \ldots \ldots \ldots \ldots \ldots \\
\text { " }{ }_{\text {fiber... }} \ldots \ldots \ldots \ldots \ldots \\
\text { Nitrogen-free extract } \ldots \ldots \ldots \ldots \\
\text { Ether extract } \ldots \ldots \ldots \ldots \ldots \ldots\end{array}$ & $\begin{array}{r}757.07 \\
636.10 \\
3874.36 \\
279.45\end{array}$ & $\begin{array}{r}750.53 \\
713.40 \\
4488.15 \\
275.43\end{array}$ & $\begin{array}{l}-\quad 6.54 \\
+\quad 77.30 \\
+613.79 \\
-\quad 4.02\end{array}$ \\
\hline & 5546.98 & 6227.51 & +680.53 \\
\hline
\end{tabular}

The work was performed in a special sweep-power which was so constructed as to act as a dynamometer. With a uniform draft of $76 \mathrm{kgs}$., the daily work in the four subdivisions of the first period consisted of $300,600,500$, and 400 revolutions respectively, while in the two subdivisions of the second period it was 800 and 600 respectively. From the daily results for live weight and urinary nitrogen and from a comparison with another period in which 1.5 $\mathrm{kgs}$. of starch was fed, Kellner concludes that the maximum amounts of work which the animal could perform without causing an increase in its proteid metabolism and a decrease in its live weight were for the first period 500 revolutions and for the second period 700 revolutions. The difference of 200 revolutions, then, represents the additional work derived from the added starch. Two hundred revolutions with a draft of $76 \mathrm{kgs}$. equaled $438,712 \mathrm{kgm}$., to which is to be added the work of locomotion, estimated by Kellner (com-

* Landw. Jahrb., 9, 670. 
pare p. 539) at $100,000 \mathrm{kgm}$., making the total additional work $538,712 \mathrm{kgm}$. Kellner compares this difference with the increased amount of nitrogen-free extract digested, 613.79 grams, neglecting the small differences in the other nutrients. As corrected in a later publication, * the results are as follows:

$$
\begin{gathered}
613.79 \text { grms. starch }=2527.601 \text { Cals. }=1,071,698 \mathrm{kgm} . \\
538,712 \div 1,071,698=50.27 \text { per cent. }
\end{gathered}
$$

If we base the calculation upon the difference in total organic matter digested, the percentage will of course be somewhat smaller.

It was discovered later that the indications of the dynamometer used in these experiments and many subsequent ones were untrustworthy, so that no value attaches to the percentage computed above, but it serves just as well to illustrate the method employed, and which was followed in the whole series of experiments. In brief, the attempt is to find in the indications of live weight and urinary nitrogen a partial substitute for the determination of the respiratory products. As Kellner and Wolff do not fail to point out, the results are but approximations, and in any single experiment may vary considerably from the truth, but on the average of a large number of experiments it was hoped that satisfactory results might be reached. In later experiments rather more importance seems to be attached to the effects upon live weight than to those upon urinary nitrogen, but it should be noted that the live weight showed remarkably small variations from day to day; under the carefully regulated conditions of the experiments, and was quite sensitive to changes in the amount of work done.

The experiments may be conveniently divided into three groups. The first of these $\dagger$ includes the years 1877 to 1886 , inclusive, in which the work done was compared with the total digested food. The second $\ddagger$ covers the experiments of $1886-1891$, in which the digested crude fiber was omitted in computing the work-equivalent of the food, while the third group $\$$ includes the experiments of 1891-1894 with a new and more accurate form of dynamometer.

* Wolff, Grundlagen, etc., p. 89.

$\uparrow$ Grundlagen für die rationelle Fütterung des Pferdes, 1886, 66-155; Neue Beiträge, Landw. Jahrb., 16, Supp. III, 1-48.

$\ddagger$ Landw. Jahrb., 16, Supp. III, 49-131, and 24, 125-192.

$\S$ Ibid., 24, 193-271. 
Experiments of $1877-1886$.-During the years named, in addition to the preliminary investigations necessary in working out the method, a large number of experiments were made on three different animals. The rations consisted largely of hay and oats in somewhat varied proportions, together with smaller amounts of other feeding-stuffs. In three experiments on starch and four on oats a comparison of the increase in digested nutrients $*$ with the increased work which could be done gave the following results: $\uparrow$

\begin{tabular}{|c|c|c|c|}
\hline & $\begin{array}{l}\text { Increase in Digested] } \\
\text { Nutrients, } \\
\text { Grms. }\end{array}$ & $\begin{array}{c}\text { Increase in Work Done } \\
\text { at } 76 \mathrm{Kg} \text {. Draft, } \\
\text { Revolutions. }\end{array}$ & $\begin{array}{l}\text { Nutrients Equivalent } \\
\text { to } 100 \text { Revolutions, } \\
\text { Grms. }\end{array}$ \\
\hline $\begin{array}{l}\text { Starch........... } \\
\text { Oats ......... }\end{array}$ & $\begin{array}{l}677.3 \\
577.0\end{array}$ & $\begin{array}{l}217 \\
175\end{array}$ & $\begin{array}{l}312 \\
318 \\
\end{array}$ \\
\hline Average....... & $\ldots$ & $\ldots$ & 315 \\
\hline
\end{tabular}

The Maintenance Requirement.-As already stated, it was discovered later that the dynamometer used was unreliable and gave too high readings, so that the above result cannot be employed to compute the utilization of the energy of the added food. It does, however, in its present form, enable us to compute the maintenance requirements of the horse by subtracting from the total digested food the nutrients equivalent to the work performed (i.e., 3.15 grams $\times$ the number of revolutions). The results of such a computation made by Wolff $\ddagger$ are given on p. 532 .

The actual live weights in these experiments were somewhat below the normal weights, which were regarded as being about $533 \mathrm{kgs}$. for No. I, $500 \mathrm{kgs}$. for No. II, and $475 \mathrm{kgs}$. for No. III. Wolff considers the maintenance requirements to be independent of minor changes in weight, and on the basis of the above "normal" weights computes the maintenance requirements per $500 \mathrm{kgs}$. live weight as follows:

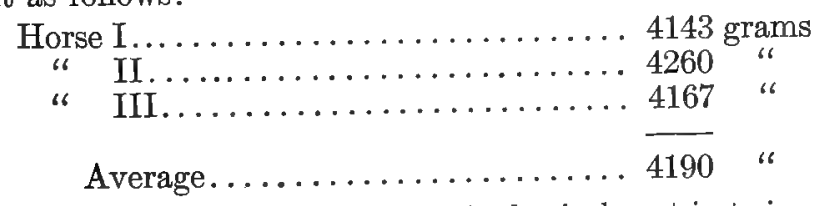

* The algebraic sum of the differences in the single nutrients is used, and in this and the succeeding comparisons the digested fat is multiplied by 2.44 .

$\dagger$ Loc. cit., pp. 125-129.

$\ddagger$ Loc cit., pp. 99 and 132. 


\begin{tabular}{|c|c|c|c|c|c|c|c|}
\hline & $\begin{array}{l}\text { No. of } \\
\text { Experi- } \\
\text { ments. }\end{array}$ & $\begin{array}{c}\text { Total } \\
\text { Nutrients, } \\
\text { Grms. }\end{array}$ & $\begin{array}{c}\text { Nutritive } \\
\text { Ratio. }\end{array}$ & $\begin{array}{c}\text { Live } \\
\text { Weight, } \\
\text { Kgs. }\end{array}$ & $\begin{array}{c}\text { No. of } \\
\text { Revolu- } \\
\text { tions. }\end{array}$ & $\begin{array}{c}\text { Equiva- } \\
\text { lent } \\
\text { Nutrients, } \\
\text { Grms. }\end{array}$ & $\begin{array}{c}\text { For } \\
\text { Mainte- } \\
\text { nance, } \\
\text { Grms. }\end{array}$ \\
\hline Horse $I . . . .$. & 4 & 6305.6 & $1: 5.79$ & 521 & 600 & 1890 & 4416 \\
\hline $\begin{array}{l}\text { Horse II: } \\
\quad 1881-82 \ldots \\
\quad 1882-83 \ldots \\
1883-84 \ldots\end{array}$ & $\begin{array}{l}7 \\
4 \\
6\end{array}$ & $\begin{array}{l}5831.1 \\
6748.3 \\
5920.2\end{array}$ & $\begin{array}{l}1: 6.64 \\
1: 6.37 \\
1: 7.26\end{array}$ & $\begin{array}{l}477 \\
486 \\
457\end{array}$ & $\begin{array}{l}546 \\
662 \\
567\end{array}$ & $\begin{array}{l}1720 \\
2085 \\
1786\end{array}$ & $\begin{array}{l}4111 \\
4663 \\
4134\end{array}$ \\
\hline Average... & 17 & 6078.4 & $1: 6.80$ & 473 & 577 & 1818 . & 4260 \\
\hline $\begin{array}{l}\text { Horse III: } \\
\quad 1881-82 \ldots \\
1882-83 \ldots \\
1883-84 \ldots \\
1885 \ldots \ldots\end{array}$ & $\begin{array}{l}6 \\
6 \\
5 \\
4\end{array}$ & $\begin{array}{l}5313.8 \\
6061.3 \\
5734.8 \\
5761.2\end{array}$ & $\begin{array}{l}1: 7.16 \\
1: 6.88 \\
1: 7.55 \\
1: 7.57\end{array}$ & $\begin{array}{l}454 \\
469 \\
473 \\
473\end{array}$ & $\begin{array}{l}404 \\
683 \\
580 \\
575\end{array}$ & $\begin{array}{l}1273 \\
2152 \\
1827 \\
1811\end{array}$ & $\begin{array}{l}4041 \\
3909 \\
3908 \\
3 \sqsupset 50\end{array}$ \\
\hline Average... & 21 & 5717.8 & $1: 7.29$ & 467 & 5,1 & 1766 & 3952 \\
\hline
\end{tabular}

By means of a comparison of the results by groups* Wolff shows that the maintenance requirement as thus computed is apparently independent of the amount of work done and of the nutritive catio, and from this uniformity concludes that the relative efficiency of the food for work production is unaffected by these factors, within the range of his experiments.

A series of similar experiments on Horse No. III in 1885-86, $\dagger$ computed in substantially the same way, gave results for the maintenance ration agreeing well with those of earlier years, viz.,

\begin{tabular}{|c|c|c|c|c|c|}
\hline \multicolumn{2}{|c|}{ Period I.. } & \multicolumn{4}{|c|}{3934 grams total nutrients } \\
\hline " & II. . & 3984 & " & " & \\
\hline "6 & III and $V$ & 4001 & " & " & “ \\
\hline " & $\mathrm{VII} b \ldots$ & 4094 & " & " & " \\
\hline " & VIII... & 4094 & “ & “ & " \\
\hline & rage & 4021 & “ & “ & " \\
\hline
\end{tabular}

with an average live weight of $475 \mathrm{kgs}$., equivalent to 4230 grams per $500 \mathrm{kgs}$. In a succeeding period (IX), however, in which hay alone was fed, a decidedly higher result was obtained, viz., 4357 grams per head, or 4586 grams per $500 \mathrm{kgs}$.

* Loc. cit., pp. 135 and 137.

† Landw. Jahrb., 16, Supp. III, 32. 
Experiments of I886-9I.-In the experiments thus far described, with the exception of the last, the proportions of grain and coarse fodder in the rations were not widely different, the latter furnishing on the average fully one half of the dry matter fed. Consequently the experiments were not calculated to bring out any difference in the nutritive value of the two such as is indicated by the results of the one trial with hay alone.

Grain vs. Coarse Fodder for Maintenance.-The results obtained by Grandeau \& LeClerc upon the maintenance ration of the horse when fed a mixture containing about 75 per cent. of grain fully confirm the indications of Wolff's trial with hay. Their experiments have been very fully discussed, and in part recalculated, by Wolff $*$ in their bearing on this question. The three horses experimented on were fed two different amounts of the same mixture in several different thirty-day periods, eighteen such periods in all being available for comparison. In all of them the animals were led daily, at a walk, over a distance of about four kilometers. Wolff estimates the amount of work of locomotion by means of the formula $\frac{1}{2}\left(\frac{W}{g}\right) v^{2}$ and by subtracting the equivalent. amount of nutrients from the total digested obtains the amount required for maintenance. The results are as follows:

\begin{tabular}{|c|c|c|c|c|c|c|}
\hline & \multirow{2}{*}{$\begin{array}{l}\text { No. of } \\
\text { Experi- } \\
\text { ments. }\end{array}$} & \multirow{2}{*}{$\begin{array}{c}\text { Live } \\
\text { Weight, } \\
\text { Kgs. }\end{array}$} & \multirow{2}{*}{$\begin{array}{c}\text { Digested } \\
\text { Nutrients, } \\
\text { Grms. }\end{array}$} & \multirow{2}{*}{$\begin{array}{c}\text { Nutrients } \\
\text { Equiva- } \\
\text { lent to } \\
\text { Work, } \\
\text { Grms. }\end{array}$} & \multicolumn{2}{|c|}{ For Maintenance. } \\
\hline & & & & & $\begin{array}{l}\text { Per } \\
\text { Head, } \\
\text { Grms. }\end{array}$ & $\begin{array}{c}\text { Per } \\
500 \text { Kgs., } \\
\text { Grms. }\end{array}$ \\
\hline 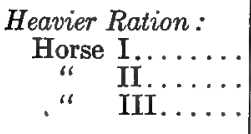 & $\begin{array}{l}3 \\
5 \\
4\end{array}$ & $\begin{array}{l}416.6 \\
405.9 \\
439.0\end{array}$ & $\begin{array}{l}3553 \\
3432 \\
3625\end{array}$ & $\begin{array}{l}110 \\
108 \\
119\end{array}$ & $\begin{array}{l}3443 \\
3324 \\
3506\end{array}$ & $\begin{array}{l}4132 \\
4078 \\
3994\end{array}$ \\
\hline Average.... & $\cdots$ & 420.5 & 3537 & 112 & 3425 & 4068 \\
\hline $\begin{array}{l}\text { Lighter Ration: } \\
\text { Horse II.... } \\
\text { III... }\end{array}$ & $\begin{array}{l}2 \\
4\end{array}$ & $\begin{array}{l}411.0 \\
441.2\end{array}$ & $\begin{array}{l}3060 \\
3310\end{array}$ & $\begin{array}{l}108 \\
119\end{array}$ & $\begin{array}{l}2952 \\
3191\end{array}$ & $\begin{array}{l}3636 \\
3617\end{array}$ \\
\hline Average. . & $\cdots$ & 426.1 & 3185 & 114 & 3071 & 3626 \\
\hline
\end{tabular}

The results, and particularly those on the lighter ration, which appeared ample for maintenance, are much lower than those com* Landw. Jahrb., 16, Supp. III, 73-81. 
puted in the previous paragraph. The difference is too great to be ascribed to experimental errors in estimating the small amount of work done, and can most reasonably be ascribed to the difference in the character of the ration. Apparently the horse, like cattle (p. 433), requires less digestible food for maintenance when the latter consists largely of grain than when it is chiefly or wholly coarse fodder.

Direct experiments by Wolff * likewise show that the digestible nutrients of concentrated feed (oats) are more valuable for work production than those of coarse feed (hay). The experiments were made in the manner already described, the draft being uniformly $60 \mathrm{kgs}$. Although the measurements of the work actually done are probably incorrect, it may be assumed to have been substantially proportional to the number of revolutions of the dynamometer. A ration of $3 \mathrm{kgs}$. of hay and $5.5 \mathrm{kgs}$. of oats served as the basal ration, to which was added in one case $4 \mathrm{kgs}$. of hay and in another $1 \frac{1}{2} \mathrm{kgs}$. of oats. The nutrients digested in each case and the equivalent amount of work secured were:

\begin{tabular}{|c|c|c|c|c|c|c|c|}
\hline \multirow[b]{2}{*}{ 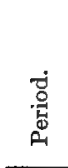 } & \multirow[b]{2}{*}{ Ration. } & \multicolumn{5}{|c|}{ Digested. } & \multirow{2}{*}{ 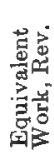 } \\
\hline & & $\begin{array}{c}\text { Protein, } \\
\text { Grms. }\end{array}$ & $\begin{array}{l}\text { Crude } \\
\text { Fiber, } \\
\text { Grms. }\end{array}$ & $\begin{array}{c}\text { Nitrogen- } \\
\text { free } \\
\text { Extract, } \\
\text { Grms. }\end{array}$ & $\begin{array}{l}\text { Ether } \\
\text { Ex- } \\
\text { tract, } \\
\text { Grms. }\end{array}$ & $\begin{array}{l}\text { Total } \\
\text { (Fat } \times \\
2.4) \text {. } \\
\text { Grms. }\end{array}$ & \\
\hline \multirow[t]{2}{*}{$\begin{array}{l}\mathrm{I}-\mathrm{III} \\
\mathrm{V} \ldots . . .\end{array}$} & \multirow{2}{*}{ 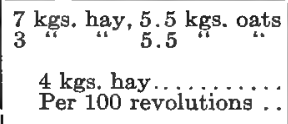 } & \begin{tabular}{|l|}
822.58 \\
626.46
\end{tabular} & & $\begin{array}{l}3889.64 \\
3068.46\end{array}$ & $\begin{array}{l}186.72 \\
184.78\end{array}$ & $\begin{array}{l}5973.62 \\
4561.13\end{array}$ & $\begin{array}{l}750 \\
350\end{array}$ \\
\hline & & 196.12 & 393.94 & 821.18 & 1.94 & $\begin{array}{r}1412.49 \\
353.12\end{array}$ & 400 \\
\hline \multirow[t]{2}{*}{$\begin{array}{l}\text { VI.... } \\
\text { V.... }\end{array}$} & \multirow{2}{*}{ 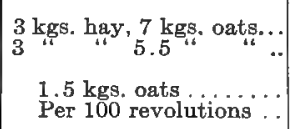 } & $\begin{array}{l}754.52 \\
626.46\end{array}$ & $\begin{array}{l}355.24 \\
393.94\end{array}$ & $\begin{array}{l}3719.24 \\
3068.46\end{array}$ & $\begin{array}{l}252.17 \\
184.78\end{array}$ & $\begin{array}{l}5434.21 \\
4561.13\end{array}$ & $\begin{array}{l}700 \\
350\end{array}$ \\
\hline & & $\begin{array}{l}128.06 \\
\ldots \ldots\end{array}$ & -67.50 & $\begin{array}{r}650.78 \\
\cdots \ldots \ldots\end{array}$ & 67.39 & $\begin{array}{l}873.08 \\
249.45\end{array}$ & 350 \\
\hline
\end{tabular}

The relative value of the digested matter of hay and of oats for work production in these trials was thus approximately as $5: 7$.

In the earlier experiments (p. 531) it was found that when oats or starch were added to a basal ration, approximately 315 grams of digested nutrients were required to produce the amount of work represented by 100 revolutions at $76 \mathrm{kgs}$. draft. Converting this result and the one just given for oats into kilogram-meters, Wolff computes that 100 grams of digested nutrients was equivalent,

$$
\text { * Loc. cit., pp. 84-95. }
$$


in round numbers, to 85,400 kilogram-meters in the earlier experiments and to 90,480 in the one just cited. While these figures are not correct absolutely, they are probably comparable, being obtained with the same apparatus. In the later experiment the work of locomotion is computed by Wolff's formula, which gives higher results than Kellner's. Taking this into account we may regard the agreement of the two equivalents as satisfactory.

Value of Crude Fiber.-In all the experiments with concentrated feeds the additional nutrients digested from the added food contained no crude fiber, the apparent difference, indeed, being in most cases, as in the above experiment, negative. When hay was added, on the other hand, over one fourth of the additional nutrients digested consisted of crude fiber. If, now, we neglect this crude fiber and compare the work and the fiber-free nutrients we have $1018.55 \div 4.00=254.64$ grams of fiber-free nutrients per 100 revolutions, or a figure corresponding almost exactly with that obtained for the fiber-free nutrients added in oats or starch. In other words, it would appear from this result that the digested crude fiber of hay is as valueless for work production as it appears to be for maintenance.

If, however, the crude fiber is valueless both for maintenance and work, then by omitting it altogether from our computations we ought to get results for the maintenance ration and for the ratio of nutrients to work which are independent of the proportion of grain to coarse fodder in the ration. Confirmatory evidence of this sort is abundantly furnished by Wolff's experiments and likewise by the results of Grandeau on maintenance. Taking first the averages of the experiments of $1877-1886$ (p. 532) we have-

\begin{tabular}{|c|c|c|c|c|c|c|c|}
\hline & \multicolumn{3}{|c|}{ Nutrients Digested. } & \multirow{2}{*}{$\begin{array}{c}\text { No. of } \\
\text { Revolu- } \\
\text { tions at } \\
76 \mathrm{Kgs} \text {. }\end{array}$} & \multirow{2}{*}{$\begin{array}{c}\text { Equiva- } \\
\text { lent } \\
\text { Nutrients, } \\
\text { Grms. }\end{array}$} & \multicolumn{2}{|c|}{$\begin{array}{l}\text { Fiber-free Nutrients } \\
\text { for Maintenance. }\end{array}$} \\
\hline & $\begin{array}{l}\text { Total, } \\
\text { Grms. }\end{array}$ & $\begin{array}{l}\text { Crude } \\
\text { Fiber, } \\
\text { Grms. }\end{array}$ & $\begin{array}{l}\text { Without } \\
\text { Crude } \\
\text { Fiber, } \\
\text { Grms. }\end{array}$ & & & $\begin{array}{c}\text { Per Head, } \\
\text { Grms. }\end{array}$ & $\begin{array}{c}\text { Per } 500^{\circ} \\
\text { Kgs.. } \\
\text { Grnas. }\end{array}$ \\
\hline \multirow{4}{*}{ 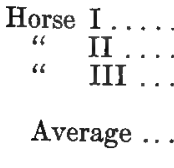 } & 6306 & 815 & 5491 & 600 & 1890 & 3601 & 3378 \\
\hline & 6078 & 978 & 5100 & 577 & 1818 & 3282 & 3282 \\
\hline & 5718 & 809 & 4909 & 561 & 1766 & 143 & 3306 \\
\hline & & & & & & & 3322 \\
\hline
\end{tabular}


The results of the series made in $1885-86$ on Horse No. III (p. 532), computed in the same way, give the following as the amounts of fiber-free nutrients required for maintenance:

\begin{tabular}{|c|c|c|}
\hline & $\begin{array}{l}\text { Per Head. } \\
\text { Grms. }\end{array}$ & $\begin{array}{c}\text { Per } 500 \text { Kgg. } \\
\text { Live Weight, } \\
\text { Grms. }\end{array}$ \\
\hline 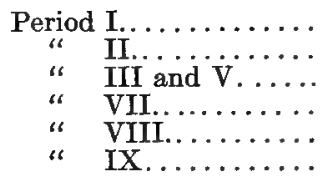 & $\begin{array}{l}3270 \\
3186 \\
3242 \\
3342 \\
3316 \\
3170\end{array}$ & $\begin{array}{l}3442 \\
3353 \\
3413 \\
3549 \\
3490 \\
3335\end{array}$ \\
\hline Average........... & 3254 & 3430 \\
\hline
\end{tabular}

From Grandeau's experiments (p. 533), by the same method, we have for the lighter ration the following:

\begin{tabular}{|c|c|c|}
\hline & $\begin{array}{l}\text { Per Head, } \\
\text { Grms. }\end{array}$ & $\begin{array}{c}\text { Per } 500 \text { Kgg. } \\
\text { Live Weight. } \\
\text { Grms. }\end{array}$ \\
\hline \multirow{2}{*}{$\begin{array}{r}\text { Horse } \\
\text { II } \ldots \ldots \ldots \ldots \\
\text { III } \ldots \ldots \ldots \ldots \\
\text { Average. } \ldots \ldots \ldots \ldots\end{array}$} & $\begin{array}{l}2732 \\
2935\end{array}$ & $\begin{array}{l}3324 \\
3328\end{array}$ \\
\hline & $\ldots \ldots \ldots \ldots \ldots$ & 3326 \\
\hline
\end{tabular}

Finally, for the series of experiments by Wolff, just discussed, upon the relative value of the digested matter of oats and of hay, and from which the conclusion as to the lack of value of the crude fiber was drawn, by computing backwards, we get figures for the fiber-free nutrients required for maintenance which not only agree with each other, as they necessarily must, but also with those of the earlier experiments. The results are:

\begin{tabular}{|c|c|c|}
\hline & Per Head, Grms. & $\begin{array}{l}\text { Per } 500 \mathrm{Kgs} \text { Live } \\
\text { Weight. Grms. }\end{array}$ \\
\hline $\begin{array}{cl}\text { Period } & \text { I-III } \\
" & \text { IV } \ldots \ldots \ldots \ldots \\
" & \text { V } \ldots \ldots \ldots \ldots \\
" & \text { VI } \ldots \ldots \ldots\end{array}$ & $\begin{array}{l}3175 \\
3275 \\
3180 \\
3196\end{array}$ & $\begin{array}{l}3342 \\
3429 \\
3329 \\
3364\end{array}$ \\
\hline Average.. & $\ldots \ldots \ldots \ldots \ldots$ & 3366 \\
\hline
\end{tabular}


Wolff's conclusions from these results * are-

1. The digested crude fiber is apparently valueless, both for maintenance and for work production.

2. The remaining nutrients may be regarded as of equal value whether derived from grain or coarse fodder.

3 . The maintenance of a $500-\mathrm{kg}$. horse requires approximately 3350 grams per day of fiber-free nutrients.

Wolff's subsequent experiments up to $1891 \dagger$ gave results confirmatory in general of the above conclusions. Particularly was this the case when the work of locomotion was computed by Kellner's formula and not by the formula $\frac{1}{2}\left(\frac{W}{g}\right) v^{2}$. The work done (expressed in number of revolutions of the dynamometer) per 100 grams of fiber-free nutrients was reasonably uniform and agreed well with the results previously obtained, while the fiber-free nutrients required for maintenance likewise agreed with the results given above. On the other hand, the inclusion of the digested crude fiber in the computations gave in-many cases strikingly discordant results. In view of the unreliability of the measurement of the work no conclusions can be drawn as to the percentage utilization of the energy of the food, and it seems unnecessary to deseribe the individual experiments.

A discussion by Wolff $t$ of the results of some of the experiments by Grandeau in which work was done, although rendered uncertain by the difficulty in estimating the work of locomotion at varying velocities, and by the changes in live weight of the animals, seems to indicate that they also confirm Wolff's conclusions.

Significance of the Results.-In drawing his conclusions Wolff is careful to say that the digested crude fiber is apparently valueless, and while calling attention to Tappeiner's then recent results on the fermentation of cellulose in the digestive tract as probably explaining its low nutritive value he points out that other ingredients of the food may also undergo fermentation. He therefore holds fast to the fact actually observed, viz., the lower nutritive value of the digested matter of coarse fodder compared

* Loc. cit., p. 95.

† Landw. Jahrb., 24, 125-192.

$\ddagger$ Ibid., 16, Supp. III, 110-126. 
with that of grain, and virtually regards the amount of crude fiber as furnishing a convenient empirical measure of the difference.

In the light of our present knowledge this reserve seems amply justified. The difference in the value of coarse fodder and grain we should now regard as arising largely from the difference in the amounts of energy consumed in digestion and assimilation. Kellner's experiments on extracted straw discussed in the previous section have shown, however, that with cattle this difference is by no means determined by the simple presence of more or less crude fiber, but is related rather to the physical properties of the feeding-stuff, while Zuntz (see p. 392) has shown that the same factor largely affects the work of mastication in the horse. That the nutritive value of the rations in Wolff's experiments was proportional to the amount of fiber-free nutrients which they contained, or, in other words, that the energy expended in digestion, etc., was proportional to the digested crude fiber, is explained by the limited variety of feeding-stuffs employed. The coarse fodder was meadow hay with, in some cases, an addition (usually relatively small) of straw, while the grain was commonly oats, part of which was in some instances replaced by maize, beans, barley, flaxseed, or oil-meal, while starch was added to the ration in a number of trials. The larger part of the work of digestion, under these circumstances, was probably caused by the coarse fodders, viz., hay and straw, while the digested crude fiber was likewise derived chiefly or entirely from these substances. Such being the case, it follows that the loss of energy through digestive work would be in general proportional to the amount of crude fiber in the ration. The essential point in Wolff's experiments is that the omission of crude fiber renders the results concordant, and this is as well explained in the manner just indicated as by the estimate of Zuntz \& Hagemann that the work of digesting and assimilating crude fiber consumes the equivalent of its metabolizable energy.

Experiments of I89I-94.-In the dynamometer employed by Wolff the resistance was produced by the friction of metallic surfaces. A copy of his dynamometer was employed by Grandeau \& LeClere in their investigations at Paris, and these experimenters found * that the measurement of the work was subject to large errors,

* Fourth Memoir, p. 49. 
particularly in experiments at a trot, owing to the continual changes in the friction. Wolff believes that in his experiments, all made at a rather slow walk, the errors are less, but admits that they are sufficient to deprive his computations of utilization of all val e.

Grandeau \& LeClerc, however, were successful in improving the dynamometer, by the addition of an integrating apparatus,* so that its measurements of the total work were satisfactory, and this apparatus was added to Wolff's dynamometer in 1891. Before that date, therefore, Wolff's experiments, while of great value in many other respects, afford no trustworthy direct data as to the utilization of the energy of the food for work production, although, as we have just seen, they afford some information on subsidiary points. From 1891, however, we may regard the measurements of the work done on the dynamometer as reasonably accurate.

Corrections. - Unfortunately, in the light of subsequent investigation, the same is not true of some of the other factors entering into the comparison, particularly the work of locomotion and the metabolizable energy of the food.

In all his later experiments Wolff computes the work of horizontal locomotion per second by means of the formula $\frac{1}{2}\left(\frac{W}{g}\right) v^{2}$, in which $W$ equals the weight of the animal, $g$ the force of gravity, and $v$ the velocity per second. Zuntz's experiments, however, appear to show that this formula gives too high results, the error increasing with the velocity, and Wolff $\dagger$ himself recognizes the truth of this for higher speeds. According to Zuntz's determinations (p. 512), Kellner's method of computation gives results agreeing quite closely with those computed from his respiration experiments. Under the conditions of Wolff's experiments this corresponds quite closely to $50,000 \mathrm{kgm}$. per 100 revolutions of the dynamometer, and in the comparisons which follow this amount has been substituted for that computed by Wolff, thus reducing materially the figures for the total work performed.

Wolff estimates the metabolizable energy of the food, on the basis of Rubner's results, by multiplying the digested fat by 2.4, adding the remaining digested nutrients, and reckoning the total

* Ann. Sci. Agron., 1881, I, 464.

† Landw. Jahrb., 16, Supp. III, 119. 
at 4.1 Cals. per gram. As we have seen, however (Chapter X), this figure is probably too high for herbivora, although exact figures for the horse are not yet fully available. Approximately, however, we may estimate the metabolizable energy of the several digested nutrients as follows (p. 332):

Protein. 3.228 Cals per gram

Crude fiber. 3.523

Nitrogen-free extract....... 4.185

Ether extract............ 8.572

Zuntz * estimates the metabolizable energy of the total nutrients (including fat $\times 2.4$ ) at 3.96 Cals. per gram. This figure is probably somewhat high, especially for rations containing much crude fiber or ether extract, but may serve the purpose of approximate calculations.

Experiments on Single Feeding-stuffs.-Comparatively few of the experiments admit of a direct computation of the utilization for a single feeding-stuff, since in most cases the amounts of two or more feeding-stuffs were varied simultaneously. As an example of the former class we may take Periods I and II of the experiments of 1892-93. In Period I the ration consisted of $7.5 \mathrm{kgs}$. of hay and $4 \mathrm{kgs}$. of oats per day, while in Period II the oats were increased to $5.5 \mathrm{kgs}$. The quantities of nutrients digested and the metabolizable energy of the difference between the two rations (computed by the use of the factors just given) were-

\begin{tabular}{|c|c|c|c|c|c|}
\hline & $\begin{array}{c}\text { Protein, } \\
\text { Grms. }\end{array}$ & $\begin{array}{l}\text { Crude } \\
\text { Fiber, } \\
\text { Grms. }\end{array}$ & $\begin{array}{c}\text { Nitrogen- } \\
\text { free } \\
\text { Extract, } \\
\text { Grms. }\end{array}$ & $\begin{array}{l}\text { Ether } \\
\text { Extract, } \\
\text { Grms. }\end{array}$ & $\begin{array}{c}\text { Total } \\
\text { Nutrients, } \\
\text { Grms. }\end{array}$ \\
\hline $\begin{array}{c}\text { Period } \\
\text { II } \\
\text { I. . . . . . . . }\end{array}$ & $\begin{array}{r}1022.4 \\
847.8\end{array}$ & $\begin{array}{l}849.6 \\
819.9\end{array}$ & $\begin{array}{l}4152.8 \\
3598.4\end{array}$ & $\begin{array}{l}175.8 \\
137.1\end{array}$ & $\begin{array}{l}6446.6 \\
5595.3\end{array}$ \\
\hline Equiv, energy... & $\begin{array}{c}174.6 \\
\text { Cals. } \\
564\end{array}$ & $\begin{array}{l}29.7 \\
\text { Cals. } \\
105\end{array}$ & $\begin{array}{l}554.4 \\
\text { Cals. } \\
2320\end{array}$ & $\begin{array}{l}38.7 \\
\text { Cals. } \\
332\end{array}$ & $\begin{array}{l}851.3 \\
\text { Cals. } \\
3321\end{array}$ \\
\hline
\end{tabular}

In Period I ( 20 days) the daily work consisted of 300 revolutions of the dynamometer. With this amount of work the live weight of the horse underwent very little change, but there was a material

* Landw. Jahrb., 27, Supp. III, 418. 
gain of nitrogen, so that Wolff estimates that the work might have been increased to 350 revolutions. In Period II (23 days) the daily work was increased to 450 revolutions and the same behavior was observed, while a further increase to 500 revolutions during the last ten days checked the gain of nitrogen without causing a decrease in live weight. Taking 350 and 500 revolutions respectively as representing the maximum amount of work that could be done on the two rations, the equivalent of the oats added may be computed as follows:

\begin{tabular}{|c|c|c|}
\hline & Revolutions. & $\begin{array}{c}\text { Equivalent } \\
\text { Work, } \\
\text { KgIn. }\end{array}$ \\
\hline 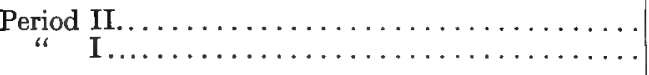 & $\begin{array}{l}500 \\
350\end{array}$ & $\begin{array}{r}1,030,687 \\
722,678\end{array}$ \\
\hline 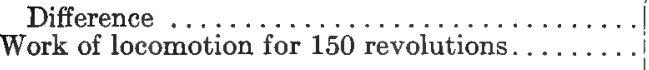 & 150 & $\begin{array}{r}308,009 \\
75,000\end{array}$ \\
\hline $\begin{array}{l}\text { Total difference. ............. } \\
\text { Equal to.................. }\end{array}$ & & $\begin{array}{l}383,009 \\
903 \text { Cals. }\end{array}$ \\
\hline
\end{tabular}

The percentage utilization was therefore $903 \div 3321=27.2$ per cent.

The above figures serve to exemplify the general method of computation and likewise to illustrate the weak points in Wolff's experiments, viz., the uncertainty in the determination of the work of locomotion and the impossibility of demonstrating the equilibrium of food and work without the use of the respiration apparatus or calorimeter.

Out of the whole number of experiments between 1891 and 1894, seven admit of a comparison of this sort, viz., four on oats, two on straw, and one on beans. Upon making the computations, however, the results are found to be so exceedingly variable (the range for oats, e.g., being from 16.89 to 63.96 per cent.) as to demonstrate that the data of Wolff's experiments are not sufficiently exact to be used in this way, and that the apparently reasonable result just computed is purely accidental.

Utilization of Fiber-Free Nutrients.-But although Wolff's results do not enable us to compute the percentage utilization of single feeding-stuffs, if we accept provisionally his conclusions regarding the non-availability of the crude fiber they afford data for numerous computations of the utilization of the fiber-free nutrients, 
and these computations in turn supply a check upon the hypothesis of the non-availability of crude fiber.

Wolff makes the comparison by deducting from the total fiberfree nutrients 3300 grams per $500 \mathrm{kgs}$. live weight for maintenance and comparing the energy of the remainder with the amount of work done. In the following tabulation of his results this method has been pursued. For the energy of the fiber-free nutrients, Zuntz's figure (3.96 Cals. per gram) has been used and the work of locomotion has been estimated at $50,000 \mathrm{kgm}$. per 100 revolutions of the dynamometer (compare p. 539).

\begin{tabular}{|c|c|c|c|c|c|c|}
\hline \multirow[t]{2}{*}{ Period. } & \multirow[t]{2}{*}{ Ration. } & \multicolumn{2}{|c|}{$\begin{array}{l}\text { Fiber-freo } \\
\text { Nutrients } \\
\text { Minus } 3300 \\
\text { Grms. }\end{array}$} & \multicolumn{2}{|c|}{ Work Done. } & \multirow{2}{*}{ 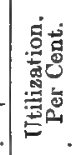 } \\
\hline & & Grms. & . Cals. & Kgm. & Cals. & \\
\hline $\begin{array}{l}\text { II } c \ldots \\
\text { III } \ldots \\
\text { IV } \ldots\end{array}$ & 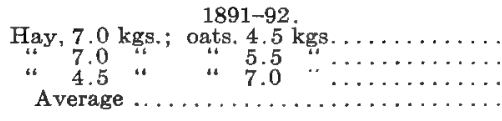 & $\begin{array}{l}1,424 \\
1,990 \\
2,259 \\
\cdots\end{array}$ & $\begin{array}{l}5,639 \\
7,881 \\
8,945\end{array}$ & $\left\{\begin{array}{r}931,676 \\
1,129,568 \\
1,094,328 \\
\cdots\end{array}\right.$ & $\begin{array}{l}2,197 \\
2,663 \\
2,581 \\
\cdots\end{array}$ & $\begin{array}{l}38.95 \\
33.79 \\
28.86 \\
33.16\end{array}$ \\
\hline $\begin{array}{l}\operatorname{I} a-d \ldots \\
\operatorname{II} b \ldots \\
\operatorname{III} \ldots \\
\operatorname{IV} \ldots\end{array}$ & 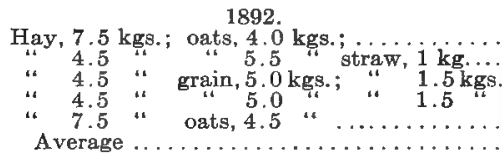 & $\begin{array}{l}1,775 \\
1.873 \\
1,521 \\
1,860 \\
1903 \\
\cdots\end{array}$ & \begin{tabular}{|l}
7,026 \\
7,416 \\
6,023 \\
7,365 \\
7,537 \\
$\cdots$
\end{tabular} & $\begin{array}{r}1,074,802 \\
1,153,813 \\
912,454 \\
1,186,577 \\
1,188,388 \\
\cdots \ldots\end{array}$ & $\begin{array}{l}2,535 \\
2,720 \\
2,152 \\
2,799 \\
2,803 \\
\cdots\end{array}$ & $\begin{array}{l}36.07 \\
36.68 \\
35.73 \\
38.00 \\
37.18 \\
36.77\end{array}$ \\
\hline $\begin{array}{l}\mathrm{I} \ldots \ldots \\
\mathrm{II} \ldots \ldots \\
\operatorname{II} \mathbf{I} \ldots \ldots \\
\mathrm{V} b \ldots \ldots\end{array}$ & 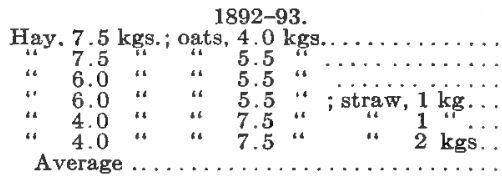 & $\begin{array}{l}1,475 \\
2,297 \\
1,670 \\
2,036 \\
2,577 \\
2,692\end{array}$ & $\begin{array}{r}5.841 \\
9.095 \\
6.613 \\
8.063 \\
10,210 \\
10,660 \\
\cdots\end{array}$ & $\begin{array}{r}897,678 \\
1,280,687 \\
905,568 \\
1,167,127 \\
1,421,285 \\
1.549,620 \\
\ldots \ldots\end{array}$ & $\mid \begin{array}{l}2,116 \\
3,024 \\
2,135 \\
2,752 \\
3,352 \\
3,655 \\
\cdots\end{array}$ & $\begin{array}{l}36.24 \\
33.20 \\
32.28 \\
34.14 \\
32.85 \\
34.28 \\
33.74\end{array}$ \\
\hline $\begin{array}{l}\mathrm{I} \\
\mathrm{I} I \mathrm{I} \\
\mathrm{V} \\
\mathrm{V}\end{array} \ldots$ & 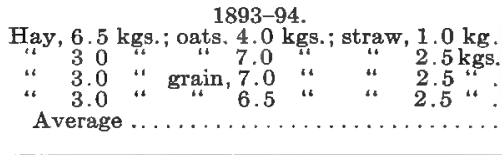 & $\begin{array}{l}1,607 \\
2,580 \\
2,560 \\
2,880\end{array}$ & $\begin{array}{r}6,362 \\
10,220 \\
10,140 \\
11,420\end{array}$ & $\begin{array}{r}900,267 \\
1,549,262 \\
1,545,702 \\
1,673,786\end{array}$ & $\begin{array}{l}2,122 \\
3,653 \\
3,645 \\
3,948 \\
\cdots\end{array}$ & $\begin{array}{l}33.36 \\
35.76 \\
35.95 \\
34.61 \\
35.05\end{array}$ \\
\hline
\end{tabular}

In every instance but one the utilization as thus computed exceeds 31.3 per cent. In other words, the energy of the body material which, according to Zuntz \& Hagemann's results, must have been metabolized to produce the amount of work done exceeds considerably the amount computed to be available from the fond. There being no reason to question the substantial accuracy of Zuntz \& Hagemann's factor, this means, of course, that if the food and work were in equilibrium our estimates of the energy available from 
the food are too low. Either 3300 grams of fiber-free nutrients $(13,068$ Cals.) is too large an allowance for maintenance, or the assumption that the energy of the digested crude fiber is substantially equivalent to the work of digestion and assimilation is erroneous, or, finally, the figure of 3.96 Cals. per gram of digested nutrients is too small. As regards the latter possibility, while it may be conceded that the energy per gram of digested matter will vary somewhat in different experiments, the difference will be too small to materially affect the result. The uncertainty regarding the maintenance requirement may be readily eliminated by a computation based on the differences between the several periods, thus affording, to a degree at least, a test of the correctness of Wolff's hypothesis regarding the crude fiber. The following table contains the results of such comparisons. In each series the period with the least amount of digested food (fiber-free) has been compared with the other periods of the same series.

\begin{tabular}{|c|c|c|c|}
\hline & $\begin{array}{c}\text { Metabolizable } \\
\text { Energy of } \\
\text { Fiber-free } \\
\text { Nutrients. } \\
\text { Cals. }\end{array}$ & $\begin{array}{l}\text { Work, } \\
\text { Cals. }\end{array}$ & $\begin{array}{l}\text { Utilization, } \\
\text { Per Cent. }\end{array}$ \\
\hline \multirow[t]{2}{*}{$\begin{array}{c}1891-92 . \\
\text { Period } \operatorname{III} \ldots \ldots \ldots \ldots \ldots \ldots \ldots \\
\text { II } c \ldots \ldots \ldots \ldots \ldots\end{array}$} & $\begin{array}{l}20,949 \\
18,707\end{array}$ & $\begin{array}{l}2663 \\
2197\end{array}$ & \multirow{3}{*}{20.79} \\
\hline & 2,242 & 466 & \\
\hline \multirow[t]{2}{*}{ Period ${ }_{\text {IV }}^{\text {IV }} \ldots \ldots \ldots \ldots \ldots \ldots$} & $\begin{array}{l}22,013 \\
18,707\end{array}$ & $\begin{array}{l}2581 \\
2197\end{array}$ & \\
\hline & 3,306 & 384 & \multirow[t]{2}{*}{11.62} \\
\hline \multirow{2}{*}{$\begin{array}{c}1892 . \\
\text { Period } \operatorname{I} a-d \ldots \ldots \ldots \ldots \ldots \\
\text { III } \ldots \ldots \ldots \ldots \ldots\end{array}$} & $\begin{array}{l}20,094 \\
19,091\end{array}$ & $\begin{array}{l}2535 \\
2152\end{array}$ & \\
\hline & 1,003 & 383 & \multirow[t]{2}{*}{38.19} \\
\hline \multirow[t]{2}{*}{$\underset{" \text { Period }}{\operatorname{II} b} \ldots \ldots \ldots \ldots \ldots \ldots \ldots$} & $\begin{array}{l}20,484 \\
19,091\end{array}$ & $\begin{array}{l}2720 \\
2152\end{array}$ & \\
\hline & 1,393 & 568 & \multirow[t]{2}{*}{40.77} \\
\hline \multirow[t]{2}{*}{ Period IV. } & $\begin{array}{l}20,433 \\
19,091\end{array}$ & $\begin{array}{l}2799 \\
2152\end{array}$ & \\
\hline & 1,342 & 647 & 48.21 \\
\hline
\end{tabular}




\begin{tabular}{|c|c|c|c|}
\hline & $\begin{array}{l}\text { Metabolizable } \\
\text { Energy of } \\
\text { Fiber-free } \\
\text { Nutrients, } \\
\text { Cals. }\end{array}$ & $\begin{array}{l}\text { Work, } \\
\text { Cals. }\end{array}$ & $\begin{array}{l}\text { Utilization, } \\
\text { Per Cent. }\end{array}$ \\
\hline 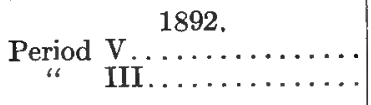 & $\begin{array}{l}20,605 \\
19,091\end{array}$ & $\begin{array}{l}2803 \\
2152\end{array}$ & \multirow[b]{2}{*}{43.00} \\
\hline \multirow[t]{2}{*}{ 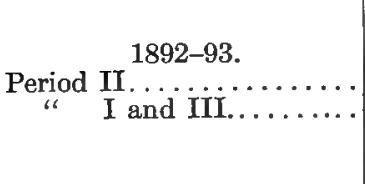 } & $\begin{array}{r}1,514 \\
22,163 \\
19,295\end{array}$ & $\begin{array}{r}651 \\
\\
3024 \\
2126\end{array}$ & \\
\hline & 2,868 & 898 & \multirow[t]{2}{*}{31.31} \\
\hline \multirow[t]{2}{*}{$\underset{" \text { Period }}{\operatorname{IV}} \underset{\text { I and }}{\mathrm{IIII}} \ldots \ldots \ldots \ldots$} & $\begin{array}{l}21,131 \\
19,295\end{array}$ & $\begin{array}{l}2752 \\
2126\end{array}$ & \\
\hline & 1,836 & 626 & \multirow[t]{2}{*}{34.10} \\
\hline \multirow[t]{2}{*}{ 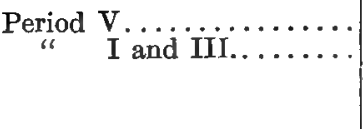 } & $\begin{array}{l}23,278 \\
19,295\end{array}$ & $\begin{array}{l}3352 \\
2126\end{array}$ & \\
\hline & 3,983 & 1226 & \multirow[t]{2}{*}{30.78} \\
\hline 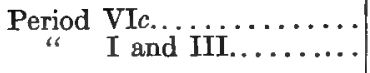 & $\begin{array}{l}23,728 \\
19,295\end{array}$ & $\begin{array}{l}3655 \\
2126\end{array}$ & \\
\hline \multirow[t]{2}{*}{ 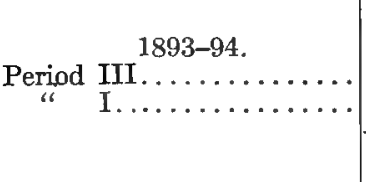 } & $\begin{array}{r}4,433 \\
\\
23,288 \\
19,430\end{array}$ & $\begin{array}{l}3653 \\
2122\end{array}$ & 34.48 \\
\hline & 3,858 & 1531 & \multirow[t]{2}{*}{39.68} \\
\hline Period $\underset{"}{\mathrm{~V}} \mathrm{I} \ldots \ldots \ldots \ldots \ldots \ldots \ldots \ldots$ & $\begin{array}{l}23,208 \\
19,430\end{array}$ & $\begin{array}{l}3645 \\
2122\end{array}$ & \\
\hline$\underset{*}{\operatorname{Period}} \mathrm{VI} \ldots \ldots \ldots \ldots \ldots \ldots \ldots$ & $\begin{array}{r}3,778 \\
\\
24,488 \\
19,430\end{array}$ & $\begin{array}{l}1523 \\
3948 \\
2122\end{array}$ & $\leq 0.31$ \\
\hline $\begin{array}{r}\text { Totals and averages, ex- } \\
\text { cluding } 1891-92 . \ldots .\end{array}$ & $\begin{array}{r}5,058 \\
31,066\end{array}$ & $\begin{array}{r}1826 \\
11,408 \\
\end{array}$ & 36.10 \\
\hline
\end{tabular}

With the exception of the experiments of 1891-92, which were the first with the new form of dynamometer and which Wolff considers unsatisfactory, we have but two cases in which the apparent utilization does not exceed 31.3 per cent. Having eliminated the uncertainty as to the maintenance ration, and the figures for the energy of the food being regarded as substantially correct, this can 
mean only one of two things, viz., that the figures for the work done are too high or that the deduction on account of the crude fiber is too great.

That a determination of the equivalence of food and work by Wolff's method is subject to considerable uncertainty in an individual case is obvious, but there seems to be no apparent reason why it should be uniformly overestimated. The measurement of the work was made with great care, and while the work of locomotion is an estimate, its close agreement with the results of Zuntz \& Hagemann (p. 539) renders it unlikely that it is seriously in error.

It would appear, then, that with the rations used in these experiments the energy required for digestion and assimilation was less than the energy of the digested crude fiber. How much less it was, however, unfortunately does not appear, and we are obliged to content ourselves for the present with this negative conclusion.

Zuntz \& Hagemann's Computations.-These investigators * have recalculated Wolff's results in a still different manner. Instead of taking for the amount of work equivalent to the ration the figures given by Wolff, which, as already explained, are to a certain extent estimates, they take the amount of work actually performed in each case and correct for the observed gain or loss of live weight. This method is in conception more scientific than Wolff's, provided the requisite correction can be accurately estimated. As the basis for such an estimate, Zuntz \& Hagemann take an early experiment by Wolff, $\dagger$ from which they compute that one gram loss of live weight is equivalent to one half revolution of the dynamiometer (at $76 \mathrm{kgs}$. draft). From the same experiment they compute the mechanical equivalent of one revolution as $2694 \mathrm{kgm}$. This, however, aside from the fact that it is the result of a single series of experiments, was obtained with the old form of dynamometer, whose indications, as we have seen, were too high, but the later experiments unfortunately are not reported in a way to permit of an estimate of the difference.

Taking the correction, then, as estimated, Zuntz \& Hagemann divide Wolff's experiments into two groups, viz., those in which the work was 400 or less revolutions and those in which it was more

* Loc. cit., pp, 412-422.

† Grundlagen, etc., o. 80. 
than 400 revolutions. Comparing the averages of these two groups, they obtain the following:

\begin{tabular}{|c|c|c|c|}
\hline & $\begin{array}{l}\text { Total } \\
\text { Digested } \\
\text { Nutrients } \\
\text { Grms. }\end{array}$ & Work, Kgm. & $\begin{array}{l}\text { Loss of } \\
\text { Live } \\
\text { Weight, } \\
\text { Grms. }\end{array}$ \\
\hline \multirow{3}{*}{ 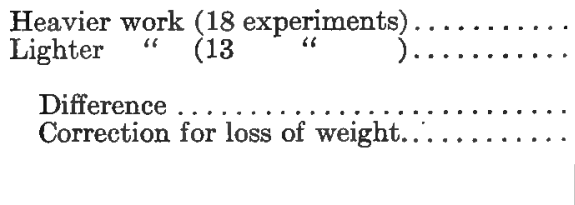 } & $\begin{array}{l}6236 \\
5851\end{array}$ & $\begin{array}{r}1,415,755 \\
995,225\end{array}$ & $\begin{array}{r}179.5 \\
7.3\end{array}$ \\
\hline & \multirow[t]{2}{*}{$\begin{array}{l}385 \\
\ldots \ldots\end{array}$} & $\begin{array}{l}420,530 \\
231,922\end{array}$ & \multirow[t]{2}{*}{172.2} \\
\hline & & 188,608 & \\
\hline
\end{tabular}

According to this computation, the 385 grams of added nutrients enabled $188,608 \mathrm{kgm}$. of work to be performed. At 3.96 Cals. per gram the metabolizable energy of the added nutrients equals 1524 Cals. From this, according to Zuntz \& Hagemann, is to be deducted 9 per cent. for the work of digestion and also 2.65 Cals. for each gram of total crude fiber in the added food. On this basis we have the following:

\begin{tabular}{|c|c|c|c|}
\hline & $\begin{array}{l}\text { Weight, } \\
\text { Grms. }\end{array}$ & \multicolumn{2}{|c|}{$\begin{array}{l}\text { Energy. } \\
\text { Cals. }\end{array}$} \\
\hline Digested nutrients. . . . . . & 385 & & 1524 \\
\hline Average crude fiber fed: & & & \\
\hline Heavier work .......... & 2338 & & \\
\hline Lighter work. . . . . . . . . . . & 2356 & & \\
\hline Difference......... & -18 & & \\
\hline Equivalent energy......... & $-\infty$ & -48 & \\
\hline Work of digestion $(1524 \times 0.9)$. & & 137 & \\
\hline Deduction & & & 89 \\
\hline Available energy . . . . . . . . & & & 1435 \\
\hline Work done $(188,608 \div 424) \ldots$ & & & 445 \\
\hline
\end{tabular}

The work done is 31 per cent. of the computed available energy of the food, a figure corresponding very closely with the 31.3 per cent. found by Zuntz \& Hagemann.

The difference in the average amount of crude fiber fed in the two groups of experiments is so small that the estimate for the 
energy required by its digestion hardly affects the computation. What the result appears to show is that the estimate of 9 per cent. for the digestion and assimilation of the fiber-free nutrients is approximately correct.

The difference in the amount of digested crude fiber was somewhat greater than that in the total amount. If we make the comparison of the two averages on the basis of the fiber-free nutrients in the same manner as in previous cases we have-

Fiber-free nutrients:

Heavier work............. 5524 grams

Lighter work............. 5086 "

Difference............... $\overline{438}$ “

Equivalent energy ............. 1735 Cals.

Energy of work.............. 445 "

Utilization.................25.65 per cent.

Apparently a considerable amount of energy was required for the work of digestion and assimilation in addition to that equivalent to the digested crude fiber, a result which seems to conflict with the conclusions drawn from a discussion of the same experiments in the preceding paragraph. The apparent discrepancy lies in the determination of the amount of external work equivalent to the added nutrients. Wolff, as we have seen, after securing an approximate constancy of live weight, corrects the measured amount of work in accordance with his judgment of the amount which would have been equivalent to the ration given and relies on the "might of averages" to overcome the inherent uncertainties of his method. Zuntz \& Hagemann, on the other hand, reckon with the measured amount of work, but are then compelled to correct their final result for the loss of live weight, and unfortunately this correction is relatively a very large one (over 50 per cent.) and rests upon a rather uncertain basis. While it would perhaps be presumptuous to attempt to decide the relative value of the two methods and the probability of the divergent conclusions based on them, one can hardly avoid feeling that the trained judgment of the actual experimenter is a safer reliance than such a relatively large correction computed by a critic. 
In any case it is obvious that while the extensive researches of Zuntz and his associates afford very reliable data as to the ratio between the energy liberated in muscular work and the amount of external work accomplished, or, in other words, as to the utilization of the net available energy of the food, we bave as yet, notwithstanding the vast amount of work done by Wolff and his co-laborers and others, but very fragmentary and uncertain data as to the utilization of the metabolizable energy of the food for work production. 


\section{APPENDIX.}

TABLE I. METABOLIZABLE ENERGY OF COARSE FODDERS.

\begin{tabular}{|c|c|c|c|c|c|c|c|c|c|c|}
\hline \multirow[b]{2}{*}{ Feed Added. } & \multirow[b]{2}{*}{ हुँ } & \multirow[b]{2}{*}{. } & \multicolumn{2}{|c|}{$\begin{array}{l}\text { Organic } \\
\text { Matter } \\
\text { Eaten. }\end{array}$} & \multicolumn{4}{|c|}{ Energy of } & \multicolumn{2}{|c|}{$\begin{array}{l}\text { Metabolizable } \\
\text { Energy. }\end{array}$} \\
\hline & & & 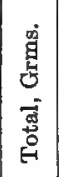 & 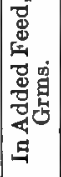 & $\begin{array}{l}\text { Food, } \\
\text { Cals. }\end{array}$ & $\begin{array}{l}\text { Feces, } \\
\text { Cals. }\end{array}$ & $\begin{array}{c}\text { Urine } \\
\text { (Cor- } \\
\text { rected), } \\
\text { Cals. }\end{array}$ & $\begin{array}{l}\text { Methane, } \\
\text { Cals. }\end{array}$ & $\begin{array}{l}\text { Total, } \\
\text { Cals. }\end{array}$ & $\begin{array}{c}\text { Per } \\
\text { Grm. } \\
\text { Or- } \\
\text { ganic } \\
\text { Mat- } \\
\text { ter, } \\
\text { Cals. }\end{array}$ \\
\hline Meadow hay $\mathrm{V}\{$ & $\mathbf{F}$ & $\begin{array}{l}1 \\
3\end{array}$ & $\begin{array}{l}9475 \\
6630\end{array}$ & 6024 & $\begin{array}{l}44821.2 \\
31327.8\end{array}$ & $\begin{array}{r}16323.7 \\
9599.2\end{array}$ & $\begin{array}{l}2113.3 \\
1530.0\end{array}$ & $\begin{array}{l}3250.6 \\
2560.7\end{array}$ & $\begin{array}{l}23133.6 \\
17637.9\end{array}$ & \\
\hline $\begin{array}{l}\text { Difference.... } \\
\text { Correction.... }\end{array}$ & & & 2845 & 2849 & $\begin{array}{r}13493.4 \\
+19.0\end{array}$ & $\begin{array}{r}6724.5 \\
+5.9\end{array}$ & $\begin{array}{l}583.3 \\
+0.9\end{array}$ & $\begin{array}{r}689.9 \\
+1.5\end{array}$ & $\begin{array}{r}5495.7 \\
+10.7\end{array}$ & \\
\hline Percentage. & & & & & $\begin{array}{r}13512.4 \\
100.00\end{array}$ & \begin{tabular}{|}
6730.4 \\
49.81
\end{tabular} & $\begin{array}{r}584.2 \\
4.32\end{array}$ & $\begin{array}{r}691.4 \\
5.12\end{array}$ & $\begin{array}{c}5506.4 \\
40.75\end{array}$ & 1.933 \\
\hline Meadow hay $\mathrm{V}\{$ & $\underset{\mathbf{G}}{\mathbf{G}}$ & $\begin{array}{l}2 \\
3\end{array}$ & $\begin{array}{l}9405 \\
6651\end{array}$ & $\begin{array}{l}5950 \\
3206\end{array}$ & $\begin{array}{l}43811.3 \\
30750.7\end{array}$ & $\begin{array}{r}15336.3 \\
9491.5\end{array}$ & $\begin{array}{l}1916.1 \\
1359.6\end{array}$ & $\begin{array}{l}3432.1 \\
2524.7\end{array}$ & $\begin{array}{l}23126.8 \\
17374.9\end{array}$ & \\
\hline $\begin{array}{l}\text { Difference.... } \\
\text { Correction... }\end{array}$ & & & 2754 & 2744 & $\begin{array}{r}13060.6 \\
-45.4\end{array}$ & $\begin{array}{r}5844.8 \\
-14.0\end{array}$ & $\begin{array}{l}556.5 \\
-2.0\end{array}$ & $\begin{array}{l}907.4 \\
-3.7\end{array}$ & $\begin{array}{l}5751.9 \\
-25.7\end{array}$ & \\
\hline Percentage. & & & & & $\begin{array}{c}13015.2 \\
100.00\end{array}$ & $\begin{array}{l}5830.8 \\
44.80\end{array}$ & $\begin{array}{r}554.5 \\
4.26\end{array}$ & $\begin{array}{r}903.7 \\
6.94\end{array}$ & $\begin{array}{c}5726.2 \\
44.00\end{array}$ & 2.087 \\
\hline Meadow hay VI \{ & H & $\begin{array}{l}2 \\
4\end{array}$ & $\begin{array}{l}9527 \\
6402\end{array}$ & $\begin{array}{l}6323 \\
3198\end{array}$ & $\begin{array}{l}45255.8 \\
30338.1\end{array}$ & $\begin{array}{r}14103.7 \\
8574.9\end{array}$ & $\begin{array}{l}2576.3 \\
1795.0\end{array}$ & $\begin{array}{l}3306.6 \\
2579.4\end{array}$ & $\begin{array}{l}25269.2 \\
17388.8\end{array}$ & \\
\hline $\begin{array}{l}\text { Difference.... } \\
\text { Correction.... }\end{array}$ & & & 3125 & 3125 & $\begin{array}{r}14917.7 \\
-8.9\end{array}$ & $\begin{array}{r}5528.8 \\
-2.5\end{array}$ & $\begin{array}{l}781.3 \\
-0.5\end{array}$ & $\begin{array}{l}727.2 \\
-0.8\end{array}$ & $\begin{array}{r}7880.4 \\
-5.1\end{array}$ & \\
\hline Percentage... & & & & & $\begin{array}{r}14908.8 \\
100.00\end{array}$ & $\begin{array}{r}5526.3 \\
37.07\end{array}$ & $\begin{array}{r}780.8 \\
5.24\end{array}$ & $\begin{array}{r}726.4 \\
4.87\end{array}$ & $\begin{array}{r}7875.3 \\
52.82\end{array}$ & 2.520 \\
\hline Meadow hayVI \{ & $\begin{array}{l}\mathrm{H} \\
\mathrm{H}\end{array}$ & $\begin{array}{l}7 \\
4\end{array}$ & $\begin{array}{l}9743 \\
6402\end{array}$ & $\begin{array}{l}6495 \\
3198\end{array}$ & $\begin{array}{l}46275.0 \\
30338.1\end{array}$ & $\begin{array}{r}14104.8 \\
8574.9\end{array}$ & $\begin{array}{l}2593.0 \\
1795.0\end{array}$ & $\begin{array}{l}3564.2 \\
2579.4\end{array}$ & $\begin{array}{l}26013.0 \\
17388.8\end{array}$ & \\
\hline $\begin{array}{l}\text { Difference.... } \\
\text { Correction... }\end{array}$ & & & 3341 & 3297 & $\begin{array}{r}15936.9 \\
-208.3\end{array}$ & $\begin{array}{r}5529.9 \\
-58.9\end{array}$ & $\begin{array}{r}798.0 \\
-12.3\end{array}$ & $\begin{array}{r}984.8 \\
-17.7\end{array}$ & $\begin{array}{r}8624.2 \\
119.4\end{array}$ & \\
\hline Percentage & & & & & $\begin{array}{c}15728.6 \\
100.00\end{array}$ & $\begin{array}{r}5471.0 \\
34.78\end{array}$ & $\begin{array}{r}785.7 \\
5.00 \\
\end{array}$ & $\begin{array}{r}967.1 \\
6.15 \\
\end{array}$ & \begin{tabular}{|c|}
8504.8 \\
54.07
\end{tabular} \mid & 2.580 \\
\hline
\end{tabular}


TABLE I (Continued).

\begin{tabular}{|c|c|c|c|c|c|c|c|c|c|c|}
\hline \multirow[b]{2}{*}{ Feed Added. } & \multirow[b]{2}{*}{ 总 } & \multirow[b]{2}{*}{$\begin{array}{l}.8 \\
.0 \\
0 \\
0.1\end{array}$} & \multicolumn{2}{|c|}{$\begin{array}{l}\text { Organic } \\
\text { Matter } \\
\text { Eaten. }\end{array}$} & \multicolumn{4}{|c|}{ Energy of } & \multicolumn{2}{|c|}{$\begin{array}{c}\text { Metabolizable } \\
\text { Energy. }\end{array}$} \\
\hline & & & 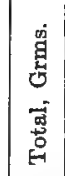 & 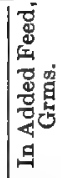 & $\begin{array}{l}\text { Food, } \\
\text { Cals. }\end{array}$ & $\begin{array}{l}\text { Feces, } \\
\text { Cals. }\end{array}$ & $\begin{array}{c}\text { Urine } \\
\text { (Cor- } \\
\text { rected), } \\
\text { Cals. }\end{array}$ & $\begin{array}{l}\text { Methane, } \\
\text { Cals. }\end{array}$ & $\begin{array}{l}\text { Total, } \\
\text { Cals. }\end{array}$ & $\begin{array}{c}\text { Per } \\
\text { Grm. } \\
\text { Or- } \\
\text { ganic } \\
\text { Mat- } \\
\text { ter, } \\
\text { Cals. }\end{array}$ \\
\hline Meadow hayVI & $\mathrm{J}$ & $\begin{array}{l}2 \\
4\end{array}$ & $\begin{array}{l}9539 \\
6458\end{array}$ & $\begin{array}{l}6340 \\
3239\end{array}$ & $\begin{array}{l}45239.6 \\
30548.5\end{array}$ & $\begin{array}{r}13218.1 \\
8171.2\end{array}$ & $\begin{array}{l}2755.1 \\
1824.6\end{array}$ & $\begin{array}{l}3620.2 \\
2722.2\end{array}$ & $\begin{array}{l}25646.2 \\
17830.5\end{array}$ & \\
\hline $\begin{array}{l}\text { Difference.... } \\
\text { Correction... }\end{array}$ & & & 3081 & 3101 & $\begin{array}{r}14691.1 \\
101.8\end{array}$ & $\begin{array}{r}5046.9 \\
+27.2\end{array}$ & $\begin{array}{r}930.5 \\
6.1\end{array}$ & $\begin{array}{r}898.0 \\
9.1\end{array}$ & $\begin{array}{r}7815.7 \\
59.4\end{array}$ & \\
\hline Percentage.. & & & & & $\begin{array}{r}14792.9 \\
100.00\end{array}$ & $\begin{array}{r}5074.1 \\
34.30\end{array}$ & $\begin{array}{r}936.6 \\
6.33\end{array}$ & $\begin{array}{r}907.1 \\
6.13\end{array}$ & $\begin{array}{r}7875.1 \\
53.24\end{array}$ & 2.540 \\
\hline Oat straw II.. & $\underset{\mathbf{F}}{\mathbf{F}}$ & $\begin{array}{l}2 \\
3\end{array}$ & $\begin{array}{l}9819 \\
6630\end{array}$ & $\begin{array}{r}3170 \\
0\end{array}$ & $\begin{array}{l}46690.1 \\
31327.8\end{array}$ & $\begin{array}{r}18296.3 \\
9599.2\end{array}$ & $\begin{array}{l}1884.2 \\
1529.8\end{array}$ & $\begin{array}{l}3239.9 \\
2560.7\end{array}$ & $\begin{array}{l}23269.7 \\
17638.1\end{array}$ & \\
\hline $\begin{array}{l}\text { Difference ... } \\
\text { Correction.... }\end{array}$ & & & 3189 & 3170 & $\begin{array}{r}15362.3 \\
-94.3\end{array}$ & $\begin{array}{r}8697.1 \\
-28.9\end{array}$ & $\begin{array}{r}354.4 \\
-4.6\end{array}$ & $\begin{array}{r}679.2 \\
-7.7\end{array}$ & $\begin{array}{r}5631.6 \\
-53.1\end{array}$ & \\
\hline Percentage... & & & & & $\begin{array}{c}15268.0 \\
100.00\end{array}$ & $\begin{array}{r}8668.2 \\
56.77\end{array}$ & $\begin{array}{r}349.8 \\
2.29\end{array}$ & $\begin{array}{r}671.5 \\
4.40\end{array}$ & \begin{tabular}{|c|}
5578.5 \\
36.54
\end{tabular} & 1.760 \\
\hline Oat straw II. . & $\stackrel{\mathrm{G}}{\mathrm{G}}$ & $\begin{array}{l}1 \\
3\end{array}$ & $\left|\begin{array}{l}9740 \\
6651\end{array}\right|$ & $\begin{array}{r}3115 \\
0\end{array}$ & $\begin{array}{l}45626.1 \\
30750.7\end{array}$ & $\begin{array}{r}17983.1 \\
9491.5\end{array}$ & $\begin{array}{l}1633.6 \\
1359.6\end{array}$ & $\begin{array}{l}3448.1 \\
2524.7\end{array}$ & $\begin{array}{l}22561.3 \\
17374.9\end{array}$ & \\
\hline $\begin{array}{l}\text { Difference.... } \\
\text { Correction... }\end{array}$ & & & 3089 & 3115 & $\begin{array}{r}14875.4 \\
+126.5\end{array}$ & $\begin{array}{r}8491.6 \\
+39.0\end{array}$ & $\begin{array}{r}274.0 \\
+5.6\end{array}$ & $\begin{array}{r}923.4 \\
+10.4\end{array}$ & $\begin{array}{r}5186.4 \\
+71.5\end{array}$ & \\
\hline Percentage... . & & & & & $\begin{array}{c}15001.9 \\
100.00\end{array}$ & $\begin{array}{r}8530.6 \\
56.86\end{array}$ & $\begin{array}{r}279.6 \\
1.86\end{array}$ & $\begin{array}{r}933.8 \\
6.23\end{array}$ & \begin{tabular}{|c|}
5257.9 \\
35.05
\end{tabular} & 1.688 \\
\hline Wheat straw I & $\stackrel{\mathrm{H}}{\mathrm{H}}$ & $\begin{array}{l}1 \\
4\end{array}$ & $\begin{array}{l}9611 \\
6402\end{array}$ & $\begin{array}{r}3195 \\
0\end{array}$ & $\begin{array}{l}45570.1 \\
30338.1\end{array}$ & $\begin{array}{r}17751.7 \\
8574.9\end{array}$ & $\begin{array}{l}2084.7 \\
1795.0\end{array}$ & $\begin{array}{l}3792.4 \\
2579.4\end{array}$ & $\begin{array}{l}21941.3 \\
17388.8\end{array}$ & \\
\hline $\begin{array}{l}\text { Difference.... } \\
\text { Correction... }\end{array}$ & & & 3209 & 3195 & $\begin{array}{r}15232.0 \\
-76.6\end{array}$ & $\begin{array}{l}9176.8 \\
-21.7\end{array}$ & $\begin{array}{r}289.7 \\
-4.5\end{array}$ & $\begin{array}{r}1213.0 \\
-6.5\end{array}$ & $\begin{array}{r}4552.5 \\
-43.9\end{array}$ & \\
\hline Percentage... & & & & & $\begin{array}{r}15155.4 \\
100.00\end{array} \mid$ & $\begin{array}{r}9155.1 \\
60.41\end{array}$ & $\begin{array}{r}285.2 \\
1.88\end{array}$ & $\begin{array}{r}1206.5 \\
7.96\end{array}$ & \begin{tabular}{|c|}
4508.6 \\
29.75
\end{tabular} & 1411 \\
\hline Wheat straw I \{ & $\mathrm{J}$ & $\begin{array}{l}1 \\
4\end{array}$ & $\begin{array}{l}9583 \\
6458\end{array}$ & $\begin{array}{r}3188 \\
0\end{array}$ & $\begin{array}{l}45365.9 \\
30548.5\end{array}$ & $\begin{array}{r}16562.1 \\
8171.2\end{array}$ & $\begin{array}{l}2237.8 \\
1824.6\end{array}$ & $\begin{array}{l}4003.2 \\
2722.2\end{array}$ & $\begin{array}{l}22562.8 \\
17830.5\end{array}$ & \\
\hline $\begin{array}{l}\text { Difference.... } \\
\text { Correction... }\end{array}$ & & & 3125 & 3188 & $\begin{array}{r}14817.4 \\
+302.4\end{array}$ & $\begin{array}{r}8390.9 \\
+80.9\end{array}$ & $\begin{array}{r}413.2 \\
+18.1\end{array}$ & $\begin{array}{r}1281.0 \\
+26.9\end{array}$ & $\begin{array}{r}4732.3 \\
+176.5\end{array}$ & \\
\hline Percentage... & & & & & $\begin{array}{r}15119.8 \\
100.00\end{array}$ & $\begin{array}{c}8471.8 \\
56.03\end{array}$ & $\begin{array}{r}431.3 \\
2.85\end{array}$ & $\begin{array}{r}1307.9 \\
8.65\end{array}$ & $\begin{array}{c}4908.8 \\
32.47\end{array}$ & 1.540 \\
\hline $\begin{array}{c}\text { Extracted rye } \\
\text { straw ..... }\end{array}$ & H. & $\begin{array}{l}\mathbf{5} \\
\mathbf{4}\end{array}$ & $\begin{array}{l}9114 \\
6402\end{array}$ & $\begin{array}{r}2665 \\
0\end{array}$ & $\begin{array}{l}41900.7 \\
30338.1\end{array}$ & $\begin{array}{l}9926.4 \\
8574.9\end{array}$ & $\begin{array}{l}1756.5 \\
1795.0\end{array}$ & $\begin{array}{l}4004.5 \\
2579.4\end{array}$ & $\begin{array}{l}26213.3 \\
17388.8\end{array}$ & \\
\hline $\begin{array}{l}\text { Difference ... } \\
\text { Correction ... }\end{array}$ & & & 2712 & 2665 & $\begin{array}{r}11562.6 \\
-232.7\end{array}$ & $\begin{array}{r}1351.5 \\
-65.8\end{array}$ & $\begin{array}{l}-38.5 \\
-13.8\end{array}$ & $\begin{array}{r}1425.1 \\
-19.8\end{array}$ & $\begin{array}{r}8824.5 \\
-133.3\end{array}$ & \\
\hline Percentage ... & & & & & $\begin{array}{r}11329.9 \\
100.00\end{array}$ & $\begin{array}{r}1285.7 \\
11.35\end{array}$ & $\begin{array}{c}-52.3 \\
-0.46\end{array}$ & $\begin{array}{r}1405.3 \\
12.40\end{array}$ & $\begin{array}{r}8691.2 \\
76.71\end{array}$ & 3.261 \\
\hline $\begin{array}{c}\text { Extracted rye } \\
\text { straw...... }\end{array}$ & $\mathbf{J}$ & $\begin{array}{l}5 \\
4\end{array}$ & $\begin{array}{l}9142 \\
6458\end{array}$ & 2659 & $\begin{array}{l}41962.6 \\
30548.5\end{array}$ & $\begin{array}{l}9799.0 \\
8171.2\end{array}$ & $\begin{array}{l}1705.8 \\
1824.6\end{array}$ & $\begin{array}{l}4147.4 \\
2722.2\end{array}$ & $\begin{array}{l}26310.4 \\
17830.5\end{array}$ & \\
\hline $\begin{array}{l}\text { Difference.... } \\
\text { Correction... }\end{array}$ & & & 2684 & 2659 & $\begin{array}{l}11414.1 \\
-113.3\end{array}$ & $\begin{array}{r}1627.8 \\
-30.3\end{array}$ & $\begin{array}{r}-118.8 \\
-6.8\end{array}$ & $\begin{array}{r}1425.2 \\
-10.1\end{array}$ & $\begin{array}{r}8479.9 \\
-66.1\end{array}$ & \\
\hline & & & & & $\begin{array}{r}11300.8 \\
10000\end{array}$ & $\begin{array}{r}1597.5 \\
14.14\end{array}$ & $=\begin{array}{c}-125.6 \\
-1.11\end{array}$ & $\begin{array}{r}1415.1 \\
12.52\end{array}$ & $\begin{array}{r}8413.8 \\
74.45\end{array}$ & 3.164 \\
\hline
\end{tabular}


TABLE II. METABOLIZABLE ENERGY OF BEET MOLASSTS.

\begin{tabular}{|c|c|c|c|c|c|c|c|c|c|c|}
\hline \multirow[b]{2}{*}{ Feed Added. } & \multirow[b]{2}{*}{ 苞 } & \multirow[b]{2}{*}{.ृّં } & \multicolumn{2}{|c|}{$\begin{array}{l}\text { Organic } \\
\text { Matter } \\
\text { Eaten. }\end{array}$} & \multicolumn{4}{|c|}{ Energy of } & \multicolumn{2}{|c|}{$\begin{array}{c}\text { Apparent } \\
\text { Metabolizable } \\
\text { Energy. }\end{array}$} \\
\hline & & & 兽 & 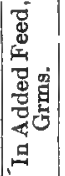 & $\begin{array}{l}\text { Food, } \\
\text { Cals. }\end{array}$ & $\begin{array}{l}\text { Feces, } \\
\text { Cals. }\end{array}$ & $\begin{array}{c}\text { Urine } \\
\text { (Cor- } \\
\text { rected), } \\
\text { Cals. }\end{array}$ & $\begin{array}{l}\text { Methane, } \\
\text { Cals. }\end{array}$ & $\begin{array}{l}\text { Total, } \\
\text { Cals. }\end{array}$ & $\begin{array}{c}\text { Per } \\
\text { Gram } \\
\text { Or- } \\
\text { ganic } \\
\text { Mat- } \\
\text { ter, } \\
\text { Cals. }\end{array}$ \\
\hline Beet mol'ses I & $\stackrel{F}{F}$ & $\begin{array}{l}6 \\
3\end{array}$ & $\left|\begin{array}{l}8262 \\
6630\end{array}\right|$ & $\begin{array}{r}1702 \\
0\end{array}$ & $\begin{array}{l}37946.2 \\
31327.8\end{array}$ & $\begin{array}{r}11365.8 \\
9599.2\end{array}$ & $\begin{array}{l}1786.1 \\
1530.0\end{array}$ & $\begin{array}{l}2397.9 \\
2560.7\end{array}$ & $\begin{array}{l}22396.4 \\
17637.9\end{array}$ & \\
\hline $\begin{array}{l}\text { Difference..... } \\
\text { Correction... }\end{array}$ & & & 1632 & 1702 & $\begin{array}{r}6618.4 \\
+330.8\end{array}$ & $\begin{array}{r}1766.6 \\
+101.3\end{array}$ & $\begin{array}{r}256.1 \\
+16.2\end{array}$ & $\begin{array}{r}-162.8 \\
+27.0\end{array}$ & $\begin{array}{r}4758.5 \\
+186.3\end{array}$ & \\
\hline Percentage... & & & & & $\begin{array}{c}6949.2 \\
100.00\end{array}$ & $\begin{array}{r}1867.9 \\
26.87\end{array}$ & $\begin{array}{r}272.3 \\
39.2\end{array}$ & $\begin{array}{r}-135.8 \\
-1.95\end{array}$ & $\begin{array}{r}4944.8 \\
71.16\end{array}$ & 2.905 \\
\hline Beet mol'ses II \{ & $\begin{array}{l}\mathbf{H} \\
\mathbf{H}\end{array}$ & $\begin{array}{l}6 \\
4\end{array}$ & $\begin{array}{l}8110 \\
6402\end{array}$ & $\left|\begin{array}{r}1611 \\
0\end{array}\right|$ & $\begin{array}{l}37544.4 \\
30338.1\end{array}$ & $\begin{array}{l}9070.0 \\
8574.9\end{array}$ & $\begin{array}{l}2035.2 \\
1795.0\end{array}$ & $\begin{array}{l}3458.8 \\
2579.4\end{array}$ & $\begin{array}{l}22980.4 \\
17388.8\end{array}$ & \\
\hline $\begin{array}{l}\text { Difference.... } \\
\text { Correction... }\end{array}$ & & & 1708 & 1611 & $\begin{array}{r}7206.3 \\
-459.4\end{array}$ & $\begin{array}{r}495.1 \\
-129.8\end{array}$ & $\begin{array}{r}240.2 \\
-27.2\end{array}$ & $\begin{array}{r}879.4 \\
-39.1\end{array}$ & $\begin{array}{r}5591.6 \\
-263.3\end{array}$ & \\
\hline Percentage... . & & & & & $\begin{array}{c}6746.9 \\
100.00\end{array}$ & $\begin{array}{r}365.3 \\
5.40\end{array}$ & $\begin{array}{r}213.0 \\
3.16\end{array}$ & $\begin{array}{c}840.3 \\
12.44\end{array}$ & $\begin{array}{r}5328.3 \\
79.00\end{array}$ & 3.308 \\
\hline Beet mol'ses II \{ & $\frac{J}{J}$ & $\begin{array}{l}6 \\
4\end{array}$ & $\begin{array}{l}8104 \\
6458\end{array}$ & $\begin{array}{r}1595 \\
0\end{array}$ & $\begin{array}{l}37461.1 \\
30548.5\end{array}$ & $\begin{array}{l}9198.7 \\
8171.2\end{array}$ & $\begin{array}{l}2017.2 \\
1824.6\end{array}$ & $\begin{array}{l}3422.7 \\
2722.2\end{array}$ & $\begin{array}{l}22822.5 \\
17830.5\end{array}$ & \\
\hline $\begin{array}{l}\text { Difference.... } \\
\text { Correction... }\end{array}$ & & & 1646 & 1595 & $\begin{array}{r}6912.6 \\
-234.3\end{array}$ & $\begin{array}{r}1027.5 \\
-62.7\end{array}$ & $\begin{array}{r}192.6 \\
-14.0\end{array}$ & $\begin{array}{r}700.5 \\
-20.9\end{array}$ & $\begin{array}{r}4992.0 \\
-136.7 \\
\end{array}$ & \\
\hline Percentage & & & & & $\begin{array}{c}6678.3 \\
100.00\end{array}$ & $\begin{array}{c}964.8 \\
14.45\end{array}$ & $\begin{array}{r}178.6 \\
2.67\end{array}$ & $\begin{array}{c}679.6 \\
10.18\end{array}$ & $\begin{array}{r}4855.3 \\
72.70\end{array}$ & 3.044 \\
\hline
\end{tabular}


TABLE III. METABOLIZABLE ENERGY OF STARCH. KUHN'S EXPERIMENTS.

\begin{tabular}{|c|c|c|c|c|c|c|c|c|c|c|}
\hline \multirow[b]{2}{*}{ Feed Added. } & \multirow[b]{2}{*}{ 悬 } & \multirow[b]{2}{*}{ 密 } & \multicolumn{2}{|c|}{$\begin{array}{l}\text { Organic } \\
\text { Matter } \\
\text { Eaten. }\end{array}$} & \multicolumn{4}{|c|}{ Energy of } & \multicolumn{2}{|c|}{$\begin{array}{c}\text { Apparent } \\
\text { Metabolizable } \\
\text { Energy. }\end{array}$} \\
\hline & & & 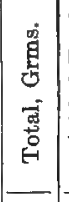 & 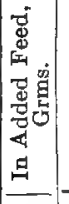 & $\begin{array}{l}\text { Food, } \\
\text { Cals. }\end{array}$ & $\begin{array}{l}\text { Feces. } \\
\text { Cals. }\end{array}$ & $\begin{array}{c}\text { Urine* } \\
\text { (Cor- } \\
\text { rected). } \\
\text { Cals. }\end{array}$ & $\begin{array}{c}\text { Methane } \\
\text { Cals. }\end{array}$ & $\begin{array}{l}\text { Total, } \\
\text { Cals. }\end{array}$ & $\begin{array}{c}\text { Per } \\
\text { Grm. } \\
\text { Or- } \\
\text { ganic } \\
\text { Mat- } \\
\text { ter, } \\
\text { Cals. } \\
\text {. } \\
\end{array}$ \\
\hline Starch I... & III & $\begin{array}{l}2 \\
1\end{array}$ & $\begin{array}{l}8839 \\
7328\end{array} \mid$ & $\begin{array}{r}1651 \\
0\end{array}$ & $\begin{array}{l}40964.5 \\
34603.2\end{array}$ & $\begin{array}{l}16615.5 \\
15505.1\end{array}$ & $\begin{array}{l}1430.3 \\
1549.6\end{array}$ & $\begin{array}{l}3225.3 \\
2670.1\end{array}$ & $\begin{array}{l}19593.4 \\
14878.4\end{array}$ & \\
\hline $\begin{array}{l}\text { Difference.. } \\
\text { Correction.. }\end{array}$ & & & 1511 & 1651 & $\begin{array}{r}6361.3 \\
+658.0\end{array}$ & $\begin{array}{r}1110.4 \\
+294.8\end{array}$ & $\begin{array}{r}-119.3 \\
+29.5\end{array}$ & $\begin{array}{r}655.2 \\
+50.8\end{array}$ & $\begin{array}{r}4715.0 \\
282.9\end{array}$ & \\
\hline Percentage. & & & & & $\begin{array}{c}7019.3 \\
100.00\end{array}$ & $\begin{array}{r}1405.2 \\
20.02\end{array}$ & $\begin{array}{r}-89.8 \\
-1.29\end{array}$ & $\begin{array}{c}706.0 \\
10.06\end{array}$ & $\begin{array}{r}4997.9 \\
71.21\end{array}$ & 3.029 \\
\hline Starch I ... & IV & $\begin{array}{c}2 \\
1 a \notin b\end{array}$ & $\begin{array}{l}8787 \\
7074\end{array}$ & $\begin{array}{r}1608 \\
0\end{array}$ & $\begin{array}{l}40725.6 \\
33405.1\end{array}$ & $\begin{array}{l}17202.1 \\
15250.6\end{array}$ & $\begin{array}{l}1434.9 \\
1481.5\end{array}$ & $\begin{array}{l}3348.0 \\
2491.3\end{array}$ & $\begin{array}{l}18740.6 \\
14181.7\end{array}$ & \\
\hline $\begin{array}{l}\text { Difference } \\
\text { Correction }\end{array}$ & & & 1713 & 1608 & $\begin{array}{r}7320.5 \\
-492.0 \\
\end{array}$ & $\begin{array}{r}1951.5 \\
-224.6\end{array}$ & $\begin{array}{l}-46.6 \\
-21.8\end{array}$ & $\begin{array}{r}856.7 \\
-36.7\end{array}$ & $\begin{array}{r}4558.9 \\
-208.9\end{array}$ & \\
\hline Percentage. & & & & & $\begin{array}{c}6828.5 \\
100.00\end{array}$ & \begin{tabular}{|}
1726.9 \\
25.29
\end{tabular} & $\begin{array}{r}-68.4 \\
-1.01\end{array}$ & $\begin{array}{c}820.0 \\
12.01\end{array}$ & $\begin{array}{r}4350.0 \\
63.71\end{array}$ & 2.705 \\
\hline Starch II. . \{ & $\begin{array}{l}\mathrm{V} \\
\mathrm{V}\end{array}$ & $\begin{array}{l}2 a \\
1\end{array}$ & $\begin{array}{l}8767 \\
7199\end{array}$ & $\begin{array}{r}1621 \\
0\end{array}$ & $\begin{array}{l}40827.5 \\
34211.5\end{array}$ & 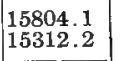 & $\begin{array}{l}1618.3 \\
1559.3\end{array}$ & $\begin{array}{l}3021.1 \\
2268.5\end{array}$ & $\begin{array}{l}20384.0 \\
15071.5\end{array}$ & \\
\hline $\begin{array}{l}\text { Difference. . } \\
\text { Correction. }\end{array}$ & & & 1568 & 1621 & $\begin{array}{r}6616.0 \\
+255.0\end{array}$ & $\begin{array}{r}491.9 \\
+114.2 \\
\end{array}$ & $\begin{array}{r}59.0 \\
+11.6\end{array}$ & $\begin{array}{r}752.6 \\
+16.9\end{array}$ & $\begin{array}{r}5312.5 \\
+112.3 \\
\end{array}$ & \\
\hline Percentage. & & & & & $\begin{array}{l}6871.0 \\
100.00\end{array}$ & $\begin{array}{r}606.1 \\
8.82\end{array}$ & $\begin{array}{c}70.6 \\
1.03\end{array}$ & $\begin{array}{c}769.5 \\
11.20\end{array}$ & \begin{tabular}{|r|}
5424.8 \\
78.95
\end{tabular} & 3.347 \\
\hline Starch II... \{ & $\begin{array}{l}\mathrm{V} \\
\mathrm{V}\end{array}$ & $\begin{array}{l}2 b \\
1\end{array}$ & $\begin{array}{l}8792 \\
7199\end{array} \mid$ & $\begin{array}{r}1663 \\
0\end{array}$ & $\begin{array}{l}40917.4 \\
34211.5\end{array}$ & $\begin{array}{l}16270.0 \\
15312.2\end{array}$ & $\begin{array}{l}1524.8 \\
1559.3\end{array}$ & $\begin{array}{l}2941.0 \\
2268.5\end{array}$ & $\begin{array}{l}20181.6 \\
15071.5\end{array}$ & \\
\hline $\begin{array}{l}\text { Difference.. } \\
\text { Correction.. }\end{array}$ & & & 1593 & 1663 & $\begin{array}{r}6705.9 \\
+334.1\end{array}$ & $\begin{array}{r}957.8 \\
+149.5\end{array}$ & $\begin{array}{l}-34.5 \\
+15.2\end{array}$ & $\begin{array}{r}672.5 \\
+22.2\end{array}$ & $\begin{array}{r}5110.1 \\
+147.2\end{array}$ & \\
\hline Percentage. & & & & & $\begin{array}{c}7040.0 \\
100.00\end{array}$ & $\begin{array}{r}1107.3 \\
15.73\end{array}$ & $\begin{array}{r}-19.3 \\
-0.27\end{array}$ & $\begin{array}{r}694.7 \\
9.86\end{array}$ & $\begin{array}{r}5257.3 \\
74.68\end{array}$ & 3.161 \\
\hline Starch II. . \{ & $\begin{array}{l}\text { VI } \\
\text { VI }\end{array}$ & $\begin{array}{l}2 b \\
1\end{array}$ & $\begin{array}{l}8861 \\
7125\end{array}$ & $\left|\begin{array}{r}1669 \\
0\end{array}\right|$ & $\begin{array}{l}41245.9 \\
33855.4\end{array}$ & $\begin{array}{l}15485.9 \\
13765.2\end{array}$ & $\begin{array}{l}1569.6 \\
1737.9\end{array}$ & $\begin{array}{l}3130.5 \\
2480.6\end{array}$ & $\begin{array}{l}21059.9 \\
15871.7\end{array}$ & \\
\hline $\begin{array}{l}\text { Difference. . } \\
\text { Correction.. }\end{array}$ & & & 1736 & 51669 & $\begin{array}{r}7390.5 \\
-320.9\end{array}$ & $\begin{array}{r}1720.7 \\
-130.5\end{array}$ & $\begin{array}{r}-168.3 \\
-16.5\end{array}$ & $\begin{array}{r}649.9 \\
-23.5\end{array}$ & $\begin{array}{r}5188.2 \\
-150.4\end{array}$ & \\
\hline Percentage. & & & & & $\begin{array}{c}7269.6 \\
100.00\end{array}$ & $\left|\begin{array}{r}1590.2 \\
22.49\end{array}\right|$ & $\begin{array}{r}-184.8 \\
-2.61\end{array}$ & $\begin{array}{r}626.4 \\
8.86\end{array}$ & $\begin{array}{r}5037.8 \\
71.26\end{array}$ & 3.018 \\
\hline Starch II... & $\begin{array}{l}\text { VI } \\
\text { VI }\end{array}$ & $\begin{array}{l}3 \\
1\end{array}$ & $\left|\begin{array}{l}9953 \\
7125\end{array}\right|$ & \begin{tabular}{r|r|}
3 & 2788 \\
5 & 0
\end{tabular} & $\begin{array}{l}45859.6 \\
33855.4\end{array}$ & $\begin{array}{l}16091.4 \\
13765.2\end{array}$ & $\begin{array}{l}1643.9 \\
1737.9\end{array}$ & $\begin{array}{l}3897.8 \\
2480.6\end{array}$ & $\begin{array}{l}24226.5 \\
15871.7\end{array}$ & \\
\hline $\begin{array}{l}\text { Difference. } \\
\text { Correction.. }\end{array}$ & & & 2828 & 82788 & $\begin{array}{r}12004.2 \\
-193.4\end{array}$ & $\begin{array}{r}2326.2 \\
-78.6\end{array}$ & $\begin{array}{r}-94.0 \\
-9.9\end{array}$ & $\begin{array}{r}1417.2 \\
-14.2\end{array}$ & $\begin{array}{r}8354.8 \\
-90.7\end{array}$ & \\
\hline Percentage.J & & & & & $\mid \begin{array}{r}11810.8 \\
100.00\end{array}$ & $\mid \begin{array}{c}2247.6 \\
19.03\end{array}$ & $\left|\begin{array}{r}-103.9 \\
-0.88\end{array}\right|$ & $\begin{array}{r}1403.0 \\
11.87\end{array}$ & $\begin{array}{r}8264.1 \\
69.98\end{array}$ & 2.964 \\
\hline
\end{tabular}

* Computed from carbon content. 
TABLE IV. METABOLIZABLE ENERGY OF STARCH. KELLNER'S EXPERIMENTS

\begin{tabular}{|c|c|c|c|c|c|c|c|c|c|c|}
\hline \multirow[b]{2}{*}{ Feed Added. } & \multirow[b]{2}{*}{ 营 } & \multirow[b]{2}{*}{ 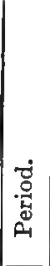 } & \multicolumn{2}{|c|}{$\begin{array}{l}\text { Organic } \\
\text { Matter } \\
\text { Eaten. }\end{array}$} & \multicolumn{4}{|c|}{ Energy of } & \multicolumn{2}{|c|}{$\begin{array}{l}\text { Apparent } \\
\text { Metabolizable } \\
\text { Energy. }\end{array}$} \\
\hline & & & 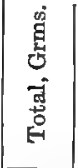 & 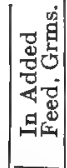 & $\begin{array}{l}\text { Food, } \\
\text { Cals. }\end{array}$ & $\begin{array}{l}\text { Feces, } \\
\text { Cals. }\end{array}$ & $\begin{array}{c}\text { Urine } \\
\text { (Cor- } \\
\text { rected), } \\
\text { Cals. }\end{array}$ & $\begin{array}{c}\text { Methane, } \\
\text { Cals. }\end{array}$ & $\begin{array}{l}\text { Total, } \\
\text { Cals. }\end{array}$ & $\begin{array}{c}\text { Per } \\
\text { Grm. } \\
\text { Or- } \\
\text { ganic } \\
\text { Mat- } \\
\text { ter, } \\
\text { Cals. }\end{array}$ \\
\hline $\begin{array}{c}\text { Starch I and } \\
\text { II . . . . . . }\end{array}$ & $\underset{\mathrm{B}}{\mathrm{B}}$ & $\begin{array}{l}2 \\
4\end{array}$ & $\begin{array}{l}11698 \\
10067\end{array}$ & 3231 & $\begin{array}{l}52928.6 \\
46129.1\end{array}$ & $\begin{array}{l}15915.8 \\
11874.4\end{array}$ & $\begin{array}{l}1740.1 \\
1958.5\end{array}$ & $\begin{array}{l}3382.7 \\
3716.3\end{array}$ & $\mid \begin{array}{l}31890.0 \\
28579.9\end{array}$ & \\
\hline $\begin{array}{l}\text { Difference ... } \\
\text { Correction .. }\end{array}$ & & & 1631 & 1624 & $\begin{array}{l}6799.5 \\
-30.9\end{array}$ & $\begin{array}{r}4041.4 \\
-8.0 \\
\end{array}$ & $\begin{array}{r}-218.4 \\
-1.3 \\
\end{array}$ & $\begin{array}{r}-333.6 \\
-2.5\end{array}$ & $\begin{array}{r}3310.1 \\
-19.1\end{array}$ & \\
\hline Percentage... & & & & & $\begin{array}{c}6768.6 \\
100.00\end{array}$ & $\begin{array}{r}4033.4 \\
59.60\end{array}$ & $\mid \begin{array}{r}-219.7 \\
-3.25\end{array}$ & $\begin{array}{r}-336.1 \\
-4.97\end{array}$ & $\begin{array}{r}3291.0 \\
48.62\end{array}$ & 2.027 \\
\hline $\begin{array}{l}\text { Starch I and } \\
\quad \text { II } \ldots . . . .\}\end{array}$ & $\stackrel{\mathrm{C}}{\mathrm{C}}$ & $\begin{array}{l}2 \\
1\end{array}$ & $\begin{array}{l}11980 \\
10407\end{array}$ & $\begin{array}{l}3193 \\
1602\end{array} \mid$ & $\begin{array}{l}54016.5 \\
47458.0\end{array}$ & $\begin{array}{l}19185.6 \\
15746.8\end{array}$ & $\begin{array}{l}1723.7 \\
1785.7\end{array}$ & $\begin{array}{l}3250.6 \\
3255.9\end{array}$ & $\begin{array}{l}29856.6 \\
26669.6\end{array}$ & \\
\hline $\begin{array}{l}\text { Difference } . . \\
\text { Correction ... }\end{array}$ & & & 1573 & 1591 & $\begin{array}{r}6558.5 \\
+70.8\end{array}$ & $\begin{array}{l}3438.8 \\
+23.5\end{array}$ & $\begin{array}{r}-62.0 \\
+2.7\end{array}$ & $\begin{array}{l}-5.3 \\
+4.9\end{array}$ & $\begin{array}{r}3187.0 \\
+39.7\end{array}$ & \\
\hline Percentage .. & & & & & $\begin{array}{c}6629.3 \\
100.00\end{array}$ & $\begin{array}{r}3462.3 \\
52.22\end{array}$ & $\begin{array}{c}-59.3 \\
-0.89\end{array}$ & $\begin{array}{l}-0.4 \\
-0.01\end{array}$ & $\begin{array}{c}3226.7 \\
48.68\end{array}$ & 2.028 \\
\hline Starch III .. & $\stackrel{D}{D}$ & $\begin{array}{l}2 \\
1\end{array}$ & $\begin{array}{r}11636 \\
9974\end{array}$ & $\begin{array}{r}1583 \\
0\end{array}$ & $\begin{array}{l}53902.2 \\
46945.4\end{array}$ & $\begin{array}{l}17817.9 \\
15718.3\end{array}$ & $\begin{array}{l}2211.2 \\
2407.0\end{array}$ & $\begin{array}{l}3381.4 \\
2957.0\end{array}$ & $\begin{array}{l}30491.7 \\
25863.1\end{array}$ & \\
\hline $\begin{array}{l}\text { Difference ... } \\
\text { Correction ... }\end{array}$ & & & 1662 & 1583 & $\begin{array}{r}6956.8 \\
-379.5\end{array}$ & $\begin{array}{r}2099.6 \\
-127.1\end{array}$ & $\begin{array}{r}-195.8 \\
-19.5\end{array}$ & $\begin{array}{r}424.4 \\
-23.9\end{array}$ & $\begin{array}{r}4628.6 \\
-209.0\end{array}$ & \\
\hline Percentage .. & & & & & $\begin{array}{c}6577.3 \\
100.00\end{array}$ & $\begin{array}{c}1972.5 \\
29.99\end{array}$ & $\left|\begin{array}{c}-215.3 \\
-3.27\end{array}\right|$ & $\begin{array}{r}400.5 \\
6.08\end{array}$ & $\begin{array}{r}4419.6 \\
67.20\end{array}$ & 2.792 \\
\hline Starch III . . & $\frac{F}{F}$ & $\begin{array}{l}4 \\
3\end{array}$ & $\begin{array}{l}8374 \\
6630\end{array}$ & $\begin{array}{r}1687 \\
0\end{array}$ & $\begin{array}{l}38608.3 \\
31327.8\end{array}$ & $\begin{array}{r}10833.9 \\
9599.2\end{array}$ & $\begin{array}{l}1594.3 \\
1530.0\end{array}$ & $\begin{array}{l}3382.7 \\
2560.7\end{array}$ & $\begin{array}{l}22797.4 \\
17637.9\end{array}$ & \\
\hline $\begin{array}{l}\text { Difference } . . \\
\text { Correction ... }\end{array}$ & & & 1744 & 1687 & $\begin{array}{r}7280.5 \\
-268.3\end{array}$ & $\begin{array}{l}1234.7 \\
-82.5\end{array}$ & $\begin{array}{r}64.3 \\
-13.2\end{array}$ & $\begin{array}{r}822.0 \\
-22.0\end{array}$ & $\begin{array}{r}5159.5 \\
-150.6\end{array}$ & \\
\hline Percentage.. & & & & & $\begin{array}{c}7012.2 \\
100.00\end{array}$ & $\begin{array}{r}1152.2 \\
16.42\end{array}$ & $\begin{array}{c}51.1 \\
0.73\end{array}$ & $\begin{array}{c}800.0 \\
11.41\end{array}$ & $\begin{array}{r}5008.9 \\
71.44\end{array}$ & 2.969 \\
\hline Starch III .. & $\stackrel{G}{G}$ & $\begin{array}{l}4 \\
3\end{array}$ & $\begin{array}{l}8380 \\
6651\end{array}$ & $\begin{array}{r}1676 \\
0\end{array}$ & $\begin{array}{l}37963.6 \\
30750.7\end{array}$ & $\begin{array}{r}10497.1 \\
9491.5\end{array}$ & $\begin{array}{l}1394.7 \\
1359.6\end{array}$ & $\begin{array}{l}3170.5 \\
2524.7\end{array}$ & $\begin{array}{l}22901.3 \\
17374.9\end{array}$ & \\
\hline $\begin{array}{l}\text { Difference ... } \\
\text { Correction... }\end{array}$ & & & 1729 & 1676 & $\begin{array}{r}7212.9 \\
-246.2\end{array}$ & $\begin{array}{r}1005.6 \\
-76.0\end{array}$ & $\begin{array}{r}35.1 \\
-10.9\end{array}$ & $\begin{array}{r}645.8 \\
-20.2\end{array}$ & $\begin{array}{r}5526.4 \\
-139.1\end{array}$ & \\
\hline Percentage .. & & & & & $\begin{array}{c}6966.7 \\
100.00\end{array}$ & $\begin{array}{r}929.6 \\
13.35\end{array}$ & $\begin{array}{c}24.2 \\
0.35\end{array}$ & $\begin{array}{r}625.6 \\
8.98\end{array}$ & $\begin{array}{r}5387.3 \\
77.32\end{array}$ & 3.214 \\
\hline Starch IV... & $\begin{array}{l}\mathrm{H} \\
\mathbf{H}\end{array}$ & $\begin{array}{l}3 \\
4\end{array}$ & $\begin{array}{l}8373 \\
6402\end{array}$ & $\left|\begin{array}{r}2013 \\
0\end{array}\right|$ & $\begin{array}{l}38562.4 \\
30338.1\end{array}$ & $\begin{array}{l}9843.8 \\
8574.9\end{array}$ & $\begin{array}{l}1588.4 \\
1795.0\end{array}$ & $\begin{array}{l}3183.9 \\
2579.4\end{array}$ & $\begin{array}{l}23946.3 \\
17388.8\end{array}$ & \\
\hline $\begin{array}{l}\text { Difference .. } \\
\text { Correction .. }\end{array}$ & & & 1971 & 2013 & $\begin{array}{r}8224.3 \\
+193.2\end{array}$ & $\begin{array}{l}1268.9 \\
+54.6\end{array}$ & $\begin{array}{r}-206.6 \\
+11.4\end{array}$ & $\begin{array}{r}604.5 \\
+16.4\end{array}$ & $\begin{array}{r}6557.5 \\
+110.8\end{array}$ & \\
\hline Percentage.. & & & & & $\begin{array}{c}8417.5 \\
100.00\end{array}$ & $\begin{array}{r}1323.5 \\
15.72\end{array}$ & \begin{tabular}{|}
-195.2 \\
-2.32 \\
\end{tabular} & $\begin{array}{r}620.9 \\
7.38\end{array}$ & $\begin{array}{r}6668.3 \\
79.22\end{array}$ & 3.313 \\
\hline Starch IV... & $\begin{array}{l}\mathrm{J} \\
\mathrm{J}\end{array}$ & $\begin{array}{l}3 \\
4\end{array}$ & $\begin{array}{l}8004 \\
6458\end{array}$ & $\begin{array}{r}1600 \\
0\end{array}$ & $\begin{array}{l}36982.6 \\
30548.5\end{array}$ & $\begin{array}{l}9096.8 \\
8171.2\end{array}$ & $\begin{array}{l}1885.4 \\
1824.6\end{array}$ & $\begin{array}{l}3492.1 \\
2722.2\end{array}$ & $\begin{array}{l}22508.3 \\
17830.5\end{array}$ & \\
\hline $\begin{array}{l}\text { Difference ... } \\
\text { Correction .. }\end{array}$ & & & 1546 & 1600 & $\begin{array}{r}6434.1 \\
+254.1\end{array}$ & $\begin{array}{r}925.6 \\
+68.0\end{array}$ & $\begin{array}{r}60.8 \\
+15.2\end{array}$ & $\begin{array}{r}769.9 \\
+22.6\end{array}$ & $\begin{array}{r}4677.8 \\
+148.3\end{array}$ & \\
\hline Percentage ... & & & & & $\begin{array}{c}6688.2 \\
100.00\end{array}$ & $\begin{array}{c}993.6 \\
14.85\end{array}$ & $\begin{array}{c}76.0 \\
1.14\end{array}$ & $\begin{array}{c}792.5 \\
11.85\end{array}$ & $\begin{array}{r}4826.1 \\
72.16\end{array}$ & 3.017 \\
\hline
\end{tabular}


TABLE V. METABOLIZABLE ENERGY OF WHEAT GLUTEN.

\begin{tabular}{|c|c|c|c|c|c|c|c|c|c|c|}
\hline \multirow[b]{2}{*}{ F'eed Added. } & \multirow[b]{2}{*}{ 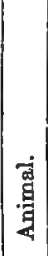 } & \multirow[b]{2}{*}{ 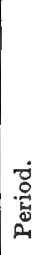 } & \multicolumn{2}{|c|}{$\begin{array}{l}\text { Organic } \\
\text { Matter } \\
\text { Eaten. }\end{array}$} & \multicolumn{4}{|c|}{ Energy of } & \multicolumn{2}{|c|}{$\begin{array}{c}\text { Apparent } \\
\text { Metabolizable } \\
\text { Energy. }\end{array}$} \\
\hline & & & 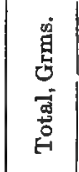 & 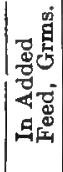 & $\begin{array}{l}\text { Food, } \\
\text { Cals. }\end{array}$ & $\begin{array}{l}\text { Feces, } \\
\text { Cals. }\end{array}$ & $\begin{array}{l}\text { Urine } \\
\text { (Cor- } \\
\text { rected), } \\
\text { Cals. }\end{array}$ & $\begin{array}{c}\text { Methane } \\
\text { Cals. }\end{array}$ & $\begin{array}{l}\text { Total, } \\
\text { Cals. }\end{array}$ & $\begin{array}{l}\text { Per } \\
\text { Grm. } \\
\text { Or- } \\
\text { ganic } \\
\text { Mat- } \\
\text { ter, } \\
\text { Cals. }\end{array}$ \\
\hline Wheat gluten ? & IIII & $\begin{array}{l}3 \\
2\end{array}$ & $\begin{array}{l}9311 \\
8839\end{array}$ & $\begin{array}{r}576 \\
0\end{array}$ & $\begin{array}{l}44025.3 \\
40964.5\end{array}$ & $\begin{array}{l}16041.5 \\
16615.5\end{array}$ & $\begin{array}{l}2048.8^{*} \\
1430.3^{*}\end{array}$ & $\begin{array}{l}3669.6 \\
3325.3\end{array}$ & $\begin{array}{l}22265.4 \\
19593.4\end{array}$ & \\
\hline $\begin{array}{l}\text { Difference... } \\
\text { Correction. . }\end{array}$ & & & 472 & 576 & $\begin{array}{r}3060.8 \\
+502.9\end{array}$ & $\begin{array}{r}-574.0 \\
+204.0\end{array}$ & $\begin{array}{r}618.5 \\
+17.6\end{array}$ & $\begin{array}{r}344.3 \\
+40.8\end{array}$ & $\begin{array}{r}2672.0 \\
+240.5\end{array}$ & \\
\hline Percentage. . & & & & & $\begin{array}{c}3563.7 \\
100.00\end{array}$ & $\begin{array}{l}-370.0 \\
-10.38\end{array}$ & $\begin{array}{r}636.1 \\
17.85\end{array}$ & $\begin{array}{l}385.1 \\
10.81\end{array}$ & $\begin{array}{r}2912.5 \\
81.72\end{array}$ & 5.057 \\
\hline Wheat gluten & IIII & $\begin{array}{l}4 \\
2\end{array}$ & $\begin{array}{r}10037 \\
8839 \\
\end{array}$ & $\begin{array}{r}1157 \\
0\end{array}$ & $\begin{array}{l}48293.6 \\
40964.5\end{array}$ & $\begin{array}{l}16593.3 \\
16615.5\end{array}$ & $\begin{array}{l}2990 \cdot 4^{*} \\
1430 \cdot 3^{*}\end{array}$ & $\begin{array}{l}3703.0 \\
3325.3\end{array}$ & $\begin{array}{l}25006.9 \\
19593.4\end{array}$ & \\
\hline $\begin{array}{l}\text { Difference... } \\
\text { Correction... }\end{array}$ & & & 1198 & 1157 & $\begin{array}{r}7329.1 \\
-171.2\end{array}$ & $\begin{array}{l}-22.2 \\
-69.4\end{array}$ & $\begin{array}{r}1560.1 \\
-6.0\end{array}$ & $\begin{array}{r}377.7 \\
-13.9\end{array}$ & $\begin{array}{r}5413.5 \\
-81.9\end{array}$ & \\
\hline Percentage .. & & & & & $\begin{array}{l}7157.9 \\
100.00\end{array}$ & $\begin{array}{l}-91.6 \\
-1.28\end{array}$ & $\begin{array}{r}1554.1 \\
21.71\end{array}$ & $\begin{array}{r}363.8 \\
5.08\end{array}$ & $\begin{array}{r}5331.6 \\
74.49\end{array}$ & 4.608 \\
\hline Wheat gluten \{ & $\begin{array}{l}\text { IV } \\
\text { IV }\end{array}$ & $\begin{array}{l}\mathbf{3} \\
\mathbf{2}\end{array}$ & $\begin{array}{l}9483 \\
8787\end{array}$ & $\begin{array}{r}582 \\
0\end{array}$ & $\begin{array}{l}44860.3 \\
40725.6\end{array}$ & $\begin{array}{l}16845.6 \\
17202.1\end{array}$ & $\begin{array}{l}2036.3^{*} \\
1434.9^{*}\end{array}$ & $\begin{array}{l}3346.7 \\
3348.0\end{array}$ & $\begin{array}{l}22631.7 \\
18740.6\end{array}$ & \\
\hline $\begin{array}{l}\text { Difference... } \\
\text { Correction... }\end{array}$ & & & 696 & 582 & $\begin{array}{r}4134.7 \\
-534.4\end{array}$ & $\begin{array}{l}-356.5 \\
-225.7\end{array}$ & $\begin{array}{r}601.4 \\
-18.8 \\
\end{array}$ & $\begin{array}{r}-1.3 \\
-43.9\end{array}$ & $\begin{array}{r}3891.1 \\
-246.0\end{array}$ & \\
\hline Percentage... & & & & & $\begin{array}{c}3600.3 \\
100.00\end{array}$ & $\begin{array}{r}-582.2 \\
-16.17\end{array}$ & $\begin{array}{r}582.6 \\
16.18\end{array}$ & $\begin{array}{r}-45.2 \\
-1.26\end{array}$ & $\begin{array}{c}3645.1 \\
101.25\end{array}$ & 6.263 \\
\hline $\begin{array}{c}\text { Wheat gluten }\{ \\
\mathbf{I} \ldots \ldots\end{array}$ & $\begin{array}{l}\mathbf{B} \\
\mathbf{B}\end{array}$ & $\frac{1}{4}$ & $\begin{array}{l}11636 \\
10067\end{array}$ & $\begin{array}{r}1746 \\
261 \\
\end{array}$ & $\begin{array}{l}54939.3 \\
46129.1\end{array}$ & $\begin{array}{l}14514.7 \\
11874.4\end{array}$ & $\begin{array}{l}3372.3 \\
1958.5\end{array}$ & $\begin{array}{l}3753.7 \\
3716.3\end{array}$ & $\begin{array}{l}33298.6 \\
28579.9\end{array}$ & \\
\hline $\begin{array}{l}\text { Difference .. } \\
\text { Correction .. }\end{array}$ & & & 1569 & 148.5 & $\begin{array}{r}8810.2 \\
-380.5\end{array}$ & $\begin{array}{r}2640.3 \\
-97.9\end{array}$ & $\begin{array}{r}1413.8 \\
-16.2\end{array}$ & $\begin{array}{r}37.4 \\
-30.7\end{array}$ & $\begin{array}{r}4718.7 \\
-235.7\end{array}$ & \\
\hline Percentage... & & & & & $\begin{array}{c}8429.7 \\
100.00\end{array}$ & $\begin{array}{r}2542.4 \\
30.16\end{array}$ & $\begin{array}{r}1397.6 \\
16.58\end{array}$ & $\begin{array}{l}6.7 \\
0.08\end{array}$ & $\begin{array}{r}4483.0 \\
53.18\end{array}$ & 3.019 \\
\hline $\begin{array}{c}\text { Wheat gluten } \\
\quad \mathbf{I} \ldots . . . .\end{array}$ & $\underset{\mathbf{B}}{\mathbf{B}}$ & $\begin{array}{l}3 \\
4\end{array}$ & $\begin{array}{l}1.1533 \\
10067\end{array}$ & $\begin{array}{r}1742 \\
261\end{array}$ & $\begin{array}{l}54469.0 \\
46129.1\end{array}$ & $\begin{array}{l}13753.4 \\
11874.4\end{array}$ & $\begin{array}{l}3092.1 \\
1958.5\end{array}$ & $\begin{array}{l}3574.9 \\
3716.3\end{array}$ & $\begin{array}{l}34048.6 \\
28579.9\end{array}$ & \\
\hline $\begin{array}{l}\text { Difference .. } \\
\text { Correction .. }\end{array}$ & & & 1466 & 1481 & $\begin{array}{r}8339.9 \\
+62.3\end{array}$ & $\begin{array}{r}1879.0 \\
+16.0\end{array}$ & $\begin{array}{r}1133.6 \\
+2.6\end{array}$ & $\begin{array}{r}-141.4 \\
+5.0\end{array}$ & $\begin{array}{r}5468.7 \\
+38.7\end{array}$ & \\
\hline Percentage .. & & & & & $\begin{array}{r}8402.2 \\
100.00\end{array}$ & $\begin{array}{r}1895.0 \\
22.55\end{array}$ & $\begin{array}{r}1136.2 \\
13.52\end{array}$ & $\begin{array}{r}-136.4 \\
-1.62\end{array}$ & $\begin{array}{r}5507.4 \\
65.55\end{array}$ & 3.719 \\
\hline $\begin{array}{c}\text { Wheat gluten } \\
\text { II ....... }\end{array}$ & $\stackrel{\mathrm{C}}{\mathrm{C}}$ & $\begin{array}{l}3 \\
1\end{array}$ & $\begin{array}{l}11994 \\
10407\end{array}$ & $\begin{array}{r}1746 \\
261 \\
\end{array}$ & $\begin{array}{l}56293.6 \\
47458.0\end{array}$ & $\left\{\begin{array}{l}17643.2 \\
15746.8\end{array}\right.$ & $\begin{array}{l}2744.1 \\
1785.7\end{array}$ & $\begin{array}{l}2973.0 \\
3255.9\end{array}$ & $\begin{array}{l}32933.3 \\
26669.6\end{array}$ & \\
\hline $\begin{array}{l}\text { Difference ... } \\
\text { Correction .. }\end{array}$ & & & 1587 & 1485 & $\begin{array}{r}8835.6 \\
-411.4\end{array}$ & $\begin{array}{r}1896.4 \\
-136.5\end{array}$ & $\begin{array}{r}958.4 \\
-15.5\end{array}$ & $\begin{array}{r}-282.9 \\
-28.2\end{array}$ & $\begin{array}{r}6263.7 \\
-231.2\end{array}$ & \\
\hline Percentage.. & & & & & $\begin{array}{r}8424.2 \\
100.00\end{array}$ & $\begin{array}{r}1759.9 \\
20.89\end{array}$ & $\begin{array}{r}942.9 \\
11.19\end{array}$ & $\begin{array}{r}-311.1 \\
-3.69\end{array}$ & $\begin{array}{r}6032.5 \\
71.61\end{array}$ & 4.062 \\
\hline $\begin{array}{c}\text { Wheat gluten } \\
\text { II. ........ }\end{array}$ & $\frac{\mathrm{D}}{\mathrm{D}}$ & $\begin{array}{l}4 \\
1\end{array}$ & $\left|\begin{array}{r}11578 \\
9974\end{array}\right|$ & $\begin{array}{r}1407 \\
\quad 0\end{array}$ & $\begin{array}{l}56053.6 \\
46945.4\end{array}$ & 17322.9 & $\begin{array}{l}3468.0 \\
\mathbf{2 4 0 7 . 0}\end{array}$ & $\begin{array}{l}3171.9 \\
2957.0\end{array}$ & $\begin{array}{l}32090.8 \\
25863.1\end{array}$ & \\
\hline $\begin{array}{l}\text { Difference .. } \\
\text { Correction .. }\end{array}$ & & & 1604 & 1407 & $\begin{array}{r}9108.2 \\
-934.1 \\
\end{array}$ & $\begin{array}{r}1604,6 \\
-312.7\end{array}$ & $\begin{array}{r}1061.0 \\
-47.9\end{array}$ & $\begin{array}{r}214.9 \\
-58.8\end{array}$ & $\begin{array}{r}6227.7 \\
-514.7\end{array}$ & \\
\hline Percentage... & & & & & $\begin{array}{r}8174.1 \\
100.00\end{array}$ & $\begin{array}{r}1291.9 \\
15.80\end{array}$ & $\begin{array}{r}1013.1 \\
12.39\end{array}$ & $\begin{array}{r}156.1 \\
1.91\end{array}$ & $\begin{array}{r}5713.0 \\
69.90\end{array}$ & 1.061 \\
\hline
\end{tabular}

* Estimated from carbon content. 
TABLE VI. METABOLIZABLE FNERGY OF PEANUT OIL.

\begin{tabular}{|c|c|c|c|c|c|c|c|c|c|c|}
\hline \multirow[b]{2}{*}{ Feed Added. } & \multirow[b]{2}{*}{ 章 } & \multirow[b]{2}{*}{. } & \multicolumn{2}{|c|}{$\begin{array}{l}\text { Organic } \\
\text { Matter } \\
\text { Eaten. }\end{array}$} & \multicolumn{4}{|c|}{ Energy of } & \multicolumn{2}{|c|}{$\begin{array}{l}\text { Apparent } \\
\text { Metabolizable } \\
\text { Energy. }\end{array}$} \\
\hline & & & 是 & 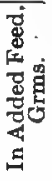 & $\begin{array}{l}\text { Food. } \\
\text { Cals. }\end{array}$ & $\begin{array}{l}\text { Feces. } \\
\text { Cals. }\end{array}$ & $\begin{array}{l}\text { Urine } \\
\text { (Cor- } \\
\text { rected), } \\
\text { Cals. }\end{array}$ & $\begin{array}{c}\text { Methane, } \\
\text { Cals. }\end{array}$ & $\begin{array}{l}\text { Total, } \\
\text { Cals. }\end{array}$ & $\begin{array}{c}\text { Per } \\
\text { Gram } \\
\text { Or- } \\
\text { ganic } \\
\text { Mat- } \\
\text { ter, } \\
\text { Cals. }\end{array}$ \\
\hline Peanut oil I.. & $\begin{array}{l}\mathrm{D} \\
\mathbf{D}\end{array}$ & $\begin{array}{l}3 \\
1\end{array}$ & $\begin{array}{r}10752 \\
9974\end{array}$ & $\begin{array}{r}709 \\
0\end{array}$ & $\begin{array}{l}54007.3 \\
46945.4\end{array}$ & $\begin{array}{r}1749.2 \\
15718.3\end{array}$ & $\begin{array}{l}2351.2 \\
2407.0\end{array}$ & $\begin{array}{l}2909.0 \\
2957.0\end{array}$ & $\begin{array}{l}31279,6 \\
25863,1\end{array}$ & \\
\hline $\begin{array}{l}\text { Difference. . . } \\
\text { Correction. . }\end{array}$ & & & 778 & 709 & $\begin{array}{r}7061.9 \\
-331.1\end{array}$ & $\begin{array}{r}1749.2 \\
-110.9\end{array}$ & $\begin{array}{l}-55.8 \\
-17.0\end{array}$ & $\begin{array}{l}-48.0 \\
-20.8\end{array}$ & $\begin{array}{r}5416.5 \\
-182.4\end{array}$ & \\
\hline Percentage .. & & & & & $\begin{array}{c}6730.8 \\
100.00\end{array}$ & $\begin{array}{r}1638.3 \\
24.34\end{array}$ & $\begin{array}{r}-72.8 \\
-1.08\end{array}$ & $\begin{array}{r}-68.8 \\
-1.02\end{array}$ & $\begin{array}{r}5234.1 \\
-77.76\end{array}$ & 7.382 \\
\hline Peanut oil II. \{ & $\frac{\mathbf{F}}{\mathbf{F}}$ & $\begin{array}{l}\mathbf{5} \\
\mathbf{3}\end{array}$ & $\begin{array}{l}7491 \\
6630\end{array}$ & $\begin{array}{r}798 \\
0\end{array}$ & $\begin{array}{l}39185.9 \\
31327.8\end{array}$ & $\begin{array}{r}14585.7 \\
9599.2\end{array}$ & $\begin{array}{l}1455.0 \\
1530.0\end{array}$ & $\begin{array}{l}1369.1 \\
2560.7\end{array}$ & $\left\{\begin{array}{l}21776.1 \\
17637.9\end{array}\right.$ & \\
\hline $\begin{array}{l}\text { Difference... } \\
\text { Correction... }\end{array}$ & & & 861 & 798 & $\begin{array}{r}7858.1 \\
-302.0\end{array}$ & $\begin{array}{r}4986.5 \\
-92.5\end{array}$ & $\begin{array}{l}-75.0 \\
-14.8\end{array}$ & $\begin{array}{c}-1191.6 \\
-24.7\end{array}$ & $\begin{array}{r}4138.2 \\
-170.0\end{array}$ & \\
\hline Percentage .. & & & & & $\begin{array}{c}7556.1 \\
100.00\end{array}$ & $\begin{array}{r}4894.0 \\
64.77\end{array}$ & $\begin{array}{l}-89.8 \\
-1.19\end{array}$ & $\begin{array}{c}1216.3 \\
-16.10\end{array}$ & $\begin{array}{r}3968.2 \\
52.52\end{array}$ & 4.973 \\
\hline Peanut oil II. \{ & $\mathrm{G}$ & $\begin{array}{l}5 \\
3\end{array}$ & $\begin{array}{l}7396 \\
6651\end{array}$ & $\begin{array}{r}798 \\
0\end{array}$ & $\begin{array}{l}38057 \cdot 3 \\
30750 \cdot 7\end{array}$ & $\begin{array}{r}12512.9 \\
9491.5\end{array}$ & $\begin{array}{l}1452.1 \\
1359.6\end{array}$ & $\begin{array}{l}2371.2 \\
2524.7\end{array}$ & $\begin{array}{l}21721.1 \\
17374.9\end{array}$ & \\
\hline $\begin{array}{l}\text { Difference... } \\
\text { Correction... }\end{array}$ & & & 745 & 798 & $\begin{array}{r}7306.6 \\
+249.5\end{array}$ & $\begin{array}{r}3021.4 \\
+77.0\end{array}$ & $\begin{array}{r}92.5 \\
+11.0\end{array}$ & $\begin{array}{r}-153.5 \\
+20.5\end{array}$ & $\begin{array}{r}4346.2 \\
+141.0\end{array}$ & \\
\hline Percentage & & & & & $\begin{array}{c}7556.1 \\
100.00\end{array}$ & $\begin{array}{r}3098.4 \\
41.00\end{array}$ & $\begin{array}{r}103.5 \\
1.37\end{array}$ & $\begin{array}{r}-133.0 \\
-1.76\end{array}$ & $\begin{array}{r}4487.2 \\
59.39\end{array}$ & 5.623 \\
\hline
\end{tabular}




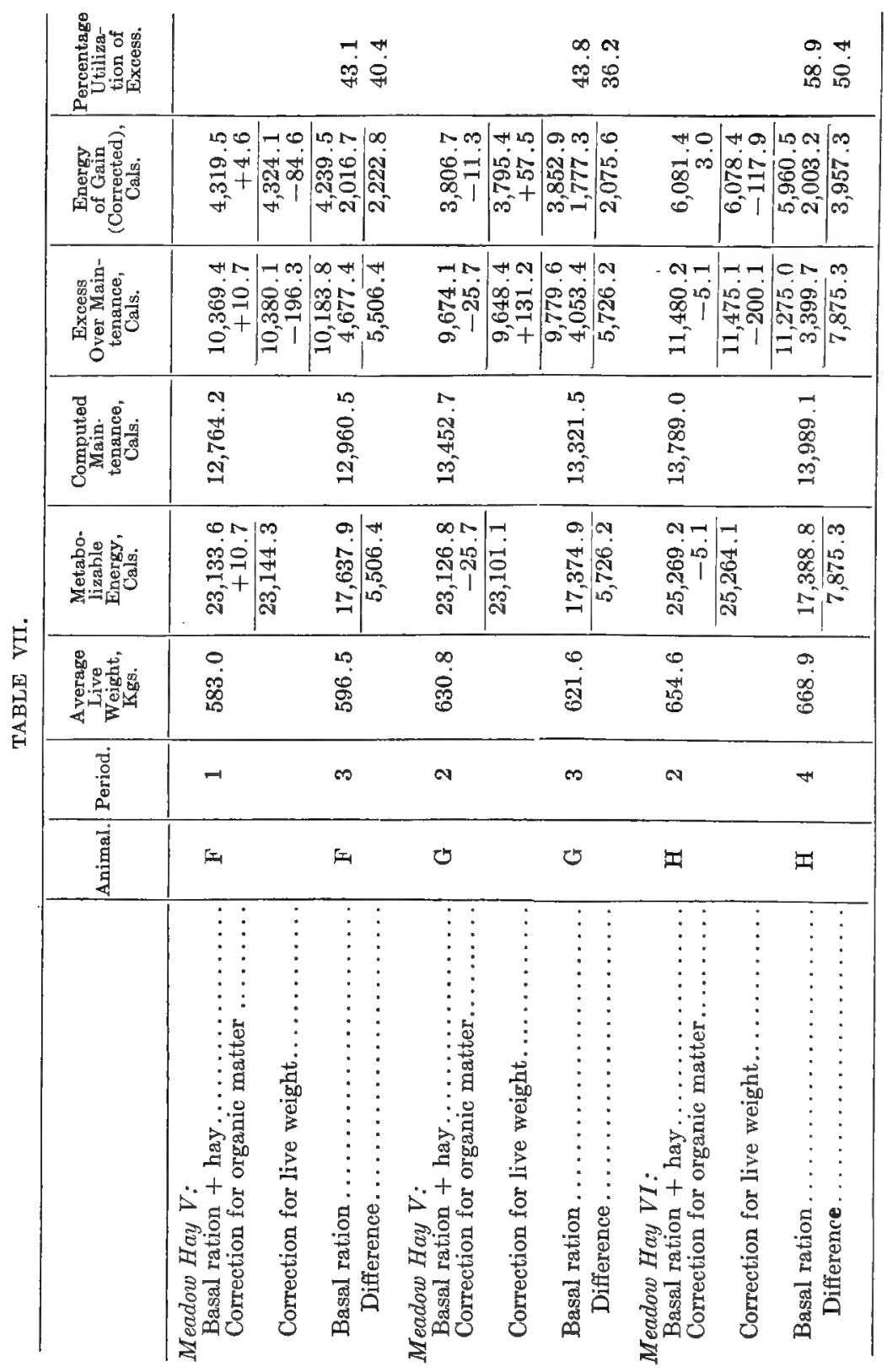




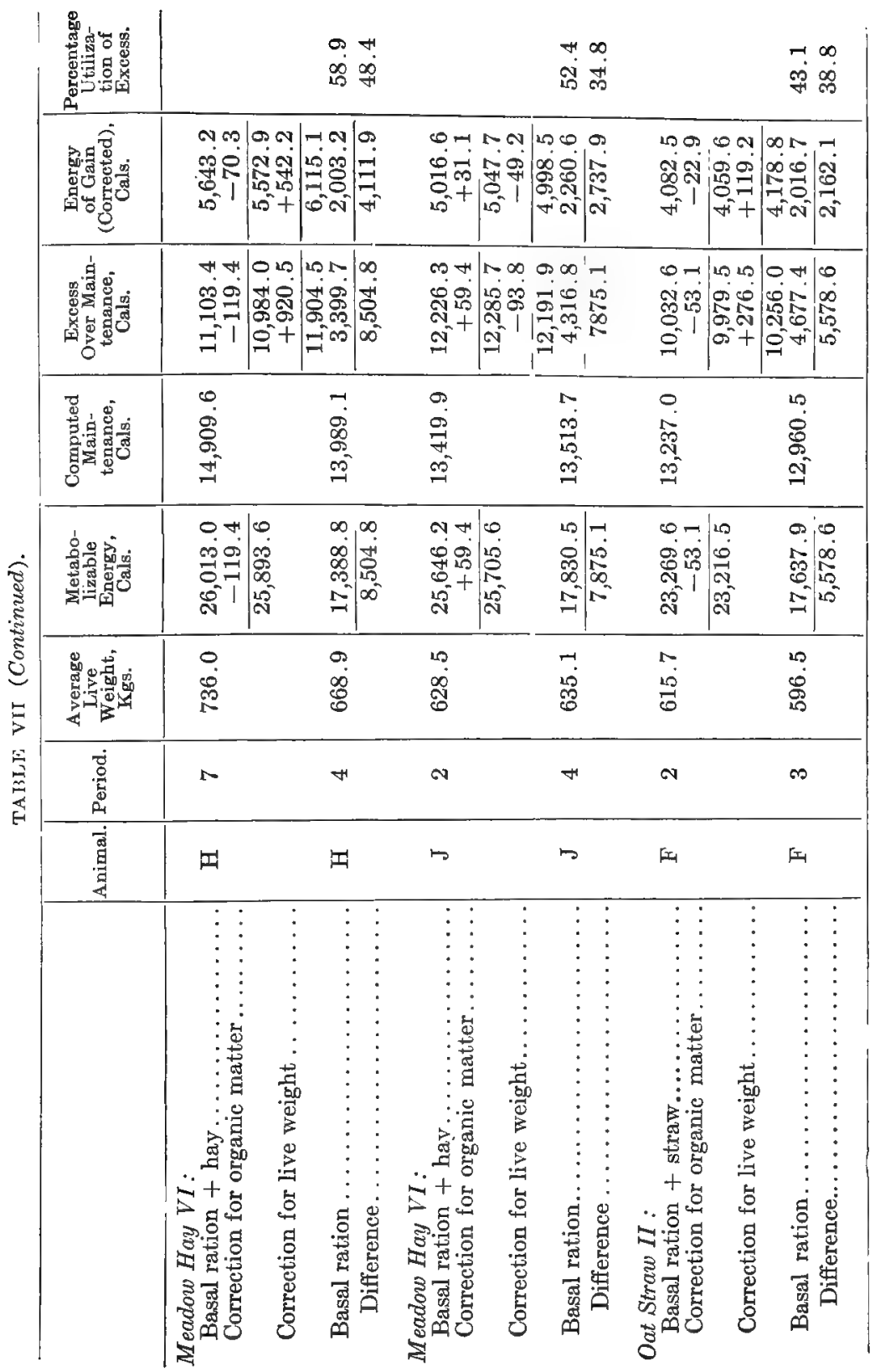




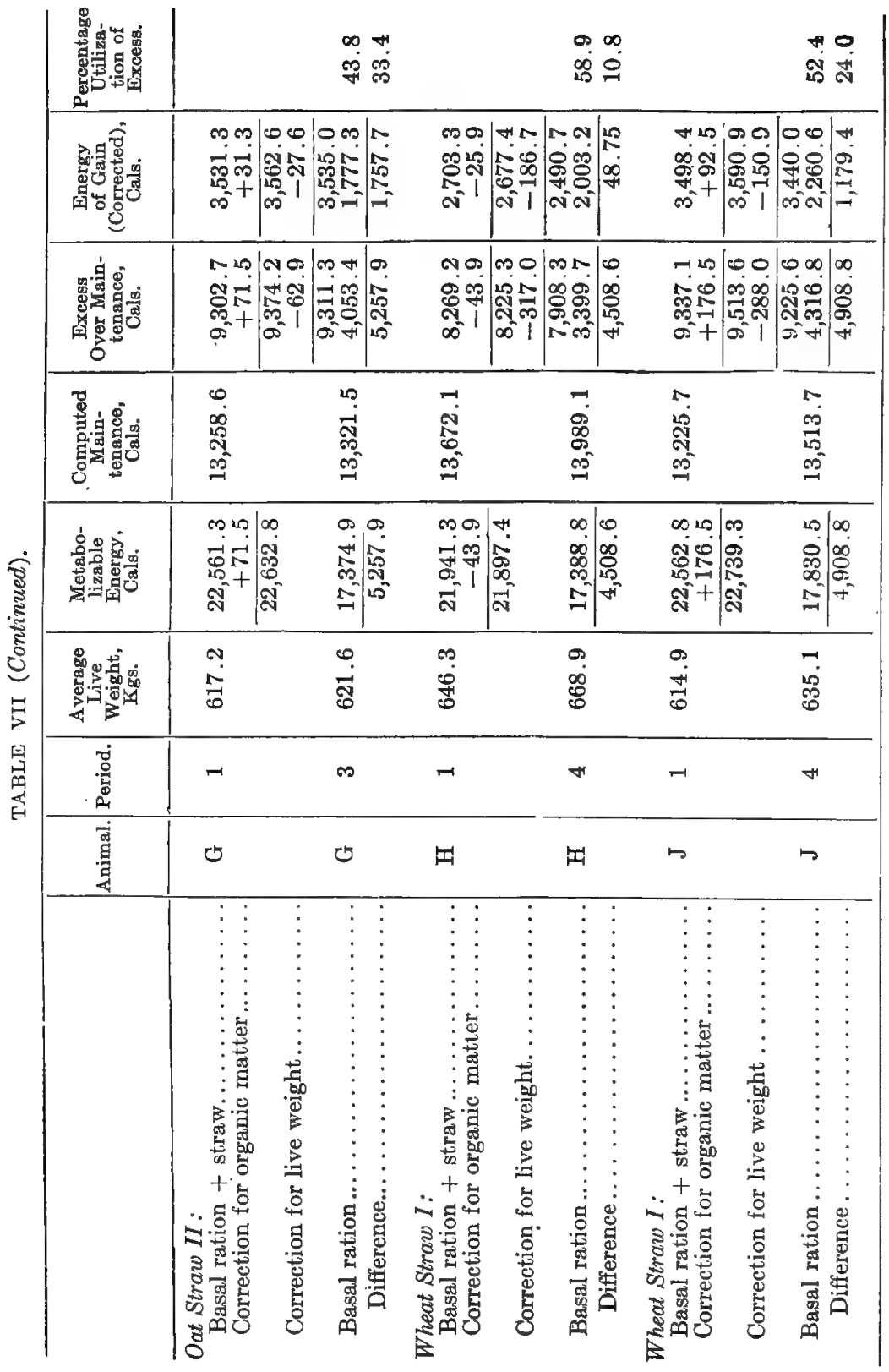




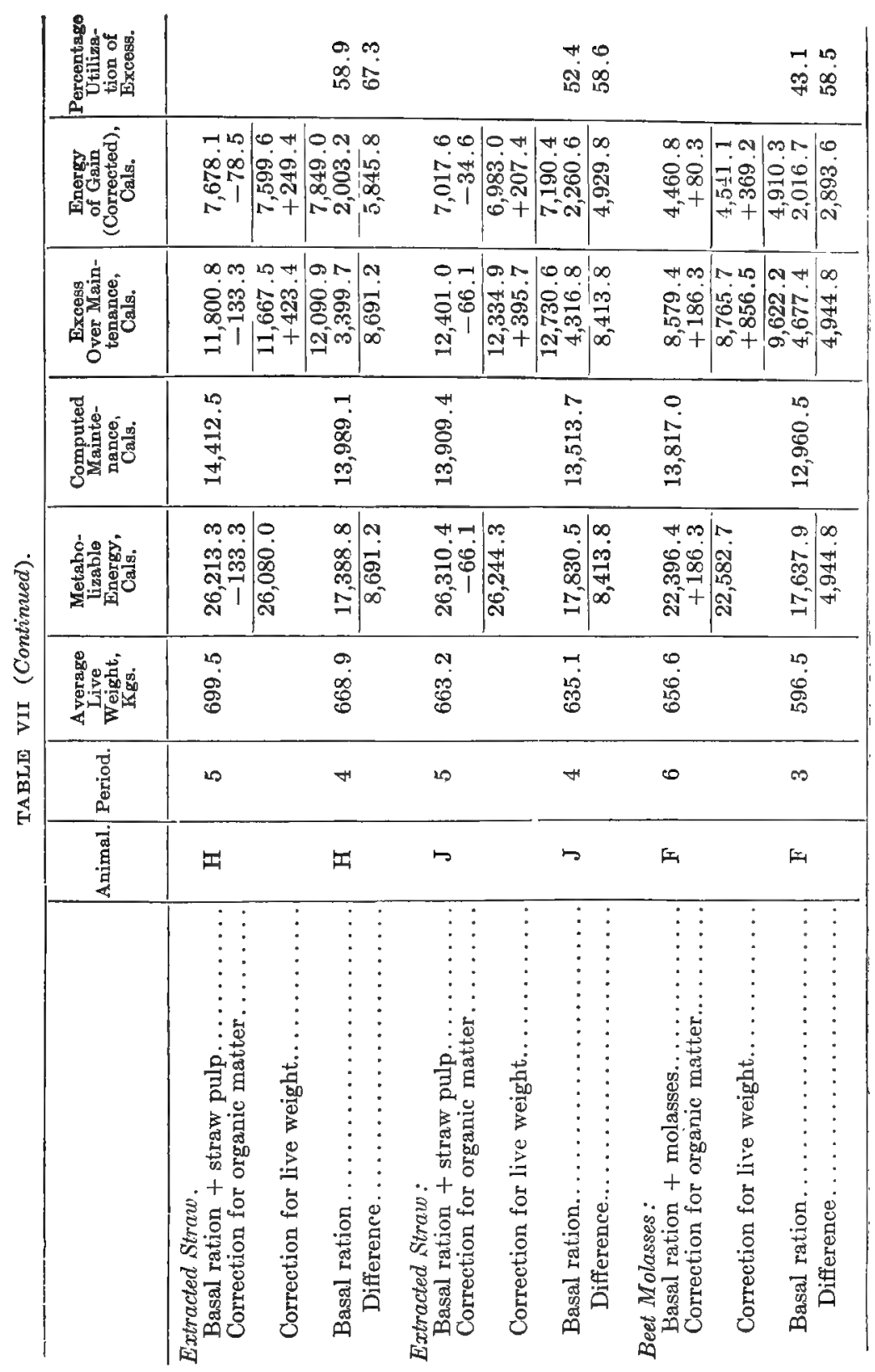




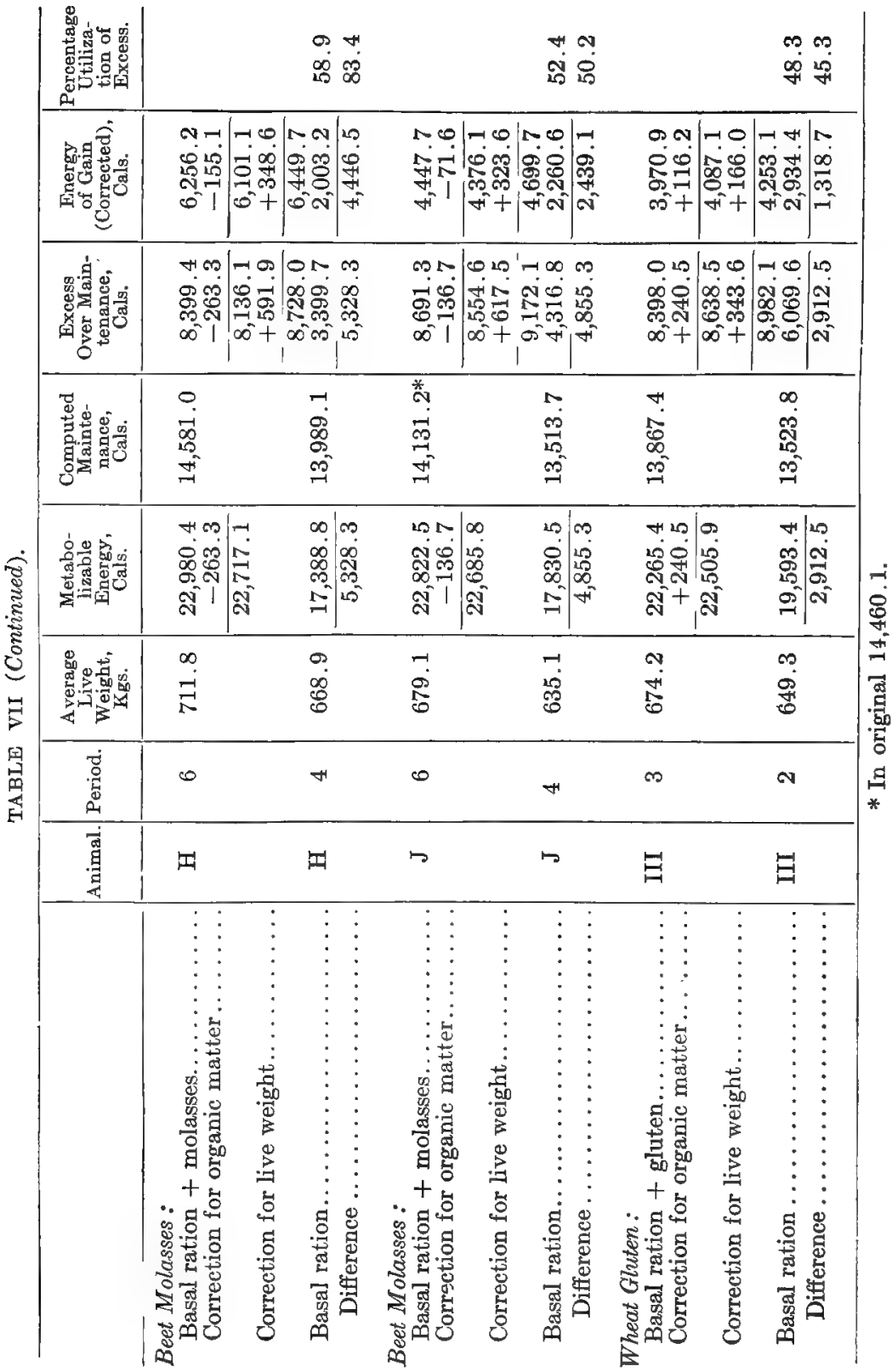




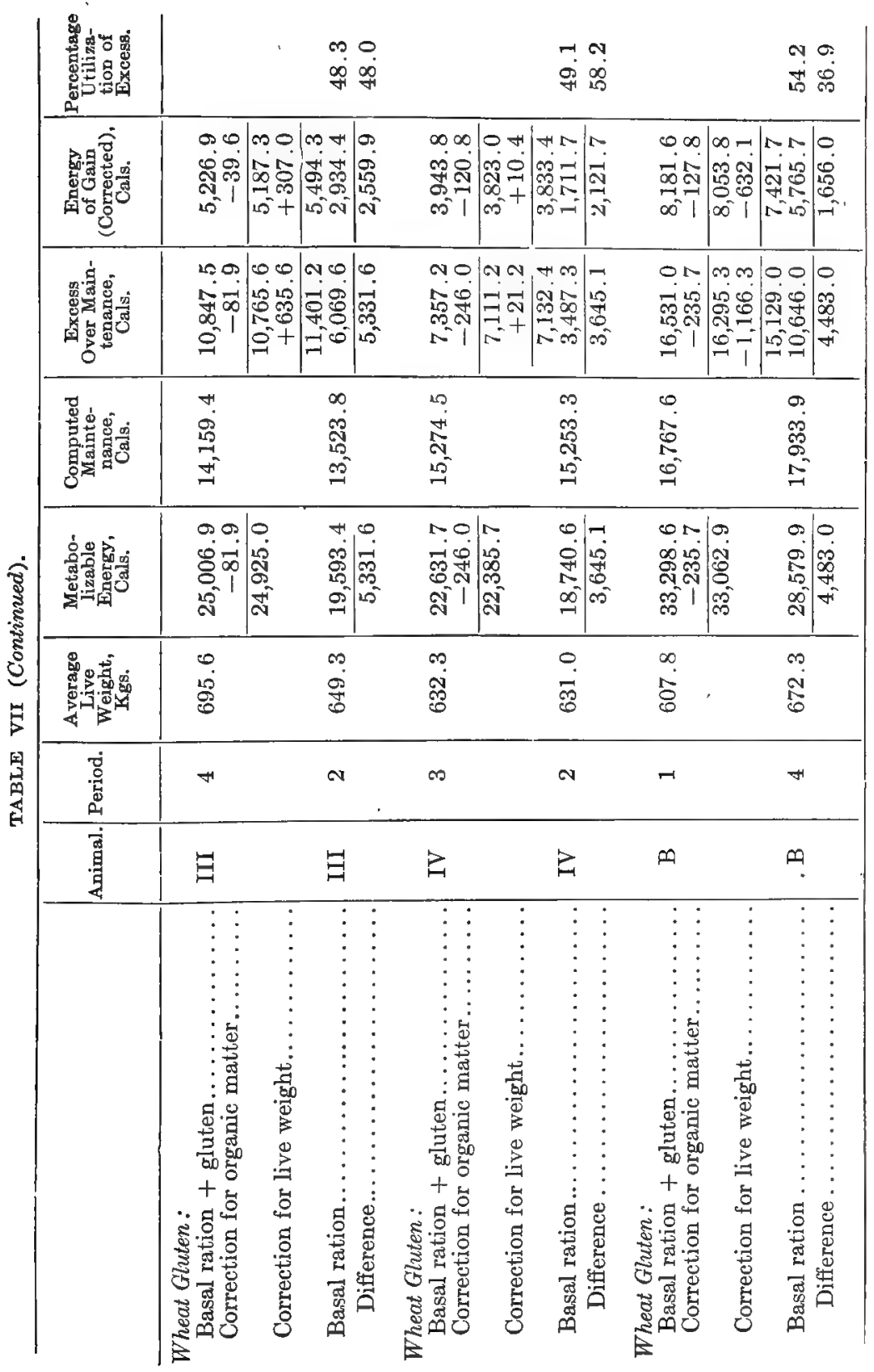




\begin{tabular}{|c|c|c|c|c|c|c|c|c|c|c|c|c|}
\hline 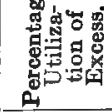 & & & & & & & تn & $\begin{array}{l}\text { N } \\
\text { ִָ }\end{array}$ & & & & $\stackrel{\infty}{\infty}$ \\
\hline 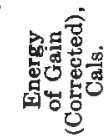 & $\begin{array}{l}\infty 0 \\
0 \\
0 \\
0 \\
\infty \\
\infty \\
\infty\end{array}$ & $\mid \begin{array}{ll}\infty & \infty \\
-1 & 5 \\
0 & 0 \\
0 & -1 \\
\infty & 1\end{array}$ & 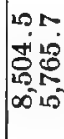 & $\mid \begin{array}{l}\infty \\
\infty \\
\infty \\
\infty \\
\sim\end{array}$ & 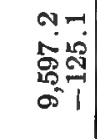 & 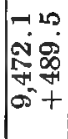 & $\left|\begin{array}{ll}0 & 0 \\
- & 0 \\
0 & 0 \\
5 & 0 \\
0 & n \\
0 & n\end{array}\right|$ & $\begin{array}{l}0 \\
\text { m } \\
8 \\
0 \\
\text { N }\end{array}$ & $\begin{array}{l}\infty \\
\infty \\
\infty \\
\infty \\
\infty \\
\infty \\
\infty\end{array}$ & $\mid \begin{array}{l}-\infty \\
-\infty \\
0 \\
25 \\
100 \\
n+\infty\end{array}$ & 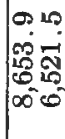 & אั \\
\hline 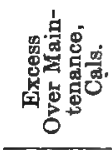 & 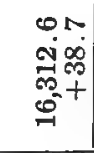 & 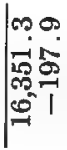 & $\begin{array}{l}40 \\
100 \\
130 \\
-10 \\
0 \\
0 \\
0\end{array}$ & 10 & 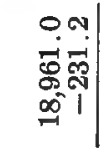 & 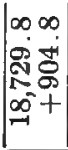 & $\mid$\begin{tabular}{cc}
0 & -1 \\
1 & 0 \\
0 & 0 \\
0 & 0 \\
0 & 0 \\
\hdashline & -1
\end{tabular} & 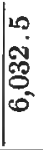 & 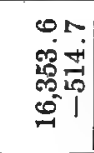 & 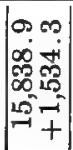 & 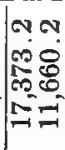 & $\begin{array}{l}0 \\
3 \\
10 \\
10\end{array}$ \\
\hline 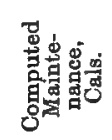 & $\begin{array}{l}0 \\
\dot{0} \\
\stackrel{0}{N} \\
\end{array}$ & & 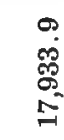 & & 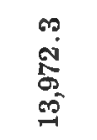 & & 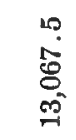 & & 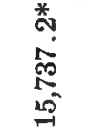 & & 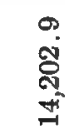 & \\
\hline 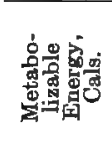 & 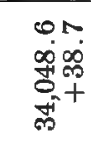 & m. & $\begin{array}{l}0 \\
\stackrel{R}{0} \\
20 \\
0 \\
0\end{array}$ & $\mid \begin{array}{l}8 \\
50 \\
05 \\
20 \\
20\end{array}$ & 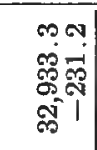 & 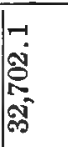 & \begin{tabular}{l}
0 \\
8 \\
8 \\
0 \\
\hdashline
\end{tabular} & مी & 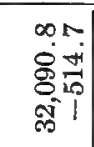 & 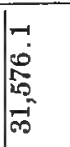 & $\begin{array}{l}\overrightarrow{1} \\
0 \\
0 \\
10\end{array}$ & $\mid \begin{array}{l}0 \\
0 \\
0 \\
10 \\
10\end{array}$ \\
\hline 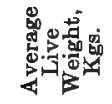 & श. & & กิ & & 5 & & ஜ̊ & & $\vec{\infty}$ & & ఝ & \\
\hline 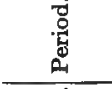 & $\infty$ & & H & & $\infty$ & & $\Rightarrow$ & & H & & -1 & \\
\hline 晋 & $\oplus$ & & 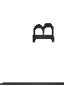 & & 0 & & 0 & & A & & $\dot{A}$ & \\
\hline & 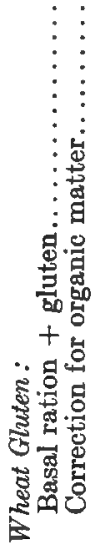 & 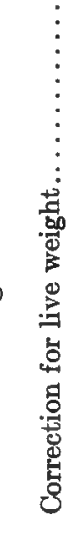 & 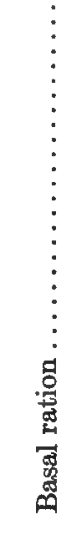 & 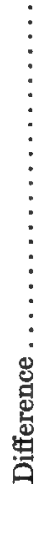 & 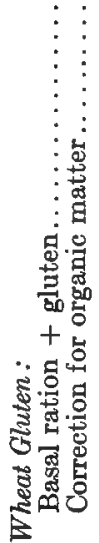 & 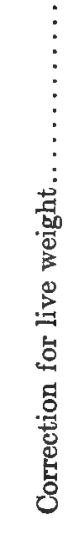 & 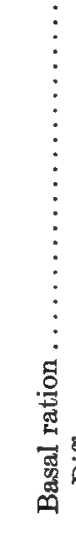 & 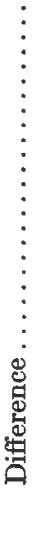 & 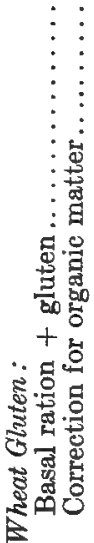 & 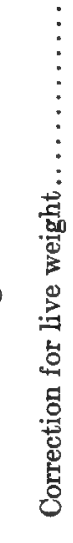 & 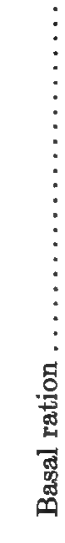 & 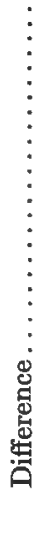 \\
\hline
\end{tabular}




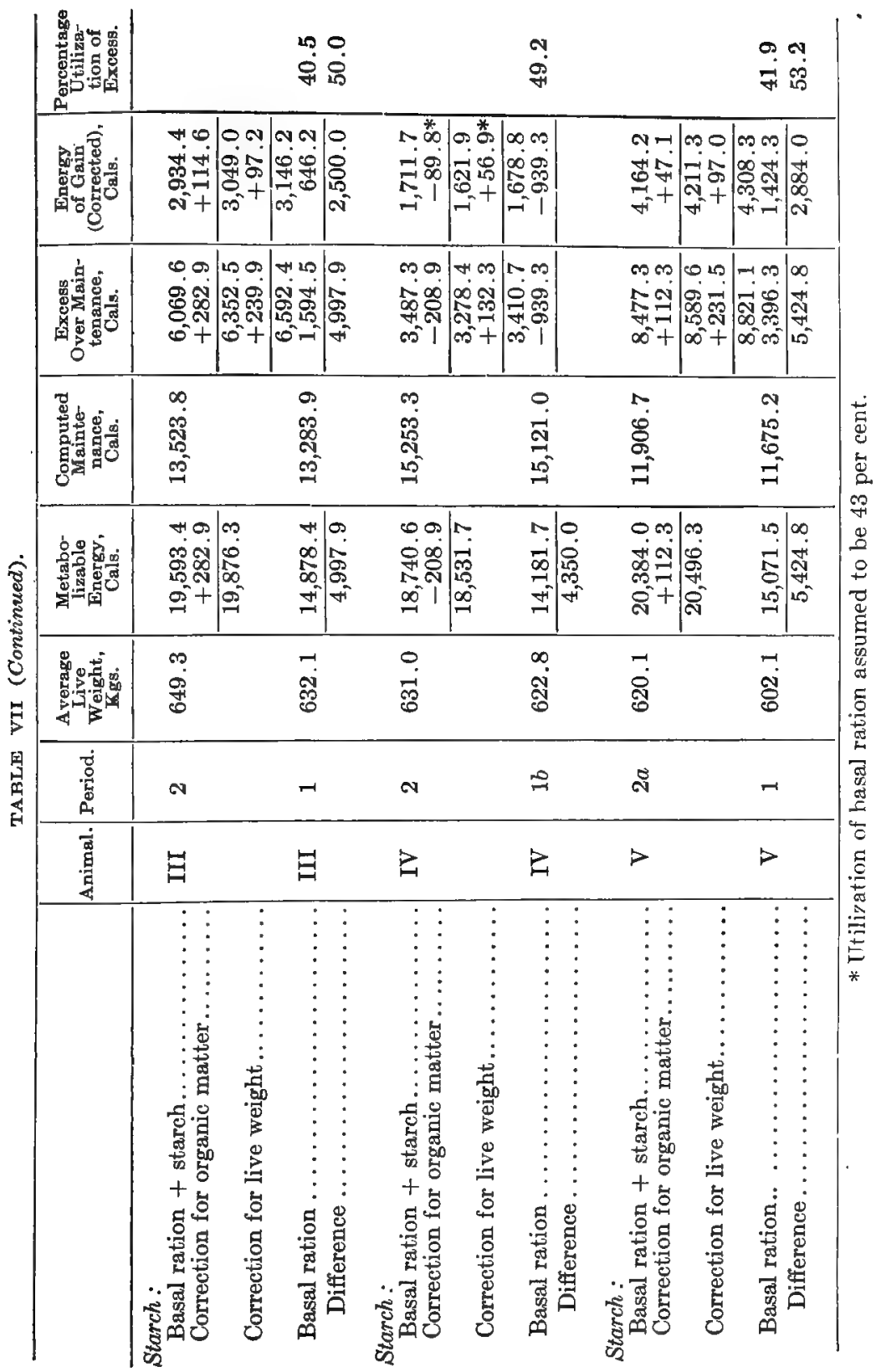




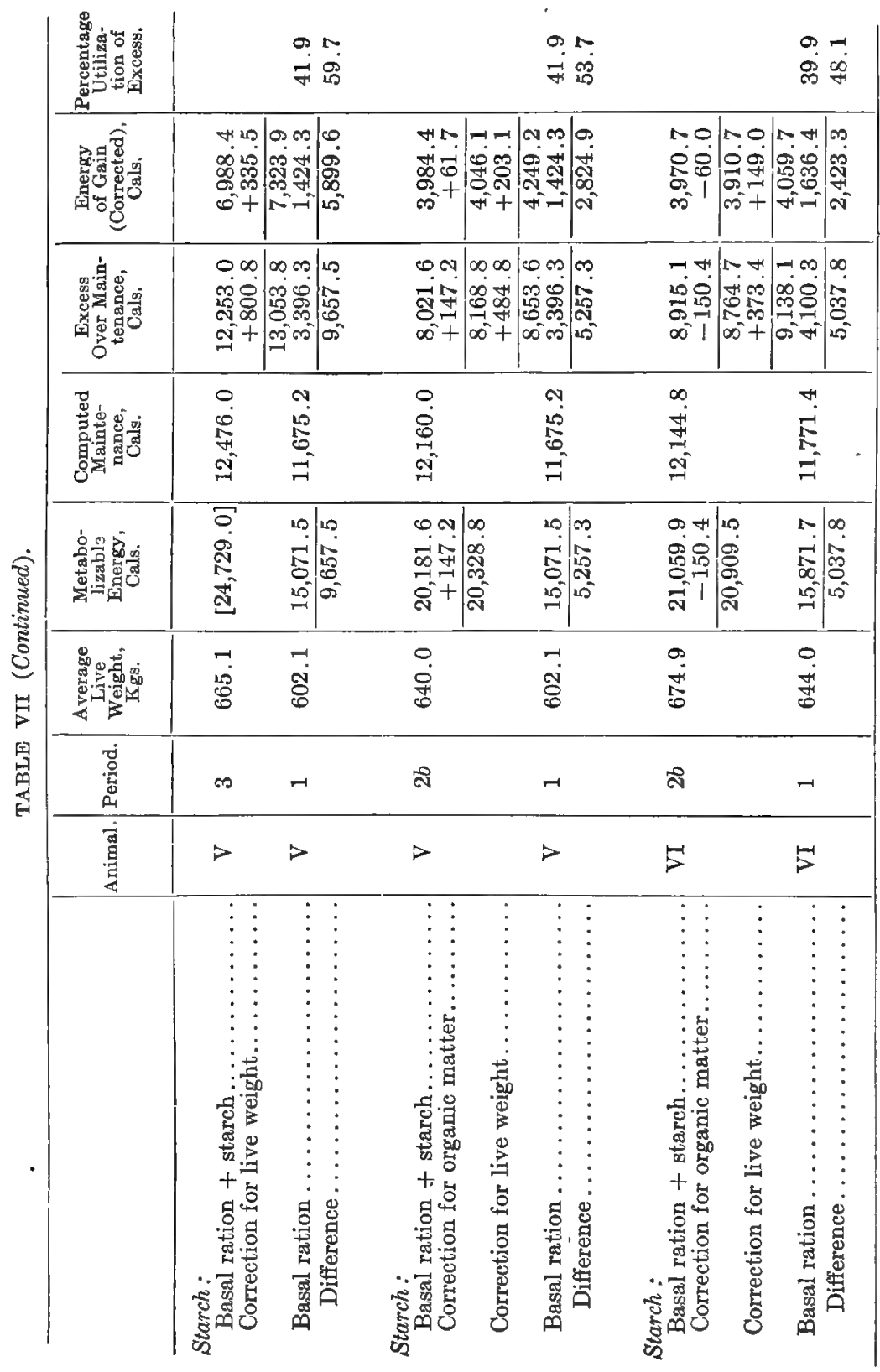




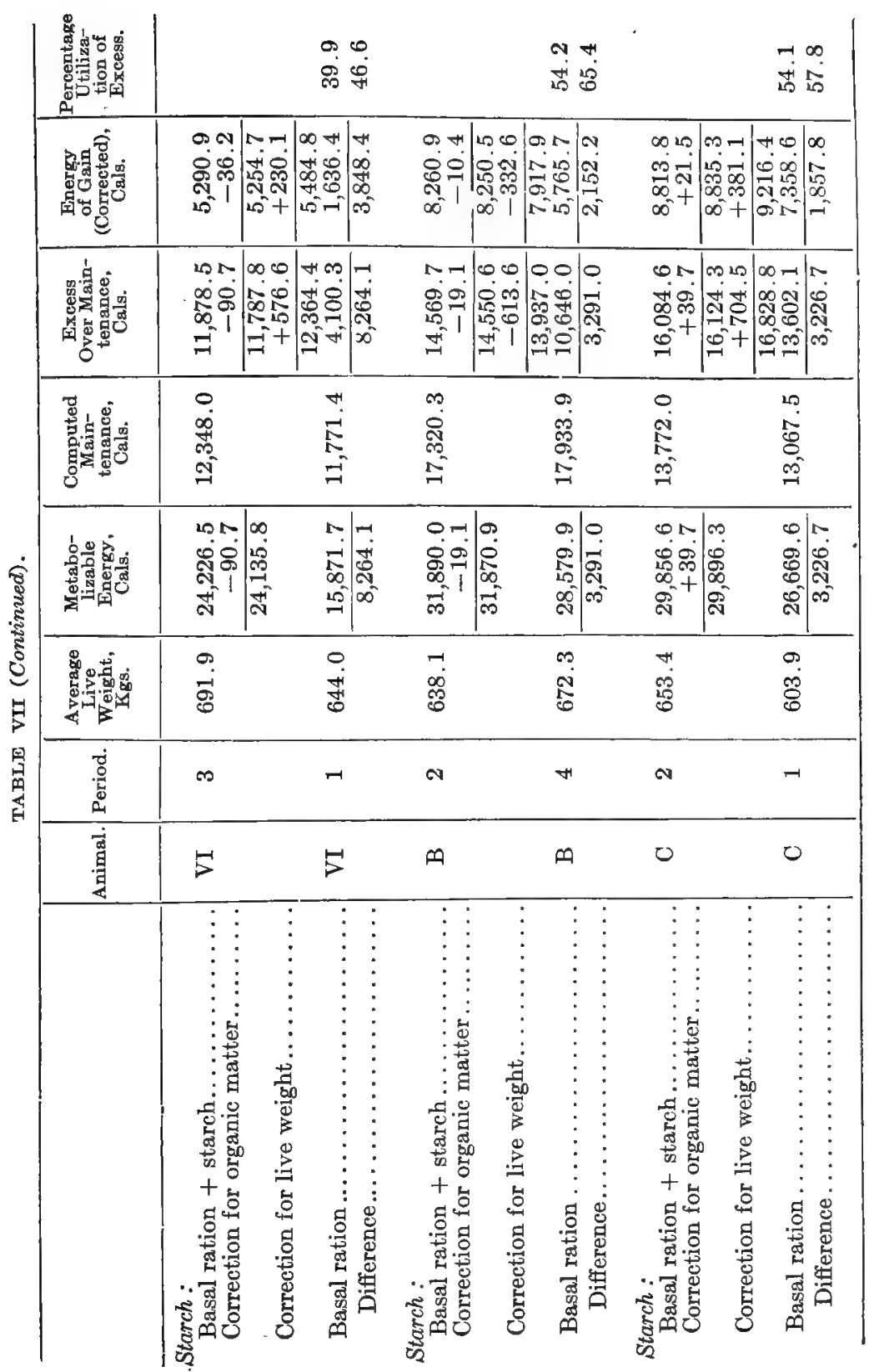




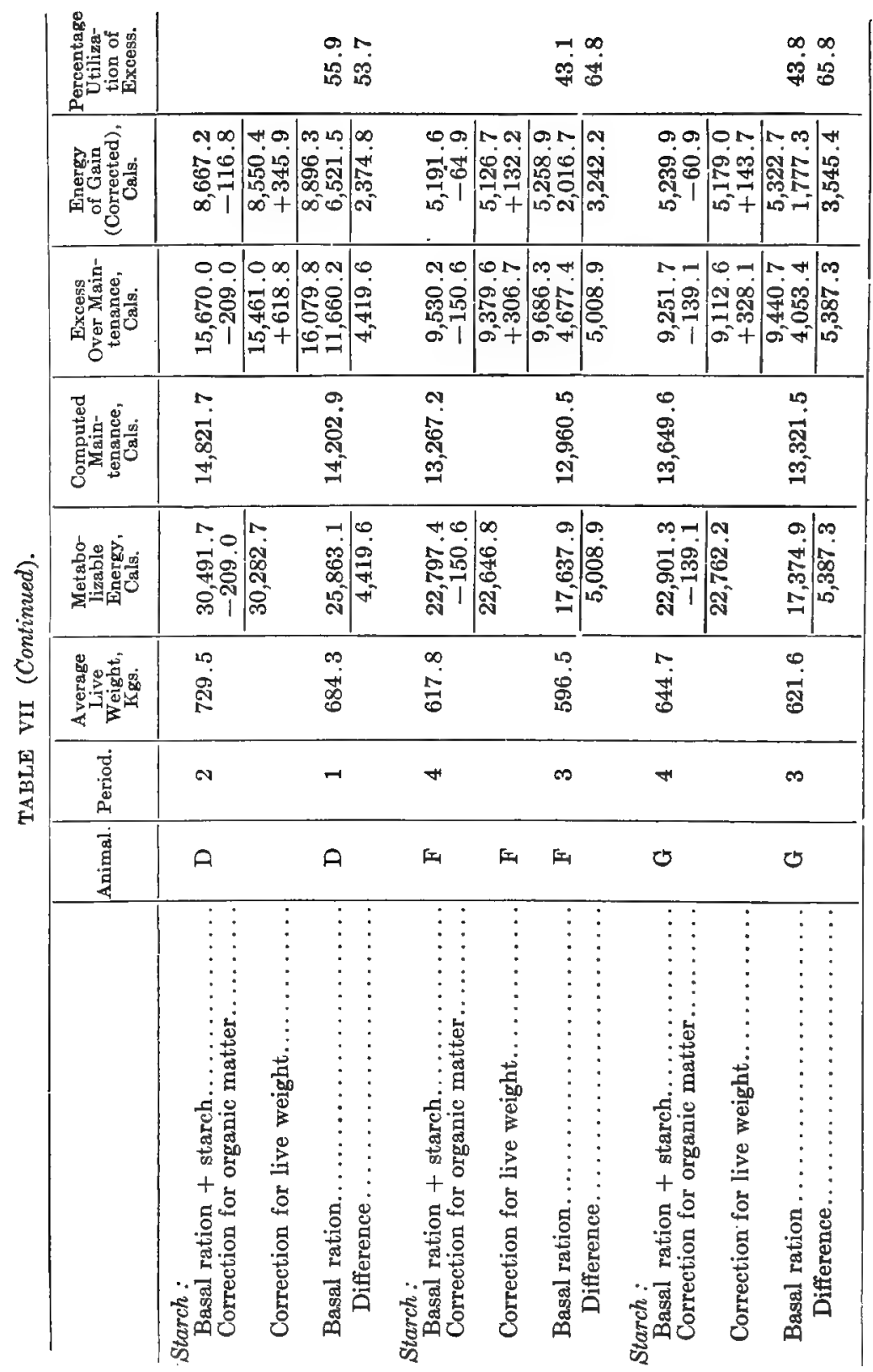




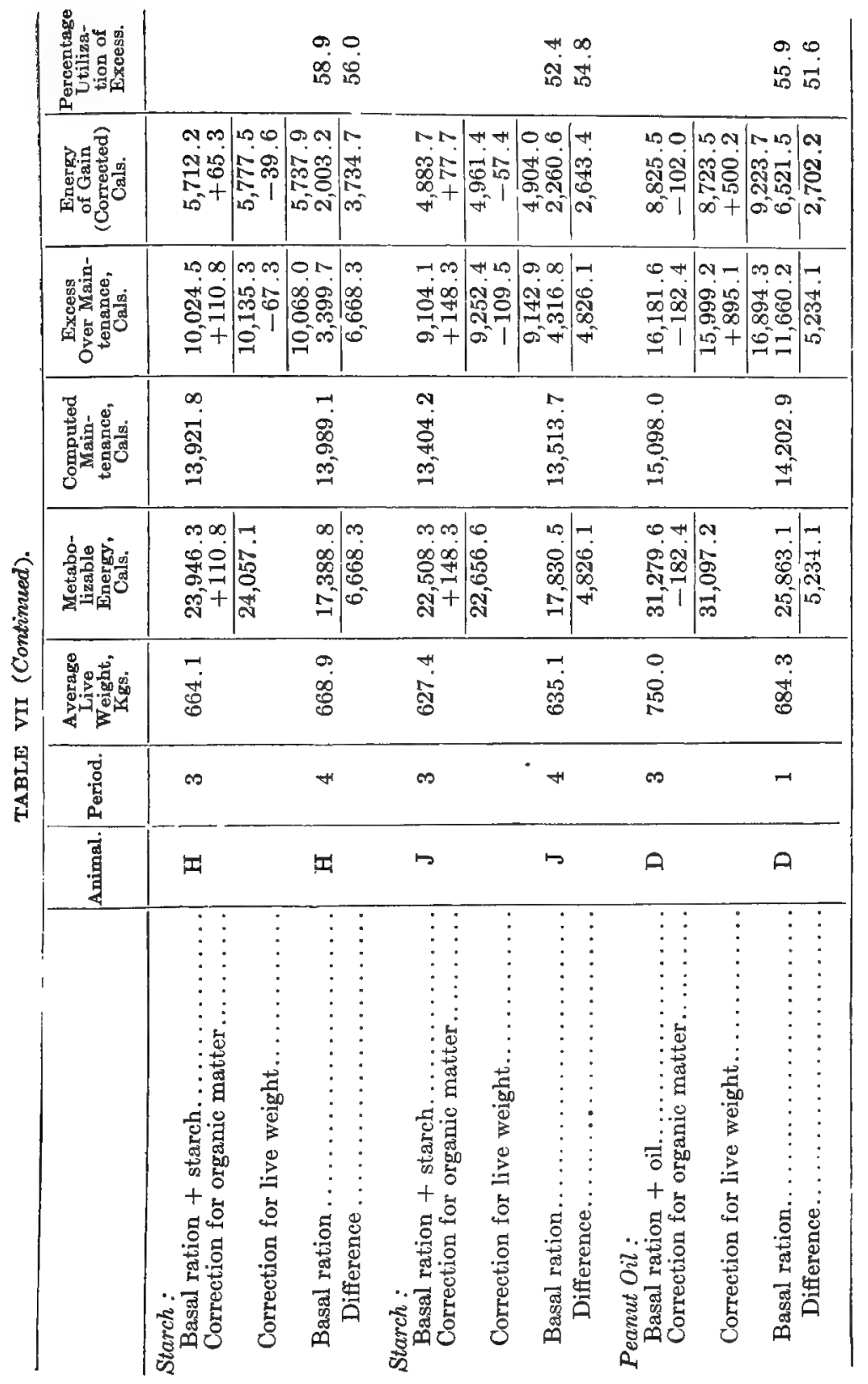




\begin{tabular}{|c|c|c|c|c|c|c|c|c|}
\hline 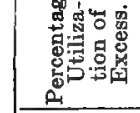 & & & $\overrightarrow{\mathfrak{g}}$ & $\overrightarrow{8}$ & & & $\stackrel{\infty}{\infty}$ & $\ddot{8}$ \\
\hline 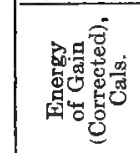 & 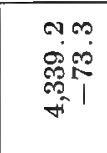 & 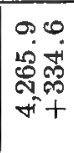 & 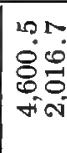 & $\mid \begin{array}{l}\infty \\
\infty \\
\infty \\
\infty \\
\infty \\
\omega\end{array}$ & 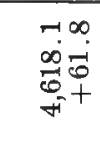 & 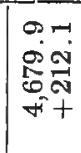 & 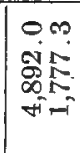 & 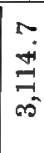 \\
\hline 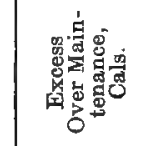 & 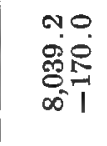 & 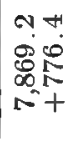 & 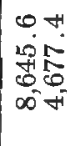 & 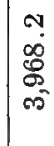 & $\begin{array}{l}m o \\
50 \\
5 \\
5 \\
5+4\end{array}$ & $\mid \begin{array}{ll}\infty & \infty \\
0 & +1 \\
0 & 0 \\
0 & 0+1 \\
\infty & +1\end{array}$ & 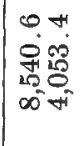 & 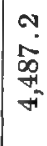 \\
\hline 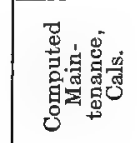 & $\begin{array}{l}\stackrel{0}{0} \\
0 \\
0 \\
0\end{array}$ & & $\begin{array}{l}28 \\
8 \\
8 \\
\text { जे }\end{array}$ & & $\begin{array}{l}\infty \\
\dot{2} \\
\infty \\
\infty \\
-1\end{array}$ & & & \\
\hline 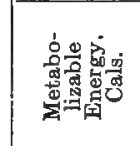 & $\begin{array}{l}-0 \\
00 \\
0.0 \\
\text { Ni } \\
\text { जi }\end{array}$ & $\begin{array}{l}-1 \\
\ddot{8} \\
\dot{0} \\
\vec{N}\end{array}$ & $\begin{array}{l}0 . \\
1 \\
0 \\
0 \\
0-1 \\
-1\end{array}$ & $\mid \begin{array}{l}\infty \\
\infty \\
\infty \\
8 \\
\infty \\
\infty\end{array}$ & 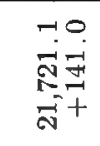 & 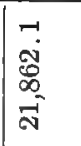 & 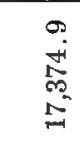 & 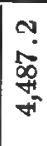 \\
\hline 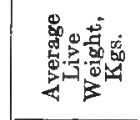 & $\ddot{8}$ & & $\begin{array}{l}10 \\
\stackrel{0}{\circ} \\
0\end{array}$ & & 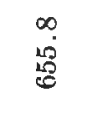 & & $\stackrel{\circ}{\overrightarrow{0}}$ & \\
\hline 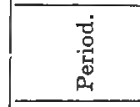 & مـ & & $\infty$ & & מתו & & $\infty$ & \\
\hline 氈 & 死 & & 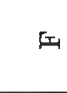 & & $\sigma$ & & ש & \\
\hline & 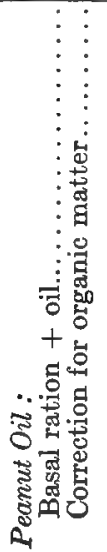 & 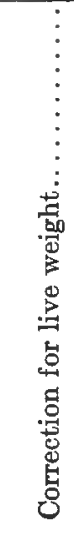 & 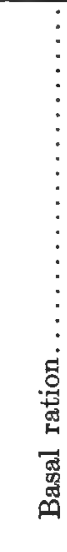 & 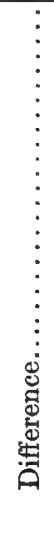 & 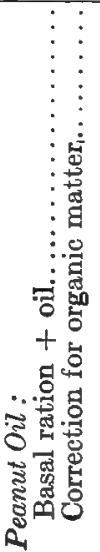 & 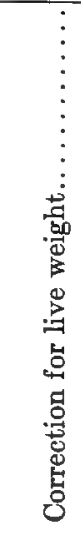 & 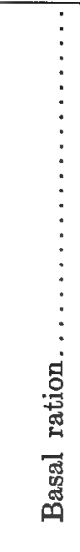 & 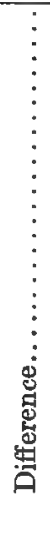 \\
\hline
\end{tabular}




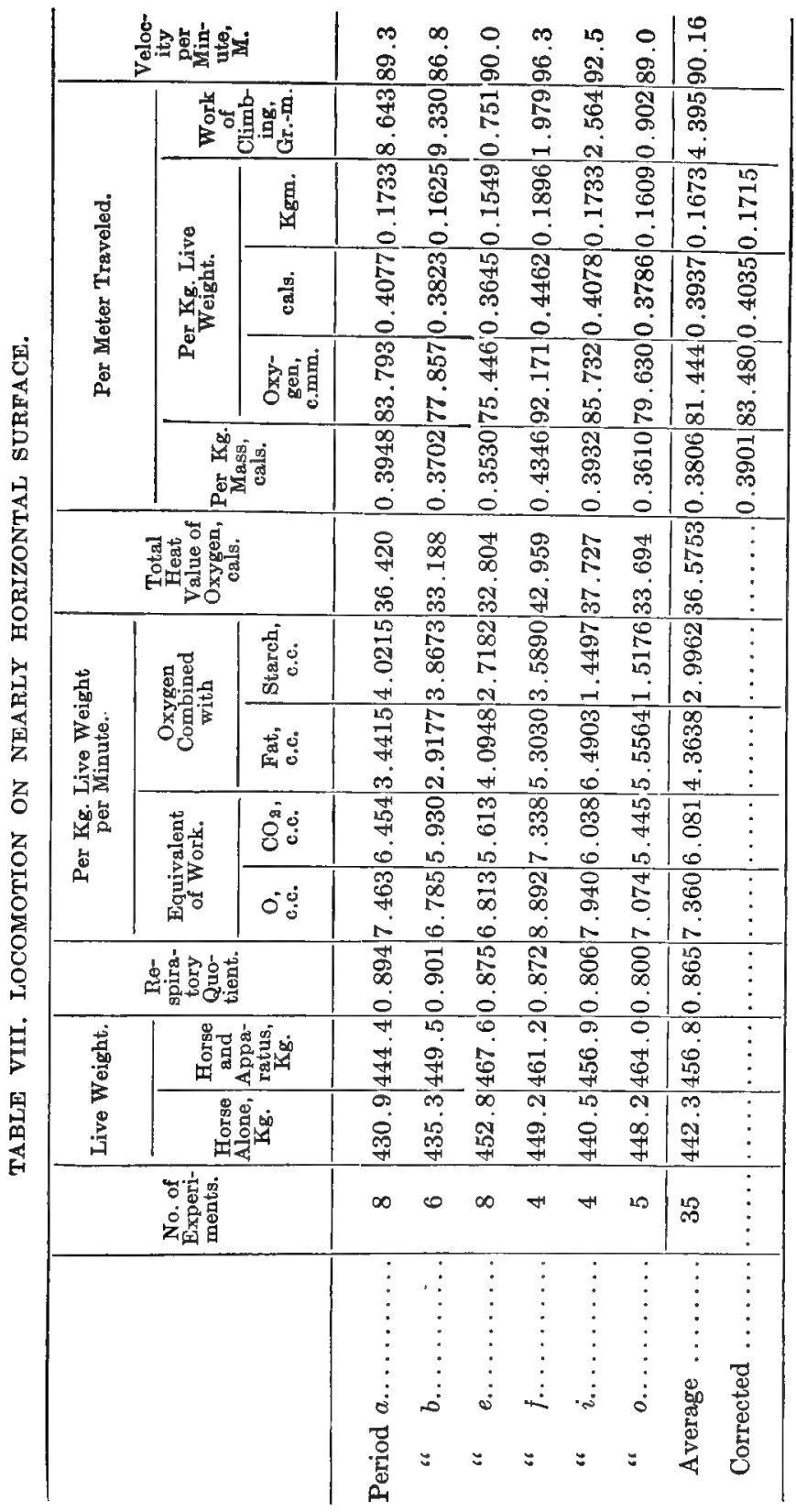




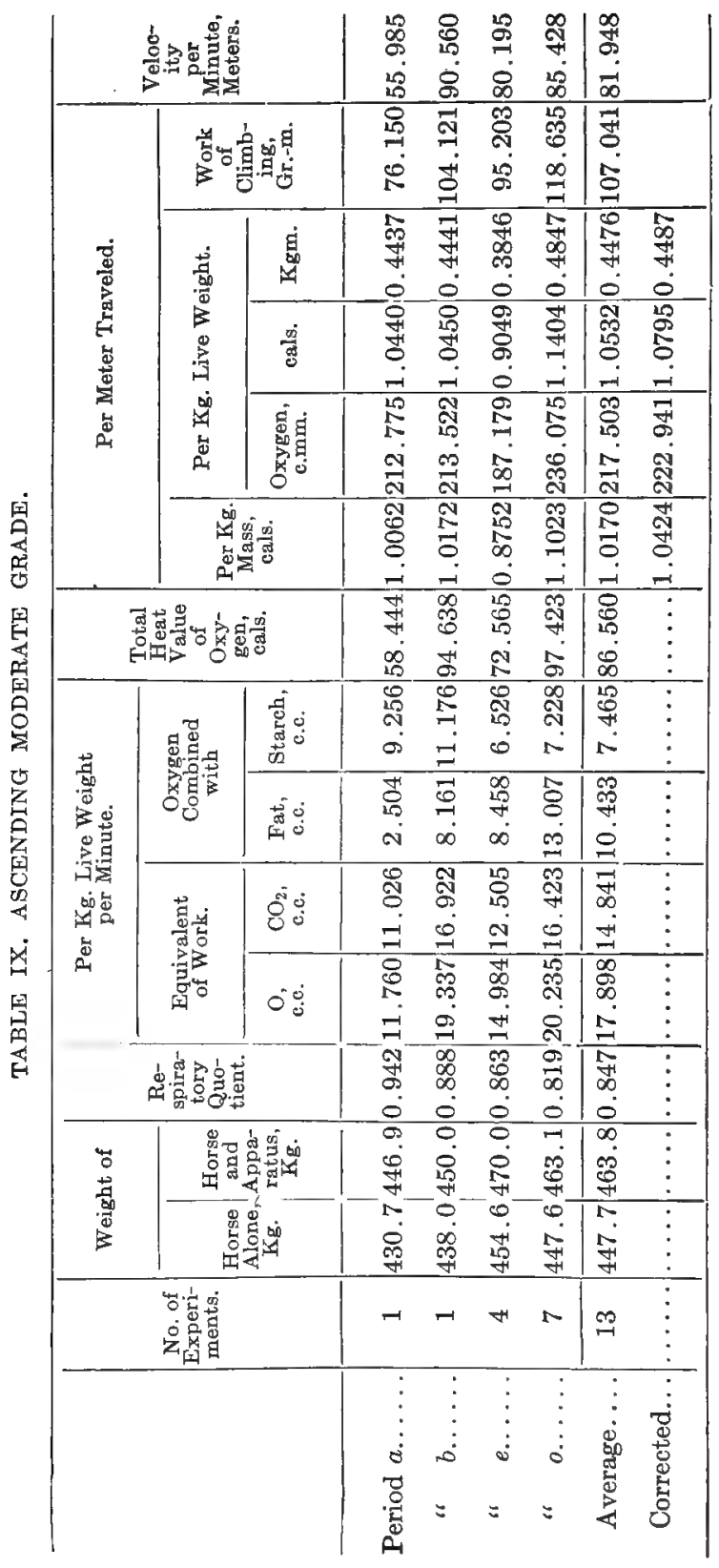




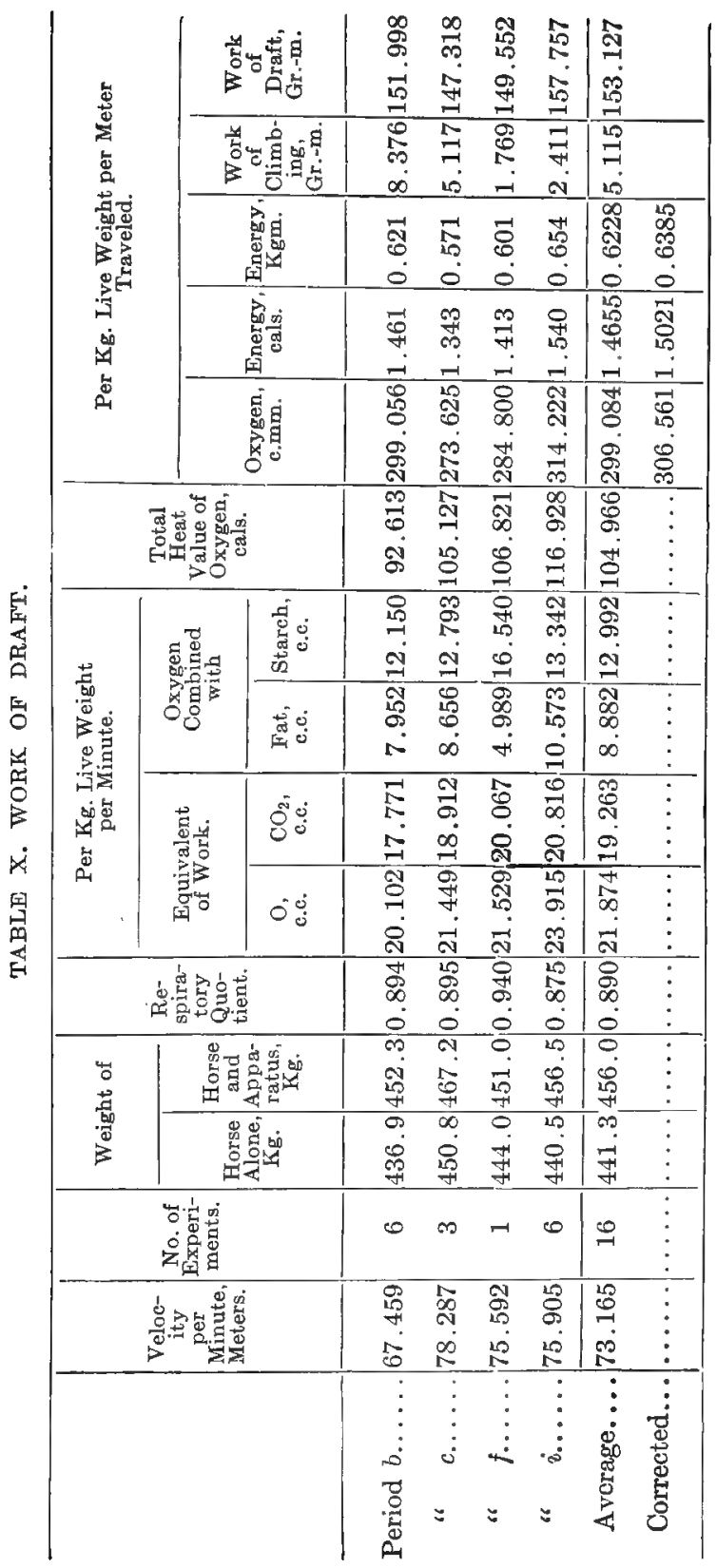


Digitized by Microsoft巴 


\section{INDEX.}

Acid, acetic, effect of, on proteid metabolism. . . . . . . . . . . . . 123

total metabolism.................... 160

replacement value of . ..................... 160

aspartic, formed from proteids..................... 39

oxidized in body ...................... 52

benzoic, formation of hippuric acid from............... 44

butyric, effect of, on total metabolism............... 158

replacement value of . ................... 158

glutaminic, formed from proteids.................. 39

hippuric, effect of proteids on formation of .............. 463

formation of, from benzoic acid .............. 44

glycocol. ................. 44

in urine. . . ......................... 44

loss of energy in.................. 313, 322

non-nitrogenous nutrients as source of ... $\ldots \ldots \ldots \ldots, 45$

origin of . . . . . . . . . . . . 44

pentose carbohydrates as source of ............ 46

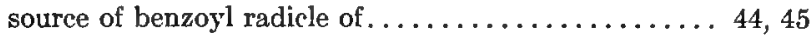

lactic, effect of, on proteid metabolism................ 123

total metabolism.................. 158

production of, in metabolism of carbohydrates........ 23

replacement value of........................ 158

Acids, organic, absent from excreta................... 27

effect of, on proteid metabolism.............. 123

total metabolism . ................ 157

net available energy of . . . . . . . . . . . . 425

metabolism of ......................... 26

oxidized in body...................... 27

produced by fermentation of carbohydrates....... 13, 26

replacement values of $\ldots \ldots \ldots \ldots \ldots \ldots \ldots \ldots \ldots \ldots . \ldots \ldots$

Acid, uric, in perspiration. . . . . . . . . . . . . . . . . 48

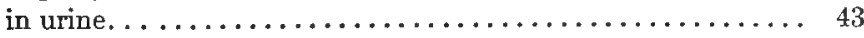

origin of. ........................... 43 
Activity, muscular, general features of . . . . . . . . . . . . 185

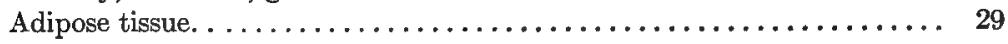

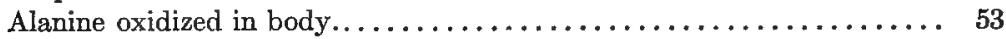

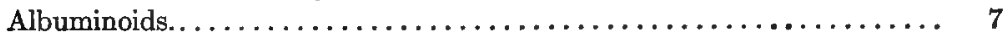

compound.......................... 7

composition of. ............... 62

derived. ........................... 7

modified. ........................... 7

simple.......................... 7

simple, composition of $\ldots \ldots \ldots \ldots \ldots \ldots \ldots \ldots \ldots \ldots \ldots \ldots \ldots \ldots \ldots \ldots \ldots \ldots \ldots \ldots \ldots \ldots \ldots \ldots \ldots \ldots \ldots \ldots \ldots \ldots$
Albumins. .

Albumoses formed from proteids................... 38, 39

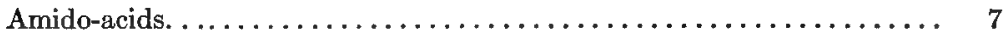

Amides...................................... 7

formed from proteids. $\ldots \ldots \ldots \ldots \ldots \ldots \ldots \ldots \ldots \ldots, 39,52$

influence of, on digestibility................. 54

of carbohydrates........... 57

crude fiber. . . ........ 57, 58

nitrogen-free extract. ........ 57

fermentation of carbohydrates........... 55

in digestive tract. .......... 54

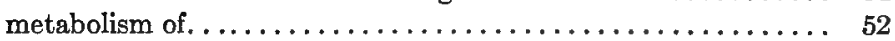

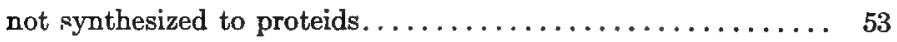

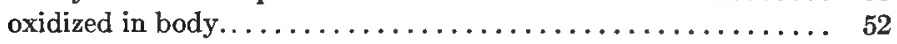

replacement of proteids by................... 53

urea formed from......................... 39

Ammonium acetate, influence of, on digestibility of carbohydrates. . . 57, 58

crude fiber. . . . . . 57

nitrogen-free extract. 57

carbonate as antecedent of urea .............. 43

lactate as antecedent of urea.................. 43

salts influence of, on digestibility of carbohydrates. . . . . . . 57

fermentation of carbohydrates...... 56

in digestive tract. . ..... 56

in perspiration. ...................... 48

Amount of food, critical. ...................... 408

influence of, on effects of muscular exertion. . . . . . . 197

net availability of energy ......... 430

utilization of energy............ 466

Anabolism. . . . . . . . . . . . . . . . . . . . . . . . 16, 17

absorption of energy in .................. 17

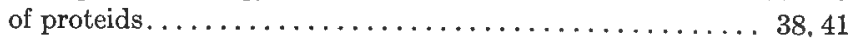

Animal, efficiency of. . ..................... $496,498,511$

as motor. . ...................... 498

conditions determining. .............. 511 
Animal, efficiency of, influence of fatigue on.................. PAGE

fatigue on................. 519

gait on. .................... 513

grade on. ................ 512

individuality on. ............ 517

kind of work on $\ldots \ldots \ldots \ldots \ldots \ldots \ldots 512$

load on.................... 515

size on.................. 515

species on. ................ 515

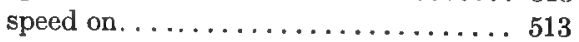

training on $\ldots \ldots \ldots \ldots \ldots \ldots \ldots \ldots 519$

method of determining............... 498

Antecedents of urea . . . . . . . . . . . . . . . . . . . . 42

Aromatic compounds in urine. .................... 46

Ascent, work of, in dog, consumption of oxygen in ........... 500

utilization of energy in. . . . . . . . . . . . 510

by $\operatorname{dog} \ldots \ldots \ldots \ldots \ldots \ldots \ldots 502$

horse............. 506

$\operatorname{man} . \ldots \ldots \ldots \ldots \ldots . \ldots 503$

effect of grade on. ........ 512

load on. . . 5 509, 510, 515

Ash ingredients, balance of . . . . . . . . . . . . 79

Asparagin, influence of, on digestibility................ 54

of carbohydrates.......... 57

crude fiber. ......... 57,58

nitrogen-free extract. ..... 57

fermentation. ................. 54

of carbohydrates. ........ 55

nutritive value of..................... 54

oxidized in body...................... 52

replacement of proteids by ................. 54

typical of non-proteids. . . . . . . . . . . . . . . 8

Aspartic acid formed from proteids. . . . . . . . . . . . . . 39

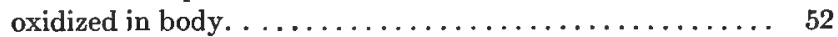

Assimilation, expenditure of energy in digestion and. ...... 372. 375

tissue building and .... 491

of fat, loss of energy in. . . . . . . . . . . . . . . 35

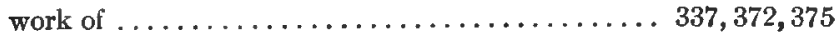

digestion and . . . . $\ldots \ldots \ldots \ldots \ldots, 80,93,406$

above critical point......... 407

of bone. . ............... 381

carbohydrates. ...... 379, 382, 384

fat. . . . . . . 378, 382. 384,385

mixed diet. . . . . . . . 382, 384

proteids. . . . . . . . 381, 382, 384

indirect utilization of heat from ... . 406 
Assimilation, work of digestion and in dog. ............... 378

horse. . . . . . . . . . . . 385

$\operatorname{man} . \ldots \ldots \ldots \ldots \ldots \ldots . \ldots . \ldots 382$

methods of determining........ 377

relation of, to surface.........4 408

Availability of energy, gross. . . . . . . . . . . . . . . 270, 395

for maintenance...... $396,406,410,413,427,497$

work. . . . . . . . . . . . . . . . 497

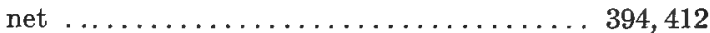

net, of energy, determination of. . . . . . . 413,427, 428

in carnivora .... 413,427, 428

herbivora..... 418, 427,428

distinction between utilization and....... 395

of carbohydrates. . . . . . . 417, $419,427,428$

crude fiber. . . . . . . . . . . . 422, 428

fat. . . . . . . . $416,419,427,428$

organic acids. . . . . . . . . . . . 423

pentoses. . . . . . . . . . . . 420,428

proteids. . . . . . . . . . 414, 427, 428

timothy hay.............. 424,428

influence of amount of food on.........4 430

character of food on......... 431

relation of maintenance ration to......... 432

Barley, utilization of energy of . . . . . . . . . . . . . . 483, 491

Beet molasses, metabolizable energy of . . . . . . . . 293, 297, 301

utilization of energy of. . . . . . . . . . . . . 4 483.490.491

Benzoic acid, formation of hippuric acid from . . . . . . . . . . . . 44

Benzoyl radicle of hippuric acid, source of $\ldots \ldots \ldots \ldots \ldots \ldots \ldots \ldots 44,45$

Blood, consumption of dextrose of. in muscles. . . . . . . . . . . 22

parotid gland. . . . . . . . . 22

dextrose of. . . . . . . . . . . . . . .

fat production from. ................ 23

percentage of ..................... 18

variations in................ 18

fate of dextrose of . . . . . . . . . . . . . . . . . . . 22

lævulose in. . . . . . . . . . . . . . . . . . . . 17

peptones absent from...................... 40

regulation of supply of dextrose to $\ldots \ldots \ldots \ldots \ldots \ldots \ldots \ldots 18,20$

Body, animal, components of. . . . . . . . . . . . . . . . . . .

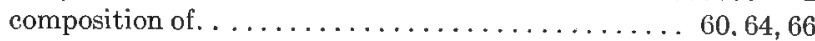

conservation of energy in . . . . . . . . . . . 228, 258

liberation of energy in..................... 1

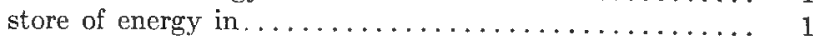

transformations of energy in $\ldots \ldots \ldots \ldots \ldots \ldots \ldots, 2$ 
Body, schematic. . ......................... 60, 66

Bone, work of digestion and assimilation of . . . . . . . . . . . . 381

Butyric acid, effect of, on total metabolism. ................ 158

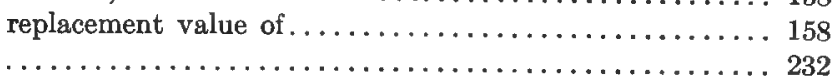

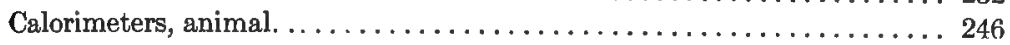

Carbohydrate radicle in proteids.................... 50

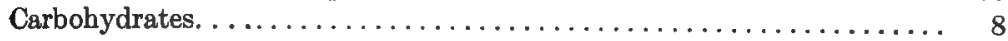

apparent digestibility of, influence of amides on...... 57

ammonium salts on. 57 .

asparagin on...... 57

as source of energy to body. ............

$\begin{array}{cr}\text { non-proteids on... } & 57 \\ \ldots \ldots \ldots \ldots \ldots & 91\end{array}$

consumption of, in muscular contraction......... 220

digestible, gross energy of. . . . . . . . . . . . 308

metabolizable energy of . . . . . . 324.327, 332

utilization of energy of . ......475, 477, 490, 491

disappearance of, in fasting. ................ 85

effects of, on minimum of proteids........... 135

proteid metabolism . . . . . . . . . 115

compared with fat.... 127

total metabolism. ................. 146

fermentation of. . . . . . . . . . . . . . . .

influence of amides on. . . . . . . . 55

ammonium salts on..... 56

asparagin on ........ 55

non-proteids on. $\ldots \ldots \ldots .55$

on nutritive value $\ldots \ldots \ldots \quad 13$

organic acids from. . . . . . . . . 13, 26

products of. ................ 13

formation of dextrose from, in liver . . . . . . 19, 20, 21

fat from. ................ 24, 30, 165

equation for.............. 24

respiratory quotient in........ 179

glycogen from. . . . . . . . . . . 20,21

milk fat from. . .............. 174

hexose. . . . . . . . . . . . . . . . .

formation of giycogen from. . . . . . . 20, 21

metaboiism of. See Metabolism ............

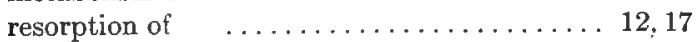

rate of................ 18

liver as reservoir of . . . . . . . . . 20

metabolism of See Melabolism

mutual replacement of fat and . .............. 151

net available energy of. $\ldots \ldots \ldots \ldots 4417,419.427,428$ 
Carbohydrates, of crude fiber $\ldots \ldots \ldots \ldots \ldots \ldots \ldots \ldots \ldots \ldots \ldots, 9$

food, replacement of proteids by............ 149

nitrogen-free extract . . . . . . . . . . . . 9

oxidized, computation of, from respiratory quotient $\quad 76$

pentose................... 8,9

assimilability of................. 25

as source of hippuric acid............ 46

determination of $\ldots \ldots \ldots \ldots \ldots \ldots \ldots \ldots \ldots, 9$

digestibility of . . ................ 24

effects of, on proteid metabolism .......... 124

total metabolism. . . . . . . . 156

formation of fat from. . . . . . . . . . . . . 183

glycogen from...........25, 26

metabolism of. See Metabolism.............

of crude fiber...................... 9

nitrogen-free extract............. 9

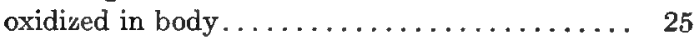

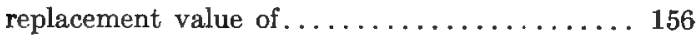

replacement value of ................ 152

resorption of........................ 12

respiratory quotient of............... 74

subdivisions of.................. \&, 9

substitution of, for body fat. ............ 146

utilization of energy of . .......461,462,473, 490, 491

value of, for maintenance.............400,402

work of digestion and assimilation of.....379, 382, 384

Carbon balance computation of fat from . . . . . . . . . . . 77

heat production from nitrogen and.... 255

dioxide, determination of, in respiration . . . . . . . . . 69,73

produced by fermentation of carbohydrates......... 13

production of, in fasting................ 84

metabolism................. 14, 15

of carbohydrates.......23, 27 ,

fat............ 36

proteids........... 42

equilibrium, amount of proteids required to produce........ 105

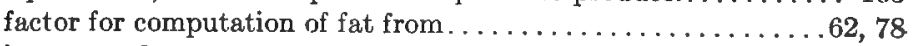

income and outgo of ......................... 69

determination of . . . . . . . . . .

of excreta, determination of . . . . . . . . . . . . . . . 69

metabolism, effect of muscular exertion on. . . . . . . . . . . . 209

Carnivora, determination of net availability of energy in. . . . .413,427,428

hippuric acid in urine of................... 44

metabolizable energy of food of ................ 272

utilization of energy by . . . . . . . . . . . . 
Cattle, excretion of methane by . . ............................

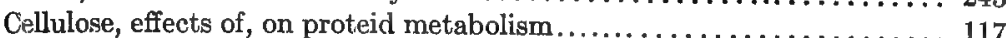

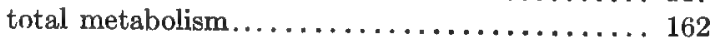

fermentation of.......................... 13

formation of fat from $\ldots \ldots \ldots \ldots \ldots \ldots \ldots \ldots \ldots \ldots \ldots \ldots \ldots$

of crude fiber.......................... 9

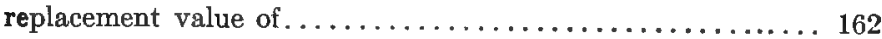

Changes, chemical, during muscular contraction. .............186, 189

thermal, during muscular contraction.............. 189

Chymosin ...................................... 40

Circulation, effects of muscular exertion on.................... 191

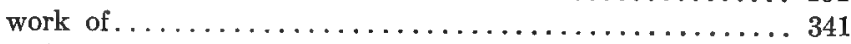

Cleavage digestive of proteids. . . . . . . . . . . . . . . . 38

purpose of ................. 38

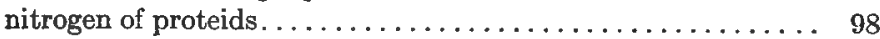

cause of . . . . . . . . . .

effects of non-nitrogenous nutrients on.... 131 independent of total metabolism........ 99

proteids in digestion. ...................... 38

purpose of .................. 38

products rebuilding of proteids from.............. 40

Cleavages, influence of, on computation of heat production........ 253

by an enzym........ 40

Coarse fodders, expenditure of energy of, in digestion, assimilation, and tissue building. . . . . . . . . . . . . . 491 metabolizable energy of. . .285, 286, $287,290,297,298,300,301$ digestible protein of.......320, 332 carbohydrates of . . 327,332

non-nitrogenous matter of urine derived from....... 28 relative value of grain and, for maintenance....433, 533, 537 work production......534, 537 utilization of energy of..............484, 490,491 Coefficient of utilization of energy $\ldots \ldots \ldots \ldots \ldots \ldots \ldots \ldots \ldots \ldots 44,498$

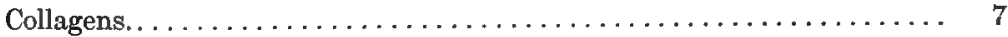

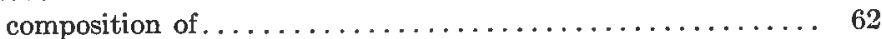

Combustion, heats of . . . . . . . . . . . . . . . . . . . 229

Concentrated feeding-stuffs, metabolizable energy of........289, 297, 299 digestible protein of .........315, 332 utilization of energy of .........472,490,491

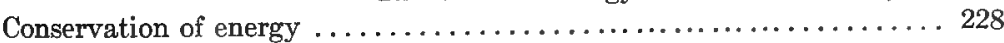

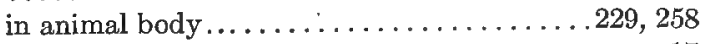

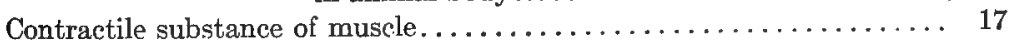

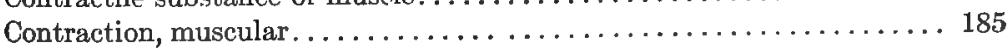


Contraction, muscular, chemical changes during $\ldots \ldots .186,189$

consumption of carbohydrates in............220

dextrose in..............220, 221

isometric..................... 495

isotonic..................... 495

oxidations in, incomplete............ 186

oxygen not essential to.............. 188

respiratory quotient of muscle in......... 187

thermal changes during. ............... 189

transformation of energy in............ 495

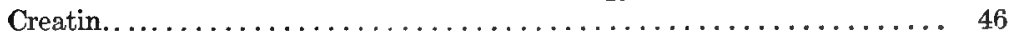

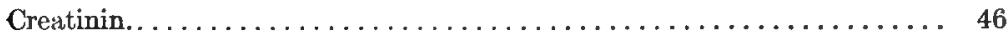

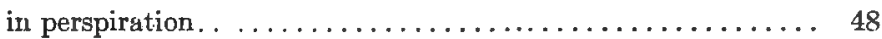

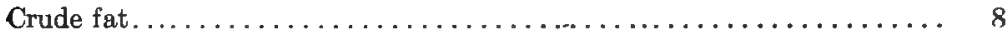

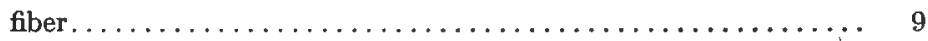

apparent digestibility of..................... 12

influence of amides on...........57, 58

ammonium acetate on . $\quad \mathbf{5 7}$

asparagin on . . . . . 57, 58

non-proteids on ..... 57, 58

carbohydrates of $\ldots \ldots \ldots \ldots \ldots \ldots \ldots \ldots \ldots \ldots \ldots . \ldots$

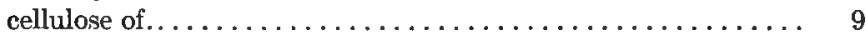

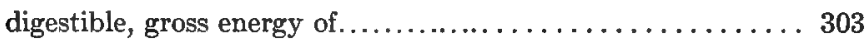

metabolizable energy of................329, 332

digestive work for. . . . . . . . . . . . . . . . . 389

effect of, on total metabolism.................. 161

expenditure of energy of, in digestion, assimilation, and tissue

building. . . . . . . . . . . . . . . . . . . . . . . 494

formation of fat from . . . . . . . . . . . . . . . 181

furfuroids of $\ldots \ldots \ldots \ldots \ldots \ldots \ldots \ldots \ldots \ldots \ldots \ldots, 9$

ligneous material of $\ldots \ldots \ldots \ldots \ldots \ldots \ldots \ldots \ldots \ldots \ldots$

modified in digestive tract. .................. 12

net available energy of . . . . . . . . . . . . . . . . 422,428

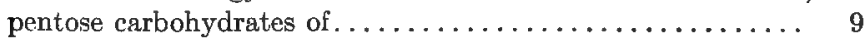

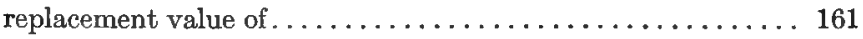

value of, for maintenance.............. $435,535,537$

work production $\ldots \ldots \ldots \ldots \ldots \ldots \ldots \ldots, 537$

Descent, work of ............................... 509

influence of grade on.................. 509

Dextrose, amount of, produced by liver................... 19

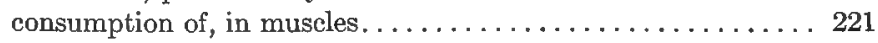

muscular contraction............220, 221

formation of fat from. . . . . . . . . . . . . . . . . 23

from carbohydrates in liver.........19, 20, 21

fat........................... 385 
Dextrose, formation of, from fat during muscular exertion. . . . . . . . 223

equation for................. 38,51

in liver................. 36,37

proteids.

$19,21,49,50$

glycogen from

$20,21,22$

in muscles. .............. 23

importance of constant supply of . . . . . . . . . . .

liver as source of. . . . . . . . . . . .

on carbohydrate diet............... 19

on proteid diet. . . . . . . . . . . . . . . 19

method of formation of, in liver................ 20

of blood. .......................... 17, 18

consumption of, in muscles. . . . . . . . . . 22

parotid gland. . . . . . . . . 22

fate of ......................... 22

fat production from. .................. 23

percentage of ........................ 18

variations in................ 18

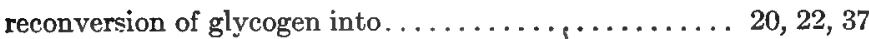

regulation of supply of, to blood.............. 18, 20

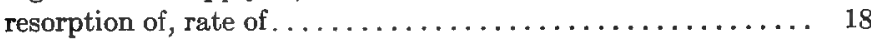

storage of, in resting muscle................. 222

Digestibility.

apparent. .......................... 10, 11

determination of. . . . . . . . . . . .

influence of metabolic prod-

ucts on............. 10

of carbohydrates, influence of amides on .... 57, 58

ammonium salts on $\quad 57$

asparagin on.... 57, 58

non-proteids on. 57,58

crude fiber.

influence of amides on......57,58

ammonium acetate on. 57

asparagin on. .....57,58

non-proteids on. . . . 5758

nitrogen-free extract ............... 12

influence of amides on... 57

ammonium

acetate on 57

asparagin on. 57

non-proteids

on...... 57

significance of results on. .............. 11

determination of. . . . . . . . . . . . . . . 9 
Digestibility, determination of, influence of products of metabolism on 10 of pentose carbohydrates................... 24

real. ........................... 10, 11

determination of . . . . . . . . . . . . . . . 10

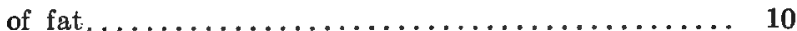

protein.......................... 10

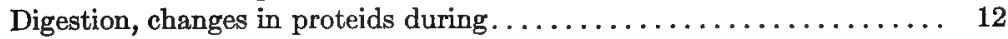

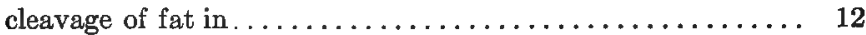

proteids in. ................ 12, 38

purpose of. .................. 38

expenditure of energy in assimilation and........ 337, 372

tissue building and. 491

influence of amides on..................... 54

asparagin on................... 54

non-proteids on. ................. 54

peptones produced during. . . . . . . . . . . . . . . . 12

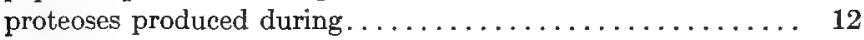

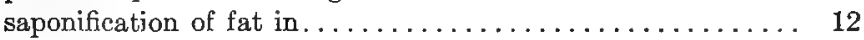

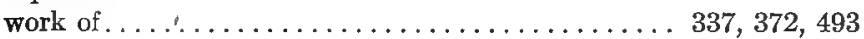

assimilation and. . ........ $80,93,337,372,376,406$

above critical point......... 407

below critical point........... 406

indirect utilization of heat from.... 406

in the $\operatorname{dog} . \ldots \ldots \ldots \ldots \ldots . \ldots . \ldots 378$

the horse............... 385

man.................. 382

methods of determining. . . . . . 377

of bone................. 384

carbohydrates........ 379, 382,384

fat....................... $378,382,385$

mixed diet........... 382,384

proteids.......... 381, 382,384

relation of, to surface.......... 408

factors of.......................... 374

for crude fiber. . . . . . . . . . . . . . . . . . 389

Digestive tract, functions of, in excretion................. 10

Dog, consumption of oxygen by, in locomotion. ............ 500

work of ascent. ............ 500

draft. .............. 501

expenditure of energy by, in locomotion.............. 502

utilization of energy by, in muscular work. . . . . . . . . . . 499

work of ascent. ............... 502

draft. .................. 502

work of ascent by, consumption of oxygen in............ 500

utilization of energy in. ............. 502 
Dog, work of digestion and assimilation in . . $\ldots \ldots \ldots \ldots \ldots$

draft by, consumption of oxygen in............ 501

utilization of energy in. . ............. 502

Draft, work of, consumption of oxygen in, by dog. ........... 501

horse. . ........... 507

utilization of energy in. . . . . . . . . 502, 507, 510, 513

by $\operatorname{dog} \ldots \ldots \ldots \ldots \ldots \ldots \ldots 2$

horse. ............ 507, 510

Dynamometer for experiments on horses. . . . . . . . . . 538, 539

Dyne. . . . . . . . . . . . . . . . . . . . . . . . . 231

Efficiency of animal. See Animal.

single muscle. . . . . . . . . . . . . . . . . . . . . 495

Emission of heat, rate of, influence of temperature on........... 350

regulation of . ..................... 349

Energy. ..................................... 226

absorption of, in anabolism................... 17

available. . . . . . . . . . . . . . . . . . . 269, 394

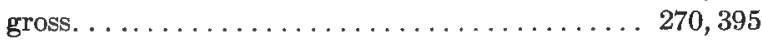

net. . . . . . . . . . . . . . . 270, 395

determination of, in carnivora. . . . . 413,427, 428

herbivora. ..... 418, 427, 428

availability of, distinction between utilization and......... 395

for maintenance..... 396, 406, 410,413, 427, 497

influence of amount of food on........... 430

character of food on...........441

relation of maintenance ration to. . . . . . . . 432

carbohydrates as source of, to body............... 91

coefficient of utilization of . . . . . . . . . . . . . . . . 440

conservation of. . . . . . . . . . . . . . . . . . . . 228

in animal body. ............... 228, 258

Atwater's and Benedict's inves-

tigations. ............. 265

early experiments.......... 261

Laulanié's experiments on.... 265

nature of evidence.......... 259

Rubner's experiments on..... 263

expenditure of, by the body............ 2, 226, 336, 339

in digestion and assimilation. . ...... 372, 375, 376 and tissue building. 491 method of determining. ....... 377

Energy, expenditure of, in locomotion. ................. 510

by $\operatorname{dog} \ldots \ldots \ldots \ldots \ldots \ldots \ldots, 502$

horse, at a trot $\ldots \ldots 509,510,514$ 
Energy, expenditure of, in locomotion, by horse, at a walk 504, 506, 508, 510

$\operatorname{man} . \ldots \ldots \ldots \ldots \ldots . \ldots 503$

influence of gait on. ........ 513

individuality on .... 517

load on. .... 509, 510, 515

size of animal on.... 516

species on........ 516

speed on........ 513

standing. . . . . . . . . . . . . . . . 499

sustaining load. . . . . . . . . . . 508,515

influence of individuality on. . 518

food as source of . . . . . . . . . . . . . . . 269

gross, of digestible crude fiber. . . . . . . . . . . . 303

ether extract. . . . . . . . . . . . . . . 304

nutrients. ................. 302,306

organic matter..................... 309

income and expenditure of . . . . . . . . . . .

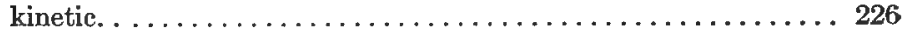

determination of . . . . . . . . . . . . . . . . 245

liberation of, in animal body .................... 1

loss of, in assimilation of fat. . . . . . . . . . . . . 35

fermentations. . . . . . . . . . . . . . . . . . 374

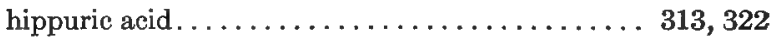

methane.......................... 310, 325, 335

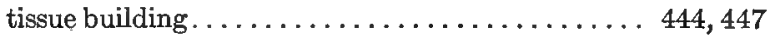

warming ingesta..................... 374

metabolizable. ...................... 269, 270

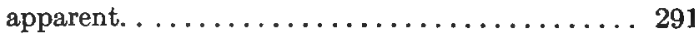

factors for. . . . . . . . . $\ldots \ldots \ldots \ldots \ldots \ldots \ldots$

Atwater's.................281

Rubner's.............. 279, 333

of coarse fodders. $285,286,287,290,297,298,300,301$

concentrated feeding-stuffs....... 289, 297, 299

digestible carbohydrates. .......... 324, 332

crude fiber. ............ 329, 332

ether extract. . ......... 323, 332

nutrients. .......... 310, 332, 333

organic matter. . . . . . . 297, 307

protein. $310,315,317,318,320,327,332$

fber-free nutrients, utilization of, in work pro-

duction. . ............. 541,543,547

food of carnivora.................. 272

herbivora. . ................. 281

$\operatorname{man} . \ldots \ldots \ldots \ldots \ldots \ldots 277,280,282$ 
Energy, metabolizable, of nutrients, utilization of, in work production. . . 545

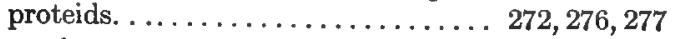
total organic matter............ 284, 285 real. .. 291

utilization of, in work production. . ..... 525, 540 methods of determination. ... 526, 528 Wolff's investigations. .... 528 muscular, fat as source of ............... 200, 223 proteids as source of............... 201, 207 source of . . . . . . . . . . . . . . . . . 196

starch as source of . . . . . . . . . . . . . . . 199 nature of demands for......................... 340 net available . . . . . . . . . . . . . . . . . . . . . . . . . . 394

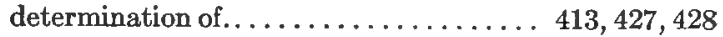
for maintenance. ...... 396, 406, 410,413, 427, 497 work. . . . . . . . . . . . . . . . . . 497 of carbohydrates. . ........ 417,419, 427, 428 crude fiber................. 422,428

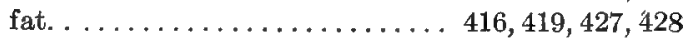
organic acids................... 423 pentoses. . . . . . . . . . . . . . 420,428 proteids................. 414,427,428

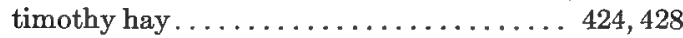
utilization of, in work. . . . . . . . . . . . . 497

of food. ..................................... 2

protein, losses of, in methane. . . . . . . . . . . . 310

urine. . . . . . . . . . . . . 312

potential . . . . . . . . . . . . . . . .

determination of. .................... 235

of combustible gases. .................. 243

excreta, computation of. . ............. 241

determination of. ................ 240

feces, computation of................ 242

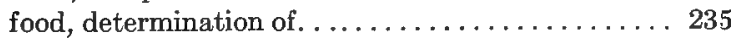

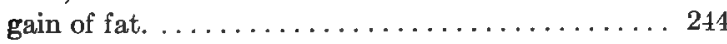

protein. . . . . . . . . . . . . . 244

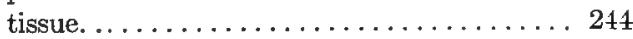

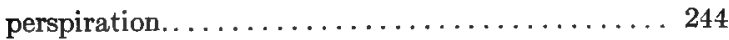

urine................ 272, 275, 278, 312

computation of . .......... 241.277, 312

store of, in animal body ......................... 1

transformation of, in animal body................. 2 
Energy, transformation of, in muscular contraction . . . . . . . . . 495

units of measurement of ................. 231, 233

utilization of, in tissue building. . . . . . . . 444, 447, 448, 461

by carnivora......... 448, 466

$\operatorname{man} \ldots \ldots \ldots \ldots \ldots \ldots 451$

ruminants. . . . 455, 461, 467

swine. . . . . . . 452, 466

earlier experiments on....... 460

effect of amount of food on.... . 466

character of food on ...4 472

differences in live

weight on.......4457

thermal environment on 471

'work. . . ................4444, 447,494

by $\operatorname{dog} \ldots \ldots \ldots \ldots \ldots \ldots \ldots \ldots 494$

horse ................... 502

at a trot. . . . . . . . . 509

walk. . . . . . . . . 504

man. .................... 502

influence of fatigue on ............ 519

individuality on. ........ 517

kind of work on......... 512

load on. . .............. 508

size of animal on........ 515

species on............ 515

speed on. ....... 507,513,514

training on . . . . . . . . 519

of ascent. . . . . . . . 502,503,506, 510

by $\operatorname{dog} \ldots \ldots \ldots \ldots \ldots \ldots . \ldots 2$

horse. . . . . . . . . . 506

$\operatorname{man} . . \ldots \ldots \ldots \ldots \ldots 5$

effect of grade on. ......... 512

load on $\ldots 509,510,515$

draft. ............... 502,510,513

by $\operatorname{dog} \ldots \ldots \ldots \ldots \ldots \ldots \ldots 2$

horse. ............ 507

locomotion, computed. $\quad \ldots \ldots \ldots \ldots 513$

of barley. . . . . . . . . . . . . . . . . . 4 483,491

beet molasses . . . . . . . . . . . 483,490.491

carbohydrates. . ....... 461, 462,473, 490,491

coarse fodders. . . . . . . . . . 484, 490, 491

concentrated feeding-stuffs. . . . . . 4 472, 490, 491

digestible carbohydrates. ..... . 475, 477, 490, 491

protein.............. 481,491

extracted straw. . . . . . . . . 4 488, 490, 491 
Energy, utilization of, of meadow hay.

PAGE mixed grains. $484,490,491$

oat straw. . . 483,491

oil. $485,490,491$ proteids.............. 463,482,491 rice................... 483,491 starch. . . . . . . . . . . 473, 490, 491 wheat gluten. . . . . . . . . . . 480,490, 491

Environment, thermal, critical. . . . . . . . . . . . . . . . 358

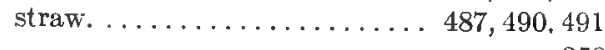

influence of, on heat production in fasting....... 347

maintenance ration........... 435

utilization of energy.........4 471

Enzym, rebuilding of proteids from cleavage products by .......... 40

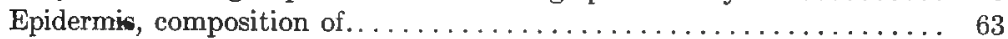

Ether extract. ............................... 8

digestible, gross energy of . . . . . . . . . . . . 304

metabolizable energy of . . . . . . . . 323,332

Exchange, gaseous, computation of heat production from......... 249

effect of load on. . . . . . . . . . . . . . . . 5 509

muscular exertion on............... 209

respiratory, determination of. .............. 73

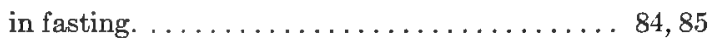

intermediary metabolism. . . . . . . . . 405

Excreta, determination of carbon of. . . . . . . . . . . . . . 69

dioxide in................ 69

hydrocarbons in. . . . . . . . . . 69,72

methane in $\ldots \ldots \ldots \ldots \ldots \ldots \ldots \ldots \ldots \ldots \ldots \ldots \ldots \ldots \ldots \ldots \ldots \ldots \ldots \ldots \ldots \ldots \ldots \ldots \ldots \ldots \ldots \ldots$
hydrogen in. .

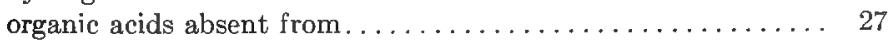

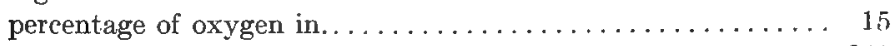

potential energy of, computation of . . . . . . . . . . . 241

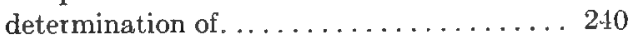

total, computation of heat production from........... 252

Fxcretion, functions of digestive tract in . . . . . . . . . . . . . . 10

nitrogen, proteid metabolism and ............. 97

rate of. .......................... 98

effect of non-nitrogenous nutrients on.... 130

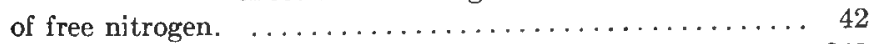

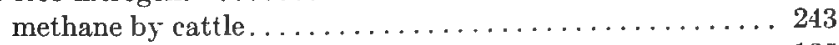

Exertion, muscular (see also $\left.W_{\text {ork }}\right) \ldots \ldots \ldots \ldots \ldots \ldots \ldots \ldots \ldots \ldots \ldots$

effects of, influence of amount of food on.... . . 197

on carbon metabolism............. 209

circulation. ................. 191

gaseous exchange. . . . . . . . . . 209 
Exertion, muscular, effects of, on metabolism. . . . . . . . . 185, 193

proteid metabolism. ......... 194, 206

respiration..................... 192

respiratory quotient. . ..... 211, 212, 216

work of heart. ................ 192

formation of dextrose from fat during. . . . . . . . 223

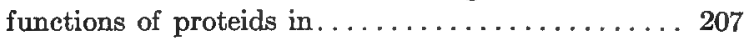

gain of proteids caused by . . . . . . . . . . 204

general features of . . . . . . . . . . . . . . 185

intermediary metabolism during........... 219

nature of non-nitrogenous material metabolized in. . 218

respiratory quotient during, conclusions from..... 218

secondary effects of.................... 191

Expenditure of energy. See Energy.

Extracted straw, metabolizable energy of. . . . . . . 290, 297, 300, 301

digestible, carbohydrates of $.327,332$ utilization of energy of............488, 490, 491

Extractives. . . . . . . . . . . . . . . . . . . . . 7

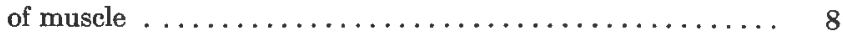

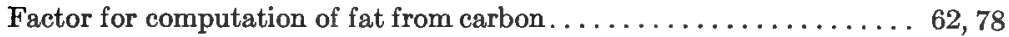

non-proteids from nitrogen.......... 8

protein from nitrogen..........667,68,77

Factors for metabolizable energy of digestible nutrients. . . . . . 302, 332, 333

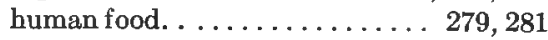

protein in human foods........................ 6

of proteid metabolism in fasting .............. 81, 90

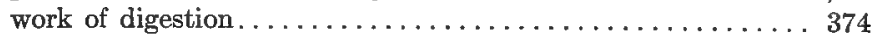

Fasting, constant loss of tissue in . . . . . . . . . . . . . . 83

disappearance of carbohydrates in. . . . . . . . . . . 85

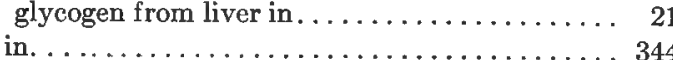

constancy of . . . . . . . . . . 345

influence of size of animal on.......... 359

thermal environment on. . . . . 347

is a minimum. ............. 347,356

measure of internal work. . . . . . . 344

metabolism in. . . . . . . .

effect of body fat on . . . . . . . . . 88,90

loss of protein on. . . . . . . . . . . 90

ratio of proteid to total. . . . . $86,88,89,90,93$

total. . . . . . . . . . . . . . . . 83,90

proportional to active tissue....... 86,93

of fat in. . . . . . . . . . . . $\ldots \ldots \ldots, 88,90$

proteids in. . ............... 81,90

minimum of proteids less than.... 136 
Fasting, metabolism of proteids in, tends to become constant.... 81,90 two factors of .............. 81,90 oxygen consumption in ................ $82,83,90,94$ production of carbon dioxide in. ................ 84 ratio of fat to protein in body in ............. $88,89,90$ respiratory exchange in $\ldots \ldots \ldots \ldots \ldots \ldots \ldots \ldots \ldots \ldots .64,85$

Fat. ................................. 8

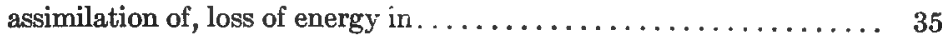
as source of muscular energy . . . . . . . . . . . . . . 200, 223 body, effect of, on fasting metabolism.............. 88, 90

formation of, from food fat. ................ 164

proteids substituted for. ...................... 104

replacement of proteids by . . . . . . . . . . . . . 149

substitution of non-nitrogenous nutrients for.......... 144

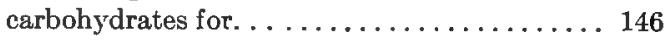

fat for. . . . . . . . . . . . . . . . . 144

cleavage of, in digestion. . . . . . . . . . . . . . . . . 12

composition of, constancy of. ................... 35

from different animals.................... 61

parts of animal............. 33

influence of feeding on................... 32

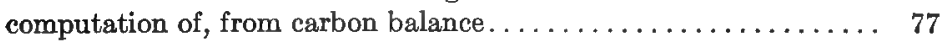

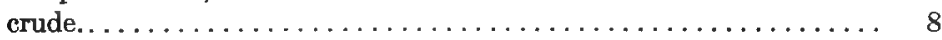

deposition of iodine addition products of . . . . . . . . . 31

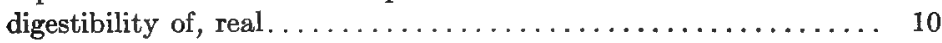

effects of, on proteid metabolism. ................... 114

compared with carbohydrates.... 127

factor for computation of, from carbon. . . . . . . . . . 62,78

food, formation of body fat from . . . . . . . . . . . . . . 164

quantitative relation of, to fat production........... 34

replacement of proteids by ................... 149

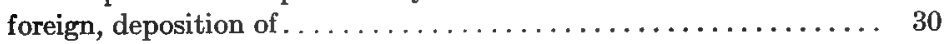

formation of dextrose from. . . . . . . . . . . . . $38 \ldots$

during muscular exertion. ......... 223

equation for. . . ........... 38, 51

in liver. . . . . . . . . . . . . 36,37

from carbohydrates. . . . . . . . . . 24, 30, 165

equation for. ............. 24

respiratory quotient in....... 179

cellulose. . . . . . ................. 181

crude fiber. . . . . . . . . . . . . . . . 181

dextrose of blood.................... 23

food fat. . . . . . . . . . . . . . . . . . 164

non-nitrogenous nutrients of feeding-stuffs. . . . . 180 
Fat, formation of, from other ingredients of food............. 163 pentose carbohydrates. .............. 183 proteids. . . . . . . .

difficulty of proof. . . . . . . . . . 113

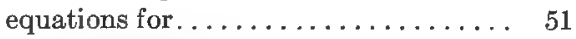

later experiments. ............. 111

Pettenkofer and Voit's experiments. . . . 108

Pflüger's recalculations. . . . . . . . . . 109

functions of food......................... 30

gain or loss of, determination of $\ldots \ldots \ldots \ldots \ldots \ldots \ldots \ldots, 77$

influence of glycogen on computation of . . . . . .66,78

potential energy of . . . . . . . . . . . . . 244

influence of on minimum of proteids . . . . . . . . . . . . 135

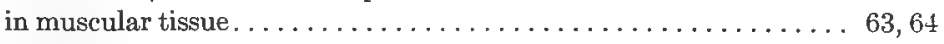

katabolism of............................. 35

loss of energy in assimilation of. . . . . . . . . . . . . . . . . 35

manufactured in body. . ................ 29, 30, 163

metabolism of. See Metabolism.

mutual replacement of carbohydrates and.............. 151

net availability of energy of. . . . . . . . . . .

of plant, nature of .......................... 8

oxidized, computation of, from respiratory quotient........ 76

production, quantitative relation of food fat to . . . . . . . . . 34

ratio of, to protein in body in fasting. . . . . . . . $\ldots \ldots \ldots, 89,90$

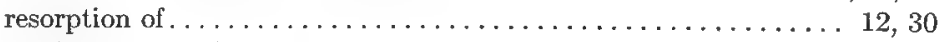

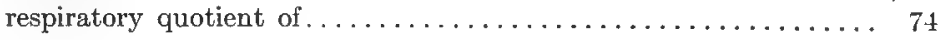

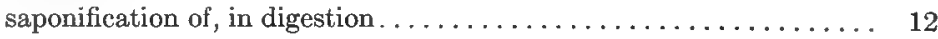

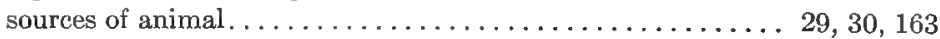

substitution of, for body fat.................... 144

value of, for maintenance. . . . . . . . . . . . 4 400, 402

work production...................... 522

work of digestion and assimilation of . . . . . . . $378,382,384,385$

Fatigue, influence of, on utilization of energy in work . . . . . . . . . . 519

Fattening, influence of, on maintenance ration. . . . . . . . . 441,458

Feces, computation of potential energy of . . . . . . . . . . . . 242

metabolic nitrogen in. ............................ 47

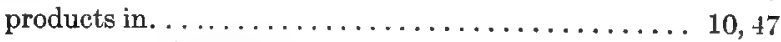

determination of. ............... 10

influence of, on determination of digesti-

bility...................... 10

nature of ....................... 47

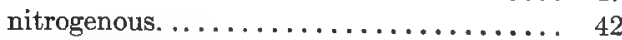

Feeding, influence of, on composition of fat............... 32

Feeding-stuffs, concentrated, expenditure of energy of, in digestion, assim-

ilation, and tissue building......... 492 
Feeding-stuffs, concentrated, metabolizable energy of. metabolizable energy of, utilization of, in work production 540 non-nitrogenous nutrients of, effects of, on total metabolism. 154 $\begin{array}{lr}\text { formation of fat from. . . } & 180 \\ \text { mutual replacement of . . } & 154 \\ \ldots \ldots \ldots \ldots \ldots \ldots \ldots \ldots & 6 \ldots \ldots\end{array}$

non-proteids in $\ldots \ldots$ mutual replacement of . . 154

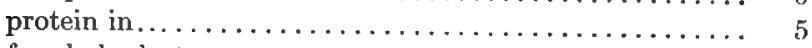

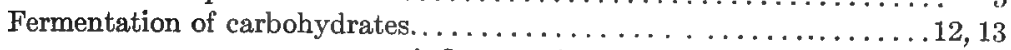

influence of amides on........... 55

ammonium salts on...... 56

asparagin on........... 55

non-proteids on ......... 55

organic acids from...............13, 26

products of ....................... 13

cellulose. . ........................... 13

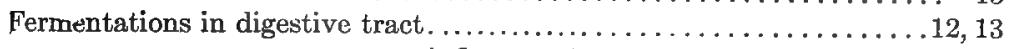

influence of amides on.......... 5.5

amnonium salts on...... 56

asparagin on...........54,55

non-proteids on........ 55

Fermentations, influence of, on nutritive value of carbohydrates...... 13

loss of energy in . . . . . . . . . . . . . . . 374

Fiber, crude. See Crude Fiber.

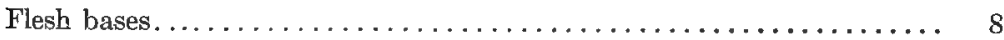

Flesh, proteid metabolism expressed in terms of ............ 68

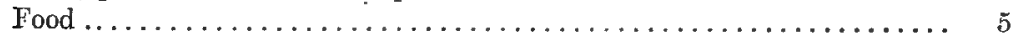

amount of, critical....................... 408

influence of, on effects of muscular exertion........ 197

net availability of energy........ 430

utilization of energy ...........4 466

as source of energy. .......................... 269

character of, influence of, on net availability of energy........ 431

utilization of energy ..........4 472

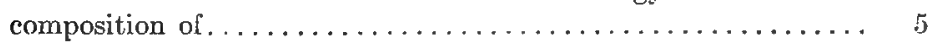

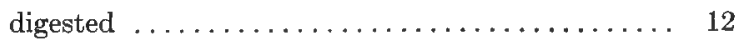

consumption, influence of, on heat production.........338, 372, 387

metabolism................ 387

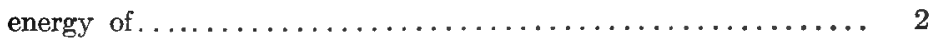

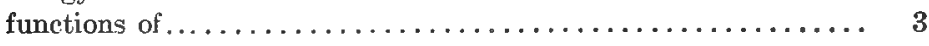

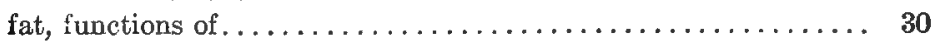


PAGE

Food, increases metabolism. . ....................... 372

ingredients, heats of combustion of . . . . . . . . . . . . . . 236

metabolizable energy of. See Energy.

nature of. ............................ 2

potential energy of, determination of . . . . . . . . . . . . 235

purposes to which applied. . . . . . . . . . . . . . . . . . 80

Foods, heats of combustion of . . . . . . . . . . . . . . . . 236, 237

Food-supply, relation of metabolism to . . . . . . . . . . . . . 93

Foot-pound. . . . . . . . . . . . . . . . . . . . . . . . 231

Force .................................... 226

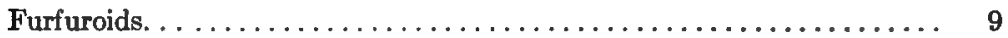

of crude fiber. . . . . . . ................... 9

nitrogen-free extract. .................... 9

Gain of fat, determination of . . . . . . . . . . . . . . . . 69,77

influence of glycogen on computation of . . . . . . . . 66, 78

potential energy of . . . . . . . . . . . . . . . . . . 244

nitrogen by body . . . . . . . . . . . . . . . . . 66, 67

protein by body. .......................... 66

during work. . . . . . . . . . . . . . . . . . . 204

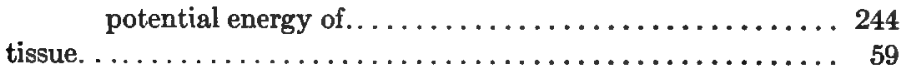

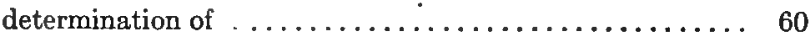

potential energy of ...................... 244

Gait, influence of, on expenditure of energy in locomotion. . . . . . . . . 513

Gases, combustible, composition of. . . . . . . . . . . . . . . . 243

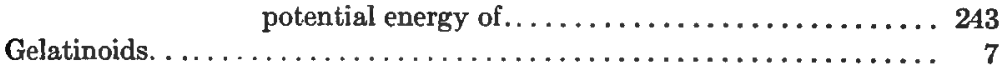

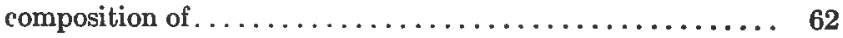

Globulin. ................................ 7

Glutaminic acid formed from proteids. . . . . . . . . . . . . . . . . 39

an intermediate product of proteid metabolism. ......... 44

Glycocol, formation of hippuric acid from .................. 44

oxidized in body. . .................... 52,53

Glycogen, amount of, in body. . . . . . . . . . . . . . . 66, 78

disappearance of, from liver in fasting. ............. 21

formation of, from artificial hexoses. ............... 20

carbohydrates, hexose........... 20, 21

pentose. ........... 25, 26

dextrose............... 20, 21, 22, 23

hexoses. . . . . . . . . . . . . . . . 20, 21

pentoses................... 25, 26

proteids. .................. 21,98

in liver. . .................. 20, 21, 22

muscles....................... 23

identical, from different hexoses.................. 20 
PAGE

Glycogen, identical, from hexoses and pentoses............... 26

influence of, on computation of gain or loss of fat......66, 78

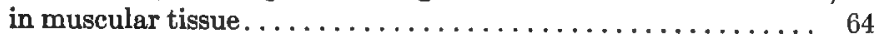

muscular, disappearance of, in work. . . . . . . . . . 23

functions of. . . . . . . . . . . . . 222, 223

reappearance of, in rest............... 23

reconversion of, into dextrose ............ 20, 22, 37

Grade, influence of, on efficiency of animal. . . . . . . . . . . 512

utilization of energy in work of ascent. . . . . . 512

work of descent. . . . . . . . . . . . . . . . 509

Grain, relative effects of hay and, on metabolism $\ldots \ldots \ldots \ldots \ldots \ldots \ldots 8$

value of coarse fodder and, for maintenance. . . . 433, 533, 537

work production. ....... 533

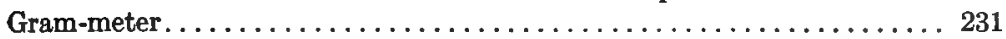

Gravity, force of . . . . . . . . . . . . . . . . . . . 231

Hair, composition of . . . . . . . . . . . . . . . . . . . . 63

Hay, relative effects of grain and, on metabolism............ 388

Heart, work of, influence of muscular exertion on. . . . . . . . . . . . 192

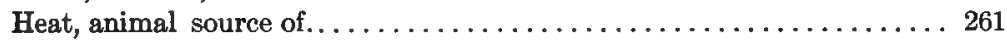

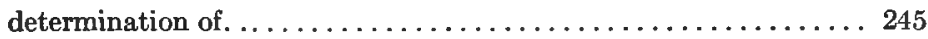

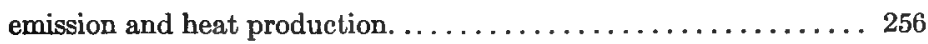

influence of insolation on. ................. 357

relative humidity on.............. 358

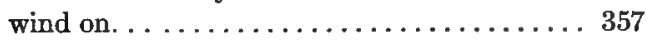

method of, above critical temperature.......... 355

rate of, influence of temperature on........... 350

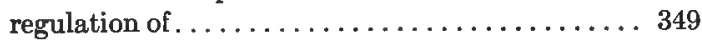

from digestive work, indirect utilization of . . . . . . . . 406

production. . . . . . . . . . . . . . . . . . . . . . 338

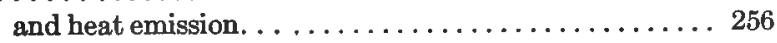

computation of. . . . . . . . . . . . . . . 249

from carbon and nitrogen balance.... 255

gaseous exchange. ......... 249

total excreta............. 252

influence of cleavages on........ 253

hydrations on. ....... 253

determination of. ...................... 245

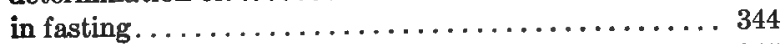

constancy of ................. 345

influence of size of animal on......... 359

thermal environment on...... 347

is a measure of internal work. . . . . . . 344

minimum. ............ 347, 356

influence of consumption of food on. . . . . 338, 372, 387

water on. . . ......... 438 
Heat production, influence of muscular tonus on ............ 191

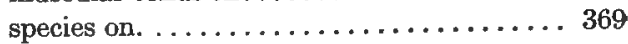

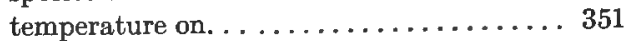

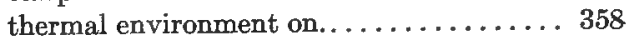

time element on............... 439

in intermediary metabolism. .............. 405

on maintenance ration............... 436, 437

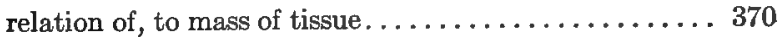

surface.................... 359

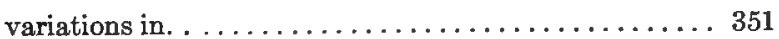

causes of. . . . . . . . . . 363

mechanism of. ................. 352

regulation of rate of emission of . . . . . . . . . . . . 349

Heats of combustion. ........................... 229

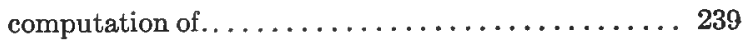

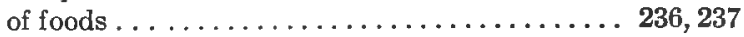

food ingredients...............236, 237

organic substances. . .............. 237

Heat, units of. . . . . . . . . . . . . . . . . . . . . 232

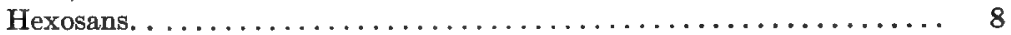

Hexoses. ..................................... 8

artificial, formation of glycogen from. . . . . . . . . . . 20

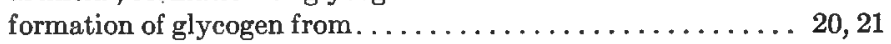

Herbivora, determination of net availability of energy in. . . . 418,427, 428

hippuric acid in urine of .................... 44

metabolizable energy of food of ................ 281

minimum of proteids for .................. 140

Hippuric acid. ............................. 44

composition of ....................... 44

formation of, from benzoic acid ............. 44

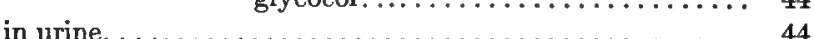

loss of energy in................. 313, 322

non-nitrogenous nutrients as source of ......... 45

origin of. . . . . . . . . . . . 44

pentose carbohydrates as source of ............ 46

source of benzoyl radicle of . . . . . . . . . . . 44,45

Hoof, composition of............................ 63

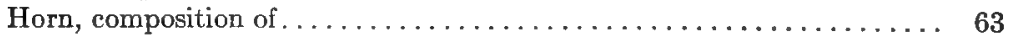

Horse, consumption of oxygen in locomotion by........504, 506, 507

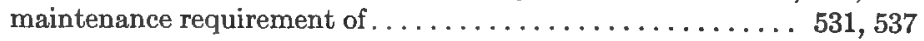

utilization of energy by, in work. . . . . . . . . . . . 502

at a trot. ............. 509

walk. . . . . . . . . . . . . 504

of ascent. ............ 506 
Horse, utilization of energy by, in work of draft.

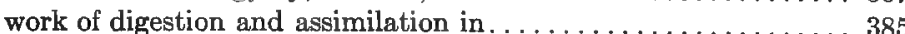

locomotion in. . . . . 5 504, 506, 508, 509, 510, 514, 535, 539

Human food, metabolizable energy of. . . . . . . . . 277, 280, 282

foods, protein factors for. . . . . . . . . . . . . . . 6

Humidity, relative, influence of, on heat emission. . . . . . . . . 358

Hydrations, influence of, on computation of heat production........ 253

Hydrocarbons of excreta, determination of . . . . . . . . . . 69,72

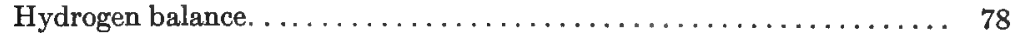

in excreta. ............................. 72

Income and expenditure of energy ................. 3, 226

matter. ..................... 5

Individuality, influence of, on expenditure of energy in locomotion..... 517

sustaining load... 518

utilization of energy in work......... 517

Indol in urine. . . . . . . . . . . . . . . . . . . . . . 46

Ingesta, warming, loss of energy in. ..................... 374

Insolation, influence of, on heat emission. . . . . . . . . . . 357

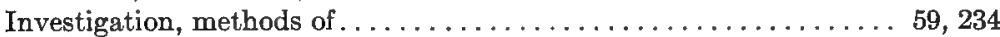

Katabolism. ............................. 16, 17

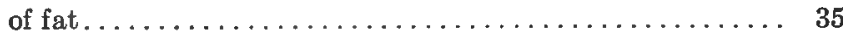

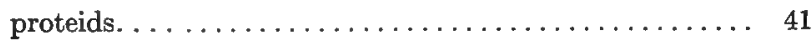

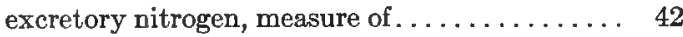

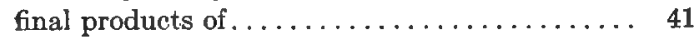

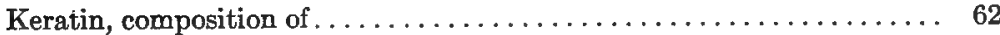

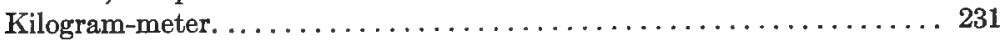

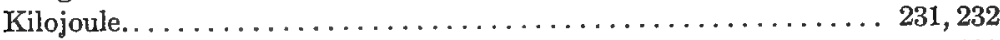

Lactic acid, effect of, on proteid metabolism............... 123

total metabolism.................. 158

production of, in metabolism of carbohydrates......... 23

replacement value of . . . . . . . . . . . . . . . 158

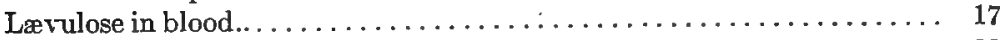

Leucin formed from proteids. . . . . . . . . . . . . . . . . . 39

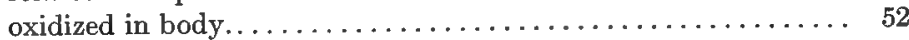

Ligneous material of crude fiber. . . . . . . . . . . . . . . . . . 9

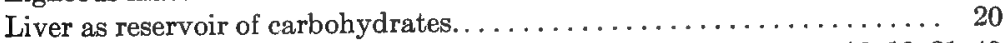

source of dextrose. ................. 18, 19, 21, 49

on carboydrate diet............. 19

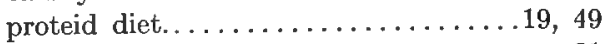

disappearance of glycogen from, in fasting. ............. 21

formation of dextrose in, from carbohydrates. . . . . . 19, 20, 21

fat. ............... 21, 36, 37

proteids............ 19, 21, 45, 50

method of .................. 20

glycogen in. . . . . . . . . . . . . 21,22 
Liver, formation of glycogen in, from dextrose............20, 21, 22

proteids................... 21

sugar in.

$18,19,21,49,50$

functions of................................ 18

in work production..................... 220

glycogenic function of . . . . . . . . . . . . . . . . . . . 21

reconversion of glycogen to dextrose in ............ 20, 22, 37

Live weight, influence of, on maintenance ration. . . . . . . . . . . 458

utilization of energy ............... 457

Load, effect of, on expenditure of energy in locomotion...... 509, 510, 515

total metabolism.................... 509, 515

utilization of energy................... 508

in work of ascent......509, 510,515

expenditure of energy in sustaining.

508,515

influence of individuality on... 518

Locomotion, consumption of oxygen in, by dog. .............. 500

horse........... 504, 506, 507

expenditure of energy in .................. 499, 510

by $\operatorname{dog} \ldots \ldots \ldots \ldots \ldots \ldots, 502$

horse at a trot. .....509, 510, 514

walk. .504, 506, 508, 510,

533,539

$\operatorname{man} . \ldots \ldots \ldots \ldots \ldots \ldots . \ldots . \ldots 3$

influence of gait on........... 513

individuality on... ... 517

load on..... 59, 510, 515

size of animal on.....516

species on......... 516

work of. See Work of Locomotion.

speed on.... 507, 508, 513

Loss of fat, determination of . . . . . . . . . . .

influence of glycogen on computation of ...........66, 78

nitrogen by body $\ldots \ldots \ldots \ldots \ldots \ldots \ldots \ldots \ldots \ldots \ldots, 66,67$

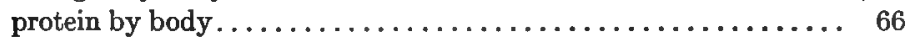

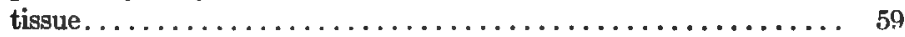

constant, in fasting........................ 83

determination of ........................... 60

Maintenance..................................... 394

availability of energy for........396, 406, $410,413,427,497$

isodynamic values for. ...................... 397

isoglycosic values for ...................... 400

ration. . ............................ 432

heat production on................ 436, 437

of horse. . ................... 531, 537

influence of consumption of water on..........438 
Maintenance ration, influence of fattening on........

fltening on.............. 441, 458

live weight on............... 458

shearing on................. 436

size of animal on ............ 440

thermal environment on.......... 435

time element on. . . . . . . . . . . . 439

relation of, to net availability of energy....... 432

relative value of grain and coarse fodder for. . . . 433, 533, 537

value of carbohydrates for. . . . . . . . . . . . . 400-402

crude fiber. ...................... 435

fat for .................... 400, 402

Man, expenditure of energy by, in locomotion. ............. 503

hippuric acid in urine of . . . . . . . . . . . . . . . . . . . . 44

metabolizable energy of food of . . . . . . . . .

utilization of energy by, in muscular work ............. 503

tissue building.............. 451

work of ascent............... 503

work of digestion and assimilation in . . . . . . . . . . . . . 382

Mastication, work of . . . . . . . . . . . . . . . . . . . . . . . 391

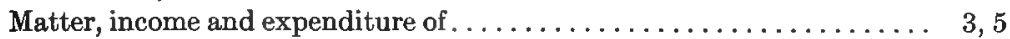

Meadow hay, metabolizable energy of . . . . . . . . 286, 290, 297, 300, 301

utilization of energy of . ............4 484, 490,491

Metabolic products, nitrogenous, in feces................ 42

Metabolism................................. 14

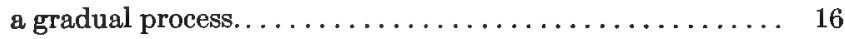

an analytic process...................... 15

a process of oxidation.................... 15

carbon dioxide produced in............... 14, 15

carbon, effects of muscular exertion on........... 209

consumption of oxygen in ................ 14, 15, 16

effects of muscular exertion on............. 185, 193

non-nitrogenous nutrients on ..........114, 125

proteid supply on . . . . . . . . . . . . 94, 104

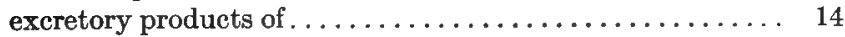

fasting.................... 80, 90, 340

effect of body fat on ............... 88,90

loss of protein on ............... 90

ratio of proteid to total. . . . . . $81,88,89,90,93$

total. . . . . . . . . . . . . . . . . 83,90

proportional to active tissue. ....... 86,93

fat, in fasting. ........................ 85, 88,90

food increases. . . . . . . . . . . . . . . . . 372

glandular, similar to muscular................. 344

influence of food consumption on ................. 387

muscular exertion upon............. 185. 193 
PAGF

Metabolism in muscular tonus. . . . . . . . . . . . . . . . 190

intermediary. .......................... 91

during muscular exertion............ 219

heat production in .............. 405

of fat. ..................... 91

protein................... 91

respiratory exchange in ............ 405

intermediate products in............... 16, 44

muscular, nature of.................... 495

of amides. .......................... 52

carbohydrates. ...................... 15, 17

hexose.................... 17

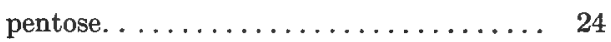

production of carbon dioxide in..... 23, 27

lactic acid in.......... 23

water in.........23, 27

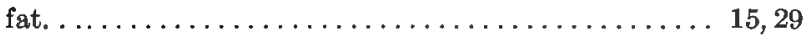

intermediary..................... 91

production of carbon dioxide in............. 36

water in ................... 36

non-proteids. . . . . . . . . . . . . . . . . . . 52

organic acids. . . . . . . . . . 26

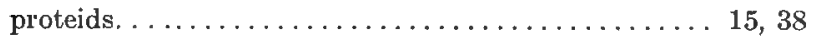

products of in feces. . . . . . . . . . .

determination of............. 10

influence of, on determination of diges-

tibility.................. 10

nature of. . . . . . . . . . . 47

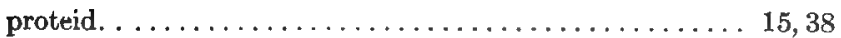

and nitrogen excretion. ............... 97

determined by supply................... 128

effects of acetic acid on................. 123

carbohydrates on. ............... 115

compared with fat.... 127

cellulose on .................. 117

excess of proteids on ............ 96

fat on................... 114

compared with carbohydrates.... 127

lactic acid on................... 123

muscular exertion on......... 194, 206

influence of amount

of food on ....... 197

non-nitrogenous nutrients on. . . . . 114, 125

duration of. . 128

magnitude of 128 
Metabolism, proteid, effects of organic acids on ......... 123

pentose carbohydrates on ........... 124

proteid supply on............... 94

starch on ................... 116

sugars on. ............... 116

expressed in terms of flesh............ 68

glycocol intermediate product of . . . . . . . 44

identity of, in different animals......... 317, 335

on different feeds. . . . . . . . . . . . . 322

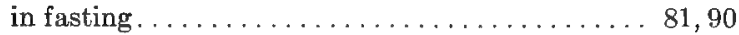

minimum of proteids less than ........ 136

tends to become constant........ 81,90

two factors of . . . . . . . . . . 81,90

intermediate products in . . . . . . . . . . 44

intermediary........................... 91

production of carbon dioxide in . . . . . . . . 42

phosphoric acid in............ 42

sulphuric acid in............ 42

urea in ................... 42

water in............... 42

ratio of, to total, in fasting. . . . . $86,88,89,90,93$

urea as measure of . . . . . . . . . . . . . 68

relations of, to food supply ................ 93

relative effects of hay and grain on............. 388

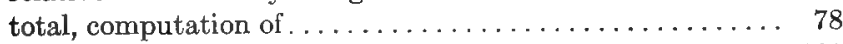

effect of acetic acid on. . . . . . . . . . . . 160

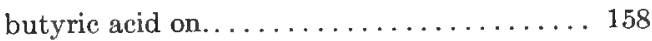

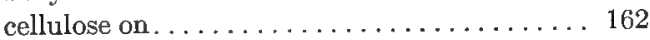

crude fiber on.................... 161

lactic acid on................ 158

load on.................. 509, 515

non-nitrogenous ingredients of feeding-stuffs

on. ............. 154

nutrients on........144, 154

organic acids on ................ 157

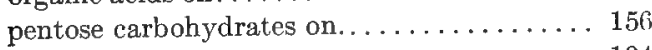

proteid supply on................ 10-1

rhamnose on. ................ 15

in fasting . . . . . . . . . . . . .

proportional to active tissue........ $86,9:$

nitrogen cleavage of proteids independent of . . . . . . 99

ratio of, to proteid, in fasting. ........ $86,88,89,90$

urea produced in. . . . . . . . . . . . . . . . . . .

water produced in......................... 14, 15

Methane, excretion of, by cattle. . . . . . . . . . . . . . . 243 
Methane in excreta, determination of.

losses of energy in . . . . . . . . . . . $310,325,328,330,335$

produced by fermentation of carbohydrates........... 13

Methods of investigation. . . . . . . . . . . . . . . . . . 234

Milk fat, formation of, from carbohydrates................ 174

Minimum demands of vital functions.................. 80

of proteids. . . . . . . . . . . . . 133

amount of non-nitrogenous nutrients required to reach. ............................ 139

effect of carboydrates on ............. 136

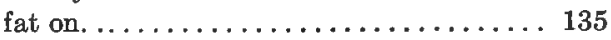

non-nitrogenous nutrients on......... 134

on health.................... 143

for herbivora...................... 140

in fasting. . . . . . . .

less than fasting metabolism............. 136

Mixed diet, work of digestion and assimilation of . . . . . . . . . 382, 384

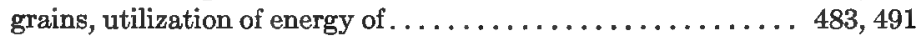

Möckern experiments......................... 281, 455

Motor, efficiency of animal as........................ 498

Mucins, composition of......................... 62

Muscle, consumption of dextrose in ................. 22, 221

contractile substance of . . . . . . . . . . . . . . . 17

efficiency of single. . . . . . . . . . . . . . . . . . 495

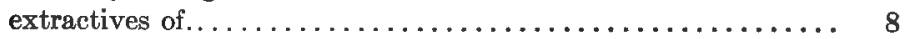

formation of glycogen in..................... 23

respiratory quotient of . . . . . . . . . . . . . . . . 187

resting, storage of dextrose in . . . . . . . . . . . . . . . 222

oxygen in . . . . . . . . . . . . . . . . 222

voluntary, work of . . . . . . . . . . . . . . . . . . 337

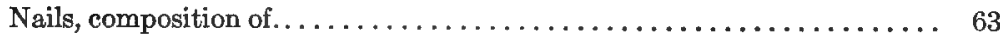

Nitrogen-free extract, apparent digestibility of. . . . . . . . . . . 12

influence of amides on... 57

ammonium

acetate on. 57

asparagin on. 57

non-proteids

on....... 57

carbohydrates of.................... 9

digestible, gross energy of . . . . . . . . . 305, 306

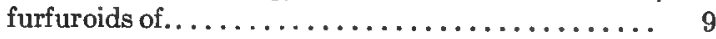

pentose carbohydrates of............... 9

Nitrogen balance, computation of heat production from carbon and. . . . 255

cleavage of proteids. . ...................... 98

cause of.............. 100,101, 103 
Nitrogen cleavage of proteids, effects of non-nitrogenous nutrients on... infepent... 131

content of proteids. . . . . . . . . . . . . . . . . . . . 39

equilibrium, amount of proteids required to reach. . . . . . . . 94

estimation of protein from. . . . . . . . . . . .

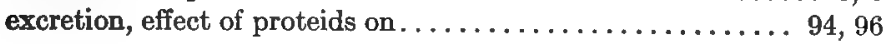

of free. ....................... 42

proteid metabolism and................. 97

rate of........................... 98

effects of non-nitrogenous nutrients on..... 130

excretory, measure of proteid katabolism............. 42

factor for computation of non-proteids from. . . . . . . . . 8

protein from..........6.67, 68, 77

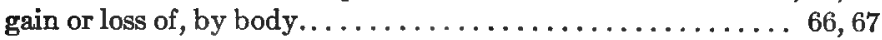

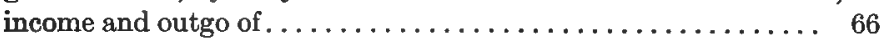

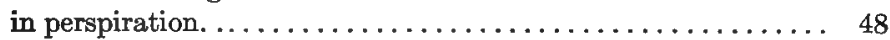

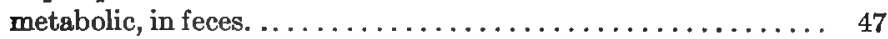

percentage of, in body protein. . . . . . . . . . . . 62,65

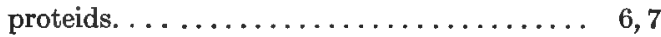

protein. . . . . . . . 6, 62,65

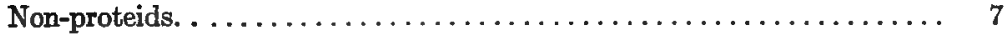

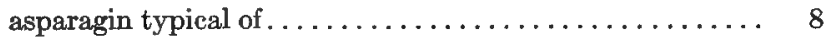

determination of..................... 8

factor for computation of, from nitrogen......... 8

in feeding-stuffs. . . . . . . . . . . . . . . . 6

influence of, on digestion. ................. 54

apparent digestibility of carbohydrates... 57

crude fiber ... 57, 58

nitrogen-free ex-

tract....... 57

fermentation of carbohydrates....... 55

fermentations in digestive tract....... 54

of animal body......................... 8

oxidized in body . . . . . . . . . . . . . . . . 52

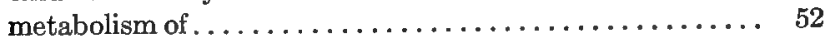

nature of. . . . . . . . . . . .

not synthesized to proteids............... 53

produced by cleavage of proteids. . . . . . . . . . 7,8

replacement of proteids by................. 53

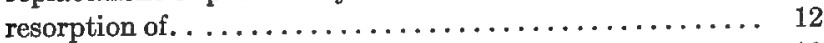

Nutrients, available. . . . . . . . . . . . . . . . . . . . . 10

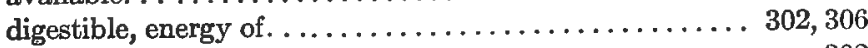

gross. . . . . . . . . . . . . . 302

metabolizable. .......... 310, 332, 333

factors for. .... 302, 332, 333 
Nutrients, fiber-free, utilization of, in worl

541,543

isoglycosic replacement of.................... 153

metabolizable energy of, utilization of, in work production. . . 545

modified in digestive tract. . . . . . . . . . . . . . . 12

mutual replacement of. . . . . . . . . . . . . . 148

non-nitrogenous, amount of, required to reach minimum of

proteids................... 139

as source of hippuric acid...........45

effects of, on metabolism.......... 114, 125

minimum of proteids........ 134

nitrogen cleavage of proteids... 131

proteid metabolism. ..... 114, 125

magnitude

of...... 128

duration of. 128

rate of nitrogen excretion. . . . 130

total metabolism. ....... 144, 154

formation of fat from............ 162

of feeding-stuffs, formation of fat from. . . . 180

mutual replacement of.............. 154

substitution of, for body fat.......... 144

utilization of excess of . . . . . . . . . 162

percentage of oxygen in...................... 5

relative values of ........................ 152

in work production. . . . . . . . . . . 522

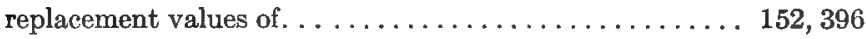

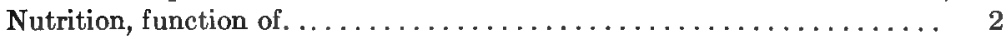

statistics of. . . . . . . . . . . . . .

Oat straw, metabolizable energy of . . . . . . . . . . 290, 297, 300301

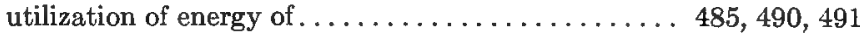

Oil, metabolizable energy of. . . . . . . . . . . . . . . 296, 323, 332

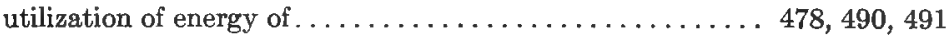

Organic acids, absence of, from excreta................ 27

net availability of energy of . . . . . . . . . . . . 423

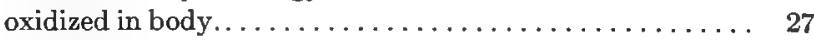

matter, digestible, gross energy of . . . . . . . . . . . 309

metabolizable energy of . . . . . . 297, 307

utilization of energy of . . . . . . . . . 490

total, metabolizable energy of . . . . . . . . 284,285

utilization of energy of . . . . . . . . 455,461, 490

substances, heats of combustion of ............... 237

Oxidations incomplete in muscular contraction.............. 186

Oxygen balance. ............................ 79 
Oxygen, consumption of, determination of.

PAGE in fasting.

$70,71,73,79$ locomotion, by $\operatorname{dog} . \ldots \ldots \ldots \ldots \ldots \ldots, 500$ horse......... 504, 506, 507 metabolism................. 14, 15, 16 work of ascent by $\operatorname{dog} . \ldots \ldots \ldots \ldots \ldots, 500$ horse..............506

draft by $\operatorname{dog} \ldots \ldots \ldots \ldots \ldots \ldots, 501$

horse.............. 507

not essential to muscular contraction. . . . . . . . . . . . . . 188

percentage of, in excreta. ....................... 15

nutrients......................... 15

storage of, in resting muscle....................... 222

Parotid gland, consumption of dextrose of blood in ............. 22

Peanut oil, metabolizable energy of $\ldots \ldots \ldots \ldots \ldots \ldots \ldots 296,323,332$

Pentosans.

oxidized in body......................... 26

Pentose carbohydrates. See Carbohydrates.

Pentoses........................................ 8

formation of glycogen from ................. 25,26

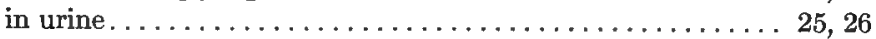

metabolism of. ........................... 24

net availability of energy of . . . . . . . . . . . . 420,428

oxidized in body............................. 25

Peptones absent from blood .......................... 40

formed from proteids.................... $12,38,39$

produced during digestion...................... 12

synthesis of, to proteids by an enzym.............. 40

Perspiration, ammonium salts in $\ldots \ldots \ldots \ldots \ldots \ldots \ldots \ldots \ldots \ldots, 48$

creatinin in $\ldots \ldots \ldots \ldots \ldots \ldots \ldots \ldots \ldots \ldots \ldots \ldots, 48$

nitrogen in. . . . . . . . . . . . . . . . . . . . 48

nitrogenous matter in....................... 42

potential energy of......................... 242

proteids in................................ 48

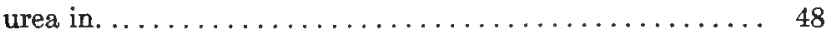

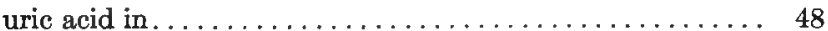

Phenols in urine.............................. 27, 46

Phosphoric acid, production of, in metabolism of proteids. . . . . . . 42

Phosphorus balance. .............................. 79

Plastein...................................... 40

Proteids. . . ......................................... 6

albumoses formed from..................... 38, 39

amides formed from $\ldots \ldots \ldots \ldots \ldots \ldots \ldots \ldots \ldots \ldots, 7,39,52$

not synthesized to...................... 53 
TsGE

Proteids, amount of, required to produce carbon equilibrium. . . . . . . . .
nitrogen equilibrium.

anabolism of. . . . . . . . . . .

aspartic acid formed from..................... 39

as source of muscular energy .............. 201, 207

body, and food proteids..................... 40

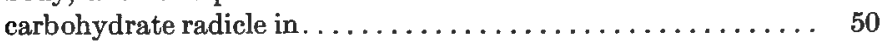

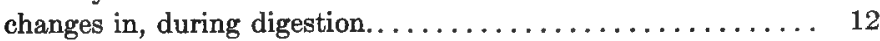

classification of ........................... 7

cleavage of, in digestion. . . . . . . . . . .

purpose of....................... 38

non-proteids produced by............ 7,8

differences in . . . . . . . . . . . . . . . . . . . . . . 39

effect of excess of, on proteid metabolism............. 96

on formation of hippuric acid............. 463

metabolism................... 94, 104

nitrogen excretion. ...............994,96

proteid metabolism. ................ 94

total metabolism. . . . . . . . . . . . . . . . . . . . . . 104

food, and body proteids . . . . . . . . . . . . . . . 40

formation of dextrose from, in liver.......... 19, 21, 49, 50

fat from. ................ 30, 50,98, 101

difficulty of proof of ............ 113

equations for.............. 51

later experiments.............. 111

Pettenkofer and Voit's experiments.... 108

Pflüger's recalculations. . . . . . . . . . . 109

glycogen from. ................ 21, 98

sugar from. . . . . . . . . . . .

functions of, in muscular exertion.................. 207

gain of, during work . . . . . . . . . . . . . . . . . 204

glutaminic acid formed from $\ldots \ldots \ldots \ldots \ldots \ldots \ldots \ldots \ldots, 39$

in perspiration. . . . . . . . . . . . . . . 48

intermediary metabolism of . . . . . . . . . . . . . 91

katabolism of. . . . . . . . . . . . . . . . . . 41

excretory nitrogen measure of . . . . . . . 42

final products of ................. 41

leucin formed from....................... 39

metabolizable energy of . . . . . . . . . . 27. 272, 277

metabolism of. See Metabolism.

minimum of . . ......................... 133

amount of non-nitrogenous nutrients required to

reach....................... 139

effect of non-nitrogenous nutrients on........ 134

effects of, on health................. 143 
Proteids, minimum of for herbivora $\ldots \ldots \ldots \ldots \ldots$ in fasting. ..... 82, 83, 90,94 influence of carbohydrates on ............ 136

fat on.................... 135

less than proteid metabolism in fasting $\ldots \ldots \ldots \ldots, 136$
molecular weight of $\ldots \ldots \ldots \ldots \ldots \ldots \ldots \ldots \ldots \ldots \ldots \ldots \ldots \ldots$

nature of. . . . . . . . . . . . . . . . . . . . . 39

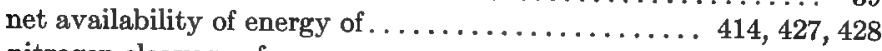

nitrogen cleavage of. . . . . . . . . . . . . . . . . 98

cause of $\ldots \ldots \ldots \ldots \ldots \ldots \ldots 100,101,103$

effects of non-nitrogenous nutrients on. . . 131 independent of total metabolism....... 97

non-nitrogenous residue of. . . . . . . . .

fate of.............. 49,98

formation of sugar from.....49, 50, 98

non-proteids not synthesized to . . . . . . . . . . . 53

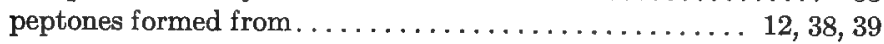

percentage of nitrogen in ...................... 6,7

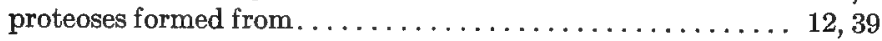

putrefaction of, in intestines. .............. 44, 46

products of ............ $44 ; 46$

rebuilding of, from cleavage products............. 40

replacement of, by amides..................... 53

asparagin.................. 54

body fat. . . . . . . . . . . . . . . . 149

fats and carbohydrates of food........ 149

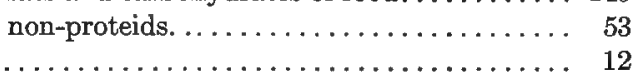

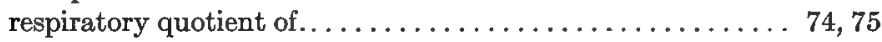

synthesis of peptones to ................... 40

substituted for body fat. . . . . . . . . . . . . . . . 104

Proteid supply, effects of, on metabolism............... 94, 104

proteid metabolism. . . . . . . . . . . 94

total metabolism. . . . . . . . . . . . . 104

Proteids, terminology of. . . . . . . . . . . . . . . .

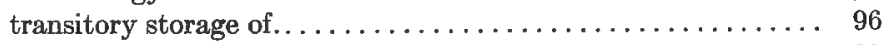

tyrosin formed from . . . . . . . . . . . . . . . . . 39

utilization of energy of.................. 482, 491

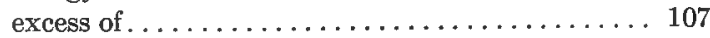

work of digestion and assimilation of......... 381, 382, 384

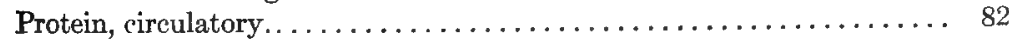

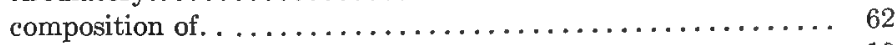

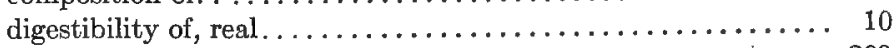

digestible, gross energy of . . . . . . . . . . . . . . 309 
Protein, digestible, metabolizable energy of .. $310,315,317,318,320,327,332$

utilization of energy of . . . . . . . . . . 481,491

estimation of, errors in. . . . . . . . . . . . . . . . . 6

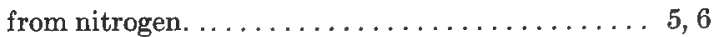

factor for computation of, from nitrogen . . . . . . $6,67,68,77$

in human foods....................... 6

gain or loss of, by body. . . . . . . . . . . . . . . . 66

potential energy of.................. 244

in feeding-stuffs. . . . . . . . . . . . . . . . . . . 5

loss of, in fasting, effect of, on metabolism............ 90

energy of, in methane. ........................ 310

urine. ........................ 312

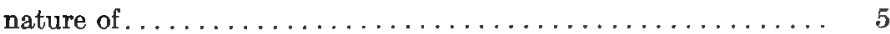

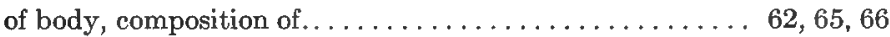

percentage of nitrogen in.............62,65

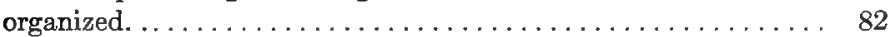

percentage of nitrogen in ............... 6, 62, 65

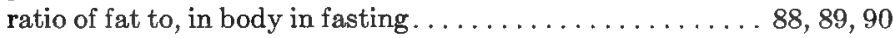

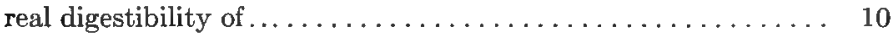

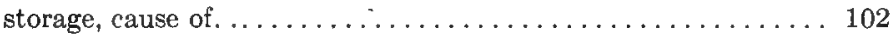

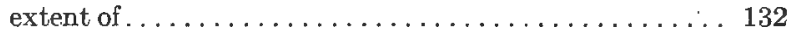

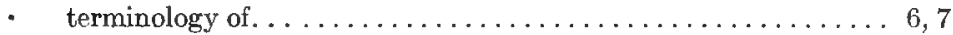

Proteoses, formed from proteids . . . . . . . . . . . . . . 12, 39

produced during digestion. ... . . . . . . . . . . . . 12

Putrefaction of proteids in intestines. . ................ 44

products of ............ 44, 46

Quotient, respiratory. .............................. 74

change in, caused by work............ 212

computation from, of carbohydrates oxidized... . 76

fat oxidized.......... 76

deductions from. .................. 75

during work, conclusions from. ........... 75

effects of muscular exertion on..............211

in fat-formation from carbohydrates. . . . . . . 179

of carbohydrates. . . . . . . . . . . . 74

fat........................ 74

muscle. . ..................... 187

influence of contraction on........ 187

proteids...................... 74,75

variations of . . . . . . . . . . . . . 211

during work.............. 216

Range, thermic .................................... 348

Rate of nitrogen excretion. ............................ 98

Ration, maintenance. See Maintenance.

Regulation of body temperature. ................ 347, 348 
Regulation of body temperature, chemical ................... BSE

means of. .................... 348

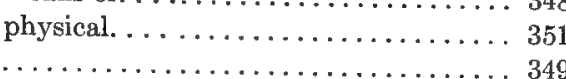

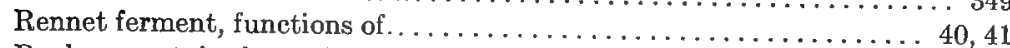

Replacement, isodynamic, law of . . . . . . . . . . . . . .

isoglycosic, law of.................. 153, 399

mutual, of fat and carbohydrates............... 151

non-nitrogenous ingredients of feeding-stuffs... 154

nutrients. . . . . . . . . . . . . . . . . 148

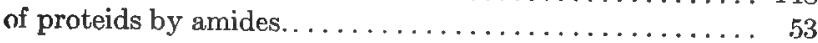

asparagin. . . . . . . $\ldots \ldots \ldots \ldots \ldots \ldots$

body fat. . . . . . . . . . . . . . . 149

carbohydrates and fat of food.......... 149

non-proteids. ................ 53

value of acetic acid..................... 160

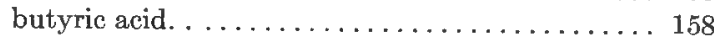

carbohydrates. . . . . . . . . . . . . . . 152

cellulose. . . . ................... 162

crude fiber. . . . . . . . . . . . . . . . . 161

lactic acid. . ................... 158

non-nitrogenous ingredients of feeding-stuffs. . . . 154

nutrients. . . . . . . . . . . . . . . . 152

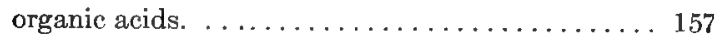

pentose carbohydrates. . ............. 156

rhamnose. . . . . . . . . . . . . . . . 156

Residue, non-nitrogenous, of proteids. . . . . . . . . . . . 48, 98

fate of............ 49,98

formation of sugar from. . . 49, 50,98

Resorption of carbohydrates. . . . . . . . . . . . . . . . . 12

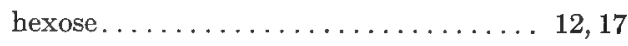

rate of. . . . . . . . . . 18

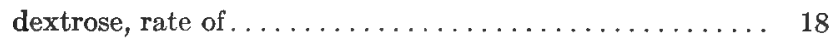

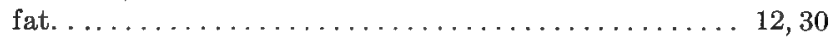

non-pioteids. . . . . . . . . . . . . . . . 12

proteids. . . . . . . . . . . . . .

Respiration apparatus. . . . . . . . . . . . . . . . . . 69

determination of water by........... 79

Petterkofer type of ............... 70

Regnault type of................ 69

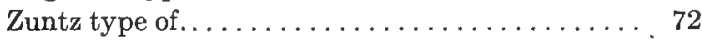

Respiration-calorimeter. . . . . . . . . . . . . . . . 246, 248

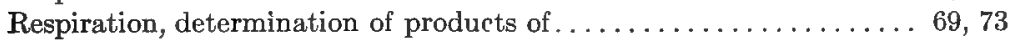

effects of muscular exertion on ............... 192

work of. . . . . . . . . . . . . . . . . 193, 341 
Respiratory exchange, determination of . ............... 73

in intermediary metabolism............. 405

Rest, reappearance of muscular glycogen in ............... 23

Rhamnose, effect of, on total metabolism ................ 156

replacement value of . . . . . . . . . . . . . . 156

Rice, utilization of energy of . . . . . . . . . . . . . . . . 483, 491

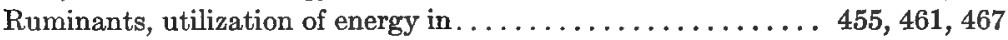

Sarkosin oxidized in body......................... 53

Saponification of fat in digestion. . . . . . . . . . . . . . . . . 12

Schematic body............................. 60, 66

Shearing, influence of, on maintenance ration.............. 436

Size of animal, influence of, on efficiency of animal. ............ 515

expenditure of energy in locomotion..... 516

heat production. ................. 359

in fasting.......... 359

maintenance ration............... 440

relation of, to physiological activities. ........... 368

Species, comparison of heat production of . . . . . . . . . . . 369

influence of, on efficiency of animal. ............. 515

expenditure of energy in locomotion.........511

Speed, correction for, in work of locomotion. . . . . . . . . 507, 508

influence of, on expenditure of energy in locomotion. . . . 507, 508, 513

utilization of energy in work........507, 513, 514

Standing, expenditure of energy in. . . . . . . . . . . . . 343, 499

Starch, as source of muscular energy . . . . . . . . . . . . . . . . 199

digestible, gross energy of . . . . . . . . . . . . . . . . 306

metabolizable energy of . . . . . . . . . . 324,332

utilization of energy of . . . . . . . . . . .

effect of, on proteid metabolism................. 116

metabolizable energy of . . . . . . . .

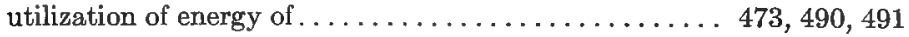

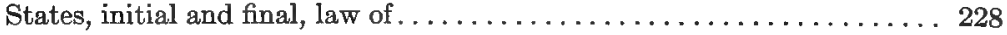

Statistics of nutrition. .......................... 3

Storage of protein, extent of......................... 132

transitory. ..................... 96

cause of.................. 102

Straw, extracted, gross energy of carbohydrates of . . . . . . . . . . 308

metabolizable energy of..........290, 297, 300, 301

carbohyydrates of. ........ 327

utilization of energy of ...........4 488,490, 491

oat, metabolizable energy of. . .......... 290, 297, 300, 301

protein of. . ................ 321

carbohydrates of. . ........... 329

utilization of energy of . . . . . . . . . . $485,490,491$

wheat, metabolizable energy of. . . . . . . . 290, 297, 300, 301 
Straw, wheat, metabolizable energy of protein of . . . . . . . . . 321

carbohydrates............. 329

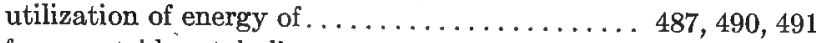

Sugar, effect of, on proteid metabolism . . . . . . . . . . . . . . . 116

formation of, from non-nitrogenous residue of proteids . . . 49,50,98

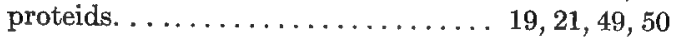

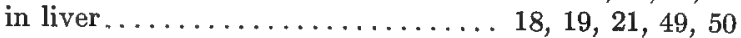

Sulphur balance. . . . . . . . . . . . . . . . . . . . . . . 79

Sulphuric acid, conjugated, in urine ................. 46

production of, in metabolism of proteids........... 42

Surface of animal, computation of. . . . . . . . . . . . . . . . . 364

relation of heat production to . . . . . . . . . . . 359

internal work to................ 366

to work of digestion and assimilation. .... 408

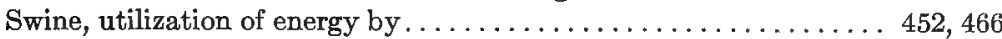

Temperature, body. . . . . . . . . . . . . . . . . . . . . . 347

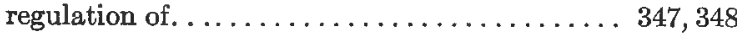

chemical. . . . . . . . . . . . . 352

means of . . .............. 348

physical. ................. 351

critical. .............................. 353

method of heat emission above. . . . . . . . 355

modification of conception of . .......... 357

influence of, on heat production . . . . . . . . . . . 351

rate of emission of heat. . . . . . . . . 350

Thermal environment, critical. . . . . . . . . . . . . . . . 358

influence of, on heat production in fasting. . . . . 347

maintenance ration. . . . . . . . 435

utilization of energy.......... 471

Thermic range. . . . . . . . . . . . . . . . . . . . . . . . . . 348

Thermo-chemistry. . . . . . . . . . . . . . . . . . . . . . . . . 228

Time element, influence of, on heat production. . . . . . . . . . . . . 439

maintenance ration. . . . . . . . . . . 439

Timothy hay, metabolizable energy of. . . . . . . . 287, 290, 297, 301 net availablity of energy of. . . . . . . . . . . 424, 428

Tissue. .................................. 59 active, fasting metabolism proportional to. ..........86,93 adipose. . .............................. 29 building, expenditure of energy in digestion, assimilation, and . . 491 loss of energy in . . . . . . . . . . . . . . 444, 447 utilization of energy in. .........4444,447,448,461

by carnivora. . . ....... 448, 466

$\operatorname{man} . . . \ldots \ldots \ldots . . . . . .451$

ruminants. . . . . 455,461, 467

swine. . . . . . . . . 452, 466 
PAGE

Tissue, building, utilization of energy in, earlier experiments on. . . . . 460

effect of amount of food on ....466

character of food on. . . 472

differences in live weight

on............4 457

thermal environment on 471

constant loss of, in fasting. ................... 83

gain of potential energy in . . . . . . . . . . . . . . . . 244

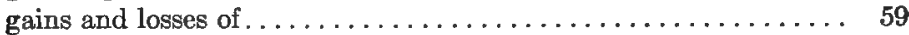

determination of.................. 60

mass of, relation of heat production to . . . . . . . . . . 370

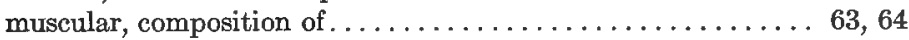

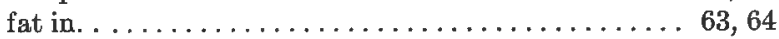

glycogen in. . . . . . . . . . 64

heat of combustion of . . . . . . . .

Tonus, muscular. . . . . . . . . . . . . . . . . . . . . 190

influence of, on heat production............. 191

metabolism in ....................... 190

work of........................... 341

Training, influence of, on utilization of energy in work. . . . . . . . 519

Transformation of energy in body ..................... 2

muscular contraction. . ............ 495

Trot, expenditure of energy in locomotion at. . . . . . . 509, 510, 514

utilization of energy in work at. . . . . . . . . . .

Tyrosin, formed from proteids....................... 39

oxidized in body......................... 52

Units of heat. . . . . . . . . . . . . . . . . . . . . 232

measurement of energy .................. 231, 233

Urea. . . . . . . . . . . . . . . . . . . . . . . . . . 42

antecedent of. .......................... 42

ammonium carbonate as.............. 43

lactate as.................. 43

as measure of proteid metabolism................. 68

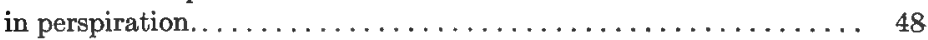

production of, from amides.................... 52

in metabolism. . . . . . . . . . . . . . 14, 15

of proteids............. 42

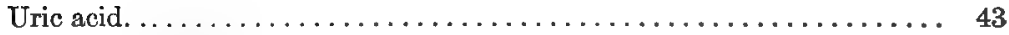

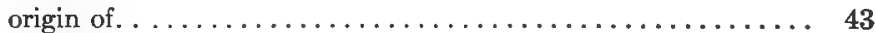

in perspiration. ......................... 48

urine. ............................ 43

Urine, aromatic compounds in........................ 46

computation of potential energy of ............. 241, 313

conjugated sulphuric acid in ................. 46 
Urine, hippuric acid in . ................................ PAGE

ind

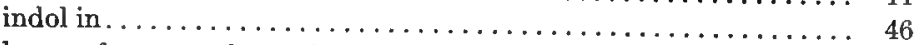

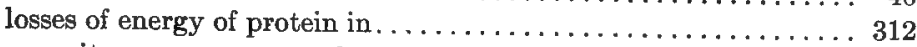

non-nitrogenous matter of. . . . . . . . . . . .

amount of ............... 28

derived from coarse fodders........ 28

non-nitrogenous matter. 321

influence of ................. 320

source of ................. 27,321

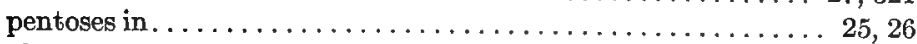

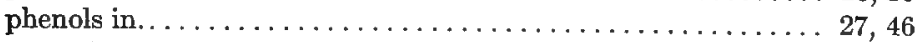

potential energy of. . . . . . . . $\ldots \ldots \ldots 272,275,278,312$

computation of ........... 241, 277, 312

uric acid in

Utilization of energy. See Energy.

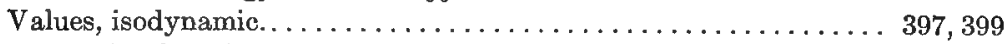

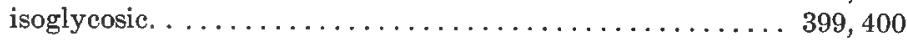

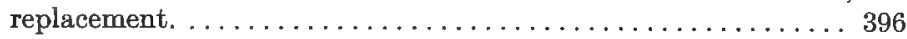

modified conception of................ 405

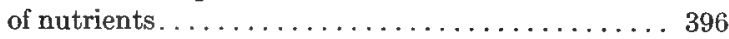

Variations in heat production, causes of . . . . . . . . . . . 363

Walking, consumption of oxygen in, by horse . . . . . . . . . . 505

expenditure of energy in, by horse.... 504, 506, 508, 510, 533, 539

utilization of energy in, by horse $\ldots \ldots \ldots \ldots \ldots \ldots \ldots .513$

Water, consumption of, influence of, on heat production. . . . . . . . 438

maintenance ration......... 438

determination of, by respiration apparatus........... 79

production of, in metabolism................. 14, 15

of carbohydrates. . ....... 23, 27

fat. ................. 36

proteids............. 42

Wheat gluten, digestible protein of, metabolizable energy of . . . . . 310, 317

gross energy of...................... 309

utilization of energy of . . . . . . 481, 491

metabolizable energy of . . . . . . . . 29. 297, 301

digestible matter of . . . . . . 301

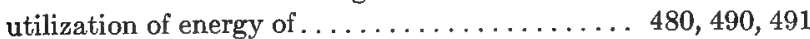

straw, digestible carbohydrates of, metabolizable energy of . . . . 329

crude fiber of, metabolizable energy of . . . 330, 332

matter of, gross energy of.............. 310

metabolizable energy of ....... 300,301

utilization of energy of. ......... 487

protein of, metabolizable energy of . . . . . . . . 321, 332

metabolizable energy of ............290, 297, 300, 301 
Wheat straw, utilization of energy of . . . . . . .

Wind, influence of, on heat emission...................... 357

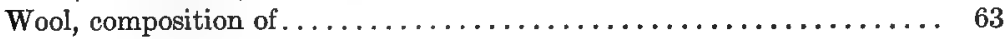

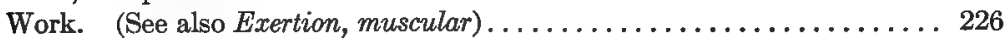

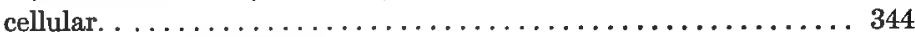

change in respiratory quotient caused by ............. 212

coefficient of utilization in. .................. 498

disappearance of muscular glycogen in . . . . . . . . . . 23

gain of proteids during ....................... 204

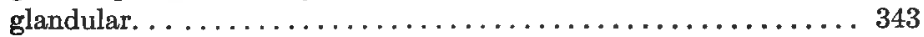

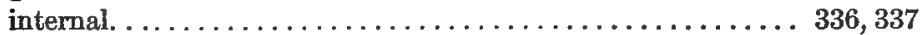

fasting heat production a measure of........... 344

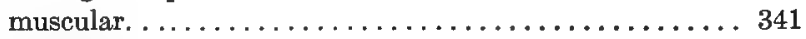

relation of, to surface.................. 366

kind of, influence of, on efficiency of animal. . . . . . . . . 512

mechanical, determination of.................... 245

muscular, disappearance of glycogen in . . . . . . . . . . 23

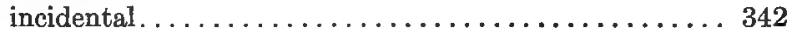

net available energy for. . . . . . . . . . . . . . . . 497

of ascent, consumption of oxygen in, by $\operatorname{dog} \ldots \ldots \ldots \ldots \ldots \ldots 500$

corrected. ............................ . 508

utilization of energy in. .......... 502,503,510

by $\operatorname{dog} . \ldots \ldots \ldots \ldots \ldots \ldots 50 \ldots \ldots$

horse............... 506

man................ 503

effect of grade on......... 512

load on. . . . . 5. 509, 510

circulation......................... 191, 341

descent. . .................................. 509

influence of grade on. .................. 509

digestion and assimilation. . ...... 80, 93, 337, 372, 376, 406, 493

above critical point.......... 407

below critical point........... 406

indirect utilization of heat from... 406

in $\operatorname{dog} . \ldots \ldots \ldots \ldots \ldots . \ldots . \ldots 378$

horse.................... 385

man.................. 382

methods of determining. ....... 377

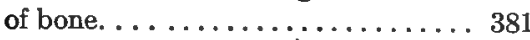

carbohydrates. .......3 379, 382,384

fat. .......... 378, $382,384,385$

mixed diet. . . . . . . . . 382. 384

proteids........... 381, 382,384

relation of, to surface..........4 408 


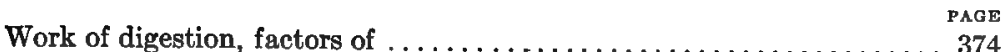

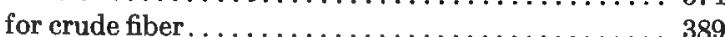

draft, consumption of oxygen in, by dog................ 501

utilization of energy in. ............... 502, 507, 513

by $\operatorname{dog} . \ldots \ldots \ldots \ldots \ldots \ldots, \ldots \ldots 2$

horse............507,513

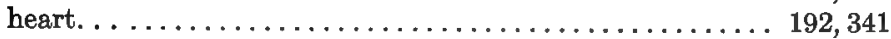

locomotion, computation of. ................... 512

consumption of oxygen in, by dog. . . . . . . . . 500

horse.......... 505

correction for speed in. . . . . . . . . . 507, 508

expenditure of energy in, by dog. . . . . . . . . 500

horse.... 504, 506, 508, 509

$510,514,533,539$

utilization of energy in, computed. . .......... 513

mastication. ................................ 391

muscular tonus................................. 341

respiration............................. 192, 341

standing. ................................. 343

voluntary muscles. . . . . . . . . . . . . . . . . . . . . . 337

physiological. . . . . . . . . . . . . . . . . . . . . . . 336

production, function of liver in $\ldots \ldots \ldots \ldots \ldots \ldots \ldots \ldots \ldots \ldots \ldots \ldots \ldots \ldots$

relative value of nutrients in............... 522

coarse fodder and grain

for $\ldots \ldots \ldots \ldots \ldots . \ldots 533$

value of crude fiber for............... 535, 537

fat for............................ 522

utilization of energy in. . . . . . . .

by $\operatorname{dog} \ldots \ldots \ldots \ldots \ldots \ldots \ldots \ldots \ldots, 499$

horse...................... 502

at a trot..................509

walk. ..............., 504

man. ..................... 502

influence of fatigue on.............. 519

individuality on.......... 517

kind of work on.......... 512

load on............... 508

size of animal on.......... 515

species on. ............. 515

speed on......... 507, 513,514

training on. . . .......... 519

metabolizable energy in .................. 525

methods of determina-

tion. ........... 526, 528 
PAGF

Work, utilization of metabolizable energy in, Wolff's investigations. . . . . 528

of feeding-stuffs in........ 510

fiber-free nutrients in 541,543 ,

545,547

net available energy in................ 497

variations of respiratory quotient during. .............. 216 
Digitized by Microsoft@ 
Digitized by Microsoft@ 


\section{SHORT-TITLE CATALOGUE \\ OF THE PUBLICATIONS}

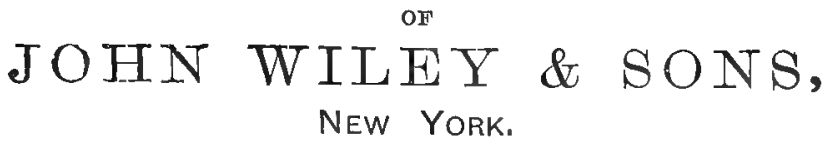

LONDON: CHAPMAN \& HALL, LIMITED.

\section{ARRANGED UNDER SUBJECTS.}

Descriptive circulars sent on application.

Books marked with an asterisk are sold at net prices only.

All books are bound in cloth unless otherwise stated.

\section{AGRICULTURE.}

Armsby's Manual of Cattle-feeding.............12mo, \$1 76 Budd and Hansen's American Horticultural Manual:

Part I.-Propagation, Culture, and Improvement....12mo, 150 Part II.-Systematic Pomology. (In preparation.)

Downing's Fruits and Fruit-trees of America...........8vo, 500 Grotenfelt's Principles of Modern Dairy Practice. (Woll.)..12mo, 200 Kemp's Landscape Gardening....................12mo, 260 Maynard's Landscape Gardening as Applied to Home Decoration.

Sanderson's Insects Injurious to Staple Crops..........12mo, i 50 “ Insects Injurious to Garden Crops. (In preparation.)

"Insects Injuring Fruits. (In preparation.)

Stockbridge's Rocks and Soils.................. 8vo, 250 Woll's Handbook for Farmers and Dairymen...........16mo, I 50

\section{ARCHITECTURE.}

Baldwin's Steam Heating for Buildings............12mo, 250 Berg's Buildings and Structures of American Railroads....4to, 500 Birkmire's Planning and Construction of American Theatres.8vo, 300

"s Architectural Iron and Steel...............8vo, 350

" Compound Riveted Girders as Applied in Buildings. $8 \mathrm{ro}, 200$

“ Planning and Construction of High Office Buildings.

$8 \mathrm{vo}, 350$

“ Skeleton Construction in Buildings...........8vo, 300

Briggs's Modern American School Buildings..........8vo, 400

Carpenter's Heating and Ventilating of Buildings....... .8vo, 400

Freitag's Architectural Engineering. 2d Edition, Rewritten.8vo, 350

" Fireproofing of Steel Buildings............8vo, 250

Gerhard's Guide to Sanitary House-inspection..........16mo, 100

"Theatre Fires and Panics.................12mo, 150

Hatfield's American House Carpenter.................... 500

Holly's Carpenters' and Joiners' Handbook............ 18mo, 75

Kidder's Architect's and Builder's Pocket-book. .16mo, morocco, 400

Merrill's Stones for Building and Decoration.............8vo, 500

Monckton's Stair-building......................4to, 400 
Patton's Practical Treatise on Foundations............8vo,

Siebert and Biggin's Modern Stone-cutting and Masonry..8vo,

Snow's Principal Species of Wood: Their Characteristic Properties. (In preparation.)

Wait's Engineering and Architectural Jurisprudence......8vo, Sheep,

" Law of Operations Preliminary to Construction in Engineering and Architecture.................8vo, Sheep,

" Law of Contracts................... 8vo,

Woodbury's Fire Protection of Mills.................. 8vo,

Worcester and Atkinson's Small Hospitals, Establishment and Maintenance, and Suggestions for Hospital Architecture, with Plans for a Small Hospital................12mo, The World's Columbian Exposition of 1893.................. 4to, 100

\section{ARMY AND NAVY.}

Bernadou's Smokeless Powder, Nitro-cellulose, and the Theory of the Cellulose Molecule..................12mo,

- Bruff's Text-book Ordnance and Gunnery................ 8vo, Chase's Screw Propellers and Marine Propulsion.........8vo, Craig's Azimuth........................4to, Crehore and Squire's Polarizing Photo-chronograph......8vo, Cronkhite's Gunnery for Non-commissioned Ufficers..24mo, mor., - Davis's Elements of Law.......................... 8vo,

- "Treatise on the Military Law of United States...8vo,

De Brack's Cavalry Outpost Duties. (Carr.) ....24mo, moroceo, Dietz's Soldier's First Aid Handbook...........16mo, moroceo,

- Dredge's Modern French Artillery..........4to, half morocco, 1500 Durand's Resistance and Populsion of Ships............8vo, 500 - Dyer's Handbook of Light Artillery............... 12mo, 300 Eissler's Modern High Wixplosives................8vo, 400

- Fiebeger's Text-book on Field Fortification........ Small 8vo, 200 Hamilton's The Gunner's Catechism...............18mo, 100

- Hoff's Elementary Naval Tactics........................... 150 Ingalls's Handbook of Problems in Direct Fire..........8vo, 400 * " Ballistic Tables............................8vo, 150

Lyons's Treatise on Electromagnetic Phenomena.........8vo, 600

- Mahan's Permanent Fortifications. (Mercur.)..8vo, half mor., 750 Manual for Courts-martial...............6mo, morocco, 150

- Mercur's Attack of Fortified Places................ 12mo, 200

- "Elements of the Art of War..................... $4 \mathrm{vo}, 40$

Metcalf's Cost of Manufactures-And the Administration of Workshops, Public and Private...........8vo, 500

- “ Ordnance and Gunnery..................12mo, 500

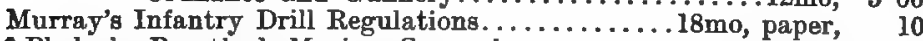

- Phelps's Practical Marine Surveying.................8vo, 250

Powell's Army Officer's Examiner........................... 400

Sharpe's Art of Subsisting Armies in War...... 18mo, moroceo, 150

Walke's Lectures on Explosives...................8vo, 400

-Wheeler's Siege Operations and Military Mining.........8.8vo, 200

Winthrop's Abridgment of Military Law.............12mo, \& 50

Woodhull's Notes on Military Hygiene...............

Young's Simple Elements of Navigation........16mo, morocco, 100 Second Edition, Fnlarged and Revised.......16mo, mor., 2 no 


\section{ASSAYING.}

Fletcher's Practical Instructions in Quantitative Assaying with the Blowpipe.....................12mo, morocco,

Furman's Manual of Practical Assaying................. . .

Miller's Manual of Assaying......................12mo,

O'Driscoll's Notes on the Treatment of Gold Ores..........8vo,

Ricketts and Miller's Notes on Assaying................8vo,

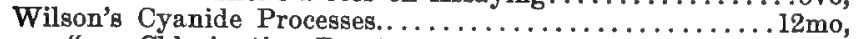
Chlorination Process.....................

\section{ASTRONONY.}

Craig's Azimuth..........................4to,

Doolittle's Treatise on Practical Astronomy............... 8vo,

Gore's Elements of Geodesy.....................8vo,

Hayford's Text-book of Geodetic Astronomy............... 8 vo,

Merriman's Elements of Precise Surveying and Geodesy.... .8vo,

- Michie and Harlow's Practical Astronomy............ 8vo,

-White's Elements of Theoretical and Descriptive Astronomy.

$12 \mathrm{mo}, 200$

\section{BOTANY.}

Baldwin's Orchids of New England.............. Small 8vo, Davenport's Statistical Methods, with Special Reference to Biological Variation.................16mo, morocco,

Thome and Bennett's Structural and Physiological Botany. $16 \mathrm{mo}, 225$

Westermaier's Compendium of General Botany. (Schneider.) 8vo, 200

\section{CHEMISTRY.}

Adriance's Laborstory CaIculations and Specific Gravity Tables.

$12 \mathrm{mo}, 125$

Allen's Tables for Iron Analysis................... 8vo. 300

Arnold's Compendium of Chemistry. (Mandel.) (In preparation.)

Austen's Notes for Chemical Student3..............12mo, 150

Bernadou's Smokeless Powder.-Nitro-cellulose, and Theory of

the Cellulose Molecule.....................

Bolton's Quantitative Analysis...................8vo, 150

Brush and Penfield's Manual of Determinative Mineralogy...8vo, 400

Classen's Quantitative Chemical Analysis by Electrolysis. (Herrick-Boltwood.) ....................... 300

Cohn's Indicators and Test-papers.................12mo, 200

Craft's Short Course in Qualitative Chemical Analysis. (Schaeffer.) ..................................... 200

Drechsel's Chemical Reactions. (Merrill.).............12mo, 125

Eissler's Modern High Explosives...................8vo, 400

Effront's Enzymes and their Applications. (Prescott.)...8vo, 300

Erdmann's Introduction to Chemical Preparations. (Dunlap.)

Fletcher's Practical Instructions in Quantitative Assaying with the Blowpipe....................12mo, morocco, 150

Fowler's Sewage Works Analyses. ...............12mo, 200

Fresenius's Manual of Qualitative Chemical Analysis. (Wells.) 8vo, 500 " Manual of Qualitative Chemical Analysis. Part I. Descriptive. (Wells.) ................ 3 vo, 300

" System of Instruction in Quantitative Chemical Analysis. (Allen.)................8vo, 600 
Fuertes's Water and Public Health...............12mo,

Furman's Manual of Practical Assaying.................. 8vo,

Gill's, Gas and Fuel Analysis for Engineers...........12mo,

Grotenfelt's Principles of Modern Dairy Practice. (Woll.)..12mo,

Hammarsten's Text-book of Physiological Chemistry. (Mandel.)

8vo,
(Helm.s

Helm's Principles of Mathematical Chemistry. (Morgan.) 12mo,

Hinds's Inorganic Chemistry. . . . . . . . . . . . . . 8vo,

* " Laboratory Manual for $\mathrm{S}$ dents................. 12mo,

Holleman's Text-book of Inorganic Chemistry. (Cooper.) ...8vo, " " "Organic " (Walker and Mott.)

(In preparation.)

Hopkins's Oil-chemists' Handbook....................8vo, 300

Keep's Cast Iron...................................... 250

Ladd's Manual of Quantitative Chemical Analysis........12mo, I 00

Landauer's Spectrum Analysis. (Tingle.)..............8vo, 300

Lassar-Cohn's Practical Urinary Analysis. (Lorenz.) (In preparation.)

Leach's The Inspection and Analysis of Food with Special Reference to State Control. (In preparation.)

Löb's Electrolysis and Electrosynthesis of Organic Compounds.

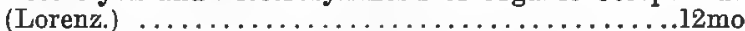

Mandel's Handbook for Bio-chemical Laboratory.......12mo,

Mason's Water-supply. (Considered Principally from a Sanitary Standpoint.) 3d Edition, Rewritten......8vo, “ Examination of water. (Chemical and Bacteriological.) ........................... 12mo,

Meyer's Determination of Radicles in Carbon Compounds.

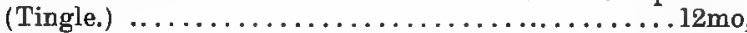

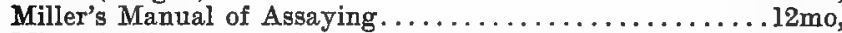

Mixter's Elementary Text-book of Chemistry............ I2mo,

Morgan's Outline of Theory of Solution and its Results. . 12mo,

Nicho Elements of Physical Chemistry.............12mo, and Sanits Stand considered mainiy from a Chemical

O'Brine's Laboratory Guide in Chemical Analysis......... . . 8vo,

O'Driscoll's Notes on the Treatment of Gold Ores..........8vo,

Ost and Kolbeck's Text-book of Chemical Technology. (Lorenz-Bozart.) (In preparation.)

- Penfield's Notes on Determinative Mineralogy and Record of Mineral Tests..................... 8vo, paper,

Pinner's Introduction to Organic Chemistry. (Austen.) 12mo,

Poole's Calorifie Power of Fuels. Ch..................

.

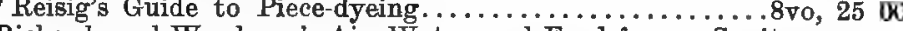

Richards and Woodman's Air, Water, and Food from a Sanitary

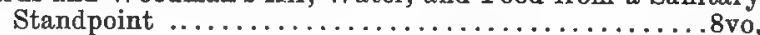

Richards's Cost of Living as Modified by Sanitary Science $12 \mathrm{mo}$,

" Cost of Food, a Study in Dietaries...........12mo,

- Richards and Williams's The Dietary Computer............

Ricketts and Russell's Skeleton. Notes upon Inorganic Chemistry. (Part I.-Non-metallic Elements.) . .8vo, moroceo,

Ricketts and Miller's Notes on Assaying.............. 8vo,

Rideal's Sewage and the Bacterial Purification of Sewage..8vo,

Ruddiman's Incompatibilities in Prescriptions........... $8 \mathrm{vo}$,

Schimpf's 'Text-book of Volumetric Analysis.............

Spencer's Handbook for Chemists of Beet-sugar Houses. $16 \mathrm{mo}$,

a Handbook for Sugar Manufacturers and their Cher.,

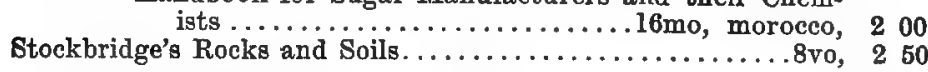

200

100

100

150 
* 'Tillman's Elementary Lessons in Heac...............8vo, 150

Descriptive General Chemistry.................

Turneaure and Russell's Public Water-supplies............

Van Deventer's Physical Chemistry for Beginners. '(Boltwood.)

Walke's Lectures on Explosives................ 150

Wells's Laboratory Guide in Qualitative Chemical Anal...8vo, 400

"s Short Cours in

sis for Hn lnorganic Quallative Chemical Analy-

Engineering Students............12mo, 150

Wipple

Wiechmann's Sugar Analysis... ........................... 8vo, 250

Wilson's Cyanide Processes... Theoretical Chemistry....12mo, 300

Wilson's Cyanide Processes............................ I 50

Wulling's Elorination Process......................... 150

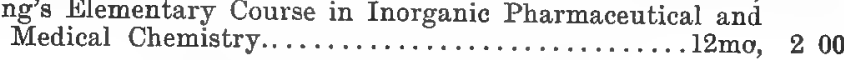

CIVIL ENGINEERING.

\section{BRIDGES AND ROOFS. HYDRAULICS. MATERIALS OF} ENGINEERING. RAILWAY ENGINEERING.

Baker's Engineers' Surveying Instruments...........12mo, 300

Bixby's Graphical Computing Table...Paper, $19 \frac{1}{2} \times 241$ inches. 25

Davis's Elevation and Stadia Tables.................. 8 vo, 100

Folwell's Sewerage. (Designing and Maintenance.).......8vo, 300

Freitag's Architectural Engineering. 2d Eid., Rewritten...8vo, 350

French and Ives's Stereotomy..................8vo, 250

Goodhue's Municipal Improvements...................... 12 mo, 75

Goodrich's Economic Disposal of Towns' Refuse.........8vo, 350

Gore's Elements of Geodesy.................... 8vo, 2 50

Hayford's Text-book of Geodetic Astronomy............... 3 vo, 300

Howe's Retaining-walls for Earth...................12mo, i 25

Johnson's Theory and Practice of Surveying...........Small 8vo, 400

" Stadia and Earth-work Tables..................8vo, 125

Kiersted's Sewage Disposal.........................

Laplace's Philosophical Essay on Probabilities. (Truscott and

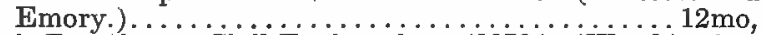

Mahan's Treatise on Civil Engineering. (1873.) (Wood.)..8vo,

- Mahan's Descriptive Geometry..................8vo,

Merriman's Elements of Precise Surveying and Geodesy....8 8vo,

Merriman and Brooks's Handbook for Surveyors....16mo, mor.,

Merriman's Elements of Sanitary Engineering............8vo,

Nugent's Plane Surveying........................8vo,

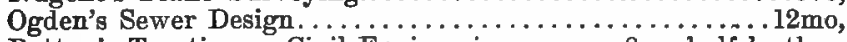

Patton's Treatise on Civil Engineering.......8vo, half leather,

Reed's Topographical Drawing and Sketching..........4to,

Rideal's Sewage and the Bacterial Purification of Sewage...8vo,

Siebert and Biggin's Modern Stone-cutting and Masonry... .8vo,

Smith's Manual of Topographical Drawing. (McMillan.) . .8vo,

"Trautwine's Civil Engineer's Pocket-book....16mo, morocco,

Wait's Engineering and Architectural Jurisprudence......8vo,

“ Law of Operations Preliminary to Construction in Engineering and Architecture.............. 8vo,

Sheep,

Wait's Law of Contracts................... 3 vo, 0

Warren's Stereotomy-Problems in Stone-cutting........8vo, 250

Webb's Problems in the Use and Adjustment of Engineering Instruments ................... 
* Wheeler's Elementary Course of Civil Engineering....... \& \&o,

Wilson's Topographic Surveying..................... 3 vo, 30

\section{BRIDGES AND ROOFS.}

Boller's Practical Treatise on the Construction of Iron Highway Bridges ..............................8vo,

- Boller's Thames River Bridge...............4to, paper,

Burr's Course on the Stresses in Bridges and Roof Trusses, Arched Ribs, and Suspension Bridges...........8vo,

Du Bois's Mechanics of Fingineering. Vol. II.......... Small 4to, 1000

Foster's Treatise on Wooden Trestle Bridges..........4to, 500

Fowler's Coffer-dam Process for Piers................8vo, 250

Greene's Roof Trusses.................................. 125

" Bridge Trusses......................8vo, 250

" Arches in Wood, Iron, and Stone.............. $8 \mathrm{vo}, 250$

Howe's Treatise on Arches.......................8vo, 400

" Design of Simple Roof-trusses in Wood and Steel. 8vo, 200

Johnson, Bryan and Turneaure's Theory and Practice in the

Designing of Modern Framed Structures...... Sraall 4to, 1000

Merriman and Jacoby's Text-book on Roofs and Bridges:

Part I.-Stresses in Simple Trusses................8vo, 250

Part II.-Graphic Staties......................

Part III.-Bridge Design. Fourth Ed., Rewritten.....8vo, 250

Part IV.-Higher Structures...................8vo, 250

Morison's Memphis Bridge.......................4to, 1000

Waddell's De Pontibus, a Pocket Book for Bridge Engineers.

" Specifications for Steel Bridges............ 12mo,

Wood's Treatise on the Theory of the Construction of Bridges

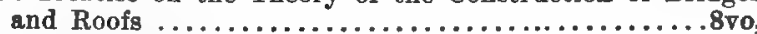

Wright's Designing of Draw-spans:

Part I.-Plate-girder Draws.................

Part II.-Riveted-truss and Pin-connected Long-span Draws.

Two parts in one volume.................. 8vo,

\section{HYDRAULICS.}

Bazin's Experiments upon the Contraction of the Liquid Vein

Issuing from an Orifice. (Trautwine.) ............ 8vo,

Bovey's Treatise on Hydraulies..................... 8vo,

Church's Mechanics of Engineering.................8vo,

" Diagrams of Mean Velocity of Water in Open Channels

Coffin's Graphical Solution of Hydraulic Problems 16 paper,

Flather's Dynamometers, and the Measurement of Power.12mo,

Folwell's Water-supply Engineering...................8vo,

Frizell's Water-power...........................8vo,

Tuertes's Water and Public Health................ 12mo,

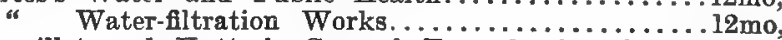

Ganguillet and Kutter's General Formula for the Uniform

Flow of Water in Rivers and Other Channels. (Her-

ing and Trautwine.)..........................8vo,

Hazen's Filtration of Public Water-supply................8vo,

Hazlehurst's Towers and Tanks for Water-works.......8vo,

Eerschel's 115 Experiments on the Carrying Capacity of Large,

Riveted, Metal Conduits....................8vo, 
Mason's Water-supply. (Considered Principally from a Sanitary Standpoint.) ........................8vo,

Merriman's Treatise on Hydraulics........................

- Michie's Elements of Analytical Mechanics..................

Bchuyler's Reservoirs for Irrigation, Water-power, and Domestic Water-supply........................ Large 8vo,

Turneaure and Russell. Public Water-supplies........... 8vo,

Wegmann's Design and Construction of Dams..............4to, Water-supply of the City of New York from 1658 to

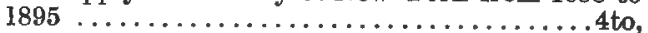

Weisbach's Hydraulics and Hydraulic Motors. (Du Bois.)..8vo, Wilson's Manual of Irrigation Engineering......... Small 8vo, Wolff's Windmill as a Prime Mover..................8vo, Wood's Turbines...................................

" Elements of Analytical Mechanies.............8vo,

\section{MATERIALS OF ENGINEERING.}

Baker's Treatise on Masonry Construction............8vo, Black's United States Publie Works.............. Oblong 4to, Bovey's Strength of Materials and Theory of Structures... 8vo, Burr's Elasticity and Resistance of the Materials of Engineering ................................8vo,

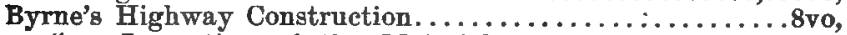
" Inspection of the Materials and Workmanship Employed in Construction..................16mo,

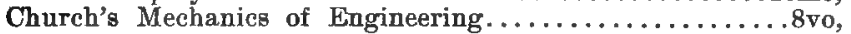
Du Bois's Mechanics of Engineering. Vol. I........... Small 4to, Johnson's Materials of Construction................ Large Rvo, Keep's Cast Iron.........................8 8vo, Lanza's Applied Mechanics....................87o, Martens's Handbook on Testing Materials. (Henning.).2 v., 8vo, Merrill's Stones for Building and Decoration...........8vo, Merriman's Text-book on the Mechanics of Materials.....8vo, Merriman's Strength of Materials.................12mo, Metealf's Steel. A Manual for Steel-users...............12mo,

Patton's Practical Treatise on Foundations.............8 8o, Rockwell's Roads and Pavements in France............12mo, Smith's Wire: Its Ure and Manufacture...........Small 4to, "Materials of Machines.................12mo,

Snow's Principal Species of Wood: Their Characteristic Properties. (In preparation.)

Spalding's Hydraulic Cement.....................12mo, " Text-book on Roads and Pavements............12mo,

Thurston's Materials of Engineering...........3 Parts, 8vo, Part I.-Non-metallic Materials of Engineering and Metal-

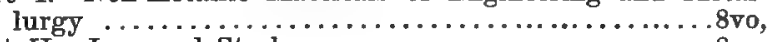

Part II.-Iron and Steel.................... 8vo,

Part III.-A Treatise on Brasses, Bronzes and Other Alloys and Their Constituents................... Thurston's Text-book of the Materials of Construction.... 8vo, Tillson's Street Pavements and Paving Materials.........8vo, Waddell's De Pontibus. (A Pocket-book for Bridge Engineers.) $16 \mathrm{mo}$, moroceo,

“ Specifications for Steel Bridges............. 12mo, 500

Wood's Treatise on the Resistance of Materials, and an Appendix on the Preservation of Timber......... 8vo, Elements of Analytical Mechanics............8vo, 


\section{RAILWAY ENGINEERING.}

Lndrews's Handbook for Street Railway/Engineers. 3x5 in. mor., 125 Serg's Buildinga and Structures of American Railroads...4to, 500

Brooks's Handbook of Street Railroad Location..16mo, morocco, 150

Butts's Civil Engineer's Field-book..........16mo, morocco, 250

Crandall's Transition Curve...............16mo, morocco, 150

" Railway and Other Earthwork Tables.......8vo, 150

Dawson's Electric Railways and Tramways. Small 4to, half mor., 1250 "Fingineering" and Electric Traction Pocket-book.

$16 \mathrm{mo}$, morocco, 400

Dredge's History of the Pennsylvania Railroad: (1879.).Paper, 500

- Drinker's Tunneling, Fxplosive Compounds, and Rock Drills. 4 to, half morocco, 2500

Fisher's Table of Cubic Yards.................. Cardboard, Godwin's Railroad Engineers' Field-book and Explorers' Guide. $16 \mathrm{mo}$, morocco,

Howard's Transition Curve Field-book........16mo, morocco, Hudson's Tables for Calculating the Cubic Contents of Excavations and Embankments.................. 8vo,

Nagle's Field Manual for Railroad Engineers. ... 16mo, morocco, Philbrick"s Field Manual for Engineers........16mo, morocco, Pratt and Alden's Street-railway Road-bed.............8vo, Bearles's Field Engineering.............16mo, morocco, " Railroad Spiral.................16mo, morocco, Taylor's Prismoidal Formulæe and Earthwork..........8vo,

- Trautwine's Method of Calculating the Cubic Contents of Excavations and Embankments by the Aid of Diagrams ............................

- 6 The Field Praetice of Laying Out Circular Curves for Railroads................. I2mo, moroceo,

Webb's Railroad Construction.............................. Proper, Wellington's Economic Theory of the Location of Railways..

Small 8vo, 500

\section{DRAWING.}

Barr's Kinematics of Machinery.................... 8vo,

- Bartlett's Mechanical Drawing...................... 8vo, Coolidge's Manual of Drawing. . . . . . . . . . . 8vo, paper, Durley's Elementary Text-book of the Kinematics of Machines.

Hill's Text-book on Shades and Shadows, and Perspective..8vo, Jones's Machine Design:

Part I. - Kinematies of Machinery............... 8vo,

Part II.-Form, Strength and Proportions of Parts. . . . .8 8vo,

MacCord's Elements of Descriptive Geometry............ . 8vo, " Kinematics; or, Practical Mechanism.........8vo,

" Mechanical Drawing....................4to,

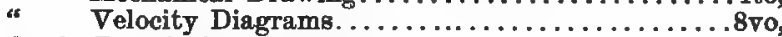

- Mahan's Descriptive Geometry and Stone-cutting........ 8vo,

Mahan's Indugtrial Drawing. (Thompson.).............8vo,

Reed's Topographical Drawing and Sketching............4to,

Reid's Course in Mechanical Drawing....................

"Text-book of Mechanical Drawing and Eilementary Machine Design.........................8vo,

Robinson's Principles of Mechanism.................. 
Bmith's Manual of Topographical Drawing. (McMillan.) .8vo, Warren's Elements of Plane and Solid Free-hand Geometrical

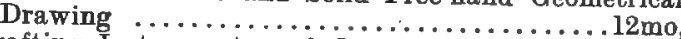

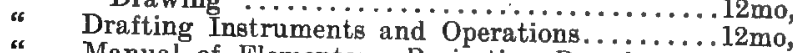

“ Manual of Elementary Projection Drawing......12mo,

Manual of Elementary Problems in the Linear Perspective of Form and Shadow.............12mo,

" Plane Problems in Elementary Geometry...........12mo, " Primary Geometry......................12mo,

"Elements of Descriptive Geometry, Shadows, and Perspective $\ldots \ldots \ldots \ldots \ldots \ldots \ldots \ldots \ldots \ldots$. . . . .

"General Problems of Shades and Shadows........ . . 8 vo,

" Elements of Machine Construction and Drawing..8vo,

" Problems, Theorems, and Examples in Descriptive Geometry .......................8vo,

Weisbach's Kinematics and the Power of Transmission. "Herrmann and Klein.) ..................... Whelpley's Practical Instruction in the Art of Letter En-

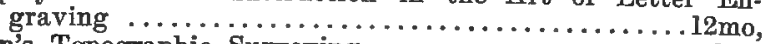

Wilson's Topographic Surveying......................

Wilson's Free-hand Perspective.......................

Woolf's Elementary Course in Descriptive Geometry. . Large 8vo,

\section{ELECTRICITY AND PHYSICS.}

Anthony and Brackett's Text-book of Physics. (Magie.)

Anthony's Lecture-notes on the Theory of Electrical Measur-

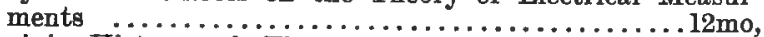

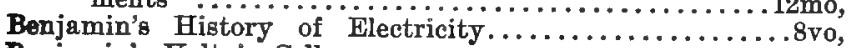

Benjamin's Voltaic Cell.............................

Classen's Qantitative Chemical Analysis by Electrolysis. Herrick and Boltwood.) .........................8vo,

Crehore and Squier's Polarizing Photo.chrono................... 300

Dawson's Electric Railways and Tramways..Small 4to, half mor., 1250

Dawson's "Engineering" and Electric Traction Pocket-book.

Flather's Dynamometers, and the Measurement of Powo, morocco,

Gilbert's De Magnete. (Mottelay.)..................8\%,

Holman's Precision of Measurements.....................

Telescopic Mirror-scale Method, Adjustments, and

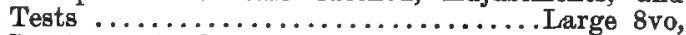

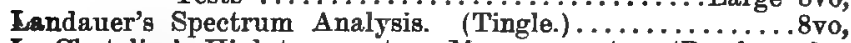
Lo Chatelier's High-temperature Measurements. (BoudouardBurgess.) ............................ 12mo,

Losb's Electrolysis and Electrosynthesis of Organic Compounds.

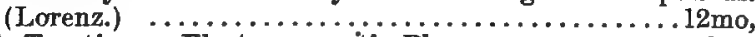

Lyons's Treatise on Electromagnetic Phenomena.......... 8vo,

- Michie. Elements of Wave Motion Relating to Sound and

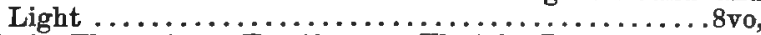
Niaudet's Elementary Treatise on Electric Batteries (Fish-

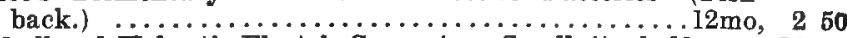

- Parshall and Hobart's Electric Generators.small 4to, half mor., 1000 Ryan, Norris, and Hoxie's Electrical Machinery. (In preparation.)

Thurston's Stationary Steam-engines............... 2 vo, 50

- Tillman. Elementary Lessons in Heat.............. 8vo, 150

Tory and Pitcher. Manual of Laboratory Physics.. Small 8vo, 200 


\section{LAW.}

- Davis. Elements of Law..................... 8vo,

Treatise on the Military Law of United States. .8vo,

Sheep,

Manual for Courts-martial $.16 \mathrm{mo}$, morocco,

Wait's Engineering and Architectural Jurisprudence..... . 8vo,

Sheep,

"Law of Operations Preliminary to Construction in Engineering and Architecture................8vo,

Sheep,

"Law of Contracts.........................8vo,

Winthrop's Abridgment of Military Law.............12mo, 250

\section{MANUFACTURES.}

Beaumont's Woollen and Worsted Cloth Manufacture....12mo, Bernadou's Smokeless Powder-Nitro-cellulose and Theory of the Cellulose Molecule..................... r2mo,

Bolland's Iron Founder.................. clotho,

" "The Iron Founder" Supplement.............12mo,

Encyclopedia of Founding and Dictionary of Foundry

Terms Used in the Practice of Moulding....12mo,

Eissler's Modern High Expiosives.................. 8vo,

Efiront's Enzymes and their Applications. (Prescott.)...8vo,

Fitzgerald's Boston Machinist.................. 18mo,

Ford's Boiler Making for Boiler Makers............18mo,

Hopkins's Oil-chemists' Handbook. . . . . . . . . . . . . .8 8 ,

Keep's Cast Iron..............................

Leach's The Inspection and Analysis of Food with Special

Reference to State Control. (In preparation.)

Metcalf's Steel. A Manual for Steel-users............12mo,

Metcalf's Cost of Manufactures-And the administration of Workshops, Public and Private............ 8 vo, 500

Meyer's Modern Loconotive Construction................4to, 1000

- Reisig's Guide to Piece-dyeing.....................8vo, 2500

Smith's Press-working of Metals.................... 300

" Wire: Its Use and Manufacture............Small 4to, 300

Spalding's Hydraulic Cement........................ 200

Spencer's Handbook for Chemists of Beet-sugar Houses.

c 16mo, morocco, ists......................16mo, moroce

Thurston's Manual of Steam-boilers, their Designs, Construetion and Operation.......................8vo,

Walke's Lectures on Explosives....................... 8vo,

West's American Foundry Practice................12mo,

" Moulder's Text-book.....................12mo,

Wiechmann's Sugar Analysis.......................... 8vo,

Wolff's Windmill as a Prime Mover................ 8vo,

Woodbury's Fire Protection of Mills.................8vo,

\section{MATHEMATICS.}

Baker's Elliptic Functions.......................8vo, 1 50

- Bass's Elements of Differential Calculus...............12mo, 400

Briggs's Elements of Plane Analytic Geometry..........12mo, 100

Chapman's Elementary Course in Theory of Equations...12mo, 150

Compton's Manual of Logarithmic Computations........12mo, 150 
Davis's Introduction to the Logic of Algebra............8vo, 150

"Dickson's College Algebra...................Large 12mo, 150

Halsted's Elements of Geometry.............................. 175

" Elementary Synthetic Geometry................8vo, 150

* Johnson's Three-place Logarithmic Tables: Vest-pocket size,

pap., $\quad 15$

100 copies for 500

* $\quad$ Mounted on heavy cardboard, $8 \times 10$ inches, 25

10 copies for 200

“ Elementary Treatise on the Integral Calculus.

“ Small 8vo, 150

"Curve Tracing in Cartesian Co-ordinates.......12mo, 100

"Treatise on Ordinary and Partial Differential Equations ................................ 8 vo, 350

"Theory of Errors and the Method of Least

- “ Squares ...........................12mo,

Laplace's _Philosophical Essay on Probabilities. (Truscott and

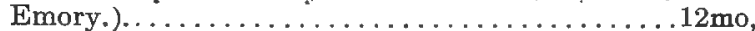

- Ludlow and Bass. Elements of Trigonometry and Logarith-

mic and Other Tables.....................8vo,

"Trigonometry. Tables published separately...Each,

Merriman and Woodward. Higher Mathematics..........8vo,

Merriman's Method of Least Squares................ 8vo,

Rice and Johnson's Elementary Treatise on the Differential Calculus ................. Small 8vo, “ Differential and Integral Calculus. 2 vols. in one.................. Small 8vo,

Wood's Elements of Co-ordinate Geometry.............. 150

\section{MECHANICAL ENGINEERING.}

\section{MATERIALS OF ENGINEERING, STEAM ENGINES AND BOILERS.}

Baldwin's Steam Heating for Buildings. .............12mo,

Barr's Kinematics of Machinery..................8vo,

- Bartlett's Mechanical Drawing.................... 8vo,

Benjamin's Wrinkles and Recipes................12mo,

Carpenter's Experimental Engineering.............. 8vo,

"Heating and Ventilating Buildings. . . . . . . . .8vo,

Clerk's Gas and Oil Engine................................

Coolidge's Manual of Drawing. ............ 8vo, paper,

Cromwell's Treatise on Toothed Gearing...................12mo,

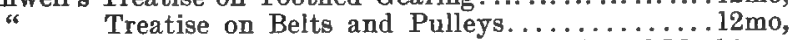

Durley's Elementary Text-book of the Kinematics of Machines. (In preparation.)

Flather's Dynamometers, and the Measurement of Power ..12mo,

" Rope Driving......................12mo,

Gill's Gas an Fuel Analysis for Engineers............ 12mo,

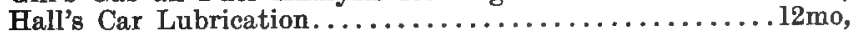
Jones's Machine Design:

Part I.-Kinematies of Machinery................8vo, 150

Part II.-Form, Strength and Proportions of Parts......8vo, 300

Kent's Mechanical Engineers' Pocket-book....16mo, morocco, 500

Kerr's Power and Power Transmission...............8vo, 200 
MacCord's Kinematics; or, Practical Mechanism........8vo, " Mechanical Drawing. .....................4to

"Velocity Diagrams...................8vo,

Mahan's Industrial Drawing. (Thompson.).............. 8vo,

Poole's Calorific Power of Fuels...................8vo,

Reid's Course in Mechanical Drawing................... 8vo,

" Text-book of Mechanical Drawing and Elementary

Machine Design...........................8vo,

Richards's Compressed Air..................... 12mo,

Robinson's Principles of Mechanism..................

Smith's Press-working of Metals...................8vo,

Thurston's Treatise on Friction and Lost Work in Machinery and Mill Work.................. 8vo, “ Animal as a Machine and Prime Motor and the Laws of Energetics...................12mo,

Warren's Elements of Machine Construction and Drawing. . 8vo, Weisbach's Kinematics and the Power of Transmission. (Herrmann-Klein.) ....................

" Machinery of Transmission and Governors. (Herrmann-Klein.) .....................8vo,

" Hydraulies and Hydraulic Motors. (Du Bois.).8vo,

Wolff's Windmill as a Prime Mover.................8vo,

Wood's Turbines.............................

\section{MATERIALS OF ENGINEERING.}

Bovey's Strength of Materials and Theory of Structures. .8vo, Burr's Elasticity and Resistance of the Materials of Engineer-

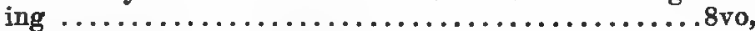

Church's Mechanics of Engineering................ Johnson's Materials of Construction.................... 8vo, Keep's Cast Iron ..........................8vo, Lanza's Applied Mechanics.....................8vo, Martens's Handbook on Testing Materials. (Henning.)....8vo, Merriman's Text-book on the Mechanies of Materials....8vo, " Strength of Materials...................12mo,

Metcalf's Steel. \& Manual for Steel-users.............12mo, Smith's Wire: Its Use and Manufacture........... Small 4to, " Materials of Machines....................12mo, Thurston'в Materials of Engineering.............. vols., 8vo,

Part II.-Iron and Steel..................... 8vo,

Part III.-A Treatise on Brasses, Bronzes and Other Alloys and their Constituents...................8vo,

Thurston's 'Text-book of the Materials of Construction....8vo, Wood's Treatise on the Resistance of Materials and an Appendix on the Preservation of Timber.........8vo,

" Elements of Analytical Mechanics.............8vo,

\section{STEAM ENGINES AND BOILERS.}

Carnot's Reflections on the Motive Power of Heat. (Thurston.)

Dawson's "Engineering" and Electric Traction Pocket-book.

Ford's Boiler Making for Boiler Makers.............. 18mo, 100 Goss's Locomotive Sparks........................ 8vo, 200 Hemenway's Indicator Practice and Steam-engine Economy.

Hutton's Mechanical Engineering of Power Plants ..... $12 \mathrm{mo}$ 
Kent's Steam-boiler Economy...................8vo, 400

Kneass's Practice and Theory of the Injector..............8vo, 1 б0

MacCord's Slide-valves...........................8vo, 200

Meyer's Modern Locomotive Construction.............4to, 1000

Peabody's Manual of the Steam-engine Indicator.........12mo, 150

Tables of the Properties of Saturated Steam and Other Vapors....................... 8vo,

"Thermodynamics of the Steam-engine and Other Heat-engines .........................

“ Valve-gears for Steam-engines................. 8vo,

Peabody and Miller. Steam-boilers......................

Pray's Twenty Years with the Indicator...................... 8ve 8vo,

Pupin's Thermodynamics of Reversible Cycles in Gases and

Saturated Vapors. (Osterberg.)..............12mo,

Reagan's Locomotive Mechanism and Engineering.......12mo,

Rontgen's Principles of Thermodynamics. (Du Bois.)....8vo,

Sinclair's Locomotive Engine Running and Management. . 12mo,

Smart's Handbook of Engineering Laboratory Practice..12mo,

Snow's Steam-boiler Practice......................8vo,

Spangler's Valve-gears.......................

Notes on Thermodynamics..............12mo,

Thurston's Handy Tables.................................. I 50

"Manual of the Steam-engine........2 vols., 8vo, 1000

Part I.-History, Structure, and Theory..............8vo, 600

Part II.-Design, Construction, and Operation.......8vo, 600

Thurston's Handbook of Engine and Boiler Trials, and the Use of the Indicator and the Prony Brake....... 8 vo,

" Stationary Steam-engines...................8.

" Steam-boiler Explosions in Theory and in Prac-

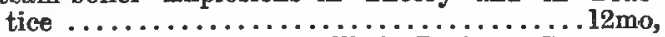

“ Manual of Steam-boilers, Their Designs, Construetion, and Operation...................8vo,

Weisbach's Heat, Steam, and Steam-engines. (Du Bois.)..8vo, Whitham's Steam-engine Design .................. Wilson's Treatise on Steam-boilers. (Flather.)........16mo, Wood's Thermodynamics, Heat Motors, and Refrigerating

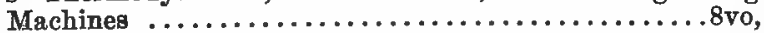

\section{MECHANICS AND MACHINERY.}

Barr's Kinematics of Machinery.................. 250 Bovey's Strength of Materials and Theory of Structures. .8vo, 750 Chordal.-Fixtracts from Letters.....................12mo, 200

Chureh's Mechanice of Engineering.......................... 600

Notes and Examples in Mechanics...............8vo, 200

Compton's First Lessons in Metal-working............. 12mo, I 50

Compton and De Groodt. The Speed Lathe............12mo, 1 60

Cromwell's Treatise on Toothed Gearing................. $12 \mathrm{mo}$, 150

" Treatise on Belts and Pulleys........................ 150

Dana's Text-book of Elementary Mechanics for the Use of Colleges and Schools..........................

Dingey's Machinery Pattern Making..................

Dredge's Record of the Transportation Exhibits Building of the

World's Columbian Exposition of 1893 .....4to, half mor.,

Du Bois's Elementary Principles of Mechanics:

Vol. I.-Kinematics .......................... 8vo, 350

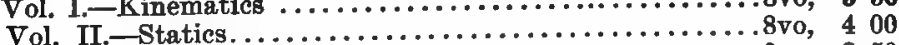

Vol. III.-Kinetics.................................... 350

Du Bois's Mechanics of Engineering. Vol. 1 .......... Small 4to, 750 " ${ }_{\text {sc }}$ " 
Durley's Elementary Text-book of the Kinematics of Machines. (In preparation.)

Fitzgerald's Boston Machinist...................... Flather's Dynamometers, and the Measurement of Power.12mo,

" Rope Driving.......................12mo,

Goss's Locomotive Sparks........................8vo,

Hall's Car Lubrication..........................12mo,

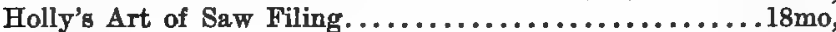

* Johnson's Theoretical Mechanies....................12mo,

Johnson's Short Course in Statics by Graphic and Algebraic Methods. (In preparation.)

Jones's Machine Design:

Part 1.-Kinematics of Machinery................8vo, 150

Part II.-Form, Strength and Proportions of Parts...8vo, 300

Kerr's Power and Power Transmission...............8vo, 200

Lanza's Applied Mechanies.....................8vo, 750

MacCord's Kinematics; or, Practical Mechanism........8vo, 500

“ Velocity Diagrams.....................8vo, 150

Merriman's Text-book on the Mechanics of Materials......8vo, 400

* Michie's Elements of Analytical Mechanics...........8vo, 400

Reagan's Locomotive Mechanism and Engineering.......12mo, 200

Reid's Course in Mechanical Drawing................8vo, 200

" Text-book of Mechanical Drawing and Elementary

Machine Design.......................8vo,

Richardes . Compressed Air .......................... 300

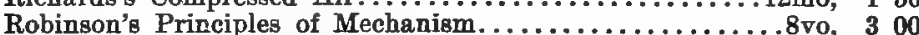

Ryan, Norris, and Hoxie's Electrical Machinery. (In preparation.)

Sinclair's Locomotive-engine Running and Management..12mo, 200

Smith's Press-working of Metals....................8vo, 00

" Materials of Machines.......................... 100

Thurston's Treatise on Friction and Lost Work in Machinery and Mill Work....................8vo, 300

"As Animal as a Machine and Prime Motor, and the Laws of Energetics..................12mo,

Warren's Elements of Machine Construction and Drawing. . 8vo, Weisbach's Kinematics and the Power of Transmission. (Herrman-Klein.) ................. 8vo, " Machinery of Transmission and Governors. (Herт(man-Klein.) ...................8.

Wood's Elements of Analytical Mechanics...............8

" Principles of Elementary Mechanics.............12mo,

" Turbines ............................8.

The World's Columbian Exposition of $1893 \ldots \ldots \ldots \ldots \ldots . . . . .4$ to,

\section{METALLURGY.}

Egleston's Metallurgy of Silver, Gold, and Mercury:

$\nabla$ ol. I.-Silver................................8vo, 7 50

Vol. II.-Gold and Mercury.....................8vo, 750

** Tes's Lead-smelting. . . . . . . . . . . . . . . . . . . . 12mo, 250

Keep's Cast Iron......................................... 250

Kunhardt's Practice of Ore Dressing in I urope..........8vo, I 50

Le Chatelier's High-temperature Measurements. (Boudouard-

Burgess.) ............................12mo,

Metealf's Steel. A Manual for Steel-users...................

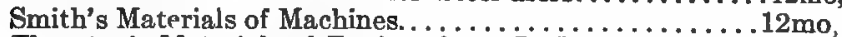

Thurston's Materials of Engineering. In Three Parts...... 8vo,

Part II.-Iron and Steel..................... 8vo,

Part III.-A Treatise on Brasses, Bronzes and Other Alloys and Their Constituents...................8vo, 


\section{MINERALOGY.}

Barringer's Description of Minerals of Commercial Value.

Oblong, moroceo,

Boyd's Resources of Southwest Virginia.............. 8vo, Map of Southwest Virginia........Pocket-book form,

Brush's Manual of Determinative Mineralogy. (Penfield.).8vo, Chester's Catalogue of Minerals................8vo, paper,

Cloth, 125

Dictionary of the Names of Minerals.........8vo, 350

Dana's System of Mineralogy.... .......Large 8vo, half leather, 1260 " First Appendix to Dana's New "System of Mineralogy."

Large 8vo

" Text-book of Mineralogy..................8vo,

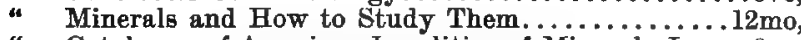

“ Catalogue of American Localities of Minerals. Large 8vo,

c Manual of Mineralogy and Petrography..........12mo,

Fgleston's Catalogue of Minerals and Synonyms............. Hussak's The Determination of Rock-forming Minerals. (Smith.) .......................... Small 8vo,

- Penfield's Notes on Determinative Mineralogy and Record of Mineral Tests.....................

Rosenbusch's Microscopical Physiography of the Rock-making Minerals. (Idding's.) .................... - Tillman's Text-book of Important Minerals and Rocks..8vo, Williams's Manual of Lithology...................

250

300

200

400

100

1 ou

400

I 50

100

200

250

200

50

500

200

300

\section{MINING.}

Beard's Ventilation of Mines....................12mo,

Boyd's Resources of Southwest Virginia............... 8vo, Map of Southwest Virginia........Pocket-book form,

- Drinker's Tunneling, Explosive Compounds, and Rock

Drills.......................4to, half moroceo, $2 \bar{\delta} 00$

Eissler's Modern High Explosives................... 4 vo, 400

Fowler's Sewage Works Analyses............................ 200

Goodyear's Coal-mines of the Western Coast of the United

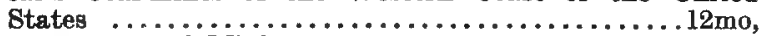

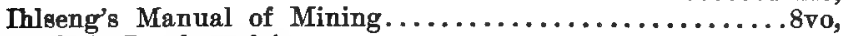

** Iles's Lead-smelting........................ 12mo,

Kunhardt's Practice of Ore Dressing in Europe............8vo,

O'Driscoll's Notes on the Treatment of Gold Ores.........8vo,

Bawyer's Accidents in Mines......................8vo,

Walke's Lectures on Explosives.................... 8vo,

Wilson's Cyanide Processes...............................

Wilson's Chlorination Process......................

Wilson's Hydraulic and Placer Mining..................

Wilson's Treatise on Practical and Theoretical Mine Ventilation $\ldots \ldots \ldots \ldots \ldots \ldots \ldots \ldots \ldots \ldots \ldots \ldots \ldots \ldots \ldots$.

\section{SANITARY SCIENCE.}

Folwell's Sewerage. (Designing, Construetion and Maintenance.)

8vo, 300

" Water-supply Engineering..............8vo, 400

Fuertes' Water and Public Health.................. 12mo, 150

" Water-filtration Works........................ \& 50 
Gerhard's Guide to Sanitary House-inspection........16mo, Goodrich's Economical Disposal of 'Towns' Refuse....Demy 8vo,

Hazen's Filtration of Public Water-supplies............8vo,

Kiersted's Sewage Disposal......................12mo,

Leach's The Inspection and Analysis of Food with Special Reference to State Control. (In preparation.)

Mason's Water-supply. (Considered Principally from a Sanitary Standpoint. 3d Edition, Rewritten.... 8vo, “ Examination of Water. (Chemical and Bacterio-

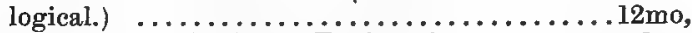

Merriman's Elements of Sanitary Eingineering..............

Nichols's Water-supply. (Considered Mainly from a Chemical and Sanitary Standpoint.) (1883.) .............8vo,

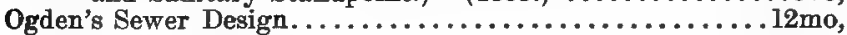

- Price's Handbook on Sanitation......................... 12mo,

Richards's Cost of Food. A Study in Dietaries............12mo, Richards and Woodman's Air, Water, and Food from a Sanitary Standpoint....................... 8vo,

Richards's Cost of Living as Modified by Sanitary Science.12mo,

- Richards and Williams's The Dietary Computer.........8vo,

Rideal's Sewage and Bacterial Purification of Sewage......8vo,

Turneaure and Russell's Public Water-supplies...........8vo, Whipple's Microscopy of Drinking-water............8vo,

Woodhull's Notes on Military Hygiene............. $16 \mathrm{mo}$,

\section{MISCELLANEOUS.}

Barker's Deep-sea Soundings................. 8vo, Emmous's Geological Guide-book of the Rocky Mountain Excursion of the International Congress of Geologists.

Terrel's Popular Treatise on the Winds............... Large 8vo, Haines's American Railway Management.................. Mott's Composition, Digestibility, and Nutritive Value of Food.

Mounted chart, " Fallacy of the Present Theory of Sound.........16mo, Ricketts's History of Rensselaer Polytechnic Institute, 1824$1894 \ldots \ldots \ldots \ldots \ldots \ldots \ldots \ldots \ldots \ldots \ldots$. . . . . . 8 . Rotherham's Emphasised New Testament...........Large 8vo, " Critical Emphasised New Testament........12mo,

Steel's Treatise on the Diseases of the Dog.............. Totten's Important Question in Metrology............. 8vo, The World's Columbian Exposition of $1893 \ldots \ldots \ldots \ldots \ldots . . . . .4$ to, Worcester and Atkinson. Small Hospitals, Establishment and Maintenance, and Suggestions for Hospital Architecture, with Plans for a Small Hospital............... 12mo,

\section{HEBREW AND CHALDEE TEXT-BOOKS.}

Green's Grammar of the Hebrew Language..........8vo, 300

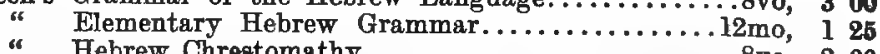

“ Hebrew Chrestomathy......................... \& 200

Gesenius's Hebrew and Chaldee Lexicon to the old Testament Scriptures. (Tregelles.)........ Small 4to, half morocco, 500 Letteris's Hebrew Bible.................... 
Digitized by Microsoft巴 
Digitized by Microsoft巴 
Digitized by Microsoft巴 

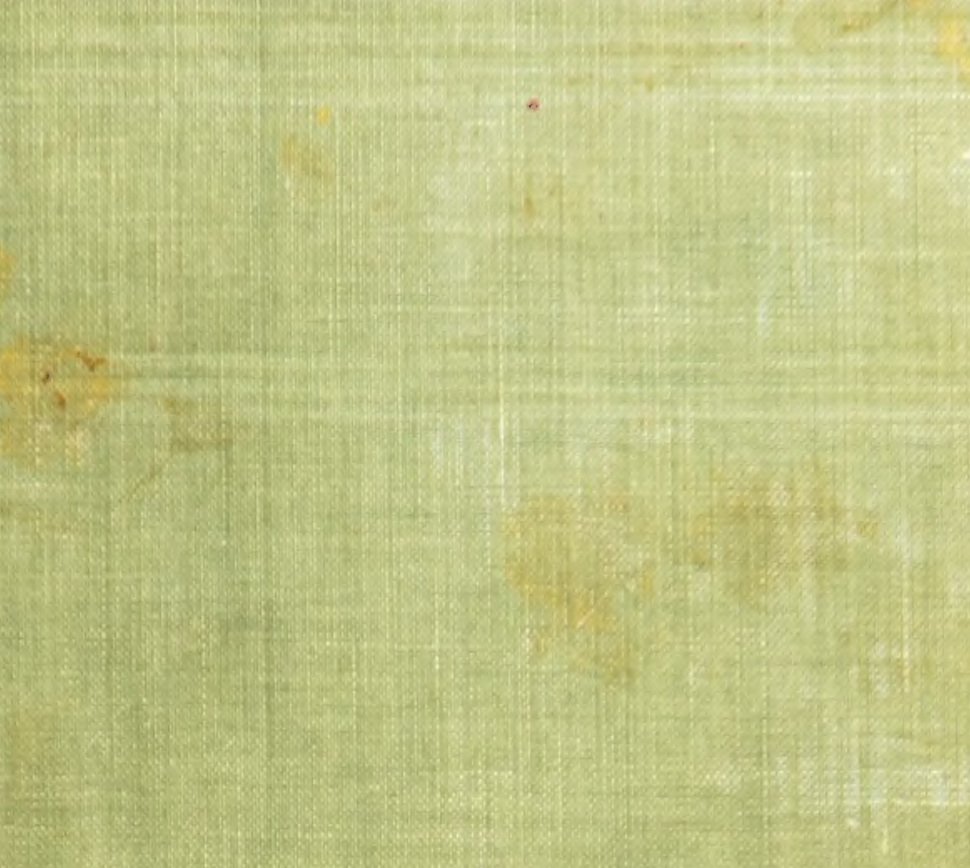